Marion Thuswald,

Elisabeth Sattler (Hg.)

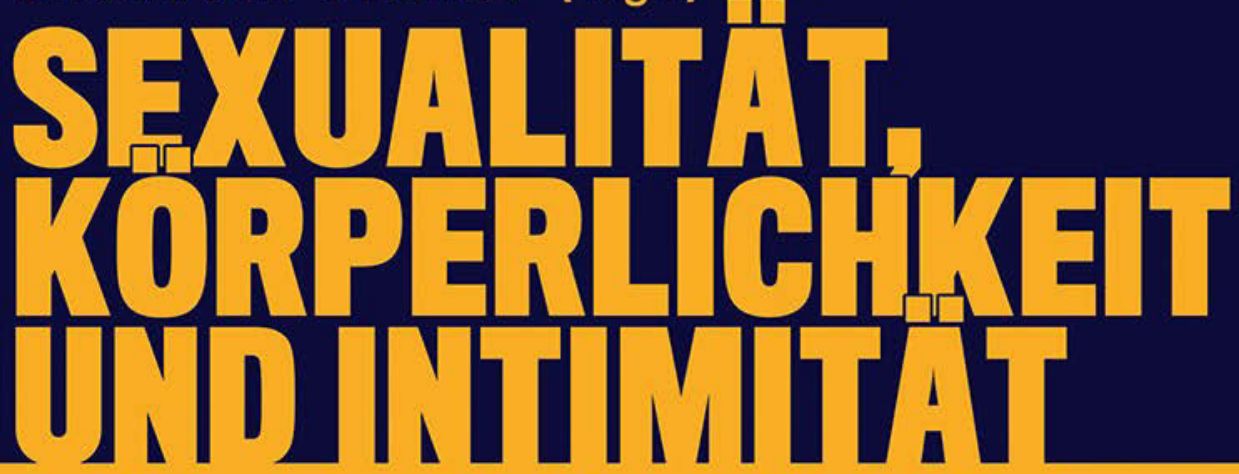

Pädagogische Herausforderungen und professionelle Handlungsspielräume in der Schule

[transcript] Pădagogik 
Marion Thuswald, Elisabeth Sattler (Hg.)

Sexualität, Körperlichkeit und Intimität

Pädagogik 
Marion Thuswald, Sozialpädagogin und Bildungswissenschaftlerin, lehrt und forscht in der Pädagog:innenbildung an der Akademie der bildenden Künste Wien. Sie arbeitet zu sexueller Bildung und Kunstpädagogik, Professionalisierung und Critical Diversity sowie in partizipativen Forschungsprojekten.

Elisabeth Sattler ist Professorin für Kunst- und Kulturpädagogik am Institut für das künstlerische Lehramt an der Akademie der bildenden Künste Wien. Sie lebt und arbeitet in Wien. Ihre Forschungsschwerpunkte liegen auf Bildungs-, Lehrund Lern-sowie Professionalisierungsprozessen in der Lehrer:innenbildung. 
Marion Thuswald, Elisabeth Sattler (Hg.)

\section{Sexualität, Körperlichkeit und Intimität}

Pädagogische Herausforderungen und professionelle Handlungsspielräume in der Schule 
Die Publikation entstand im Rahmen des Projekts Imagining Desires, das im Förderprogramms Sparkling Science aus Mitteln des Bundesministeriums für Bildung, Wissenschaft und Forschung gefördert wurde. Wir danken der Akademie der bildenden Künste Wien für die Kofinanzierung.
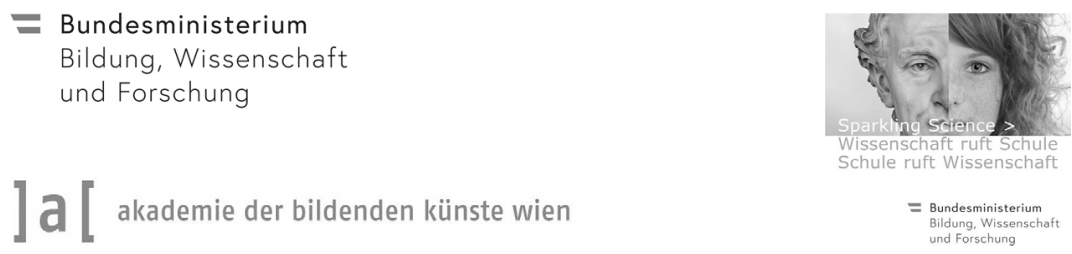

\section{Bibliografische Information der Deutschen Nationalbibliothek}

Die Deutsche Nationalbibliothek verzeichnet diese Publikation in der Deutschen Nationalbibliografie; detaillierte bibliografische Daten sind im Internet über http://dnb.d-nb.de abrufbar.

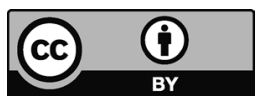

Dieses Werk ist lizenziert unter der Creative Commons Attribution 4.o Lizenz (BY). Diese Lizenz erlaubt unter Voraussetzung der Namensnennung des Urhebers die Bearbeitung, Vervielfältigung und Verbreitung des Materials in jedem Format oder Medium für beliebige Zwecke, auch kommerziell. (Lizenztext:

https://creativecommons.org/licenses/by/4.o/deed.de)

Die Bedingungen der Creative-Commons-Lizenz gelten nur für Originalmaterial. Die Wiederverwendung von Material aus anderen Quellen (gekennzeichnet mit Quellenangabe) wie z.B. Schaubilder, Abbildungen, Fotos und Textauszüge erfordert ggf. weitere Nutzungsgenehmigungen durch den jeweiligen Rechteinhaber.

\section{Erschienen 2021 im transcript Verlag, Bielefeld (๖) Marion Thuswald, Elisabeth Sattler ( $\mathrm{Hg}$.)}

Umschlaggestaltung: Maria Arndt, Bielefeld

Lektorat: Else Rieger

Druck: Majuskel Medienproduktion $\mathrm{GmbH}$, Wetzlar

Print-ISBN 978-3-8376-5840-8

PDF-ISBN 978-3-8394-5840-2

https://doi.org/10.14361/9783839458402

Buchreihen-ISSN: 2703-1047

Buchreihen-eISSN: 2703-1055

Gedruckt auf alterungsbeständigem Papier mit chlorfrei gebleichtem Zellstoff. Besuchen Sie uns im Internet: https://www.transcript-verlag.de Unsere aktuelle Vorschau finden Sie unter www.transcript-verlag.de/vorschau-download 


\section{Inhalt}

\section{Sexuelle Bildung weiterdenken}

Differenzreflektierende Perspektiven und emanzipatorische Ansätze im Handlungsfeld Schule

Elisabeth Sattler und Marion Thuswald

9

\section{Jugendsexualität und sexuelle Bildung}

Öffentliche Diskurse, empirische Befunde und die Frage

nach pädagogischer Professionalität

Christin Sager

\section{Zu intim?}

Eine Gesprächsrunde mit Lehrer:innen zu Fragen rund um

Sexualität, Beziehungen und Intimität in Unterricht und Schulalltag

Anna Pritz, Elisabeth Sattler und Marion Thuswald

\section{Diskriminierungsreflektierte Sexualpädagogik}

Katharina Debus 69

\section{Wie über Sexualität sprechen?}

Zur Lust auf Begriffsarbeit in der sexualpädagogischen Praxis

Marion Thuswald

Intimität

Anna Vida.... 


\section{Sexualerziehung - für wen?}

Die Reproduktion weißer Mittelklasse-Normen

durch Sexualpädagogik in der Schule

Dorottya Rédai

\section{Imagining Subjectivities}

Eine Reflexion über eigene Geschlechtervorstellungen

und ihre Bedeutung für sexualpädagogisches Handeln

Magdalena Rest

\section{Körperspuren}

Erinnerungsgeschichten an Körperlichkeit in der Schule

Kerstin Witt-Löw

\section{Von blutigen Tampons und Penis-Zeichnungen}

Stanislaus Medan

\section{Digitale Intimitäten}

Orlinder Krinkel

"Nacktfotos auf's Handy kriegen ist doch ganz normal!"

Einblicke in die Arbeit mit Jugendlichen

zu Sexualität und digitalen Medien

Michaela Moosmann, Katharina Buhri, Ariane Grabherr und Angelika Atzinger

\section{Auf Community bauen und in Vernetzungen lernen}

Eine Gesprächsrunde über rassismuskritische Ansätze

in der Bildungsarbeit zu Sexualität und Vielfalt

Rosemarie Ortner

\section{Sexuelle Bildung in der Schule braucht Vielfalt}

Gregor Steininger

Geschlechtliche, amouröse und sexuelle Vielfalt in der Sexualpädagogik 


\section{Im Schwimmbad}

Anna Vida....

Sexuelle Übergriffe unter Kindern

Eine Herausforderung für den Schulalltag

Ulli Freund

\section{Einvernehmlichkeit und Zustimmungsunfälle}

Orlinder Krinkel 253

Nähe in pädagogischen Beziehungen gestalten

Von der Notwendigkeit eines integrierten Prozesses

von Professionalisierungsstrategien und

Organisationsentwicklung für Schulen

Alexandra Retkowski

Queer in der Schule?

Rosa Wiesauer 269

\section{LGBTIOA*-Jugendliche in der Schule}

Ansätze für einen bestärkenden Umgang mit sexueller

und geschlechtlicher Vielfalt im Arbeitsfeld Schule

Paul Haller und Anton Cornelia Wittmann

Sexualbiografische Reflexionsarbeit als Element pädagogischer Professionalisierung

Julia Kerstin Maria Siemoneit. 299

Wie hast du dir in deiner Kindheit Sexualität vorgestellt?

Erfahrungen und Zeichnungen aus einem Workshop

zu Körper, Sexualität und Intimität

Christine Aebi. 313

Das Ungreifbare greifbar machen

Handlungsmöglichkeiten von Pädagog:innen

gegen sexuelle Gewalt an Schüler:innen

Stefanie Vasold .333 
Sexualpädagogik - betrifft mich das?

Eva Zürcher...

Rechtliche Aspekte in der Sexualpädagogik

Heidemarie König und Adriane Krem

Sexualpädagogische Elternarbeit in und rund um Schule

Eine Gesprächsrunde zu Haltungen, Herausforderungen

und Rahmenbedingungen in der Elternarbeit

Claudia Schneider

Masturbate

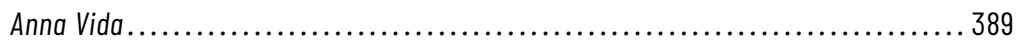

Körperlichkeiten

Anna Vida 390

Autor:innenverzeichnis ......................................... 393 


\section{Sexuelle Bildung weiterdenken Differenzreflektierende Perspektiven und emanzipatorische Ansätze im Handlungsfeld Schule}

Elisabeth Sattler und Marion Thuswald

Der vorliegende Band geht von der These aus, dass viele pädagogisch Tätige in ihrem schulischen Alltag Herausforderungen rund um Sexualität, Körperlichkeit und Intimität wahrnehmen - auch dann, wenn sie nicht explizit dazu unterrichten. Die Herausforderungen mögen oft implizit bleiben und betreffen etwa die Frage der angemessenen (körperlichen) Nähe und Distanz zwischen Schüler:innen und Lehrer:innen. Bisweilen wird jedoch der Bedarf an (sexual-)pädagogischen Interventionen sehr explizit, wenn beispielsweise über den Klassen-Chat Dick Pics verschickt wurden oder Schüler:innen angesichts von Verhütungspannen Beratung bei Lehrer:innen suchen.

Selbst wenn im Unterricht die Wissensvermittlung $\mathrm{zu}$ fachspezifischen Themen im Fokus steht, werden dabei immer auch Fragen von Nähe und Distanz, vom Umgang mit der Körperlichkeit aller Beteiligten, von Geschlechter- und Körpernormen u.Ä.m. berührt. Mit Blick auf die Entwicklungsthemen von Schüler:innen in der Sekundarstufe sind die Auseinandersetzungen mit dem eigenen Begehren, mit Körperlichkeit und sexueller Lust, mit Verlieben und außerfamiliären Beziehungen in der Jugendphase als virulent einzuschätzen und auch aus diesem Grund in der Schule (omni-)präsent (vgl. Retkowski/Thole 2012: 291).

Angehende Lehrer:innen werden über die Lehramtsstudien für die Sekundarstufe vorrangig auf fachlichen Unterricht vorbereitet. Viele sogenannte Querschnittsmaterien - von der Konfliktbearbeitung unter Schüler:innen und Kolleg:innen, der Elternarbeit und der Organisation von Schulveranstaltungen bis zur Unterstützung von Schüler:innen in schwierigen Lebenslagen und der Beteiligung an Schulentwicklungsprozessen - kommen im schuli- 
schen Alltag dann seinfach so dazu und können herausfordern, manchmal vielleicht auch überfordern.

In der Annahme, dass (Lehramts-)Studierende wie auch im Beruf stehende Pädagog:innen bisweilen stark beansprucht sind, zielen wir mit diesem Band darauf $\mathrm{ab}$, sie in der theoriebasierten Deutung und im professionellen Umgang mit Herausforderungen zu stärken, mit denen sie im schulischen Alltag konfrontiert sein können, und gleichzeitig auch proaktiv Handlungsspielräume für sexuelle Bildung zu eröffnen.

\section{Sexualität, Körperlichkeit und Intimität}

Mit den Begriffen Sexualität, Körperlichkeit und Intimität, die der Band im Titel trägt, wird ein Bereich schulischer Herausforderungen benannt, der in Erzählungen von Unterrichtspraktikant:innen und Junglehrer:innen wiederholt auftaucht und auch erfahrene Lehrer:innen und Schulleiter:innen beschäftigt. Unter diesen Chiffren können etwa der Umgang mit sexualisierten und queerfeindlichen Beschimpfungen, der Beratungsbedarf von Schüler:innen zu Liebes- und Sexualitätsthemen, die Frage nach Körpernormen und Bekleidungspolitiken in der Schule sowie der Umgang mit dem Tabuthema Verlieben und Anziehung zwischen Schüler:innen und Lehrer:innen gefasst werden. Bei diesen sehr unterschiedlichen Herausforderungen und Handlungsbedarfen, mit denen pädagogische Tätige in der Schule zu tun haben, setzen die hier versammelten Beiträge an: Sie zeigen Problemlagen und Spannungsfelder auf, beschreiben diese, bieten begriffliche Analyse- und Interpretationswerkzeuge an und zielen darauf $a b$, professionelle Handlungsspielräume für sexuelle Bildung zu eröffnen. Dabei liegt der Fokus der unterschiedlichen Einsätze sowohl auf dem im Schulalltag oft nötigen reaktiven Handeln (etwa bei Übergriffen) als auch auf proaktivem (sexual-)pädagogischen Tun im Horizont emanzipatorisch orientierter sexueller Bildung. ${ }^{1}$

$1 \quad$ Als Bildungswissenschaftler:innen wissen wir freilich um die theoretischen wie methodischen Problematiken, in denen sich heute - lange nach dem Ende der großen Erzählungen - eine auf Emanzipation fokussierende wissenschaftliche Position befindet. Weder lässt sich Emanzipation als telos letztbegründen noch ist von einem identitätslogischen, sich aus sich selbst einfach >entwickelnden< Selbst erkenntnistheoretisch auszugehen, was angesichts des Bezugs auf Selbstbestimmung, die in den Beiträgen immer wieder in den Blick gerückt wird, möglicherweise angenommen werden könnte. Angesichts dessen eröffnen wir mit dem Band einen Raum, sexuelle Bildung, 
Die Beiträge des Bandes eröffnen über die drei Chiffren Sexualität, Körperlichkeit und Intimität eine breite und differenzierte Perspektivität auf den Themenbereich, ohne seine Vielschichtigkeit und Vielfalt ausschließlich unter dem Begriff der Sexualität zu verhandeln und damit entweder alle beschriebenen Phänomene zu sexualisieren oder den Begriff Sexualität ins Vage auszudehnen (vgl. Thuswald). ${ }^{2}$

Freilich kann auch der vorliegenden Band bei weitem nicht alle Themen und Fragen abdecken, die zu Sexualität, Körperlichkeit und Intimität im Kontext Schule aufgerufen werden. Auf Basis unseres Wissens - und auch der Grenzen desselben - legen wir als Herausgeber:innen eine auf wissenschaftliche und pädagogisch-professionell Diskurslagen rückbezogene systematische Auswahl und Zusammenstellung von schulrelevanten Beiträgen vor. Diese bearbeiten sowohl sexualpädagogische >Dauerbrenner<, wie etwa empirische Ergebnisse zu Jugendsexualität (vgl. Sager), oder Ansätze der Gewaltprävention (vgl. Freund, Vasold) als auch neuere Herausforderungen, etwa Social Media und sexualitätsbezogene Medienpädagogik (vgl. Moosman u.a., Krinkel), und bisher vernachlässigte Themen, z.B. rassismuskritische Perspektiven (vgl. Ortner), Elternarbeit (vgl. Schneider) oder rechtliche Grundlagen der sexualpädagogischen Arbeit in Schule (vgl. Krem/König). ${ }^{3}$

Subjektivität und Emanzipation - auch angesichts von epistemologischen Unwägbarkeiten - weiterzudenken.

2 Zum Begriff Intimität lässt sich etwa auf die Soziologin Viviana Zelizer verweisen, die Intimität durch Vertrauen, das Teilen eines spezifischen Wissens übereinander und als gegenseitige Aufmerksamkeit charakterisiert (vgl. Zelizer 2010 zit n. Buschmeyer/Tolasch 2014). In kritischem Bezug darauf gehen Anna Buschmeyer und Eva Tolasch davon aus, dass Intimität in Interaktionen ausverhandelt wird. In ihrer Studie zu körpernahen Berufen unterscheiden sie zwischen privater Intimität und einer professionellen Intimität. Letztere ist durch ein geteiltes Wissen und gemeinsame Erfahrungen geprägt und wird von professionell Handelnden für ihre Klient:innen hergestellt. Sie ist somit Teil des professionellen Handelns (vgl. Buschmeyer/Tolasch 2014).

Die Philosophin Carolin Emcke weist in einem Interview darauf hin, dass Intimität mit Verletzbarkeit und Empathie verwoben ist und eine Form von Verbundenheit darstellt, die bedeutet sich »verletzbar zu machen« (Meßmer/Villa 2014).

3 Eine weitere inhaltliche Leerstelle in der sexualpädagogischen Fachliteratur bearbeitet der Band »Sexuelle Einvernehmlichkeit gestalten. Theoretische, pädagogische und künstlerische Perspektiven auf eine Leerstelle sexueller Bildung«(Dalhoff u.a. i.E.), der ebenfalls aus dem Projekt Imagining Desires heraus entwickelt wurde (siehe dazu auch den künstlerischen Beitrag »Die Wippe«von Krinkel i.d.B.). 


\section{Sexuelle Bildung weiterdenken ...}

Der Band reagiert damit auf einen Bedarf an theoriebasierten und gleichzeitig handlungsorientierenden Texten zum Themenbereich Sexualität und Schule. Bisher liegen zum einen wissenschaftliche Forschungsarbeiten vor, die spannende und differenzierte Erkenntnisse liefern, aber nicht an den Interessen und Fragen pädagogischer Praktiker:innen im System Schule orientiert sind. ${ }^{4}$ Zum anderen wurden in den letzten Jahren handlungsanleitende Publikationen veröffentlicht, die jeweils ein Schwerpunktthema erörtern oder den Fokus auf sexualpädagogische Inhalte und methodische Settings legen. ${ }^{5}$ Letztere konzentrieren sich vor allem auf die didaktische Gestaltung von Unterrichtseinheiten oder Workshops schulexterner Sexualpädagog:innen. ${ }^{6}$ Der vorliegende Band ist $\mathrm{zu}$ den bisher erschienenen Publikationen als komplementär zu verstehen, d.h. sie decken unterschiedliche Bereiche ab, die sich ergänzen. Die in diesem Band vorgelegten Beiträge greifen didaktische Fragen auf (vgl. etwa Debus, Moosmann u.a., Rest, Thuswald, Zürcher), gehen aber auch darüber hinaus. Sie nehmen vor allem die vernachlässigten Herausforderungen und Handlungsspielräume in den Blick, die sich jenseits expliziter sexualpädagogischer Settings im schulischen Alltag ergeben und für alle pädagogisch Tätigen in und rund um Schule von Bedeutung sind - unabhängig von ihren Unterrichtsfächern und ihren spezifischen Aufgabenbereichen.

Erfreulicherweise hat die Dichte an deutschsprachigen sexualpädagogischen Publikationen in den letzten Jahren zugenommen. Wir knüpfen mit diesem Buch an die bereits vorhandenen Ansätze und Einsätze differenzre-

Vgl. etwa Schetsche/Schmidt 2013; Blumenthal 2014; Kleiner 2015; Hoffmann 2016; Urban 2019.

$5 \quad \mathrm{Zu}$ themenspezifischen Publikationen rund um geschlechtliche und sexuelle Vielfalt vgl. etwa Huch/Lücke 2015; queerformat o.].; Busche u.a. 2018; Debus/Laumann 2018a; b. Zu Methodensammlungen und handlungsorientierenden Publikationen für den schulischen Kontext vgl. etwa Valtl 1998; Selbstlaut 2020; Tuider u.a. 2012; Thuswald/Sattler 2016; Martin/Nitschke 2017; Kahrer/Wagner 2018; Rose 2018; Schmutzer/Thuswald 2019.

6 Publikationen, die sich dem Feld der Schule widmen, bieten neben kurzen Einführungen vor allem Methodenpools für die schulische Sexualpädagogik an, wie das bereits gut 20 Jahre alte Buch »Sexualpädagogik in der Schule«von Karlheinz Valtl (1998) oder die Neuerscheinungen »Sexuelle Bildung in der Schule« (Martin/Nitschke 2017), »Sexualerziehung mit Generation Z« (Rose 2018) oder »Sex, was?« (Kahrer/Wagner 2018). 
flektierender Sexualpädagogik ${ }^{7}$ an und zielen auf ein kritisches emanzipatorisch orientiertes Weiterdenken. Der Titel der Einleitung "Sexuelle Bildung weiterdenken« rekurriert auf den 2004 veröffentlichen Sammelband "Sexualpädagogik weiterdenken« von Stefan Timmermanns, Elisabeth Tuider und Uwe Sielert, der in der sexualpädagogischen Fachdiskussion wichtige Impulse gesetzt hat. ${ }^{8}$

Im vorliegenden Band finden sich Beiträge von Autor:innen versammelt, die über sexuelle Bildung im Kontext Schule erfahrungsbasiert und theorieorientiert nachdenken. Neben wissenschaftlichen Beiträgen und Ergebnissen empirischer Studien enthält der Band auch erfahrungsbasierte Texte aus der Perspektive von Lehramtsstudierenden (vgl. Medan, Rest, Steininger, Wiesauer, Zürcher) sowie theoriebasierte Praxisreflexionen, die etwa in Form von Gesprächsrunden erfahrener Praktiker:innen gestaltet sind (vgl. Ortner, Pritz u.a., Schneider). Ergänzt werden die Texte durch künstlerische Beiträge, die nochmals andere Zugangsweisen zum Themenfeld erschließen (vgl. Aebi, Krinkel, Vida).

Der Band richtet sich vorrangig an pädagogisch Tätige in der Sekundarstufe, also etwa Lehrer:innen, Schulleiter:innen, Sexualpädagog:innen, Psycholog:innen und Schulsozialarbeiter:innen, wobei diese Verortung nicht bedeutet, dass die Beiträge nicht auch für pädagogische Tätige in anderen Bereichen, etwa der Sozialpädagogik, der außerschulischen Jugendarbeit, der Kulturvermittlung oder der Primarstufe, anregend sein können. Das Buch adressiert explizit auch Lehrende und Studierende im Lehramtsstudium. ${ }^{9}$ Es zielt darauf ab, gut lesbare und nachvollziehbare theorie- und reflexionsgeleitete Texte für die Lehrer:innenbildung bereitzustellen, die die gegenwärtigen wissenschaftlichen und professionellen Diskussionen widerspiegeln, kritisch kommentieren, weiterdenken und bisweilen auch durchkreuzen. Dabei geht

7 Wir beziehen uns hier auf Ansätze, wie sie etwa von Jutta Hartmann (2004), Elisabeth Tuider und Kolleg:innen (2012), Mart Busche u.a. (2018) sowie Katharina Debus (2016 sowie i.d.B.) entwickelt wurden.

8 Wir halten mit dem Sammelband zudem Denkbewegungen in Cang, die wir bereits 2016 mit dem Band »Teaching Desires. Möglichkeitsräume sexueller Bildung im künstlerisch-gestalterischen Unterricht« (Thuswald/Sattler 2016) begonnen haben.

9 Erfreulicherweise hat in den letzten Jahren das sexualpädagogische Angebot in der Aus- und Fortbildung von Lehrer:innen in Deutschland und Österreich etwas zugenommen (vgl. Henningsen 2016; Thuswald 2021). 
es uns auch darum, die Lust an theoretischer Auseinandersetzung anzuregen und sie als Teil professioneller pädagogischer Praxis zu verstehen (vgl. Schähle 2012).

\section{Grenzachtend, lustvoll und differenzreflektierend}

Die Idee zu diesem Band entwickelte sich im Laufe des Projektes Imagining Desires (2017-2019), das wir am Fachbereich Kunst- und Kulturpädagogik ${ }^{10}$ an der Akademie der bildenden Künste Wien gemeinsam mit der Fachstelle Selbstlaut konzipiert und durchgeführt haben. ${ }^{11}$ In Rahmen dieses transdisziplinären Forschungs- und Bildungsprojekt forschten Lehrer:innen, Wissenschaftler:innen, Sexualpädagog:innen, Künstler:innen sowie Schüler:innen und Studierende des künstlerischen Lehramts gemeinsam zu Fragen von Sexualität, visueller Kultur und Pädagogik. ${ }^{12}$ Aus der forschenden und pädagogischen Zusammenarbeit dieser unterschiedlichen Personengruppen heraus entstanden mehrere Publikationsprojekte, die theoretische Perspektiven der Critical Studies - also etwa geschlechterreflektierende und rassismuskritische ${ }^{13}$ - mit handlungspraktischen pädagogischen Fragen verbinden und diese in emanzipatorischer Absicht bearbeiten. ${ }^{14}$ Für pädagogisch-professio-

10 Dieser Fachbereich, in dem wir arbeiten, ist für die Lehre der allgemeinen bildungswissenschaftlichen Crundlagen im künstlerischen Lehramtsstudium zuständig. Das Institut für das künstlerische Lehramt der Akademie der bildenden Künste Wien bildet für die Unterrichtsfächer Bildnerische Erziehung (in Deutschland: Kunst) und Technisches und textiles Werken in der Sekundarstufe aus.

11 Wir bedanken uns bei Barbara Rothmüller und Sabine Ziegelwanger für ihre Vorschläge und Rückmeldungen in der Konzeptionsphase dieses Bandes.

12 Das zweijährige Projekt wurde in Kooperation zwischen dem Fachbereich Kunst- und Kulturpädagogik an der Akademie der bildenden Künste Wien und der Fachstelle Selbstlaut-Fachstelle gegen sexualisierte Gewalt an Kindern und Jugendlichen geplant und durchgeführt (vgl. dazu https://www.imaginingdesires.at). Zudem waren an dem Projekt auch das Hernalser Gymnasium und Realgymnasium Geblergasse, die Neue Mittelschule der Sir-Karl-Popper-Schule in der Schweglerstraße sowie die Pädagogische Hochschule Wien beteiligt.

13 Zu rassismuskritischen Perspektiven auf und für Sexualpädagogik vgl. die Beiträge von Maisha-Maureen Auma, Jaqueline Ejiji und Rafaela Siegenthaler in der online zugänglichen Textsammlung »Bilder befragen - Begehren erkunden« (Pritz u.a. 2020).

14 Bereits publiziert wurde die Monografie »Mit Bildern zu Lust und Begehren arbeiten« (Schmutzer/Thuswald 2019), die online veröffentliche Textsammlung »Bilder befragen - Begehren erkunden« (Pritz u.a. 2020), der Band »Sexuelle Einvernehmlichkeit 
nelles Handeln rund um Sexualität, Körperlichkeit und Intimität bedeutet dies konkret etwa die Weiterentwicklung von grenzachtend-gewaltpräventiven und gleichzeitig lustfreundlichen und lustvollen sowie macht-und differenzreflektierenden Zugängen, wie sie in den Beiträgen dieses Bandes ausgearbeitet werden (vgl. insbesondere Debus, Freund, Haller/Wittmann, Rédai, Retkowski, Ortner, Steininger, Vasold, Wiesauer). Deutlich wird dabei auch die Notwendigkeit, Sexualpädagogik und die Präventionsarbeit von sexualisierter Gewalt, die historisch aus unterschiedlichen Bewegungen heraus entstanden sind, stärker zusammenzudenken und mit macht- und differenzreflektierten Perspektiven zu verbinden (vgl. etwa Debus, Haller/Wittmann, Rédai, Vasold). ${ }^{15}$

Kinder und Jugendliche verstehen wir dabei - nicht nur, aber insbesondere auch in Bezug auf Sexualität, Körperlichkeit und Intimität - als eigenständig handlungsfähige Menschen, die in ihren je eigenen Bedürfnissen, Sichtweisen, Grenzen und Ausdruckformen anzuerkennen sind und die gleichzeitig auch Begleitung von Erwachsenen und Schutz vor Diskriminierung und Gewalt benötigen. Vielerorts wird betont, dass der Schule in der sexuellen Bildung und Gewaltprävention eine wichtige Aufgabe zukommt - ist sie doch jene pädagogische Institution, die nahezu alle Kinder und Jugendlichen erreicht. Ob sie sie auch tatsächlich erreicht, also ob die dort bereitgestellten Formate und Inhalte die Bedarfe der Heranwachsenden treffen, ist unserer Wahrnehmung nach eine durchaus berechtigte Frage. So arbeitet etwa Dorottya Rédai in ihrem Beitrag zu diesem Band heraus, dass Sexualpädagogik oft an weißen Mittelklasse-Normen orientiert ist, und Katharina Debus weist in ihren Beiträgen darauf hin, dass die Interessen mancher Heranwachsender aufgrund von Un_Sichtbarkeitsdynamiken unberücksichtigt bleiben.

Die Bedingungen für emanzipatorische sexuelle Bildung sind in der Schule wahrscheinlich nicht immer günstig oder gar optimal: Vorgegebene und

gestalten« (Dalhoff u.a. 2021), das Online-Heft »Porno: Behind the Scenes« (Danner 2021) sowie einige Artikel in Zeitschriften und Sammelbänden, vgl. etwa Rothmüller 2019; Sattler/Thuswald 2019; Thuswald/Sattler 2020.

15 Sexualpädagogik kann als ein Teil von Präventionsarbeit angesehen werden, geht aber gleichzeitig über diese hinaus. Ebenso kann Präventionsarbeit als ein Teil von Sexualpädagogik betrachtet werden. Sie umfasst aber mehr als Bildungsarbeit mit Heranwachsenden und pädagogisch Tätigen, insbesondere auch die Prävention auf der institutionellen und gesellschaftlichen Ebene (vgl. dazu Cameron-Lewis/Allen 2013; Dalhoff/Eder 2016). 
zum Teil rigide Zeitstrukturen, Leistungs- und Notendruck, Jahrgangsklassen, die wenig soziale Flexibilität ermöglichen, sowie fehlende Vorbereitung auf sexualpädagogische Arbeit in der Ausbildung der Lehrpersonen können es erschweren, einen guten und grenzachtenden Rahmen für das Sprechen über Sexualität zu etablieren (vgl. dazu die Beiträge von Debus, Siemoneit, WittLöw). ${ }^{16}$

Schulische Bildungsarbeit rund um Sexualität, Körperlichkeit und Intimität bewegt sich in vielfältigen Spannungsfeldern: Trotz ihrer medialen Präsenz gilt Sexualität als etwas Intimes und Privates und steht so in einem Spannungsfeld zur Schule als öffentliche Einrichtung und der Schulklasse mit ihrer Klassenöffentlichkeit. Wenn Schüler:innen im Unterricht sprechen, dann sprechen sie nicht nur als Beitrag zum Unterrichtsgeschehen, sondern auch vor ihren Peers, also ihren Gleichaltrigen, d.h. sie orientieren sich auch an den Normen ihrer Gleichaltrigen-Gruppe. Dies gilt nicht nur für sexualpädagogische Themen, ist aber für das Sprechen über Sexualität in besonderer Weise relevant, da Sexualität als peerkulturell hochrelevantes Thema einzuschätzen ist. Es gilt daher, Formen und Formate zu (er-)finden, die in genau diesem Kontext passend sind, um die Schüler:innen vor Abwertung oder Beschämung zu schützen und gleichzeitig die peerkulturelle Tabuisierung bestimmter Themen nicht zu reproduzieren (vgl. Debus).

Hinzu kommt, dass Menschen in der Schule (auch) in professionellen >Rollen miteinander agieren - etwa als Lehrer:innen und Schüler:innen -, die mit bestimmten Aufgaben und Verantwortlichkeiten wie etwa Notengebung, Anwesenheits- und Aufsichtspflicht u.Ä.m. verbunden sind. Diese Rollen können es manchmal erschweren, über Sexualität zu sprechen, wenn das Thema als privat und (zu) intim gilt (vgl. Pritz u.a.). Gleichzeitig stellen die professionellen Rollen einen wichtigen Schutz für die Beteiligten dar (vgl. dazu Retkowski, Siemoneit). ${ }^{17}$

Vgl. dazu auch die etwas ältere Arbeit von Walter Müller zu den Möglichkeiten und Grenzen schulischer Sexualerziehung (1992).

17 Die professionellen Rollen zu verlassen kann Verwirrung erzeugen, wie Markus Hoffmann herausarbeitet. Er konstatiert in einer Studie mit Lehrpersonen, dass in allen geführten Interviews das Sprechen über Sexualität im Rahmen des offiziellen Schulunterrichts als grundlegendes Problem der Sexualerziehung benannt wird (vgl. Hoffmann 2016). 
Aus dieser Problemskizze heraus wird deutlich, dass es sinnvoll ist, unterschiedliche und für den jeweiligen Kontext stimmige Formate und Formen der Auseinandersetzung zu entwickeln. Üblicherweise wird die Aufgabe der expliziten Sexualkunde in der Schule dem Biologieunterricht zugeschrieben, der sich häufig auf biologisches Wissen beschränkt (vgl. Sager) und/oder es werden externe Sexualpädagog:innen ${ }^{18}$ eingeladen, die in Workshops von wenigen Stunden Fragen rund um Sexualität und Liebe mit den Jugendlichen besprechen (vgl. Moosmann u.a., Ortner).

Sowohl differenzierte biologische Kenntnisse als auch die Gelegenheit, mit schulexternen Personen zu sprechen, können als wichtiger Teil sexueller Bildung eingeschätzt werden. Es bestehen über den Biologieunterricht und sexualpädagogische Workshops hinaus sowohl Bedarfe als auch Anlässe und Möglichkeitsräume sexueller Bildung: Sexualpädagogik wird - vielfach begründet und ausformuliert ${ }^{19}$ - fächerübergreifend gedacht, da sich in zahlreichen Unterrichtsfächern Anknüpfungspunkte dafür finden (vgl. Zürcher). In den letzten Jahren sind Publikationen entstanden, die etwa zeigen, wie das Thema sexuelle Vielfalt in unterschiedlichen Unterrichtsfächern aufgegriffen werden kann, ${ }^{20}$ oder welche Möglichkeitsräume sexueller Bildung der künstlerisch-gestalterische Unterricht bietet. ${ }^{21}$

Zudem sehen wir Potenzial in fächer- und/oder klassenübergreifenden Projekten, Projekttagen und Lehrausgängen $\mathrm{zu}$ sexualpädagogischen Themen. Es erscheint nicht immer notwendig - und auch nicht immer zielführend -, Fragen rund um Körperlichkeit, Begehren, Sinnlichkeit, Liebe, Familienplanung, Erotik und unterschiedliche Beziehungen unter der Überschrift Sexualpädagogik zu verhandeln, weil der Begriff Sex(ualität) in vielfältiger Weise aufgeladen ist. Diese Themen können beispielsweise in einer Projektwoche zum Thema Erwachsenwerden (vgl. Brommer in

Antje Langer (2017) arbeitet anhand von Gesprächen mit externen Sexualpädagog:innen heraus, dass es in den schulischen Workshops die alltäglichen Grenzen des Besprechbaren zu erweitern und gleichzeitig konventionelle und individuelle Grenzen zu wahren gilt, um Möglichkeitsräume des Sprechens in Schulen zu eröffnen. Es sei also etwa ein »Umgang mit Scham zu finden, ohne dabei zu beschämen« (Langer 2017: 171). Zu Scham in der schulischen Sexualerziehung vgl. auch Blumenthal 2014.

19 Siehe dazu etwa den Crundsatzerlass Sexualpädagogik in Österreich (vgl. BMBF 2015).

20 Vgl. dazu etwa Huch/Lücke 2015; Busche u.a. 2018; Debus/Laumann 2018 a; b; queerformat o.].

21 Vgl. dazu etwa die Arbeiten von Angelika Beck 2013; 2016 sowie Thuswald/Sattler 2016; Schmutzer/Thuswald 2016. 
Pritz u.a.) oder in Projekttagen zu sozialem Lernen, zu politischer Bildung, Gesundheitserziehung oder kultureller Bildung aufgegriffen werden; möglicherweise auch in Kooperation mit außerschulischen Akteur:innen oder in der Zusammenarbeit von Schüler:innen und Lehramtsstudierenden. ${ }^{22}$

Als Möglichkeitsräume sexueller Bildung verstehen wir auch Initiativen zur Stärkung einer grenzachtenden und sexualfreundlichen Schulkultur, beispielsweise die Entwicklung von sexualpädagogischen und gewaltpräventiven Schulkonzepten sowie Fortbildungen für das Kollegium (vgl. Retkowski), den Aufbau von themenbezogener Peer Education oder Maßnahmen zu einer inklusiv-queerfreundlichen ${ }^{23}$ und diskriminierungsreflektierten Schule (vgl. dazu Haller/Wittmann). Auch die Etablierung niederschwelliger Beratungsmöglichkeiten zu körperlichen und sexuellen Fragen kann im Horizont sexueller Bildung unterstützend für Schüler:innen sein (vgl. Rédai, Haller/Wittmann).

Nicht zu unterschätzen sind auch die scheinbar `kleinen< Handlungsspielräume in der Begleitung von Bildungsprozesse, die durch alltägliche schulische Situationen entstehen oder auch geplant realisiert werden können (vgl. Medan, Rest, Witt-Löw): Manchmal sind es beiläufig geäußerte, den Prozess öffnende Nebenbemerkungen, das Durchkreuzen von Geschlechternormen, Hinweise auf Informations- und Beratungsangebote, eine klar formulierte oder deutlich ausgedrückte Haltung zu diskriminierenden Äußerungen oder eine humorvoll-kritische Nachfrage bei sexuell konnotierten Kommentaren, die dazu beitragen, dass Heranwachsende sich wahr- und ernstgenommen fühlen und gleichzeitig in ihren Bildungsprozessen herausgefordert werden (vgl. u.a. Debus, Rest).

\section{Gesellschaftliche Debatten und professionelle Handlungsspielräume}

Die Möglichkeitsräume sexueller Bildung in der Schule sind vielfältig und der sexualerzieherische Auftrag, der in Deutschland und Österreich seit 1968/1970

\footnotetext{
22 Vgl. zu Letzterem etwa die Publikation von Schmutzer und Thuswald (2019), die Möglichkeiten einer solchen Kooperation beschreiben.

23 Vgl. dazu etwa Projekte zu »Schule der Vielfalt« von der HOSI Salzburg (https://www.schule-der-vielfalt.de/projekt.htm) oder von »Ausgesprochen! LCBTIQ-Lehrer:innen (www.verein-ausgesprochen.at).
} 
besteht, ist weitergehend unbestritten. Dennoch wurden in den letzten Jahren immer wieder kontroverse gesellschaftspolitische Debatten um Sexualität als pädagogisches Thema geführt, z.B. darüber, wer für die Sexualerziehung von Heranwachsenden zuständig sein soll und welche Inhalte Teil schulischer Sexualpädagogik sein sollen.

Es lässt sich beobachten, dass sexualpädagogische Fragen stärker als andere pädagogische Themen emotionalisieren. Dies zeigt sich prägnant etwa daran, dass die Neuformulierung des österreichischen Grundsatzerlasses Sexualpädagogik 2015 für eine breitere öffentliche Diskussion gesorgt hat als der zeitgleich überarbeitete Grundsatzerlass Politische Bildung, der kaum medial wahrgenommen wurde (vgl. Der Standard 22.06.2015). Sexualpädagogik scheint gegenwärtig politisch brisanter als die politische Bildung: Sie erweist sich noch immer als ein symbolisches Kampffeld, in dem öffentlichkeitswirksam um hegemoniale Deutungsmacht gerungen wird. ${ }^{24}$

Die Kritik an sexualpädagogischer Arbeit geht in den letzten Jahren vor allem von politisch rechten und religiös-konservativen Akteur:innen aus, die vielfaltsorientierter Sexualpädagogik eine >Frühsexualisierung` oder >Verwirrung von Kindern vorwerfen (vgl. Sager, Thuswald). Eine Ausnahme stellte die Debatte um den Verein TeenStar 2018/19 in Österreich dar, dessen vielfaltsfeindlicher Zugang in die Kritik geriet: Dem Verein wurde vorgeworfen, in seinen Schulungsunterlagen Homosexualität als sheilbares Identitätsproblem< darzustellen und Masturbation als schädlich zu bezeichnen (vgl. Tóth 2018). Die von der HOSI Salzburg initiierte Diskussion führte zu einer Empfehlung von Bildungsminister Heinz Faßmann an die Schulen, mit dem Verein nicht mehr zu kooperieren (vgl. Tóth 2019).

Die Debatte um die Arbeit des Vereins TeenStar wurde jedoch kurz drauf von Mitte-rechts-Parteien für einen Vorstoß genutzt, externe sexualpädagogische Vereine zur Gänze aus den Schulen zu verbannen. In dem breiten Protest gegen den entsprechenden Entschließungsantrag im Parlament betonten viele Akteur:innen die Bedeutung externer Sexualpädagog:innen an Schulen als Ergänzung zur Arbeit der Lehrpersonen: Dabei wurde etwa argumentiert, dass Schüler:innen manche Fragen und Themen lieber nicht mit ihren Lehrer:innen besprechen wollen - und vice versa. Externe Sexualpädagog:innen hätten den professionsbasierten Vorteil, nur zeitlich begrenzt in die Klassen

24 Vgl. dazu etwa Schmincke 2015; Henningsen u.a. 2016; Vasold 2016; Rothmüller/Scheibelhofer 2016. 
zu kommen und keine Noten zu geben. Ein solches Setting mache es für Schüler:innen leichter, als unangenehm erlebte Erfahrungen zu besprechen oder tabuisierte Fragen zu stellen. ${ }^{25}$ Für externe Workshopleiter:innen erscheine es bisweilen einfacher, auf solche Fragen einzugehen und mit möglichen Projektionen der Schüler:innen umzugehen. Als sexualpädagogische Expert:innen verfügten sie darüber hinaus über ein breites und aktuelles sexualpädagogisches Fach- und Handlungswissen. ${ }^{26}$

Lehrer:innen nehmen jedoch als langjährige Bezugspersonen eine wichtige Rolle in der Begleitung Jugendlicher ein: Sie können wichtige erste Ansprechpersonen sein, etwa bei sexuellen Übergriffserfahrungen oder Unsicherheiten mit dem eigenen körperlichen Entwicklungsprozess. Lehrer:innen kennen und unterrichten die Schüler:innen über einen längeren Zeitraum und haben daher die Möglichkeit Veränderungen und Prozessschritte wahrzunehmen, zu benennen und Lern- und Entwicklungsprozesse kontinuierlich professionell zu begleiten.

Nicht zu vergessen ist, dass neben Lehrer:innen und externen Sexualpädagog:innen auch andere professionell Tätige in der Schule eine wichtige Aufgabe in der sexuelle Bildungs- und Präventionsarbeit übernehmen können, wie Dorottya Rédai am Beispiel von Schulkrankenpfleger:innen in Ungarn beschreibt. ${ }^{27}$

25 Der Grundsatzerlass »Reflexive Geschlechterpädagogik und Gleichstellung«, der im Oktober 2018 erlassen wurde, formuliert treffend, was auch für die Sexualpädagogik gilt: »Das Beiziehen von externen Fachkräften, die frei sind von der Rolle einer benotenden Autoritätsperson, kann für derartige Lernräume von großem Vorteil sein bzw. sogar notwendig erscheinen.«(Vgl. BMBWF 2018: 9).

26 Vgl. dazu etwa die Stellungnahme der Plattform Sexuelle Bildung (2019) sowie weitere Stellungnahmen, die auf deren Website https://www.redmadrüber.jetzt [Zugriff: 21.08.2020] veröffentlicht wurden.

27 Louisa Allen schlussfolgert in ihrer Studie zu schulischer Sexualpädagogik aus Perspektive von Jugendlichen in Neuseeland, dass es für diese weniger wichtig sei, welche Personengruppen die schulische Sexualpädagogik übernehmen, sondern welche professionellen Qualitäten diese Personen in der Interaktion mit den Schüler:innen zeigen (vgl. Allen 2011). 


\section{Lust auf Lesen - Dank an die Beitragenden}

Die Autor:innen, die wir für diesen Band um Beiträge gebeten haben, stehen an verschiedenen professionellen Schnittstellen zur Schule, viele von ihnen arbeiten sowohl wissenschaftlich als auch pädagogisch, als Lehrer:innen, Forscher:innen, Sexualpädagog:innen, Fort- und Weiterbildner:innen, Berater:innen, Künstler:innen, Lehramtsstudierende, Bildungswissenschaftler:innen oder Medienpädagog:innen und bringen so unterschiedliche Perspektiven und Expertisen zu Sexualität und Schule ein, die einen differenzierenden Blick auf die pädagogischen Herausforderungen und die professionellen Handlungsspielräume ermöglichen.

Wir haben das vorliegende Buch nicht zuletzt auch im eigenen Forschungs- und Lehrfokus angedacht, konzipiert, mehrfach abgeändert und schließlich realisiert. Als Bildungswissenschaftler:innen, die im Lehramtsstudium arbeiten, sind wir stets auf der Suche nach Texten, die Lust auf Lesen und Verstehen, auf diskursive Auseinandersetzung und kritisches Weiterdenken machen; nach Texten, die auch ohne einem bereits absolvierten Studium gut lesbar sind und die sowohl pädagogische Analysen ermöglichen als auch Handlungsspielräume eröffnen. Insofern erfüllen wir uns mit diesem Buch selbst einen Wunsch und hoffen, dass der Band darüber hinaus auch die Fragen und Interessen vieler Leser:innen trifft.

Als Herausgeberinnen bedanken wir uns ganz herzlich bei den Beiträger:innen, insbesondere auch für die Stimmen aus den Lehramtsstudien, die den Band nochmals deutlich vielstimmiger werden lassen. Allen Autor:innen möchten wir insbesondere für die intensive Arbeit an den Texten und ihre Kooperation mit uns als Herausgeberinnen danken. Wie arbeitsintensiv sich der Weg bis zu einem publikationsfertigen Text gestaltet - insbesondere unter dem Anspruch, theoretische und praxisbezogene Perspektiven zu verschränken, - bleibt ja häufig unsichtbar.

Vielen Dank auch an alle Gesprächsrunden-Teilnehmer:innen, die ihre Perspektiven aus der pädagogischen Praxis eingebracht haben. Ein herzliches Dankeschön auch an die Beiträger:innen der künstlerischen Arbeiten, die über visuelles Material nochmals Anderes in den Blick rücken.

Ein großer Dank geht auch an unsere Lektorin Else Rieger für die fachkundige Zusammenarbeit sowie an Laura Ettl und Christina Theny, die uns im Rahmen ihrer Studienassistenz im Fachbereich Kunst- und Kulturpädago- 
gik am Institut für das künstlerische Lehramt bei der Realisation des Bandes unterstützt haben.

Danke an den transcript Verlag, der in der Zusammenarbeit hilfreich und unterstützend diesen Band ermöglicht hat. Ohne der Finanzierung unseres Projekts Imagining Desires über das Sparkling Science-Programm des Bundesministeriums für Bildung, Wissenschaft und Forschung wäre dieses Buch nicht entstanden; ein Danke auch der Akademie der bildenden Künste Wien, durch deren Ko-Finanzierungen dieser Band den Leser:innen in Print und Open Access zur Verfügung gestellt werden kann.

\section{Literatur}

Allen, Louisa (2011): Young People and Sexuality Education. Rethinking Key Debates. Hampshire: Palgrave Macmillian.

Auma, Maisha-Maureen (2020): Zwischen Kulturalisierung und Empowerment. Sexualpädagogische Repräsentationen von Schwarzen Menschen und People-of-Color im deutschsprachigen Raum. In: Pritz, Anna/Siegenthaler, Rafaela/Thuswald, Marion (Hg.): Bilder befragen - Begehren erkunden. Repräsentationskritische Einsätze in der Bildungsarbeit. Textsammlung in der Zeitschrift Kunst Medien Bildung. Online unter: ht tp://zkmb.de/zwischen-kulturalisierung-und-empowerment-sexualpaed agogische-adressierungen-von-schwarzen-menschen-und-people-of-col or-im-deutschsprachigen-raum [Zugriff: 02.12.2020].

Beck, Angelika (2013): Kunstpädagogik in der sexuellen Bildung. In: Schmidt, Renate-Berenike/Sielert, Uwe (Hg.): Handbuch Sexualpädagogik und sexuelle Bildung. Weinheim/Basel: Beltz Juventa, S. 726-736.

Beck, Angelika (2016): Let's switch! Mit Jugendlichen unterwegs zwischen Sex und Kunst. In: Thuswald, Marion/Sattler, Elisabeth (Hg.): teaching desires. Möglichkeitsräume sexueller Bildung im künstlerisch-gestalterischen Unterricht. Wien: Löcker, S. 33-56.

Blumenthal, Sara-Friederike (2014): Scham in der schulischen Sexualaufklärung. Eine pädagogische Ethnographie des Gymnasialunterrichts. Wiesbaden: Springer VS.

BMBF - Bundesministerium für Bildung und Frauen (2015): Grundsatzerlass Sexualpädagogik. Online unter: https:/www.bmbwf.gv.at/Themen/schu le/schulrecht/rs/1997-2017/2015_11.html [Zugriff: 22.08.2020]. 
BMBWF - Bundesministerium für Bildung und Forschung der Republik Österreich (2018): Grundsatzerlass »Reflexive Geschlechterpädagogik und Gleichstellung. Online unter: https://www.bmbwf.gv.at/Themen/schule/ schulrecht/rs/2018_21.html [Zugriff: 22.08.2020].

Busche, Mart/Hartmann, Jutta/Nettke, Tobias/Streib-Brzic, Uli (2018): Heteronormativitätskritische Bildungsarbeit. Reflexionen am Beispiel eines museumspädagogischen Modellprojekts. Bielefeld: transcript.

Buschmeyer, Anna/Tolasch, Eva (2014): »Ein Löffelchen für dich und eins für mich« - (Ver-)Handlungen von professioneller Intimität durch Vermeidung von Privatheit. In: Meßmer, Anna-Katharina/Schmidbaur, Marianne/Villa, Paula-Irene (Hg.) (2014): Intimitäten. Feministische Studien, Vol. 32(1)/2014. Online unter: https://doi.org/10.1515/fs-2014-0103.

Cameron-Lewis, Vanessa/Allen, Louisa (2013): Teaching pleasure and danger in sexuality education. In: Sex Education. Sexuality, Society and Learning, Bd. 13, 2/2013, S. 121-132.

Dalhoff, Maria/Eder, Sevil (2016): Für eine sexuelle Bildung der Unbequemlichkeiten. Über die Notwendigkeit, die Prävention von sexualisierter Gewalt mit Diversity Education zusammen zu denken. In: Thuswald, Marion/Sattler, Elisabeth (Hg.): teaching desires. Möglichkeitsräume sexueller Bildung im künstlerisch-gestalterischen Unterricht. Wien: Löcker, S. 83-96.

Dalhoff, Maria/Hempel, Stefanie/Önsür-Oluğ, Zeynep/Rauch, Bianca/Thuswald, Marion (Hg.) (2021): Sexuelle Einvernehmlichkeit gestalten. Theoretische, pädagogische und künstlerische Perspektiven auf eine Leerstelle sexueller Bildung. Hannover: fabrico.

Danner, Rosa (2021): Porno: Behind the Scenes. Begleitheft für Pädagog:innen $\mathrm{zu}$ einem Video für Jugendliche. Herausgegeben von Eliabeth Sattler und Marion Thuswald. Online unter: https://doi.org/10.21937/porno.behindth escenes.

Debus, Katharina (2016): Nicht-diskriminierende Sexualpädagogik. In: Scheer, Albert/El-Mafaalani, Aladin/Yüksel, Emine Gökçen (Hg.): Handbuch Diskriminierung. Wiesbaden: Springer VS, S. 811-833.

Debus, Katharina/Laumann, Vivien (2018a): LSB-was? Geschlechtliche, amouröse und sexuelle Vielfalt - Einführung und Spannungsfelder. In: Debus, Katharina/Laumann, Vivien: Pädagogik geschlechtlicher, amouröser und sexueller Vielfalt. Zwischen Sensibilisierung und Empowerment. Berlin: Dissens - Institut für Bildung und Forschung, S. 12-70. Online 
unter: https://interventionen.dissens.de/materialien/handreichung [Zugriff: 13.07.2020].

Debus, Katharina/Laumann, Vivien (2018b): Pädagogik geschlechtlicher, amouröser und sexueller Vielfalt. Zwischen Sensibilisierung und Empowerment. Berlin: Dissens - Institut für Bildung und Forschung. Online unter: https://interventionen.dissens.de/materialien/handreichung [Zugriff: 13.07.2020].

Der Standard (22.06.2015): Heinisch-Hosek veröffentlicht Erlass zu neuer Sexualerziehung. In: Der Standard 22.06.2015. Online unter: https:/ww w.derstandard.at/story/2000017856103/heinisch-hosek-veroeffentlicht-e rlass-zu-neuer-sexualerziehung [Zugriff 22.08.2020].

Ejiii, Jaqueline (2020): Wenn Popkultur überfordert und Körperlichkeit mit Scham besetzt wird. Rassismus in den (sexual-)pädagogischen Blick nehmen. In: Pritz, Anna/Siegenthaler, Rafaela/Thuswald, Marion (Hg.): Bilder befragen - Begehren erkunden. Repräsentationskritische Einsätze in der Bildungsarbeit. Textsammlung der Onlinezeitschrift Kunst Medien Bildung. Online unter: http://zkmb.de/wenn-popkultur-ueberfordert -und-koerperlichkeit-mit-scham-besetzt-wird-rassismus-in-den-sexual -paedagogischen-blick-nehmen [Zugriff 23.12.2020].

Hartmann, Jutta (2004): Dynamisierung in der Triade Geschlecht - Sexualität - Lebensform: dekonstruktive Perspektiven und alltägliches Veränderungshandeln in der Pädagogik. In: Timmermanns, Stefan/Truider, Elisabeth/Sielert, Uwe (Hg) (2004): Sexualpädagogik weiter denken. Postmoderne Entgrenzungen und pädagogische Orientierungsversuche. Weinheim u.a.: Juventa, S. 59-78.

Henningsen, Anja (2016): Professionalität und Zuständigkeit sexualpädagogischer Expert_innen. In: Henningsen, Anja/Tuider, Elisabeth/Timmermans, Stefan (Hg.): Sexualpädagogik kontrovers. Weinheim/Basel: Beltz Juventa, S. 46-68.

Henningsen, Anja/Tuider, Elisabeth/Timmermans, Stefan (Hg.) (2016): Sexualpädagogik kontrovers. Weinheim/Basel: Beltz Juventa.

Hoffmann, Markus (2016): Schulische Sexualerziehung. Deutungsmuster von Lehrenden. Opladen: Verlag Barbara Budrich.

Huch, Sarah/Lücke, Martin (Hg.) (2015): Sexuelle Vielfalt im Handlungsfeld Schule. Konzepte aus Erziehungswissenschaft und Fachdidaktik. Bielefeld: transcript.

Imagining Desires (o.J.): Online unter: https://www.imaginingdesires.at [Zugriff: 22.08.2020]. 
Kahrer, Bettina/Wagner, Salva (2018): Sex, was? Lehr-, Lern- und Methodenbuch zur sexuellen und reproduktiven Bildung. Herausgegeben von der Österreichischen Gesellschaft für Familienplanung. Wien.

Kleiner, Bettina (2015): subjekt. bildung. Heteronormativität. Rekonstruktion schulischer Differenzerfahrungen lesbischer, schwuler, bisexueller und Trans*Jugendlicher. Opladen: Verlag Barbara Budrich.

Kowalski, Marlene/Hildebrand, Julia/Marks, Svenja/Retkowski, Alexandra/Thole, Werner/Schäfer, Dorothee/Behnisch Michael (2018): Pädagogische Intimität. Zur Untersuchung von Mustern der Gestaltung pädagogischer Beziehungen in unterschiedlichen Handlungsfeldern. Interdisziplinäre Fachzeitschrift für Prävention und Intervention (DGfPI), 21/2, S. 188-201.

Langer, Antje (2017): Arbeit an und mit Widersprüchen - Zur Herstellung und Aufrechterhaltung einer sexualpädagogischen Situation. In: Klein, Alexandra/Tuider, Elisabeth (Hg.): Sexualität und Soziale Arbeit. Baltmannsweiler: Schneider Verlag Hohengehren, S. 149-165.

Martin, Beate/Nitschke, Jörg (2017): Sexuelle Bildung in der Schule. Themenorientierte Einführung und Methoden. Stuttgart: Kohlhammer.

Meßmer, Anna-Katharina/Villa, Paula-Irene (2014): »Einfach nur privatistisch Intimitäten ausplaudern, kann nicht zielführend sein«. Anna-Katharina Meßmer und Paula-Irene Villa im Gespräch mit Carolin Emcke. In: Feministische Studien, Nr. 32, 1/2014, S. 78-85.

Plattform Sexuelle Bildung (2019): Statement Sexualpädagogik externer Vereine in Schulen. Online unter: https://sexuellebildung.at/aktuelles/statem ent-sexualpadagik-externer-vereine-in-schulen [Zugriff: 23.12.2020].

Pritz, Anna/Siegenthaler, Rafaela/Thuswald, Marion (Hg.) (2020): Bilder befragen - Begehren erkunden. Repräsentationskritische Einsätze in der Bildungsarbeit. Textsammlung in der Zeitschrift Kunst Medien Bildung. Online unter: http://zkmb.de/sammlung/bilder-befragen-begehren-erku nden [Zugriff: 23.12.2020].

Queerformat (o.J.): Diverse Materialien. Online unter: https://www.queerfor mat.de [Zugriff: 06.05.2019].

Retkowski, Alexandra/Thole, Werner (2012): Professionsethik und Organisationskultur. In: Thole, Werner/Baader, Meike/Helsper, Werner/Kappeler, Manfred/Leuzinger-Bohleber, Marianne/Reh, Sabine/Sielert, Uwe/Thompson, Christiane (Hg.): Sexualisierte Gewalt, Macht und Pädagogik. Opladen u.a.: Verlag Barbara Budrich, S. 291-316. 
Ricken, Norbert (2012): Macht, Gewalt und Sexualität in pädagogischen Beziehungen. In: Thole, Werner/Baader, Meike/Helsper, Werner/Kappeler, Manfred/Leuzinger-Bohleber, Marianne/Reh, Sabine/Sielert, Uwe/Thompson, Christiane (Hg.): Sexualisierte Gewalt, Macht und Pädagogik. Opladen u.a.: Verlag Barbara Budrich, S. 103-117.

Rosen, Ursula (2018): Sexualerziehung mit Generation Z: Zeitgemäßer Biologieunterricht nach den aktuellen Richtlinien in den Klassen 5-10. Hamburg: Auer Verlag.

Rothmüller, Barbara (2018): Embodying and Resisting Racialised Desires in Young People's Sexual Imagery. In: Sex Education 19(2), S. 195-211. Online unter: https://doi.org/10.1080/14681811.2018.1540345.

Rothmüller, Barbara/Scheibelhofer, Paul (2016): Pornografisierung durch Sexualpädagogik? Über problematische Vorwürfe und komplizierte Auseinandersetzungen. In: Barberi, Alessandro/Danner, Rosa/Sonderegger, Ruth (Hg.): Internet und digitale Medien als sexualisierte Räume, 2/2016. Online unter: https:/www.medienimpulse.at/articles/view/959 [Zugriff: 22.08.2020].

Sager, Christin (2015): Das aufgeklärte Kind. Zur Geschichte der bundesrepublikanischen Sexualaufklärung (1950-2010). Bielefeld: transcript.

Sattler, Elisabeth/Thuswald, Marion (2019): Sparkling Student Research? Vom Forschen Lernen Studierender des künstlerischen Lehramts im Rahmen von partizipativen Forschungsprojekten. In: Kunz, Ruth/Peters, Maria (Hg.): Der professionalisierte Blick. Forschen im Lehramtsstudium der Kunstpädagogik. München: Kopaed, S. 530-546.

Schähle, Anna (2012): Wie werden theoretische Auseinandersetzung und der Anspruch an eine professionelle pädagogische Praxis in Vermittlungszusammenhängen vereinbart? Wien: Diplomarbeit an der Akademie der bildenden Künste Wien.

Schetsche, Michael/Schmidt, Renate-Berenike (2013): Intime Kommunikation in der Schule. In: Schmidt, Renate-Berenike/Sielert, Uwe (Hg.): Handbuch Sexualpädagogik und sexuelle Bildung. Weinheim/Basel: Beltz, S. 601-609.

Schmidt, Renate-Berenike/Sielert, Uwe (Hg.) (2008): Handbuch Sexualpädagogik und sexuelle Bildung. Weinheim: Beltz.

Schmidt, Renate-Berenike/Sielert, Uwe (2012): Sexualpädagogik in beruflichen Handlungsfeldern. Köln: Bildungsverlag EINS.

Schmincke, Imke (2015): Das Kind als Chiffre politischer Auseinandersetzung am Beispielneuer konservativer Protestbewegungen in Frank- 
reich und Deutschland. In: Hark, Sabine/Villa, Paula-Irene (Hg.): AntiGenderismus: Sexualität und Geschlecht als Schauplätze aktueller politischer Auseinandersetzungen. Bielefeld: transcript, S. 93-107.

Schmutzer, Karla/Thuswald, Marion (2019): Mit Bildern zu Lust und Begehren arbeiten. Kunst- und sexualpädagogischen Methoden und Materialien für Schule und Lehrer*innenbildung. Hannover: fabrico.

Selbstlaut (2020): Ganz schön intim. Sexualerziehung für 6-12 Jährige [sic!]. Unterrichtsmaterialien zum Download. Erstellt im Auftrag des Bundesministeriums für Unterricht, Kunst und Kultur. Aktualisierte Auflage. Online unter: https://selbstlaut.org/publikationen-und-materialien/unserepublikationen [Zugriff: 23.12.2020].

Siegenthaler, Rafaela (2020:): Zu einer Dekolonisierung der Sexualpädagogik. Re-Präsentationen von Liebe, Lust und Begehren aus rassismuskritischer Perspektive. In: Pritz, Anna/Siegenthaler, Rafaela/Thuswald, Marion (Hg.): Bilder befragen - Begehren erkunden. Repräsentationskritische Einsätze in der Bildungsarbeit. Textsammlung in der Zeitschrift Kunst Medien Bildung. Online unter: http://zkmb.de/zu-einer-dekolonisierung -der-sexualpaedagogik-re-praesentationen-von-liebe-lust-und-begehre n-aus-rassismuskritischer-perspektive [Zugriff: 23.12.2020].

Sielert, Uwe (2015): Einführung in die Sexualpädagogik. Weinheim/Basel: Beltz.

Sielert, Uwe/Marburger, Helga/Griese, Christiane (Hg.) (2017): Sexualität und Gender im Einwanderungsland. Öffentliche und zivilgesellschaftliche Aufgaben: Ein Lehr- und Praxishandbuch. Oldenburg: De Gruyter.

Thole, Werner/Baader, Meike/Helsper, Werner/Kappeler, Manfred/LeuzingerBohleber, Marianne/Reh, Sabine/Sielert, Uwe/Thompson, Christiane (Hg.) (2012): Sexualisierte Gewalt, Macht und Pädagogik. Opladen: Verlag Barbara Budrich.

Thompson, Christiane (2012): Die Gewalt der Sprachlosigkeit. In: Thole, Werner/Baader, Meike/Helsper, Werner/Kappeler, Manfred/LeuzingerBohleber, Marianne/Reh, Sabine/Sielert, Uwe/Thompson, Christiane (Hg.): Sexualisierte Gewalt, Macht und Pädagogik. Opladen u.a.: Verlag Barbara Budrich, S. 118-128.

Thuswald, Marion (2021): Sexuelle Bildung ermöglichen. Sprachlosigkeit, Lust, Verletzbarkeit und Emanzipation als Herausforderungen pädagogischer Professionalisierung. Bielefeld: trancript.

Thuswald, Marion/Sattler, Elisabeth (2019): Critical Diversity in der kulturellen und sexuellen Bildung. Einblicke in das kunst- und sexualpädagogi- 
sche Projekt Imagining Desires. In: schulhelft 175. Innsbruck: Studienverlag, S. 82-96.

Thuswald, Marion/Sattler, Elisabeth (Hg.) (2016): teaching desires. Möglichkeitsräume sexueller Bildung im künstlerisch-gestalterischen Unterricht. Wien: Löcker.

Tóth, Barbara (2018): Die Teenstar-Leaks. Der Verein Teenstar bietet in ganz Österreich Aufklärungskurse an Pflichtschulen an. Interne Schulungsunterlagen zeigen erstmals, dass Fundamentalchristen am Werk sind. In: Falter 47/18. Online unter: https:/www.falter.at/zeitung/20181120/die-te enstar-leaks?ref=nav [Zugriff: 22.08.2020].

Tóth, Barbara (2019): Ministerium verbannt Teenstar. Nach FALTERRecherchen: Der fundamentalchristliche Aufklärungsverein darf an Schulen nicht mehr lehren. Online unter: https:/www.falter.at/zeitung/20190 401/ministerium-verbannt-teenstar [Zugriff: 22.08.2020].

Tuider, Elisabeth/Müller, Mario/Timmermanns, Stefan/Bruns-Bachmann, Petra/Koppermann, Carola (2012): Sexualpädagogik der Vielfalt. Praxismethoden zu Identitäten, Beziehungen, Körper und Prävention für Schule und Jugendarbeit. Weinheim/Basel: Juventa.

Urban, Maria (2019): Sexuelle Bildung und sexualisierte Gewalt in Schulen. Zwischen Anspruch und Wirklichkeit. Bd. 21 der Reihe Angewandte Sexualwissenschaft. Gießen: Psychosozial-Verlag.

Valtl, Karlheinz (1998): Sexualpädagogik in der Schule. Didaktische Analysen und Materialien für die Praxis. Primar- und Sekundarstufe. Herausgegeben von pro familia Nürnberg. Weinheim/Basel: Beltz.

Vasold, Stefanie (2016): Hemmungslos gegen Gleichheit. Wie reaktionäre Gruppen das Feld der sexuellen Bildung zum Austragungsort gegen gesellschaftlichen Fortschritt inszenieren. In: medienimpulse-online, Bd. 64, 2/2016. Online unter: http://medienimpulse.at/articles/view/935 [Zugriff: 22.08 .2020$]$. 


\section{Jugendsexualität und sexuelle Bildung Öffentliche Diskurse, empirische Befunde und die Frage nach pädagogischer Professionalität}

Christin Sager

Die kindliche und jugendliche Sexualität ist spätestens seit dem 18. Jahrhundert Gegenstand pädagogischer Theorien und Programmatiken. Kindliche Sexualäußerungen wurden beobachtet, kontrolliert und pädagogisch reglementiert. Die Pädagogisierung der kindlichen Sexualität ist damit ein Produkt der Moderne. ${ }^{1}$ Im Anschluss an Rousseaus »Emile«, in dem der Philosoph das Kind als von >Natur aus gut bezeichnete und damit die Grundlage der romantischen Vorstellung von der >Unschuld des Kindes« legte, wurde in der Pädagogik das Augenmerk auf deren Bewahrung gerichtet, was sich insbesondere in einem Kampf gegen die kindliche Masturbation niederschlug. Der Anti-Onanie-Diskurs wurde von theologischer, medizinischer und pädagogischer Seite geführt und ist vor allem im Kontext der Ausbildung des bürgerlichen Sozialcharakters zu sehen. Zugleich breitete sich ein sSchweigen über die Sexualität des Kindes aus, das erst Freud zu Beginn des 20. Jahrhunderts mit seiner Studie über die »infantile Sexualität« aufbrach, mit der er die Idee der Romantik von der skindlichen Unschuld als Täuschung entlarvte (vgl. Freud 1910/2009: 140).

100 Jahre später leben wir in einer scheinbar ssexualitätsdurchtränkten Gesellschaft, in der es kaum noch Tabus zu sexuellen Fragen zu geben scheint. Nicht so jedoch, wenn es um kindliche und jugendliche Sexualität geht. In Kindertagesstätten und Schulen stellt die kindliche Sexualität Pädagog:innen noch immer vor Herausforderungen - sowohl in Bezug auf die pädagogische Reaktion auf sexuelle Wünsche, Fragen und Praktiken der Kinder als auch in

Das Sexualverhalten und die Sexualität des Kindes - insbesondere die kindliche Onanie - wurden auch in der Vormoderne wahrgenommen - jedoch nicht als pädagogisches Problem, wie Michael Schetsche in seiner Studie zeigt (vgl. Schetsche 1993: 21). 
Bezug auf die Frage nach sexualpädagogischen Konzepten sowie institutionellen Richtlinien zum Schutz vor sexualisierter Gewalt. Am Übergang zum 21. Jahrhundert entstanden in der BRD neue Diskursstränge, so z.B. über die >sexuelle Verwahrlosung der Jugend oder auch die >Frühsexualisierung der Kinder<, die von den sogenannten Besorgten Eltern forciert und von anderen u.a. rechtspopulistischen, Akteur:innen aufgegriffen wurden.

Im ersten Kapitel werden diese Diskurse nachgezeichnet und theoretisch vor dem Hintergrund von Foucaults Sexualitätsdispositiv diskutiert. Dass die Sorge bezüglich eines ausschweifenden jugendlichen Sexualverhaltens unbegründet ist, belegen empirische Studien zur Jugendsexualität. Besorgniserregend erscheinen jedoch die Leid- und Diskriminierungserfahrungen von LSBTIQ* ${ }^{*}$-Schüler:innen und Lehrer:innen, ${ }^{2}$ die Ausdruck der binären Geschlechts- und Sexualitätsvorstellungen und Normierungsdiskurse unserer Gesellschaft sind. Diese Thematik ist Gegenstand des zweiten Kapitels. Was diese Studienergebnisse und öffentlichen Diskurse für die pädagogische Praxis bedeuten und vor welche Herausforderungen Pädagog:innen gestellt sind, wird im dritten Kapitel aufgezeigt. Im Fazit wird schließlich für eine sexuelle Bildung plädiert, die sich einer politischen Bildung verpflichtet sieht.

Foucault versteht unter Diskursen gesellschaftliche Strukturen und Wissensordnungen, also das gesamte Feld kulturellen Wissens, das sich in Aussagen und Texten, aber auch in Bildern, in Institutionen und in der Architektur niederschlägt. Diskurse sind Schauplätze der Macht und regulieren, was als Norm bzw. Normalität und was als Abweichung gilt. Sie produzieren und reproduzieren Macht- und Herrschaftsverhältnisse, indem sie unser Denken und Wahrnehmen beeinflussen, indem sie die Möglichkeiten von Aussagen zu einem bestimmten Gegenstand, also das Sag- und Denkbare, regulieren und

LSBTIQ* steht für Lesben, Schwule, Bisexuelle, Trans*, Inter* und Queers, das Sternchen markiert eine Öffnung respektive einen Platzhalter für weitere, nicht benannte Identitäten. Einschränkend sei darauf hingewiesen, dass dieses Akronym »westliche Definitionen zusammenfasst, die einen einschränkenden, uniformierenden und hegemonialen (vorherrschenden) Charakter haben « und diese Bezeichnungen nicht implizieren, dass es sich hierbei um »lebenslang[e] und universell gültige Identitätsformen handelt. Sie werden vielmehr strategisch benutzt, um über die unterschiedlichsten sexuellen Orientierungen und Geschlechtsidentitäten über alle kulturellen, geographischen, sprachlichen und zeitlichen Grenzen hinweg sprechen zu können« (Sauer 2018 , Stichwort LSBTI). Auch diese Bezeichnungen produzieren damit Ausschlüsse und Normierungen. 
normieren. Damit sind sie produktiv: Sie bringen die Wirklichkeit überhaupt erst hervor, indem sie die Gegenstände, von denen sie sprechen, formen. Ihre Macht drückt sich vor allem darin aus, dass sie präreflexiv wirken - sie strukturieren unser Denken so stark, dass alternative Bedeutungen im Vorfeld bereits ausgeschlossen werden (vgl. Foucault 2010 [1970]). Sie regulieren damit auch die Vorstellungen von Geschlecht, Begehren und Sexualität - auch jene von Kindern und Jugendlichen und über sie (vgl. Bublitz 2003: 60; Landwehr 2009: 21). In diesem Beitrag wird im Anschluss an Ina Kerner vor allem die epistemische Dimension dieser Diskurse rekonstruiert, also die Wissensformationen, die den Diskursen zugrunde liegen, während die institutionellen und personalen Dimensionen nicht im Fokus stehen (vgl. Kerner 2009a: 14).

\section{1. Öffentliche Diskurse über `Frühsexualisierung، und Sexuelle Verwahrlosungs}

$\mathrm{Zu}$ Beginn des 21. Jahrhunderts entwickelten sich in der BRD zwei Diskursstränge, von denen der eine die kindliche Sexualentwicklung in den Fokus nahm, der andere jugendliches Sexualverhalten. 2007 publizierte die christlich-konservative Autorin Gabriele Kuby 2007 in der Jungen Freiheit - einer Wochenzeitung, die als Sprachrohr der Neuen Rechten gilt - einen Beitrag, in dem sie vor einer "Sexualisierung « der Kinder warnte, die durch neue Tendenzen in der Sexualpädagogik hervorgerufen würde. Sie führte als Argument u.a. Textstellen aus den Aufklärungsbroschüren der Bundeszentrale für gesundheitliche Aufklärung (BZgA) an, in denen das autoerotische Körpererkunden von Kindern sowie die Bedeutsamkeit von körperlicher Nähe zwischen Kindern und Eltern beschrieben wurden (Kuby 2007). In der Folge wurden diese Broschüren auch von weiteren Kritiker:innen zerrissen und als Belege für eine Frühsexualisierung der Kinder ins Feld geführt sowie als Anleitungen zu sexualisierter Gewalt diskreditiert. Nach einer Anzeige bei der Kölner Staatsanwaltschaft, die den Vorwurf des sexuellen Missbrauchs von Kindern nach $\$ 176$ StGB zurückwies und der Broschüre anstelle dessen bescheinigte, sexuelle Übergriffe unterbinden zu wollen, nahm die BZgA die Broschüre dennoch vom Markt.

Flankiert wurden diese Diskussionen von dem sich parallel ausbreitenden Diskurs über die »sexuelle Verwahrlosung « der Jugend (Siggelkow/Büscher 2008), die auch als "Generation Porno« etikettiert wurde (Gernert 2010). Hier war das zentrale Argument, dass Jugendliche - insbesondere jene aus der so- 
genannten >bildungsfernen « Schicht - nicht mehr lernen würden, »was Liebe sei« (vgl. Siggelkow/Büscher 2008). Stattdessen praktizieren sie ein promiskuitives und unkontrolliertes Sexualleben, das sich an den Inhalten der exzessiv konsumierten Pornografie orientieren und sich schließlich in zunehmenden Teenagerschwangerschaften niederschlagen würde (vgl. ebd.). Obwohl Studien zum Sexualverhalten der Jugend und zur Zahl von Teenagerschwangerschaften diese Aussagen von Beginn an widerlegt haben, erlebte dieser Diskursstrang mehrere Jahre ein hohes mediales Interesse.

Abgelöst wurden diese Debatten, als ein Arbeitspapier zur Novellierung des Bildungsplans der Landesregierung Baden-Württemberg im Jahr 2013 veröffentlicht wurde, aus dem hervorging, das künftig sexuelle Vielfalt Teil des Lehrplans werden sollte. Unter dem Motto »Keine Ideologie unter dem Regenbogen « formierte sich von Seiten verschiedener Familienorganisationen und christlicher Fundamentalist:innen umfassender Protest, etwa in Form der Besorgten Eltern und Demos für alle. Sie initiierten Petitionen und Demonstrationen, um eine >Homosexualisierung und >Frühsexualisierung - hier wurde dieser Begriff dann erneut ins Feld geführt - ihrer Kinder zu unterbinden. Unterstützt wurden diese Gruppierungen schnell von rechtsextremistischen Akteur:innen sowie Mitgliedern und Anhänger:innen der AFD, die in ihr Bundestagswahlprogramm 2017 als politisches Ziel u.a. die Bewahrung der >normalen`, also weißen, heterosexuellen, Kleinfamilie und die Abschaffung einer Sexualpädagogik der Vielfalt formulierte und zwar unter der Überschrift »Gender-Ideologie raus aus den Schulen Frühsexualisierung stoppen« (AFD 2017: 41).

All diese Diskursstränge, sowohl jene über die ssexuelle Verwahrlosung der Jugend , der 〉Frühsexualisierung der Kinder $<$ als auch die Forderung nach Abschaffung einer Sexualpädagogik der Vielfalt, können im Anschluss an Michel Foucault als Ausdruck des »Sexualitätsdispositivs« verstanden werden. Dieses bildete sich im 18. Jahrhundert neben dem bis dahin gültigen »Allianzdispositiv« heraus. ${ }^{3}$ Standen in Ersterem Verwandtschaftsbeziehungen,

Als Dispositiv beschreibt Foucault eine »heterogene Cesamtheit, bestehend aus Diskursen, Institutionen, architektonischen Einrichtungen, reglementierenden Entscheidungen, Gesetzen, administrativen Maßnahmen, wissenschaftlichen Aussagen, philosophischen, moralischen und philanthropischen Lehrsätzen, kurz, Cesagtes ebenso wie Ungesagtes [...].« (Foucault 2003: 292f.). Im Dispositiv verbinden sich Macht und Wissen, schlagen sich im Körper nieder und wirken subjekt- und identitätskonstituierend (vgl. Foucault 1983: 129). 
die Weitergabe von Besitz und die Reproduktion im Fokus, so richtet sich das Interesse des Sexualitätsdispositivs auf den Körper, auf dessen Kontrolle und Empfindungen (Foucault 1983: 128f.). Dieses zunehmende Interesse am Sex und dessen Regulierung versteht Foucault als Ausdruck einer Bio-Macht, einer »Macht zum Leben« (ebd.: 166), die sich auf die Demografie richtet. Innerhalb dieses Machttypus stellt die Sexualität das Scharnier zwischen dem Individuum und der Gesellschaft dar, da sie sowohl die Disziplinierung des individuellen Körpers als auch die Regulierung der Bevölkerung tangiert.

»Die Fortpflanzung, die Geburten- und Sterblichkeitsrate, das Gesundheitsniveau, die Lebensdauer, die Langlebigkeit mit allen ihren Variationsbedingungen wurden zum Gegenstand eingreifender Maßnahmen und regulierender Kontrollen: Biopolitik der Bevölkerung.«(Ebd., Hervorh. i. O.)

Das Sexualitätsdispositiv umfasst vier strategische Komplexe, »die um den Sex spezifische Wissens- und Machtdispositive« entfalten (ebd.: 125). Diese sind, so Foucault, produktiv und auf die Generativität ausgerichtet - also auf eine heterosexuelle, reproduktive und damit zugleich erwachsene Sexualität. Hierbei handelt es sich zum einen um die »Sexualisierung des Kindes«, deren Ziel vor allem in der Bekämpfung der Onanie bestand, aber auch in der Einschreibung der Zweigeschlechtlichkeit in die nächste Generation. Der zweite Aspekt, die »Hysterisierung der Frauen«, verweist auf die den Frauen zugeschriebene Verantwortung, Sorge zu tragen für die Gesundheit ihrer Kinder, für den Bestand der Familie und der Gesellschaft. Die »Psychiatrisierung der perversen Lust « sowie die "Sozialisierung des Fortpflanzungsverhaltens« als dritter und vierter Aspekt dienen ebenfalls der biopolitischen Normalisierung der Sexualität, die ihrerseits allein auf das Ziel der Fortpflanzung ausgerichtet ist (vgl. Foucault 1983: 126-138). Damit wurde eine Vorstellung von Sexualität und Geschlecht entwickelt, die »anatomische Elemente, biologische Funktionen, Verhaltensweisen, Empfindungen und Lüste in einer künstlichen Einheit« zusammenfasst und diese »fiktive Einheit als ursächliches Prinzip, als allgegenwärtigen Sinn [...] funktionieren [...] lässt « (Foucault 1977: 184), so dass das Geschlecht als »Verknüpfung von Sex, Gender und Begehren« zu einer "naturalisierten, als biologisch und damit allem Sozialen vorgängig erscheinenden Entität« gerann (Kerner 2009a: 32).

Im Sexualitätsdispositiv wurden also biologische und physiologische Aspekte $\mathrm{zu}>$ Normalitätsprinzipien der menschlichen Sexualität< erklärt, die bis heute in unseren Vorstellungen wirksam sind. Im Zuge der sSexuellen Revoluti- 
on` der 1960er Jahre wurde der Sexualitätsdiskurs erweitert: Sexualität, sexuelles Begehren werden seither - mehr oder weniger - öffentlich diskutiert und sexuelle Normen aufgebrochen. In der Auseinandersetzung mit dem Faschismus und dem Holocaust bestand eines der Ziele der sogenannten 68erBewegung darin, neue Lebens-, Partizipations- und Demokratieformen zu etablieren, um nicht weniger als den >Neuen Menschen eine neue Gesellschaft $\mathrm{zu}$ entwickeln, die resistent gegen autoritäre Versuchungen und Systeme sein könnte. Hierzu wurde in der bundesdeutschen 68er-Bewegung vor allem die Erziehung der nächsten Generation fokussiert. Kinder und Jugendliche sollten u.a. Körperwissen erlangen und wurden als sexuelle Subjekte adressiert, die sich ihrer sexuellen Lust und deren Befriedigung gewahr werden sollten. Auch wenn sich die Hoffnungen der 68er, dass sowohl die Sexualität als auch darüber das Subjekt und die Gesellschaft >befreit « werden könnten, bereits wenige Jahre später zerschlugen, so hat die Bewegung dennoch auf der soziokulturellen, aber auch auf der pädagogischen Ebene zu nachhaltigen Veränderungen geführt. Patriarchale, hierarchische Gesellschafts- und Familienstrukturen wurden ebenso in Frage gestellt wie rigide Moralvorstellungen und damit der Weg zur Etablierung der Vorstellung von selbstbestimmtem Leben sowie neuen Geschlechter- und Generationenbeziehungen, mit denen zugleich neue Familien- und Lebensformen einhergingen, geebnet.

Diese Liberalisierungstendenzen, die mit der gesetzlichen Gleichstellung der homosexuellen Ehe sowie der Änderung des Personenstandsrechts auch juristischen Niederschlag gefunden haben, werden von den Besorgten Eltern, der Neuen Rechten und christlich konservativen Kreisen in ihren Petitionen, Demonstrationen und politischen Zielsetzungen abgelehnt und angegriffen. ${ }^{4}$

Ins Feld geführt wird dabei immer wieder die Figur des >reinen<, >unschuldigen und damit asexuellen Kindes, um das die Akteur:innen >besorgt< seien, und das vor sexueller Vielfalt und sexueller Aufklärung beschützt werden müsse, um seine Entwicklung nicht zu gefährden. Unabhängig von wissenschaftlichen Erkenntnissen zur Sexualität des Kindes und der Sexualpraxis

Mit dieser Cesetzesnovellierung sind mitnichten Diskriminierungserfahrungen von trans* oder inter* Personen ausgemerzt. Auch rechtliche Fragen der Cleichstellung sind damit noch nicht beantwortet, geschweige denn Fragen zur Attestpflicht von inter* Personen, so dass der Bundesverband intersexueller Menschen das Cesetz nur als »einen ersten Schritt in die richtige Richtung « bezeichnet (vgl. Intersexuelle Menschen e.V. Bundesverband 2018a, b). 
der Jugend, die von diesen Gruppen ignoriert und/oder negiert werden, wird die Figur des >unschuldigen Kindes` von ihnen instrumentalisiert.

Neben der Infragestellung sexualpädagogischer Konzepte für Kindergärten und Schulen werden vor allem der Gender-Begriff und damit verbunden Gendertheorien und die Genderforschung diskreditiert. »Gender stehe für eine nicht-natürliche, damit also post-essentialistische Fassung von Geschlecht (und Sexualität)« (Hark/Villa 2015: 7) und gefährde die >normale<, heterosexuelle >Kleinfamilie und damit zugleich die Zukunft des Nationalstaates (vgl. Notz 2016: 2), so diese ideologischen Ausrichtungen, die Gisela Notz als familialistischen Anti-Feminismus bezeichnet. Eine Auseinandersetzung mit den theoretischen Grundlagen der Gendertheorien findet hierbei mitnichten statt, vielmehr handelt es sich um eine Instrumentalisierung des Begriffs, um Gleichstellungspraxen zu kritisieren. ${ }^{5}$

Dieser anti-feministische Diskurs ist europaweit $\mathrm{zu}$ beobachten. So gab es in Frankreich bereits 2012 Bewegungen, die gegen das geplante Adoptionsrecht homosexueller Paare protestierten und die Manif pour tous, den Vorgänger der Demo für alle, gründeten (vgl. Schmincke 2015: 94f.). In Ungarn wurden 2018 Gender-Studies von den Universitäten verbannt, aktuell wird in Polen das Verbot von Sexualkundeunterricht angestrebt (vgl. Spiegel-Online 2018; 2019).

Längst geht es also nicht mehr nur um Fragen der Sexualaufklärung, vielmehr wird dieses Thema genutzt, um es mit weiteren Positionen $\mathrm{zu}$ verbinden, die sich gegen eine Gleichstellung der Geschlechter sowie vielfältiger Begehrens-, Familien- und Lebensformen richten. Darüber hinaus verschaffen sich in diesem Diskurs auch rassistische Positionen einen Platz, die mit anti-feministischen, sexistischen und xenophoben Positionen verschränkt werden. Mit Foucault können sowohl »Rassismen als auch Sexismen [...] als komplexe Machtphänomene verstanden werden, die im Zusammenhang kategorialer Differenzzuschreibungen operieren. Oft [...] funktionieren sie unter Rekurs auf körperliche Merkmale, und damit unter Rekurs auf vermeintliche biologische Gewissheiten«, so Ina Kerner.

»Egal, ob es sich um Behauptungen >rassentypischer Charaktereigenschaften handelt, um die These, ethnische Konflikte seien unvermeidliche Folge 
ethnischer Kontakte, oder um Annahmen >normaler Ceschlechtsidentitäten und >normaler Sexualität - im Falle von rassistischen und sexistischen Zuschreibungen dominiert die Vorstellung, sie gründeten in der menschlichen Natur; weshalb rassistische und sexistische Differenzzuschreibungen eben meist naturalisierte Differenzzuschreibungen sind, die überzeitliche oder wenigstens langfristige Gültigkeit beanspruchen.« (Kerner 2009b: 37)

Es werden Gefährdungspotenziale heraufbeschworen, Polarisierungen und Normalitätskonstruktionen vorgenommen sowie Essentialisierungen und ethnische Ein- und Ausgrenzungsprozesse, um ein >Wir-Gefühl im Sinne einer nationalstaatlich ausgerichteten >Identität in Abgrenzung zu (ethnisch) >Anderen $\mathrm{zu}$ produzieren und $\mathrm{zu}$ reproduzieren. Im Kern geht es damit um gesellschaftliche Positionierungen, um »unterscheiden und herrschen« (Hark/Villa 2018: 31f.).

\section{Empirische Befunde zur Jugendsexualität: Gibt es Grund zur Sorge?}

Zum Sexualitätsdispositiv gehört neben diesen Machtverhältnissen, die Foucault als Bio-Macht beschrieben hat, auch ein »Wille zum Wissen« (Foucault 1983). Dieser drückt sich in einem Interesse am Sexualverhalten aus, das sich in einer zunehmenden empirischen Verwissenschaftlichung der Sexualität niederschlägt, die Ende der 1950er Jahre einsetzte. Sexualpraktiken, sexuelle Phantasien und Einstellungen wurden empirisch erhoben, statistisch ausgewertet sowie >Normalverteilungen <ermittelt und damit sexuelle Empfindungen und sexuelle Praktiken normiert und kategorisiert.

In der BRD richtete sich der Fokus der empirischen Sexualforschung seit 1966 verstärkt auf die jugendliche Sexualität. Zunächst erhob die Abteilung für Sexualforschung der Universität Hamburg empirische Daten, seit 1980 führt die Bundeszentrale für gesundheitliche Aufklärung (BZgA) in regelmäßigen Abständen Studien zu den Einstellungen und Verhaltensweisen von Jugendlichen in Bezug auf Aufklärung, Sexualität und Verhütung durch. Die letzte, bislang achte Studie dieser Trendreihe wurde im August 2014 durchgeführt. ${ }^{6}$ 
Befragt wurden Jugendliche und junge Erwachsene im Alter von 14 bis 25 Jahren.

Die Wirksamkeit des Sexualitätsdispositivs schlägt sich auch in diesen Studien nieder, geht die BZgA doch in der Fragebogenanlage grundsätzlich von eindeutigen, binären und cisgeschlechtlichen ${ }^{7}$ Geschlechtsidentitäten aus. Zwar wurde erstmals nach nicht heterosexuellen Aktivitäten gefragt, dennoch stehen heterosexuelle Perspektiven nach wie vor im Fokus. Die BZgA begründet dies mit dem gesetzlichen Auftrag der BZgA, der in der Erforschung und Optimierung des Kontrazeptionsverhaltens besteht (vgl. Heßling/Bode 2015: 97). Damit werden jedoch - gemäß dem Sexualitätsdispositiv - heterosexuelle Perspektiven weiter reproduziert, die der Alltagsrealität nur begrenzt entsprechen, bezeichneten sich doch immerhin $4 \%$ der männlichen Befragten und $2 \%$ der weiblichen Befragten als homosexuell und $5 \%$ der Mädchen und $2 \%$ der Jungen als bisexuell (vgl. ebd.: 118). Keine Auskunft gibt die Studie über den Anteil von Jugendlichen, die sich keiner cis-normativen Identität zuschreiben.

Ebenso fällt eine Dichotomie in den Ergebnisdarstellungen bezüglich der Herkunft der untersuchten Jugendlichen auf. Hier unterscheidet die Studie zwischen Jugendlichen »deutscher Herkunft « und Jugendlichen »mit Migrationshintergrund «. Zu letzterer Kategorie zählt die BZgA Jugendliche, die keine deutsche Staatsangehörigkeit besitzen, oder jene, bei denen mindestens ein Elternteil »bei der (eigenen) Geburt eine nichtdeutsche Staatsangehörigkeit« besaß (ebd.: 213). Auch in dieser Dichotomie werden Homogenisierungen innerhalb beider Gruppen vorgenommen, die der Alltagsrealität nicht gerecht werden können. Auch scheint bei moralischen Einstellungen zur Sexualität

Volkmar Sigusch führte den Begriff >cissexuell/cisgender als Cegenbegriff zu >transsexuelk ein, um auf die heteronormative Ausrichtung der Ceschlechtszuordnung aufmerksam zu machen. Cissexuell/cisgender steht für Personen, bei denen »Körpergeschlecht und Geschlechtsidentität scheinbar natural zusammenfallen«. »Das lateinische cis- bedeutet als Vorsilbe: diesseits. [...] Das lateinische trans- bedeutet als Vorsilbe: hindurch, quer durch, hinüber, jenseits, über - hinaus. [...] Zissexuelle befinden sich folglich (vom Körpergeschlecht und damit vom kulturellen Bigenus aus gesehen) diesseits, Transsexuelle jenseits. [...] Indem der Transsexualismus beweist, dass auch die Ceschlechtlichkeit ein kulturell Zusammengesetztes und psychosozial Vermitteltes ist, fallen Körpergeschlecht und psychosoziale Geschlechtsidentität bei den >Normalen«, die bisher die einzig `Gesunden` waren, nicht mehr fraglos zusammen.«(Sigusch 2013: 244) 
weniger die Herkunft eine Rolle zu spielen als vielmehr die Religiosität der Jugendlichen, unabhängig von der Frage, welcher Religion sie sich selbst zuordnen. Derartige Differenzierungen werden in der Studie aber nur an wenigen Stellen explizit gemacht. Die sich im Folgenden auf die Studie beziehenden Ergebnisse sind vor dem Hintergrund dieser Beschränkungen zu lesen.

Jugendliche sammeln ihre ersten sexuellen Erfahrungen im Alter von 14 bis 17 Jahren. Hierbei handelt es sich vor allem um erste Kuss- und Pettingerfahrungen. Den ersten Sexualverkehr erleben sie - wie auch in den letzten Studien aufgezeigt - meist im Alter von 16 und 17 Jahren (vgl. ebd.: 107f.). Eine Ausnahme bilden Mädchen mit Migrationshintergrund: Hier geben mehr als $50 \%$ an, ihren ersten Geschlechtsverkehr erst mit 19 Jahren erlebt zu haben (vgl. ebd.: 107f.). Als Gründe für das Nicht-Eingehen des Geschlechtsverkehrs werden allgemein vor allem das Fehlen der >richtigen Partner:innen angegeben sowie das Gefühl von Schüchternheit (vgl. ebd.: 101). Relevant ist auch die Religiosität: Jugendliche, »die sich ihrer Religion stark verbunden fühlen«, haben »doppelt so oft keine Beziehung zum anderen Geschlecht aufgenommen wie diejenigen mit nur loser Religionsbindung« (ebd.: 94f.). Mädchen mit Migrationshintergrund geben mit jeweils mehr als $40 \%$ als Grund für die sexuelle Abstinenz moralische Argumente an sowie die Angst davor, die »Eltern könnten davon erfahren« (ebd.: 96f.).

Der erste Sexualkontakt findet in den meisten Fällen im Rahmen einer festen Partnerschaft statt. Rund sechs von zehn Mädchen haben ihr serstes Mal in einer festen Partnerschaft praktiziert, bei $6 \%$ der Mädchen mit Migrationshintergrund hatte diese Beziehung bereits den Status einer Verlobung bzw. einer Ehe. Von den Jungen gaben knapp die Hälfte an, mit der Partnerin >fest zusammen gewesen zu sein (vgl. ebd.: 128). Im Langzeittrend der letzten zehn Jahre fällt auf, dass der Anteil der Jungen, die ihren ersten Geschlechtsverkehr innerhalb einer festen Partnerschaft erlebt haben, gestiegen ist (vgl. ebd.: 130). Sexuelle Treue ist für $80 \%$ der Mädchen und $67 \%$ der Jungen »unbedingt notwendig« (ebd.: 148). Dies widerlegt erneut die diskursive Figur der:des >sexuell verwahrlosten Jugendlichen`, derzufolge Jugendliche immer früher sexuell aktiv werden und wahllos Sexualkontakte eingehen. Der Anteil der sexuell aktiven 14-Jährigen liegt deutlich im einstelligen Bereich. Ein Anstieg auf zweistellige Werte, die zwischen zehn und 12 \% lagen, wurde lediglich in den Untersuchungen von 1998 bis 2005 festgestellt, seither sinken die Zahlen wieder (ebd.: 109-113). Dass das Alter beim sersten Mak darüber hinaus wenig über die Qualität der Beziehungen oder das Sexualverhalten selbst aussagt, zeigt etwa die Studie von Klein/Zeiske/Oswald (2008), in der »Früh- 
erfahrene« im jungen Erwachsenenalter ihre Kompetenzen in der sexuellen Kommunikation als besser einschätzen als »Spätstartende«.

Jugendliche Sexualität findet heute in überwiegendem Maße mit elterlichem Einverständnis und unter dem elterlichen Dach statt, so dass eher von einer »Familialisierung« als von einer »Verwahrlosung« der Jugendsexualität gesprochen werden kann (vgl. Sigusch 2013: 444).

Im Durchschnitt haben $8 \%$ der Befragten beim sersten Mal nicht verhütet. Mädchen verhüten wesentlich häufiger als Jungen (vgl. ebd.: 150). Im Langzeittrend sinken die Zahlen der nicht verhütenden Jungen jedoch seit zehn Jahren (vgl. ebd.: 153). Die Wahrscheinlichkeit, dass an Verhütung gedacht wird, ist innerhalb fester Partnerschaften höher. Ebenfalls steigt die Wahrscheinlichkeit, je älter die Jugendlichen beim ersten Geschlechtsverkehr sind und je höher der angestrebte Bildungsabschluss ist (vgl. ebd.: 151). Gefragt nach den Gründen, warum sie nicht verhütet haben, antworten mehr als die Hälfte der Jugendlichen, dass das serste Mak $\mathrm{zu}$ »spontan passierte«, gefolgt von der Angabe, dass »schon nichts passieren wird « (26\% der Mädchen, $29 \%$ der Jungen) (vgl. ebd.: 163). Auch wenn ab dem >zweiten Mak die Wahrscheinlichkeit, dass an Verhütung gedacht wird, steigt, zeigt sich hier noch immer Aufklärungsbedarf.

Insgesamt zieht die BZgA in ihrer Studie eine positive Bilanz hinsichtlich des Aufklärungsstatus der Jugendlichen. Für Mädchen stellt nach wie vor das Elternhaus die favorisierte Informationsquelle dar, dicht gefolgt von der Schule und >besten Freund:innen/Partner:innen<. Jungen geben in dieser Studie erstmals die Schule als wichtigste Anlaufstelle für sexuelle Fragen an. Insbesondere für Jugendliche aus Familien, in denen wenig über Sexualität gesprochen wird, nimmt die Schule eine bedeutende Rolle für die Informationsbeschaffung ein (vgl. ebd.: 13-15). Über $90 \%$ der befragten Jugendlichen gab an, schulischen Sexualkundeunterricht erfahren zu haben (vgl. ebd.: 34). Zugleich zeigt sich dessen nach wie vor biologistische Ausrichtung. »Geschlechtsorgane « wurden $\mathrm{zu}$ fast $100 \%$ als behandeltes Thema angegeben, die Werte für die Bereiche »Empfängnisverhütung, Geschlechtskrankheiten, körperliche Entwicklung, Regel, Eisprung, Schwangerschaft und Geburt« liegen zwischen $70 \%$ und fast $90 \%$. Bei den Themen »Homosexualität, Schwangerschaftsabbruch, Zärtlichkeit und Liebe, sexuelle Gewalt, Missbrauch« sinken die Zahlen dagegen auf ca. 40 \% (ebd.:36). Diese Fokussierung auf biologische Themen und die Einbindung des Sexualkundeunterrichts in Fächer wie Biologie oder Gesundheitserziehung finden sich auch in anderen europäischen Ländern (vgl. BZgA 2018: 32). 
Findet die Vermittlung biologistischer Informationen also weitestgehend statt, so stellt die Frage nach der Vermittlung sozialer und kultureller Aspekte der Sexualität, von Informationen über Selbstbefriedigung, nicht-heteronormative Sexualität und Pornografie, aber auch nach der Adressierung der Jugendlichen und dem Umgang mit den eigenen, subjektiven Wertvorstellungen Lehrende vor große Herausforderungen, wie Markus Hoffmann in seiner Studie über Deutungsmuster von Lehrer:innen in Bezug auf die Vermittlung sexualpädagogischen Wissens zeigt (vgl. Hoffmann 2016).

Diese Ergebnisse korrespondieren mit Studien zur Verankerung der Themen Sexualität, sexuelle Bildung, Kinder- und Jugendsexualität, sexuelle Vielfalt und sexualisierte Gewalt in der Lehrer:innenausbildung in der BRD, die ebenfalls nicht strukturell festgeschrieben sind, sondern meist nur sporadisch angeboten werden (vgl. Kollender 2016: 193; Glammeier 2019; Urban 2019). Lehrer:innen werden in Folge dessen in ihrer Ausbildung unzureichend darauf vorbereitet, in angemessener pädagogischer Form mit Schüler:innen über das Thema Sexualität im Unterricht umfassend und über die biologische Dimension hinaus zu sprechen und damit Sexualkundeunterricht professionell umzusetzen.

Dies führt auf mehreren Ebenen zu verpassten Chancen. Durch die gesetzliche Schulpflicht kann die Schule alle Heranwachsenden erreichen. Das ist einerseits zentral für jene Kinder und Jugendliche, die im Elternhaus keine ausreichende Sexualaufklärung erhalten, andererseits vor dem Hintergrund des Schutzauftrages und der damit verbundenen Möglichkeiten der Prävention und Intervention bei sexualisierten Übergriffen.

Obwohl die Vielfalt von Begehrens- und Lebensformen für viele Kinder alltäglich erfahrbar ist und spätestens durch Gesetzesänderungen zunehmend in das öffentliche Bewusstsein rückt, erscheint der schulische Alltag meist an der heterosexuellen Matrix orientiert. Dies betrifft sowohl die nach außen repräsentierte Geschlechtlichkeit und sexuelle Orientierung der dort agierenden Personen als auch die behandelten Themen, die Einstellungen vieler Lehrer:innen und schließlich die Materialien, auf die zurückgegriffen wird (vgl. Bittner 2011; Hess/Retkowski 2019). ${ }^{8}$ Würde die Vielfältigkeit gelebter Familienformen, sexueller Lustempfindungen, sexueller Praktiken und Paarkon-

8 Dies betrifft ebenso den Bereich der Heimerziehung und der offenen Jugendarbeit (vgl. Domann/Rusack 2016). Auch in Kinderbüchern dominieren heteronormative, geschlechterstereotype Darstellungen (vgl. Burghardt/Klenk 2016). Zugleich werden sowohl in Bilderbüchern, in Schulmaterialien als auch auf der Ebene der Lehrpersonen 
stellationen dargestellt, könnten Orientierungsmuster, aber auch Normalisierungsformen für Jugendliche geschaffen werden, die den heteronormativen Perspektiven nicht entsprechen wollen oder können. Dies würde zu einer Sensibilisierung für Diskriminierungs- und Gewalterfahrungen - auch im schulischen Kontext - führen und diesen im besten Fall entgegenwirken und damit den Leidensdruck mindern, von dem vor allem LSBTIQ*-Jugendliche in diversen Studien berichten, wie z.B. jenen, die die Antidiskriminierungsstelle des Bundes 2017 in der Bundesrepublik durchführte. ${ }^{9}$

Die Schule ist besonders wichtig für (LSBTIQ*-)Jugendliche, die mit ihren Fragen und Themen zuhause kein Gehör finden. Um sie zu erreichen, darf sich schulische Sexualerziehung nicht auf die Vermittlung biologischen Wissens allein beziehen, das meist begrenzt ist auf heterosexuelle, penetrative Sexualität sowie die Prävention sexuell übertragbarer Krankheiten und ungewollter Schwangerschaften, sondern sollte als sexuelle Bildung »Begehren in seiner Pluralität und Diversität [...] lehren und dabei auch die wahrnehmbaren Ambivalenzen « einbeziehen (Thuswald/Sattler 2016: 12), um Heranwachsende bei der Entwicklung sexueller Selbstbestimmung zu unterstützen.

Eine bedeutsame Rolle kommt der Schule auch bei Verdachts- und Disclosure-Fällen in Bezug auf sexualisierte Gewalt zu. In der BZgA-Studie gab jede fünfte der befragten Mädchen und Frauen an, bereits sexualisierte Gewalt erfahren zu haben, unter den sexuell Aktiven liegt der Wert sogar bei rund jeder vierten Befragten. ${ }^{10}$ Bei den männlichen Jugendlichen liegt der Wert deutlich niedriger (4\%). Diese Zahlen ändern sich jedoch, wenn die sexuelle Orientierung miteinbezogen wird: Homo- oder bisexuell orientierte Jungen (21\%) sind fast genauso häufig von Übergriffen betroffen wie Mädchen, homo- und bisexuell orientierte Mädchen berichteten noch häufiger

Mehrheitsverhältnisse repräsentiert, während Differenzkategorien wie race, class und body marginalisiert werden.

10 In der Studie wurde gefragt: »Hat ein Junge oder Mann schon einmal versucht, Sie gegen Ihren Willen zu Sex oder Zärtlichkeiten zu bringen, indem er Sie unter Druck gesetzt hat?« (ebd.: 196). Die Studie gibt keine Auskunft über weibliche Täter:innen und geht auch nicht auf betroffene heterosexuelle Jungen/junge Männer ein, sondern fokussiert weibliche Betroffene, die Gewalt durch Männer erfahren haben. Statistisch ist die Anzahl weiblicher Täter:innen sehr gering, jedoch werden von Frauen verübte Gewaltformen noch stärker tabuisiert als Gewalttaten durch Männer. 
(32\%) von sexuellen Gewalterfahrungen (vgl. Heßling/Bode 2015: 196). ${ }^{11}$ Für Mädchen und junge Frauen erhöht sich das Risiko, wenn sie »den Partner beim ersten Geschlechtsverkehr kaum oder gar nicht kannten«, ebenso steigt es, je jünger die Mädchen beim ersten Geschlechtsverkehr sind, nicht zuletzt weil mit einer potenziell zunehmenden Anzahl von Sexualkontakten generell das Risiko steigt, sexualisierte Gewalt zu erfahren (vgl. ebd.: 197).

\section{Herausforderungen für die (sexual-)pädagogische Professionalität}

Obwohl die aktuellen Zahlen zur Jugendsexualität also einerseits zeigen, dass Jugendliche in den letzten Jahrzehnten viele Fortschritte im Bereich des sexuellen Wissens und des verantwortungsvollen Umgangs in der sexuellen Praxis gemacht haben, wird zugleich die Bedeutsamkeit der Sexualaufklärung deutlich. Pädagogisch Tätige - sowohl in der Schule als auch in außerschulischen Handlungsfeldern - tragen eine besondere Verantwortung bezüglich der Vermittlung und Gewährleistung von »Sexuellen Rechten«, die im Jahr 2002 durch die Weltgesundheitsorganisation (WHO) $\mathrm{zu}$ einem Bestandteil der allgemeinen Menschenrechte erhoben wurden. $\mathrm{Zu}$ diesen Rechten zählt sowohl das Recht auf freie Partner:innenwahl und einvernehmliche, gewaltfreie sexuelle Beziehungen als auch das Recht auf sexuelle Informationen und sexuelle Aufklärung. Sexualaufklärung wird zugleich als wesentliche Voraussetzung "Sexueller Gesundheit« verstanden, die wiederum als Zustand eines körperlichen, emotionalen, geistigen und sozialen Wohlbefindens in Bezug auf Sexualität definiert wird und das Recht auf lustvolle und sichere sexuelle Erfahrungen - ohne Unterdrückung, Diskriminierung und Gewalt - impliziert (vgl. WHO-Regionalbüro für Europa und BZgA 2011: 19f.). Um diese Rechte umsetzen und sexuelle Gesundheit gewährleisten zu können, sei Sexualaufklärung unabdingbar, damit Kinder und Jugendliche Informationen »sowohl über die Risiken als auch die Potenziale der Sexualität« erhalten, um sie $\mathrm{zu}$ »einem verantwortungsvollen Umgang nicht nur mit sich selbst, sondern auch gegenüber den anderen Mitgliedern der Gesellschaft « zu befähigen (ebd.: 5). Zur Umsetzung hat die BZgA zusammen mit der WHO eine Matrix für eine altersgerechte Sexualaufklärung vorgelegt, in der zentrale Ziele und 
Inhalte für Kinder unterschiedlicher Altersstufen von vier bis zu zwölf Jahren formuliert sind (vgl. ebd.: 42-49).

Pädagog:innen haben in ihren spezifischen Handlungsfeldern eine große Verantwortung zu tragen, um dieses Wissen zu vermitteln sowie für sexuelle Rechte und sexuelle Gesundheit, die die Prävention von und Intervention bei sexualisierter Gewalt beinhaltet, zu sensibilisieren und Jugendliche bei der Entwicklung ihrer sexuellen Selbstbestimmung zu unterstützen.

Alle pädagogischen Einrichtungen haben im Rahmen des Erziehungsauftrages die Aufgabe, Kinderschutz zu gewährleisten. Die Einrichtungen müssen innerhalb der eigenen Institution organisationale Maßnahmen ergreifen, um Machtverhältnisse aufzudecken, die (sexualisierte) Gewalt gegenüber den ihnen anvertrauten Kindern und Jugendlichen ermöglichen, um Übergriffe durch pädagogische Professionelle möglichst im Vorfeld zu verhindern. $\mathrm{Zu}$ gleich muss das pädagogische Personal geschult und sensibilisiert sein, um Präventions- und Interventionsarbeit leisten und auf Disclosure-Prozesse reagieren zu können.

Dies tangiert zugleich das Verhältnis von Nähe und Distanz, von Intimität und Autonomie, das jeder pädagogischen Beziehung inhärent ist. Die Reflexion dieser Doppelorientierung muss sowohl auf der Ebene der institutionellen Strukturen erfolgen als auch auf der intersubjektiven Ebene sowohl bezogen auf das Gegenüber als auch bezogen auf das eigene, subjektive Empfinden und die eigenen Deutungsmuster (vgl. Dörr 2017: 203). Bleibt dieses Verhältnis unreflektiert, eröffnen sich neue Gefahren für Gewalt- und Abhängigkeitskonstellationen sowie Grenzverletzungen.

\section{Fazit: Sexuelle Bildung als politische Bildung}

Vor dem Hintergrund dieser Herausforderungen und Aufgaben, aber auch der aktuellen Instrumentalisierung dieser Thematik durch die Neue Rechte und rechts-konservative Diskurse ist eine umfassende sexuelle Bildung notwendig, die sich auch der politischen Dimension verpflichtet fühlt, wie sie im Konzept von Karlheinz Valtl angelegt ist. Sexuelle Bildung betont den Aspekt des selbstbestimmten Bildungsprozesses und fokussiert damit nicht nur intendierte Sexualerziehungsangebote, sondern auch sexuelle Sozialisationsprozesse. Akzentuiert wird mit diesem Begriff die Herausbildung eines reflexiven, sexuellen Subjekts (vgl. Valtl 2008: 128). Sexualität wird diesem Ansatz entsprechend ein eigener Wert zugesprochen, was impliziert, dass »umfas- 
sende Lehrangebote $\mathrm{zu}$ allen Themen der Sexualität« bereitgestellt (ebd.: 131) und diese zugleich weltoffen gestaltet werden sollen. Sexuelle Bildung vertritt einen ganzheitlichen Anspruch, der alle Lebensalter adressiert und schließlich als eine politische Bildung verstanden werden soll (vgl. ebd.: 133-139).

»Da Sexualität unser ganzes Leben durchzieht, lässt sich sexuelle Bildung nicht auf einen engen sprivaten Raum< reduzieren, sondern wird zum integrierten Bestandteil einer selbstbestimmten, informierten und praktisch kompetenten Lebensführung mündiger Bürger in einer demokratischen $\mathrm{Ce}$ sellschaft.«(Ebd.: 139)

Um diesem Anspruch gerecht zu werden, sollte sie eingebunden sein in ein umfassendes Bildungskonzept. Nur so sei »eine demagogische Instrumentalisierung sexueller Themen unmöglich « (ebd.: 138), wie sie aktuell in Bezug auf eine Sexualpädagogik der Vielfalt zu beobachten ist.

Wenn sexuelle Bildung als Bestandteil politischer Bildung verstanden wird, geht es auch darum, Macht- und Ungleichheitsstrukturen zu diskutieren, auf sexistische und rassistische Strukturen innerhalb unserer Gesellschaft aufmerksam zu machen sowie auf damit einhergehende Ausgrenzungen und Diskriminierungen, aber auch auf Instrumentalisierungen des Sexuellen und der Sexualerziehung etwa durch rechtspopulistische Akteur:innen.

Notwendig für diese Aufgaben und (Selbst-)Ansprüche ist jedoch, dass sexuelle Bildung bereits im pädagogischen Studium verankert wird - sowohl im Lehramtsstudium als auch in anderen pädagogischen Studiengängen. Es müsse, so der Abschlussbericht des $>$ Runden Tisches gegen sexuellen Kindesmissbrauch im Bachelorstudium ein Basiswissen vermittelt werden, zu dem der professionelle Umgang mit Nähe und Distanz zählt sowie »Wissen über Sexualität und Gewalt«, aber auch »Handlungskompetenzen, die Prävention und Intervention in der pädagogischen Praxis erfolgreich machen« (BMJ, BMFSFJ, BMBF 2011: 39). Wie groß diese Herausforderung ist, wird deutlich, wenn man sich vergegenwärtigt, an wie wenigen universitären Standorten sexualwissenschaftliche und -pädagogische Lehrstühle vertreten sind. Ohne diese Verankerung in der Hochschullehre kann sexuelle Bildung in ihrem ganzheitlichen und zugleich politischen Anspruch für pädagogische Handlungsfelder nicht gelingen, sondern wird nur weiterhin zu einer Überforderung der pädagogisch Tätigen führen. Auch die Vermittlung dieser Voraussetzungen im öffentlichen Diskurs wäre ein Anspruch an eine an politischer Bildung orientierten sexuellen Bildung. 


\section{Literatur}

Adler-Klausner, Evangeline/Jauk, Daniela/Mayer, Stefanie/Scambor, Elli (2017): Gleichberechtigte Wissenschaft, fundiert argumentieren für Genderstudies. Hg. von der Koordinationsstelle für Geschlechterstudien und Gleichstellung, Universität Graz, für die GENDERPLATTFORM. Online unter: https://www.aau.at/wp-content/uploads/2017/02/Gleichberechtigt e-Wissenschaft.pdf [Zugriff: 28.04.2020].

AFD (2017): Programm für Deutschland. Wahlprogramm der Alternative für Deutschland für die Wahl zum Deutschen Bundestag am 24. September 2017. Online unter: https://cdn.afd.tools/wp-content/uploads/sites/111/2 017/06/2017-06-01_AfD-Bundestagswahlprogramm_Onlinefassung.pdf [Zugriff: 28.04.2020].

Antidiskriminierungsstelle des Bundes (Hg.) (2017): LSBTIQ*-Lehrkräfte in Deutschland. Diskriminierungserfahrungen und Umgang mit der eigenen sexuellen und geschlechtlichen Identität im Schulalltag. Berlin: Antidiskriminierungsstelle des Bundes.

Bittner, Melanie (2011): Geschlechterkonstruktionen und die Darstellung von Lesben, Schwulen, Bisexuellen, Trans* und Inter* (LSBTI) in Schulbüchern. Eine gleichstellungsorientierte Analyse. Frankfurt a.M.: GEW.

Bublitz, Hannelore (2003): Diskurs. Bielefeld: transcript.

Bundesministerium der Justiz, das Bundesministerium für Familie, Senioren, Frauen und Jugend sowie das Bundesministerium für Bildung und Forschung (2011): Abschlussbericht Runder Tisch Sexueller Kindesmissbrauch in Abhängigkeits- und Machtverhältnissen in privaten und öffentlichen Einrichtungen und im familiären Bereich. Berlin 2011: Bundesministerium der Justiz; Bundesministerium für Familie, Senioren, Frauen und Jugend; Bundesministerium für Bildung und Forschung.

Burghardt, Lars/Klenk, Florian Cristobal (2016): Geschlechterdarstellungen in Bilderbüchern. Eine empirische Analyse. In: Gender. Zeitschrift für Geschlecht, Kultur und Gesellschaft. 8. Jg., Heft 3/2016, S. 61-80.

Domann, Sophie/Rusack, Tanja (2016): Wie sehen Jugendliche Gender und Sex in öffentlicher Erziehung? Rekonstruktionen der Perspektiven von Adressat_innen der Kinder- und Jugendhilfe. In: Gender. Zeitschrift für Geschlecht, Kultur und Gesellschaft, 8. Jg., Heft 3/2016, S. 81-97.

Dörr, Margret (2017): Nähe und Distanz in professionellen pädagogischen Beziehungen. In: Kessl, Fabian/Kruse, Elke/Stövesand, Sabine/Thole, Wer- 
ner (Hg.): Soziale Arbeit - Kernthemen und Problemfelder. Opladen/Toronto: Verlag Barbara Budrich, S. 202-210.

BZgA - Bundeszentrale für gesundheitliche Aufklärung (Hg.) (2018): Sexuality Education. Lessons Learned and Future Developments in the WHO European Region. Conference Report. Online unter: https://www.bzgawhocc.de/fileadmin/user_upload/Dokumente/BZgA_Conference_Report _Online.pdf [Zugriff: 28.04.2020].

Foucault, Michel (2010 [1970]): Die Ordnung des Diskurses. Mit einem Essay von Ralf Konersmann. 11. Auflage. Frankfurt a.M.: Fischer Verlag.

Foucault, Michel (1983): Sexualität und Wahrheit. Bd. 1: Der Wille zum Wissen. Frankfurt a.M.: Suhrkamp.

Foucault, Michel (2003): Das Spiel des Michel Foucault (Gespräch). In: Ders.: Schriften in vier Bänden. Dits et Ecrits. Bd. III 1976-1979. Frankfurt a.M.: Suhrkamp, S. 391-429.

Freud, Sigmund (2009 [1910]): Über Psychoanalyse. Fünf Vorlesungen, gehalten zur zwanzigjährigen Gründungsfeier der Clark University in Worcester, Mass., September. In: Sigmund Freud: Abriss der Psychoanalyse. Einführende Darstellungen. Frankfurt a.M.: Fischer, S. 105-183.

Gernert, Johannes (2010): Generation Porno. Jugend, Sex, Internet. Köln: Fackelträger.

Glammeier, Sandra (2019): Sexuelle Gewalt und Schule. In: Wazlawik, Martin/Voß, Heinz-Jürgen/Retkowski, Alexandra/Henningsen, Anja/Dekker, Arne (Hg.): Sexuelle Gewalt in pädagogischen Kontexten. Aktuelle Forschungen und Reflexionen. Wiesbaden: VS Verlag, S. 197-209.

Hark, Sabine/Villa, Paula-Irene (2015): »Anti-Genderismus« - Warum dieses Buch? In: Dies. (Hg.): Anti-Genderismus. Sexualität und Geschlecht als Schauplätze aktueller politischer Auseinandersetzungen. Bielefeld: transcript, S. 7-13.

Hark, Sabine/Villa, Paula-Irene (2018): Unterscheiden und herrschen. Ein Essay zu den ambivalenten Verflechtungen von Rassismus, Sexismus und Feminismus in der Gegenwart. 2. Auflage. Bielefeld: transcript.

Hess, Johanna/Retkowski, Alexandra (2019): Berufsbiographische Identitätskonstruktionen und Sexualität. In: Wazlawik, Martin/Voß, HeinzJürgen/Retkowski, Alexandra/Henningsen, Anja/Dekker, Arne (Hg.): Sexuelle Gewalt in pädagogischen Kontexten. Aktuelle Forschungen und Reflexionen. Wiesbaden: VS Verlag, S. 231-245.

Heßling, Angelika/Bode, Heidrun (2015): Jugendsexualität 2015. Die Perspektive der 14- bis 25-Jährigen. Ergebnisse einer aktuellen Repräsentativen 
Wiederholungsbefragung. Köln: Bundeszentrale für gesundheitliche Aufklärung.

Intersexuelle Menschen e.V., Bundesverband (2018a): Gemeinsame Pressemitteilung von Intersexuelle Menschen e.V., Bundesverband und Intersexuelle Menschen Landesverband Niedersachsen e.V. zum Gesetzentwurf zur Eintragungsmöglichkeit einer weiteren Option im Personenstand. Online unter: https://www.im-ev.de/pdf/2018_Gemeinsame_Pressemitte ilung.pdf [Zugriff: 08.03.2020].

Intersexuelle Menschen e.V., Bundesverband (2018b): Stellungnahme zum Gesetz zur Änderung der in das Geburtenregister einzutragenden Angaben. Online unter: https://www.im-ev.de/pdf/Stellungnahme_zum_Gese tz_zur_Aenderung_der_in_das_Geburtenregister_einzutragenden_Anga ben.pdf [Zugriff: 08.03.2020].

Kerner, Ina (2009a): Differenzen und Macht. Zur Anatomie von Rassismus und Sexismus. Frankfurt a.M.: Campus.

Kerner, Ina (2009b): Alles intersektional? Zum Verhältnis von Rassismus und Sexismus. In: Feministische Studien, 27, 1, S. 36-50.

Klein, Alexandra/Schweitzer, Jann (2018): Besorgte Eltern, die Kinder und die Anderen: Leidenserfahrungen und Zumutungen in schulischer Sexualerziehung. In: Widersprüche. Verlag Westfälisches Dampfboot, Heft 148, 38. Jg., Nr. 2, S. 31-55.

Klein, Alexandra/Zeiske, Anja/Oswald, Hans (2008): Früh übt sich ... Sexuelle Handlungsfähigkeit und das Alter beim sersten Mak. In: Ittel, Angela/Stecher, Ludwig/Merkens, Hans/Zinnecker, Jürgen (Hg.): Jahrbuch Jugendforschung 2007. Wiesbaden: VS Verlag, S. 93-114.

Kleiner, Bettina (2015): subjekt bildung heteronormativität. Rekonstruktion schulischer Differenzerfahrungen lesbischer, schwuler, bisexueller und Trans*Jugendlicher. Opladen/Berlin/Toronto: Verlag Barbara Budrich.

Klocke, Ulrich/Küppers, Carolin (2017): Zur Situation lesbischer, schwuler, bisexueller und queerer Menschen: Von der Diskriminierung zur Inklusion durch Sichtbarkeit und flexiblere Geschlechternormen. In: Diehl, Elke (Hg.): Teilhabe für alle?! Lebensrealitäten zwischen Diskriminierung und Partizipation. Bonn: Bundeszentrale für politische Bildung, S. 180-205.

Kocher, Eva/Porsche, Stefanie (2015): Sexuelle Belästigung im Hochschulkontext. Eine Expertise. Berlin: Antidiskriminierungsstelle des Bundes.

Krell, Claudia/Oldemeier, Kerstin (2018): Coming-out - und dann ...?! Coming-out-Verläufe und Diskriminierungserfahrungen von lesbischen, 
schwulen, bisexuellen, trans" und queeren Jugendlichen und jungen Erwachsenen in Deutschland. Bonn: Bundeszentrale für politische Bildung. Kuby, Gabriele (2007): Auf dem Weg zum neuen Menschen. In: Junge Freiheit, 27/07, 29.06.2007. Online unter: https://www.jf-archiv.de/archiv07/2007 27062957.htm [Zugriff: 13.07.2020].

Küpper, Beate/Klocke, Ulrich/Hoffmann, Lena-Carlotta (2017): Einstellungen gegenüber Lesben, Schwulen und Bisexuellen in Deutschland. Ergebnisse einer bevölkerungsrepräsentativen Umfrage. Hg. von der Antidiskriminierungsstelle des Bundes. Baden-Baden: Nomos.

Landwehr, Achim (2009): Historische Diskursanalyse. 2. Auflage. Frankfurt a.M.: Campus.

Notz, Gisela (2016): Brauchen wir einen neuen Familismus? Kritische Anmerkungen zu einem ideologisierten Familienverständnis. Vortrag auf der Tagung: »Gegner*innenaufklärung - Informationen und Analysen $\mathrm{zu}$ AntiFeminismus" im Gunda-Werner-Institut in der Heinrich-BöllStiftung am 31.05.2016. Online unter: https://www.gwi-boell.de/sites/de fault/files/uploads/2016/08/brauchen_wir_einen_neuen_familismus_gise la_notz.pdf [Zugriff: 07.12.2019].

Sauer, Arn (2018): LSBTIQ-Lexikon. Grundständig überarbeitete Lizenzausgabe des Glossars des Netzwerkes Trans"Inter*Sektionalität. Online unter: http://transintersektionalitaet.org/?page_id=36. Bundeszentrale für politische Bildung: Bonn. [Zugriff: 08.03.2020].

Schetsche, Michael (1993): Das »sexuell gefährdete Kind«. Kontinuitäten und Wandel eines sozialen Problems. Pfaffenweiler: CentaurusVerlagsgesellschaft.

Schmidt, Gunter (Hg.) (2004): Kinder der sexuellen Revolution. Kontinuität und Wandel studentischer Sexualität 1966-1996. Eine empirische Untersuchung. Gießen: Psychosozial Verlag.

Schmincke, Imke (2015): Das Kind als Chiffre politischer Auseinandersetzung am Beispiel neuer konservativer Protestbewegungen in Frankreich und Deutschland. In: Hark, Sabine/Villa, Paula-Irene (Hg.): AntiGenderismus. Sexualität und Geschlecht als Schauplätze aktueller politischer Auseinandersetzungen. Bielefeld: transcript, S. 93-107.

Schutzbach, Franziska/Pühl, Katharina/von Bargen, Henning (2018): Gender Raus. Zwölf Richtigstellungen zu Antifeminismus und Gender-Kritik. Hg. von der Heinrich-Böll-Stiftung und der Rosa-Luxemburg-Stiftung. 2. aktualisierte Auflage. Online unter: https://www.gwi-boell.de/sites/default /files/gender_raus_epdf_2.pdf [Zugriff: 28.04.2020]. 
Sigusch, Volkmar (2013): Sexualitäten. Eine kritische Theorie in 99 Fragmenten. Frankfurt a.M.: Campus.

Spiegel-Online (2018): Ungarn schafft das Fach Gender Studies ab. Online unter: https://www.spiegel.de/lebenundlernen/uni/ungarn-schafft-das-f ach-gender-studies-ab-a-1233500.html [Zugriff: 24.11.2019].

Spiegel-Online (2019): EU kritisiert Polen wegen Gesetzentwurf zur Sexualkunde. Online unter: https:/www.spiegel.de/lebenundlernen/schule /polen-eu-parlament-kritisiert-plaene-fuer-sexualkunde-a-1296488.html [Zugriff: 24.11.2019].

Thuswald, Marion/Sattler, Elisabeth (2016): Teaching Desires - sexuelle Bildung herausfordern. In: Dies. (Hg.): teaching desires. Möglichkeitsräume sexueller Bildung im künstlerisch-gestalterischen Unterricht. Wien: Erhard Löcker.

Urban, Maria (2019): Sexuelle Bildung und sexualisierte Gewalt an Schulen. Zwischen Anspruch und Wirklich. Gießen: Psychosozial Verlag.

Valtl, Karlheinz (2008): Sexuelle Bildung: Neues Paradigma einer Sexualpädagogik für alle Lebensalter. In: Schmidt, Renate-Berenike/Sielert, Uwe (Hg.): Handbuch Sexualpädagogik und sexuelle Bildung. Weinheim/München: Beltz, S. 125-140.

WHO-Regionalbüro und Bundeszentrale für gesundheitliche Aufklärung (2011): Standards für die Sexualaufklärung in Europa: Rahmenkonzept für politische Entscheidungsträger, Bildungseinrichtungen, Gesundheitsbehörden, Expertinnen und Experten. Köln: Bundeszentrale für gesundheitliche Aufklärung. 



\section{Zu intim? \\ Eine Gesprächsrunde mit Lehrer:innen zu Fragen rund um Sexualität, Beziehungen und Intimität in Unterricht und Schulalltag}

Anna Pritz, Elisabeth Sattler und Marion Thuswald

Der Beitrag gibt Einblicke in eine Gesprächsrunde mit Marlies Brommer, Tobias Dörler und Caro Estrada-Steiger, drei Lehrer:innen für künstlerischgestalterische Fächer in der Sekundarstufe. ${ }^{1}$ Das Gespräch zeigt, wie unterschiedlich Sexualität, Beziehungen und Körperlichkeit im Unterricht und im Schulalltag Thema sind und pädagogisch zum Thema gemacht werden können: Des Öfteren wird von Schüler:innen darauf angespielt oder die Thematik wird explizit aufgeworfen, häufig kommt sie angesichts von anderen Inhalten auf und bisweilen wird sie im Sinne des Unterrichtsprinzips Sexualpädagogik klar intendiert ins schulische Lehren und Lernen eingebracht. Die folgende Gesprächsrunde, die von Rafaela Siegenthaler und Marion Thuswald geleitet wurde, gibt Einblicke in den schulischen Alltag mit seinen inhärenten sexualpädagogischen Fragen und Herausforderungen. Aufgegriffen wird etwa die Frage, welche Gespräche wann als >zu intim`wahrgenommen werden, wie im Unterricht nebenbei über Beziehungen und Sexualität gesprochen werden kann, oder wie ein professioneller Umgang mit Anziehung und Verlieben zwischen Schüler:innen und Lehrer:innen aussehen kann.

Das Gespräch zeigt, welche Balance-Akte Lehrende in der Schule herausfordern, welche reaktiven und proaktiven Handlungsstrategien im Fachunterricht, in Projekten, im pädagogischen Alltag sowie in unterschiedlichen Formaten als good practice eingeschätzt werden können und auch, wo kollegialer Austausch als hilfreich erlebt wird und welche offenen Fragen die schulische und Textiles Werken. 
Begleitung von Jugendlichen rund um Sexualität, Beziehung und Körperlichkeit mit sich bringt. ${ }^{2}$

\section{Erwachsenwerden $=$ Sex?}

Marlies Brommer: Ich war dieses Jahr in das Schulprojekt erwachsen werden bei uns an der Schule eingebunden. In diesem Rahmen beschäftigten sich alle Fächer eine ganze Woche lang mit dem Erwachsenwerden. Dabei konnte ich mich in dreierlei Weise einbringen: Zum einen haben wir im Fach Bildnerische Erziehung zur Thematik Beziehungen und Social Media gearbeitet. Zum anderen war ich im Fach Biologie als Assistentin dabei, als es um den Körper ging. Ich fand mich unerwartet in der Situation wieder, mit einer Jungengruppe Arbeitsblätter zum Körper zu bearbeiten, die nicht mehr ganz aktuell waren. Das war schwierig, aber auch interessant, da die Gruppendynamik im Unterschied zu sonstigen Situationen für mich unerwartet angenehm und wertschätzend war. Und zum Dritten war ich in einer sogenannten Vertrauensrunde, die für Mädchen und Jungen getrennt stattfand. Dort konnten die Mädchen mit einer Lehrerin, also mit mir, über selbstgewählte Themen und Fragen sprechen.

Tobias Dörler: Wie alt waren die Schüler:innen?

Marlies Brommer: Das war eine dritte Klasse [7. Schulstufe]. Dieses Erwachsen werden-Projekt ist bei uns an der Schule institutionalisiert. Das machen alle Lehrer:innenteams in der dritten oder vierten Klasse und es existiert schon sehr lange. Deshalb gibt es bereits Material und diese Vertrauensrunde ist ein Element, das etabliert ist. Die Schüler:innen freuten sich total auf diese Woche, weil sie dann auch keine Hausaufgaben bekommen. Sie waren aufgeregt, weil es ums Erwachsenwerden geht und sie meinten, es gehe endlich um Sex - und sie wollten jetzt alles wissen. schließend gekürzt und in Absprache mit den Teilnehmer:innen sprachlich bearbeitet. Der Text folgt im Wesentlichen der thematischen Chronologie des Cesprächs. Eine längere Fassung wurde unter dem Titel »Let's talk ... «von Anna Pritz und Marion Thuswald in der Textsammlung »Bilder befragen - Begehren erkunden« (Pritz u.a. 2020) unter http://zkmb.de/lets-talk-about-eine-gespraechsrunde-zu-koerperlichkeit-intimitaet -und-sexualitaet-in-unterricht-und-schulalltag publiziert. 
Marion Thuswald: Die Schüler:innen haben das Thema Erwachsenwerden mit Sex assoziiert?

Marlies Brommer: Ja, obwohl wir es offengelassen haben, aus der Erfahrung heraus, dass sonst Kinder manchmal zu Hause bleiben, weil ihre Eltern sehr skeptisch sind. Wir haben nur ins Mitteilungsheft geschrieben: „Nächste Woche Projekt erwachsen werden «. Diese Offenheit hat das Projekt noch interessanter gemacht für die Schüler:innen. Sie waren dann teilweise auch enttäuscht, dass nicht alles in der ersten Stunde erklärt wurde, was sie wissen wollten. Die Erwartungshaltung war: Es geht um Sex und die Lehrer:innen erzählen uns jetzt darüber. Und die Enttäuschung war dann, dass wir über Instagram gesprochen haben, das fanden sie langweilig. Wir haben dann aber besprochen, was das miteinander zu tun hat.

\section{Nebenbei über Beziehungen und Sexualität sprechen}

Tobias Dörler: Während du erzählt hast, ist mir eingefallen, dass das Thema Sexualität bei uns öfters nebenbei in der Holzwerkstätte auftaucht. Weil die Schüler:innen so intensiv den ganzen Nachmittag arbeiten und vielleicht auch, weil wir viele Gruppen mit ausschließlich Mädchen haben, wird das Thema von ihnen selbst eingebracht. Es kommt in den Gesprächen während der Arbeit auf.

Insbesondere am Anfang der Oberstufe, wenn sie viel in den Werkstätten arbeiten, sind Themen wie Sexualität, Beziehungen und so weiter oft Thema in Gesprächen untereinander. Es besteht bei uns in den Werkstätten oft ein großes Vertrauensverhältnis, sodass sie auch vor mir und mit mir solche Inhalte thematisieren. Sie wollen wissen, wie ich dazu stehe, oder loten Grenzen aus.

Marlies Brommer: Ich glaube, das macht der andere Rahmen. Ich kenne das auch vom Unterrichtsfach Werken. Da sind ja nur etwa zwölf Schüler:innen in einer Gruppe. Da binden sie mich in Themen ein, die sie untereinander besprechen, oder stellen eine Frage. Ich glaube, das wird durch diese andere Arbeitsweise ermöglicht, die nicht frontal ist. Die Atmosphäre ist irgendwie beweglicher; sie arbeiten, sprechen dazu und dabei kommen alle möglichen Alltagsthemen auf. 
Tobias Dörler: Oft werden Begrifflichkeiten eingeworfen, die Schüler:innen irgendwo aufgeschnappt haben. Am Anfang der Oberstufe wissen sie oft gar nicht genau, was diese bedeuten. Es gibt meist ein paar Schüler:innen, die die Themen vorgeben, und manchmal geht es auch um das Ausloten, ob ich als Lehrperson dieses Thema selber kenne und ob ich ihnen auch Informationen geben könnte.

Marlies Brommer: Es geht um das Einordnenkönnen und gelegentlich auch um Provokation. Ich kenne das vom Musikhören. Manchmal dürfen sie beim Arbeiten Musik hören und wenn sexistische Rap-Texte kommen, warten sie auf meine Reaktion und sind gespannt auf die Diskussion. Manchmal rede ich mit ihnen über die Sprache, den Text, die Beschimpfungen. Oft ziehen sich solche Themen über Wochen. Bis dann wieder jemand einen solchen Song einschaltet und jemand anders sagt, »Ah, dreh das ab, das ist mir doch zu sexistisch!« Manchmal ändert sich ihre Sichtweise sogar innerhalb einer Stunde, das ist interessant zu beobachten und zeigt, wie wichtig es ist, wie Lehrer:innen reagieren.

Marion Thuswald: Und was sind das für Worte und Themen, welche die Schüler:innen einbringen?

Tobias Dörler: Oft sind es Worte, die ich in dem Moment wirklich auch gar nicht einordnen kann, also Jugendausdrücke, bei denen ich dann merke, dass ich einen Jugenddiskurs verpasst habe. Ich bin mir dann nicht ganz sicher, ob das Wörter sind, mit denen sie mir nur eine kurze Reaktion entlocken wollen, oder etwas, zu dem sie wirklich Informationen haben bzw. länger darüber diskutieren wollen. Also Beziehungsmöglichkeiten in verschiedenster Form, sexuelle Orientierungen in verschiedenster Form bzw. eigene Erlebnisse, die sie zum Thema machen und bei denen dann nicht so ganz klar ist, ob sie wirklich passiert sind. Manchmal kommt angesichts der Erzählungen bei mir die Frage auf, ob ich die Schüler:innen schützen muss oder ob sie prahlen. Ich finde das schwierig abzuwägen.

Marion Thuswald: Meinst du, sie erzählen von Grenzüberschreitungen, die sie selber machen, oder die ihnen passieren? 
Tobias Dörler: Beides. Ich muss ihnen dann klar machen, dass - wenn manches wirklich passiert ist - dies besprochen werden und auch Konsequenzen haben muss. Schwierig ist da die Balance. Ich find es ja auch schön, wenn es nicht so ernst sein muss, sondern einfach mal so grundsätzlich Thema sein kann, aber trotzdem sind manche Themen zu ernst, um nichts dazu zu sagen.

Marlies Brommer: Worum geht es da? Um sexuellen Missbrauch?

Tobias Dörler: Das ist manchmal schwierig einzuschätzen in der Oberstufe. Sie sind in einem Alter, in dem sie grundsätzlich selbst entscheiden können, ob und mit wem sie Sex haben wollen. Die Frage ist also: Wie sind die sexuellen Handlungen zustande gekommen? Wie alt war die andere Person? Ohne kontrollierend sein zu wollen, ist es wichtig herauszufinden, ob sie Hilfe brauchen. Es ist in jedem Fall sehr zu begrüßen, dass sie es aktiv thematisieren.

In einem Semester hat z.B. eine Schüler:innengruppe sexuelle Orientierung und Körperlichkeit als gemeinsames Thema für mein Unterrichtsfach gewählt und wir haben Comics gezeichnet. Eine Schülerin hat dargestellt, wie sich ein Tanzlehrer der Protagonistin im Rahmen des Tanzens nähert und wie furchtbar sie das empfindet und wie sehr es sie ekelt, wenn er sie berührt.

Marion Thuswald: Oh! Das klingt recht klar nach Grenzüberschreitung, nach Übergriffen in einer pädagogischen Beziehung, wo es nicht nur um das Alter der Beteiligten geht, sondern auch um die Frage des Ausnützens eines Abhängigkeitsverhältnisses.

Marlies Brommer: Bei der erwähnten Vertrauensrunde mit den Mädchen ging es oft um das Gedrängtwerden durch gleichaltrige Jungen und darum, dass sie zu sexuellen Handlungen überredet werden. Das sind natürlich Grenzüberschreitungen und wir haben intensiv darüber gesprochen, was eigentlich wollen heißt, welche Gefühle es gibt, was sie (mir) signalisieren und wie ich drüber sprechen kann. Das sind Fragen, die unter den Gleichaltrigen wichtig sind. 


\section{Körperlichkeit, Geschlecht und sexuelle Orientierung als Projektthemen}

Tobias Dörler: Im Fach Mediale Darstellungsverfahren taucht jedes Jahr das Thema Körperlichkeit auf: Sie wählen das entweder in Kleingruppen als Thema oder als ganze Gruppe und dann arbeiten wir gemeinsam daran. Im Bereich der digitalen Medien, also Video oder Fotografie, arbeiten die Schüler:innen oft in Kleingruppen zu zweit, zu dritt, zu viert oder manchmal auch als ganze Klasse an einem Video. Dort gebe ich oft ein Rahmenthema; meistens, dass sie zu einem gesellschaftskritischen Thema arbeiten sollen. Dann wählen die Schüler:innen für sich in der Kleingruppe meist ein spezifisches Thema, bei dem es um die Verhandlung von Körpern in Medien geht. Wenn ich am Anfang des Schuljahrs mit ihnen Themen sammle, zu denen sie arbeiten wollen, nennen sie auch manchmal sexuelle Orientierung, geschlechtliche Vielfalt und Körperlichkeit. Wenn diese Themen das Überthema für alle sind, hat das den Vorteil, dass ich sie mit allgemeinem Input für alle verknüpfen kann. Das Interesse ist da meist groß und die Schüler:innen sind sehr bereit, zu diesen Themen zunächst einmal Informationen zu bekommen und dann darauf basierend zu diskutieren.

Ich nehme Themen wie etwa Gender oder sexuelle Orientierung auch als Querschnittsmaterie wahr, die immer wieder auftauchen, wenn wir gerade an anderen Themen dran sind.

Caro Estrada-Steiger: Ganz am Anfang meiner Unterrichtstätigkeit wollte ich mit einer Gruppe aus dem Medienzweig in eine Fotoausstellung gehen. Das Semester hatte gerade begonnen und ich hatte sie erst zwei, drei Mal gesehen. Ich wusste inzwischen, dass in dieser siebten Klasse Jungen dabei waren, die sich sehr mit ihrer Religion beschäftigten. Ich habe sie gefragt, ob es ein Problem für sie sei, wenn wir in die Ausstellung des chinesischen Fotografen Ren Hang gehen, der für seine Nacktfotos bekannt und in China deshalb mehrmals verhaftet worden ist. (Die Ausstellung gab es noch vor seinem Suizid 2017.) Ich dachte damals, dass es eine spannende Auseinandersetzung mit dem Thema hätte sein können. Sie meinten, das wäre für sie schon ein Problem, es sei harām nackte Körper zu sehen. Ich weiß nicht, ob sie privat hingegangen wären, aber mit mir als Lehrerin wollten sie nicht hingehen. Sie waren sehr solidarisch untereinander und der Rest der Gruppe wollte dann auch nicht hingehen. Schließlich sind wir in eine andere Fotoausstellung gegangen. 
Tobias Dörler: Es ist spannend, wie das Thema Religion eine Barriere darstellt, wenn man über sexuelle Orientierung oder Sexualität generell spricht. Mir kommt vor, dass es bei allen Religionen ähnlich ist. Ich denke an das Schulbuchprojekt zurück, bei dem wir uns in verschiedenen Schulen mit Schulbüchern beschäftigt haben und die Bücher daraufhin untersucht haben, wie Personengruppen und ihre Lebenssituation in Schulbüchern diskriminiert werden. Ich erinnere mich an eine Gruppe von Schüler:innen, bei der es um die Darstellung von schwulen Personen ging. Für manche der Mädchen war das Thema aufgrund ihres katholischen Hintergrunds schwierig zu behandeln. Mir scheint, es ist bei allen Religionen ähnlich: Wenn jemand sehr strenggläubig ist, taucht schnell diese Grenze auf.

Das heißt nicht unbedingt, dass man nicht trotzdem an dem Thema arbeiten kann oder dass nicht auch der Wunsch von Schüler:innen da ist, diese Grenze doch zu überschreiten oder zu verschieben oder zumindest drüber zu reden.

Marlies Brommer: Ich frage mich manchmal, ob manche Altersgruppen weniger voreingenommen sind als andere. Ich habe das Gefühl, dass die Jüngeren (zwischen zehn und 14 Jahren) Sachen oft leichter annehmen oder weniger Ablehnung zeigen. Ich habe z.B. in einer dritten Klasse Unterstufe zwei Jungen, die sich sehr zugewandt sind. Sie sitzen gegenseitig auf dem Schoß und berühren sich immer wieder mal. Irgendwer hat mal gesagt, das sei schwul und die ganze Klasse hat sich darüber aufgeregt und gemeint: »Das ist ganz normal, jeder darf sein, wie er will.«Es scheint mir bei den Jüngeren weniger festgefahren. Kontroversen und Diskussionen finden sich eher bei älteren Schüler:innen ab etwa 15 Jahren.

Caro Estrada-Steiger: Es kann sein, dass sie dann erst an ihre Grenzen stoßen, wenn sie älter werden und merken, was für sie vorgesehen ist und wie sie sich dagegen wehren oder wie sehr sie sich damit identifizieren können.

Marlies Brommer: Vielleicht auch, weil das Thema Sexualität dann präsenter wird für die Schüler:innen.

Tobias Dörler: Mir scheint, dass mit der Pubertät die Suche nach Identität zum Thema wird und diese mit extremer Unsicherheit verbunden ist. Grenzen werden plötzlich Thema. Die Schüler:innen finden, dass irgendwas gar nicht geht, pervers erscheint oder Ähnliches. Grenzziehungen machen es 
leichter, sich auf eine klare eigene Position zu beziehen. Im Sinne von »So bin ich und das sind meine Dinge und das Andere ist schlecht «. Erst später, Richtung Matura ${ }^{3}$ hin, wenn sie ihre eigene Identität gefunden haben, scheint es leichter, dass Themen anders verhandelt werden untereinander, die Akzeptanz ist größer.

\section{Visuelles Material - Vielfalt und Repräsentationskritik}

Marion Thuswald: Wie arbeitet ihr mit visuellem Material? Und wie geht ihr damit um, dass viele Darstellungen von einem männlichen Blick auf Frauen geprägt sind?

Marlies Brommer: Das Spannende in der klassischen Kunstgeschichte ist ja, dass bestimmte Motive ganz oft auftauchen. Hier kann man das ganz gut zeigen: den weiblichen Blick und den männlichen Blick, der sich so oft wiederholt. Z.B. gibt es das Motiv Susanna im Bade, das ist eine klassische Szene, die ganz oft vorkommt. Beispielsweise die Darstellung von Tintoretto: Die Frau ist nackt und schön, die Männer beobachten sie, eigentlich >spannen`sie. Das Bild zeigt stark die männliche Perspektive. Artemisia Gentileschi hingegen hat dieselbe Szene aus anderer Perspektive gemalt: Die Frau ist angeekelt. Man sieht es an ihrem Blick. Es geht in diesem Bild nicht darum, wie sie aussieht, sondern auch um die Gefühlsebene, also wie sie sich vielleicht fühlt, wenn sie bespannt wird. An solchen unterschiedlichen Bildern zu gleichen Motiven kann man viel vergleichen. Das ist ein gutes Beispiel, weil es aus derselben Zeit ist und dasselbe Motiv hat, aber einmal aus männlicher und einmal aus weiblicher Perspektive. Es gibt jedoch aus dieser Zeit viel weniger weibliche Perspektiven.

Tobias Dörler: Das ist ein Riesenproblem, wenn man kunstgeschichtlich arbeitet, dass man merkt, wie Frauen sukzessive aus der Geschichtsschreibung rausgeschrieben worden sind. Über Künstlerinnen gibt es noch immer wenige Bücher. Es ist weiterhin schwierig, zu Material und Bildern zu kommen. Spannend ist auch, wenn man merkt, wie Frauen, also Künstlerinnen, den männlichen Blick kopiert haben. Darum braucht es eine überlegte Auswahl der Bilder. Manche Frauen haben ja auch diesen männlichen Blick kopiert, 
um überhaupt Eingang in die jeweilige männlich dominierte Kunstcommunity zu bekommen.

Marion Thuswald: Es gibt ja in der Kunstgeschichte auch viele Darstellungen, die Sexualität und Beziehungen mit Gewalt verbinden, die also etwa Entführungen, Vergewaltigungen oder Zwangsheiraten darstellen. Arbeitet ihr damit und wenn ja wie?

Tobias Dörler: Also ich finde das schon wichtig, diese gewaltvollen Darstellungen zu thematisieren. Gerade in Bezug auf klassische Motive. Die Venusdarstellungen stellen ja eigentlich oft Vergewaltigungsszenen dar. Es ist zwar hart, aber wesentlich, das zu benennen und den Schüler:innen bewusst zu machen, wie etwa in antiken Sagen und deren künstlerischen Darstellungen sexuelle Gewalt an Frauen normalisiert wird.

Marion Thuswald: Aus heutiger Perspektive wird in diesen Bildern Gewalt ästhetisiert.

Tobias Dörler: Ja, das gewaltvolle Motiv wird etwa verpackt in irgendeinen Goldregen und muss gemeinsam dekonstruiert werden. Dazu braucht es am Anfang Informationen von uns als Lehrpersonen.

Rafaela Siegenthaler: Gibt es Bilder, die ihr bewusst einspeist als affirmative, positive, andere Repräsentationsformen oder Identifikationsangebote im Sinne von Empowerment? Habt ihr da Bilder, mit denen ihr gerne arbeitet? ${ }^{4}$

Tobias Dörler: Es ist nicht in allen Bereichen der Kunst leicht, solche Werke zu finden. In der Fotografie ist es leichter, da gibt es unendlich viel und es ist sehr spannend, Beispiele zu zeigen, die diverse Darstellungen von Körpern und auch nicht-heterosexuelle Beziehungen repräsentieren. Bei Malerei

4 Zu Fragen der Repräsentationskritik und affirmativer Repräsentationsformen vgl. auch die online verfügbare Textsammlung »Bilder befragen - Begehren erkunden « von Anna Pritz, Rafaela Siegenthaler und Marion Thuswald (2020), veröffentlicht unter http ://zkmb.de/sammlung/bilder-befragen-begehren-erkunden, sowie die didaktische Publikation »Mit Bilder zu Lust und Begehren arbeiten« von Karla Schmutzer und Marion Thuswald (2019). 
finde ich das viel schwieriger, da tue ich mir oft schwer, alternative Darstellungen zu Hetero-, Cis- und gängigen Körpernormen zu zeigen. Es gibt weniger Alternativen, die man den Schüler:innen zeigen kann, weil viele bekannte Künstler:innen für diese Alternativen in den letzten 50 Jahren bewusst andere Medien gewählt haben.

Spannend finde ich, wenn man ihnen verschiedene Bilder anbietet und sie mit dem Auftrag auswählen lässt: »Beschäftigt euch zu zweit mit den Bildern und analysiert eines dieser Bilder.«In so einem Pool von Bildern können auch welche sein, die nicht den Normen entsprechen, die sie im Kopf haben. Es ist spannend zu beobachten, welche Bilder sie dann auswählen. Und wie sie darauf kommen, was das Bild bedeutet.

Wenn das nicht so offensichtlich ist, dann wählen sie es auch aus, und erst in der Analyse merken sie, was da überhaupt dargestellt ist. Ein spannendes Beispiel ist Pete and the Wolfman von Elizabeth Peyton. Die Darstellung ist androgyn und die Schüler:innen haben ziemlich lang gemeint, das sei ein heterosexuelles, küssendes Paar, bis sie irgendwann darauf gekommen sind: »Moment, dieser Titel!« Es war ein spannender Moment, als sie entdeckt haben, dass das überhaupt kein heterosexuelles Paar ist. Sie hätten das Bild wahrscheinlich nicht ausgewählt, aber auf diese Weise haben sie so ein Beispiel analysiert.

Marion Thuswald: Ihr habt auch von Alltagsbildern gesprochen. Welches visuelle Material verwendet ihr neben Kunst sonst noch?

Tobias Dörler: Also ich finde es wichtig, über Werbung zu reden, diese zu analysieren. Auch Eigendarstellungen in den sozialen Medien, auf Plattformen wie Instagram oder YouTube beschäftigen sie sehr.

Marlies Brommer: Ich greife Phänomene auf, die sie vielleicht aus den Online-Medien kennen, oder Personen, die sie da vielleicht kennen. Ich finde aber auch Dokumentarisches wichtig. Pressefotografie fällt bei mir etwa auch unter Alltagsbilder.

Tobias Dörler: Ich wollte noch einen Zugang erwähnen: Wenn man an Themen arbeitet, die im Kunstunterricht vorkommen, finde ich es manchmal ganz spannend, wenn Dinge einfach offenbleiben, z.B. eine Gender-Identität plötzlich vage wird. Wenn Uneindeutigkeit nicht gleich in eine neue Eindeutigkeit umschlägt, sondern mal offenbleiben kann. Das löst manchmal 
Verwirrung aus. Ich finde es okay, wenn sie verwirrt sind in dem Moment. Ich versuche das mit einem gewissen Spaß zu verknüpfen, also ihnen meinen Spaß an der aktuell auftretenden Ungewissheit zu vermitteln. Vielleicht ist es im Moment absurd für sie, aber ich schlage dann vor, dass wir uns mal verschiedene Kunstwerke ansehen und Unklarheit dazu so stehen lassen oder möglichst viele verschiedene Interpretationsmöglichkeiten der Schüler:innen dazu sammeln. Also nicht, dass ich als Lehrer:in sofort erzähle, in welchem Kontext ein Kunstwerk entstanden ist und was die Künstler:in darstellen möchte. Sondern erst später - als eine mögliche Interpretation.

\section{Zu intim?}

Marion Thuswald: Wie geht ihr mit Fragen von Intimität um? Das ist ja in der Schule auch die Frage: Wie viel Persönliches wollen die Schüler:innen von sich zeigen oder auch nicht?

Caro Estrada-Steiger: Ich habe mit Bildanalysen gute Erfahrungen gemacht. Ich lasse meine Schüler:innen gerne Analysen zu Bildern schreiben, die sie selber aussuchen. Bei den Bildern, die ich zur Auswahl anbiete, achte ich dabei immer auf mindestens 50 Prozent Frauenanteil bei den Künstler:innen. Ich habe oft den Eindruck, dass sich Schüler:innen sehr schwer tun zu artikulieren, was sie überhaupt sehen. Ich habe einen einfachen Fragenkatalog, mit dem sie arbeiten: Was siehst du? Was kannst du noch entdecken, das du vorher nicht gesehen hast? Beschreibe das Bild jemandem, der das Bild nicht sehen kann. Was glaubst du, könnte die Künstlerin oder der Künstler gemeint haben? usw. Da schreiben sie oft sehr persönliche Dinge. Öfters ist es insbesondere für Schüler:innen, die sich sonst sehr wenig artikulieren im Unterricht, einfacher, sich schriftlich zu äußern. Manchmal gibt es dann Anknüpfungspunkte, um aus der Analyse Projekte oder Referate zu entwickeln oder auch Gruppenarbeiten. Aber es geht mir vor allem darum, dass sie üben, genau hinzusehen und eigene Assoziationen und Gefühle auch in Worte zu fassen.

Marlies Brommer: Ich habe das auch schon gemacht, dass sie Fragen an sich selbst gestellt und beantwortet haben, ohne es jemandem zu zeigen. Ich finde die Frage, was zu intim ist, eine Gratwanderung. Manchmal kann es schon schwierig sein, ganz einfache Fragen vor der ganzen Klasse zu beantworten. Bei dem Projekt erwachsen werden gab es am Anfang Fragen wie etwa: Was be- 
deutet mir Freundschaft? oder Wer bin ich? Fragen, zu denen man sich vielleicht in diesem Alter nicht mit/in der Klasse auseinandersetzen will, aber bei denen es schon gut ist, darüber nachzudenken. Und als ich sie nach der Einzelarbeit gefragt habe, war die Rückmeldung, dass sie es schön gefunden haben, sich zu solchen Fragen etwas zu überlegen, ohne es mit den Anderen zu besprechen.

Sie mussten die Fragen und Antworten aber schon aufschreiben, weil das Verschriftlichen, glaub ich, etwas macht.

Marion Thuswald: Seid ihr auch mit intimen Fragen von Schüler:innen an euch konfrontiert? Welche Fragen sind das, wie geht ihr damit um?

Marlies Brommer: Ja, solche Fragen kommen vor allem von Jüngeren. Sie fragen alles Mögliche: Ob ich schon Sex gehabt habe? Mit wem? Wann? Wie? Da sag ich dann einfach: "Das möchte ich jetzt nicht beantworten. « Ich erkläre, dass ich es zu privat finde oder sage dann auch manchmal, warum ich es nicht sinnvoll finde, wenn ich die Frage beantworte. Manchmal frage ich sie auch, ob sie sicher sind, dass sie von meinen Antworten so viel profitieren würden. Sie antworten dann manchmal: »Ich wollt's nur probieren, ob Sie was drauf sagen.«

Tobias Dörler: Wenn es um eine Positionierung geht, also um meine Einschätzung zu einem intimen Thema, dann habe ich manchmal im Nachhinein bereut, dass ich ein Gespräch abgebrochen habe. Sie reden dann untereinander weiter und ich denke mir dann, dass es doch wichtig gewesen wäre, etwas dazu zu sagen. Es geht ja nicht darum, persönlich zu antworten, sondern Themen können auch allgemeiner besprochen werden.

Marlies Brommer: Das finde ich auch. Wenn sie mich fragen; "Wann haben Sie das erste Mal Sex gehabt? «, dann spreche ich mit ihnen darüber, was >das richtige Alter bedeutet und warum es den Schüler:innen wichtig ist, wann ich oder andere Sex hatten. Das ist als Antwort ausreichend. Es geht ja nicht unbedingt um mich.

Caro Estrada-Steiger: Ich glaube, es ist auch ein Unterschied, in welcher Situation man solche Sachen gefragt wird, ob die ganze Klasse zuhört oder ob das ein Gespräch ist, bei dem ich vor einer kleinen Gruppe von Schüler:innen gefragt werde. Also ich versuche schon, möglichst offen und authentisch 
mit solchen Fragen umzugehen und diese auch zu beantworten. Auch wenn Fragen vielleicht provokant gemeint waren und ich den Verdacht habe, dass sie sich gar keine Antwort darauf erwartet hätten. Manchmal ist es auch so, dass sie dann überrascht sind von dieser ehrlichen und direkten Antwort und ich dadurch dann die Chance habe, das Blatt zu wenden von einer provokanten Frage hin zu einer ernsthaften Auseinandersetzung mit dem Thema. Jedenfalls finde ich es wichtig, auf solche Fragen möglichst authentisch zu reagieren. Wenn mir eine Frage wirklich zu weit geht, dann sage ich das auch einfach. Das ist in dem Fall dann meine authentische Antwort darauf.

Marlies Brommer: Manchmal braucht es das. Vielleicht geht es nicht nur um die Fragen, sondern auch um die Klärung der Grenze. Die richtige Distanz zu den Schüler:innen - das finde ich schon wichtig.

\section{Anziehung und Verlieben zwischen Schüler:innen und Lehrer:innen}

Marion Thuswald: Du spricht schon Themen an, über die ich auch gerne sprechen würde: Grenzen in der pädagogischen Beziehung und Anziehung zwischen Schüler:innen und Lehrer:innen und Verliebtsein von Schüler:innen in Lehrpersonen. Das sind Herausforderungen, die in der Schule präsent sind, und mit denen ein professioneller Umgang gefunden werden muss.

Tobias Dörler: Ja, Grenzen zu ziehen ist wichtig. Wenn Lehrer:innen beispielsweise in ihrer Beziehung labil sind, dann können sie sehr offen dafür sein, dass Schüler:innen private Dinge fragen. Wenn von Schüler:innen positives Feedback auf Persönliches kommt, werden Grenzen vielleicht schneller überschritten. Also nicht wirklich krasse Grenzen, aber der Eindruck entsteht, dass das zu weit geht und die professionelle Ebene abhandenkommt, wenn etwa Schüler:innen Lehrer:innen bei ihren Beziehungsproblemen beraten und sie bestätigen.

Manche Schüler:innen finden es absurd, wenn Lehrer:innen so agieren, andere spielen bis zu einem gewissen Grad mit, oder sie fühlen sich verpflichtet, den Lehrer:innen Anerkennung zu zeigen. Für manche Schüler:innen erscheint es spannend, wenn man der Lehrperson diese Bestätigung geben kann, an der sie sich anhalten kann - und man sie umgekehrt auch fallen lassen kann. So ein Spiel können Schüler:innen auch spielen. Das passiert nicht auf sexueller Ebene, sondern es geht darum, Zuwendung zu geben und 
wieder zu entziehen. Es ist absurd, wenn Lehrer:innen sich darauf einlassen, das sollte nicht vorkommen. Denn wer emotional von der Zuwendung von Schüler:innen abhängig ist, kann von ihnen auch verletzt werden. Und sei es etwa auch nur, dass Schüler:innen sich aus dieser Rolle - Lehrer:innen privat Anerkennung oder Zuwendung zu geben - wieder auf eine professionelle Schüler:innen-Rolle zurück begeben wollen. Das allein kann bei Lehrer:innen in dieser anfälligen Situation schon eine Verletzung hervorrufen.

Und was dann auch schon Thema geworden ist, sind Lehrpersonen, die eine Liebesbeziehung mit einer sehr jungen erwachsenen Person haben, die also ähnlich alt ist wie die Schüler:innen. Es beschäftigt die Schüler:innen, wenn ihr Alter mit dem von Lehrpersonen oder deren Beziehungspartner:innen überlappt. Da wollen einzelne Schüler:innen sehr direkt Liebesbeziehungsdetails erfragen. Auch wenn sie selber eine Beziehung zu einer viel älteren Person haben, wird das zum Thema und sie wollen von mir wissen, wie ich mich dazu positioniere.

Marlies Brommer: Ich kenne das auch, dass Schüler:innen in Lehrer:innen verliebt sind. Ich habe das auch mitbekommen bei Kolleginnen, die Liebesbriefe von Schülern bekommen. Da muss man immer wieder Grenzen ziehen und den Schüler:innen klarmachen, dass man als Lehrerin nicht Teil ihrer Gruppe ist, weder vom Alter her noch von der Rolle. Man muss klar kommunizieren ohne sie zu beleidigen. Manchmal können sie noch gar nicht wirklich einordnen, welches Gefühl das ist, das sie empfinden, ob das jetzt Liebe ist oder einfach Wertschätzung für eine Person. Bei den Jüngeren, die z.B. etwas für mich zeichnen, ist es vielleicht Bewunderung oder einfach Zuneigung, vielleicht auch Verliebtsein. Bei den Größeren ist das manchmal ein Spaß, dass sie mir flirtend begegnen. Ich habe schon erlebt, dass sie mich angetanzt haben. Ich spüre da keine Anziehung und finde das dann eher komisch.

Marion Thuswald: Gibt es eine Kommunikation unter Kolleg:innen über diese Erfahrungen?

Marlies Brommer: Es gibt schon Kolleg:innen, die fragen, wie sie tun sollen. Ich habe das Gefühl, es kommt bei jüngeren Kolleginnen öfter vor. Die sind vielleicht noch mehr Projektionsfläche. Männliche Kollegen haben teilweise Angst und wissen nicht, wie sie sich verhalten sollen, weil ihnen vorgeworfen werden könnte, sie hätten das provoziert. Das kommt mir schwieriger vor als 
vielleicht unter weiblichen Kolleginnen, die das Thema eher humorvoll verhandeln. Für männliche Kollegen ist es schwieriger, wenn Schülerinnen in sie verliebt sind oder Briefe schreiben. Es gibt ja auch Fälle von Übergriffen in Schulen, wo Schülerinnen von Lehrern belästigt werden. Kollegen haben Angst, dass - wenn Schülerinnen ihnen Liebesbriefe schreiben -, die Situation quasi umgedreht wird und ihnen Übergriffigkeit vorgeworfen wird.

Caro Estrada-Steiger: Mir ist mal etwas sehr Unangenehmes passiert. Als ich anfangs noch Nachmittagsbetreuung gemacht habe, hat ein Junge bei der Weihnachtsfeier Gitarre gespielt. Das war der Sohn einer Frau, die auch Betreuung an der Schule gemacht hat. Als ich mich für seinen Einsatz bedankt habe, habe ich auch zu ihm gesagt, dass er ganz toll Gitarre spielt. Daraufhin hat er sich laut seiner Mutter leider in mich verliebt. Sie hat es mir erzählt und das sehr lustig gefunden. Ich war jedoch ziemlich schockiert, es war eine skurrile und beschämende Situation für mich. Als es sich dann mal ergeben hat, habe ich mit ihm geredet und erklärt, dass ich um vieles älter und außerdem glücklich verheiratet und seine Lehrerin bin. Ich wollte ihn nicht verletzen oder traurig machen, aber es war mir sehr wichtig, dass er diese Grenze versteht.

Tobias Dörler: Es ist immer auch die Frage, wie viel Anerkennung man welchen Schüler:innen zukommen lässt und wann sie plötzlich auf diese Anerkennung extrem ansprechen, wie in deinem Beispiel. Es gibt Situationen, in denen man merkt, dass Schüler:innen ein großes Bedürfnis nach Anerkennung haben. Sie ordnen diese dann aber anders ein, als sie gemeint ist. Sie nehmen sie nicht als Anerkennung, die man von einer Lehrperson bekommt, sondern es spricht sie auf einer anderen Ebene an.

\section{Unterwegs zu sexualpädagogischer Professionalisierung ...}

Marion Thuswald: Ihr habt die professionellen Rollen thematisiert. Was wäre eurer Einschätzung nach sinnvoll, um angehende Lehrpersonen gut auf solche Herausforderungen vorzubereiten?

Tobias Dörler: Aus meiner Erfahrung heraus scheinen mir rechtliche Fragen und Fragen der professionellen Zusammenarbeit wichtig, die im Studium kaum vorkommen: An wen kann ich mich wenden, im Voraus oder während 
der Umsetzung eines sexualpädagogischen Unterrichtskonzeptes, etwa um die Involvierung der Eltern oder von den Schüler:innen thematisierte Übergriffigkeiten zu besprechen? Wo bin ich selber zu stark gefordert und brauche andere Personen, die mich unterstützen usw.?

Marlies Brommer: Das Beziehungsgeflecht in der Schule ist einfach kompliziert: Schüler:innen, Kolleg:innen, Eltern, Direktion - man hat so viele unterschiedliche Beziehungen.

Tobias Dörler: Manche Themen bräuchte es dann in der Fortbildung oder Supervision und nicht während des Studiums.

Marlies Brommer: Es muss nicht alles im Studium vorkommen, ja, das stimmt. Viele Themen sind erst in der Praxis interessant. Wen interessiert im dritten Semester des Studiums so ein Thema wie Elternarbeit?

Tobias Dörler: Ich finde es wichtig, Rahmenbedingungen im Studium mitzubekommen, weil ich glaube, dass viele Lehrer:innen das Thema Sexualpädagogik abblocken - aus Angst irgendwelche Grenze zu verletzen. Beispielsweise ein Thema zu behandeln, wo die Eltern hätten einbezogen werden sollen und es das Risiko gibt, dass sich Eltern beschweren. Je mehr mir im Studium die Rahmenbedingungen klar werden, desto mehr kann ich das dann später auch aktiv thematisieren.

Marion Thuswald: Vielleicht ist es entlastend, sich klar zu machen, dass wir in der Schule nicht nicht-sexualerzieherisch agieren können. Wenn wir sexualpädagogische Themen negieren oder verweigern - was ohnehin schwer möglich ist -, dann ist das auch eine sexualerzieherische Botschaft an die Schüler:innen. Und wir verweigern ihnen damit möglicherweise auch Unterstützung und wichtige Lern- und Bildungsprozesse.

Marlies Brommer: Es gibt Eltern, die ihre Kinder zu Hause lassen, wenn sexuelle Themen behandelt werden, das finde ich schwierig. Ich halte das für eine Frage der Kommunikation. Es muss erklärt werden, dass dies ein Lehrinhalt ist, genauso wie die binomische Formel, und es eine Teilnahmeverpflichtung für alle gibt. Es ist besser, dass wir uns als Lehrpersonen mit den Eltern konfrontieren, als dass die Kinder dann plötzlich nicht kommen. 
Tobias Dörler: Manche Schüler:innen sind auch abgeschreckt, weil sie meinen, dass das Thema Konfliktpotenzial mit den Eltern darstellen könnte.

Marlies Brommer: Ich habe den Eindruck, viele Eltern können sich auch nicht vorstellen, was Sexualpädagogik bedeutet. Viele denken, es geht um Sexstellungen. Sie wissen nicht, dass wir eigentlich ganz viel über Gefühle sprechen, über Wollen und Nicht-Wollen und dass wir Präventionsarbeit machen, um Übergriffe zu verhindern.

Marion Thuswald: Was Eltern oft gut verstehen, ist der Schutz der eigenen Kinder, also der Präventionsgedanke: Wenn Sexualität insgesamt von einem Schleier des Gefährlichen oder Verbotenen umgeben wird, dann ist es schwer für die Schüler:innen, ein Gefühl für ihre Grenzen zu entwickeln und zwischen Einvernehmlichkeit und Übergriffen zu unterscheiden. Wenn sie keinen positiven Bezug zu Sexualität haben, dann können sie schwerer klar spüren, was übergriffig ist. Deshalb braucht es eigentlich auch aus dem Präventionsgedanken heraus - und der ist ja den meisten Eltern zugänglich - eine Sprache für Körperteile, für Genitalien, für Empfindungen und für sexuelle Handlungen. Wenn das Thema Sexualität insgesamt von sehr viel Scham umgeben ist, ist es viel schwieriger, Übergriffe zu benennen.

Caro Estrada-Steiger: An unserer Schule gab es einen Schüler aus der 5. Klasse [9. Schulstufe], der eine Ausbildung bei Jugend gegen Aids gemacht hat und Peer-to-Peer-Workshops an unserer Schule anbieten wollte. Anfangs war man seitens der Schulleitung von dem Projekt sehr begeistert, dann machte sich jedoch große Verunsicherung breit und diese Workshops wurden an unserer Schule vorerst nicht genehmigt. Der Schüler begann daraufhin mit Schüler:innen anderer Schulen Workshops in mehreren anderen Schulen zu halten. Es hat zwei Jahre gedauert, bis der Schüler einen dieser Peer-to-Peer Workshops bei uns halten durfte. Das Konzept dieser Workshops sah vor, dass die Schüler:innen zwar bis zu einem gewissen Grad beaufsichtigt wären, die Lehrperson sich jedoch phasenweise in einen Nebenraum zurückziehen würde, sodass die Schüler:innen ungestört miteinander diskutieren und Fragen stellen könnten. Das wurde nicht gerne gesehen. Es gab diese Angst, die Schüler:innen könnten miteinander über ein heikles Thema sprechen, ohne dass sie dabei von einer Lehrperson beaufsichtigt wären. Die Befürchtung 
war, glaube ich, tatsächlich, dass die Schüler:innen miteinander über Sex reden, noch dazu im Schulgebäude, ohne Beaufsichtigung.

Marion Thuswald: Die Schüler:innen reden ja sowieso miteinander.

Marlies Brommer: Im besten Fall reden sie drüber! 


\title{
Diskriminierungsreflektierte Sexualpädagogik
}

\author{
Katharina Debus
}

Körper, Sexualität, Intimität, Partnerschaft, Familie und Fortpflanzung sind wichtige Themen in der Pädagogik - als explizite Lerngegenstände, Fragen oder implizite Unterströmungen. Sie sind bedeutsam für die Förderung der persönlichen Entwicklung, für Teilhabe und die psychische sowie physische Gesundheit der Adressat:innen. Darüber hinaus sind sie zentral für Gewaltund Diskriminierungsprävention. Wenn in diesem Artikel von Sexualpädagogik geschrieben wird, dann ist jegliche Form der pädagogischen Auseinandersetzung mit diesen Themen, insbesondere mit dem Thema Sexualität, gemeint. $^{1}$

Sexualpädagogik kann entweder diskriminierende Normalitätsannahmen reproduzieren oder inklusiv zur Akzeptanz von Vielfalt und zum Abbau von Diskriminierung beitragen. Jenseits von vorsätzlicher Diskriminierung ${ }^{2}$ kommt es vor dem Hintergrund der gesellschaftlichen Allgegenwärtigkeit von Diskriminierung sowie begrenzter Zeit, Materialien, Qualifikation und Reflexionsräume auch bei inklusiven Absichten zu diskriminierenden Bewertungen, Ausblendungen und Schwerpunktsetzungen. Dieser Artikel will für Diskriminierungsrisiken in der Sexualpädagogik sensibilisieren und Orientierungspunkte für einen diskriminierungsreflektierten Umgang vorstellen.

Zunächst gehe ich auf Diskriminierungsrisiken in der Sexualpädagogik ein (1). Im Anschluss skizziere ich verschiedene Diskriminierungsverhältnisse in ihrer Relevanz für Sexualpädagogik (2) und beschäftige mich dann ergänzend mit den Bedarfen sexuell traumatisierter Teilnehmender (3). Abschlie-

1 Dieser Artikel ist weiterentwickelt aus Debus 2017 .

2 Exemplarisch für eine Sexualpädagogik, die bewusst bestimmte Lebensweisen diskriminiert, vgl. die Berichte über den Verein TeenStar: Tóth 2018; 2019; queer.de 2018. Zu vielfaltsfeindlichen Angriffen auf Sexualpädagogik vgl. u.a. Voß 2014; Hark/Villa 2015; Tuider/Timmermanns 2015; Tuider 2016; Laumann/Debus 2018. 
ßend diskutiere ich verschiedene Spannungsfelder diskriminierungsreflektierten sexualpädagogischen Handelns (4).

\section{Diskriminierungsreflektierte Sexualpädagogik}

Laut den WHO-Standards für Sexualaufklärung in Europa haben »[a]lle Kinder und Jugendlichen [...] das Recht auf Zugang zu altersgerechter Sexualaufklärung« u.a. in Form

- (altersgerechter) Vermittlung von »Informationen, Fähigkeiten und positiven Werten«

- der Befähigung, »ihre Sexualität zu verstehen und zu genießen, sichere und erfüllende Beziehungen einzugehen sowie verantwortlich mit ihrer eigenen sexuellen Gesundheit und der ihres Partners umzugehen«

- der Befähigung zu Entscheidungen, »die zu einer von Mitgefühl und Gerechtigkeit geprägten Gesellschaft beitragen« (WHO-Regionalbüro für Europa/BZgA 2011: 22)

Aufbauend auf diesen Rechten schlage ich in Anlehnung an Doris Liebscher und Heike Fritzsche (2010: 27ff.) folgende Definition von Diskriminierung in der Sexualpädagogik vor:

Unmittelbare Diskriminierung liegt vor, wenn in der Sexualpädagogik bestimmte Identitäten, Zugehörigkeiten, Körper, Lebensweisen, Neigungen oder Erfahrungen abgewertet bzw. tabuisiert werden. Darunter fallen auch Einschränkungen der Teilhabe, wenn (zu befürchtende) Abwertungen, Belästigungen, Drohungen und/oder Grenzüberschreitungen durch Mitlernende, Pädagog:innen etc. die Möglichkeit der aktiven Teilhabe bestimmter Adressat:innen einschränken.

Mittelbare Diskriminierung liegt vor, wenn ohne direkte Abwertung, sondern beispielsweise durch das Vorenthalten von Informationen und Auseinandersetzungsangeboten bestimmte Adressat:innengruppen nicht ausreichend in den genannten Fähigkeiten gefördert werden.

Othering-Praktiken ${ }^{3}$ verbesondern bestimmte Identitäten, Lebensweisen, Neigungen oder Erfahrungen, stellen sie als fremd oder andersartig dar 
und gliedern sie damit aus der snormalen« Sexualpädagogik aus, z.B. durch Peinlichkeits- und Distanzierungssignale in Wortwahl, Stimme oder Körpersprache oder durch Verlagerung in exotisierende oder problematisierende Extra-Einheiten (z.B. unter dem Stichwort >Minderheiten). Letzteres ist zumindest ambivalent: Solche Einheiten können unter Umständen ein geringeres Übel gegenüber mittelbarer Diskriminierung durch Verschweigen oder Tabuisierung darstellen. Gleichzeitig hat Othering immer eine direkt diskriminierende Wirkung, da dadurch bestimmte Gruppen aus der Normalität ausgegliedert werden.

In einer auf Selbstbestimmung und Grenzachtung ausgerichteten Sexualpädagogik gilt dabei der Gleichbehandlungsanspruch ausschließlich für Lebensweisen, Neigungen und Handlungen, die die Selbstbestimmung anderer Menschen respektieren. Übergriffige und gewalttätige Handlungen bzw. sexuelle Handlungen an nicht einwilligungsfähigen Menschen (z.B. von Erwachsenen an Kindern vor dem Hintergrund des Machtverhältnisses zwischen den Generationen und der unterschiedlichen psychosozialen Entwicklung) sollten problematisiert, als verboten benannt und unterbunden werden.

Für die Frage, ob Sexualpädagogik diskriminierend ist, ist der Effekt entsprechend der oben genannten Kriterien relevant. Hierfür sind nicht immer die einzelnen Pädagog:innen verantwortlich, sondern auch strukturelle Faktoren wie eingeschränkte Verfügbarkeit von Materialien, mangelnder Zugang zu Qualifizierung, Zeitknappheit oder diskriminierende rechtliche Vorgaben.

Die Intention der Pädagog:innen ist nicht entscheidend für die Beurteilung, ob eine Handlung, Methode oder ein Konzept diskriminierend wirkt. Sie ist dennoch wichtig, da Pädagog:innen, denen inklusives und nicht-diskriminierendes Handeln wichtig ist, bereit sind, ihr Vorgehen kritisch zu reflektieren und sich um eine Erweiterung ihrer Handlungsspielräume zu bemühen. 
Der Begriff diskriminierungsreflektierte Sexualpädagogik ${ }^{4}$ beschreibt in diesem Sinne eine Pädagogik, die sich darum bemüht, möglichst inklusiv zu handeln.

\section{Dimensionen von Diskriminierung}

In allen mir bekannten Gesellschaften finden wir verschiedene große Achsen der Diskriminierung vor, also der Ungleichbehandlung verschiedener Menschengruppen bezüglich ihres Zugangs zu Ressourcen, Bildung, Teilhabe und gesellschaftlicher Anerkennung. Ich greife in den folgenden Abschnitten einige dieser Achsen heraus ${ }^{5}$ und gebe Einblicke in ihre sexualpädagogische

Der Begriff diskriminierungsreflektierte Sexualpädagogik soll keine neue sSchule der Sexualpädagogik begründen. Er ist beschreibend gemeint und soll als sensibilisierende Analysefolie und zur Motivation dienen. Er lässt sich mit anderen diskriminierungskritischen und inklusiven Ansätzen verbinden (z.B. Timmermanns 2016).

Ich habe verschiedene Begriffe abgewogen: In Debus 2017a schreibe ich von snichtdiskriminierender Sexualpädagogik‘. Der Begriff war als Handlungsziel gemeint, verspricht allerdings zu viel, da es in einer von Diskriminierung durchzogenen Cesellschaft kaum möglich ist, diskriminierende Situationen zu verhindern (exemplarisch für Rassismus: Terkessidis 2012, https://www.youtube.com/watch?v=EK4ZKnmBf UI [Zugriff: 11.04.2020]). Der Begriff `diskriminierungssensibel< ist m. E. zu sehr auf das Innenleben der Pädagog:innen fokussiert anstatt auf ihr Handeln. Der Begriff `diskriminierungskritisch< beschreibt m.E. eine Pädagogik, die Diskriminierung explizit zum zentralen Thema macht - dies ist sehr sinnvoll und überschneidet sich, ist aber nicht deckungsgleich mit dem hier verwendeten Begriff.

Der Begriff `diskriminierungsreflektierte Sexualpädagogik« soll analog zur geschlechterreflektierten Pädagogik (Debus 2012; 2017b) dazu anregen, pädagogische Vorgehensweisen nach ihrem Potenzial auszuwählen, inklusiv die Entwicklungsmöglichkeiten und Selbstbestimmung der Adressat:innen zu fördern. Oft heißt das, in diskriminierungsreflektierter Weise an übergreifenden Themen wie Körper, Sexualität, Verlieben, Begehren, Fortpflanzung, Risikobewusstsein, Partner:innenschaft, Grenzen, Kommunikation etc. zu arbeiten, ohne immer explizit über Diskriminierung zu sprechen. Zusätzlich wägen die Pädagog:innen ab, wann und in welcher Weise eine explizite Beschäftigung mit Diskriminierung hilfreich bzw. notwendig für die genannten Ziele ist. Das Konzept von Dramatisierung, Entdramatisierung und Nicht-Dramatisierung von Ceschlecht (ebd.) kann hierfür anregend sein und auf andere Themenfelder übertragen werden.

5 Darüber hinaus schränken Bodyismus (online: IDA e.V. o.J.) bzw. Lookismus (online: Schmid u.a. 2017, Leber 2016), also Diskriminierung aufgrund von Körper-/Aussehensnormen, individuelle Cestaltungsspielräume bezüglich Liebe, Partnerschaft, Erotik, 
Relevanz. Einleitend benenne ich einige Aspekte von Diskriminierung, die mehrere Achsen der Ungleichheit betreffen:

Beeinträchtigung des Rechts auf Information: Oft fallen sexualpädagogische Angebote hinter die Bedarfe der Adressat:innen zurück u.a. durch Zeitmangel, Qualifikationslücken oder Kommunikationstabus. Dies gilt verstärkt für die Bedarfe von Angehörigen diskriminierter Gruppen.

Sexuelle Stigmatisierung und Verunsichtbarung von Ressourcen: Diskriminierte Gruppen werden oft mit einem Defizit-Blick betrachtet, d.h. es wird ein Problematisierungsscheinwerferlicht auf sie geworfen ${ }^{6}$ und ihre Ressourcen und Kompetenzen werden unsichtbar gemacht. Einerseits wird dies jenen nicht gerecht, die stigmatisiert werden und deren Ressourcen in den Schatten rücken. Andererseits wird es auch den Mehrheitsangehörigen bzw. Privilegierten nicht gerecht, deren Bedarfe nicht als spezifisch erkannt und bearbeitet werden.

Relevanz diskriminierungsbedingter Belastungen in der Sexualpädagogik: Diskriminierung kann bei Betroffenen zu Belastungen und Traumatisierung führen u.a. durch erlebte oder beobachtete sexualisierte, körperliche oder psychische Gewalt oder akkumulierte Micro-Aggressionen. ${ }^{7}$ Die sexuelle, partnerschaftliche oder familiäre Ebene wird einerseits oft direkt angegriffen, kann aber auch durch Belastungen aus anderen Lebensbereichen beeinträchtigt werden. Zur Förderung der sexuellen Entwicklung sind entsprechend qualifizierte therapeutische und Empowerment-Räume nötig. Aufgabe von Schule und Jugendhilfe kann es sein, solche Angebote anzuregen, bekannt zu machen und gegebenenfalls Finanzierung dafür zu organisieren.

Sexualität, Fortpflanzung und Familie ein. Auch Ageismus, die Diskriminierung alter Menschen, ist relevant (online: Cewirtz-Meydan u.a. 2018, wahnsinnigverflochten o.J.). Die Bedeutung von Antisemitismus und weiteren Diskriminierungsformen für Sexualpädagogik ist noch zu untersuchen.

6 Dieses Bild ist inspiriert von Angela McRobbies (2010) Anwendung des Begriffs der Luminositäten von Deleuze.

7 Zum Thema Microagressionen vgl. Peşmen 2018, Moghimi o.].; Pierce 1970; Sue 2010 sowie die Videoclips Fusion Comedy 2016 (https://www.youtube.com/watch?v=hDd3bz A7450 [Zugriff 09.04.2020]); Niemann 2017 (https://www.youtube.com/watch?v=Zahtl xW2CIQ [Zugriff: 09.04.2020]). 


\subsection{Adultismus}

Der Begriff Adultismus (Ritz o.J.) beschreibt das Machtverhältnis zwischen Erwachsenen und Kindern. In den Debatten um >Frühsexualisierung (kritisch dazu Diskursatlas Antifeminismus o.J.; Tuider 2016) wird Kindern ihr Recht auf Information über Sexualität abgesprochen und dem Erziehungsrecht der Eltern untergeordnet. Kinder werden zudem zu Projektionsflächen für Unsicherheiten von Erwachsenen. Für viele Erwachsene ist es schwer vorstellbar, dass Kinder gelassen, kompetent oder interessiert mit etwas umgehen könnten, das sie selbst beschämt. Darüber hinaus ist es für sie aufgrund von Souveränitäts- und Kompetenzanforderungen an Erwachsene leichter, eigene Verunsicherungen auf Kinder oder Jugendliche zu projizieren, anstatt Informationsbedarfe oder Schamgefühle zuzugeben.

Dabei bewegen wir uns in einem Spannungsfeld: Die Einschränkung des Informations- und Selbstbestimmungsrechts von Kindern und Jugendlichen ist diskriminierend. Gleichzeitig wurde die einfache Gleichsetzung von Kindern mit Erwachsenen unter Ausblendung von Macht-, Verantwortungs- und Entwicklungsunterschieden von pädosexuellen Täter(:inne)n genutzt, um sexualisierte Gewalt an Kindern zu legitimieren. Darüber hinaus ist es im pädagogischen Machtverhältnis besonders wichtig, Schamgrenzen zu respektieren. Es muss also darum gehen, das Interesse von Kindern und Jugendlichen an Sexualität anzuerkennen und bedarfsgerecht und grenzachtend zu bearbeiten.

Dies kann in heterogenen Gruppen eine Herausforderung darstellen. Ein möglicher Zugang sind anonyme Interessenabfragen und bedarfsgerechte Binnendifferenzierung.

\section{Individuelle Grenzregulierung per Kopfhörer}

Idealerweise beginnen Pädagog:innen in der Sexualpädagogik mit einem oder mehreren Impulsen zum Einstieg ins Thema und zum Abbau von Kommunikationshemmungen. Danach sammeln sie anonym Fragen und Interessen sowie Grenzen der Teilnehmenden. ${ }^{8}$ Aus den Rückmeldungen entwickeln sie eine gruppenspezifische Kombination aus einem Pflichtteil mit BasisWissen für alle und frei wählbaren Vertiefungsthemen.

Oft erlauben die Ressourcen ein solches Vorgehen nicht. Es kann passieren, dass manche Teilnehmende Fragen haben, deren Bearbeitung die Schamgrenzen anderer Teilnehmender verletzen könnte. Daher sollte in Einheiten 
wie der Fragenkiste grundsätzlich das Freiwilligkeitsprinzip gelten. Oft lassen Sexualpädagog:innen darüber hinaus einen höheren Geräuschpegel zu, da Lachen und Herumalbern entlastend wirken und zur akustischen Distanzierung genutzt werden können.

Seit einiger Zeit gebe ich Teilnehmenden zusätzlich die Möglichkeit, in dieser Einheit Kopfhörer zu tragen und Musik zu hören. Dies erlaubt einerseits eine bessere akustische Distanzierung und gleichzeitig die Möglichkeit, unbemerkt aufmerksam zuzuhören.

\subsection{Ableismus}

Der Begriff Ableismus (vgl. Arnade 2016; Maskos 2011) beschreibt die Hierarchisierung zwischen Menschen, die als gesund gelten, und Menschen mit Behinderungen bzw. gesundheitlichen Einschränkungen. Dabei werden Behinderungen nicht einfach als etwas natürlich Gegebenes betrachtet, sondern der Blick wird darauf gelenkt, wie bestimmte Menschengruppen in unseren Gesellschaften behindert werden. Durch Ideale körperlicher, kognitiver und psychischer Leistungs- und Funktionsfähigkeit geraten alle Menschen unter Druck - Menschen mit Behinderungen erfahren darüber hinaus massive Diskriminierung.

Viele Menschen, die behindert werden, erleben Einschränkungen selbstbestimmter Sexualität und Familienplanung. Oft wird ihnen zudem wenig Privatsphäre zur Selbsterkundung zugestanden. Nicht zuletzt sind Menschen mit Behinderungen in höherem Maße sexualisierter Gewalt ausgesetzt.

In der außerschulischen Sexualpädagogik wird dieses Thema seit Jahrzehnten bearbeitet. Es existiert ein relativ breites Angebot an Materialien und Artikeln zur sexualpädagogischen Arbeit mit Menschen mit (verschiedenen) Behinderungen. Einige Autor:innen diskutieren auch Diskriminierung entlang sexualisierter Gewalt oder der Behinderung sexueller und reproduktiver Selbstbestimmung (z.B. Specht 2013).

Eine diskriminierungsreflektierte Sexualpädagogik mit Menschen, die behindert werden, sollte diese auch in ihren Ressourcen stärken, sich gegen Einschränkungen ihres Selbstbestimmungsrechts $\mathrm{zu}$ wehren und sie im dissens.de/materialien/methoden [Zugriff: 09.04.2020]. Methode Stop \& Go zur Abfrage von Grenzen und Bedürfnissen auf Nachfrage: kd@katharina-debus.de. 
Umgang mit den Barrieren stärken, auf die sie bei der Anbahnung von Sexualität, Partnerschaft und Familie stoßen. Darüber hinaus besteht großes Potenzial darin, in der Arbeit mit allen Schüler:innen zu berücksichtigen und thematisieren, wie Ideale von Gesundheit und Leistungsfähigkeit die Sexualität aller Menschen beeinträchtigen.

\subsection{Rassismus}

Rassistische ${ }^{9}$ Logiken unterteilen Menschen in natio-ethno-kulturell-religiös ${ }^{10}$ konstruierte Gruppen. Dabei wird zwischen der seigenen $<$ Gruppe und Menschen unterschieden, die $\mathrm{zu}>$ Anderen $<$ bzw. >Fremden $\diamond$ gemacht werden (Othering). Eine Person gehört demzufolge immer nur einer dieser beiden Gruppen an. Der Gruppe der >Anderen sprochen - Mehrfachzugehörigkeit, Hybridität und Transnationalität werden ebenso ignoriert wie kontroverse Debatten innerhalb der als sanders< konstruierten Kulturen. Diese Gruppen werden hierarchisiert: Zumindest am Ort des Geschehens (hier: Deutschland, Österreich oder die Schweiz) werden der Gruppe der >Eigenen $\ll$ mehr Wert bzw. mehr Rechte zugesprochen. Dabei wird aktuell seltener auf genetische Unterschiede verwiesen, sondern oft mit einem verengten Kulturbegriff operiert. Kultur ist dann ein (zumindest bei den >Anderen $\prec$ homogenes statisches Gebilde und bestimmte Kulturen scheinen nicht $\mathrm{zu}>$ uns $<\mathrm{zu}$ passen, werden als minderwertig, rückständig oder bedrohlich konstruiert (vgl. zu Kulturkonzepten Sarma 2012; zu Kulturalisierung Kalpaka 2005; 2011). Damit wird eine Entrechtung, Diskriminierung und oft auch Belehrung der jeweils als sanders` Konstruierten gerechtfertigt.

Sexualpädagogik greift oft auf kulturalisierende Bilder zurück (zur Kritik daran vgl. Auma 2020; Timmermanns 2013) und reproduziert einen solchen verengten Kulturbegriff. So nennt beispielsweise Uwe Sielert zwar die Vielfalt der Einstellungen von Jugendlichen mit Migrationsgeschichte zu Sexualität. Diese führt er jedoch auf unterschiedliche Grade der Integration zurück, wobei er individualistische und gleichstellungsorientierte Positionen der »deutschen Hauptkultur« zuschreibt (Sielert 2015: 131f.).

Entsprechend liegt der Fokus in sexualpädagogischen Veröffentlichungen und Diskussionen zu Bedarfen von Jugendlichen >mit Migrationshinter-

9 Einführend zu Rassismus vgl. etwa Mecheril u.a. 2010.

10 Diese Formulierung verwende ich in Anlehnung an Paul Mecherils Begriff der natioethno-kulturellen Zugehörigkeit. 
grund oft auf Themen wie >Jungfräulichkeit</>Jungfernhäutchen</Scheidenkranz, Scham, Analsex als Einstiegssexualität zum Schutz des >Jungfernhäutchens $\triangleleft,{ }^{11}$ Beschneidungen, Ehrkultur, Geschlechterkonservatismus und leichter Sprache.

All dies können wichtige Themen sein, auch für mehrheitsangehörige Jugendliche. Problematisch wird es zum einen, wenn alle Jugendlichen mit bestimmten Migrationsgeschichten versämtlicht werden und Heterogenität sowie Individualität u.a. in der Werteorientierung und Lebensgestaltung innerhalb jeder Nation, Ethnie oder Religion unsichtbar werden. Zudem werden so besondere Herausforderungen der Entwicklung von Sexualität in z.B. mehrheitsdeutschen protestantisch geprägten bürgerlich-konservativen Milieus oder völkischen Siedlungsgemeinschaften ebenso vernachlässigt wie die Ressourcen vieler Menschen mit verschiedenen Migrationsgeschichten und Rassismuserfahrungen. ${ }^{12}$

Im Sinne eines erweiterten Kulturbegriffs ${ }^{13}$ ist immer davon auszugehen, dass jede (auch nationale) Gruppe kulturell heterogen ist und kulturelle Normen immer dynamisch und umstritten sind. Für adressat:innengerechte Pädagogik ist es daher hilfreich, eigene Bilder und Materialien kritisch zu reflektieren. Nicht zuletzt haben viele rassismuserfahrene ${ }^{14}$ Kinder und Jugendliche Empowerment-Bedarfe, da sie ihren Zugang zu ihren Körpern, ihrer Sexualität und Partnerschaften unter rassistischen Bedingungen entwickeln müssen. ${ }^{15}$ Um allen Adressat:innen die Teilhabe an sexualpädagogischen Angeboten zu ermöglichen, sollten darüber hinaus diskriminierende Aussagen konsequent problematisiert werden.

Die Relevanz für fundamentalistisch christlich aufwachsende Jugendliche wird selten thematisiert.

Das SEEDS-Collective arbeitet daran, »die sexualpädagogische Expertise aus Schwarzen und POC-Communitys aus der Unsichtbarkeit [zu] holen und [zu] bündeln«, vgl. Ceneration Adefra u.a. o.].

Ein erweiterter Kulturbegriff versteht Kultur als grundsätzlich dynamisch, kontrovers und hybrid und nimmt verschiedene Cruppenkulturen in den Blick, die in einem Individuum zusammenlaufen und vom Individuum je unterschiedlich angeeignet bzw. ver-/bearbeitet werden, u.a. ethnische, religiöse, nationale, regionale, institutionelle, politische, Milieu-, Familien-, Peer- oder Ceschlechter-Kultur. Wie diese in einem Menschen zusammenlaufen, kann nicht von außen diagnostiziert werden. Es empfiehlt sich eine Haltung interessierten Nicht-Wissens statt der Suche nach Rezepten. Ich verdanke diesen Begriff Maisha-Maureen Auma.

15 Zu Rassismus im Dating: Ku/Baden 2018; Rautenberg 2019. Zur rassistischen Konstruktion bedrohlicher Männlichkeit IDA-NRW 2016. 


\subsection{Klassismus}

Klassistische ${ }^{16}$ Logiken diskriminieren Menschen aus Milieus mit weniger Zugang zu Geld, sozialem Status und formaler Bildung. So haben z.B. Menschen aus bildungsbürgerlichen Familien wesentlich bessere Chancen auf schulischen Erfolg als Menschen aus prekarisierten oder proletarischen $\mathrm{Mi}$ lieus. Kulturelle Aspekte, die mit Letzteren in Verbindung gebracht werden, werden oft problematisiert, während bildungsbürgerliche Kultur normalisiert oder idealisiert wird. Probleme klassistisch diskriminierter Menschen werden oft als individuelles Versagen personalisiert, während kapitalistische und institutionelle Strukturen, die diese Probleme hervorbringen, ausgeblendet werden.

Auch die Sexualpädagogik wirft in Bezug auf Milieu bzw. Klasse oft einen Problematisierungsscheinwerfer auf Jugendliche aus proletarischen bzw. prekarisierten Milieus (exemplarisch: Sielert 2015: 113f., 126) u.a. bezüglich Geldmangel, (Geschlechter-)Konservatismus oder Teenager-Schwangerschaften. Diese Themen sind potenziell für alle Jugendlichen wichtig, sollten aber nicht versämtlichend auf Jugendliche aus proletarischen bzw. prekarisierten Milieus projiziert werden.

Spuren von Ressourcen sexueller Selbstbestimmung dieser Jugendlichen ${ }^{17}$ werden dagegen ebenso selten verfolgt wie spezifische Schwierigkeiten bürgerlicher Sexualkultur(en), denen meist mehr Progressivität im Vergleich zu weniger formal gebildeten Menschen zugeschrieben wird (z.B. Sielert 2015: 84, 127, 134). Dabei wird die Tatsache missachtet, dass die heute als traditionell geltenden Geschlechterbilder historisch eine bürgerliche Erfindung waren (Maihofer 1995: 98-108) und heute proletarische und prekarisierte Milieus eine große Bandbreite an Lebensweisen aufweisen. Ohne reale Schwierigkeiten eines begrenzten Zugangs zu Ressourcen $\mathrm{zu}$ bestreiten, verspricht eine klassismuskritische Analyse sexualpädagogischer Theoriebildung und Praxis Weiterentwicklungspotenzial (vgl. dazu Rédai i.d.B.). ${ }^{18}$

16 Einführend: Kemper/Weinbach 2009.

17 Z.B. referiert Böhm (2013: 308), dass Hauptschülerinnen Solosexualität positiver bewerten als Gymnasiastinnen.

18 Vgl. für Verweise auf die Abwertung von ArbeiterInnensexualität Kemper und Weinbach 2009; für einen persönlichen Bericht über klassistische Verletzungen und institutionelle Gewalt im Kontext von Sexualität Clara Rosa 2012. 


\subsection{Diskriminierung im Kontext von Geschlechterverhältnissen und sexueller Vielfalt}

Geschlechterverhältnisse sowie amouröse und sexuelle Vielfalt sind zentrale Themen der Sexualpädagogik und oft diskriminierungsrelevant. ${ }^{19} \mathrm{Da}$ ich hierzu einen weiteren Artikel in diesem Band veröffentliche (Debus i.d.B.), soll an dieser Stelle nur ein kurzer Einblick gegeben werden.

Mit dem Begriff Sexismus beschreibe ich die Hierarchisierung von Männern bzw. Jungen über Frauen bzw. Mädchen und berücksichtigt darüber hinaus auch die Benachteiligung von Jungen und Männern, die als >unmännlich beschreiben werden (Debus 2015a). In der Sexualpädagogik zeigt sich dies u.a. in begrenzten Informationen über die Klitoris; in Schlampenbildern, die es Mädchen erschweren, Interesse an Sexualität zu äußern; in sexistischen Kommentaren von Mitschüler:innen; Ekelbekundungen gegenüber verschiedenen Merkmalen und Funktionen weiblich gelesener Körper; der versämtlichenden Annahme, dass Jungen >immer nur das eine< wollten und hormongesteuert seien, sowie in Scham bei Erektionsproblemen.

Der Begriff Endosexismus beschreibt die Diskriminierung intergeschlecht

licher Menschen, also von Menschen mit körperlichen Variationen, die in der biologisch-medizinisch konstruierten Binarität von Männlich oder Weiblich marginalisiert werden. Dies zeigt sich in der Sexualpädagogik u.a., wenn binär von sogenannten Männer- und Frauenkörpern gesprochen wird und andere Varianten körperlicher Entwicklungen gar nicht auftauchen oder stigmatisierend als $>$ Mutationen $<$ oder $>$ Zwitter $<$ besprochen werden.

Der Begriff Cis-Sexismus beschreibt die Diskriminierung transgeschlechtlicher Menschen, also von Menschen, deren geschlechtliches Selbstverständnis nicht der Geschlechtszuweisung bei der Geburt entspricht. In der Sexualpädagogik wird dies u.a. relevant, wenn von Männer- oder Frauenkörpern gesprochen wird, ohne zu benennen, dass nicht alle Menschen mit Vagina Frauen sind und nicht alle Menschen mit Penis Männer. Auch Geschlechtertrennungen sind oft für trans Menschen sehr problematisch. Dies stellt ein 
Dilemma dar, weil sie in Anbetracht von Sexismus und Schamempfinden in der Sexualpädagogik für andere Adressat:innen oft notwendig sind. ${ }^{20}$

Der Begriff Heterosexismus beschreibt die Privilegierung heterosexueller Menschen und die Diskriminierung u.a. lesbischer, schwuler, bisexueller, pansexueller, asexueller und queerer Menschen. Dies zeigt sich in der Sexualpädagogik u.a. in der heterosexuellen Vorannahme, dass alle Menschen, die sich nicht entsprechend outen, heterosexuell seien. Oft werden Sexualität und Fortpflanzung nur über Penis-in-Vagina-Sex beschrieben und andere Varianten werden vernachlässigt oder nur im Kontext der Prävention sexuell übertragbarer Infektionen besprochen. Außerdem wird meist davon ausgegangen, dass alle (gesunden) Menschen sexuell begehren. Dabei werden asexuelle Menschen unsichtbar gemacht bzw. pathologisiert. Sex unter Männern wird oft auf Analsex reduziert und Sex unter Frauen gar nicht besprochen. Homofeindliche Beschimpfungen sind in den meisten Schulklassen Alltag.

Weitere Themen rund um sexuelle und amouröse Vielfalt in einem erweiterten Sinne, die in der Sexualpädagogik diskriminierungsrelevant werden können, wie Polyamorie und BDSM, bespreche ich in meinem anderen Artikel in diesem Band (Debus i.d.B.).

All dies ist einerseits für die jeweils Betroffenen problematisch, weil ihre Bedarfe vernachlässigt werden und der Unterricht oft angstbesetzt ist. Darüber hinaus engen Geschlechternormen alle Menschen in ihren Entwicklungsmöglichkeiten ein. Diese Themen können daher so bearbeitet werden, dass sie für alle/die meisten Adressat:innen interessant sind und ihre sexuelle und reproduktive Selbstbestimmungsfähigkeit fördern.

\subsection{Intersektionalität}

Alle Diskriminierungsverhältnisse sind intersektional verschränkt. ${ }^{21}$ So unterscheiden sich z.B. rassistische Bilder zu Sexualität meist nach Geschlecht. Menschen mit bestimmten Behinderungen wird oft ihre Geschlechtlichkeit abgesprochen, sie werden also in erster Linie als behindert und erst in zweiter Linie als z.B. Männer oder Frauen wahrgenommen. Die heterosexuelle

20 Vgl. zur Problematisierung zweigeschlechtlicher Cruppenteilungen Hornstein 2018; zu didaktischen Abwägungen Debus 2017; zu Abwägungen im Kontext von Mädchen_arbeit Pohlkamp 2010; Busche/Wesemüller 2010.

21 Vgl. Walgenbach 2012 sowie portal-intersektionalitaet.de [Zugriff: 12.04.2020]. 
Vorannahme verschärft sich bei Menschen, die als proletarisch, prekarisiert, schwarz, muslimisch oder ssüdländisch`wahrgenommen werden. Kindern bildungsbürgerlicher, weißer Eltern wird in der Pädagogik oft mehr Selbstbestimmungsrecht zugesprochen, während die Lebensentscheidungen rassistisch und klassistisch markierter Jugendlicher schneller abgewertet werden. Menschen im ländlichen Raum und mit Sinnes- und Gehbeeinträchtigungen haben oft erschwerten Zugang zu Empowerment-Angeboten zu allen genannten Diskriminierungsthemen. Selbst bei thematischen Schwerpunktsetzungen sollte daher die Verschränkung mit anderen Themenfeldern berücksichtigt werden.

\section{Grenzachtung und Betroffene sexualisierter Gewalt in der Sexualpädagogik}

Grenzachtung und die Prävention von Partnerschafts ${ }^{-22}$ und sexualisierter Gewalt $^{23}$ sind bedeutende Themen der Sexualpädagogik.

Im Sinne einer gewaltpräventiven Sexualpädagogik ist es wichtig, dass Pädagog:innen einen achtsamen Umgang mit den (Scham-)Grenzen der Adressat:innen vorleben und diese dazu ermutigen, Grenzen gegenüber Gleichaltrigen und Autoritäten zu setzen. Dies fördert die Fähigkeit, Übergriffe zu erkennen, und das Vertrauen, bei Offenlegung Unterstützung zu finden (vgl. Enders 2012; Scambor u.a. 2016). U.a. bedeutet dies, dass Adressat:innen selbst den Grad der aktiven Mitwirkung an sexualpädagogischen Angeboten bestimmen können sollten (vgl. Tuider/Timmermanns 2015: 45).

Diskriminierungsreflektierte Sexualpädagogik beachtet im Sinne inklusiver Teilhabemöglichkeiten zudem die (individuell unterschiedlichen) Bedarfe von Kindern und Jugendlichen, denen sexualisierte Gewalt widerfahren ist. Bei einer Beschäftigung mit Sexualität sowie bei Methoden mit Körperkontakt sind Retraumatisierungsrisiken nicht auszuschließen (vgl. Rieske 2016). Für Kinder und Jugendliche, denen sexualisierte Gewalt widerfahren ist, sind daher Grenzachtung und Freiwilligkeit besonders wichtig. Gleichzeitig ist es für sie sehr hilfreich, eine Sprache für ihren Körper und die Körper anderer Menschen sowie zur Benennung und Differenzierung zwischen einer-

22 Für Materialen vgl. https://www.1st.gear-ipv.eu/de/node/56 [Zugriff: 13.04.2020].

23 Vgl. Enders (2012); https://www.zartbitter.de; https://www.tauwetter.de; https://www. dgfpi.de [Zugriff: 13.04.2020]. 
seits einvernehmlicher Intimität und Sexualität und andererseits Grenzverletzungen, sexuellen Übergriffen und sexualisierter Gewalt zu lernen (vgl. Enders 2012). Dazu gehört auch die Förderung der Kompetenz, eigene Grenzen wahrzunehmen und zu setzen. All dies kann die Fähigkeit zum Aufdecken von Grenzverletzungen, sexuellen Übergriffen und sexualisierter Gewalt sowie das Unrechtsbewusstsein stärken (Scambor u.a. 2016).

Präventionsangebote haben Aufdeckungscharakter, wie der Bundesverein zur Prävention von sexuellem Missbrauch an Mädchen und Jungen e.V. schreibt (2003), d.h. sie können Betroffene von (sexualisierter) Gewalt dabei unterstützen, sich bewusst zu werden, dass das Erlebte Unrecht war, und sie ermutigen, sich Unterstützung zu suchen. Daher sollten allen Beteiligten sexualpädagogischer Angebote Hilfeoptionen und lokale Ansprechstellen bekannt gemacht werden. Damit Sexualpädagogik gewaltpräventiv und inklusiv für Betroffene sexualisierter und Partnerschaftsgewalt wirkt, bedarf es fachkundiger Qualifizierung u.a. zu angemessenen Reaktionen und Interventionen nach einer Offenlegung sexualisierter Gewalt (vgl. Enders 2012: 187-218; Vasold und Freund i.d.B.), sowie einer kritischen Beschäftigung mit Spannungsfeldern sexualpädagogischen Handelns (vgl. Laumann/Debus 2018).

\section{Spannungsfelder diskriminierungsreflektierter Sexualpädagogik}

Diskriminierungsreflektierte Sexualpädagogik bewegt sich in einer Vielzahl von Spannungsfeldern, bei denen es oft eher um ein Ausbalancieren der Spannung geht als um einfache Rezepte zu deren Auflösung. Die Auseinandersetzung mit diesen Spannungsfeldern fördert professionelles Handeln durch einen bewussten Umgang mit Balance-Akten und kann von personalisierenden Gefühlen des Scheiterns entlasten.

\subsection{Zuschreibungswissen, Ausblendungen und Reflexionskompetenz}

Oft wird in pädagogischen Diskursen nahegelegt, möglichst viel über spezifische Zielgruppen zu lernen in der Hoffnung auf Rezepte für zielgruppengerechte Pädagogik. Diese sollen von Komplexität entlasten und lenken von 
einem kritischen Umgang mit Arbeitsbedingungen ab, die subjektorientierte $^{24}$ Pädagogik verhindern.

Ein solches Zuschreibungswissen über die Adressat:innen ist meist ver-sämtlichend, verbesondernd und/oder defizitorientiert und damit diskriminierend. Es wird ihren heterogenen Bedarfen und Ressourcen nicht gerecht und bietet nur Scheinlösungen für pädagogische Herausforderungen.

Am anderen Pol des Trugschlusspaares steht die Behauptung, nur das Individuum zu sehen. Gesellschaftliche Ungleichheit durchdringt das Leben in unseren Gesellschaften und schafft Realitäten - in den Leben unserer Adressat:innen, in unseren eigenen (un-)bewussten Wahrnehmungsmustern, Haltungen, Wissensbeständen und in unseren Methoden und Materialien. Diese Realität zu leugnen führt schnell zur Reproduktion gesellschaftlich nahegelegter Normalitätsannahmen. ${ }^{25}$

Im Umgang mit diesem Spannungsfeld finde ich eine Differenzierung zwischen Zuschreibungswissen und Reflexionskompetenz hilfreich (Debus 2015b). Reflexionskompetente Pädagog:innen wissen um mögliche Wirkungen sozialer Ungleichheiten, biografischer Faktoren etc. Mit diesem Hintergrundwissen gehen sie subjektorientiert und ergebnisoffen mit ihren Adressat:innen in den Dialog. Das Hintergrundwissen wirkt sensibilisierend u.a. für mögliche Kommunikationstabus und unterschwellige Dynamiken. Es hilft bei der Auswahl thematischer Angebote, Entscheidungen zwischen freiwilligen und verpflichtenden Formaten, diskriminierungsreflektierter Sprache, Materialauswahl und -entwicklung. Dabei empfiehlt sich eine Haltung aufmerksamen Nicht-Wissens.

\subsection{Un_Sichtbarkeits-Dynamiken und Teilnehmenden-Orientierung}

Manche Diversitäts-Ebenen scheinen offensichtlich, z.B. Alter, zugeschriebenes Geschlecht, Hautfarbe, Sprachbeherrschung, Körperform und manche Behinderungen - allerdings sind auch hier Fehlzuschreibungen möglich. Andere Diversitäts-Ebenen sind weniger offensichtlich, z.B. Gewaltwiderfahrnisse, psychische Erkrankungen, manche Behinderungen, Intergeschlechtlichkeit, (eigene oder familiäre) queere Lebensweisen, Transgeschlechtlich-

24 Eine subjektorientierte Pädagogik geht immer von den einzelnen Adressat:innen aus und begleitet diese entlang ihrer Ressourcen, Bedarfe und Interessen in einem dialogischen Lernprozess.

25 Bezüglich Geschlechterverhältnissen vgl. Hagemann-White 2006. 
keit vor dem Beginn äußerlich sichtbarer Transitionen, Jüdisch-Sein bzw. jüdische Familienbezüge sowie Armutserfahrungen und (familiäre) Klassismuserfahrungen. Wenn sie mit Bedeutung aufgeladen werden durch Verletzlichkeit, Scham und/oder Diskriminierung, ist die Wahrscheinlichkeit hoch, dass Betroffene entsprechende Persönlichkeitsanteile, Zugehörigkeiten, Diskriminierungswiderfahrnisse und Bedarfe verbergen. Dies kann eine sinnvolle Selbstschutzstrategie sein (vgl. Klemm 2018), führt aber oft zu einer pädagogischen Unterschätzung der Bedarfe. Wir haben dafür im Projekt Interventionen für geschlechtliche und sexuelle Vielfalt ${ }^{26}$ den Begriff Un_SichtbarkeitsDynamiken entwickelt (Laumann 2018).

Un_Sichtbarkeits-Dynamiken fordern das Prinzip der TeilnehmendenOrientierung heraus. Es ist wichtig, von den Bedarfen der Adressat:innen auszugehen. Dabei sollten wir allerdings um mögliche Kommunikationstabus in unseren Themen wissen (z.B. Fragen queerer Menschen, NichtEntsprechung mit Körpernormen, Traumatisierungen, Wissenslücken, Scheiternserfahrungen, sexuelles Interesse etc.). Hier gibt es einen Bedarf an Lernangeboten, die nicht dem Gruppenprozess überlassen werden und die in Anspruch genommen werden können, ohne sich zu outen oder (auch durch anonyme Fragen) Nachforschungen bzw. verächtliche Kommentare zu riskieren.

\subsection{Sensibilisierung und Empowerment}

Alle Lerngruppen sind entlang von Diskriminierungsthemen und biografischen Faktoren gemischt und selbst in Lerngruppen, die zu einem Thema homogen erscheinen, haben die Teilnehmenden verschiedene Umgangsweisen mit ihren Erfahrungen entwickelt.

Daraus folgt, allen Lerngruppen unabhängig von Coming-outs einerseits sensibilisierende Lernangebote zur Prävention von Diskriminierung (z.B. Debus 2018b) und Grenzüberschreitungen und andererseits empowernde Lernangebote (z.B. Klemm 2018) zu machen. Das kann heißen, über Diskriminierung zu sprechen und damit Teilnehmende in ihrer Analyse- und Empörungsfähigkeit sowie Wehrhaftigkeit und Zivilcourage zu stärken. Zusätzlich zur Beschäftigung mit belastenden Themen heißt Empowerment aber auch, Teilnehmende durch ermutigende Perspektiven und Erfahrungen, positive Lern- 
modelle ${ }^{27}$ und utopische Momente zu stärken, ohne Barrieren und Ungerechtigkeiten zu dethematisieren (vgl. Debus 2018).

\subsection{Schutz und transformatives Lernen}

In diskriminierungskritischen Debatten hat sich in den letzten Jahren der Anspruch verstärkt, Teilnehmende, die Diskriminierung und Gewalt erlebt haben, vor Erfahrungen zu schützen, die Schmerz aufrufen. Dabei ist jedoch zu bedenken, dass es im Umgang mit Verletzungen, Diskriminierungen und Traumatisierungen verschiedene Bewältigungsstrategien gibt. Diese unterscheiden sich individuell und im Verlauf eines Verarbeitungsprozesses. Während für manche Betroffene Vermeidung (zur Zeit) die richtige Strategie ist, ist für andere Konfrontation stärkender. Wie Iven Saadi und ich herausgearbeitet haben, können Safer-Space-Konzepte, die Sicherheit vor Schmerz priorisieren, für Erstere stärkend und für Letztere schwächend sein. Das Umgekehrte gilt für Braver-Space-Konzepte, die eher eine Erweiterung von Handlungsfähigkeit im Umgang mit Angst oder Schmerz fokussieren (Saadi/Debus i.E.). Letztere können durch stärkende Gegenerfahrungen im Umgang mit Belastung, Angst, Trauer etc. transformative Lernprozesse ermöglichen. Dafür muss der Raum eine - individuell variierend - angemessene Mischung aus Sicherheit und Herausforderung bieten.

Bildungsangebote in und rund um Schule stoßen durch strukturelle Rahmenbedingungen (u.a. Klassengröße, fehlende Freiwilligkeit, Zwangsgemeinschaft Schulklasse, fehlende Qualifizierung) an Grenzen, die Vorsicht bei der Gestaltung transformativer Lernprozesse gebieten. Vor allem geht es mir an dieser Stelle darum, sich auch bezüglich der Bedürfnisse Betroffener von Zuschreibungswissen zu verabschieden und achtsam zwischen Risiken und Lern- und Wachstumspotenzialen gerade auch für marginalisierte und belastete Adressat:innen abzuwägen. Es gibt derzeit m.E. keine Alternative dazu, reflexiv mit diesem Dilemma umzugehen und den Adressat:innen möglichst viel Selbstbestimmung innerhalb der Bildungsangebote $\mathrm{zu}$ ermöglichen.

27 Ich ziehe diesen Begriff dem `Vorbild ` vor, da er einen aktiveren Lernprozess nahelegt. 


\subsection{Diskriminierungsreflektierte Pädagogik und schwierige Rahmenbedingungen}

Die eingangs formulierten Diskriminierungsrisiken in der Sexualpädagogik eignen sich dazu, regelmäßig das eigene Handeln zu prüfen und weiterzuentwickeln. Dabei geht es um eine Mischung aus

- Vermeidung diskriminierender Bilder und Handlungsweisen,

- Sensibilität für (auch unausgesprochene) Bedarfe im Sinne von Reflexionskompetenz statt Zuschreibungswissen,

- inklusiver Wissens- und Kompetenzvermittlung (u.a. Kommunikations-, Wahrnehmungs- und Aushandlungskompetenzen, vielfaltsorientiertes Wissen, Diskriminierungssensibilität, Empowerment, Zivilcourage gegen Diskriminierung und Gewalt) sowie

- konsequenten Interventionen bei Diskriminierungen.

Dies ist herausforderungsreich und stößt an Grenzen pädagogischer Rahmenbedingungen. Zur Unterstützung einer diskriminierungsreflektierten (Sexual-)Pädagogik ist es daher notwendig, die sexualpädagogischen und diskriminierungsreflektierten Kompetenzen aller (Sexual-)Pädagog:innen durch Aus-, Fort- und Weiterbildung zu stärken und Reflexionsräume im Sinne von Supervision und Intervision in der alltäglichen Arbeit zu schaffen. Zudem braucht es eine Verbesserung sexualpädagogischer Rahmenbedingungen in Bezug auf Zeit, Personalschlüssel, Qualifizierung, Vernetzung, Kooperation sowie ökonomische Absicherung. Nicht zuletzt ist eine Verbreitung und (Weiter-)Entwicklung diskriminierungsreflektierter und vielfaltsorientierter sexualpädagogischer Materialien notwendig - und auch deren ausführliche Kommentierung bezüglich Potenzialen, Risiken und Nebenwirkungen (vgl. Debus 2018c). 
Für alle am pädagogischen Prozess Beteiligten ist es hilfreich, eine Haltung lebenslangen Lernens ${ }^{28}$ sowie Ambiguitätstoleranz ${ }^{29}$ (weiter) $\mathrm{zu}$ entwickeln. Gelingt dies, bietet Sexualpädagogik Potenziale für Empowerment; Förderung von Selbstwert, Glückserlebnissen und selbstbestimmten Lebensentwürfen; Gewalt- und Diskriminierungsprävention; transformative Lernprozesse; adressat:innengerechte pädagogische Angebote und für eine gelungene pädagogische Beziehungsarbeit.

\section{Literatur und Videoclips}

Arnade, Sigrid (2018): Ableismus erkennen und begegnen. Strategien zur Stärkung von Selbsthilfepotenzialen. Berlin: Interessenvertretung Selbstbestimmt Leben in Deutschland e.V. - ISL. Online unter: https:// www.isl-ev.de/attachments/article/1527/Able-Ismus-bf_2018-bf.pdf [Zugriff: 10.04.2020].

Auma, Maisha-Maureen (2020): Zwischen Kulturalisierung und Empowerment. Sexualpädagogische Repräsentationen von Schwarzen Menschen und People-of-Color im deutschsprachigen Raum. Pritz, Anna/Siegenthaler, Rafaela/Thuswald Marion (Hg.): Bilder befragen - Begehren erkunden. Repräsentationskritische Einsätze in der Bildungsarbeit. Textsammlung der Zeitschrift Kunst Medien Bildung. Online unter: http: //zkmb.de/zwischen-kulturalisierung-und-empowerment-sexualpaedag ogische-adressierungen-von-schwarzen-menschen-und-people-of-color -im-deutschsprachigen-raum [Zugriff: 18.06.2021].

Böhm, Maika (2013): Solosexualität. In: Schmidt, Renate-Berenike/Sielert, Uwe (Hg.): Handbuch Sexualpädagogik und sexuelle Bildung. Weinheim/Basel: Beltz Juventa, S. 301-310.

28 Hiermit ist nicht die neoliberale Anforderung gemeint, sich ein Leben lang unentgeltlich für den Arbeitsmarkt zu optimieren. Es geht um eine Haltung, die davon ausgeht, dass Pädagogik so komplex ist, dass wir nie ausgelernt haben können, dass Unsicherheiten und Fehler daher nicht bedeuten, dass wir inkompetent oder unprofessionell sind, sondern dass vielmehr ein professioneller Umgang mit Unsicherheit, NichtWissen und Fehlern ein Merkmal von Professionalität ist (vgl. dazu Mecheril 2013).

Ambiguitätstoleranz beschreibt die Fähigkeit, Spannungsverhältnisse und Mehr-bzw. Uneindeutigkeiten als selbstverständlichen Bestandteil von Realität mindestens auszuhalten, besser noch zu akzeptieren und produktiv mit ihnen umzugehen. 
Bundesverein zur Prävention von sexuellem Missbrauch an Mädchen und Jungen e.V. (2003): Empfehlungen für Qualitätskriterien in der Präventionsarbeit im Bereich der sexualisierten Gewalt an Mädchen und Jungen. Online unter: https://www.dgfpi.de/tl_files/bundesverein/flyer/oo_Gesamta usgabe.pdf [Zugriff: 12.04.2020].

Busche, Mart/Wesemüller, Ellen (2010): Mit Widersprüchen für neue Wirklichkeiten. Ein Manifest für Mädchen_arbeit. In: Busche, Mart/Maikowski, Laura/Pohlkamp, Ines/Wesemüller, Ellen (Hg.): Feministische Mädchenarbeit weiterdenken. Zur Aktualität einer bildungspolitischen Praxis. Bielefeld: transcript, S. 309-324.

Clara Rosa (2012): I am not a... Online unter: http://clararosa.blogsport.de/20 12/09/13/i-am-not-a [Zugriff: 12.04.2020].

Debus, Katharina (2012): Dramatisierung, Entdramatisierung und NichtDramatisierung in der geschlechterreflektierten Bildung. Oder: (Wie) Kann ich geschlechterreflektiert arbeiten, ohne geschlechtsbezogene Stereotype zu verstärken? In: Dissens e.V./Debus, Katharina/Könnecke, Bernard/Schwerma, Klaus/Stuve, Olaf (Hg.): Geschlechterreflektierte Arbeit mit Jungen an der Schule. Texte zu Pädagogik und Fortbildung rund um Jungen, Geschlecht und Bildung. Berlin: Dissens e.V., S. 149-158. Online unter: http://www.jungenarbeit-und-schule.de/material/abschlusspublik ation [Zugriff: 12.04.2020].

Debus, Katharina (2015a): Du Mädchen! Funktionalität von Sexismus, Postund Antifeminismus als Ausgangspunkt pädagogischen Handelns. In Hechler, Andreas/Stuve, Olaf (Hg.): Geschlechterreflektierte Pädagogik gegen Rechts. Opladen: Verlag Barbara Budrich, S. 79-99. Online unter: https://www.oapen.org/search?identifier=1004470 [Zugriff: 12.04.2020].

Debus, Katharina (2015b): >Ein gutes Leben! - Ansätze, Stolpersteine und Qualitätsmerkmale einer intersektionalen geschlechterreflektierten Pädagogik integrierter Berufs- und Lebensorientierung. In: Micus-Loos, Christiane/Plößer, Melanie (Hg.): Des eigenen Glückes Schmied_in!? Geschlechterreflektierende Perspektiven auf berufliche Orientierungen und Lebensplanungen von Jugendlichen. Wiesbaden: Springer VS, S. 115-134.

Debus, Katharina (2017a): Nicht-diskriminierende Sexualpädagogik. In: Scherr, Albert/El Mafaalani, Aladin/Gökcen Yüksel, Emine (Hg.): Handbuch Diskriminierung. Wiesbaden: Springer VS, S. 811-834.

Debus, Katharina (2017b): Dramatisierung, Entdramatisierung und NichtDramatisierung von Geschlecht und sexueller Orientierung in der geschlechterreflektierten Bildung. Oder: (Wie) Kann ich geschlechterre- 
flektiert arbeiten, ohne Stereotype zu verstärken? In: Glockentöger, Ilke/Adelt, Eva (Hg.): Gendersensible Bildung und Erziehung in der Schule. Grundlagen - Handlungsfelder - Praxis. Münster: Waxmann, S. 25-42.

Debus, Katharina (2018a): Was heißt das für die Praxis? Konzeptionelle und didaktische Zugänge zu geschlechtlicher, amouröser und sexueller Vielfalt in der Pädagogik. In: Debus, Katharina/Laumann, Vivien: Pädagogik geschlechtlicher, amouröser und sexueller Vielfalt. Zwischen Sensibilisierung und Empowerment. Berlin: Dissens - Institut für Bildung und Forschung, S. 87-120. Online unter: https://interventionen.dissens.de/mater ialien/handreichung [Zugriff: 12.04.2020].

Debus, Katharina (2018b): Diskriminierungskritische Sensibilisierung und Förderung von Zivilcourage in heterogenen Lerngruppen. In: Debus, Katharina/Laumann, Vivien: Pädagogik geschlechtlicher, amouröser und sexueller Vielfalt. Zwischen Sensibilisierung und Empowerment. Berlin: Dissens - Institut für Bildung und Forschung, S. 131-144. Online unter: https://interventionen.dissens.de/materialien/handreichung [Zugriff: 12.04.2020].

Debus, Katharina (2018c): Irgendwas zu Vielfalt ... Anregungen für eine reflektierte Methodenauswahl. In: Debus, Katharina/Laumann, Vivien: Pädagogik geschlechtlicher, amouröser und sexueller Vielfalt. Zwischen Sensibilisierung und Empowerment. Berlin: Dissens - Institut für Bildung und Forschung, S. 121-127. Online unter: https://interventionen.dissens.de/m aterialien/handreichung [Zugriff: 12.04.2020].

Debus, Katharina/Laumann, Vivien (2018): LSB-was? Geschlechtliche, amouröse und sexuelle Vielfalt - Einführung und Spannungsfelder. In: Dies.: Pädagogik geschlechtlicher, amouröser und sexueller Vielfalt. Zwischen Sensibilisierung und Empowerment. Berlin: Dissens - Institut für Bildung und Forschung, S. 12-70. Online unter: https://interventionen.disse ns.de/materialien/handreichung [Zugriff: 12.04.2020].

Diskursatlas Antifeminismus (o.J.): Frühsexualisierung. Online unter: $h$ ttps://www.diskursatlas.de/index.php?title=Frühsexualisierung [Zugriff: 09.04.2020].

Enders, Ursula (2012): Grenzen achten. Schutz vor sexuellem Missbrauch in Institutionen. Ein Handbuch für die Praxis. Köln: Kiepenheuer \& Witsch. Fusion Comedy (2016): How microaggressions are like mosquito bites (2016). Online unter: https://www.youtube.com/watch?v=hDd3bzA7450 [Zugriff: 09.04.2020]. 
Generation Adefra, Black Women in Germany in Cooperation with EOTO, Each One Teach One, and MSO Inklusiv (o.J.): We are the seeds collective! Online unter: https://www.adefra.de/index.php/component/tags/ta g/21-sexualpaedagogisches-empowerment [Zugriff: 11.04.2020].

Gewirtz-Meydan, Ateret/Hafford-Letchfield, Trish/Benyamini, Yael/Phelan, Amanda/Jackson, Jeanne/Ayalon, Liat (2018): Ageism and Sexuality. In: Ayalon, Liat/Tesch-Römer, Clemens (Hg.): Contemporary Perspectives on Ageism. Cham: Springer Open, S. 149-162. Online unter: https://link.spri nger.com/book/10.1007/978-3-319-73820-8 [Zugriff: 11.04.2020].

Hagemann-White, Carol (2006): Sozialisation - zur Wiedergewinnung des Sozialen im Gestrüpp individualisierter Geschlechterbeziehungen. In: Bilden, Helga/Dausien, Bettina (Hg.): Sozialisation und Geschlecht. Theoretische und methodologische Aspekte. Opladen/Farmington Hills: Verlag Barbara Budrich, S. 71-88.

Hark, Sabine/Villa, Paula-Irene (Hg.) (2015): Anti-Genderismus. Sexualität und Geschlecht als Schauplätze aktueller politischer Auseinandersetzungen. Bielefeld: transcript.

Hornstein, René_(2018): Trans und nicht-binäre Schüler*innen unterstützen. In: Spahn, Annika/Wedl, Juliette (Hg.): Schule lehrt/lernt Vielfalt. Praxisorientiertes Basiswissen und Tipps für Homo-, Bi-, Transund Inter*freundlichkeit in der Schule. Göttingen: Edition Waldschlösschen, S. 106-111. Online unter: http://akzeptanz-fuer-vielfalt.de [Zugriff: 12.04.2020]

IDA e.V. - Informations- und Dokumentationszentrum für Antirassismusarbeit (o.J.): Bodyismus. Glossar. Online unter: https://www.idaev.de/r echerchetools/glossar?tx_dpnglossary_glossary\%5B\%40widget_0\%5D\%5 Bcharacter\%5D=B\&cHash=c9c5b2bfacd7e7535b24591b5b2e788e [Zugriff: 25.04.2021].

IDA-NRW (2016): Zur Ethnisierung der Genderdebatte. Zeitschrift des Informations- und Dokumentationszentrums für Antirassismusarbeit in Nordrhein-Westfalen 22, 1. Online unter: https://www.ida-nrw.de/filead $\mathrm{min} /$ user_upload/ueberblick/Ueberblick_1_16.pdf [Zugriff: 12.04.2020].

Institute for Art Education (o.J.): Othering. Online unter: https://www.zhdk. $\mathrm{ch} /$ forschung/ehemalige-forschungsinstitute-7626/iae/glossar-972/other ing-5894 [Zugriff: 09.04.2020].

Kalpaka, Annita (2005): Pädagogische Professionalität in der Kulturalisierungsfalle - Über den Umgang mit »Kultur« in Verhältnissen von Differenz und Dominanz. In: Leiprecht, Rudolf/Kerber, Anne (Hg.): Schule 
in der Einwanderungsgesellschaft. Ein Handbuch. Schwalbach: Wochenschau, S. 387-405.

Kalpaka, Annita (2011): Institutionelle Diskriminierung im Blick - Von der Notwendigkeit Ausblendungen und Verstrickungen in rassismuskritischer Bildungsarbeit zu thematisieren. In: Leiprecht, Rudolf/Scharathow, Wiebke (Hg.): Rassismuskritik. Bd. 2: Rassismuskritische Bildungsarbeit. Schwalbach: Wochenschau, S. 25-40.

Kemper, Andreas/Weinbach, Heike (2009): Klassismus. Eine Einführung. Münster: Unrast.

Klemm, Sarah (2018): Empowerment von queeren Jugendlichen in heterogenen Lerngruppen. In: Debus, Katharina/Laumann, Vivien: Pädagogik geschlechtlicher, amouröser und sexueller Vielfalt. Zwischen Sensibilisierung und Empowerment. Berlin: Dissens - Institut für Bildung und Forschung, S. 145-155. https://interventionen.dissens.de/materialien/handre ichung [Zugriff: 12.04.2020].

Ku, Marvin Xin/Baden, Rebecca (2018): Nicht-Weiße erzählen, wie sie beim Daten Rassismus erleben. Online unter: https://www.vice.com/de/articl e/qved7p/nicht-weisse-erzahlen-wie-sie-beim-daten-rassismus-erleben [Zugriff: 12.04.2020].

Laumann, Vivien/Debus, Katharina (2018): >Frühsexualisierung< und >Umerziehung? Pädagogisches Handeln in Zeiten antifeministischer Organisierungen und Stimmungsmache. In: Lang, Juliane/Peters, Ulrich (Hg.): Antifeminismus in Bewegung. Aktuelle Debatten um Geschlecht und sexuelle Vielfalt. Hamburg: Marta-Press, S. 275-301.

Leber, Sebastian (2016): Lookismus. Die Bevorzugung der Schönen. Online unter: https://www.tagesspiegel.de/politik/lookismus-die-bevorzugungder-schoenen/14659816-all.html [Zugriff: 11.04.2020].

Liebscher, Doris/Fritzsche, Heike (2010): Antidiskriminierungspädagogik. Konzepte und Methoden für die Bildungsarbeit mit Jugendlichen. Wiesbaden: Springer VS.

Maskos, Rebecca (2011):»Bist Du behindert oder was?!« Behinderung, Ableism und souveräne Bürger_innen. Vortrag im Rahmen der Ringvorlesung "Jenseits der Geschlechtergrenzen« der AG Queer Studies und der Ringvorlesung »Behinderung ohne Behinderte!? Perspektiven der Disability Studies«, Universität Hamburg, 14.12.2011. Online unter: https://www.ze dis-ev-hochschule-hh.de/files/maskos_14122011.pdf [Zugriff: 10.10.2020]. McRobbie, Angela (2010): Top Girls. Feminismus und der Aufstieg des neoliberalen Geschlechterregimes. Wiesbaden. Springer VS. 
Mecheril, Paul (2013): »Kompetenzlosigkeitskompetenz«. Pädagogisches Handeln unter Einwanderungsbedingungen. In: Auernheimer, Georg (Hg.): Interkulturelle Kompetenz und pädagogische Professionalität. Wiesbaden: Springer VS, S. 15-34.

Mecheril, Paul/Castro Varela, María do Mar/Dirim, İnci/Kalpaka, Annita/Melter, Klaus (2010): Migrationspädagogik. Weinheim/Basel: Beltz.

Moghimi, Floria (o.J.): Wie wirken sich Mikroaggressionen aus? Online unter: https://blog.floriamoghimi.de/2019/05/13/wie-wirken-sich-mikroagg ressionen-aus [Zugriff: 09.04.2020].

Niemann, Yolanda Flores (2017): Microaggressions in the classroom. Online unter: https://www.youtube.com/watch?v=ZahtlxW2CIQ [Zugriff: 09.04.2020].

Peşmen, Azadê (2018): Rassismus macht den Körper krank. Wie Tausende kleine Mückenstiche. Online unter: https://www.deutschlandfunkkultur. de/rassismus-macht-den-koerper-krank-wie-tausende-kleine.976.de.ht ml?dram:article_id=422167 [Zugriff: 09.04.2020].

Pierce, Chester (1970): Offensive Mechanisms. In: Barbour, Floyd B.: The Black Seventies. Boston: Porter Sargent, S. 265-282.

Pohlkamp, Ines (2010): TransRäume. Mehr Platz für geschlechtliche Nonkonformität! In: Busche, Mart/Maikowski, Laura/Pohlkamp, Ines/Wesemüller, Ellen (Hg.): Feministische Mädchenarbeit weiterdenken. Zur Aktualität einer bildungspolitischen Praxis. Bielefeld: transcript, S. 37-58.

Queer.de (2018): »Christliche« Sexualpädagogik. Österreich: Werbung für Homo-»Heilung« an Schulen?. Online unter: https:/www.queer.de/detai l.php?article_id=31538 [Zugriff: 09.04.2018].

Rautenberg, Charlene (2019): »Ich will nicht deine Schokolade sein.« Online unter: https://sz-magazin.sueddeutsche.de/liebe-und-partnerschaft/rass ismus-dating-liebe-88200 [Zugriff: 12.04.2020].

Rieske, Thomas Viola (2016): Junge $\neq$ Opfer? Zur (These der) Verleugnung männlicher Betroffenheit von sexualisierter Gewalt im pädagogischen Feld. In: Mahs, Claudia/Rendtorff, Barbara/Rieske, Thomas Viola (Hg.): Erziehung - Gewalt - Sexualität. Zum Verhältnis von Geschlecht und Gewalt in Erziehung und Bildung. Opladen: Verlag Barbara Budrich, S. 7994.

Ritz, ManuEla (o.J.): Kindsein ist kein Kinderspiel. Adultismus - (un)bekanntes Phänomen. Online unter: https://amyna.de/amyna-medien/dokume nte/prog/Kind-sein_ist_kein_Kinderspiel.pdf [Zugriff: 09.04.2020]. Gekürzt veröffentlicht in: Wagner, Petra (2008): Handbuch Kinderwelten. 
Vielfalt als Chance - Grundlagen einer vorurteilsbewussten Bildung und Erziehung. Freiburg: Herder, S. 128-136.

Saadi, Iven/Debus, Katharina (i.E.): Verletzlichkeit und Lernen zu Diskriminierung. Anregungen und Gedanken. Online unter: https://katharina-de bus.de/material.

Sarma, Olivia (2012): KulturKonzepte. Ein kritischer Diskussionsbeitrag für die interkulturelle Bildung. Frankfurt a.M.: Amt für multikulturelle Angelegenheiten. Online unter: https://amka.de/publikationen/kulturkonze pte [Zugriff: 11.04.2020].

Scambor, Elli/Wittenzellner, Ulla/Puchert, Ralf/Rieske, Thomas Viola/Könnecke, Bernard (2016). "... dass die Leute da auch genauer hingucken.«Wie kann die Aufdeckung von sexualisierter Gewalt bei männlichen Kindern und Jugendlichen in der Jugendarbeit gefördert werden? In: sozialmagazin $41,7-8$, S. 60-67.

Schmid, Lea/Diamond, Darla/Pflaster, Petra (2017): Lookismus. Normierte Körper - Diskriminierende Mechanismen - (Self-)Empowerment. Münster: unrast.

Sielert, Uwe (2015): Einführung in die Sexualpädagogik. Weinheim: Beltz.

Specht, Ralf (2013): Sexualität und Behinderung. In: Schmidt, RenateBerenike/Sielert, Uwe: Handbuch Sexualpädagogik und sexuelle Bildung. Weinheim/Basel: Beltz Juventa, S. 288-300.

Sue, Derald Wing (2010): Microaggressions, Marginality and Oppression: An Introduction. In: Ders.: Microaggressions and Marginality: Manifestation, Dynamics, and Impact. New Jersey: John Wiley \& Sons, S. 3-23.

Terkessidis, Mark (2012): „Gehört der Rassismus zu Deutschland? Und wenn ja: Warum?«. Videomitschnitt. Online unter: https:/www.youtube.com/ watch?v=EK4ZKnmBfUI [Zugriff: 11.04.2020].

Timmermanns, Stefan (2013): Materialien der Sexualerziehung. In: Schmidt, Renate-Berenike/Sielert, Uwe: Handbuch Sexualpädagogik und sexuelle Bildung. Weinheim/Basel: Beltz Juventa, S. 708-716.

Timmermanns, Stefan (2016): Vielfalt erwächst aus Freiheit. Zur theoretischen Verortung einer Sexualpädagogik der Vielfalt. In: Henningsen, Anja/Tuider, Elisabeth/Timmermanns, Stefan (Hg.): Sexualpädagogik kontrovers. Weinheim: Beltz Juventa, S. 17-31.

Tóth, Barbara (2018): Die Teenstar-Leaks. Der Verein Teenstar bietet in ganz Österreich Aufklärungskurse an Pflichtschulen an. Interne Schulungsunterlagen zeigen erstmals, dass Fundamentalchristen am Werk sind. In: 
Falter 47/18. Online unter: https://www.falter.at/zeitung/20181120/die-te enstar-leaks?ref=nav [Zugriff: 09.04.2020].

Tóth, Barbara (2019): Ministerium verbannt Teenstar. Nach FALTERRecherchen: Der fundamentalchristliche Aufklärungsverein darf an Schulen nicht mehr lehren. Online unter: https://www.falter.at/zeitung/20190 401/ministerium-verbannt-teenstar [Zugriff: 09.04.2020].

Tuider, Elisabeth (2016): Diskursive Gemengelagen. Das Bild vom >unschuldigen, reinen Kind in aktuellen Sexualitätsdiskursen. In: Henningsen, Anja/Tuider, Elisabeth/Timmermanns, Stefan (Hg.): Sexualpädagogik kontrovers. Weinheim: Beltz Juventa, S. 176-193.

Tuider, Elisabeth/Timmermanns, Stefan (2015): Aufruhr um die sexuelle Vielfalt. In sozialmagazin 40, 1-2, S. 38-47.

Voß, Heinz-Jürgen (2014): Wenn rechtspopulistische Kreise gewinnen. Zu den Debatten um Sexualpädagogik und Antidiskriminierung. Online unter: https://dasendedessex.de/wenn-rechtpopulistische-kreise-gewinnen -zu-den-debatten-um-sexualpaedagogik-und-antidiskriminierung $\mathrm{Zu}$ griff: 13.04 .2020 ].

wahnsinnigverflochten (o.J.): Ageism und Gerontophobie. Online unter: https://wahnsinnigverflochten.wordpress.com/theoretisches/ageism -und-gerontophobie [Zugriff: 11.04.2020].

Walgenbach, Katharina (2012): Intersektionalität - eine Einführung. Online unter: https://www.portal-intersektionalität.de [Zugriff: 12.04.2020].

WHO-Regionalbüro für Europa/BZgA - Bundeszentrale für gesundheitliche Aufklärung (2011): Standards für Sexualaufklärung in Europa. Rahmenkonzept für politische Entscheidungsträger, Bildungseinrichtungen, Gesundheitsbehörden, Expertinnen und Experten. Köln: Bundeszentrale für gesundheitliche Aufklärung. Online unter: https:/www.bzga-whocc.de/p ublikationen/standards-sexualaufklaerung [Zugriff: 12.04.2020]. 


\section{Wie über Sexualität sprechen? Zur Lust auf Begriffsarbeit in der sexualpädagogischen Praxis}

Marion Thuswald

"Sex is more than merely coitus."

(WHO/BZgA 2010: 49)

»Please Use Sex Only When Speaking of Fucking. [...] It doesn't work to call gender by the same word we use for fucking and so on."

(Bornstein 2013: 49f.)

Der Begriff Sexualität wirkt wohl für viele auf den ersten Blick alltagssprachlich verständlich. Auch wenn er in der pädagogischen Arbeit benutzt wird, scheint zumeist nicht fraglich, was damit gemeint ist. Wenn wir aber genauer hinhören, zeigt sich, dass mit dem Begriff auf recht Unterschiedliches verwiesen wird.

Wie Sexualität verstanden wird und welche Vorstellungen damit verbunden sind, hat Einfluss auf die Gestaltung pädagogischer Praxis, so die Ausgangsthese dieses Artikels. Der Beitrag diskutiert unterschiedliche Zugänge zu Sexualität, die in sexualpädagogischer Fachliteratur und Praxis im Einsatz sind, und fragt nach ihren Effekten sowie nach ihrem inklusiven und normenkritischen Potenzial. ${ }^{1}$ Der Text zielt darauf ab, Lust auf (sexual-)päd-

1 Sexualerziehung wurde in Deutschland und Österreich bereits vor 50 Jahren zu einer schulischen Aufgabe erklärt, dennoch kam den »mit der ssexuellen Frage ‘verbundenen Herausforderungen in den pädagogischen Reflexionen«lange keine systematische Beachtung zu (Retkowski/Thole 2012: 292). In den letzten Jahren hat sich die Debatte um Sexualität als pädagogisches Thema etwas intensiviert, wobei den Anlass dafür vor allem 
agogische Begriffsarbeit zu machen, und ist auch als Anregung zur Reflexion und zum Austausch über (eigene) Vorstellungen und Zugänge zu Sexualität und das Sprechen darüber in der Schule gedacht.

Die Perspektive, aus der der Beitrag geschrieben ist, kann als macht- und differenzreflektierend oder auch als normenkritisch bezeichnet werden. Damit ist zum einen gemeint, dass die untersuchten Sexualitäts-Verständnisse und Sprechweisen auf implizite wie explizite Normen und Ausschlüsse hin befragt werden. Zum anderen ist eine solche Perspektive dadurch gekennzeichnet, dass Anknüpfungspunkte für eine möglichst inklusive, also vielfaltsorientierte und gleichzeitige grenzachtende sexualpädagogische Arbeit herausgearbeitet werden. Sie zielt darauf $a b$, in gewaltpräventiver und emanzipatorischer Absicht Machtverhältnisse zu reflektieren und auf den Abbau von Diskriminierung und sozialer Ungleichheit hinzuwirken. ${ }^{2}$ Der Artikel richtet sich damit insbesondere an alle, die dem »Begehren nach einer anderen pädagogischen Praxis«, einer »weniger gewaltvollen Praxis«, auf der Spur sind, wie die Bildungwissenschaftlerin Rosemarie Ortner es formuliert (vgl. Ortner 2019: 135).

\section{Sexualität: haben oder tun?}

Während der englische Begriff sex bereits im 14. Jahrhundert für Geschlecht benutzt wurde, ist der Begriff Sexualität relativ jung. Einer der Ersten, der ihn verwendete, war der Botaniker August Henschel in seinem Buch »Von der Sexualität der Pflanzen« im Jahr 1820 (vgl. Sager 2015: 47ff.). Zunächst für Pflanzen eingesetzt, wird er bald auch für Tiere und Menschen verwendet (vgl. ebd.). In seiner Begriffsgeschichte ist Sexualität also stark mit Fortpflanzung und (Zwei-)Geschlechtlichkeit verbunden.

Während das Verständnis von Sexualität gegenwärtig zumeist nicht mehr auf Fortpflanzung reduziert ist, spielt Geschlecht im alltagssprachlichen Verständnis von Sexualität noch immer eine wichtige Rolle: Begriffe

die öffentlichen Debatten um sexualisierte Cewalt in pädagogischen Einrichtungen geliefert haben (vgl. etwa Thole u.a. 2012; Retkowski u.a. 2018; Wazlawik u.a. 2019).

2 Für solche Zugänge werden etwa auch Begriffe wie diskriminierungsreflektiert (vgl. etwa Debus i.d.B.) oder Critical Diversity verwendet (vgl. etwa Auma 2017; Mörsch 2018; Thuswald/Sattler 2019). 
wie Geschlechtsverkehr für Koitus oder Geschlechtskrankheiten für sexuell übertragbare Krankheiten sind weit verbreitet. Unterscheidungen wie Hetero-, Homo- und Bisexualität machen nur Sinn, wenn von der Annahme ausgegangen wird, dass Geschlecht ein zentrales Kriterium für die Wahl von Sexual- und Liebespartner:innen darstellt. ${ }^{3}$

Im Dickicht um Sex(ualität) und Geschlecht(lichkeit) eine klare und präzise Sprache $\mathrm{zu}$ (er-)finden, die einschränkende und ausschließende Geschlechter- und Sexualnormen nicht reproduziert, erweist sich als herausfordernd, weil damit vielerlei bezeichnet wird und ihre Verwobenheit komplex ist. ${ }^{4}$ Der Begriff Geschlecht ${ }^{5}$ wird in Alltags- und Fachdiskursen sowohl für Sexualorgane benutzt als auch für das sogenannte biologische Geschlecht oder Körpergeschlecht, das seinerseits nochmals nach unterschiedlichen Aspekten wie Genen, Gonaden, Hormonen, äußeren Genitalien und sogenannten sekundären Geschlechtsmerkmalen differenziert werden kann (vgl. dazu auch Debus i.d.B.). Mit dem Begriff Geschlecht wird zudem auf

Cegenwärtig lässt sich eine Ausdifferenzierung von (Selbst-)Bezeichnungen für sexuelle Orientierungen bzw. Begehrensweisen ausmachen, die darüber hinausgehen, Ceschlecht zentral zu setzen, oder die als Ergänzungen zu geschlechtsbezogenen Orientierungen verwendet werden, wie etwa pansexuell, saphiosexuell, kinky u.a.

4 Diane Richardson arbeitet in ihrem Beitrag »Patterned Fluidities: (Re)Imagining the Relationship between Cender and Sexuality« (2007) fünf verschiedene Weisen heraus, wie das Verhältnis von Ceschlecht und Sexualität gegenwärtig theoretisiert wird. Richardson selbst plädiert dafür, die Verbindung zwischen Geschlecht und Sexualität als »complex, dynamic, contingent, fluid and unstable«zu verstehen (ebd.: 464). Sie argumentiert, dass die Verbindung von gender and sexuality auf vier Ebenen analysiert werden kann: »[...] at the level of social structure through institutions such as the law, education, the state and the media; at the level of social and cultural meaning; at the level of everyday interactions and routine practices; and at the level of individual subjectivities. At any one of these intersecting levels she suggests that the relationship between sexuality and gender may be different « (ebd.: 467). Zu feministischen Perspektiven auf das Verhältnis von Sexualität und Geschlecht vgl. auch Gruber/Ott (2014).

Bisweilen wird der Komplexität oder auch der scheinbaren `Natürlichkeit‘von Ceschlecht durch die Verwendung des englischen Begriffs gender zu entkommen versucht. Wiewohl ich dieses Anliegen verstehe, bringt die Unterscheidung von sex und gender meiner Einschätzung nach mehr problematische als hilfreiche Effekte mit sich (siehe etwa das Zitat von Kate Bornstein zu Beginn des Artikels), und es scheint mir im Deutschen sinnvoller beim Begriff Ceschlecht zu bleiben und anlassbezogen klarzustellen, wovon gesprochen wird; also etwa von Geschlechtsidentität, geschlechtlich konnotierten Ausdrucksformen etc. 
Geschlechtsidentität, auf geschlechtlich konnotierte Ausdrucksweisen (etwa durch Kleidung, Styling etc.) und vergeschlechtlichte Verhaltensnormen verwiesen oder eine machtvolle Differenzkategorie moderner Gesellschaften benannt. Sehr häufig ist eine Mischung von all dem gemeint.

Sexualität bezeichnet in Alltags- und auch Fachdiskursen bisweilen etwas, das Menschen haben (einen Trieb, eine Energie, eine Identität oder auch eine Geschlechtlichkeit), oder aber etwas, das Menschen tun (also erotisch konnotierte Handlungen und Interaktionen). Sexualität wird auch als ideologisches System (vgl. Rubin 2012 [1984]) oder moderne Subjektivierungsweise (vgl. Foucault 1983 [1976]) verstanden. Oder der Begriff wird als Sammelbegriff für ein Konglomerat an vielfältigen Aspekten benutzt (siehe etwa Offit 1979 in Abb. 1).

Was an unterschiedlichen Zugängen aus Perspektive macht- und differenzreflektierender Pädagogik als hilfreich bzw. problematisch eingeschätzt werden kann, wird im Folgenden noch Thema werden. Davor soll jedoch ein kurzer Blick auf gegenwärtige gesellschaftliche Kämpfe rund um Geschlechter- und Sexualpolitiken geworfen werden, die Einfluss auf sexualpädagogische Theoriebildung und Praxis haben.

\section{Gesellschaftliche Kämpfe um Geschlechter- und Sexualpolitiken}

In den letzten Jahrzehnten sind akademische und politische Bewegungen beobachtbar, die auf die "Denaturalisierung von Geschlecht und Sexualität« ausgerichtet sind (vgl. Engel/Schuster 2007). Theoretiker:innen wie Adrienne Rich, Gayle Rubin, Michel Foucault oder Judith Butler haben bedeutsame Beiträge dazu geleistet, die Naturhaftigkeit der engen Koppelung von Geschlechtlichkeit, Subjektivierung, Begehren und sexuellen Praktiken zu dekonstruieren.

Mit Foucault kann Sexualität als ein diskursives Phänomen begriffen werden und damit als etwas, das sich nicht direkt beobachten lässt, sondern in Diskursen entsteht. Sexuelle Praxis - oder anders gesagt Sex - ist beobachtbar und die Begriffe Begehren, Lust und Erregung benennen etwas, das sich wahrnehmen und spüren lässt. Sexualität kann hingegen als eine bestimmte, historisch und sozio-kulturell spezifische Form verstanden werden, »anatomische Elemente, biologische Funktionen, Verhaltensweisen, Empfindungen und Lüste in einer künstlichen Einheit zusammenzufassen und diese fikti- 


\section{WIE KANN SEXUALITÄT VERSTANDEN WERDEN? Zitatsammlung}

\begin{abstract}
„With sexuality we refer to „all erotically significant aspects of social life and social being - desires, pratices, relationships and identities." (Jackson/Scott 2010: 83)
\end{abstract}

\begin{abstract}
„Sexualität kann begriffen werden als allgemeine auf Lust bezogene Lebensenergie, die sich des Körpers bedient, aus vielfältigen Quellen gespeist wird, ganz unterschiedliche Ausdrucksformen kennt und in verschiedenster Hinsicht sinnvoll ist." (Sielert 2015: 40)
\end{abstract}

„Sexualität ist wie eine Sprache, nur mit dem Körper. Und wie in jeder neuen Sprache, braucht es einige Zeit bis Menschen einander verstehen."

(Axster/Aebi 2012)

Sexualität ist die "Gesamtheit der im Geschlechtstrieb begründeten Lebensäußerungen, Verhaltensweisen, Empfindungen." (Duden 2020)

,Unter Sexualität wird nicht nur der hetero- (zwischen verschiedenen Geschlechtern) und homosexuelle (zwischen gleichen Geschlechtern) Geschlechtsverkehr verstanden, sondern alle spezifisch männlichen und weiblichen Verhaltensweisen, unabhängig davon, ob sie durch biologische Unterschiede, Sexualtrieb oder durch soziokulturelle Faktoren verursacht wurden." (Michel/Novak 1994: 350)
,Sexualität ist, was wir daraus machen. Eine teure oder eine billige Ware, Mittel zur Fortpflanzung, Abwehr gegen Einsamkeit, eine Form der Kommunikation, ein Werkzeug der Aggression [...], eine Form der Zärtlichkeit, eine Art der Regression, eine Quelle der Freiheit, Pflicht, Vergnügen, Vereinigung mit dem Universum [...], eine juristische Streitsache, eine Form, Neugier und Forschungsdrang zu befriedigen, eine Technik, eine biologische Funktion, Ausdruck psychischer Gesundheit oder Krankheit oder einfach eine sinnliche Erfahrung." (Offit 1979: 16)

„Sexualität bezieht sich auf einen zentralen Aspekt des Menschseins über die gesamte Lebensspanne hinweg, der das biologische Geschlecht, die Geschlechtsidentität, die Geschlechtsrolle, sexuelle Orientierung, Lust, Erotik, Intimität und Fortpflanzung einschließt. Sie wird erfahren und drückt sich aus in Gedanken, Fantasien, Wünschen, Überzeugungen, Einstellungen, Werten, Verhaltensmustern, Praktiken, Rollen und Beziehungen." (WHO 2006 zit n. WHO/BZgA 2011: 18)

Abb. 1: ( ) Marion Thuswald, Grafik Christina Theny 
ve Einheit als ursächliches Prinzip, als allgegenwärtigen Sinn und allerorts zu entschlüsselndes Geheimnis funktionieren zu lassen«, und ist als solche nicht beobachtbar (Foucault 1983: 148ff.). Sexualität, so analysiert Foucault, ist dann die Vorstellung, dass dieses Vielgestaltige - also Anatomisches, Verhaltensweisen, Empfindungen, Lüste - zusammengehört und von großer Bedeutung für das menschliche Selbstverständnis ist (vgl. dazu auch Sager i.d.B.). ${ }^{6}$ In anderen Worten lässt sich sagen, dass Sexualität gegenwärtig als ein wichtiges gesellschaftliches »Deutungsmuster« (Bollenbeck 1996) gefasst werden kann. Damit ist gemeint, dass der Verweis auf Sexualität sich für Vielerlei und ganz Unterschiedliches als Erklärung anzubieten scheint: für Glück und Unglück, für das Scheitern von Liebesbeziehungen, für Identitätskrisen oder die Entstehung psychischer Störungen, für staatliche Repression und hate crimes, für den moralischen Verfall von Gesellschaften oder deren Überlegenheit, für die >Verwahrlosung، der Jugend sowie für die soziale Ungleichheit zwischen Männern und Frauen.

In Wissenschaften, sozialen Bewegungen und pädagogischer Praxis lassen sich emanzipatorisch orientierte Anstrengungen ausmachen, das zu entnaturalisieren und zu dynamisieren, was Foucault unter dem Dispositiv Sexualität als eng miteinander verknüpft analysiert. ${ }^{7}$ Dazu zählen etwa theoretische Zugänge, die unter Begriffen wie doing gender die soziale (Re-)Produktion geschlechtlich konnotierter Zuschreibungen betonen. Dazu können auch Konzepte wie heterosexuelle Matrix oder Heteronormativität gezählt werden, die darauf abzielen, die Normalisierung und Institutionalisierung bestimmter Formen von Beziehungen - nämlich bürgerlicher weißer heterosexueller monogamer und romantischer Zweierbeziehungen - zu analysieren und gewaltvolle Ausschlüsse dieser hegemonialen Normen herauszuarbeiten.

$\mathrm{Zu}$ den politischen Bewegungen der »Dynamisierung « rund um »Geschlecht, Sexualität und Lebensform« (vgl. Hartmann 2004) können etwa die Kämpfe um Rechte für trans, inter* und nicht-binäre Personen gerechnet

Dabei thematisiert Foucault in Bezug auf Sexualität insbesondere das Verhältnis von Machtmechanismen, Institutionen und mit Wahrheit aufgeladenen Diskursen, wie er im Vorwort zur deutschen Ausgabe schreibt (1983: 0.S.).

7 Die Ausdifferenzierung zwischen geschlechtersensibler bzw. geschlechterreflektierter Pädagogik und Sexualpädagogik - die vor allem bis in die 1960er Jahre auch Ceschlechtserziehung genannt wurde (vgl. DGC o.].) - kann als Ausdruck dieser Transformation angesehen werden. 
werden, die auf die Anerkennung von Selbstbestimmung und Geschlechtervielfalt abzielen. ${ }^{8} \mathrm{Zu}$ diesen Bewegungen kann auch der Einsatz für die (sexuelle) Selbstbestimmung von Frauen, Mädchen, Sexarbeiter:innen oder auch behinderten Menschen gezählt werden (vgl. dazu Abb. 2). ${ }^{9}$ Die Entwicklung von Ansätzen wie einer »Pädagogik vielfältiger Lebensweisen« (Hartmann 2002) oder einer "Sexualpädagogik der Vielfalt« (Tuider u.a 2012) kann als pädagogischer Beitrag zu den Dynamisierungs-Bewegungen verstanden werden. ${ }^{10}$

An den Diskreditierungskampagnen und Gegenbewegungen, die von konservativ-religiösen und rechten Akteur:innen gegen Gender Studies, progressive Sexualpädagogik und Gleichstellungsmaßnahmen gestartet werden, zeigt sich, dass diese Dynamisierungs- und SelbstbestimmungsBewegungen als bedrohlich für patriarchale, heterosexuelle (und weiße) Vorherrschaft eingeschätzt werden (vgl. etwa Hark/Villa 2017; Sager i.d.B.). Oder anders gesagt: Neben der Liberalisierung sexueller Politiken, die von Selbstbestimmungs-Bewegungen erkämpft wurden (vgl. Schmidt 2014 und Abb. 2) ${ }^{11}$ lassen sich vielerorts auch Tendenzen der Retraditionalisierung

8 Vergleiche dazu etwa Baumgartinger 2017, VIMÖ o.].

9 Diese Bewegungen haben nicht alle dieselben Anliegen und Zugänge, sondern stehen oftmals im Konflikt oder Widerspruch zueinander, wie sich etwa an den Debatten um Schutzräume, den unterschiedlichen Forderungen von trans und inter* Aktivist:innen oder den Kontroversen um Sexarbeit zeigt (vgl. dazu etwa Grenz 2018; Kokits/Thuswald 2015; Serano 2013).

10 Ich sehe diese Bewegungen der Entkoppelung und analytischen Trennung von Geschlecht und Sexualität, wie sie die Feministin Gayle Rubin schon 1984 forderte, als theoretisch wichtige und tendenziell emanzipatorische Bewegungen an. Dabei halte ich es aber für bedeutsam, dass dabei nicht aus dem Blick gerät, inwiefern Sexualität vergeschlechtlicht gedacht und Geschlecht von heternormativen Vorstellungen durchzogen ist (vgl. Jackson/Scott 2010). Wichtig ist aus meiner Sicht auch, dass eine Denaturalisierungs-Bewegung nicht die Ebene von Körperlichkeit negiert oder übersieht (vgl. dazu etwa Serano 2013).

11 Der Sexualwissenschaftler Cunther Schmidt spricht angesichts der Veränderungen rund um Sexualität von einer neuen Sexualmoral, die er »Verhandlungsmoral « (2014 [2004]: 8) nennt. Die alte Sexualmoral von Kirche und Staat, so schreibt er, war eine »Moral der Akte«, d.h. bestimmte sexuelle Handlungen - z.B. vorehelicher oder außerehelicher Sex oder Homosexualität - wurden als moralisch verwerflich angesehen bzw. waren verboten. Im Gegensatz zu einer Moral der Akte fokussiert eine Verhandlungsmoral nicht vorrangig die sexuellen Handlungen, sondern die Art und Weise ihres Zustandekommens. Es geht also nicht mehr darum, wer mit wem was macht, sondern dass und wie unter den Beteiligten ausgehandelt wird, was sie ge- 
beobachten, die traditionelle Geschlechtervorstellungen propagieren, die Rechte von LGBTIQ-Personen einschränken wollen und feministische Errungenschaften wie die Straffreiheit von Schwangerschaftsabbrüchen in Frage stellen (vgl. Hark/Villa 2015; Henningsen u.a. 2016).

Parallel zu Entwicklungen der Liberalisierung und Retraditionalisierung lässt sich auch eine Ökonomisierung sexueller Verhältnisse und intimer Beziehungen ausmachen. Damit ist nicht nur gemeint, dass es rund um die Gestaltung von Sexualität jede Menge Waren und Dienstleistungen zu kaufen gibt und sexuelle Inszenierungen eine wichtige Werbestrategie darstellen. ${ }^{12}$ Vielmehr wird mit Ökonomisierung auch markiert, dass sexuelle und emotionale Beziehungen zunehmend einer neoliberalen Logik von Selbstoptimierung und Leistungsorientierung unterworfen werden, wie die Soziologin Eva Illouz mit Blick auf Beziehungen der Mitte- und Oberschicht in den USA und Europa herausarbeitet (2006; 2012; vgl. dazu auch Debus 2015).

Angesichts der widersprüchlichen gesellschaftlichen Prozesse scheint es mir äußerst bedeutsam eine machtreflektierende pädagogische Arbeit zu stärken, die Diskriminierung abbaut und sexuelle Selbstbestimmung und solidarische Beziehungen stärkt. Gleichzeitig gilt es, (sexual-)pädagogische Zugänge, ihre Vorannahmen, Zielsetzungen und Praktiken immer wieder kritisch zu reflektieren und auf ihre Effekte hin zu befragen (vgl. dazu auch den Text von Debus »Diskriminierungsreflektierte Sexualpädagogik«i.d.B.).

meinsam machen; dass also Einvernehmlichkeit hergestellt wird. Der Wandel, den Gunther Schmidt beschreibt, bedeutet nicht unbedingt, dass diese Moral in den sexuellen Interaktionen und Beziehungen gelebt wird. Vielmehr ist damit ausgedrückt, dass bestimmte Argumentationen an Legitimität verloren haben und andere an Legitimität gewinnen (vgl. ebd.), wie sich etwa an den Debatten um sexualisierte Übergriffe rund um \#metoo zeigt.

Eine wichtige Ausnahme ist der gegenwärtigen Rede von der Verhandlungsmoral (zumeist) eingeschrieben: Jegliche sexuelle Handlung zwischen Erwachsenen und Kindern gilt gegenwärtig aufgrund des unterschiedlichen Entwicklungsstandes und des Machtgefälles als nicht einvernehmlich (vgl. Debus und Vasold i.d.B.).

12 Aktuelle feministische Kontroversen um die Ökonomisierung des Sexuellen drehen sich neben Sexarbeit und den Auswirkungen neoliberaler Prekarisierung von Lebensbedingungen auf sexuelle Selbstbestimmung auch um sex dolls bzw. Sexroboter oder um Reproduktionstechnologien wie etwa Leihmutterschaft (vgl. Gučanin 2020; Kahlert/Blome 2020). 


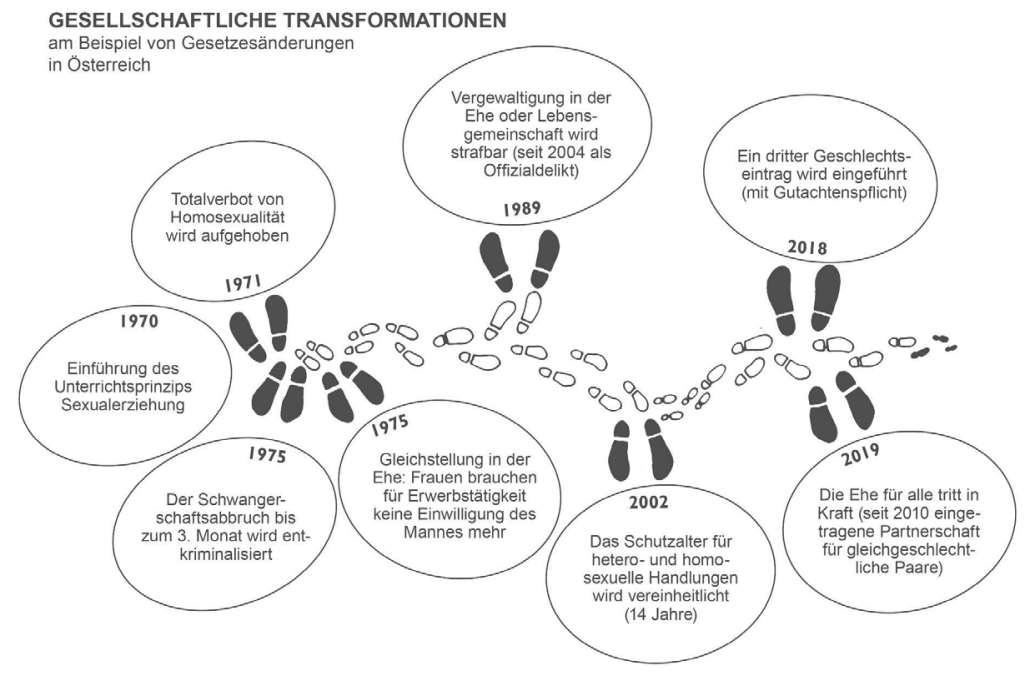

Abb. 2: ๑ Marion Thuswald, Grafik Christina Theny

\section{Verständnisse von Sexualität in der Sexualpädagogik}

Wie wird nun Sexualität in der Sexualpädagogik verstanden? Inwiefern knüpfen sexualpädagogische Theorie und Praxis an die erwähnten Bewegungen an? Und wie lassen sich die gegenwärtigen sexualpädagogischen Zugänge vor dem Hintergrund historischer Entwicklungen rund um Sexualität als pädagogisches Thema verstehen? Der folgende Abschnitt skizziert historisch bedeutsame Verschiebung rund um Sexualität als pädagogisches Thema und fragt davon ausgehend nach den gegenwärtigen Zugängen in der sexualpädagogischen Fachliteratur.

\subsection{Vom Trieb zur Energie? Repressive und emanzipatorische Sexualpädagogik}

Bis ins 17. Jahrhundert wurden Kinder aus der Erwachsenenwelt des Sexuellen nicht ausgeschlossen und kindliches Sexualverhalten wurde nicht als pädagogisches Problem betrachtet, schreibt Christin Sager (2015: 29ff.). Letzteres geriet erst im Rahmen der Familie des Wirtschafts- und Bildungsbürgertums 
unter pädagogische Beobachtung und Kontrolle (vgl. ebd.). ${ }^{13}$ Die moderne Naturwissenschaft und die Philosophie der Aufklärungszeit trugen dazu bei, das Sexuelle in die »niedrige Welt des Körperlichen « zu verbannen (vgl. Müller 1992: 105ff.). Die ersten zwei Jahrhunderte direkter und planvoller Sexualerziehung, so Walter Müller, waren daher davon beherrscht, das Individuum aus den »Fesseln der natürlichen Triebhaftigkeit zu befreien« (ebd.: 105). Dies bedeutete zumeist einen repressiven Zugang, also ein möglichst langes Fernhalten und Ablenken der Heranwachsenden von der Sphäre des Sexuellen (vgl. ebd.).

Nach ersten sexualreformerischen Bemühungen Anfang des 20. Jahrhunderts $^{14}$ entwickelte sich ab den späten 1960ern eine emanzipatorische Sexualpädagogik, die nicht auf die Beherrschung des Triebs, sondern auf die Emanzipation von äußeren und verinnerlichten Unterdrückungsmechanismen abzielte. Ein solches Denken war nur möglich, so Müller, weil dem Sexualtrieb eine neue Bedeutung beigemessen wurde: Galt Sexualität in der repressiven Sexualerziehung eher als kulturfeindlich und tendenziell zerstörerisch, so wurden ihr in der gesellschaftskritisch orientierten Sexualpädagogik Qualitäten wie Freiheit, Frieden, Humanität, Glück und Solidarität zugeschrieben. Der Sexualtrieb wurde also nicht mehr als negative, sondern als positive Kraft begriffen (vgl. Müller 1992: 115). Heranwachsende sollten unterstützen werden, ihre Sexualleben angst- und schamfrei und in Respekt und Verantwortung zu gestalten.

Gegenwärtig sind die meisten sexualpädagogischen Materialien in Deutschland und Österreich als »sexualfreundlich« einzuschätzen, schreiben Renate-Berenike Schmidt und Uwe Sielert (2012: 25). Sexualfreundlich ist nicht mit emanzipatorisch gleichzusetzen, auch wenn als gemeinsamer Nenner ein grundsätzlich positiver Zugang zu Sexualität erkennbar ist, der nicht

13 Die zunehmende Trennung von kapitalistisch orientierter Erwerbsarbeit und Privatleben, zu dem auch die Hausarbeit gehörte, führte zu einer Intimisierung der familiären Beziehung, zu einer zunehmenden Trennung kindlicher und erwachsener Sphären und der Konzeption von Kindheit als >Schonraum<. Das sich entwickelnde Bild vom Kind wurde - insbesondere durch die Romantik - »idealisiert und virgilisiert «, wie Sager formuliert (2015: 38). Die Konfrontation mit dem Sexuellen sollte so lange wie möglich hinausgezögert und durch Ablenkung verhindert werden, was nicht zuletzt zu einem Kampf gegen die kindliche Onanie führte (vgl. ebd. 38ff.).

14 Ein Beispiel dafür sind die sexualreformerischen Initiativen von Annie Reich (vgl. dazu Reich 2016 [1932]; Sager 2016). 
auf die Verhinderung sexueller Aktivität von Heranwachsenden abzielt, sondern Wissen als Grundlage für verantwortungsvolles Handeln vermittelt (vgl. Thuswald 2021). ${ }^{15}$ Abseits dieses weitgehenden professionellen Konsenses unterscheiden sich sexualpädagogische Zugänge gegenwärtig etwa dadurch, ob und in welcher Weise geschlechtliche und sexuelle Vielfalt einbezogen wird, inwieweit Geschlechter- und Sexualnormen reflektiert werden, Grenzachtung praktiziert wird und sexualisierte Übergriffe thematisiert werden (vgl. ebd.). Anders formuliert: Sie unterscheiden sich dahingehend, inwieweit sie machtreflektiert und gewaltinformiert ausgerichtet sind und die Bewegungen der Dynamisierung von Geschlecht, Sexualität und Lebensform aufgreifen.

\subsection{Sexualität als Bedürfnis, Energie, Identität oder Praxis?}

Wie wird nun Sexualität in der sexualfreundlichen zeitgenössischen Fachliteratur verstanden bzw. definiert?

Explizit ausformulierte Sexualitäts-Verständnisse findet sich im Feld der Sexualpädagogik zum einen in programmatischen Texten, wie etwa Richtlinien, Grundsatzerlässen und Empfehlungen (vgl. etwa BMBF 2015; WHO/BZgA 2011), und zum anderen in manchen theoretischen Texten (vgl. exemplarisch Sielert 2015). In der Überarbeitung des Grundsatzerlasses Sexualpädagogik in Österreich, der die programmatische Grundlage für schulische Sexualerziehung bildet, wird Sexualität »als ein positives, dem Menschen innewohnendes Potential« und ein "ganzheitliches und in die emotionale und soziale Entwicklung eingebettetes Phänomen« verstanden (BMBF 2015: o.S.). Im Rahmenkonzept der Bundeszentrale für gesundheitliche Aufklärung (BZgA), die in Deutschland für den gesetzlichen Sexualaufklärungs-Auftrag zuständig ist, wird Sexualität als »ein existenzielles Grundbedürfnis des Menschen und ein zentraler Bestandteil seiner Identität und Persönlichkeitsentwicklung« verstanden, der »sowohl biologische als auch psychosoziale und emotionale Dimensionen« umfasst (BZgA 2016: 5).

15 Während in manchen Ländern wie den USA abstinence-based sex education noch immer sehr verbreitet ist (vgl. Scott 2013), finden sich in Deutschland und Österreich nur wenige sexualpädagogische Akteur:innen, die einen solchen Ansatz vertreten. Vielmehr sind Zugänge präsent, die im englischsprachigen Diskurs als comprehensiv sex education bezeichnet werden (vgl. dazu etwa UNESCO 2018). Zur Frage, was (neo-)emanzipatorische von sexualfreundlichen Zugängen unterscheidet, vgl. Thuswald 2021. 
In der sexualpädagogischen Fachliteratur wird häufig auf ein Verständnis von Sexualität als Lebensenergie Bezug genommen, das von Uwe Sielert formuliert wurde (2015 [2005]). Der Sexualpädagoge definiert Sexualität als »allgemeine auf Lust bezogene Lebensenergie, die sich des Körpers bedient, aus vielfältigen Quellen gespeist wird, ganz unterschiedliche Ausdrucksformen kennt und in verschiedenster Hinsicht sinnvoll ist.« (Ebd.). Sielert spricht von vier Sinnaspekten der Sexualität, die als Identität, Lust, Beziehung und Fortpflanzung bezeichnet werden (vgl. ebd.: 47f.). ${ }^{16}$ An der Formulierung seiner Definition fällt auf, dass Sexualität als Akteurin erscheint: Sie bedient sich des Körpers, sie wird gespeist und kennt unterschiedliche Ausdrucksformen. Es wird dabei nicht klar, ob Sexualität als Lebensenergie in Personen verortet gedacht wird oder ob sie als etwas verstanden wird, das zwischen Personen oder in den Interaktionen situiert ist.

Als Begründung für seinen Zugang führt Sielert an, dass mit dem Begriff Lebensenergie die »Alltagserfahrung der drängenden Lust, das ungerichtete Leidenschaftliche, Lustvolle« benannt werde (ebd.: 42). Sexualität als Lebensenergie zu bezeichnen sei auch ein Versuch, sie vor der "Dramatisierung $\mathrm{zu}$ bewahren, die im Triebbegriff enthalten ist « (ebd.) und sie gleichzeitig vor den »blutleeren Begriffen des Verhaltens, der Motivation, der Kommunikation zu bewahren, die vordergründig wissenschaftlich korrekter erscheinen, letztlich aber an der >Entsexualisierung des Sexuellen [...] mitarbeiten « (ebd.).

Diese Argumentation ist insofern nachvollziehbar, als eine »Lebensenergie« in ihrer Nicht-Beobachtbarkeit tatsächlich von einer geheimnisvolleren Aura umgeben ist als der Begriff des Verhaltens oder der Kommunikation. Mir scheint jedoch fraglich, ob sich ein Verständnis von Sexualität als Lebensenergie als gesellschaftskritisch tauglicher Zugang eignet, bleibt doch vage, was mit Energie gemeint ist, wo sie situiert ist und wie sie mit gesellschaftlichen Verhältnissen zusammengedacht wird.

Die bis dato besprochenen Begriffsverständnisse begreifen Sexualität sehr umfassend und streichen ihre positive Bedeutung heraus. Diese breiten und gleichzeitig vagen Definitionen mögen als >gemeinsamer Nenner für Programmatiken sinnvoll sein und können in ihrem Bemühen, Sexualität in ihren unterschiedlichen Dimensionen und über die gesamte Lebensspanne hin-

16 Sielert spricht von einer »treibenden organischen Basis« von Sexualität, die im psychoanalytischen Jargon als Trieb bezeichnet wurde und heute »vorsichtiger als allgemeine Lebensenergie bezeichnet werden kann« (Sielert 2015 [2005]: 40). 
weg zu fassen, als durchaus progressiv eingeschätzt werden. Sie scheinen mir aber nicht hilfreich, wenn es darum geht, Geschlecht und Sexualität zu differenzieren, Machtverhältnisse rund um Sexualität zu analysieren oder mit Kindern und Jugendlichen über Sex(ualität) zu sprechen.

Ich plädiere deshalb in Anlehnung an die feministischen Soziologinnen Stevi Jackson und Sue Scott dafür, Sexualität als eine erotisch konnotierte soziale Praxis zu begreifen, die durch körperliche ebenso wie durch soziokulturelle Faktoren und gesellschaftliche Machtverhältnisse beeinflusst wird (vgl. Jackson/Scott 2010). ${ }^{17}$ Wie kann nun ein solcher Zugang im Sprechen über Sexualität mit Heranwachsenden eingesetzt werden?

\section{Mit Heranwachsenden über Sexualität sprechen}

Die Frage, wie mit Kindern und Jugendlichen über Sexualität gesprochen werden kann, steht im Zentrum vieler sexualpädagogischer Aus- und Fortbildungsveranstaltungen für pädagogisch Tätige. ${ }^{18} \mathrm{Im}$ folgenden Abschnitt stehen ausgewählte Vorschläge, die sexualpädagogische Fortbildner:innen zu dieser Frage anbieten, im Fokus der Analyse. Die Ausführungen basieren auf einer ethnografischen Studie, in deren Rahmen zwischen 2013 und 2017 rund 20 sexualpädagogische Aus- und Fortbildungsveranstaltungen für Lehrer:innen in Österreich beobachtet wurden (vgl. Thuswald 2021). ${ }^{19}$

Jackson und Scott schreiben: »[W]e reserve the term >sex and ssexual< for all that pertains to the erotic. While >sex denotes carnal acts, ssexuality< is a broader term referring to all erotically significant aspects of social life and social being-desires, practices, relationships and identities. «(2010: 83). Die feministischen Autorinnen unterscheiden zwischen Sexualität als einer Sphäre sozialen Lebens (»sphere of social life«) und Geschlecht als einer sozialen Differenzordnung bzw. einer kulturellen Unterscheidungskategorie (»a social division and a cultural distinction «) (vgl. ebd.: 83f.). Zum besseren Verständnis ziehen sie eine Analogie zu den Begriffen Arbeit und soziale Klasse: Arbeit sei - wie Sexualität - eine Sphäre sozialen Lebens, die durch Differenzordnungen, wie etwa Klasse oder auch Geschlecht, strukturiert wird (vgl. ebd.).

Die Vielfalt an Zugängen, mit Kindern und Jugendlichen über Sexualität zu sprechen, sprengt den Rahmen des Artikels, weshalb ich mich hier auf Möglichkeiten des sexualpädagogischen Umgangs mit den Fragen >Wie geht Sex? und >Wie entstehen Babys?؛ konzentriere.

19 Die folgenden Erklärungen zu Sexualität werden von den Referent:innen dieser Veranstaltungen bereits für jüngere Kinder in der Grundschule vorgeschlagen (vgl. Thuswald 2021). 
Manche Teilnehmer:innen dieser Bildungsveranstaltungen wünschen sich von den Fortbildner:innen sehr klare Handlungsorientierung, insbesondere dahingehend, wie (auch kleineren) Kindern erklärt werden kann, >wie Sex geht $<$ bzw. auch, >wie Babys entstehen Sprechweisen vorgestellt, die sexualpädagogische Aus- und Fortbildner:innen für das Sprechen mit Heranwachsenden vorschlagen. Ich wähle dabei zwei Sprechweisen aus, die sich sehr stark unterscheiden, um so unterschiedliche Perspektiven auf die Frage deutlich zu machen. ${ }^{20}$

\subsection{Sexualität als Ausdruck von Liebe}

Eine häufig vorgeschlagene Herangehensweise, mit Kindern über Sexualität zu sprechen, erklärt Sexualität als einen besonderen Ausdruck von Liebe zwischen zwei Erwachsenen. ${ }^{21}$ Auf diese Sprechweise wird häufig zurückgegriffen, wenn es um mögliche Antworten auf die Frage geht, wie Babys entstehen. Manche Aus- und Fortbildner:innen schlagen vor, die Antwort auf >die Frage nach den Babys in eine Geschichte zu verpacken. In diesen Geschichten kommen Ei- und Samenzellen sowie Penis und Scheide vor, im Zentrum steht jedoch die Liebe zwischen zwei Erwachsenen bzw. zwischen einem Mann und einer Frau. Sexualität wird als ein besonderer, nämlich koitaler Ausdruck von Liebe

20 Zwei weitere Zugänge sollen hier noch kurz benannt werden: Zum einen das Verständnis von Sexualität als Ceschlechtlichkeit, also die Gleichsetzung von Sexualität mit >Frau- oder Mann-Sein<. Dieses Verständnis kann als heteronormativ bezeichnet werden. Es beruht auf der Annahme von natürlicher und komplementär gedachter Zweigeschlechtlichkeit.

Ein zweiter Zugang, der mit Bezug auf das Kinderbuch »Das machen?« (Axster/Aebi 2012) in den Bildungsveranstaltungen eingebracht wird, beschreibt Sexualität in Analogie zu Sprache: »Sexualität ist wie eine Sprache, nur mit dem Körper. Und wie in jeder neuen Sprache, braucht es einige Zeit, bis Menschen einander verstehen. «(Axster/Aebi 2012 o.S.) Dieses Verständnis betont den Aspekt der Kommunikation, der Körperlichkeit und des Lernens und kann so einen guten Anknüpfungspunkt für das Sprechen über Sexualität bieten. Das Verständnis macht aber nicht deutlich, wie Sexuelles von anderen Formen körperlicher Interaktion (wie z.B. nicht-sexueller Zärtlichkeit, Kitzeln, Kuscheln, Körperpflege etc.) unterschieden werden kann.

21 In den Erklärungen und Geschichten sind diese Erwachsenen so gut wie immer ein Mann und eine Frau. Da aber die Generationsdifferenz zwischen Erwachsenen und Kindern stärker betont wird als die Geschlechterdifferenz und bisweilen auch die Möglichkeit der Liebe zwischen gleichgeschlechtlichen Partner:innen erwähnt wird, spreche ich hier von zwei Erwachsenen. 
dargestellt. Beim Sex, so eine Fortbildnerin, würden die beiden Erwachsenen sich streicheln, küssen und verwöhnen, was dazu führe, dass der Penis steif werde. Dieser würde dann genau in die Scheide passen und weil beide sich liebhaben, sei Sex angenehm, so die Erzählung.

Sex wird also in eine Liebesgeschichte verpackt und zumeist auch mit einem Kinderwunsch des Paares verbunden. Dabei wird betont, dass diese Liebe zwischen zwei Erwachsenen etwas anderes sei als etwa die Liebe zwischen Mutter und Kind. Sexualität als besonderer Ausdruck dieser Liebe sei nur etwas für Erwachsene. ${ }^{22}$

Diese Antwort auf die Frage nach der Entstehung von Babys ist insofern bemerkenswert, als es für Zeugung und Empfängnis - und das ist ja die Ausgangsfrage - weder Liebe noch einen Kinderwunsch braucht. Als Antwort auf die Frage, wie Babys entstehen, ist diese Antwort also sachlich nicht richtig. ${ }^{23}$ Vielmehr werden Aspekte von romantischer Liebe, Familienplanung und dem Fortpflanzungsaspekt von Sexualität im Sinne des Ideals der bürgerlichen heterosexuellen Kleinfamilie zu einer Geschichte verwoben.

\subsection{Sexualität als Wahrnehmung von Wollen, Anziehung und Erregung}

Eine andere Sprechweise, die in den Fort- und Weiterbildungen seltener beobachtbar ist, stellt Sexualität weder in den Kontext von Zeugung und Empfängnis, noch wird diese an Liebe oder Heterosexualität gekoppelt. ${ }^{24}$ In dieser Herangehensweise wird Sexualität als ein Tun verstanden und als eine Praxis erklärt, die durch die Wahrnehmung von Wollen, Anziehung und Erregung geprägt ist. Eine Fortbildnerin nützt für die Erklärung von Sexualität in diesem Sinne das Modell der Ampel. ${ }^{25}$ Grün steht dabei für Zustimmung, für das Ja zu einer

22 Dieser Zugang ist auch in vielen sogenannten Aufklärungsbüchern für Kinder zu finden.

23 Der BildungsRahmenPlan für elementarpädagogische Bildungseinrichtungen hält auch im Bereich der Elementarpädagogik »sachrichtige Antworten« auf Fragen von Kindern zu Sexualität für wichtig u.a. für die Prävention von sexuellem Missbrauch (Ämter der Landesregierungen der österreichischen Bundesländer u.a. 2009:17).

24 Eine Fortbildnerin, die diese Herangehensweise praktiziert, schlägt vor, die >Frage nach den Babys، erst im Anschluss an eine umfassendere Erklärung von Sexualität zu thematisieren.

25 Die Ampel wird in der sexualpädagogischen Arbeit bisweilen als Modell eingesetzt, wenn über Zustimmung, Einvernehmlichkeit und Grenzen gesprochen wird. Zu Einvernehmlichkeit in der sexuellen Bildung vgl. auch Debus i.d.B. sowie Dalhoff u.a. (2021). 
Handlung. Rot steht für Ablehnung, für ein Nein, während Gelb den Bereich des Unklaren und Unentschiedenen dazwischen symbolisiert. Die erwähnte Fortbildnerin benutzt das Ampelmodell, um Kindern Sex zu erklären: Sie hält fest, dass zum Sex drei Dinge gehören würden. Der Kopf müsse »ja« zum Sex sagen, also die Ampel im Kopf müsse auf Grün stehen, was als Wollen bezeichnet werden kann. Weiters müsse es im Bauch, wo das Nähe-Distanz-Gefühl verortet sei, eine grüne Ampel geben, die sich als Anziehung bzw. Nähewunsch benennen lässt. Die dritte grüne Ampel brauche es an den Genitalien, wo die Referentin in ihrem Modell die Erregung situiert. Damit also eine Praxis als Sex zu bezeichnen sei, müssen bei allen Beteiligten drei Ampeln grün anzeigen. $^{26}$

Bei diesem Erklärungsansatz zu Sexualität geht es also nicht um die Frage des Geschlechts, nicht um Fortpflanzung und nicht um die Art der Handlung oder die Anzahl der Beteiligten. Dieses Verständnis koppelt Sex auch nicht an Liebe. ${ }^{27}$ Vielmehr wird Sexualität als eine Praxis besprochen, die durch eine bestimmte Wahrnehmung gekennzeichnet ist, nämlich die Wahrnehmung von Wollen (Kopf), Anziehung (Bauchgefühl) und Erregung (Genitalien) bei allen Beteiligten.

\subsection{Sprechweisen kritisch befragen}

Wie sind diese beiden sexualpädagogischen Sprechweisen zu Sexualität aus einer macht- und differenzreflektierenden Perspektive einzuschätzen?

Wenn Sexualität als besonderer Ausdruck von Liebe zwischen zwei Erwachsenen verstanden wird, schließt dies nicht-heterosexuelle Interaktionen nicht per se aus. Es steht jedoch zumeist die Frage nach den Babys und damit Koitus und Fortpflanzung im Fokus. Das Verständnis ist also tendenziell heteronormativ und penetrationsorientiert. Geschlechtliche, sexuelle und amouröse Vielfalt (vgl. Debus i.d.B.) bleibt dabei unsichtbar - auch die Vielfalt heterosexueller Ausdrucksformen sowie unterschiedliche Zugänge zur Familienplanung. ${ }^{28}$

Wenn zwei Menschen Sex haben, so ergänzt die Referentin, müssen also sechs Ampeln auf Grün stehen, bei drei Personen seien es bereits neun Ampeln usw. (vgl. Thuswald 2021).

27 Die Fortbildnerin erwähnt zwar, dass Sex besonders schön sein kann, wenn die Beteiligten sich lieben, doch ist Liebe in ihren Ausführungen kein konstitutiver Teil des Sexualitäts-Verständnisses. 
Auch aus der Perspektive der Prävention von sexualisierter Gewalt betrachtet scheint es nicht sinnvoll, Sexualität normativ an Liebe zu koppeln, weil dies erschwert, die Unterscheidung zwischen Sex und Übergriffen zu besprechen und Erfahrungen von Übergriffen einordnen und benennen zu können. Ein Grund dafür liegt darin, dass Personen, die Kinder sexuell ausbeuten, diesen bisweilen sagen, dass sie sie lieben bzw. von diesen verlangen, dass sie bestimmte Dinge für sie aus Liebe tun. Die Fachstelle Selbstlaut, die in der Prävention von sexualisierter Gewalt an Kindern und Jugendlichen arbeitet, weist darauf hin, dass Kinder, die sexualisierte Gewalt gegen sich, ihre Geschwister oder ihre Mutter erlebt haben, den Satz »Wenn man sich liebt, wollen beide Geschlechtsverkehr« nicht mit ihrer Lebensrealität in Verbindung bringen können. Anders sei dies mit der Botschaft "Sex kann sich gut und angenehm anfühlen, wenn beide es wollen « (Selbstlaut 2020: 6f.). Diese sei offener und ehrlicher und könne auch von einem Kind mit Gewalterfahrung in Bezug zur eigenen Erfahrung gebracht werden (vgl. ebd.).

Die Sprechweise von Sexualität als Ausdruck von Liebe zwischen Erwachsenen ist demnach aus Präventionsperspektive problematisch und bietet nur insofern eine Anschlussstelle, als sie von Handlungen unter Erwachsenen spricht und daran anknüpfend thematisieren werden kann, dass sexuelle Handlungen zwischen Erwachsenen und Kindern aufgrund des Machtgefälles und der fehlenden Einwilligungsfähigkeit von Kindern nicht in Ordnung sind.

Dieser Zugang schließt durch den Fokus auf Erwachsene jedoch sexuelle Erfahrungen zwischen Jugendlichen sowie Solosex bzw. Selbstbefriedigung aus und bietet durch die Fokussierung auf Liebe auch keine Klarheit für die Unterscheidung zwischen Übergriffen und einvernehmlichem Sex ohne Liebe. $^{29}$

Der zweite vorgestellte Ansatz, über Sexualität als Wahrnehmung von Wollen, Anziehung und Erregung zu sprechen, ermöglicht es, von geschlechtlicher, sexueller und amouröser Vielfalt auszugehen. Diese Sprechweise lässt offen, welches Geschlecht die Beteiligten haben, wie viele Personen beteiligt sind

tät beziehen, also homosexuelle Beziehungen erwähnen, nicht jedoch eine Vielfalt an Begehrens-, Liebens- und Lebensweisen einbeziehen.

29 Es ist beobachtbar, dass in Aus- und Fortbildungen, in denen dieses SexualitätsVerständnis dominant ist, kaum Wissen über sexualisierte Übergriffe bzw. sexuelle Ausbeutung vermittelt wird. 
und was sie genau miteinander machen. Auf Basis eines solches Verständnis liegt es nahe, darauf hinzuweisen, dass Menschen verliebt sein können, ohne Sex zu wollen, oder Sex wollen können, ohne verliebt zu sein. ${ }^{30}$ Diese Sprechweise ist also inklusiver als Erstere und bietet größere Spielräume für den Einbezug unterschiedlicher Lebensrealitäten. Eine solche Herangehensweise knüpft zudem an der Lebensrealität von Kindern und Jugendlichen an, insofern Wollen und Nicht-Wollen sowie Anziehung, Ablehnung und körperliches Lustempfinden Erfahrungen sind, die Teil der Lebenswelt von Heranwachsenden sind. ${ }^{31}$

Das Sprechen von Sexualität als Wahrnehmung von Wollen, Anziehung und Erregung regt dazu an, die eigenen Gefühle und Empfindungen wahr und ernst zu nehmen sowie auf die grünen Ampeln der anderen Person(en) zu achten. Diese Herangehensweise unterstützt damit Fähigkeiten und Haltungen, die der Prävention von sexualisierten Übergriffen förderlich sind (vgl. dazu Vasold, Freund und Schneider i.d.B.). Eine solche Herangehensweise bietet auch Anknüpfungspunkte, um darüber zu sprechen, dass auf die eigene Wahrnehmung zu hören zumeist dann am schwersten fällt, wenn der Wunsch nach Intimität und Sex von einer geliebten Person ausgeht - oder wenn die andere Person als viel mächtiger wahrgenommen wird. Auch die Unterscheidung von Anziehung und Erregung kann für die Vorbeugung bzw. das Erkennen von sexuellen Übergriffen bedeutsam sein: Es kommt vor, dass sexualisierte Übergriffe Erregung bei der betroffenen Person hervorrufen, was von dieser als sehr verwirrend erlebt werden kann. Deshalb ist es wichtig, Heranwachsende darin zu bestärken, das `komische oder unangenehme Gefühl ernst zu nehmen, das Betroffene am Beginn von sukzessiven Grenzüberschreitungen zumeist wahrnehmen. ${ }^{32}$ Dieses >komische Gefühk kann als Bauchgefühl, das für Nähe- und Distanzwünsche steht, mit Kindern und Jugendlichen besprechbar gemacht werden.

30 In Veranstaltungen, die ein solches Verständnis vertreten, lässt sich beobachten, dass Referent_innen im Sprechen über Sexualität Ceschlechter- und Sexualnormen durchkreuzen; etwa indem sie Sexualität nicht nur auf zwei Personen beschränkt darstellen oder indem sie von gleichgeschlechtlichem Verlieben oder Sex sprechen, ohne dies in >besondernder Weise als shomosexuell zu markieren.

31 Dabei wird, so lässt sich beobachten, klar zwischen kindlichen und erwachsenen sexuellen Ausdrucksformen unterschieden, was in dieser knappen Ausarbeitung jedoch nicht so deutlich wird.

32 Ernst nehmen kann dann etwa heißen, die eigenen Grenzen klarer deutlich zu machen oder sich Hilfe zu holen. 
Einen guten Anknüpfungspunkt für Präventionsarbeit bietet auch die Ampelfarbe Gelb, mit Hilfe derer auf den Bereich zwischen einem klaren Ja und einem klaren Nein verwiesen werden kann. So können Ambivalenzen, Widersprüchlichkeiten und Verletzbarkeiten besprochen und mögliche Umgangsformen damit thematisiert werden.

Sexualität als Wahrnehmung von Wollen, Anziehung und Erregung zu besprechen, bietet also eine handlungspraktische Basis für Gewaltprävention und ermöglicht einen selbstverständlichen Einbezug von vielfältigen Liebens-, Begehrens- und Lebensformen, ohne diese im Sinne eines Otherings zu >besondern<.

Auch diese Herangehensweise hat jedoch wie jedes Modell ihre Grenzen: So vernachlässigt sie etwa Reproduktions-, Identitäts- und TranszendenzAspekte von Sexualität und verortet Erregung vorrangig genital. Diesem $\mathrm{Zu}$ gang mag auch vorgeworfen werden, dass Sexualität sehr individuell gefasst wird und die gesellschaftliche Dimension vernachlässigt wird. Meiner Einschätzung nach bietet dieser Zugang jedoch eine gute Ausgangsbasis, um zu besprechen, dass die Wahrnehmung von Wollen, Anziehung und Erregung emotional und sozial komplex und von (vergeschlechtlichten) sozialen Normen und Machtverhältnissen durchzogen ist.

\section{Sexualität als soziale Praxis besprechbar machen}

Meine kritische Befragung unterschiedlicher Zugänge zielt darauf ab, gängige Sprechweisen über Sexualität auf ihre impliziten Annahmen und mögliche Effekte in der pädagogischen Arbeit zu untersuchen und gleichzeitig auch Anknüpfungspunkte für machtreflektierende und normenkritische Zugänge herauszuarbeiten. ${ }^{33}$ An meiner Forschung zu sexualpädagogischen Aus- und Fortbildungen (vgl. Thuswald 2021) lässt sich - um ein weiteres Beispiel zu nennen - auch zeigen, wie unterschiedlich ein und dieselbe Übung gestaltet werden kann: Bei einer sexualpädagogisch häufig eingesetzten Übung Wörter-Battle, Penivagitus oder Sex-Quiz genannt - werden in einen Gruppenwettbewerb Synonyme für Sex und für Genitalien gesammelt. Diese Methode kann in einer Weise angeleitet werden, die Geschlechtervielfalt unsichtbar

33 Es geht mir dabei nicht darum, das >beste<Verständnis oder >den richtigen Zugang zu Sexualität zu finden. 
macht, heteronormative Vorstellungen reproduziert und die Klitoris als wichtiges Sexualorgan ausblendet. Eine solche Variante der Übung findet sich insbesondere in Veranstaltungen, die Sexualität mit Geschlechtlichkeit gleichsetzen oder als Ausdruck von Liebe verstehen. Dieselbe Übung kann jedoch auch so gestaltet werden, dass Geschlechter- und Sexualnormen besprechbar gemacht und durchkreuzt werden, was verstärkt in Veranstaltungen zu beobachten ist, die von Sexualität als einer sozialen Praxis ausgehen, die von Wollen, Anziehung und Erregung geprägt ist, oder die mit Sexualität als Sammelbegriff arbeiten (vgl. ebd.).

Aus einer macht- und differenzreflektierenden Perspektive und auf Basis der Ergebnisse meiner ethnografischen Forschung scheint mir für sexualpädagogische Praxis ein Zugang sinnvoll, der Sexualität als Praxis ins Zentrum stellt, anstatt von Sexualität als Identität oder Energie zu sprechen. Als theoretischer Bezugspunkt für einen solchen Zugang bietet sich etwa die obengenannte Arbeit von Jackson und Scott an (2010), die von Sexualität als erotisch konnotierter sozialer Praxis ausgeht, die durch körperliche ebenso wie durch soziokulturelle Faktoren beeinflusst wird und von Machtverhältnissen durchzogen ist. ${ }^{34}$ Ein praktisches sexualpädagogische Beispiel für das Sprechen über Sexualität als erotische soziale Praxis ist der bereits vorgestellte Zugang über das Modell der Kopf-, Bauch- und Genitalien-Ampeln. Dieser bietet Anknüpfungspunkte für das Einbeziehen von geschlechtlicher, sexueller und amouröser Vielfalt wie auch für Gewaltprävention.

Darüber hinaus scheint es mir sowohl für Forschung als auch für sexualpädagogische Praxis sinnvoll, den Begriff Sexualität weniger zentral zu setzen und, wo immer möglich, präzisere Begriffe zu verwenden. Sexualität mag als Sammelbegriff (alltags-)tauglich sein. Ich schlage aber vor, ihn nur dort zu verwenden, wo er als solcher treffend ist und ansonsten nach jenen Begriffen $\mathrm{zu}$ suchen, die genauer benennen, was gemeint ist - sei es nun Lust, Famili-

34 Zur Erklärung von geschlechtlicher, sexueller und amouröser Vielfalt können die Arbeiten von Julia Serano hilfreiche Anknüpfungspunkte bieten. Die Biologin und Transaktivistin benutzt die Analogie zum Ceschmackssinn, um die Verwobenheit von biologischen und sozialen Faktoren in der Herausbildung von geschlechtlichen Selbstverständnissen und Begehrensweisen zu erklären (vgl. Serano 2013: 155ff.). 
enplanung, Liebe, Erregung, Begehren ${ }^{35}$, romantische Beziehung, Intimität oder die Präferenz für Sexualpartner:innen eines bestimmten Geschlechts.

Damit soll nicht negiert werden, dass Liebe, Lust, Intimität, Beziehungen usw. im Alltag miteinander verflochten sind und fließend ineinander übergehen. Vielmehr halte ich es für wichtig, die unterschiedlichen Aspekte und ihre Verflechtungen in der pädagogischen Praxis besprechbar zu machen, wofür es differenzierte Begriffe und eine klare Sprache braucht. Dies ist - wie ich exemplarisch an der Unterscheidung von Sex und Liebe gezeigt habe - nicht zuletzt wichtig für die Prävention von sexualisierter Gewalt.

Eine differenzierte Wahrnehmung und eine ebensolche Sprache für Körper und sexuelle Praxis, für individuelle Erfahrungen und gesellschaftliche Normen zu entwickeln, scheint mir wesentlich für emanzipatorische sexuelle Bildung. ${ }^{36}$ Schule als »logozentrische« Institution (vgl. Müller 1992: 129ff.) mit ihren bisweilen recht einengenden Strukturen mag hinsichtlich emanzipatorischer sexueller Bildung ihre Grenzen haben (vgl. ebd.; Sattler/Thuswald i.d.B.). Sie kann aber jedenfalls Spielräume für Begriffsarbeit im weiten Sinne eröffnen: für das Aussprechen und Besprechen sexuell konnotierter Wörter, für das Befragen dominanter Sprache und damit verbundener Vorstellungsbilder, für das Anbieten und lustvolle Entwickeln von alternativen Begriffen und Sprechweisen sowie nicht-sprachlichen Ausdruckformen.

Damit dies gelingen kann, scheint es mir wichtig, etwa in der Aus- und Fortbildung oder der kollegialen Beratung Denk-, Gesprächs- und Bildungs-

35 In der Publikation »Teaching Desires« (Thuswald/Sattler 2016) und zwei Forschungsund Bildungsprojekten am Institut für das künstlerischer Lehramt (Imagining Desires und Reflecting Desires) wurde damit experimentiert, den Begriff desires ins Zentrum sexualpädagogischen Nachdenkens zu stellen. Anders als der deutsche Begriff des Begehrens ist dieser inhaltlich breiter und hat einen erkennbaren Plural. Sowohl desires als auch Begehren sind jedoch Begriffe, die nicht ohne weiteres an Alltagsdiskurse anschließen. Dennoch meine ich, dass sie Potenzial bergen, das noch nicht entfaltet wurde. Interessanterweise plädieren auch Markus Hoffmann und Matthias Proske in ihrem Artikel »Der pädagogische Blick auf Sexualität« (2017) für die Verwendung des Begriffs Begehren. Vgl. dazu auch die englischsprachige Debatte um Politics of Desires/Pleasure in Sexuality Education (siehe etwa Fine 1988; Tolman 2005; Allen u.a. 2014).

36 So hat etwa Bini Adamczak als Alternative zu einer penetrationsorientierten Sprache den Begriff der Circlusion vorgeschlagen, der das aktive Moment des Umschließens betont (vgl. 2016). Carolin Emcke sucht in ihrem Buch »Wie wir begehren« (2013) angesichts der »eingeübten Formen des Sprechens über Homosexualität« eine eigene Sprache, die »tatsächlich so genau, so präzise, so zart, so leise ist, dass ich darin mein Begehren ausdrücken kann.« (vgl. Emcke zit.n. Meßmer/Villa 2014). 
räume zu öffnen, in denen (angehende) Lehrer:innen, die eigene Lust an Begriffsarbeit erkunden und Möglichkeitsräume sexualpädagogisches Handeln entdecken und entwickeln können.

\section{Literatur}

Adamczak, Bini (2016): Come on. Über ein neues Wort, das sich aufdrängt und unser Sprechen über Sex revolutionieren wird. In: Missy Magazine. Magazin für Pop, Politik und Feminismus, 08.03.2016. Online unter: http s://missy-magazine.de/blog/2016/03/08/come-on [Zugriff: 24.08.2020].

Allen, Lousia (2011): Young People and Sexuality Education. Rethinking Key Debates. Hampshire: Palgrave Macmillian.

Allen, Lousia/Rasmussen, Mary Lou/Quinlivan, Kathleen (Hg.) (2014): The politics of pleasure in sexuality education. Pleasure bond. New York: Routledge.

Ämter der Landesregierungen der österreichischen Bundesländer/Magistrat der Stadt Wien/Bundesministerium für Unterricht, Kunst und Kultur (2009): Bundesländerübergreifender BildungsRahmenPlan für elementare Bildungseinrichtungen in Österreich. Online unter: https://www .bmbwf.gv.at/Themen/schule/bef/sb/bildungsrahmenplan.html [Zugriff: 06.02.2020].

Auma, Maisha-Maureen (2017): Kulturelle Bildung in pluralen Gesellschaften. Diversität von Anfang an! Diskriminierungskritik von Anfang an! In: Schütze, Anja/Maedler, Jens (Hg.): Weiße Flecken. Diskurse und Gedanken über Diskriminierung, Diversität und Inklusion in der kulturellen Bildung. München: kopaed, S. 61-76.

Axster, Lilly/Aebi, Christine (2012): DAS machen? Projektwoche Sexualerziehung in der Klasse 4c. Gumpoldskirchen: de'A Verlag.

Baumgartinger, Persson Perry (2017): Trans Studies. Historische, begriffliche und aktivistische Aspekte. Wien: Zaglossus.

BMBF - Bundesministerium für Bildung und Frauen (2015): Grundsatzerlass Sexualpädagogik. Online unter: https://www.bmbwf.gv.at/Themen/schu le/schulrecht/rs/1997-2017/2015_11.html [Zugriff: 22.08.2020].

Bollenbeck, Georg (1996): Bildung und Kultur. Glanz und Elend eines deutschen Deutungsmusters. Frankfurt a.M.: Suhrkamp. 
Bornstein, Kate (2013): My new Gender Workbook. A Step-by-Step Guide to Achieving World Peace Through Gender Anarchy and Sex Positivity. Oxford: Taylor \& Francis.

BZgA - Bundesszentrale für gesundheitliche Aufklärung (2016): Rahmenkonzept zur Sexualaufklärung der Bundeszentrale für gesundheitliche Aufklärung in Abstimmung mit den Bundesländern. Online unter: $h$ ttp://publikationen.sexualaufklaerung.de/index.php?docid=224 [Zugriff: 17.03.2017].

Dalhoff, Maria/Hempel, Stefanie/Önsür-Oluğ, Zeynep/Rauch, Bianca/Thuswald, Marion (Hg.) (2021): Sexuelle Einvernehmlichkeit gestalten. Theoretische, pädagogische und künstlerische Perspektiven auf eine Leerstelle sexueller Bildung. Hannover: fabrico.

Debus, Katharina (2015): Von Neoliberalismus und vom Zaubern. Plädoyer für utopische Momente. In: Hechler, Andreas/Stuve, Olaf(Hg.): Geschlechterreflektierte Pädagogik gegen Rechts. Opladen u.a.: Verlag Barbara Budrich, S. 383-385.

DGG - Deutsche Gesellschaft für Geschlechtserziehung (o.J.): Wir über uns. Online unter: http://dgg-ev-bonn.de [Zugriff: 18.01.2020].

Duden (o.J.): Sexualität. Online unter: https://www.duden.de/rechtschreibun g/Sexualitaet [Zugriff: 02.05.2020].

Emcke, Carolin (2013a): Wie wir begehren. Frankfurt a.M.: Fischer.

Engel, Antke/Schuster, Nina (2007): Die Denaturalisierung von Geschlecht und Sexualität. Queer/feministische Auseinandersetzungen mit Foucault. In: Anhorn, Roland/Bettinger, Frank/Stehr, Johannes (Hg.): Foucaults Machtanalytik und Soziale Arbeit. Eine kritische Einführung und Bestandsaufnahme. Wiesbaden: Springer VS, S. 135-153.

Federici, Silvia (2014): Caliban und die Hexe. Frauen, der Körper und die ursprüngliche Akkumulation. Übersetzt von Max Henninger. Wien: Mandelbaum.

Fine, Michelle (1988): Sexuality, schooling, and adolescent females: The missing discourse of desire. Harvard Educational Review, 58/1, S. 29-54.

Foucault (1983 [1976]): Der Wille zum Wissen. Sexualität und Wahrheit, Bd. 1. Frankfurt a.M.: Suhrkamp.

Grenz, Sabine (2018): Sex-Arbeit. Ein feministisches Dilemma. In: Femina Politica, 1/2018, S. 101-108. Online unter: https://doi.org/10.3224/feminapoli tica.v27i1.09. 
Gruber, Barbara/Ott, Veronika (2014): Sexualität und Geschlecht. Feministische Annäherungen an ein unbehagliches Verhältnis. Sulzbach/Taunus: Ulrike Helmer Verlag.

Gučanin, Jelena (2020): „Die Puppen haben alle Namen«. In: an.schläge, das feministische Monatsmagazin, 04/2020, S. 30-31.

Hark, Sabine/Villa, Paula-Irene (Hg.) (2015): Anti-Genderismus: Sexualität und Geschlecht als Schauplätze aktueller politischer Auseinandersetzungen. Bielefeld: transcript.

Hartmann, Jutta (2002): Vielfältige Lebensweisen. Dynamisierungen in der Triade. Geschlecht - Sexualität - Lebensform. Kritisch-dekonstruktive Perspektiven für die Pädagogik. Opladen: Leske + Budrich.

Hartmann, Jutta (2004): Dynamisierung in der Triade Geschlecht - Sexualität - Lebensform: dekonstruktive Perspektiven und allägliches Veränderungshandeln in der Pädagogik. In: Timmermanns, Stefan/Truider, Elisabeth/Sielert, Uwe (Hg.) (2004): Sexualpädagogik weiterdenken. Postmoderne Entgrenzungen und pädagogische Orientierungsversuche. Weinheim/München: Beltz Juventa, S. 59-78.

Henningsen, Anja/Timmermanns, Stefan/Tuider, Elisabeth (Hg.) (2016): Sexualpädagogik kontrovers. Weinheim/München: Beltz Juventa.

Hoffmann, Markus/Proske, Matthias (2017): Der pädagogische Blick auf Sexualität. In: engagement - Zeitschrift für Erziehung und Schule, Jg. 37, 3/2017, S. 121-130.

Illouz, Eva (2006): Gefühle in Zeiten des Kapitalismus. Frankfurt a.M.: Suhrkamp.

Illouz, Eva (2012): Warum Liebe weh tut. Berlin. Frankfurt a.M.: Suhrkamp.

Jackson, Stevi/Scott, Sue (2010): Theorizing Sexuality. London: Open University Press.

Kahlert, Heike/Blome, Agnes (2020): Politiken der Generativität und Reproduktive Rechte. Eine Einleitung. In: Femina Politica - Zeitschrift für feministische Politikwissenschaft, 2/2020, S. 9-23.

Kokits, Maya Joleen/Thuswald, Marion (2015): gleich sicher? sicher gleich? Konzeptionen (queer) feministischer Schutzräume. In: Femina Politica, 1/2015, S. 83-93. Online unter: https://www.ssoar.info/ssoar/handle/docu ment/43647 [Zugriff: 19.03.2020].

Meßmer, Anna-Katharina/Villa, Paula-Irene (2014): »Einfach nur privatistisch Intimitäten ausplaudern, kann nicht zielführend sein«. Anna-Katharina Meßmer und Paula-Irene Villa im Gespräch mit Carolin Emcke. In: Feministische Studien, Nr. 32, 1/2014, S. 78-85. 
Michel, Christian/Novak, Felix (1994 [1975]): Kleines Psychologisches Wörterbuch. Freiburg: Herder.

Mörsch, Carmen (2018): Critical Diversity Literacy an der Schnittstelle Bildung/Kunst: Einblicke in die immerwährende Werkstatt eines diskriminierungskritischen Curriculums. In: Kulturelle Bildung online. Online unter: https://www.kubi-online.de/artikel/critical-diversity-literacy -schnittstelle-bildung-kunst-einblicke-immerwaehrende-werkstatt [Zugriff: 09.06.2020].

Müller, Walter (1992): Skeptische Sexualpädagogik. Möglichkeiten und Grenzen schulischer Sexualerziehung. Weinheim: Deutscher Studien Verlag.

Offit, Avodah K. (1979): Das sexuelle Ich. Stuttgart: Ullstein.

Ortner, Rosemarie (2019): »Ich erlebe das schon als sehr gewaltvoll, dass ich so viele Zuschreibungen mache.« - Nachdenken über Gender und Zugehörigkeit in pädagogischer Weiterbildung zum Deutsch als ZweitspracheUnterricht. Heinemann, Alisha M.B./Khakpour, Natascha (Hg.): Pädagogik sprechen. Die sprachliche Reproduktion gewaltvoller Ordnungen in der Migrationsgesellschaft. Wiesbaden: Springer VS, S. 115-137.

Reich, Annie (2016 [1932]) Wenn dein Kind dich fragt ... Gespräche, Beispiele und Ratschläge zur Sexualerziehung. In: Zeitschrift für psychoanalytische Sozialpsychologie, 19/1, S. 17-41.

Retkowski, Alexandra/Thole, Werner (2012): Professionsethik und Organisationskultur. In: Thole, Werner/Baader, Meike/Helsper, Werner/Kappeler, Manfred/Leuzinger-Bohleber, Marianne/Reh, Sabine/Sielert, Uwe/Thompson, Christiane (Hg.): Sexualisierte Gewalt, Macht und Pädagogik. Opladen u.a.: Verlag Barbara Budrich, S. 291-316.

Retkowski, Alexandra/Treibel, Angela/Tuider, Elisabeth (2018): Handbuch Sexualisierte Gewalt und pädagogische Kontexte. Theorie, Forschung, Praxis. Weinheim/Basel: Beltz Juventa.

Richardson, Diane (2007): Patterned Fluidities: (Re)Imagining the Relationship between Gender and Sexuality. In: Sociology 41/3, S. 457-474. Online unter: https://doi.org/10.1177/0038038507076617.

Rubin, Gayle S. (2012 [1984]): Thinking Sex: Notes for a Radical Theory of the Politics of Sexuality. In: Dies.: Deviations. A Gayle Rubin Reader. Durham: Duke University Press.

Sager, Christin (2015): Das aufgeklärte Kind. Zur Geschichte der bundesrepublikanischen Sexualaufklärung (1950-2010). Bielefeld: transcript. 
Sager, Christin (2016): Annie Reichs sexualaufklärerische und -politische Arbeit im historischen Kontext. Ein Kommentar. In: Zeitschrift für psychoanalytische Sozialpsychologie, Jg. 19, 1/2016, S. 93-97.

Schmidt, Gunter (2014 [2004]): Das neue DerDieDas. Über die Modernisierung des Sexuellen. Gießn: Psychosozial-Verlag.

Scott, Brigitte C. (2013): Positioning sex educators. A critical ethnography of a professional development workshop. In: Sex Education. Sexuality, Society and Learning, Bd. 16, 6/2013, S. 660-673. Online unter: http://dx.doi.org/ 10.1080/14681811.2013.801013.

Selbstlaut (2020): Ganz schön intim. Sexualerziehung für 6-12 Jährige [sic!]. Unterrichtsmaterialien zum Download. Erstellt im Auftrag des Bundesministeriums für Unterricht, Kunst und Kultur. Aktualisierte Auflage. Online unter: https://selbstlaut.org/publikationen-und-materialien/unserepublikationen [Zugriff: 23.12.2020].

Serano, Julia (2013): Excluded. Making feminist and queer movements more inclusive. Berkely: Seal Press.

Sielert, Uwe (2003): Einführung in die Sexualpädagogik. Weinheim/Basel: Beltz.

Thole, Werner/Baader, Meike/Helsper, Werner/Kappeler, Manfred/LeuzingerBohleber, Marianne/Reh, Sabine/Sielert, Uwe/Thompson, Christiane (Hg.) (2012): Sexualisierte Gewalt, Macht und Pädagogik. Opladen u.a.: Verlag Barbara Budrich.

Thuswald (2021): Sexuelle Bildung ermöglichen. Sprachlosigkeit, Lust, Verletzbarkeit und Emanzipation als Herausforderungen pädagogischer Professionalisierung. Bielefeld: transcript.

Thuswald, Marion/Sattler, Elisabeth (2019): Critical Diversity in der kulturellen und sexuellen Bildung. Einblicke in das kunst- und sexualpädagogische Projekt Imagining Desires. In: Schulheft 175 (hg. von Eveline Christof und Julia Köhler). Wien/Innsbruck: Studienverlag, S. 82-96.

Thuswald, Marion/Sattler, Elisabeth (Hg.) (2016): teaching desires. Möglichkeitsräume sexueller Bildung im künstlerisch-gestalterischen Unterricht. Wien: Löcker.

Tolman, Deborah L. (2005): Dilemmas of desire: Teenage girls talk about sexuality. Cambridge: Harvard University Press.

UNSECO (Hg.): International technical guidance on sexuality education. An evidence-informed approach. Online unter: https://unesdoc.unesco.org/images/0026/002608/260840E.pdf $\quad[\mathrm{Zu}-$ griff: 27.02.2018]. 
VIMÖ (o.J.): Online unter: https://vimoe.at [Zugriff: 24.08.2020].

Wazlawik, Martin/Voß, Heinz-Jürgen/Retkowski, Alexandra/Henningsen, Anja/Dekker, Arne (Hg.) (2019): Sexuelle Gewalt in pädagogischen Kontexten. Aktuelle Forschungen und Reflexionen. Wiesbaden: Springer VS. WHO (2006): Defining sexual health. Report of a technical consultation on sexual health. 28-31 January 2002, Geneva. Online unter: https://www .who.int/reproductivehealth/topics/sexual_health/sh_definitions/en [Zugriff: 29.06.2020].

WHO Regional Office for Europe/BZgA (2010): Standards for Sex Education in Europe. A framework for policy makers, educational and health authorities and specialists. Online unter: https://www.bzga-whocc.de/fileadmin /user_upload/WHO_BZgA_Standards_English.pdf [Zugriff: 11.02.2020]. WHO-Regionalbüro für Europa/BZgA (2011): Standards für die Sexualaufklärung in Europa. Rahmenkonzept für politische Entscheidungsträger, Bildungseinrichtungen, Gesundheitsbehörden, Expertinnen und Experten. Online unter: https://www.bzga.de/infomaterialien/sexualaufklaeru ng/who-regionalbuero-fuer-europa-und-bzga-standards-fuer-die-sexua laufklaerung-in-europa [Zugriff: 18.01.2020]. 


\title{
Intimität
}

\author{
Anna Vida
}

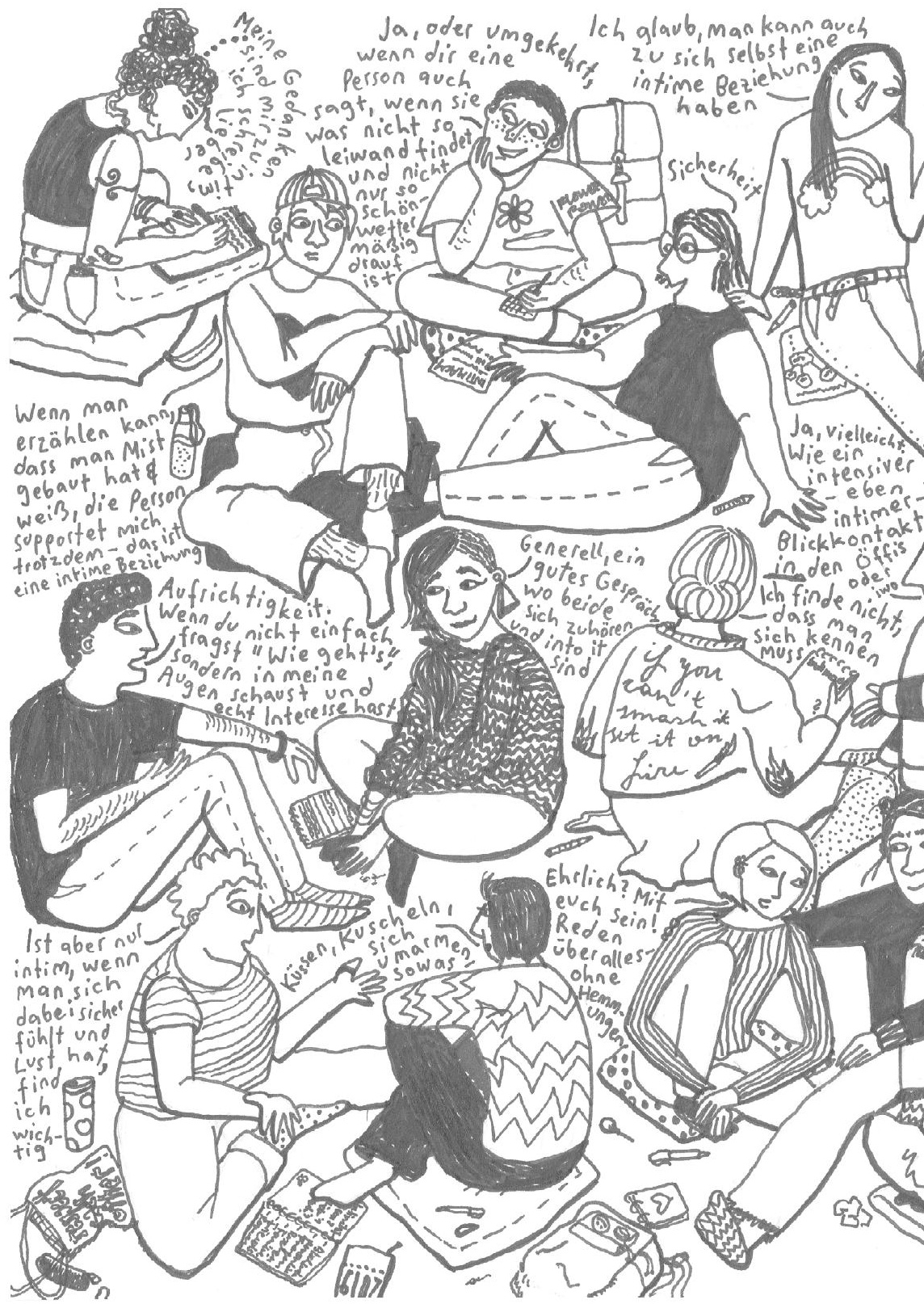


Fix.die urodistanzierte... Gefühle Arg guter lonpuy

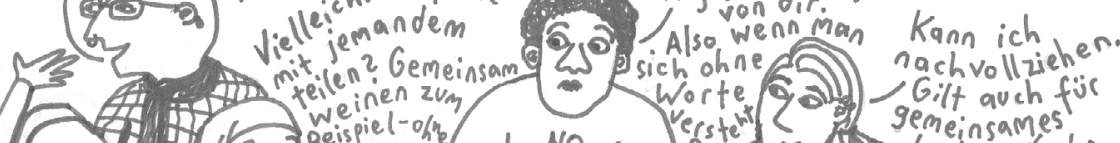

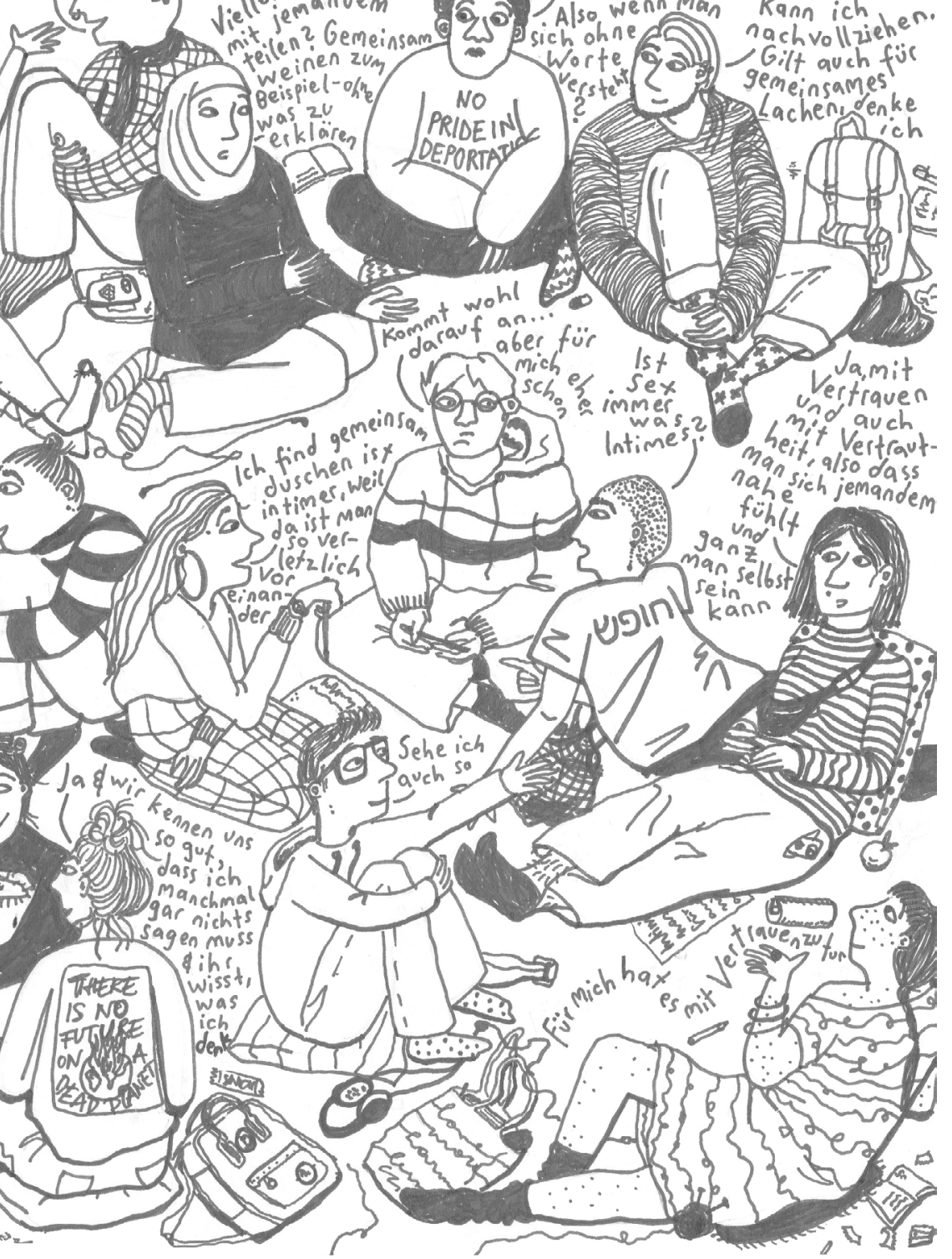





\section{Sexualerziehung - für wen? Die Reproduktion weißer Mittelklasse-Normen durch Sexualpädagogik in der Schule}

Dorottya Rédai

Die schulische Sexualerziehung kann für die sexuelle Bildung junger Menschen eine wichtige Rolle spielen. Aber welche Botschaften werden Schüler:innen durch Sexualpädagogik vermittelt? Welches Bild von Sexualität wird durch die pädagogischen Inhalte und Formate transportiert und in welchem Verhältnis stehen diese zu den sexuellen Erfahrungen von Jugendlichen? Wessen Lebensbedingungen werden adressiert und wessen bleiben außen vor? ${ }^{1}$

In diesem Beitrag untersuche ich, welche Botschaften über beginnende sexuelle und romantische Beziehungen von Heranwachsenden in der Sexualerziehung vermittelt werden. Ich werde dabei argumentieren, dass die Inhalte in der Schule häufig auf ein enges, normatives Verständnis von Sexualität rekurrieren, während Sexualität als komplex und zutiefst von Gender, Ethnizität und Klasse (class) beeinflusst zu verstehen ist. Ein solcherart eng ausgelegtes Konzept von Sexualität, das nur einen Beziehungstypus und ein Familienmodell als akzeptabel darstellt, spricht jene jungen Menschen nicht an, die keine Mittelschicht-Herkunft und/oder nicht den ethnisch-kulturellen Hintergrund der Mehrheit aufweisen oder deren Erfahrungen weder mit engen Modellen von Sexualität, Beziehung und Familie noch mit den an sie gerichteten kulturellen Erwartungen korrespondieren. Folglich bleiben im Sexualkunde-Unterricht die Erfahrungen, Probleme und Fragen vieler Jugendlicher womöglich unsichtbar oder/und unberücksichtigt. 
Grundlage meiner Analyse ist eine ethnografische Studie, durchgeführt zwischen 2009 und 2011 an einer Sekundarschule ${ }^{2}$ mit einem berufsbildenden, einem technischen und einem gymnasialen Zweig sowie einer angeschlossenen weiterführenden Fachschule für Chemietechnik in einer größeren Stadt in Ungarn. Ich beziehe mich unter dem Pseudonym Marzipanbäcker- und Konditor:innen-Schule (im Folgenden Marzipan) auf diese Einrichtung. ${ }^{3}$ Zur Zeit meiner Forschung kamen die meisten Schüler:innen dieser Schule aus der Arbeiterklasse und der unteren Mittelschicht. Ungefähr $20 \%$ der interviewten Schüler:innen bezeichneten sich als Roma oder ihre Herkunft als gemischt Roma-ungarisch; die übrigen Schüler:innen bezeichneten sich als ethnische Ungar:innen. Die Lehrenden waren allesamt weiße Ungar:innen.

Ich führte semi-strukturierte Interviews in kleinen - nach Geschlechtern getrennten - Gruppen mit ungefähr 90 zwischen 15 und 21 Jahren alten Schüler:innen und Einzelinterviews mit fünf Lehrenden, dem Schuldirektor und der Schul-Krankenpflegerin. Letztere war verantwortlich für die Durchführung des Sexualkunde-Unterrichts (szexuális nevelés) an der Schule. Ich beobachtete viele ihrer Unterrichtseinheiten für unterschiedliche Altersgruppen von der 9. bis zur 13. Schulstufe. In diesem Beitrag fokussiere ich meine Analyse auf Klasse und Ethnizität, weil die Überschneidungsbereiche dieser Kategorien mit Gender und Sexualität in der sexualpädagogischen Forschung wenig untersucht sind. Auch wenn meine Daten zehn Jahre alt sind und aus einem spezifischen lokalen Kontext stammen, schätze ich die Probleme, die sie sichtbar werden lassen, auch in der Gegenwart für relevant ein und gehe davon aus, dass in anderen Ländern durchgeführte Forschung zu ähnlichen Ergebnissen führen würde. ${ }^{4}$

2 Die Sekundarbildung umfasst in Ungarn Schüler:innen zwischen 14 und 18 bzw. 19 Jahren. Sie beginnt im neunten Schuljahr (nach acht Jahren Grundschule für 6- bis 14Jährige). Eine Schulpflicht besteht bis zum Alter von 16 Jahren, d.h. der Abschluss einer Sekundarschule ist nicht verpflichtend.

3 In dieser Schule können berufliche Qualifikationen für die Lebensmittelindustrie erworben werden: Bäcker:innen, Konditor:innen, Süßwarenerzeuger:innen, Bäckerei-/Konditoreitechniker:innen, Lebensmitteltechniker:innen.

4 Die vollständige Studie findet sich in meiner Monografie »Exploring Sexuality in Schools. The Intersectional Reproduction of Inequality« (Rédai 2019). 


\section{Sexualität von Kindern und Jugendlichen}

Ich arbeite mit einer breiten Definition des Begriffs Sexualität. Sexualität ist mehr als sexuelle Orientierung, Identität, Begehren, Verhalten, Praxis oder jegliche Kombination des Genannten. Sie umfasst biologische, psychologische, materielle, soziale, kulturelle, ethische und beziehungsmäßige Aspekte. Ich betrachte Sexualität nicht als individuelles Vermögen, sondern vielmehr als eine Praxis. Menschen pflegen von frühester Kindheit an sexuelle Praktiken, und Zuschreibungen geschlechtsspezifischer Sexualität spielen eine wichtige Rolle in der Sozialisierung und Identitätskonstruktion von Kindern in Kindergarten und Volksschule (vgl. Renold 2005; Epstein u.a. 2001), wenngleich Kinder selbstverständlich nicht dasselbe Verständnis von Sexualität haben wie Erwachsene. Sexualität ist nichts, das nur in der Schule präsent ist, sondern ihr kommt eine bedeutende Funktion in der Konstitution vieler Differenzachsen (insbesondere Gender, Ethnizität und Klasse) zu, die in Subjektivierungsprozessen junger Menschen und bei der (Re-)Produktion von sozialer Ungleichheit eine wichtige Rolle spielen (Rédai 2019).

Unter adoleszenten Heranwachsenden in den Sekundarschulen treten explizitere Formen von Sexualität auf. Dabei gilt es im Blick zu behalten, dass die Adoleszenz ein medikalisiertes und pathologisiertes Konzept darstellt. Dieser Lebensabschnitt gilt als turbulent und schwierig, als Phase des psychologischen und biologischen Übergangs hin zu einem >stabilen sensein (Waites 2005; Talburt 2004). Adoleszente Sexualität - als Bestandteil dieser >schwierigen Phase - - wird oft als problematisch bewertet; als etwas, das notwendigerweise von Erwachsenen kontrolliert und reguliert werden muss. Nachdem adoleszenter Sex überwiegend als gefährlich, gewaltsam und amoralisch konzeptualisiert wird, ist der dominanteste Diskurs in der Sexualerziehung ein >Risikodiskurs«, wie viele Autor:innen argumentieren (vgl. etwa Jackson/Weatherall 2010; Allen 2007; Alldred/David 2007). Dieser Diskurs suggeriert - so argumentiert beispielsweise Allen -, dass junge Menschen vor den potenziellen negativen Konsequenzen des Sexhabens geschützt werden müssen: »Ein solcher Diskurs verweist auf essentialistische Konzepte von Sexualität als biologisch determiniert und hormonell gesteuert, mit einer als gefährlich konstituierten Sexualität von Schüler:innen, denn sie kann junge Menschen dazu treiben, auf Arten und Weisen zu handeln, die ihrer Gesundheit schaden« (Allen 2007: 225).

Die Sexualität junger Menschen in der Schule wird nicht nur durch die Institution und die Lehrkräfte reguliert (intentional und nicht intentional), 
sondern auch über den Einfluss der Peers. Youdell (2005) beobachtete in ihrer schulethnografischen Arbeit etwa, wie aktiv Mädchen in der Konstituierung und Regulierung ihrer eigenen Heterofeminität sowie jener ihrer Peers partizipieren, indem sie untereinander eine moralische Hierarchie installieren, die auf sexuellem Verhalten und sexuellen Erfahrungen basiert. Viele Studien (vgl. etwa Pascoe 2007) analysieren die aggressive Konstruktion von Heteromaskulinität, durch die Burschen einander hinsichtlich ihres Verhaltens kontrollieren, um die außerordentlich engen Grenzen von Heteromaskulinität nicht zu überschreiten.

Mehrere Forscher:innen argumentieren, dass Sexualerziehung nicht nur heteronormativ, sondern auch ethnisierend und klassistisch wirkt und soziale Ungleichheiten verstärkt: Rahimi und Liston (2009) kamen für die USA zu dem Ergebnis, dass Lehrende aus der weißen Mittelklasse afroamerikanische und aus unteren Schichten stammende oder arme Mädchen anders behandelten - und zwar auf Basis rassistischer und schichtspezifischer Stereotype von adoleszenter Sexualität. In den Studien von Fields (2005) und Froyum (2010) zu Programmen Abstinenz-fokussierter Sexualerziehung, die auf Töchter aus schwarzen geringverdienenden Familien abzielten, ${ }^{5}$ porträtierten Lehrkräfte arme afroamerikanische Mädchen als hypersexualisiert und dem Risiko von Teenager-Schwangerschaften ausgesetzt. García (2009) erforschte, dass im Sexualkunde-Unterricht an manchen US-amerikanischen Schulen LatinaMädchen auf ähnliche Weise klassifiziert wurden. Sie galten als hypersexualisiert und -reproduktiv, während Latino-Burschen als Sexualtäter dargestellt wurden. Die heteronormativen, sexistischen und rassistischen Aussagen von Sexualkunde-Lehrkräften beschränkten für jugendliche Latinas den Zugang zu Informationen über Sexualität und verstärkten existierende genderbezogene, sexuelle und ethnische Ungleichheit.

Auch in der Marzipan vertraten manche Lehrpersonen ethnisierte Ansichten in Bezug auf die Sexualität von Schüler:innen. In solchen Diskursen wurden Roma-Mädchen - die meisten von ihnen gehörten auch unteren Sozialschichten an - als »Wohlfahrtsköniginnen« (welfare queens) und »Teenie-Mütter« bezeichnet, deren Reproduktionsmuster von ihren ethni-

Auf Abstinenz fokussierte Sexualerziehungs-Programme sind in den USA weitverbreitet. Deren Kernbotschaft lautet, dass der beste Weg, um sexuell übertragbare Krankheiten und frühe Schwangerschaften zu vermeiden, der Verzicht auf Sex vor der Ehe sei. 
schen Traditionen und ihrer sozialen Klassenzugehörigkeit definiert würden (vgl. Rédai 2019: 88).

\section{Doing sex education in der Marzipan}

Sexualkunde (szexuális nevelés) ist in Ungarn kein eigenständiges Unterrichtsfach. Die Art und Weise ihrer Erwähnung in bildungspolitischen Dokumenten lässt Ambivalenz und Unbehagen hinsichtlich des Umgangs mit Sexualität an Schulen sichtbar werden. Im zur Zeit meiner Feldarbeit gültigen Nationale Kernlehrplan 2007 wird Sexualerziehung in einem Satz erwähnt: »Es ist eine unvermeidliche Aufgabe der Schule, sich mit Themen der Sexualkultur und des Sexualverhaltens, mit der Vorbereitung auf ein Familienleben und auf verantwortungsvolle, freudvolle Beziehungen $\mathrm{zu}$ befassen " (A Nemzeti Alaptanterv 2007: 16). Den Umgang mit Sexualkultur und Sexualverhalten als schulische Notwendigkeit $\mathrm{zu}$ sehen, bedeutet ein Anerkennen der Existenz der Sexualität von Kindern und Adoleszenten, doch zugleich wird - wie das Bezugssystem »Vorbereitung auf ein Familienleben« nahelegt - Sexualität in die Zukunft verschoben. Im derzeit gültigen Nationalen Kernlehrplan von 2012 ist Sexualerziehung ähnlich knapp unter der Rubrik »Bildung für das Familienleben« angeführt (A Nemzeti Alaptanterv 2012: 10642)

Das 2013 eingeführte und derzeit gültige Rahmencurriculum ${ }^{6}$ diskutiert Sexualität in den Lehrplänen für Biologie und der Erziehung zum Familienleben. ${ }^{7}$ Die Lehrpläne für beide Fächer fassen Sexualität als heteronormativ, Eheschließung und Reproduktion als deren Voraussetzungen, Familie und Mutterschaft als ultimative Existenzweisen und Frauen und Männer als

6 In Ungarn ernhält der nationale Kernlehrplan generelle Leitlinien und Ziele für die Grundschulen und die weiterführenden (Sekundar-)Schulen. Das Rahmencurriculum regelt die Inhalte sowie die messbaren Inputs und Outputs von Schulfächern für jede Schulstufe. Schulen müssen ihren eigenen Lehrplan entwerfen, der inhaltlich nicht mehr als $10 \%$ vom Rahmencurriculum abweichen darf. Zur Zeit meiner Forschung war das Rahmencurriculum 2008 in Kraft. Sexualkunde schien im Fach Biologie auf und zwar als Teil einer längeren Passage, die auch die Prävention von Suchtgewohnheiten (Rauchen, Trinken, Drogen, ungesunde Ernährung) als Entwicklungsaufgabe festschrieb.

7 Erziehung zum Familienleben ist ein Querschnittfach, das 2013 für die 1. bis 12. Schulstufe eingeführt wurde. 
homogene, dichotome und komplementäre Gruppen. Sexuelle Minderheiten bleiben gänzlich unsichtbar. Diese Inhalte spiegeln den Zugang der aktuellen neokonservativen politischen Führung Ungarns zu Geschlecht und Sexualität. $^{8}$

Sexualkunde gilt in Ungarn allgemein als Teil der Gesundheitserziehung. Das Gesetz zum öffentlichen Gesundheitswesen 1997 (\$38.1, \$ 38.2) schreibt den Schulen vor, für Gesundheitserziehung zu sorgen. Aufgrund fehlender präziser Regelungen ist es an jeder einzelnen Schule, zu entscheiden, ob Sexualkunde angeboten wird. Es gibt dazu keine Daten - etwa was die Schultypen betrifft, die Anzahl der Schulen, der Wochenstunden, die der Sexualerziehung gewidmet werden, oder dazu, wer diesen Unterricht in Schulen durchführt. In manchen Schulen sind Biologielehrer:innen, Schulpsycholog:innen oder externe Expert:innen zuständig. In der Marzipan - wie an vielen anderen Schulen - kam die Lehrer:innenschaft überein, dass die Sexualkunde Aufgabe der Schulkrankenpflegerin sein soll.

Sexualkunde war in der Marzipan Teil eines GesundheitserziehungsModuls, dessen Curriculum die Schulkrankenpflegerin Vera entwickelt hatte. Gesundheitserziehung war nicht verpflichtend, weshalb Vera das zehnstündige Modul nur in Schulklassen durchführte, deren Lehrkräfte sie dazu einluden. Das Modul inkludierte für gewöhnlich fünf bis sechs Stunden Sexualkunde; die Themen der übrigen vier bis fünf Stunden waren Substanzmissbrauch und Abhängigkeiten (Drogen, Rauchen, Alkohol) sowie gesunder Lebensstil (Sport, Ernährung, Körperpflege). Der Unterricht umfasste dabei meist die folgenden Bereiche: den Prozess biologischer Reife und die psychosexuelle Entwicklung; den Beginn des Sexuallebens; adoleszente/jugendliche Beziehungen und monogame, langfristige Erwachsenen-Beziehungen; Familiengründung; Empfängnisverhütung und Prävention von sexuell übertragbaren Infektionen; Schwangerschaft, Geburt und Kinderpflege.

Vera beschwerte sich wiederholt über den Mangel an Handbüchern und anderen Unterrichtsmaterialien/Unterrichtsmitteln. Sie verwendete eine Kompilation visueller Materialien, zusammengestellt von ihren Kolleg:innen, und einige der wenigen verfügbaren audiovisuellen Medien. Sie zog auch ihre Erfahrungen mit der Sexualkunde in der eigenen Schulzeit und mit den Interessen von Heranwachsenden heran und versuchte das Material - ihren schen (und ebenso tschechischen und österreichischen) Lehrplänen vgl. Rédai/Sáfrány 2019. 
Erfahrungen im Unterricht entsprechend - alljährlich zu aktualisieren. Sie betonte mir gegenüber, dass zu ihrer Studienzeit Schulkrankenpfleger:innen keine methodische Ausbildung erhalten hatten, und dass sie der Frage von Pädagogik/Didaktik und Unterrichtsressourcen ratlos gegenüberstehe.

Das Format für Sexualkunde in der Marzipan waren 45-minütige Unterrichtseinheiten, wöchentlich oder alle zwei Wochen. Diese Einheiten wurden in ein Zeitfenster der Klassenlehrerin oder des Klassenlehrers geschoben, üblicherweise in die letzte Unterrichtsstunde, um zwei oder drei Uhr nachmittags, wenn die Schüler:innen müde waren und nur noch so schnell wie möglich raus aus der Schule wollten. Die Klassen bestanden aus rund 30 Schüler:innen, Burschen und Mädchen gemischt, und Frontalunterricht überwog. Die Schulstunden endeten mitunter chaotisch, speziell in den Klassen mit Schüler:innen im Alter von 15, 16 Jahren und mit Burschen, die aktiv den Unterricht störten.

Sexualität im Klassenverband zu diskutieren, bereitete den Schüler:innen gemischte Gefühle: Danach befragt, äußerten viele das Bedürfnis, über Begehren, Lust und Intimität zu sprechen, fanden das Setting des SexualkundeUnterrichts in der Schulklasse aber dazu nicht wirklich geeignet. Neben den Sexualkunde-Stunden bot Vera den Schüler:innen Beratungsstunden an und war während ihrer Bürozeiten immer für sie erreichbar. Ungefähr fünf bis zehn Schüler:innen pro Woche nutzten diese Möglichkeit der Beratung, Mädchen und Burschen gleichermaßen, manche regelmäßig, andere gelegentlich oder nur für ein Schwätzchen. So schien der größte Nutzen des SexualkundeUnterrichts darin zu liegen, dass Vera dabei das Vertrauen vieler Schüler:innen gewann und jene, die Hilfe und Unterstützung brauchten, zu ihr kommen und mit ihr sprechen konnten. In diesem Sinne erscheint individuelle Beratung unter den gegebenen Umständen die bessere Methode zu sein, weil sie vertraulich ist und die persönlichen Probleme rund um Sexualität adressiert und aufgreift. Freilich sind die Kapazitäten limitiert; eine einzelne Schulkrankenpflegerin hat - neben ihren sonstigen Verpflichtungen - nicht die Zeit, vielen Schüler:innen individuelle Beratung anzubieten.

\section{Aussagen über den Beginn eines Sexuallebens und zu romantischen Beziehungen}

Im Folgenden werde ich Diskurse über die Aufnahme eines aktiven Sexlebens sowie über adoleszente romantische und sexuelle Beziehungen untersuchen 
und dabei herausarbeiten, wie solche Diskurse zur Reproduktion schichtspezifischer und ethnischer Ungleichheit im Bildungsbereich beitragen können.

\section{Sex im eigenen Schlafzimmer?}

Was den Beginn eines aktiven sexuellen Lebens betrifft, enthalten pädagogische Botschaften häufig folgende idealisierte Vision: Jugendliche entdecken zuerst ihren eigenen Körper, dann treten sie nach und nach in eine intime Beziehung mit einer Person des anderen Geschlechts und erleben ihre erste sexuelle Begegnung, basierend auf gegenseitigem Begehren und Einvernehmlichkeit, einer Vereinbarung hinsichtlich der Verhütungsmethode und an einem ungestörten, intimen Ort. Ein signifikanter Teil der Schüler:innen in meinem Sample machte demgegenüber am Beginn des Sexuallebens völlig andere Erfahrungen. Etliche Mädchen verknüpften mit der ersten sexuellen Begegnung sexuelle Belästigung durch ein männliches Familienmitglied, Nötigung oder Vergewaltigung durch den ersten intimen Partner oder Freund. Mädchen wie Burschen berichteten von ersten sexuellen Begegnungen in betrunkenem Zustand oder unter Drogeneinfluss, und manche Burschen erzählten, vom Vater oder Onkel zu Prostituierten gebracht worden zu sein, um >zu lernen, wie man Sex hat.$^{9}$

Vera zufolge ist eine ruhige Umgebung beim Sex sehr wichtig: »Beim ersten Mal ist es sehr wichtig, dass du in einer Umgebung bist, in der dich niemand stört, dass du nicht fürchten musst, dass dich jemand überrascht.« (Vera, Schulkrankenpflegerin, Sexualkunde, 10. Schulstufe)

Aus den Interviews wurde deutlich, dass viele der Schüler:innen an dieser Schule in Familien lebten, deren finanzielle Lage und Wohnsituation keine ruhige Umgebung erlaubten.

»Wir sind zu sechst und wohnen in einem Apartment mit zwei Schlafzimmern. Die beiden Zimmer haben Hochbetten, ich teile ein Zimmer mit meiner jüngeren Schwester, und wenn mein Freund bei mir schlief - und das ist der Grund, warum er nicht mehr bei mir übernachtet - dann mussten wir das arme Mädchen rausschmeißen, sogar, wenn wir gar nichts machten. Das war weder für sie noch für uns lustig, wir konnten nicht einmal kuscheln [...].« (Nati, 18, weißes Mädchen, Gruppeninterview) 
Dieses Zitat zeigt, dass >Sex im eigenen Schlafzimmer der Vorstellung privilegierter Mittelschicht-Angehöriger entspricht. Für arme und/oder der Arbeiterklasse oder der unteren Mittelschicht zuzurechnende Familien ist es nicht selbstverständlich, dass jede:r Sex im eigenen Schlafzimmer haben kann, nachdem Geschwister sich oft ein Zimmer teilen. In der Tat hatten viele der Schüler:innen der Marzipan kein eigenes Schlafzimmer, manche lebten in staatlichen Institutionen, zusammen mit anderen in gemeinsamen Zimmern. Darüber hinaus steht das Thema sSex im eigenen Schlafzimmer auch in Bezug zum Alter, denn sogar manche jener, die über ein eigenes Schlafzimmer verfügten, fühlten sich unwohl, Sex zu haben, während die Eltern zuhause waren. Zudem sagten mehrere der Interviewpartner:innen, dass ihre Eltern dachten, sie seien noch jjungfräulichı, und sie sich vor Bestrafung fürchteten, falls ihre sexuelle Aktivität zutage träte. Sex zuhause lag also jenseits der Möglichkeiten. Infolgedessen ist Sex außerhalb des Schlafzimmers für diese jungen Leute eher eine Notwendigkeit als ein Mittel, dem Sexleben mehr Würze zu verleihen. Sie wählen für gewöhnlich öffentliche oder halböffentliche Orte, um Sex zu haben - dazu gehören Parks, Wälder, Saunen, Vororte-Linien, Einkaufszentren oder Treppenhäuser. In solchen Fällen ist die Wahl der Örtlichkeiten sexueller Aktivität nicht an der Trennlinie von öffentlich/privat situiert, sondern an der Verfügbarkeit der Räume orientiert.

\section{Sexuelles Experimentieren oder jungfräuliche Ehen?}

Die heteronormativen Perspektiven, entsprechend derer sexuelle Beziehungen nur innerhalb langfristiger, monogamer, heterosexueller romantischer Beziehungen akzeptabel sind, prägen vielfach die Vorstellungen von Lehrpersonen über sexuelle Beziehungen unter Teenagern. Demgegenüber vertrat Vera eine liberalere Haltung, der zufolge One-Night-Stands, sexuelle Experimente und wechselnde Partner:innen in Ordnung waren - solange man jung war. Später, so ihre Überzeugung, würde ein junger Mensch im Rahmen von stabilen, langfristigen, monogamen Beziehungen mit einer gegengeschlechtlichen Partnerin:einem ebensolchen Partner zur Ruhe kommen. In dieser Sichtweise erscheint die Adoleszenz als zeitlich begrenzte Phase, die stürmisch, chaotisch, der Selbsterkundung gewidmet und sexuell promiskuitiv sein kann. Dies sah die Schulkrankenpflegerin als akzeptabel (»fine«) an, solange die Heranwachsenden sich um Empfängnisverhütung und den Schutz vor sexuell übertragbaren Krankheiten kümmerten: 
»Sicherlich sind da Zeiten - besonders in der Adoleszenz und in den 2oern und 3oern, wenn man noch nicht zur Ruhe gekommen ist - in denen man experimentiert, nach einer Partnerin:einem Partner sucht oder einfach nur sich und der:dem anderen sexuelles Vergnügen und sexuelle Erfahrungen bereiten möchte. Ich denke an One-Night-Stands. Wenn für probate Sicherheit gesorgt ist, ich meine, eine adäquate Methode der Empfängnisverhütung und des Schutzes vor sexuell übertragbaren Infektionen, dann ist es deine Sache, was du willst ...«(Vera, Schulkrankenpflegerin, SexualkundeUnterricht, 10. Schulstufe)

Diese Aussagen sprechen möglicherweise Mädchen aus Familien nicht an, in denen es noch immer lebendige Tradition ist, als Jungfrau in die Ehe zu gehen, oder wo von den Mädchen wenigstens erwartet wird, ihren ersten Partner später zu heiraten. In meinen Forschungen war das meistens für Mädchen aus Roma-Familien der Fall; solche Traditionen finden sich jedoch in vielen ethnischen, kulturellen oder religiösen Gruppen. Viele der RomaMädchen werteten Jungfräulichkeit als den größten Schatz eines Mädchens, den es nicht $\mathrm{zu}$ >verlieren`, sondern vielmehr jemanden $\mathrm{zu}$ `geben gelte, den sie dafür als würdig erachteten. Sie sprachen davon, ihre Freunde monatelang warten zu lassen, bevor es zu Sex käme, denn sie wollten ihren Schatz jemanden geben, der ihn verdiente. ${ }^{10}$ Jene, die ihre Jungfräulichkeit schon jemanden >gegeben hatten - im Alter von 15 bis 17 Jahren -, dachten oft, dass sie gerne mit dieser Person eine Familie gründen und den Rest ihres Lebens verbringen würden:

»Ich habe einen Freund und ich glaube, ich werde mit ihm zusammenbleiben. Wir sind seit einem Jahr zusammen, er ist mein erster Freund. Ich hätte gerne zwei Kinder, nicht jetzt, später, ungefähr mit 22. Wir planen auszuziehen und eine Wohnung zu mieten, wenn ich 18 werde.« (Edina, 16, RomaMädchen, Gruppeninterview)

Diese Werten und Erfahrungen werden mit der oben zitierten Idee vom freien, aber sicheren sexuellen Experimentieren nicht angesprochen. Für diese Mädchen wäre es relevanter, über die möglichen Konsequenzen früher Bindung und frühen Kinderkriegens zu sprechen und darüber, was es bedeutet, wenn die Familie einen Partner für sie auswählt, oder welche Optionen sie

10 Für eine ausführliche Analyse dieses Themas vgl. Rédai 2019: Kapitel 6. 
haben, wenn sie einem anderen Muster der Partnersuche oder des Kinderkriegens folgen wollen.

\section{Sexuelle Doppelmoral}

Sexuelle Doppelstandards sind unter Heranwachsenden weit verbreitet: Mädchen, die ein aktives Sexualleben führen, bereits mehrere Partner hatten und Sex nicht nur innerhalb stabiler Beziehungen ausprobieren, werden oft negativ beurteilt - von Burschen wie Mädchen gleichermaßen (vgl. z.B. Fasula/Carry/Miller 2014). Geht es hingegen um Burschen, wird dasselbe sexuelle Verhalten eher positiv bewertet, besonders unter den Jungen, die versuchen, sich als >Sexprotze den Respekt der anderen Burschen zu sichern.

»Für einen Burschen ist es etwas ganz anderes. [...] Für ein Mädchen ist es eine Schande und für einen Burschen ... [...]. Ich habe nie verstanden, warum. Mein Papa hat das auch immer gesagt. [...] Und mein jüngerer Bruder macht es ebenso - ein Mädchen, dann ein anderes, er verpflichtet sich nie [...]. Und ich frage: >Papa, ärgert es dich nicht, dass da jede Woche ein anderes Mädchen bei ihm ist? Mich ärgert es! «Und Papa [sagt], >Naja, er ist ein Bursche.« (Vali, 17, Roma-Mädchen, Gruppeninterview)

»Es ist so, dass viele Burschen das tun, sie wechseln die Mädchen täglich, wöchentlich, und dann nennen sie die Mädchen Schlampen, weil sie mit jedem schlafen.«(Adél, 17, weißes Mädchen, Gruppeninterview)

Neben dieser Doppelmoral in Bezug auf monogame Beziehungen und unverbindliche sexuelle Affären vermittelten die Gespräche meiner Gesprächspartner:innen auch doppelte Standards hinsichtlich des Verlusts der Virginität/'Jungfräulichkeit‘. Festgelegt wurden diese Standards von den Burschen: Abgesehen davon, dass sie sich berechtigt fühlten, $\mathrm{zu}$ entscheiden, welche Mädchen sich für welche Art von sexueller Beziehung eignen würden, konzipierten sie auch eine Dichotomie zwischen jungfräulichen Mädchen und >Huren` (vgl. Youdell 2005). So erklärte etwa Levi:

»Für Männer ist es umso besser, je früher sie sie verlieren und für Mädchen, denke ich, umso besser, je später. [...] Denn für Männer gilt, je früher du sie verlierst, umso cooler bist du [...]. Bei Mädchen, denke ich, je früher sie sie verlieren, umso größere Huren sind sie.« (Levi, 17, Roma-Bursche, Cruppeninterview) 
Im selben Gespräch spezifizierte Tibi die Umstände, die Jungfräulichkeit zu verlieren und (nicht) $\mathrm{zu}$ einer $>$ Hure $\mathrm{zu}$ werden:

»[W]enn sie sie in einer Disko oder so verliert, aber z.B., ich war mit ihr zusammen für - ich weiß nicht, wie lange - und dann [...], wenn wir mit zwölf zusammenkommen sind und wir für ein Jahr zusammen waren, dann - warum nicht? Weil dann wäre es so, dass ich sie wollte, dass sie sie verliert. (Tibi, 16, weißer Bursche, Gruppeninterview)

Tibi zufolge ist es okay für ein Mädchen, die Virginität früh zu verlieren, wenn es sich nicht um eine zufällige sexuelle Begegnung handelt, sondern sie schon lange Zeit in einer Beziehung mit ihm stand - weil er dann wollen würde, dass sie das tut. Das impliziert, dass ein Freund und seine Wünsche den Faktor des jungen Alters überschreiben, sodass ein Mädchen, das seine Virginität mit einem Freund verliert, nicht zu einer >Hure wird, wohingegen eben dies passiert, wenn sie ihre Virginität bei Gelegenheitssex verliert.

Während sexuelle Doppelstandards entlang der Gender-Teilung zum Tragen kommen, scheinen sie entlang ethnischer Unterschiede innerhalb gleichgeschlechtlicher Gruppen nicht $\mathrm{zu}$ bestehen, insbesondere bei den Burschen nicht. In anderen Worten: Bei Roma-Burschen und -Männern lassen sich keine ethnisierten Traditionen hinsichtlich Virginität ausmachen; weder von Roma noch von weißen Burschen wird erwartet, dass sie ihre Jungfräulichkeit bewahren. Wenn die Gesprächspartner:innen in Bezug auf Virginität über Burschen sprachen, fehlte jegliche ethnische Unterscheidung und die geschlechtsspezifische sexuelle Doppelmoral dominierte ungeachtet der ethnischen Zugehörigkeit, die die Sprechenden jeweils für sich geltend machten. Für Burschen waren Jungfräulichkeit zugeordnete Bedeutungen nicht konstitutiv für Ethnizität, sondern für kompetitive Männlichkeit. War ein Bursche sexuell erfahren, wurde er von den anderen Burschen respektiert, egal welcher Ethnizität er angehörte.

Viele Mädchen äußerten in den Interviews Unmut darüber, dass Mädchen und Burschen nicht nach denselben Maßstäben bewertet werden, dennoch internalisieren sie eben diese Einstellung (vgl. Moran 2017). Das trifft insbesondere auf Roma-Mädchen zu, für die - wie bereits erwähnt - besonders wichtig war, ihre Virginität für den geeigneten Partner zu bewahren. Für sie ist es - aus kulturellen und sozioökonomischen Gründen - besonders bedeutsam, auf der >richtigen S Seite der Jungfrau/Hure-Dichotomie zu bleiben. Die mit dem Verlust der Virginität einhergehende Entmachtung reflektiert die Unterschichtsposition vieler Roma-Frauen: Sie verfügen über kein oder 
wenig kulturelles, soziales oder materielles Kapital, das von der Mehrheitsgesellschaft als wertvoll angesehen wird. Nachdem Virginität der größte, de facto der einzige shandelbare Vermögenswert vieler Roma-Mädchen ist, ist, sie dem `falschen Burschen zu geben, gleichbedeutend damit, den einzigen, nie mehr wiederkehrenden Moment von Handlungsfähigkeit und Macht über ihre Sexualität verschwendet zu haben. »Du kannst es nur einmal verlieren«, wie eines der Mädchen sagte. Aus dieser Perspektive ist Virginität ein symbolisches Kapital für sozioökonomisches Investment: Mit diesem einen guten Handel zu treiben, kann einem Roma-Mädchen aus einer benachteiligten sozioökonomischen Position zu einem sichereren sozialen und ökonomischen Status verhelfen.

Während Vera die Gegenseitigkeit und Gleichberechtigung in sexuellen Beziehungen betonte, wurde das Problem der sexuellen Doppelstandards nicht offen angesprochen. Es wäre wichtig, die stereotypen und geschlechtsspezifischen Erwartungen rund um sexuelles Verhalten und sexuelle Beziehungen im Sexualkunde-Unterricht zu diskutieren, um auf Gleichberechtigung und gegenseitigem Respekt basierende Beziehungen zu unterstützen und wertzuschätzen sowie an sexuelle Aktivitäten geknüpfte soziokulturelle Werte offenzulegen.

\section{Resümee: Reproduktion sozialer Ungleichheit durch Sexualerziehung}

Der Beitrag zielte darauf ab, Einblicke in die Sexualerziehung an einer ausgewählten ungarischen Sekundarschule zu geben, Einsichten in das diesbezügliche pädagogische Wissen, die vermittelten Informationen und die Sichtweisen und Erfahrungen der jungen Menschen. Das gezeichnete Bild bleibt unvollständig, nachdem ich nur einige Themen aufgreifen konnte. Die Heranwachsenden, die in Sexualkunde unterrichtet werden, scheinen mehr oder weniger mit den biologischen Aspekten von Sexualität vertraut gemacht zu werden, ebenso mit Methoden der Empfängnisverhütung und der Prävention von sexuell übertragbaren Infektionen. Dieses Wissen ist überaus wichtig und nützlich, jedoch scheinen die Heranwachsenden auch noch Bedarf an anderem sexualitätsbezogenen Wissen zu haben.

Es ist offensichtlich, dass Gender, sexuelle Orientierung und GenderIdentität, ethnische und kulturelle Zugehörigkeit sowie sozialer Hintergrund sexuelle Erfahrungen beeinflussen. Sexualkunde scheint auf imaginäre nor- 
mative Teenager:innen abzuzielen. Da es Vertreter:innen dieses Typus in der untersuchten Schule kaum gibt, bleibt die Mehrheit der jungen Menschen in ihrer Lebensrealität unadressiert. Darüber hinaus haben die übermittelten Informationen oft nichts mit den realen Erfahrungen der jungen Menschen zu tun. Sie können in einem solchen Unterrichts-Setting nicht geteilt werden und bleiben unsichtbar.

Das hat auch mit den Unterrichtsmethoden $\mathrm{zu}$ tun. Um Themen wie Beziehung und sexuelle Gewalt, sexuelle Orientierungen und GenderIdentitäten, Geschlechterrollen, kulturelle Modelle von Sexualität und Familiengründung zu diskutieren oder die soziokulturellen, emotionalen, sexuellen und praktischen Fragen, die eine Liebesbeziehung mit sich bringt, anzusprechen, ist es besser, interaktiven, eher nicht geschlechtergemischten Unterricht und Kleingruppenarbeit zu organisieren, die Schüler:innen die Chance bieten, ihre Erfahrungen auszutauschen. Ungeachtet der Präsenz dieser Themen im Alltag werden junge Menschen Fragen zu solchen Zusammenhängen eher nicht vor der gesamten Klasse stellen - sie wissen nur zu gut, dass sie Zielscheibe von Spott oder Bullying werden können, oder wollen ihre Probleme schlichtweg nicht mit der Klasse teilen.

Darüber hinaus fühlen sich Sexualkunde-Lehr:innen und Schulkrankenpfleger:innen oft nicht gewappnet, mit Themen umzugehen, die über ihr primäres Fachwissen, d.h. die biologischen und medizinischen Aspekte von Sexualität, hinausführen. Hier kann es hilfreich sein, einschlägige externe Schulprogramme zu nutzen, wie sie oft von zivilgesellschaftlichen Organisationen angeboten werden, Lehrenden und Schulkrankenpfleger:innen Weiterbildung anzubieten und/oder eine gut qualifizierte Person an der Schule zu beschäftigen, von der die Schüler:innen wissen, dass sie sich in Beziehungs- und sexuellen Angelegenheiten an sie wenden können. Wichtig ist auch zu berücksichtigen, dass für junge Menschen - neben der Schule das Internet, die Medien und sozialen Medien, Freunde und Gleichaltrige die Hauptinformationsquellen sind, es jedoch die Schule ist, die sie einen kritischen Umgang mit Informationen lehren kann. Das ist nicht unbedingt allein eine Aufgabe der Sexualerziehung, sondern auch anderer Gegenstände, etwa der Medienkunde, der geisteswissenschaftlichen Fächer, des Ethikunterrichts oder der Gesellschaftskunde.

Sexualerziehung spielt eine Rolle bei der Reproduktion sozialer Ungleichheit, besonders indem sie nicht-normative sexuelle Subjektivitäten und Erfahrungen ignoriert oder nicht zu Wort kommen lässt. Die Erfahrungen, Probleme und Fragen jener, die nicht aus der sozialen Mittelklasse 
kommen und/oder nicht den ethnischen/kulturellen Hintergrund der Mehrheit aufweisen oder deren Erfahrungen nicht mit den festgeschriebenen Modellen von Sexualität, Beziehung, Familie und den kulturellen Erwartungen korrespondieren, bleiben für die Sexualkunde-Lehrer:innen womöglich unsichtbar.

Basierend auf den Sichtweisen und Erfahrungen, die junge Menschen mit mir in der Marzipan geteilt haben, möchte ich argumentieren, dass Sexualerziehung von den Erfahrungen der Heranwachsenden ausgehen und ihnen Raum bieten sollte, sich über diese Erfahrungen auszutauschen. Sexualerziehung sollte diese Erfahrungen ernst nehmen, nicht moralisieren, den Schüler:innen nicht die Einstellung der Lehrperson zu Sexualität aufdrängen und die jungen Menschen nicht als sirrationale Wesen $<$ auffassen, die sich in einer Phase befänden, in der sie nicht zu autonomen Entscheidungen fähig wären. Eine solche Sexualerziehung sollte auch nicht-normative Identitäten, sexuelle Orientierungen und diverse sozioökonomische Situationen sowie ethnischkulturelle Hintergründe ansprechen, anstatt unachtsam soziale Ungleichheit zu reproduzieren, indem sie soziale Diversität ignoriert.

\section{Literatur}

Alldred, Pam/David, Miriam E. (2007): Get Real About Sex. The Politics and Practice of Sex Education. London: Open University Press.

Allen, Louisa (2007): Denying the Sexual Subject: Schools' Regulation of Student Sexuality. In: British Educational Research Journal 33, 2, S. 221-234.

Epstein, Debbie/O'Flynn, Sarah/Telford, David (2001): »Otheringく education: sexualities, silences, and schooling. «In: Review of Research in Education 25, S. 127-179.

Fasula, Amy M./Carry, Monique/Miller, Kim S. (2014): A Multidimensional Framework for the Meanings of the Sexual Double Standard and its Application for the Sexual Health of Young Black Women in the U.S. In: Journal of Sex Research 51, 2, S. 170-183. Online unter: https://doi.org/10.1080/00 224499.2012.716874

Fields, Jessica (2005): »Children Having Children«: Race, Innocence, and Sexuality Education. In: Social Problems 52, 4, S. 549-571.

Froyum, Carissa M. (2010): Making »Good Girls«: Sexual Agency in the Sexuality Education of Low-Income Black Girls. In: Culture, Health \& Sexuality 12, 1, S. 59-72. Online unter: https://doi.org/10.1080/13691050903272583. 
García, Lorena (2009): »Now why do you want to know about that? « Heteronormativity, Sexism, and Racism in the Sexual (Mis)Education of Latina Youth. In: Gender and Society 23, S. 520-541. Online unter: https ://doi.org/10.1177/0891243209339498.

Gesetz zum öffentlichen Gesundheitswesen (1997): évi CLIV. törvény az egészségügyről [Gesetz zum öffentlichen Gesundheitswesen] (154/1997). Online unter: http://net.jogtar.hu/jr/gen/hjegy_doc.cgi?docid=99700154.TV [Zugriff: 03.07.2020].

Jackson, Sue/Weatherall, Ann (2010): The (Im)Possibilities of Feminist School Based Sexuality Education. In: Feminism \& Psychology 20, S. 166-185. Online unter: https://doi.org/10.1177/0959353509349603.

Kerettanterv az általános iskola 5-8. évfolyamára [Rahmencurricula für die 5.-8. Grundschulstufe] (2012-2013). Online unter: http://kerettanterv.ofi. hu [Zugriff: 03.07.2020].

Kerettanterv a szakközépiskolák 9-12. évfolyama számára [Rahmencurriculum für die 9.-12. Schulstufe Technischer Schulen. Ministerium für Humanressourcen] (2008). Online unter: http://www.nefmi.gov.hu/kozokta tas/tantervek/oktatasi-kulturalis [Zugriff: 03.07.2020].

Moran, Claire (2017): Re-positioning Female Heterosexuality within Postfeminist and Neoliberal Culture. In: Sexualities 20,1-2, S. 121-139. Online unter: https://doi.org/10.1177/1363460716649335.

A Nemzeti Alaptanterv 2007 [Nationaler Kernlehrplan 2007]. Online unter: https://www.nefmi.gov.hu/kozoktatas/tantervek/nemzeti-alaptanter v-nat [Zugriff: 03.07.2020].

A Nemzeti Alaptanterv 2012 [Nationaler Kernlehrplan 2012]. In: Magyar Közlöny 2012, S. 66. Online unter: https://www.budapestedu.hu/data/cms149 320/MK_12_66_NAT.pdf [Zugriff: 03.07.2020].

Pascoe, C.J. (2007): Dude, you're a Fag. Masculinity and Sexuality in High School. Berkeley: University of California Press.

Rahimi, Regina/Liston, Delores (2009): What does she expect when she dresses like that? Teacher Interpretation of Emerging Adolescent Female Sexuality. In: Educational Studies 45, S. 512-533. Online unter: https://doi .org/10.1080/00131940903311362.

Rédai, Dorottya (2019): Exploring Sexuality in Schools. The Intersectional Reproduction of Inequality. Houndmills u.a.: Palgrave Macmillan. Online unter: https://doi.org/10.1007/978-3-030-20161-6.

Rédai, Dorottya (2011): "Sok újat nem mondtak. Mi már mindent tudunk erről.«Az iskolai szexuális nevelés és a középiskolás lányok szexualitá- 
sa. [»Sie sagten nichts Neues. Wir wussten schon alles darüber.« Sexualerziehung in Schulen und Sexualitäten von Sekundarschul-Mädchen.] In: Kozma, Tamás/Perjés, István (Hg.): Új Kutatások a Neveléstudományokban [Neue Studien in den Bildungswissenschaften]. Budapest: MTA Pedagógiai Tudományos Bizottsága, ELTE Eötvös Kiadó.

Rédai, Dorottya/Sáfrány, Réka (Hg.) (2019): Gender in National Education Documents and Teaching Resources, and in Teachers' Pedagogical Approaches and Everyday Teaching Practices in Austria, the Czech Republic and Hungary. Comparative Report for the Erasmus+ project $» T o w a r d s$ Gender Sensitive Education«, Project Nr. 2017-1-CZo1-KA201-035485. Online unter: http://gendersensed.eu/outputs [Zugriff: 03.07.2020].

Renold, Emma (2005): Girls, Boys and Junior Sexualities. Exploring Children's Gender and Sexual Relations in the Primary School. Abingdon/New York: Routledge.

Talburt, Susan (2004): Intelligibility and Narrating Queer Youth. In: Rasmussen, Mary Louise/Rofes, Eric/Talburt, Susan (Hg.): Youth and Sexualities. Pleasure, Subversion and Insubordination in and out of Schools. Houndmills u.a.: Palgrave Macmillan.

Waites, Matthew (2005): The Age of Consent: Young People, Sexuality and Citizenship. Houndmills u.a.: Palgrave Macmillan.

Youdell, Deborah (2005): Sex-Gender-Sexuality: How Sex, Gender and Sexuality Constellations are Constituted in Secondary Schools. In: Gender and Education 17, 3, S. 249-270. 



\section{Imagining Subjectivities \\ Eine Reflexion über eigene Geschlechtervorstellungen und ihre Bedeutung für sexualpädagogisches Handeln}

Magdalena Rest

An einem Dienstagvormittag im Mai stehe ich mit Arbeitsblättern vor einigen Schülern einer Wiener $\mathrm{NMS}^{1}$, sie sind zwischen 13 und 15 Jahre alt. Es ist unser zweiter Besuch im Rahmen eines sexualpädagogischen Unterrichtsprojekts und der erste Termin mit der kleinen Gruppe Jugendlicher, die mit uns arbeiten werden. Meine Studienkollegin hat eben unser Vorhaben erklärt wir wollen gemeinsam einen Stop-Motion-Film produzieren -, nun bin ich kurz davor, unseren ersten Arbeitsauftrag zu erteilen. Da kommen mir heftige Zweifel ...

Für unser Projekt haben wir uns viel vorgenommen: Wir wollen uns mit dem Thema Pornografie beschäftigen, in der vorangegangenen KennenlernEinheit mit der ganzen Klasse haben dazu einige Klassenkolleginnen assoziativ schon viele Begriffe gesammelt (von »anal« bis »zuschauen«). Daran anknüpfend wollen wir mit unserer Gruppe sexualpädagogische Inhalte erarbeiten, uns dabei an der Neugier, den Interessen und den Bedürfnissen der Schüler:innen orientieren, ihre (Scham-)Grenzen achten, aber ihre Normvorstellungen, wo notwendig, auch herausfordern. ${ }^{2}$ Wir haben uns überlegt,

NMS ist die gebräuchliche Abkürzung für eine österreichische Schulform, die »Neue Mittelschule«. Sie wurde 2012 als Kompromiss des jahrzehntealten Konflikts zwischen Verfechter:innen eines differenzierten Schulsystems und Proponent:innen einer gemeinsamen Schule für (zumindest) alle Zehn- bis 14-Jährigen geschaffen. Die gymnasiale Unterstufe - und damit die Differenzierung von Schüler:innen ab dem 10. Lebensjahr - blieb allerdings bestehen. Im Schuljahr 2020/21 wurde die Neue Mittelschule in Mittelschule (MS) umbenannt.

2 Zwei Hinweise von Katharina Debus waren für uns in diesem Zusammenhang wichtig: Zum einen betont sie in ihrem programmatischen Aufsatz zu nicht-diskriminierender Sexualpädagogik (2016) den »präventiven Charakter gegen sexualisierte Ge- 
als Angelpunkt dieser Auseinandersetzung immer wieder auf alltägliche, schöne und lustvolle (Selbst-)Erfahrungen zurückzukommen. Der kurze Film, den wir im Prozess kreieren wollen, soll dann auch auf diese Art sexualisierte Sprache und dazugehörige Vorstellungen reflektieren: Bestimmte Begriffe (z.B. »blasen«, »lecken«) sollen mit alltäglichen und positiv besetzten Bildern (z.B. Kaugummi, Eis) dargestellt werden und dabei körperlichen Genuss ins Zentrum stellen. So erhoffen wir uns, weitgehend unabhängig vom sexuellen Erfahrungshintergrund der einzelnen Jugendlichen, ein Nachdenken über positives Körper- und Selbsterleben zu ermöglichen und dabei eine Sprache für den Austausch über Sexualität und Begehren zu entwickeln, die Ressourcen für eine selbstbestimmte Erfahrung bewusst machen und mitgeben kann. Darüber hinaus stellen wir uns vor, so - abseits von Moralisierung - kritische Perspektiven auf zeitgenössische Pornografie und daran anknüpfende Ideen über Sexualität hereinholen zu können. In der Konzeption haben wir uns nicht zuletzt vor diesem Hintergrund Gedanken gemacht über unsere eigenen Zugänge zu den - auch in diesem Text benutzten und relevanten - dichotomen Geschlechterkategorien, ihrer sozialen Wirkmächtigkeit und unserem damit verbundenen Auftrag, die Realität jenseits der gesellschaftlich verordneten Zweigeschlechtlichkeit mitsamt den zugehörigen Geschlechterrollen und Begehrensformen nicht $\mathrm{zu}$ unterschlagen.

Für den Einstieg, den ich nun anleiten soll, wollen wir die Schüler:innen auffordern, Fotos zu machen. Sie sollen sich mit positiven Bezügen zu sich selbst auseinandersetzen und Teile des eigenen Körpers abbilden, die ihnen gut gefallen oder die sie mit Eigenschaften, die sie an sich selber schätzen, verbinden. Das Arbeitsblatt, das ich dazu in den Händen halte, macht mich plötzlich unsicher. Es beginnt so: »Was ist toll an mir? Es gibt Dinge, die wir nicht so gern an uns selber mögen, und manchmal denken wir viel über sie nach. Glücklicherweise gibt es aber auch Dinge, die wir gern an uns mögen,

walt«, den eine grenzachtende Sexualpädagogik haben kann: »sowohl in Bezug auf das Erkennen von Übergriffigkeit als auch in Bezug auf das Vertrauen, Unterstützung zu finden, wenn Grenzverletzungen, sexuelle Übergriffe und sexualisierte Cewalt offengelegt werden« (826). Zum anderen gibt sie zu bedenken, dass sich nicht-diskriminierende Sexualpädagogik zwischen Schamgrenzen und Normvorstellungen in einem "Spannungsfeld« bewegt: »Einerseits ist Freiwilligkeit im Sinne von Grenzachtung ein wichtiges Prinzip der Sexualpädagogik. Andererseits verknüpfen sich Scham und/oder Desinteresse z.T. mit diskriminierenden Strukturen«- beispielsweise in Bezug auf gesellschaftlich abgewertete Begehrensformen (828). 
und Sachen, für die uns andere besonders toll finden: was wir alles im Kopf haben ... wie gern wir essen und wie viel in unseren Bauch passt ... wie konzentriert wir zuhören können." Gedacht war das Arbeitsblatt vor allem da$\mathrm{zu}$, die Schüler:innen zu einer individuellen Reflexion anzuregen, bevor sie sich ans Fotografieren machen (im Anschluss an die Aufforderung, die Dinge aufzuschreiben oder -zeichnen, die ihnen einfallen, ist vermerkt: »Der Zettel gehört nur dir!«). Entworfen habe ich den Text als Unterstützung beim Nachdenken und im Bewusstsein, dass wir wahrscheinlich koedukativ arbeiten werden - aber durchaus mit den Mädchen vor Augen, mit denen wir in der ersten Einheit vor ein paar Wochen ein ganzes Plakat mit Wörtern befüllt hatten. Nun sitzen nur Jungs vor mir - ihre beiden Kolleginnen, die Teil unserer Projektgruppe sind, fehlen heute - und meine Vorstellungen zu geschlechterspezifischer Subjektivität gehen mit mir durch: Ich habe Sorge, die jungen Männer werden nichts mit meinen Sätzen anfangen können. Mir fällt auf, wie sehr ich sie auf meine eigene, weibliche Sozialisationserfahrung zugeschnitten hatte: ein positiver Bezug zu Klugheit und Intelligenz zum Einstieg, eine Referenz auf das häufig eng mit schwierigen Körperbildern verbundene Thema Essen, und schließlich die Reproduktion einer klassischen Sozialisationserwartung an Frauen - eine gute Zuhörerin zu sein. Zudem erscheint mir plötzlich, unsere Übung wäre insgesamt zu intim.

Ich löse das Problem auf die Schnelle damit, den Arbeitsauftrag mündlich vorzutragen und die Zettel zum Aufschreiben von Ideen erst auszuteilen, als schon alles geklärt ist. Mein Kalkül ist, dass die Schüler nicht so genau nachlesen werden, wenn sie schon wissen, was zu tun ist. Ich versuche also, die Auseinandersetzung mit dem geschriebenen Text zu verhindern - und bin erfolgreich: Sie gehen gleich gemeinsam an die Arbeit und erfüllen in der folgenden Stunde engagiert unseren Auftrag, machen viele Fotos (und löschen vermutlich einige wieder). Aber: Sie scheinen alle ziemlich dieselben Dinge an sich zu mögen. Oberarme und Waden stehen hoch im Kurs.

Später werde ich mich deshalb über mein Handeln ärgern - es hatte einen Sinn gehabt, den Auftrag zu verschriftlichen. Das Lesen hätte die Gelegenheit geboten, sich der Aufgabe einzeln, individuell, vielleicht auch ungestört anzunähern. Ich hatte unsere Übung mit meinem trouble shooting zu einer Gruppensituation gemacht. Wäre sie wie geplant abgelaufen, hätte sie womöglich Raum gegeben, sich auf sich selbst zu konzentrieren.

Zwar hatten meine Kollegin und ich uns zurechtgelegt, dass wir unseren mit Bedacht gewählten Ausgangspunkt (Raum für eine individuelle Annähe- 
rung schaffen; sich über als >wertvolk erachtete Teile des eigenen Selbst bewusst werden; einen empathischen, liebevollen Zugang zu sich finden) für alle Jugendlichen gleichermaßen für sinnvoll halten. In der pädagogischen Situation und ihrer Reflexion fiel mir dann aber auf, welch unterschiedliche und stereotype Subjektivitäten ich jungen Frauen und Männern in ihrem Selbst- und Körperbezug nach wie vor zuschreibe. Wichtig erscheint mir das vor allem in Bezug auf die Konsequenzen, die diese Vorstellungen für mein pädagogisches Handeln und für die Räume, die ich zur Verfügung stelle, haben können: Ich hatte die feinen Unterschiede, die ich mir für die Anschlussmöglichkeiten verschiedener Jugendlicher vorstell(t)e, beim Verfassen des Textes wenig bedacht und unterstellte dann, ihn mit Jungs durchzuarbeiten würde zu Irritationen, Komplikationen und >Verzögerungen , führen, sie würden den angedeuteten Selbstbezug peinlich finden - eine Sorge, die mir im Nachhinein nicht mehr so berechtigt erscheint.

Unser Projekt ist an dieser Konfrontation mit meinen Geschlechterbildern nicht gescheitert. Wir haben viele spannende Gespräche geführt und einen tollen Kurzfilm produziert, den die Schüler:innen am Ende stolz ihren Kolleg:innen präsentieren konnten. Außerdem haben sie die beschriebene Situation wahrscheinlich ganz anders erlebt als ich. Mir hat sie allerdings vor Augen geführt, dass mein Nachdenken nicht abgeschlossen ist: darüber, wie vielfältig unsere Ideen über Geschlechterdifferenzen in (sexual-)pädagogischen Kontexten wirken. Wie kleinteilig sie sich in Absichten, Haltungen und Handlungen niederschlagen und wie sehr unsere Vorannahmen über Räume, Grenzen, Möglichkeiten und Unmöglichkeiten entscheiden können. Darüber, dass ich Mädchen ein problematischeres Verhältnis zu ihrem Körper unterstelle als Jungs; darüber, dass ich wohl davon ausgehe, jungen Frauen mehr Intimität >zumuten zu können als jungen Männern - und darüber, was das für sexuelle Bildung heißt.

\section{Literatur}

Debus, Katharina (2016): Nicht-diskriminierende Sexualpädagogik. In: Scherr, Albert/El-Mafaalani, Aladin/Yüksel, Gökcen (Hg.): Handbuch Diskriminierung. Wiesbaden: Springer, S. 811-833. 


\section{Körperspuren \\ Erinnerungsgeschichten an Körperlichkeit in der Schule}

Kerstin Witt-Löw

»Der Professor ${ }^{1}$ wiederholte die Frage lautstark und unfreundlich. Sie spürte, wie sich das Blut in ihrem Kopf ansammelte, ihre Ohren sahen wahrscheinlich aus wie zwei Scheiben einer überreifen feuerroten Tomate. Ihr wurde heiß und kalt zugleich. Sie wusste keine Antwort auf die gestellte Frage.«

Dieses Zitat stammt aus einer Erinnerungsgeschichte, die von einer Studentin zum Thema »Prüfungssituation in der Schule» geschrieben wurde. Seit über acht Jahren verfassen Lehramtsstudierende der Universität Wien ${ }^{2}$ Erinnerungsgeschichten über eine Episode ihrer Schulzeit, die mit der Methode der Kollektiven Erinnerungsarbeit ${ }^{3}$ nach Frigga Haug bearbeitet werden. Seit wenigen Jahren werden auch Situationen aus der Perspektive als Junglehrer:in beschrieben. Durch die intensive Auseinandersetzung mit Themen, die aus den Geschichten herausgearbeitet und mit pädagogischer Literatur vertieft werden, sollen - so das Ziel der Lehrveranstaltung - die Handlungsmöglichkeiten als (zukünftige) Lehrer:innen erweitert werden.

Fast 200 dieser Erinnerungsgeschichten wurden von den Autor:innen für weitere Forschungszecke zur Verfügung gestellt. Auf diese Geschichten be-

Im österreichischen Schulalltag werden Lehrer:innen von Schüler:innen als Professor:innen bezeichnet und mit »Herr/Frau Professor:in« angesprochen.

2 Diese Lehrveranstaltungen wurden im Diplomstudium in den Bereichen »Erziehen und Beraten« und »Pädagogische Professionalität« angeboten und sind im MasterStudium dem Modul »Forschungsmethoden: Professionsverantwortung, Evaluation und Practitioner Research «zugeordnet.

3 Die Methode der Kollektiven Erinnerungsarbeit wurde von Frigga Haug und anderen feministisch-sozialistischen Forscher:innen in den 1970er Jahren entwickelt und in zahlreichen Publikationen beschrieben, etwa Haug (1990). 
zieht sich der folgende Text, der als Erkundung von Körperspuren in diesen Erinnerungen, von Auffälligkeiten, Mustern und Leerstellen in den Geschichten $\mathrm{zu}$ verstehen ist, an die sich Überlegungen für die Lehrer:innenbildung anschließen.

Die Arbeit mit Erinnerungsgeschichten geht von folgenden Annahmen aus: Erinnerungen werden als ein Konstrukt verstanden, durchzogen von Leerstellen, Verfälschungen und nachträglich hergestellten Bezügen. In der Analyse der Geschichten interessiert genau dieses Konstrukt, es geht nicht um eine >objektive Wahrheit<. Erinnert werden Ereignisse, die eine besondere Bedeutung für die Autor:innen haben. Oft sind dies Situationen, die mit heftigen Emotionen verbunden waren. In vielen Geschichten werden Gefühle der Angst und Scham beschrieben. Es gibt aber auch positiv überraschende Situationen mit Stolz und Beglückung sowie Kombinationen ambivalenter Gefühle.

In den individuellen Erlebnissen und Erfahrungen zeigen sich kollektive Muster, in denen die Lernverhältnisse zum Ausdruck kommen, die (auch) in der Institution Schule tradiert werden, wie etwa die informellen und formellen Regeln, die Rollen von Schüler:innen und Lehrer:innen, Rituale, Lernsettings oder Modi der Definition, Überprüfung und Bewertung von Leistungen.

\section{Körper außer Kontrolle}

Die Studierenden einigen sich in ihren Arbeitsgruppen jeweils auf eine Überschrift, zu der passend sie ihre Schulgeschichten schreiben. »Körperlichkeit« war in all den Jahren nur einmal das gewählte Thema, es gibt aber in allen Geschichten Körperspuren, die im Folgenden herausgearbeitet werden.

\subsection{Angst und Stress}

Das Thema »Eine Prüfungssituation«, aus dem das einleitende Zitat stammt, wurde hingegen außerordentlich häufig gewählt. Diese auffällige Häufung sowie die sich ähnelnden Beschreibungen der Situation lassen vermuten, dass hier Erfahrungen gemacht werden, die in unserem Schulsystem verbreitet sind und die sich in besonderer Weise negativ in der Erinnerung halten. Dabei werden diese sehr unangenehmen Situationen durch körperliche Reaktionen beschrieben, die die Leser:innen nachvollziehen können und die sie in Gefühlslagen übersetzen. Angst und Stress drücken sich etwa durch Schweiß, 
Herzrasen, Zittern oder Erröten aus. Die Dramatik der Situation ist durch die Unmittelbarkeit der Beschreibung von Körperlichkeit erhöht.

Gemeinsam ist den Geschichten, dass sich die Autor:innen als vereinzelt, allein und sprachlos beschreiben - ausgeliefert der Situation und den eigenen bizarren Körperreaktionen. Diese werden bildhaft geschildert und wirken wie in einem Film der Selbstbeobachtung des eigenen Körpers. Die Beschreibungen enthalten eine Kombination aus Gespürtem und Gefühltem und den nach außen sichtbaren körperlichen Zeichen, die von der Umgebung wahrgenommen werden können.

Die Geschichten zeigen auch, dass beruhigende und bestätigende Interventionen der Lehrpersonen oder von Mitschüler:innen in diesen Situationen hilfreich sein können. Obwohl Prüfungssituationen ein wichtiger und regelmäßig vorkommender Bestandteil des Schullebens sind, wird - so die Erfahrung der Studierenden als ehemalige Schüler:innen - der Umgang mit Aufregung, Stress und Angst und den körperlichen Symptomen im Schulunterricht nicht thematisiert. Zukünftige Lehrer:innen sollten daher lernen, welche Unterstützung sie ihren Schüler:innen im Umgang mit und in schulischen Stresssituationen geben können und wie sie in der Gestaltung ihres Unterrichts angsterzeugende Situationen vermeiden können. Denn, wie viele Geschichten zeigen: Angst verhindert Lernen.

\subsection{Tränen}

»Sie hörte die strenge Stimme des Professors: >B, wieder mal ein Fünfer. Du wirst es bei mir nie schaffen, du bist einfach zu blöd für Englischく. Stille! Der nächste Augenblick verlief wie in Trance. Ganz langsam sank sie in den Untergrund. [...] Sie wollte nur noch weg, weg aus der Klasse, weg aus der Stadt, weg vom Planeten Erde, wo es keine gemeinen, ungerechten, unfairen Englischlehrer mehr gibt. Die Tränen schossen ihr wie ein Wasserfall aus den Augen und während sie aus der Klasse hinauslief, hörte sie nur noch die Stimme eines Mitschülers, der für sie Partei ergriff.«

Aufregung, Stress, Angst, aber auch Scham werden körperlich beschrieben, als ungewohnte und unangenehme Sensationen. Einen emotionalen Höhepunkt bilden Tränen, sie sind der körperliche Gau. Erinnerungsgeschichten, in denen Tränen vorkommen, werden als Drama konstruiert, es sind oft Situationen, in denen mit den Tränen die völlige Hilflosigkeit und Handlungsunfähigkeit ausgedrückt wird. Beschrieben werden Tränen nach einem Ver- 
sagen, Tränen der Verzweiflung, Tränen der unterdrückten Wut (etwa über eine empfundene Ungerechtigkeit), aber auch Tränen, wenn (unerwartet) Anteilnahme ausgedrückt wird.

Tränen führen in den Geschichten oft zu einem Wendepunkt. Denn auf Tränen wird häufig reagiert. Tränen lösen Gefühle bei den direkt Beteiligten oder indirekt Beobachtenden aus. Mitgefühl, Mitleid, manchmal auch Scham, dass eine Situation so eskalieren konnte, werden in den Geschichten beschrieben. Den Autor:innen selbst sind ihre Tränen unangenehm und peinlich, ebenso wie mitleidige Reaktionen. Flucht aus der Situation, wie in der Textpassage beschrieben, ist ein verständlicher Impuls. In der Schule wird die Toilette zum einzigen Ort, an den sich Schüler:innen zurückziehen können.

Tränen fordern eine Reaktion von Lehrpersonen, sie führen $\mathrm{zu}$ Interventionen. In den Geschichten, die Junglehrer:innen aus ihrer Sicht als verantwortliche Pädagog:innen beschreiben, stechen Tränen als Thema ebenfalls heraus. Zentral dabei ist die eigene Hilflosigkeit im Umgang mit Tränen bei Schüler:innen. Oft ist der erste Impuls zu trösten, gleichzeitig gilt es aber in der Rolle als Lehrer:in, körperliche und persönliche Grenzen zu beachten. Dabei sind es in den Geschichten meist nicht Situationen im eigenen Unterricht, die zu den Tränen führen, sondern Mobbing-Erlebnisse, Probleme in der Familie oder Ungerechtigkeiten von anderen Lehrer:innen - Situationen also, die Interventionen außerhalb des Klassenzimmers erfordern, die gleichzeitig oft unübersichtlich und unklar sind. Zeit und ein geschützter Raum sind hier notwendige Rahmenbedingungen, damit weinende Schüler:innen sich wieder sammeln und sich gegebenenfalls aussprechen können.

\subsection{Blut}

Körperflüssigkeiten erscheinen in den Geschichten als ein Tabu und werden als schambesetzt erlebt. Dies gilt für Tränen und auch für Menstruationsblut, ${ }^{4}$ wie die folgende Zusammenfassung einer Geschichte zeigt:

Die Autorin schreibt konzentriert eine Geografie-Prüfung. Als alle Schüler:innen ihre Arbeiten vorne im Klassenzimmer abgeben müssen, bemerkt sie, dass ihre noch dazu helle Hose von Blut nass ist. Sie schämt sich und weiß nicht, was sie tun soll. Sie bleibt sitzen und sucht verzweifelt in ihrer 
Tasche nach einem Schal. Der Lehrer schreit sie an - er interpretiert ihr Verhalten wohl als Schummel-Versuch -, nimmt ihr die Arbeit weg und geht. Sie weint. Erst in der Pause, als auch die meisten Buben die Klasse verlassen haben, kann sie sich einen Schal umwickeln, sie erhält Taschentücher von Mitschülerinnen und wechselt in der Toilette in ihre Sportleggins. Von ihrer Klassenvorständin, ${ }^{5}$ die sie zufällig trifft, wird sie angesprochen, ihr kann sie die Situation schildern. Die Lehrerin beruhigt sie und verspricht, mit dem Geografie-Lehrer zu reden. Dieser spricht die Autorin allerdings in Folge nie auf die Situation an. Für die Autorin bleibt damit die tiefe Abneigung gegen die Geografie-Stunden.

Die Körperscham, die sich in dieser Geschichte auf das Menstruationsblut bezieht, ist so groß, dass die Schülerin ihre Problemlage nicht erklären kann und sogar unberechtigte Anschuldigungen in Kauf nimmt. Die Sprachlosigkeit bezieht sich auf den männlichen Lehrer, aber auch die Mitschüler sollen das wirkliche Problem nicht bemerken. Im Gegensatz dazu erhält die Autorin von Mitschülerinnen und der Klassenvorständin Hilfe und Verständnis. Menstruation - so die Geschichte - ist ein Tabu und kann nur als Thema unter Frauen angesprochen werden. Der Lehrer hält, auch nach einer Information durch die Klassenvorständin, die Sprachlosigkeit aufrecht und löst die Situation für die Schülerin nicht auf.

Noch eine zweite Geschichte thematisiert die Verständnislosigkeit männlicher Lehrpersonen beim Thema Menstruation: In einer Geschichte hat die Schülerin starke Menstruationsbeschwerden und möchte daher beim Sportunterricht nicht mitturnen. Der Lehrer glaubt ihr die Menstruationsschmerzen nicht und bezeichnet sie als Ausrede. Die Schülerin ist »wütend und irritiert«, versucht noch mitzuturnen, verlässt dann aber unter Schmerzen die Turnhalle. Sie bekommt Unterstützung von einer Lehrerin und wird nach Hause gebracht. Auch hier bleiben die Sprachlosigkeit und »keinerlei Motivation mehr für den Sportunterricht bei diesem Lehrer«. Beide Geschichten verknüpfen die negative Erfahrung mit einer anhaltenden Abneigung gegenüber dem jeweiligen Lehrer und dem Unterrichtsfach.

In jedem Klassenzimmer mit Jugendlichen wird es Mädchen geben, die gerade menstruieren - und besonders in der Pubertät erst einen Umgang dalehrer:innen, also jene Lehrperson, die über mehrere Jahre hinweg für eine Klasse und ihre Schüler:innen zuständig ist. 
mit entwickeln. Die selbstverständlichen, als solidarisch erlebten Reaktionen der Mitschülerinnen sowie der Lehrerinnen zeigen, dass hier auf Basis der gemeinsamer Erlebniswelt eine Sensibilität besteht (oder zumindest bestehen kann), die sich männliche Lehrpersonen erst aneignen müssen. Die Thematisierung von Sexualität und Körperlichkeit in der Lehrer:innenausbildung ist daher ein wichtiger erster Schritt.

\section{Körper unter Beobachtung}

Wie werden die Körper von Lehrpersonen und von Mitschüler:innen erinnert und wie wird der eigene Körper in den Geschichten beschrieben? Der folgende Textabschnitt fokussiert auf die Wahrnehmung von Lehrer:innen- und Schüler:innenkörpern in den Erinnerungsgeschichten und thematisiert dabei Körpernormen und Beschämung.

\subsection{Lehrer:innenkörper}

»Der Lehrer war ein älterer Herr und roch permanent und ziemlich penetrant nach billigem Rasierwasser, Zigaretten und Kaffee. Er erinnert sich noch heute an die üble Ceruchskombination, wenn sich der Lehrer über ihn beugte, um die Hausübung ${ }^{6}$ zu kontrollieren.«

Lehrer:innen, das zeigen viele Geschichten, werden von Schüler:innen sehr genau wahrgenommen, optisch, akustisch und olfaktorisch. Erinnert werden optische Eindrücke: ein Kleidungsstil, Frisur, Größe und Gestalt und vor allem Blicke (manchmal durch eine Brille), die sich auf die Schüler:innen richten: forschend, prüfend, streng, stechend, freundlich. Beschrieben werden auch die Stimme, Gesten und das Bewegungsmuster der Lehrperson - zusammen mit den Blicken werden so die Kommunikationsmuster und Unterrichtspraktiken ausgedrückt, also das, was für die Schüler:innen zur Einschätzung dessen, was sie erwartet, wichtig ist.

Wird der Geruch eines Lehrers hervorgehoben, so bisher in den Geschichten immer als unangenehmer Faktor, besonders in unerwünschten körperlichen Nahesituationen, denen sich Schüler:innen nicht entziehen können.

6 »Hausübung« bezeichnet im Österreichischen die Hausaufgaben. 
Lehrpersonen erscheinen auch als Projektionsflächen für Fantasien. Ein Student schildert in seiner Geschichte unter dem Titel »Ablenkungen«, wie in einer als »Hölle« erlebten Französisch-Stunde seine Gedanken auch zum gelegentlich sichtbaren »weißen Stringtanga« der »dominanten« jungen Französisch-Lehrerin wandern - es ist eine der wenigen Geschichten mit erotischer Konnotation und dementsprechendem Vokabular. Der Autor präsentiert dabei seine erotischen Gedanken als Widerstandsform, die Sexualisierung der Lehrerin erscheint als Reaktion auf die erlebte Ohnmacht als Schüler.

Angehenden Lehrpersonen sollte bewusst sein, dass sie genau beobachtet werden: Wie sie sich kleiden, welche körperlichen Eigenheiten sie haben, wie sie sprechen und wie sie sich bewegen - all das wird aufmerksam verfolgt. Sie sind den Blicken der Schüler:innen ausgesetzt, das Klassenzimmer ist aber auch eine Bühne für die Lehrer:innen. Selten bekommen sie allerdings eine Rückmeldung auf ihre Wirkung. Im Lehramtsstudium sollte die eigene Körperlichkeit reflektiert und der bewusste Einsatz von körperlichen Elementen zur Unterstützung pädagogischer Intentionen geübt werden, etwa die eigene Positionierung und Bewegung im Raum. Ein Stimmtraining könnte ebenso hilfreich sein wie konstruktives Feedback zur körperlichen Präsenz beim Erproben von pädagogischen Gestaltungmöglichkeiten.

\subsection{Körpernormen und Beschämung}

»Wie üblich am Dienstagnachmittag steht sie in der Turnsaalgarderobe ${ }^{7}$ : die weite Hose, die bei Hintern und Bauch gerade so passt und die stämmigen Beine gut umspielt; das weiße T-Shirt >wurfzeltartig` über ihre schwammigen Kurven und die füllige Oberweite geschlagen. So weit, so fett. Sie ist bereit für die heutige Turnstunde. Es ist die letzte vor den Weihnachtsferien und somit die letzte vor der dreiwöchigen Erlösung - nämlich jene Erlösung von den Blicken, dem Gespött und der Cewalt; also all den Dingen, denen sie im Turnunterricht sonst so ausgesetzt ist.«

In dieser Textpassage beschreibt die Autorin ihren Körper, den sie als abweichend von der Norm wahrnimmt, Körperteil-sezierend genau, mit einem abwertenden, selbstquälerischen Vokabular, das aus weiblichen Schönheits- und

7 »Turnsaal« ist der österreichische Terminus für »Turnhalle«. 
Diätdiskursen vertraut klingt. ${ }^{8}$ Nicht zufällig spielt die Szene im Turnunterricht, denn hier steht die Körperlichkeit im Zentrum. Neben den sportlichen Leistungen werden auch die Körper und die Kleidung betrachtet und kommentiert. Die oben beschriebene Geschichte enthält eine positive Wendung für die Autorin: Die neue Turnlehrerin kritisiert nämlich eines der »Models«, also eine schlanke Mitschülerin in »knappen Hot Pants«, für ihre Sportkleidung, was die Autorin als ein Zeichen von »Gerechtigkeit« wertet: »Noch nie hat sie sich so gut in einer Turnstunde gefühlt. [...] An ihr ist heute einmal nichts falsch. Nein, heute ist es mal das Model selbst, an dem etwas nicht passt.«

Allein durch das unerwartete und Muster durchbrechende Verhalten der Lehrerin veränderten sich die Gruppendynamik und damit die MobbingSituation für die Autorin.

Körperscham wird auch in anderen Geschichten beschrieben. Sie kann durch Beschämung entstehen, wenn ein Körperaspekt - in den Geschichten häufig das Aussehen, also etwa Körperumfang, Hautunreinheiten oder Kleidung - öffentlich abwertend benannt wird. Dabei können diese Bemerkungen von Mitschüler:innen kommen, aber auch - und das wird als besonders beschämend erlebt - von Lehrpersonen. Körperscham kann sprachlos und einsam machen und mit einer negativen Wahrnehmung des eigenen Körpers einhergehen, wie in vielen Geschichten eindringlich geschildert wird.

Eine weitere Geschichte zum Sportunterricht stammt von einem Studenten, der diesen Unterricht »automatisch mit Gefühlen der Erniedrigung und des Versagens verbindet.« Dazu gehören »schnippische Kommentare bezüglich seines Gewichts « und die Demütigung, wenn er als Letzter in eine Mannschaft gewählt wird. Diese Episode gipfelt in einer Bemerkung des Sportlehrers, während der Autor sich »schweißgebadet und zitternd« am Barren stützt: "Ihr seht, wie viel die (Hölzer des Barrens) aushalten. Da kann man schon gscheit blad sein und die brechen trotzdem ned. «9 - Wie häufig in Erinnerungsgeschichten wird die einzige wörtliche Rede zu einem emotionalen Höhepunkt, der Autor selbst bleibt sprachlos, er »sackt am Barren zusammen« und flüchtet »mit letzter Kraft« auf die Toilette.

$8 \quad$ Haug beschreibt die Aneignung gesellschaftlicher Maßstäbe den weiblichen Körper betreffend: »Erst wenn ich mich als abweichend erkenne, entfalten die Maßstäbe wirkliche Kraft.« (Haug 1983: 73)

9 Dieser Satz im Wienerischen Dialekt bedeutet im Hochdeutschen sinngemäß: »Man kann auch sehr dick sein, die Hölzer des Barrens brechen trotzdem nicht." 
Gerade Sportlehrer:innen benötigen eine große Sensibilität für einen respektvollen Umgang mit der Körperlichkeit der Schüler:innen. Zeichen des Unbehagens, die sich in Kleidungsstil (»wurfzeltartiges T-Shirt«) und Verhalten (»gebückt und mit verschränkten Armen«) ausdrücken, werden von den Autor:innen der Geschichten gut beschrieben. Sie sollten von Lehrer:innen wahrgenommen und abwertende Kommentare zur Körperlichkeit von Mitschüler:innen selbstverständlich gestoppt werden. Das Erkennen von Mobbing und das Wissen um Interventionsmöglichkeiten in der Schule muss Teil der professionellen Ausbildung aller Lehrer:innen sein. Dazu gehört auch die schwierige, aber wichtige Aufgabe, Kolleg:innen darauf hinzuweisen, wenn sie diskriminierende oder demütigende Aussagen treffen.

\title{
3. Schule: Orte der Körperlichkeit
}

Die Erinnerungsgeschichten erzählen uns über Orte in der Schule, in denen unterschiedliche Regeln zur Anordnung und zum Verhalten der anwesenden Körper gelten. Ritualisierte Bewegungsmuster und restriktive Bewegungsräume zeichnen den Lernort Schule aus und vermitteln Normen und Machtverhältnisse. Dabei wird je nach Ort Körperlichkeit unterschiedlich gezeigt und wahrgenommen. Ich beziehe mich hier nur auf die drei Orte, die in den bereits angeführten Geschichten beschrieben werden: das Klassenzimmer, die Turnhalle und die Toilette.

\subsection{Klassenzimmer}

\begin{abstract}
»Wenn er (der Lehrer) unterrichtete, dann schritt er gewöhnlich die Tafel entlang, von der einen Seite zur anderen und wieder zurück. Manchmal bewegte er sich auch durch die Tischreihen, er war beinahe immer in Bewegung. Ganz selten hielt er inne, meist nur um eine mathematische Formel oder Rechnung auf die Tafel zu schreiben. Stets spielte er dabei mit einem Stück Kreide, das er von der linken Hand in die rechte fallen ließ, dann wieder nahm und wieder fallen ließ.«
\end{abstract}

Das Klassenzimmer fungiert als Bühne mit starren und ritualisierten Bewegungsmustern. Hier ist es nur den Lehrpersonen erlaubt, sich im Raum zu bewegen, sie können Nähe und Distanz bestimmen. Schüler:innen dürfen während der Unterrichtsstunde ihren Platz nur auf Aufforderung oder 
mit explizit erteilter Erlaubnis verlassen. Auch der akustische Raum wird von der Lehrperson bestimmt: Wenn sie spricht, dann sollen die Schüler:innen schweigen (und zuhören), die Lehrperson fordert Schüler:innen zum Reden auf. Diese Rituale der Machthierarchie werden in den Geschichten als unausgesprochene Selbstverständlichkeit angenommen. Erwähnt werden aus der Schüler:innenperspektive nur Episoden, in denen durch die unerwünschte Aufforderung zum Reden oder Bewegen unangenehme Situationen entstehen, etwa die schon beschriebenen Prüfungen, mit den begleitenden Gefühlen von Aufregung und Angst. Ein Beispiel: »Die Stunden bei N. liefen immer nach dem gleichen Schema ab. Er suchte sich aus der Namensliste ein Opfer aus, das sich sodann mit Sessel zum LehrerInnentisch begeben musste und dann quasi zum Abschuss freigegeben wurde."

Situationen, in denen sich Schüler:innen allein »nach vorne«, zur Lehrperson, begeben müssen, um dann exponiert und unter Beobachtung der ganzen Klasse eine Aufgabe zu lösen, werden zumeist als unangenehm beschrieben. Aufgelöst und beendet werden können diese Situationen wiederum nur durch die Lehrperson. Aus der Perspektive eines Schülers stellt sich das so dar: »Er wollte einfach nur die Zeit durchstehen, um sich wieder auf seinen Platz setzen zu dürfen und so in der wohligen Anonymität des Klassenverbands abermals untertauchen zu können.«

Wie sehr die Hoheit über den physischen und akustischen Raum im Klassenzimmer als Machtfrage interpretiert wird, zeigen auch Geschichten, die von Junglehrer:innen über ihre ersten Unterrichtserfahrungen geschrieben wurden, denn »Unterrichtsstörungen « sind ein häufig gewähltes Thema. Als eine solche wird eine Situation erlebt, in der ein oder mehrere Schüler unaufgefordert reden, es in der Klasse laut ist, wenn Papierkugeln geworfen werden oder Schüler sich selbsttätig von ihren Plätzen erheben. Wird das unausgesprochene Regelwerk der Rollen von Schüler:innen durchbrochen, so verunsichert das, denn dieses Verhalten wird schnell als Provokation, Machtprobe und Infragestellung der Autorität der Rolle als Lehrer:in interpretiert - zumindest von den Junglehrer:innen, die die Geschichten geschrieben haben. Sie beschreiben diese Situation als Stresssituation. Durch eine veränderte und erweiterte Wahrnehmung der Situationen - nicht jede unautorisierte Bewegung ist eine Provokation - ergeben sich neue Handlungsperspektiven. Denn Lehrer:innen können ihren Unterricht gestalten und etwa die starren Bewegungsmuster durchbrechen, und sei es durch gemeinsame Konzentrationsoder Bewegungsübungen. 


\subsection{Turnsaal}

Der Turnsaal ist, wie bereits beschrieben, ein Ort, an dem Körperlichkeit im Zentrum steht. Es geht um die körperliche Leistungsfähigkeit, beschrieben wird in den Geschichten aber auch eine veränderte, intensivere Wahrnehmung der Körper, besonders des eigenen Körpers und der Körper der anderen Schüler:innen. Situationen mit Körperscham traten in den Erinnerungsgeschichten besonders häufig im Sportunterricht auf. Sportkleidung betont die Körperformen, beim Umkleiden in gemeinschaftlichen Räumen werden Körper genauer sichtbar, in der Nacktheit mit Haut und Haaren.

Der Sportunterricht und dessen Orte betonen zudem binäre Geschlechtszuschreibungen. Umkleideräume sind nach Jungen und Mädchen getrennt, meist auch der Sportunterricht, mit dementsprechend unterschiedlichen Sport-Schwerpunkten. Es werden in den Erinnerungsgeschichten daher Situationen innerhalb einer geschlechtlich zugeordneten Gruppe beschrieben, der Vergleich der Körper bezieht sich nicht nur auf Körpernormen, sondern auch auf Geschlechternormen. Ein Autor beschreibt, wie er als einziger Schüler mit langen Haaren deswegen von seinen Mitschülern spöttisch »Mädchen" genannt wird. Der Sportlehrer verlangt von ihm, dass er, wie die Mädchen mit langen Haaren, seine Haare mit einem Gummiband zusammenbinden muss und kommentiert das mit »schick«. Der Autor diskutiert in der Geschichte die Farbe der Zopfbänder - rosa ist besonders schambesetzt und wird vom Autor abgelehnt, schwarz wird akzeptiert und vom Schüler, wenn auch zögernd, in Kauf genommen. Denn eigentlich, so auch die Einleitung der Geschichte, turnt der Protagonist gerne und hat sich sogar für eine Sportklasse entschieden. Diese Motivation verliert sich, wenn schambesetzte Körperlichkeit in den Vordergrund rückt.

\subsection{Toilette}

Auffällig ist das häufige Vorkommen der Toilette in den Geschichten, nämlich als Fluchtziel in ausweglosen Situationen. In der Schule ist dies der einzige Ort für Privatheit, ein Ort, um zumindest kurz allein sein zu können, ein Rückzugsort mit einer Tür, die geschlossen werden kann. Häufig endet eine Geschichte mit dieser Fluchtbewegung, nur selten wird beschrieben, wie sich Schüler:innen wieder sammeln, wie sie sich wieder aus dem Ort Toilette herausbewegen. Für die Gestaltung der Toilettenräume in Schulen wäre es wichtig, diese Funktion als Schutz- und Rückzugsort mitzudenken. 
Noch eine weitere Funktion der Toilettenräumlichkeit wird in den Erinnerungsgeschichten erwähnt: Als Ort außerhalb der Kontrolle von Lehrpersonen wird sie zur Vorbereitung fürs Schummeln genutzt.

Erst in der Diskussion von Toiletten-Geschichten im Rahmen einer Lehrveranstaltung wurde deutlich, dass Toiletten als >Raum außerhalb der Beobachtung nicht nur als Schutz- und Rückzugsraum wahrgenommen werden: Ein Student beschrieb die Toilette als Ort der Gewalt von Mitschülern. Er hatte es daher während seiner Schulzeit bewusst vermieden, das WC in der Schule $\mathrm{zu}$ benutzen.

\section{Leerstelle Körperlust?}

Aufregung, Angst, Scham - von den Körpersensationen, die diese Gefühle begleiten, wird in den Geschichten zum Lernen in der Schule gehäuft erzählt, diese Erinnerungen halten sich sozusagen körperlich verankert. Während die negativen Körpersensationen in den Erinnerungsgeschichten ausführlich und plastisch beschrieben werden, tauchen Empfindungen wie Lust oder auch Erotik, abgesehen von zarten Verweisen auf Verliebtheit und Anziehung, nur selten auf.

Nur einige wenige Geschichten aus dem Sportunterricht beschreiben ein lustvolles, positives Körpererleben. Sie handeln von der Freude an der Bewegung und der Meisterung körperlicher Herausforderungen oder sie schildern das angenehme Erleben des Gehalten- und Unterstützt-Werdens. Körperlichkeit kann gerade im Sportunterricht auch positiv erlebt werden.

Lernen wird in den Erinnerungsgeschichten nur sehr selten als körperlich lustvolle Erfahrung beschrieben. Dass es möglich ist, zeigt ein Beispiel: Eine Schülerin »hasst « Mathematik. Dennoch findet sie sich an einem Freitagabend mit der ganzen Klasse zusammen in der Schule ein, um nach einer schlecht gelaufenen Schularbeit ${ }^{10}$ kurz vor der Matura ${ }^{11}$ mit dem Mathelehrer mathematische Gleichungen zu besprechen: Denn dieser hatte die schlechte Schularbeit als sein Versagen gesehen und deshalb einen Zusatztermin organisiert. »Der Raum war wie elektrisiert«, »die Leidenschaft des Lehrers und die Liebe und Fürsorge zu seinen Schülern motivierte sie«, schreibt die Autorin, und »obwohl es ihr so schwer fiel, fokussiert und konzentriert zu blei-

10 »Schularbeit« ist der österreichische Begriff für Klassenarbeiten.

11 »Matura«ist der österreichische Ausdruck für Abitur. 
ben, gab sie sich Mühe«. »Der Raum füllte sich mit Motivation, Hingabe und Wissen.«»Es fühlte sich an wie das gemeinsame Stellen eines Drachens, das gemeinsame Bezwingen eines Berges«, »alles mit Hilfe eines kompetenten Anführer«. Es wurde ein "glorreicher Freitagabend«.

Wie in den lustvollen Sportgeschichten geht auch hier um Selbstüberwindung, um Herausforderungen, die gemeistert werden, und um Anstrengungen, die zu einem Erfolg führen - ausgedrückt in körperlichen Metaphern. Worte wie Leidenschaft, Liebe und Hingabe verweisen darauf, dass hier lustvolles Lernen in einem sozialen Kontext stattfindet und eine (nicht-sexuelle) erotische Komponente beinhaltet. Erst durch das Miteinander ist dieses lustvolle Lernen - angeleitet durch den Lehrer - möglich.

Schule kann also lustvolles Lernen ermöglichen, in den bisher gesammelten Erinnerungsgeschichten zeigt sich dieses jedoch nur selten. Die Studierenden wählten zum Thema »Lernen in der Schule« um vieles häufiger Erlebnisse des Nicht-Lernens und der Lernverhinderung als positive Erfahrungen. In der Lehrer:innenbildung benötigen angehende Lehrer:innen Beispiele, wie Lernen in der Schule als lustvoll, auch körperlich lustvoll, erlebt werden kann, damit nicht die eigenen (negativen) Erinnerungen der Maßstab bleiben.

Wie zu Beginn meines Beitrags erläutert sind Erinnerungsgeschichten als Konstrukte zu verstehen, sie bilden keine Realität ab. Sie können aber gerade für zukünftige Lehrer:innen ein Ausgangspunkt sein, um scheinbare Selbstverständlichkeiten und Normalitäten des Schullebens, die in jeder Geschichte stecken, in Frage zu stellen und Handlungsalternativen anzudenken - und dies mit der Methode der Kollektiven Erinnerungsarbeit in einem lustvollen Lernprozess.

\section{Literatur}

Haug, Frigga (1990): Erinnerungsarbeit. Hamburg: Argument Verlag. Haug, Frigga (Hg.) (1983): Frauenformen 2: Sexualisierung der Körper. Berlin: Argument Verlag.

Witt-Löw, Kerstin (2020): Collective Memory-Work for Teacher Training. In: Other Education: The Journal of Educational Alternatives, Bd. 9, 1/2020. 



\title{
Von blutigen Tampons und Penis-Zeichnungen
}

\author{
Stanislaus Medan
}

In meiner dreijährigen Lehrtätigkeit in der Schule hat eine Thematik viel Raum eingenommen, die selten explizites Thema von Unterricht ist, aber doch ständig in der Schule präsent scheint: die Beschäftigung mit Sexualität. Sie äußerte sich auf verschiedenen Ebenen. So gab es deutlich sexuell motivierte Dynamiken zwischen Jungen und Mädchen im Klassenzimmer und in den Pausenräumen. Da die dominante Gruppe, insbesondere die Jungen, meine Aufmerksamkeit bewusster forderten als jene Schüler:innen, die sich nicht sexuell äußerten, war ich in meiner Reaktion zumeist auf diese Gruppe konzentriert.

Es war eine große Herausforderung für mich, klug und differenziert auf die Äußerungen zu reagieren, von denen ich manche als, umgangssprachlich gesagt, seinfach idiotisch ich einmal einen Schüler einen anderen ohne Unterbrechung »Du Schwuchtel« nennen. Ich sprach ihn darauf an, woraufhin sich eine Diskussion entwickelte. Die Klasse, die bis dahin konzentriert an ihren Zeichnungen gesessen war, schien begeistert zu sein, dass ein Lehrer sich in eine Diskussion darüber einlässt. Alle wollten mitdiskutieren und ich erlebte eine der motiviertesten Unterrichtsstunden meiner drei Unterrichtsjahre. Viele der Schüler:innen argumentierten religiös: Einer meinte etwa, dass Eva aus der Rippe von Adam entstanden sei, ein anderer, dass Gott sicher nicht gewollt hätte, dass man »sich gegenseitig in den Hintern fickt«. Die vorrangig männlichen Schüler stellten sich Menschen mit homosexueller Orientierung wie extrem sexualisierte Wesen vor, die nur darauf warten, dass sich jemand irgendwo mal zu Boden beugt. Das homophobe Denken war von den Religionsgemeinschaften geprägt, denen die Schüler:innen angehören und auf die sie sich beziehen.

Darauf war ich nicht vorbereitet gewesen, und mir fiel dazu aus dem Stehgreif keine besonders schlagfertige Antwort ein. Ich erzählte ihnen also von meinen homosexuellen Freund:innen. Meine Erzählung bewirkte, dass der 
Junge, der zuvor seinen Kollegen eine sSchwuchtek genannt hatte, mir nach dem Unterricht hinterherlief und ungläubig fragte, ob ich wirklich nichts gegen Schwule hätte. Ich kann mir vorstellen, dass sich sein Denken darüber durch dieses Gespräch geändert hat. Meiner Einschätzung nach wird Schule ihrem Bildungsauftrag in Sachen sexueller Bildung nicht gerecht und es bleiben sichtbare Lücken.

Für sexuelle Bildung, an der Jugendliche außerschulisches Interesse haben, füllt meiner Erfahrung nach auch das Internet die Lücke. So riefen mir Jungen manchmal zu, ich sollte doch mal diese und jene pornografische Website öffnen. Das verstehe ich als Hinweis, dass qualitative sexuelle Bildung in der Schule fehlt und offenbar durch das Internet kompensiert wird. Zugleich verstehe ich diese Zurufe als eine Aufforderung an mich, Sexualität stärker in den Unterricht einzubinden.

Ein weiterer alltäglicher Anknüpfungspunkt für sexuelle Bildung ist das Thema Penisgröße. Die Zielsetzung, dem Mythos der herausragenden Bedeutung der Größe des Penis entgegenzuwirken, wäre eine Gelegenheit, Biologie mit Bildnerischer Erziehung ${ }^{1}$ u verbinden. Penisse sind die am häufigsten anzutreffenden zeichnerischen Erzeugnisse im Schulhaus. Überall wurde ich ihrer fündig, am häufigsten waren sie im Moment der Ejakulation dargestellt. Haben diese Symbole eine Botschaft? Ich verstehe sie jedenfalls als Aufforderung, Sexualität $\mathrm{zu}$ thematisieren und nicht einfach $\mathrm{zu}$ ignorieren, wie ich es von Kolleg:innen empfohlen bekommen hatte. Von einer Mentorin bekam ich beispielweise den Tipp, jede Diskussion über sexuelle Begrifflichkeiten zu unterbinden und die Angelegenheit damit zu beschließen, dass ihre Verwendung im Unterrichtsraum nicht erlaubt sei. Da Schüler:innen Sexualität so häufig thematisieren, bin ich mir nicht sicher, ob ich bloß mit Verboten antworten möchte.

Einmal wurde ich mit einem rot bemalten Tampon beworfen, während ich mich gerade zur Tafel gedreht hatte. Ich finde, auf diese zugleich bemerkenswert kreative wie freche Aktion kann nicht mit bloßen Verboten geantwortet werden. Ihr sollte Unterricht folgen, der sich differenziert mit sexuellen Thematiken beschäftigt, etwa performativ, malerisch oder filmisch. Bloß, wie reagierte ich spontan in der Situation auf den »Tamponbewurf«? Ich dachte im ersten Moment, dass es echtes Blut sei, und sagte, "Das ist ja ekelhaft»

1 Im österreichischen Schulsystem wird Kunsterziehung »Bildnerische Erziehung« genannt. 
und »Bitte in den Abfalleimer werfen « und machte dann vermeintlich unbeeindruckt mit dem Unterricht weiter.

Beeindruckt hat mich dieses so wie andere Ereignisse dieser Art jedoch schon: Es blieb nicht ohne emotionale Konsequenzen, ständig mit einer pubertär artikulierten Sexualität konfrontiert zu werden und in einem fort $a b-$ wertend von Wixern, Schwuchteln und Hurensöhnen zu hören. In der Anfangszeit meines Unterrichtens bemerkte ich, dass sich das auf meine persönliche Gefühlswelt auswirkte. Im Prozess des Erwachsenwerdens haben sich meine Vorstellungen davon, was sexuelles Verhalten umfasst, verfeinert und ausdifferenziert. Ein breites und tiefes Spektrum an Gefühlen, Vorgängen und Prozessen findet darin Platz. Das Ausmaß dieses Prozesses der Ausdifferenzierung wurde mir erst als Lehrer mit dem Eintritt in die Schule bewusst. In diesem Raum erlebte ich Sexualität als gleichbedeutend mit »Ficken«, was für mein Verständnis eine deutliche inhaltliche Reduktion darstellt. Zermürbend wirkte auf mich außerdem das Macht- und Gewaltvolle, das in den zuvor genannten Wörtern steckt. Die negativen Konnotationen im Bedeutungsraum der Sexualität werden durch die formalisierten Ausrufe und Schimpfwörter immer wieder bestätigt und festigen so ein Bild von Sexualität als etwas Negativem und Gewaltvollem.

Ich bin der Überzeugung, dass Sprache Realität formt, und tendiere dazu, Wahrheit als ein zerbrechliches und veränderbares Gefüge zu verstehen. $\mathrm{Zu}$ einem guten Teil liegt es dieser Ansicht nach an den Menschen, welche Realität sie gemeinsam erschaffen möchten. Wenn ich wieder unterrichte, ist es mir daher ein Anliegen, die schönen und vielfältigen Seiten von Sexualität zu thematisieren und hervorzuheben. 


\section{Digitale Intimitäten}

\section{Orlinder Krinkel}

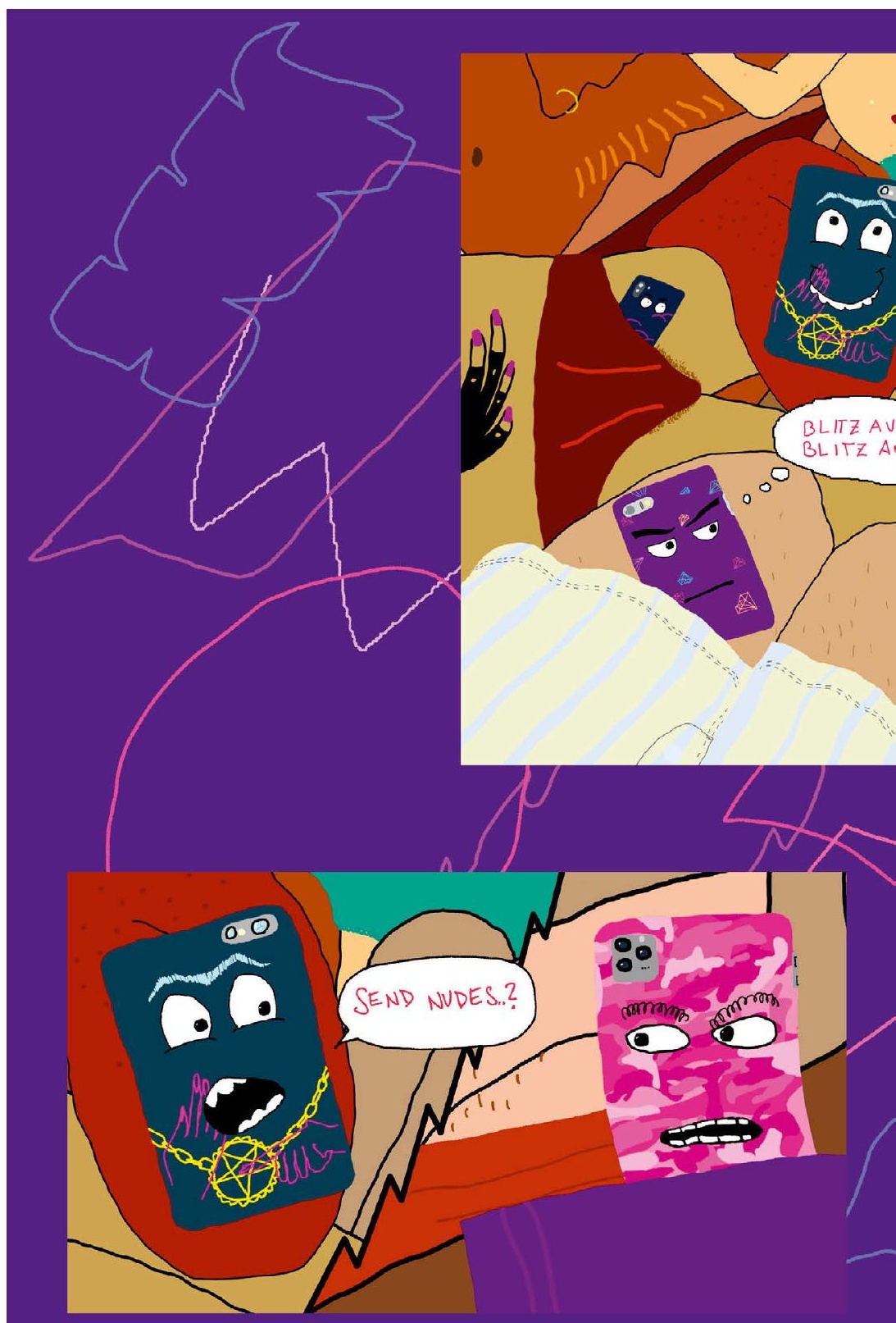





\section{"Nacktfotos auf's Handy kriegen ist doch ganz normal!» Einblicke in die Arbeit mit Jugendlichen zu Sexualität und digitalen Medien}

Michaela Moosmann, Katharina Buhri, Ariane Grabherr und Angelika Atzinger

Das titelgebende Zitat dieses Beitrags ist eine Aussage einer 13-jährigen Teilnehmerin in einem vom Verein Amazone ${ }^{1}$ durchgeführten Workshop. Sie gibt exemplarisch Einblick in die Lebensrealitäten von Jugendlichen heute, die ohne digitale Welten nicht mehr denkbar sind. In sexualpädagogischen Workshops, die der Verein Amazone seit fast 20 Jahren durchführt, sind Veränderungen im Bewusstsein und den Lebensrealitäten von Jugendlichen spürbar. Beispielsweise sind LGBTIQ-relevante Themen mittlerweile stärker ins Bewusstsein von Jugendlichen gerückt - wohl nicht zuletzt aufgrund des Internets und der sozialen Medien. Jugendliche sprechen über Transfrauen (auch) aufgrund von Germany's Next Topmodel oder Orange is the new black, sie kennen das Wort pansexuell vielleicht auch durch Miley Cyrus, sie wissen von homosexuellen Promis, die Kinder adoptiert oder über Leihmutterschaft bekommen haben.

Aber auch Phänomene wie Sexting, Cyber-Grooming ${ }^{2}$, Pornografie, Körper- und Schönheitsbilder sowie Gewalt und Sexismus im Netz sind Bestandteile digitaler Lebenswelten von Jugendlichen heute. Das Versenden

Der Verein Amazone in Bregenz arbeitet seit 1998 an der Vision einer geschlechtergerechten Welt. Dies erfolgt über die Zugänge Bildung und Förderung in den Schwerpunkten Arbeit, Ausbildung, Gesundheit, Gewalt, Kultur, Multimedia, Politik und Sexualität in drei Säulen: dem Mädchenzentrum, der Mädchenberatung und der Fachstelle Gender.

2 (Männliche) Erwachsene bauen im Internet Vertrauen zu Minderjährigen auf, um diese in der Folge sexuell zu belästigen oder zu missbrauchen (vgl. Safer Internet o.].). 
von sogenannten $\mathrm{Nudes}^{3}$, die Allgegenwärtigkeit von perfekten Bildern aus dem Alltag von Influencer:innen oder der Konsum pornografischer Videos sind selbstverständliche Bestandteile der Alltagskultur - nicht nur von Jugendlichen. Unserer Einschätzung nach gilt es diese veränderten Lebensbedingungen und Fragstellungen in der sexualpädagogischen Arbeit nicht nur zu berücksichtigen, sondern mit Jugendlichen zu diskutieren und Handlungsstrategien im Umgang damit zu erarbeiten.

Der vorliegende Beitrag basiert auf den Erfahrungen des Vereins Amazone in der pädagogischen Arbeit zu Sexualität und digitalen Medien, zeigt anhand dieser und ausgewählter Forschungsergebnisse jugendrelevante Themen rund um Sexualität und Medien auf und stellt pädagogische Handlungsmöglichkeiten vor.

Seit 2003 arbeitet der Verein Amazone im Rahmen des Projekts Klipp \& Klar mit Jugendlichen ab zehn Jahren zu sexualpädagogischen Inhalten. Durch dieses Projekt möchte der Verein selbstbestimmte und verantwortungsbewusste Sexualität bei Mädchen und Jungen fördern. ${ }^{4}$

Das Projekt \#followme - Sicherheit durch Medienkompetenz entwickelt Ansätze aus Klipp $\&$ Klar weiter, stellt aber die vielfältigen Erfahrungen Jugendlicher in digitalen und sozialen Medien in den Mittelpunkt und bezieht gewaltpräventive Ansätze mit ein. Es geht von der Annahme aus, dass die in Medien transportierten Vorstellungen von und Erwartungen an Körper, Sexualität, Liebe und Beziehungen Druck und Ängste bei Jugendlichen erzeugen können. Darauf reagiert das Projekt mit Unterstützungsangeboten, die darauf abzielen, Wissen zu Phänomenen und Realitäten im Netz zu vermitteln, Handlungsspielräume etwa bei Cybermobbing oder Sexismus in sozialen Medien zu erweitern, Selbstbestimmung und das Setzen eigener Grenzen zu stärken, positive und alternative Körperbilder sichtbar zu machen, die kritische Reflexion digitaler Inhalte zu fördern, Geschlechterrollenbilder im Netz zu

$3 \quad$ Nudes sind Nacktbilder.

$4 \quad$ Klipp \& Klar - das sexualpädagogische Projekt des Vereins Amazone arbeitet mit Mädchen*, Jungen* und Multiplikator:innen in ganz Vorarlberg und versteht sich als jugendparteiliches, wissenschaftlich fundiertes und professionelles sexualpädagogisches Angebot. Über die Verortung in der Offenen Jugendarbeit werden niederschwellige Zugänge geschaffen, die auf einem umfassenden Verständnis der Sexualpädagog:innen für jugendkulturelle Kontexte basieren. Teil des Projekts sind sowohl Workshops für Jugendliche als auch Fachberatungen, Weiterbildungen und Methodensammlungen für in der Jugendarbeit Tätige. 
diskutieren sowie in der Folge gesellschaftliche Teilhabe und Mitbestimmung zu ermöglichen.

Im folgenden ersten Teil wird das Medienverhalten von Jugendlichen anhand von Studien und Erfahrungen aus der Workshop-Praxis des Vereins beschrieben, um Tendenzen in der Nutzung digitaler Medien zu skizzieren - in dem Bewusstsein, dass Nutzungsverhalten sehr schnell wandelbar und sehr unterschiedlich ist. Im zweiten Abschnitt wird kurz dargestellt, welche Akteur:innen und welche Medien für die sexuelle Bildung Jugendlicher heute wesentlich sind. Im dritten Teil wird auf die Rolle von Lehr- und Bezugspersonen eingegangen und deren Möglichkeiten und Grenzen werden aufgezeigt. Der abschließende vierte Abschnitt stellt konkrete didaktische Zugänge vor, die Impulse für themenspezifischen Unterricht geben können.

\section{Einblicke in das Medienverhalten von Jugendlichen}

Die Erfahrungen, die AmazoneMitarbeiterinnen in ihrer Arbeit mit Jugendlichen in Bezug auf Medien machen, stimmen mit Ergebnissen und Befunden aktueller Studien ${ }^{5}$ weitestgehend überein: Jugendliche beziehen digitale Inhalte mittlerweile großteils über Smartphones, zu denen die meisten Jugendlichen Zugang haben. Jugendliche sehen nach wie vor gerne Filme, Serien und andere bewegte Bilder, wobei sie immer häufiger das Smartphone nutzen, nach wie vor den Computer und immer seltener das Fernsehgerät. Im Anstieg begriffen ist auch die Präsenz von Computerspielen, die nach wie vor von Jungen deutlich häufiger genutzt werden als von Mädchen. Social Media wie WhatsApp, YouTube, Instagram und SnapChat werden immer beliebter, Facebook spielt kaum noch eine Rolle. Hinzu kommen andere Plattformen, etwa TikTok, Twitch und Discord. In Online-Communitys folgen Jugendliche einerseits Freund:innen, die sie persönlich kennen, andererseits Influencer:innen und Stars. Dennoch: Die wichtigste Freizeitbeschäftigung von Jugendlichen ist nach wie vor die Zeit mit Freund:innen (vgl. etwa Wagner/Hochreiter 2016; Education Group GmbH 2019; Saferinternet 2019). Trotz dieser

Vgl. etwa: bitkom (2019); Bode/Heßling (2015); Education Group GmbH (2019); Internationales Zentralinstitut für das Jugend- und Bildungsfernsehen (2020); Medienpädagogischer Forschungsverbund Südwest (2018); Saferinternet (2019); Wagner/Hochreiter (2016). 
relativ eindeutigen Tendenzen zeigen sich in den Workshops Unterschiede und eine hohe Heterogenität im Medienverhalten. Diese kann, laut Einschätzungen von Workshopleiter:innen und Pädagog:innen, mit unterschiedlichen Faktoren wie Alter, Geschlecht, Wohnort oder sozialem Hintergrund in $\mathrm{Zu}$ sammenhang stehen. Relevant können hierbei sicherlich auch Faktoren wie alternative Freizeitangebote im Sozialraum der Jugendlichen, Akzeptanz von Internet-/Smartphonenutzung und sozialen Medien in der Familie oder persönliche Affinitäten sein.

Wenn es um die Frage geht, wer bzw. welche Medien für die sexuelle Bildung Jugendlicher heute relevant sind, zeigt die Jugendbefragung von Bode und Heßling, dass Erziehungsberechtigte hier immer noch eine sehr wichtige Rolle spielen, aber an Bedeutung verlieren (Bode/Heßling 2015: 5). Diese Funktion wird sowohl durch Peers als auch durch professionelle Aufklärung im schulischen Rahmen immer mehr ergänzt. Lehrpersonen werden - wenn es um Sexualität und Verhütung geht - von Jugendlichen kaum als Vertrauensperson angesehen (von $1 \%$ der Mädchen und $2 \%$ der Jungen), die Schule nimmt aber, laut Bode und Heßling, hinsichtlich der Vermittlung von Basisfakten eine sehr wesentliche Position ein. So geben über $80 \%$ der Jugendlichen an, ihre Kenntnisse zu diesen Themen stammten überwiegend aus dem Schulunterricht (vgl. ebd.: 34).

Das Internet ist für die sexuelle Bildung Jugendlicher eine Informationsquelle, deren Bedeutung zunimmt, auch wenn es andere Quellen nicht gänzlich ablöst (vgl. ebd.: 5-6, 57-61): »Es ist das mit Abstand beliebteste Medium, wenn es darum geht, sich zu Sexualität, Verhütung und anderen Themen weiter kundig zu machen, und dies quer durch alle Bevölkerungsgruppen « (ebd.: 61). Dies hängt wohl auch mit der Vielfalt der Formate zusammen, die es bietet: Mädchen und junge Frauen informieren sich auf Wikipedia und anderen Nachschlagewerken (ca. $50 \%$ ), auf Aufklärungs- und Beratungsseiten zum Thema (ca. $50 \%$ ), in Internetforen (ca. 50 \%) oder in Chats (ca. $20 \%$ ). Nur $16 \%$ der Mädchen und jungen Frauen geben an, Sexfilme als Informationsmedium zu nutzen. Demgegenüber stehen $50 \%$ der Jungen und jungen Männer, die dies berichten. Nur ein Drittel der Jungen und jungen Männer sucht Aufklärungs- oder Beratungsseiten auf (vgl. ebd.: 61). Chats spielen in der sexuellen Bildung vor allem für Minderjährige und hier vor allem für homooder bisexuell orientierte Jugendliche eine Rolle. Und: Mit zunehmendem Bildungsgrad wächst die Vielzahl der Quellen, die Jugendliche nutzen (vgl. ebd.: 62-63). 
Die zitierten Studien legen die Interpretation nahe, dass sich Jugendliche sehr viel unbeschadeter in ihrer sexualisierten Umwelt und den Medien bewegen, als Erwachsene denken: Daten widersprechen einer medial häufig dargestellten »Übersexualisierung« und zeigen, dass Erfahrungswelten von Jugendlichen sehr heterogen sind (Hummert 2018: 24). So kann etwa »von einer ungebrochenen Entwicklung zu immer mehr sexuell Erfahrenen im jugendlichen Alter [...] keine Rede sein, in den letzten Jahren scheint vielmehr eine leichte Rückentwicklung einzusetzen« (Bode/Heßling 2015: 8).

\section{Schlussfolgerungen für eine an den Bedürfnissen von heutigen Jugendlichen orientierte Sexualpädagogik}

Der vorangegangene Absatz macht deutlich, dass die Vermittlung eines kritischen und reflektierten Umgangs mit Quellen im Internet zentraler Bestandteil des Unterrichts allgemein sein muss. Jugendliche sind in Hinblick auf Liebe, Sexualität und Körper mit einer Vielzahl von für sie neuen Frage- und mitunter auch Problemstellungen konfrontiert. Dazu kommt, dass diese Themen gesellschaftlich nach wie vor tabubehaftet und schambesetzt sind, folglich erwachsene Bezugspersonen, etwa Erziehungsberechtigte und Lehrpersonen, nur sehr begrenzt als geeignete Ansprechpersonen empfunden werden. Parallel dazu sind Informationen im Internet rund um die Uhr, anonym und schnell zugänglich - was erklärt, dass Jugendliche häufig darauf zurückgreifen. Dies berücksichtigend und wissend, dass die Beurteilung, welche Informationen verlässlich oder richtig sind und wie diese eingeordnet werden sollen, Wissen und Kompetenzen erfordert, muss es Aufgabe einer umfassenden Sexualpädagogik sein, dieses Wissen und diese Kompetenzen zu vermitteln, um auf die Bedürfnisse von Jugendlichen heute eingehen und sie in ihrer Entwicklung gezielt unterstützen zu können.

Digitale Medien sind als zusätzliches, aber nicht alleiniges Angebot der sexuellen Bildung zu betrachten - Sexualpädagogik in der Schule muss diese Medien miteinbeziehen und diskutieren, damit Jugendliche sexualitätsbezogene Bilder, mit denen sie konfrontiert sind, einordnen und korrigieren können und sich somit Möglichkeiten für freie und selbstbestimmte Weiterentwickelung eröffnen können. ${ }^{6}$ 
In der Arbeit des Vereins Amazone werden in den Workshops jeweils jene Themen und Fragestellungen behandelt, die für die teilnehmenden Jugendlichen relevant sind - so kann etwa das Versenden von Nacktbildern in einer Gruppe sehr präsent sein, während in anderen Gruppen YouTubeChannels weit mehr genutzt und diskutiert werden. Darüber hinaus sind Möglichkeiten für Einzelgespräche nach den Workshops oder Hinweise auf Beratungs- und Unterstützungsangebote wesentlich, da besonders schambehaftete Frage- und Problemstellungen im Gruppensetting weniger angesprochen werden. Themenfelder, in die viele Aspekte mit hineinspielen und auf Basis derer viele Netzphänomene diskutiert werden können, sind Körperund Schönheitsbilder, Pornografie oder Hass im Netz. Offensichtlich ist, dass sich Pädagog:innen hier in einem Spannungsfeld von schnellebigen Trends und Phänomenen, rechtlichen und gesetzlichen Bestimmungen und ihren eigenen pädagogischen Möglichkeiten und Grenzen bewegen müssen.

Wie im ersten Abschnitt dargestellt, sind Personen, denen Jugendliche vertrauen und mit denen sie sich über ihre Unsicherheiten und Ängste auf Augenhöhe austauschen können, für Prozesse sexueller Bildung nach wie vor ausschlaggebend, während Neue Medien zunehmend an Bedeutung gewinnen. Diese sind als Chance und Herausforderung zugleich begreifbar und bilden neben einer unendlichen Informationsfülle auch einen virtuellen Experimentierraum für Jugendliche, in dem sie verschiedene Rollen ausprobieren und Kommunikation trainieren können, in dem sie in Kontakt treten, ihren Selbstwert stärken und Zugehörigkeitsgefühle in einer Community aufbauen können. Gleichzeitig müssen Darstellungen auf ihre Alltagstauglichkeit überprüft, Rollenzuschreibungen hinterfragt, Überforderungen und Gruppendruck sichtbar gemacht und Selbstbestimmung gesteigert werden können.

Weil Genderunterschiede das Medienverhalten bereits früh beeinflussen und einschränkende Geschlechterstereotype in sozialen Medien sehr präsent sind, braucht es entsprechende Sensibilität und Bewusstsein bei Pädagog:in-

cher Rahmenbedingungen, Hinweise zu Anlaufstellen, Informationsportalen und Unterstützungsangeboten, Vermittlung differenzierter und vielfältiger Bilder sexueller Wirklichkeit, Erweiterung von Handlungskompetenzen, insbesondere in Hinblick auf Grenzen, die Verfügbarkeit kompetenter Vertrauenspersonen, Sprache und Ausdruck (vgl. Geiser 2017: 28). 
nen, die dies in ihrer Arbeit mit Jugendlichen berücksichtigen, Rollenbilder kritisch hinterfragen und offen diskutieren.

Die Berücksichtigung von (Mehrfach-)Diskriminierungserfahrungen ist zudem unerlässlich für eine zeitgemäße Sexualpädagogik - zumal sowohl das Medienverhalten, die Möglichkeiten sexueller Bildung und die Spielräume für selbstbestimmte Sexualität stark von Faktoren wie ökonomischen oder sozialen Hintergründen, Bildung oder kulturellen/religiösen Zugehörigkeiten geprägt sind. Intersektionale Ansätze und die Berücksichtigung von Diversity in Methodik und Didaktik sind somit sowohl in der Vorbereitung der Inhalte als auch in der sexualpädagogischen Arbeit mit Jugendlichen nicht zu vernachlässigen.

Den Erfahrungen des Vereins Amazone nach haben sich die wahrnehmbaren Bedürfnisse von Jugendlichen in Hinblick auf sexuelle Bildung durch die zunehmende Medialisierung nicht grundlegend verändert: Jugendliche wollen nach wie vor ernst genommen werden, Antworten auf Fragen und Unsicherheiten bekommen und in ihrer vielfältigen und vielschichtigen Persönlichkeit und Identität wahrgenommen werden. Jugendliche brauchen auch gegenwärtig Personen - sowohl Erwachsene als auch andere Jugendliche -, denen sie

vertrauen und mit denen sie sich über ihre Unsicherheiten und Ängste auf Augenhöhe austauschen können.

\section{Zu den unterschiedlichen Handlungsmöglichkeiten von Lehrpersonen und externen Sexualpädagog:innen}

Die pädagogischen Umgangsweisen von Lehrer:innen mit digitalen Medien reichen von absoluten Verboten bis zu einem euphorischen, aber oft leichtfertigen Umgang mit den neuen Möglichkeiten. Der Umgang und Miteinbezug von digitalen Medien in den Unterricht obliegt häufig der Eigeninitiative und Selbsteinschätzung der Lehrpersonen. Dabei wird für viele Lehrpersonen sowohl die Schnelllebigkeit der Inhalte als auch die Verantwortung, die sie für ihre Schüler:innen tragen, wenn sie Komponenten und Aspekte digitaler Medien in den Unterreicht miteinbeziehen, zum Thema. Zweiteres wird insbesondere relevant, wenn es um Datenschutz und -sicherheit geht. Für Lehrpersonen ergibt sich daraus ein Spannungs- und Anforderungsfeld, das häufig ihre Ressourcen übersteigt. Aus diesem Grund ist es wichtig, mit der eigenen Rolle bewusst umzugehen, Grenzen zu erkennen und beim Aufbau der Medi- 
enkompetenzen der Schüler:innen Expert:innen für das jeweilige Themenfeld hinzuzuziehen.

Bei Frage- und Problemstellungen von Jugendlichen hinsichtlich Sexualität, Körper und Beziehung muss somit für Lehrpersonen zentral sein, welche Stärkung, welche Informationen und welches Wissen Jugendliche heute brauchen, um in einer vielfältig sexualisierten Umwelt selbstbestimmt Grenzen ziehen und ihre eigene sexuelle Identität abseits von Zuschreibungen, Medieneinfluss und Gruppendruck finden zu können. Als wesentlich kann folglich eine grundsätzliche Offenheit der Lehrpersonen gegenüber den für Jugendlichen relevanten Themen und Inhalten sowie ein verantwortungsvoller Umgang damit erachtet werden. Um einen solchen Umgang und eine Sicherheit im Thema gewährleisten zu können, ist die Verfügbarkeit von Weiterbildungs- und Beratungsangeboten für Lehrpersonen aufgrund der Schnelllebigkeit der Themen unerlässlich. Weiters kann es sinnvoll sein, externe Expert:innen miteinzubeziehen, insbesondere bei sensiblen und schambehafteten Themen. Externe Sexualpädagogik soll, wie auch im Grundsatzerlass Sexualpädagogik von 2015 (BMBF 2015) festgelegt, als ergänzendes Mittel Eltern und Lehrpersonen unterstützen.

Im Gegensatz zu Lehrpersonen haben externe Sexualpädagog:innen durch ihre einschlägige Ausbildung und den Schwerpunkt ihrer Arbeit auf Sexualität und Gewaltprävention eine vertiefte themenspezifische Expertise, die auch die Auseinandersetzung mit Netzphänomenen und Pornografie umfasst. Das Setting, in dem sie mit Jugendlichen arbeiten, unterscheidet sich klar vom Unterrichtssetting: Die Sexualpädagog:innen kennen die Jugendlichen nicht persönlich und öffnen einen Raum für anonyme Fragen und Probleme. Externe Expert:innen sind nach dem Workshop swieder weg und stehen in keinem Machtverhältnis zu den Jugendlichen, sie geben keine Noten und stehen nicht mit Erziehungsberechtigten in Kontakt. So können Jugendliche Fragen stellen, die sie einer Lehrperson nicht stellen würden, und sie können Themen ansprechen, mit denen sie nach dem Workshop nicht mehr in Verbindung gebracht werden möchten. Des Weiteren sind Sexualpädagog:innen geschult auf Gewaltprävention, Prävention sexuell übertragbarer Krankheiten und ungewollter Schwangerschaften und sensibel bei Verdacht auf Missbrauch und sexualisierter Gewalt. Sie unterstützen ihrem Verständnis nach Eltern und Lehrpersonen und sehen sich gemeinsam mit Eltern und Lehrpersonen als Akteur:innen der sexuellen Bildung. 


\section{Beispiele aus der sexualpädagogischen Arbeit zu digitalen Medien}

Im Folgenden sollen Themen und Phänomene skizziert werden, die in den Workshops des Vereins Amazone sehr präsent sind, und Beispiele aus der Workshop-Praxis aufgezeigt werden, die Sexual- und Medienpädagogik zusammendenken. Für den folgenden Abschnitt wurden Aktivitäten aus den Workshops des Vereins Amazone herausgegriffen, die Themen behandeln, die viele Jugendliche beschäftigen, und die einfach veränder- und bearbeitbar sind. Die geschilderten Beispiele können als Impulse oder Ideen für Unterrichtskonzepte dienen.

\subsection{Körper- und Schönheitsbilder in sozialen Medien}

Die Präsenz unrealistischer Körper- und Schönheitsidealen ist unumstritten - ob in Zeitschriften, Fernsehen, Werbung oder sozialen Medien wie Instagram oder Facebook. Die Darstellungen von Frauen und Männern haben sich in den letzten Jahren zwar ausdifferenziert, sind allerdings in fast allen Bereichen nach wie vor geschlechterstereotyp ausgerichtet. So spielen etwa Jugendlichkeit und das äußere Erscheinungsbild bei Frauen eine bedeutende Rolle, während Männern hier nach wie vor ein deutlich größerer Spielraum zugestanden wird.

Die Häufung an bearbeiteten Fotos und Fake-Bildern führt $\mathrm{zu}$ einer Verschiebung der Wahrnehmung. So werden etwa retuschierte Bilder von Körpern als real angesehen und oft nicht in Frage gestellt. Dadurch werden Schönheitsideale eindimensionaler und unerreichbarer. Eine kritische Auseinandersetzung und Diskussion ist daher von großer Relevanz. Durch genaue Betrachtung und spielerische Analyse können etwa Bilder und Darstellungen hinterfragt, in ihrer Konstruiertheit durchschaut und dadurch entmachtet werden. Wichtig sind dabei erlebnisorientierte Zugänge, denn Jugendliche wissen in der Theorie, dass Bilder bearbeitet und retuschiert werden.

Um deutlich zu machen, wie wenig Realitätsbezug bearbeitete Bilder aufweisen, können Aktivitäten mit Photoshop Fails hilfreich sein. Geeignete Bilder aus Werbung und Medien sind einfach im Internet zu finden. Gemeinsam werden die Bilder genau angesehen. Fehler in Proportionen, Körpergröße, Haut - fehlende Muttermale oder Hautunebenheiten - und Haaren werden aufgedeckt und diskutiert. In der Folge können idealerweise Apps mit Filtern 
und Bildbearbeitungstools ausprobiert werden. Dies schafft ein Bewusstsein darüber, dass diese Tools heute nicht mehr Profis vorbehalten, sondern mit jedem Smartphone anwendbar sind. Auch das Nachahmen von Modelposen aus der Werbung kann Aha-Effekte ermöglichen und spielerisch ein Bewusstsein dafür schaffen, wie übertrieben und künstlich Posen, Körperhaltungen und somit auch Bilder sind. Auf große Resonanz stoßen in der Regel auch Videoclips von Photoshop-Bearbeitungen (etwa "Photoshop turns pizza into woman«) mit anschließender Diskussion und Reflexion. Sie ermöglichen weitere Einblicke in Möglichkeiten der Bildbearbeitung und machen deutlich, wie weit Körperdarstellungen in der Werbung von der Realität entfernt sind.

\subsection{Sexistische Werbung}

Setzt man sich in der Arbeit mit Jugendlichen mit sexistischer Werbung auseinander, sind stereotype Betonungen von Geschlechterunterschieden, $\mathrm{Ab}$ wertungen von Frauen und eine Befürwortung traditioneller Geschlechterrollen grundlegende Themen. Wesentlich ist hier sicherlich, sichtbar zu machen, dass sich Sexismus in vielen gesellschaftlichen Bereichen zeigt, etwa in Witzen, Sprüchen und Begriffen, in seelischen und körperlichen Übergriffen, in ungerechten Arbeitsbedingungen und schlechterer Entlohnung, in der Sprache, in traditionellen Normen zu Rollenverteilung und sehr plakativ eben auch in der Werbung.

Um ein Bewusstsein dafür zu erzeugen, können Bilder sexistischer Werbeplakate und Anzeigen in Gruppen angesehen, diskutiert und infrage gestellt werden. In Kleingruppensettings und/oder im Zuge von spielerischen Aktivitäten in Teamkonstellationen können Jugendliche sich gegenseitig dafür sensibilisieren, was als verletzend und grenzüberschreitend empfunden wird und warum. Auch Perspektivenwechsel können hier helfen, um Inhalte breiter diskutieren zu können. Die Frage Was ist sexistisch und was nicht? kann etwa anhand von Abbildungen von Frauen und Männern in Unterwäsche in der Werbung geklärt werden. Fragen wie Braucht es für die Präsentation des Produktes die Nacktheit oder die Pose der abgebildeten Person? oder Geht es um Werbung für Unterwäsche oder für ein Auto/eine Bohrmaschine/ein Möbelstück? helfen, Sexismus schnell und einfach zu erkennen. Auch Werbetexte und -slogans können in Hinblick auf Sexismus analysiert und diskutiert werden. Darüber hinaus kann eine breite Diskussion zu Themen wie Sprache und Gewalt oder gerechte Sprache sinnvoll sein: Wer wird in Schulbüchern, in Aushängen im Schulgebäude oder in Beiträgen in sozialen Medien genannt und angesprochen und wer ist 
(mit-)gemeint? Fühlen sich »mitgemeinte« Personen auch angesprochen? Außerdem kann es für Jugendliche interessant sein, Institutionen wie den Österreichischen Werberat oder die Werbemelder:in-App von PINKSTINKS kennenzulernen. Jugendliche können Infos zu Aktivitäten und aktuellen Beschwerden einholen und diese anschließend diskutieren. In der Folge können sie selbst aktiv werden und sexistische Werbung melden. In Kombination dazu können Infos und Fakten für Sicherheit vor Sexismus im Netz geteilt und Informationen zu Unterstützungsangeboten und Anlaufstellen zur Verfügung gestellt werden.

\subsection{Sexting}

Sexting - zusammengesetzt aus Sex und Texting - ist mittlerweile ein weit verbreiteter Trend: Immer mehr Jugendliche (und Erwachsene) machen von sich selbst oder anderen erotische Fotos oder Nacktaufnahmen und versenden diese per Smartphone an Freund:innen und Bekannte. Nicht selten landen die Bilder auch im Internet, etwa in Sozialen Netzwerken oder FotoCommunitys und können so eine unüberschaubare Zahl von Menschen serreichen . Erfahrungen in der Arbeit mit Jugendlichen zeigen: Die möglichen Risiken von Sexting sind Jugendlichen in der Regel durchaus bewusst. In konkreten Situationen fällt es ihnen aber schwer, riskantes Verhalten zu vermeiden. Jugendliche brauchen klare Handlungsstrategien, wie sie ihre Privatund Intimsphäre und die von anderen schützen können. Pädagog:innen sind sich bewusst, dass das Versenden von Nacktbildern reizvoll sein und durchaus ein altersadäquates Verhalten von Jugendlichen darstellen kann, etwa um Reaktionen und Aufmerksamkeit zu erhalten und mit eigenen Grenzen und Möglichkeiten zu experimentieren.

Die österreichische Studie von saferinternet.at zu Sexting (Saferinternet 2015) stellt grundlegende Informationen zum Thema zur Verfügung und zeigt Möglichkeiten sowie rechtliche Schritte/Folgen auf. Gemeinsam können typische, scheinbar unbedenkliche Bilder besprochen werden. In einem zweiten Schritt kann die rechtliche Situation gemeinsam abgesteckt werden. Diese Aktivität macht Jugendliche auf eine Diskrepanz aufmerksam, der sie sich häufig nicht bewusst sind. Gerade in Hinblick auf Fotos, die ohne das Wissen oder Einverständnis der abgebildeten Person geteilt oder veröffentlicht werden, ist es wichtig, Solidarität und Verständnis für Betroffene zu erzeugen. In einem weiteren Schritt können Möglichkeiten und Strategien erarbeitet werden, wie eine betroffene Person unterstützt werden kann. Da bei Jugend- 
lichen in der Regel bereits viele Ressourcen und Ideen dazu vorhanden sind, bietet es sich an, in Kleingruppen Vorschläge und Möglichkeiten erarbeiten $\mathrm{zu}$ lassen und dann gemeinsam zu diskutieren. Hier sind auch Informationen zur Meldung oder zum Blockieren von Personen wesentlich.

\subsection{Pornografie}

In Hinblick auf Pornografie zeigen sowohl Praxis als auch Studien, dass männliche Jugendliche eher Erfahrung im Konsum pornografischer Inhalte haben als Mädchen (vgl. Geiser 2017: 26). In polemischen gesellschaftlichen Diskursen ist oft von einer pauschal als negativ bewerteten >Pornografisierung von Jugendlichen die Rede, die zu hinterfragen ist. Die Verfügbarkeit und vermehrte Nutzung pornografischer Inhalte sowie Pornografie-Bezüge in der Populärkultur müssen auf differenzierte Art und Weise in die Arbeit mit Jugendlichen miteinbezogen werden (vgl. Kurzmann 2015). Dabei geht es in der Sexualpädagogik keinesfalls darum, eine Anti-Porno-, Anti-Zensuroder Pro-Porno-Position (vgl. Döring 2015: 33) einzunehmen und die Wirkung von Pornografie zu dramatisieren oder zu bagatellisieren (vgl. Geiser 2017: 26), sondern darum, jugendliche Realitäten auch in diesem Hinblick ernst zu nehmen und $\mathrm{zu}$ respektieren.

Pornografische Inhalte können mit Jugendlichen gemeinsam reflektiert, diskutiert und kritisch hinterfragt werden. Hier können etwa Erwartungshaltungen von Jugendlichen thematisiert und in der Folge Realitätsbezügen gegenübergestellt werden. Auch Fragestellungen in Hinblick auf Körperideale und Geschlechterrollen können von Bedeutung sein. Weiters kann es sinnvoll sein, Porno-Bezüge in der Populärkultur in für Jugendliche relevanten Formaten wie Castingshows oder Werbung zu thematisieren. Die Frage nach entwicklungsbeeinträchtigenden Auswirkungen auf Jugendliche stellt sich hier ebenso wie bei expliziteren Inhalten (vgl. ebd.: 26f.). Insbesondere die hier häufig inszenierte Abwertung von Frauen ist ein Thema, das mit Jugendlichen beispielsweise anhand von Bildmaterial aus der Werbung gut behandelt und diskutiert werden kann. Jugendliche sollen dafür sensibilisiert werden, dass Pornos keine Lehrfilme für gelebte Sexualität, sondern in der Regel Fantasiefilme für die Selbstbefriedigung von Männern sind. Dazu eignet sich etwa der Film »Sex, we can?!« (Episode 3) und eine anschließende Diskussion.

Die Erfahrungen aus der praktischen Arbeit des Vereins Amazone zeigen, dass Sexual- und Medienpädagogik konsequent zusammengedacht und interdisziplinär umgesetzt werden müssen. Digitale wie analoge Identitäten, 
Lebens- und Liebenswelten, Beziehungen und Kommunikation stehen in einem kontinuierlichen Wechselspiel miteinander. Dies spricht auch dafür, dass die sexuelle Bildung Jugendlicher auf viele Akteur:innen verteilt sein muss: Eltern, Bezugspersonen, Lehrpersonen, Sexualpädagog:innen und Medien - sie alle spielen eine wichtige Rolle und tragen Verantwortung dafür, wie Jugendliche sich in diesem Hinblick bestmöglich und frei entwickeln können. Dabei sind ein klares Rollenverständnis und eine ständige Reflexion von Möglichkeiten und Grenzen dieser Rolle von höchster Wichtigkeit. Die Rolle von Sexualpädagog:innen, die für einen Workshop an eine Schule kommen, die Schüler:innen und ihre Lebensumstände nicht kennen und auch keine Noten geben, können Lehrpersonen unmöglich erfüllen. Sie sind jedoch Schlüsselpersonen für fächerübergreifende Wissensvermittlung und die längerfristige Begleitungs- und Bildungsarbeit und $\mathrm{zu}$ in diesem Kontext relevanten Themenstellungen.

\section{Literatur}

bitkom (2019): Kinder und Jugendliche in der digitalen Welt. Online unter: $h$ ttps:/www.schau-hin.info/fileadmin/content/Downloads/Sonstiges/Bit kom_Studie_Kinder_und_Jugendliche_2019.pdf [Zugriff: 21.05.2020].

Bode, Heidrun/Heßling, Angelika (2015): Jugendsexualität 2015. Die Perspektive der 14- bis 25-Jährigen. Ergebnisse einer aktuellen Repräsentativen Wiederholungsbefragung. Köln: Bundeszentrale für gesundheitliche Aufklärung.

Döring, Nicola (2011): Pornografie im Internet: Fakten und Fiktionen. In: tv diskurs. Verantwortung in audiovisuellen Medien 2/2011, S. 32-37. Online unter: https://fsf.de/data/hefte/ausgabe/57/doering032_tvd57.pdf [Zugriff: 09.10.2019].

BMBF - Bundesministerium für Bildung und Frauen (2015): Grundsatzerlass Sexualpädagogik. Online unter: https://www.bmbwf.gv.at/Themen/schu le/schulrecht/rs/1997-2017/2015_11.html [Zugriff: 21.01.2020].

Education Group GmbH (2019): Oberösterreichische Jugend-Medien-Studie 2019. Das Medienverhalten der 11- bis 18-Jährigen. Online unter: https:// www.edugroup.at/innovation [Zugriff: 02.10.2019].

Geiser, Lukas (2017): Sexualität im Digitalzeitalter. Neugier und Normalität bei Jugendlichen. In: Pädiatrie 3/2017, S. 25-29. Online unter: https://www 
.rosenfluh.ch/paediatrie-2017-03/sexualitaet-im-digitalzeitalter [Zugriff: 09.10.2019].

Hummert, Michael (2018): Geile Zeit. Sexualitätsbegleitung in der Kinderund Jugendhilfe. Vortragsskript der Einrichtungsleitungskonferenz LVR Landesjugendamt. Institut für Sexualpädagogik, Köln. Online unter: http://docplayer.org/108847679-Geile-zeit-sexualitaetsbegleitung-inder-kinder-und-jugendhilfe-koeln-25-april-michael-hummert.html [Zugriff: 21.10.2019].

Internationales Zentralinstitut für das Jugend- und Bildungsfernsehen (2020): Grunddaten Jugend und Meiden 2020. Online unter: https://www. br-online.de/jugend/izi/deutsch/Grundddaten_Jugend_Medien.pdf [Zugriff: 21.05.2020].

Kurzmann, Michael M. (2015): Buben- und Burschenarbeit: PornografieKompetenz als ein Beitrag zur Gewaltprävention? Online unter: https://w ww.gewaltinfo.at/themen/2015_08/buben-und-burschenarbeit.php [Zugriff: 09.10.2019].

Medienpädagogischer Forschungsverbund Südwest (2018): JIM-Studie 2018. Jugend, Information, Medien. Basisuntersuchung zum Medienumgang 12- bis 19-Jähriger. Online unter: https://www.schau-hin.info/ fileadmin/content/Downloads/Sonstiges/JIM_2018_Gesamt.pdf [Zugriff: 21.05.2020].

Saferinternet (2015): Sexting in der Lebenswelt von Jugendlichen. Online unter: https:/www.saferinternet.at/news-detail/aktuelle-studie-ver sand-von-eigenen-nacktaufnahmen-unter-jugendlichen-nimmt-zu [Zugriff: 23.11.2019].

Saferinternet (2019): Jugend-Internet-Monitor. Online unter: https://www.sa ferinternet.at/services/jugend-internet-monitor [Zugriff: 02.10.2019].

Safer Internet o.J.: Cyber-Grooming. Online unter: https://www.saferinterne t.at/faq/jugendarbeit/cyber-grooming [Zugriff: 28.05.2020].

Wagner, Petra/Hochreiter, Bea (2016): Digitales Medienverhalten von Jugendlichen eine Genderfrage?. In: soziales_kapital. wissenschaftliches journal österreichischer fachhochschul-studiengänge soziale arbeit 15, S. 125-136. Online unter: https://soziales-kapital.at/index.php/sozialeskapital/article /view/445/793 [Zugriff: 02.10.2019]. 


\section{Auf Community bauen und in Vernetzungen lernen Eine Gesprächsrunde über rassismuskritische Ansätze in der Bildungsarbeit zu Sexualität und Vielfalt}

Rosemarie Ortner

Rassismuskritische Zugänge zu sexueller Bildung sind im sexualpädagogischen Fachdiskurs ${ }^{1}$ noch wenig präsent und ausgearbeitet. Sie werden aber in der Praxis auf Basis der Erfahrungen im pädagogischen Handeln entwickelt und erprobt. Um dieses Feld zu explorieren und Bausteine zusammenzutragen, luden die Herausgeberinnen Personen aus der sexualpädagogischen Praxis zu einem Gruppengespräch. Der Einladung gefolgt sind Zeynep ÖnsürOluğ und Maria Dalhoff von der Wiener Fachstelle Selbstlaut-Gegen sexualisierte Gewalt an Kindern und Jugendlichen ${ }^{2}$, Rafaela Siegenthaler von dem von der Akademie der bildenden Künste Wien initiierten Bildungs- und Forschungs-

In der sexualpädagogischen Fachliteratur finden sich durchaus Ansätze, die sich mit Diskriminierung und Diversität kritisch auseinandersetzen (vgl. Debus/Laumann 2018; Debus 2016; Sielert u.a. 2017) oder Handreichungen für eine interkulturelle Einbettung sexualpädagogischer Praxis anbieten (vgl. Fachstelle Selbstlaut 2017). Unter Bedingungen der Migrationsgesellschaft stellt sich aber auch die Frage nach einem spezifischen Fokus auf Rassismus.

2 Die Fachstelle Selbstlaut - Cegen sexualisierte Gewalt an Kindern und Jugendlichen bietet Präventionsarbeit mit Kindern, Eltern und Pädagog:innen, Workshops mit Schulklassen zur Ausstellung »Ganz schön intim«, Beratung und Verdachtsbegleitung sowie Fort- und Weiterbildung für Pädagog:innen und arbeitet auch in der Beratung und Supervision von Teams und Einrichtungen (https://selbstlaut.org [Zugriff: 15.06.2020]). 
projekt Imagining Desires ${ }^{3}$ und Kristin Lerch vom ebenfalls in Wien angesiedelten queeren Bildungsprojekt queerconnexion ${ }^{4}$. Das Gespräch moderierte Nina Wlazny, Bildungswissenschaftlerin und Erwachsenenbildnerin.

Dem Text liegt das Transkript des Gesprächs zugrunde, das von Rosemarie Ortner in Absprache mit den Teilnehmenden für die Publikation gekürzt und verdichtet wurde. Er folgt in weiten Teilen der Chronologie des Gesprächsverlaufs, die jedoch an manchen Stellen zugunsten thematischer Kohärenz verlassen wird.

\section{Was uns wichtig ist - sexualpädagogische Grundhaltungen}

Nina Wlazny: Ich beginne mit der Frage nach den Zugängen zu euren eigentlich doch sehr verschiedenen Tätigkeiten. Welche Haltungen sind euch in eurer Arbeit besonders wichtig?

Kristin Lerch: Die queerconnexion ist im Kontext von LGBTI-Aktivismus entstanden, daher kommt die Idee der Selbstvertretung. Wir haben nicht den Anspruch ganze Gruppen zu repräsentieren, weil das als einzelne Person nicht möglich ist, aber wir sind schon dafür da, unsere eigene Erfahrung als queere Personen zu teilen, und arbeiten auch autobiografisch. Die Teilnehmenden können auch persönliche Fragen stellen. Alle Teams bestehen aus queeren Menschen und unsere Idee ist, dass nicht über queere Menschen gesprochen wird, sondern mit ihnen. Wir möchten, dass die Teilnehmenden queere Menschen kennenlernen, die ein Gegenüber sind, so dass die Kommunikation auf Augenhöhe stattfinden kann. Natürlich haben wir persönliche Grenzen. Wir

3 Im Forschungs- und Bildungsprojekt Imagining Desires arbeiten Schüler:innen, Lehrer:innen, Lehramtsstudierende, Wissenschaftler:innen, Sexualpädagog:innen und Künstler:innen gemeinsam zu Fragen rund um Sexualität, visuelle Kultur und Pädagogik. Als erste Ergebnisse wurden Materialien für die Bildungsarbeit erstellt (vgl. Schmutzer/Thuswald 2019). Weitere Materialien siehe https://www.imaginingdesires. at [Zugriff: 15.06.2020]. queerconnexion bietet Workshops zu sexuellen Orientierungen und Ceschlechtsidentitäten an Schulen und Jugendzentren an. Dabei werden Homosexualität, Bisexualität, Transgeschlechtlichkeit und Intergeschlechtlichkeit behandelt und Heteronormativität, vielfältige Lebensweisen und Sexualitäten thematisiert (siehe https://queerconne xion.at [Zugriff: 15.06.2020]). 
sprechen über unsere Erfahrungen mit Coming-out und Selbstfindung, aber nicht über unser Sexleben.

Zeynep Önsür-Oluğ: Bei Selbstlaut haben wir einen etwas anderen Schwerpunkt. Wir versuchen - vor allem wenn Jugendliche und Kinder zu uns kommen - ihnen Räume zu geben, wo sie über sich selbst sprechen können, wo sie sich selbst beschreiben und auch Fragen stellen können. Wichtige Haltungen in unserer Arbeit sind Freiwilligkeit und Grenzachtung, denn die Schamgrenzen bei den einzelnen Teilnehmenden sind ja ganz unterschiedlich. Z.B. hat einmal eine Gruppe von Schüler:innen die ganze Zeit über S.E.X. gesprochen - sie haben das Wort Sex nie aussprechen können, weil es Gelächter gegeben hat und manche sich geschämt haben. Und dann gibt es Gruppen, bei denen genaue und vertiefende Auseinandersetzung gewünscht ist. Das ist ganz unterschiedlich, und das ist total okay.

Maria Dalhoff: Ich möchte hier noch zwei Selbstlaut-Grundhaltungen ergänzen. Die eine besteht darin, sexuelle Gewalt in der sexuellen Bildung mitzudenken, und zwar in einer sex-positiven sexuellen Bildung: also weder Panikmache vor Vergewaltigung und Krankheiten $\mathrm{zu}$ betreiben und Sex vor allem als gefährlich darzustellen noch zu sagen: »Sex ist so toll und alle können es genießen, wenn sie nur wollen."Wir wollen vermitteln, dass es ein großes Spektrum an Sexualitäten, Begehren, Körpern, Zugängen zu Sexualität und Lebensformen gibt. Und dann aber auch Gewalt zu benennen und sie von den vielfältigen Formen von Sexualität so gut wie möglich zu unterscheiden. Es braucht Klarheit, was Sexualität und was Gewalt ist.

Eine zweite Grundhaltung zielt darauf ab, Optionenvielfalt anzubieten. Maisha-Maureen Auma hat mit diesem Begriff in ein Wort gefasst, was schon lange ein - wenn nicht sogar der - Ansatz von Selbstlaut ist. ${ }^{5}$ Durch das Eröffnen von unterschiedlichen Optionen kann ich - aus der Präventionsperspektive gedacht - Sortierhilfe für Kinder, Jugendliche und auch Erwachsene den Titel: »Sexualpädagogisches Empowerment für Schwarze Menschen und People of Color im deutschsprachigen Raum. Intersektionale, rassismuskritische, traumaorientierte Selbstbildungsansätze des Berliner SEEDS Collective.« (vgl. https://imagi ningdesires.at/sexualpaedagogisches-empowerment-maisha-maureen-auma [Zugriff: 15.06.2020]). 
geben, die sonst in den Materialien nicht vorkommen, nicht abgebildet werden. Das bedeutet Folgendes aufzuzeigen: Es gibt dies, es gibt das, und dann gibt es noch ganz viel anderes und davon nenne ich euch vielleicht noch fünf Optionen. Am Beispiel der sexuellen Orientierung bedeutet das zu benennen, dass manche Personen pansexuell begehren, andere sich als bisexuell oder lesbisch, schwul, asexuell oder aromantisch bezeichnen - und dass heterosexuelles Begehren eine Option von vielen ist, eine häufig gelebte, aber trotzdem eben nur eine Option. Es geht darum, unterschiedliche Möglichkeiten nebeneinanderzustellen ohne den moralischen Zeigefinger zu erheben und $\mathrm{zu}$ fordern, dass alle sich zu etwas bekennen.

Rafaela Siegenthaler: Da Selbstlaut ein Kooperationspartner in unserem Projekt Imagining Desires ist, gibt es sehr viele Überschneidungspunkte bezüglich der Zugänge. Bei Imagining Desires handelt es sich um ein Forschungsprojekt, deshalb könnte man auch von Forschungsansätzen sprechen anstatt von Haltungen in der pädagogischen Arbeit. Wir verfolgen jedenfalls einen kritischen Diversitätsansatz ${ }^{6}$ und einen möglichst repräsentationskritischen Zugang. Mit einem repräsentationskritischen Zugang versuchten wir die Dominanz bestimmter Darstellungen in den Blick zu nehmen. Besonders wichtig ist mir der partizipative Forschungsansatz, also der Versuch mit den CoForschenden - in diesem Fall Jugendliche oder Schüler:innen - zusammen $\mathrm{zu}$ forschen und dabei mit einem möglichst hohen Maß an Mitbestimmung $\mathrm{zu}$ arbeiten.

\section{Rassismuskritische Strategien (er-)finden}

Nina Wlazny: Ich würde gerne einige bisher angesprochene Aspekte bündelnd aufgreifen und daran anschließend nach einer Lücke im Diskurs fragen: Kritische Diversität und Repräsentationskritik wurden erwähnt, der Begriff Optionenvielfalt, Vielfalt generell in der sexuellen Bildung sowie das Thema sexuelle Gewalt. Alle diese Themen haben mit Macht- und Herrschaftsverhältnissen, Ungleichheitsverhältnissen und Diskriminierung zu tun. Dazu

Ein kritischer Diversitäts-Ansatz versucht soziale Ungleichheit und Ausschlüsse entlang von Differenzlinien nicht nur in ihrer alltäglichen Form aufzudecken, sondern auch in ihrer strukturellen Dimension zu erfassen (vgl. Mecheril/Plößer 2011; Eggers 2015; Auma 2017; Diallo/Erni 2017; Thuswald/Sattler 2019). 
gibt es in der Fachliteratur mittlerweile zunehmend mehr Publikationen. Interkulturelle Sexualpädagogik, nicht-diskriminierende Sexualpädagogik wären Schlagwörter - oder Sexualpädagogik der Vielfalt. ${ }^{7}$ Aber zu explizit rassismuskritischer oder auch ungleichheitskritischer Sexualpädagogik gibt es nicht viel. Ist das in euren Teams ein Thema? Und wenn ja, wie reflektiert ihr das und welche Begriffe verwendet ihr?

Rafaela Siegenthaler: Ich habe im Rahmen des Projekts mit Jugendlichen aus der Schwarzen Frauen Community ${ }^{8}$ zusammengearbeitet. Bei der Recherche zu einem rassismuskritischen Zugang in der Sexualpädagogik habe ich tatsächlich nicht viel gefunden. Ich meine Zugänge, die einen dekolonialen Ansatz verfolgen und nicht solche, die eigentlich mehr Rassismus reproduzieren als sie in Frage stellen, wenn z.B. nicht-weiße $e^{9}$ Männer stereotypisiert und stigmatisiert werden.

In der Arbeit mit den Jugendlichen fand ich es spannend, dass das Thema Sexualität gar nicht so schnell zur Sprache kam. Wenn es um Sexualität gehen soll, müssen zuerst ganz andere Themen besprochen werden. Am Anfang war ich irritiert, weil ich dachte, wir müssen zum Thema Sexualität kommen, weil ich ja rechtfertigen muss, warum wir diese Arbeit im Rahmen des Projekts machen (lacht). Ich habe aber schnell gemerkt: Man kann nicht über Sexualität ins Sprechen kommen, wenn man nicht Rassismuserfahrungen dazunimmt, Erfahrungen mit Körpernormen, Gendernormen, Selbstliebe. Im Rahmen des Projekts gab es den institutionellen und finanziellen Rückhalt,

7 Vgl. Debus (2016); Tuider u.a. (2012).

8 Die Schwarze Frauen Community (SFC) ist eine Selbstorganisierung Schwarzer Frauen in Wien und organisiert neben Gruppen und Beratungsangeboten auch Öffentlichkeitsarbeit und Communityevents, um den gesellschaftlichen Problemlagen einen Empowermentansatz entgegenzustellen (siehe https://www.schwarzefrauencommunity. at [Zugriff: 15.06.2020]).

9 Die Attribute »weiß « und »schwarz«stellen koloniale Konstruktionen dar; diese Begriffe benennen keine ethnischen oder gar biologischen Zugehörigkeiten, sondern die Positionierung in von Rassismus geprägten gesellschaftlichen Verhältnissen. Um dies in schriftlicher Sprache deutlich zu machen, setzen wir weiß kursiv. In Selbstorganisationen Schwarzer Menschen wird die Schreibweise Schwarz benutzt, um auf die Bedeutung der Möglichkeit zur Selbstbenennung marginalisierter Menschen aufmerksam zu machen. Daher übernehmen wir diese Schreibweise, wenn das Wort in diesem Sinne benutzt wird. 
das auch zu machen - und zuallererst die Möglichkeit, diese Kooperation einzugehen.

Nina Wlazny: Fallen euch weitere Beispiele aus der Praxis ein, bei denen sich rassistische Ungleichheitsverhältnisse zeigen?

Kristin Lerch: In vielen unserer Workshops haben wir mit dem Diskurs zu tun, dass Queer-Sein etwas ist, das vom Westen in die Welt getragen wird. Manchmal nehmen Jugendliche an, vor allem ausländische Menschen seien queerfeindlich, transfeindlich, homofeindlich - eher als österreichische Leute. Und zum anderen wird Queer-Sein oft als etwas gesehen, das für Menschen aus anderen Gegenden der Welt oder mit Migrationsbiografien nicht in Frage kommt. Aber historisch wurden nicht queere Lebensweisen, sondern die Unterdrückung von queeren Menschen in vielen Ländern erst mit der Kolonisation etabliert. Weil das in der Geschichte nicht aufgezeigt wird, scheint es aber, als wären queere Lebensweisen etwas Westliches. Das ist problematisch, denn es ist nicht so, dass weiße Österreicher:innen alle queerfreundlich wären und es keine Diskriminierung gäbe. Wir erleben oft, dass wir diese Vorstellung ein bisschen aufbrechen müssen.

Die Sichtbarkeit von queeren Menschen, die nicht österreichisch bzw. nicht weiß sind, ist da sehr wichtig. Wir versuchen zu erklären, dass Menschen queer und muslimisch sein können. Oder wir sprechen über Fluchterfahrungen oder Migrationserfahrungen von LGBTI-Personen. Gerade in Gruppen, in denen nicht nur weiße Österreicher:innen teilnehmen, können viele etwas damit anfangen, wenn ich erkläre, inwiefern trans zu sein und Transitionsprozesse $\mathrm{zu}$ machen schwierig ist für Migrant:innen, die nicht auf österreichische Gesetze zu Namens- und Personenstand, also Geschlechtseintragsänderung, zugreifen können, oder inwiefern Migrant:innen und Menschen ohne österreichischen Ausweis viel mehr Diskriminierung erfahren. Manche Jugendliche sagen dann, dass sie das kennen, also nachvollziehen können.

Zeynep Önsür-Oluğ: Ich teile deine Erfahrung, dass Jugendliche, die selbst Diskriminierungserfahrung gemacht haben, oft mehr Verständnis zeigen und sagen: Ich kenne das, selbst zum sogenannten Anderen gemacht oder auch abgewertet zu werden. Wenn eigene biografische Erfahrungen existieren, dann verstehen sie, dass homophobe Aussagen verletzen. Wir versuchen auch, diese gängigen »Othering«-Praxen in den Fortbildungen für Pädagog:innen anzusprechen. Wenn man mit Kindern und Jugendli- 
chen arbeitet, ist es notwendig, sich mit Themen wie Diskriminierung und Rassismus auseinanderzusetzen und die eigene Praxis daraufhin kritisch zu reflektieren. Teile ich die ganze Klasse in »ausländische Kinder« oder »Kinder mit Migrationserfahrung« und »einheimische Kinder« ein? Was für einen Nutzen habe ich davon? Und was für ein Signal sende ich den Kindern dadurch? Aus Präventionsperspektive macht es einfach keinen Sinn, ein Kind zum >Anderen $<\mathrm{zu}$ machen bzw. zu selektieren und zu labeln. Das Kind bekommt so einen Stempel und erfährt: Ich bin irgendwie anders und meine Bedürfnisse haben wenig Wert, ich darf auch weniger darüber sprechen, ich gehöre nicht zu den sogenannten Normalen. Ein geschwächtes Selbstvertrauen ist ein potenzielles Einfallstor für Täter:innen. Wir überlegen mit den Pädagog:innen gemeinsam, wie man diese Praxen kritisch reflektierend verändern und im besten Fall verhindern kann.

Kristin Lerch: Ich bin ein bisschen unsicher, wenn Jugendliche, ein »Bei euch okay, nicht bei uns «-Denken mitbringen. Ja, so reagieren Menschen oft. Aber meine Beschreibung kann auch zugleich das Klischee von den homo- oder queerfeindlichen ausländischen Jugendlichen verstärken. Da ist es nicht leicht, Stereotypisierungen zu vermeiden.

Nina Wlazny: Was sind Empfehlungen, wenn man in dieses Fahrwasser kommt?

Kristin Lerch: Was am meisten hilft, ist, die Realitäten queerer Menschen zu vermitteln, die nicht österreichisch, nicht-weiß, nicht christlich sind. Damit diese auch repräsentiert werden. Wir haben eine Methode, in der es um berühmte queere Personen geht. Sie ist zur Zeit sehr US-orientiert, damit die Teilnehmenden viele Personen schon kennen, aber ich fände es gut, z.B. queere Aktivist:innen, die geflüchtet sind oder in anderen Teilen der Welt leben und dort Aktivismus machen, zu zeigen. Wenn Teilnehmende sagen, dass es >das« nicht gibt, wenn sie ihre Verwandten da und dort besuchen, dann hilft es oft zu zeigen, dass es überall auf der Welt queere Menschen gibt. Eine weitere ganz konkrete Strategie ist folgende: Am Ende der Workshops stellen wir Organisationen und Beratungsstellen vor. Und da sprechen wir auch 
über $A R A^{10}$, die Queer Base ${ }^{11}$, ORQOA - Oriental Queer Organisation ${ }^{12}$. Es ist nicht immer möglich über eigene Erfahrungen zu sprechen, da einige Menschen eben keinen Migrationshintergrund haben, aber diese Organisationen vorstellen können eigentlich alle.

Maria Dalhoff: Eine unserer Methoden für sexuelle Bildung ist mitunter, nur indirekt über Sexualität oder sexuelle Orientierung zu sprechen, sondern alle möglichen anderen Themen drum herum, die in der klassischen Aufklärungsarbeit nicht zu einem engeren Sexualverständnis zählen, mit aufzunehmen. Wir thematisieren etwa Sprache, unterschiedliche Körper oder unterschiedliche geografische Orte und wie das alles in Körperlichkeit und Sexualität hineinspielt. Welche Kosenamen sage ich in welcher Sprache oder welche Genitalausdrücke kommen mir in der einen Sprache über die Lippen und in der anderen gar nicht? Oder welche Bezeichnungen habe ich für Gewalterfahrung? Ich würde das als rassismus- oder ungleichheitskritische Methode verstehen, aber im allerweitesten Sinne unter der größeren Überschrift von Barrierefreiheit fassen, d.h. auf unterschiedlichsten Ebenen Zugang zu Lebensthemen zu schaffen.

10 Afro Rainbow Austria (ARA) ist die erste Organisation von und für LCBTQI+Migrant:innen aus afrikanischen Ländern in Österreich und bietet eine Plattform für Sichtbarkeit, Kommunikation und Veränderung. ARA will eine Community aufbauen, die von Vertrauen und gegenseitiger Hilfestellung getragen wird. Diese Community thematisiert alltäglichen und strukturellen Rassismus, aber auch Homophobie sowohl aus der österreichischen Mehrheitsgesellschaft wie vonseiten der afrikanischen Communitys (siehe https://afrorainbow.at [Zugriff: 15.06.2020]).

11 Queer Base - Welcome and Support for LCBTIQ Refugees unterstützt Ceflüchtete, die in ihren Herkunftsländern aufgrund ihrer sexuellen Orientierung oder Ceschlechtsidentität verfolgt wurden, im Asylprozess und beim Ankommen in der LCBTIQCommunity in Österreich (siehe http://queerbase.at, http://friends.queerbase.at [Zugriff: 15.06.2020]).

12 ORQOA - Oriental Queer Organisation Austria ist Anlaufstelle für Menschen, die auf Grund ihrer sexuellen Orientierung oder Geschlechtsidentität in Ländern des Nahen Ostens und auch auf der Flucht oder in Wien in der migrantischen Community belästigt, isoliert und diskriminiert werden. Da in einigen Ländern Homosexualität eine strafbare Handlung darstellt und Homophobie auch zum Ausschluss von LCBTIQPersonen aus der Familie führt, sind sie, wenn sie flüchten, allein auf sich gestellt und finden bei ORQOA Unterstützung (siehe https://www.orqoa.at [Zugriff: 15.06.2020]). 
Rafaela Siegenthaler: Den Zugang über Sprache finde ich sehr gut. In der Arbeit mit Schwarzen Jugendlichen oder mit Jugendlichen aus der Schwarzen Diaspora sehe ich allerdings immer wieder eine Grenze darin, dass es im linguistischen Sinn keine gemeinsame Sprache und auch oft nur wenige gemeinsame positive Bezüge gibt - das Verbindende ist dann, auf den ersten Blick, die Rassismuserfahrung. Da ist es schwierig, über Sexualität zu reden. Man muss woanders anfangen, das Gemeinsame muss erst sichtbar gemacht oder auch gemeinsam erschaffen werden. Das ist ja gerade, was diesen schmerzhaften Moment ausmacht, dass du kaum Referenzen hast oder fast nichts, woran du dich positiv orientieren kannst.

Ich halte es aber für schwierig, wie schon gesagt, das nach außen zu rechtfertigen - sexualpädagogisch zu arbeiten und nicht über Sexualität zu sprechen. Ich hatte jetzt Glück in unserem Projekt mit dem Team und mit den Zugängen, dass das anerkannt und auch gefördert und unterstützt wurde, aber das geht nicht in jedem Kontext. Wenn du als Schwarze Person oder Person of Color dann quasi einen Auftrag nicht erfüllen kannst - nämlich >richtige< Sexualpädagogik zu machen - dann wirkt da noch eine Diskriminierungsstruktur, die deine Arbeit delegitimiert. Dann wird genauer hingeschaut, wie du arbeitest, weil dir implizit unterstellt wird, dass du nicht professionell genug arbeitest. Und dann ist verstärkt ein exzellentes Auftreten gefragt, und zwar auf verschiedenen Ebenen: wie man sich präsentiert, wie man spricht, auf welche Referenzen man sich bezieht, um Professionalität noch mehr zu unterstreichen. Man muss dieses professionelle Narrativ ständig reproduzieren, damit man überhaupt gehört und anerkannt wird.

Nina Wlazny: Das ist ein besonderer Legitimationsdruck, den du beschreibst. Es macht eben einen Unterschied, wer da vorne steht und nicht nur zu Adressat:innen spricht, sondern auch verhandeln muss und mit Lehrenden spricht.

Rafaela Siegenthaler: Genau. Es gibt im deutschsprachigen Raum wenig Theorie $\mathrm{zu}$ antirassistischer Sexualpädagogik. Auf theoretisches Wissen zurückzugreifen, mich damit zu legitimieren und auszuweisen, das ist in diesem Themenfeld einfach nicht möglich. Diese Strategie steht nicht zur Verfügung. Aber was ich wirklich hilfreich fand als antirassistische Strategie ist die Zusammenarbeit mit der Community. Es ist sicher ein großes Potenzial, wenn ihr in der queerconnexion versucht, mit verschiedenen Initiativen zusammenzuarbeiten. 
Kristin Lerch: Das habe ich etwas optimistisch dargestellt, weil es mir ein Anliegen ist, aber wie und ob das dann in all unseren Workshops umgesetzt wird, ist eine andere Frage.

Rafaela Siegenthaler: Aber es ist zumindest das Wissen da und ihr bemüht euch darum.

\section{Zuschreibungen dekonstruieren und zurückweisen}

Maria Dalhoff: Für mich ist im Zusammenhang mit rassistischen Diskursen noch ein weiteres Thema wichtig: zugeschriebene Täterschaft. Wem wird überhaupt zugetraut, sexuelle Gewalt auszuüben? Und wem wird zugetraut, ein adäquates Verständnis von sexueller Einvernehmlichkeit zu haben und das auch leben zu können? Diese Zuschreibungen wirken der Präventionsarbeit entgegen: Denn da wo Kinder geschwächt werden durch Diskriminierung, rassistischer oder anderer Art, entsteht ein potenzielles Einfallstor für Täter(:innen), die Kinder dadurch einweben, dass sie Selbstvertrauen und Anerkennung geben. Mit Kindern würde ich das nicht besprechen, mit Jugendlichen nach Möglichkeit, aber mit Erwachsenen auf jeden Fall. In den Beratungen, die ich mache, und in den Fällen, die ich kenne, geht es hauptsächlich um weiße österreichische Männer in angesehenen Berufsfeldern, die sexuelle Gewalt ausüben. Aber es dominiert das Bild, dass dies in einer weißen Mittelschicht der Bildungsgesellschaft nicht vorkommt. Das ist sehr problematisch. In den Zuschreibungen von Täterschaft kommen Rassismus und Klassismus zusammen. Und es ist wichtig zu thematisieren, dass Rassismus dadurch am Laufen gehalten wird, dass Täterschaft bestimmten Personen zugeschoben wird.

Zeynep Önsür-Oluğ: Ja, genau. Und auch andere Zuschreibungen kommen sehr häufig vor, z.B.: Wem wird zugestanden, Sexualität zu leben? Zuletzt hatten wir viele Workshops mit Jugendlichen mit Lernschwierigkeiten. Sie haben weder in der Schule noch woanders die Möglichkeit über Sexualität, Liebesbeziehungen, Begehren zu sprechen. Sie möchten Sexualität und Beziehung leben, allerdings gibt es kaum Räume dafür. Wo gibt es solche Räume, wo sie jemanden kennenlernen können?

Wichtig finde ich auch die Frage, wem zugetraut wird, über sich selbst zu 
sprechen. Bei einem Workshop in einer Schule hat die Lehrerin permanent über einen Jungen gesprochen und gemeint, dass er ganz neu sei und sich noch nicht auf Deutsch ausdrücken könne. Sie meinte, wir sollten ihm keine Fragen stellen, er sei noch nicht bereit. Im Workshop war es dann ganz anders. Er hat genau zugehört und mitgemacht. Er hat über sich gesprochen und er hat dutzende Fragen gestellt. Wir haben ihn verstanden, er hat uns verstanden.

Kristin Lerch: Solche Situationen mit Lehrpersonen kenne ich, und ich finde sie schwierig. Ich habe z.B. erlebt, dass die Lehrperson vor allen anderen und vor dem Kind zu mir sagt: „Das Kind dort hat eine Lernschwäche und es ist überhaupt ein bisschen schwierig. « Und alles was ich machen kann, ist dieses Kind genauso zu inkludieren wie alle anderen. Oder habt ihr noch andere Strategien?

Zeynep Önsür-Oluğ: Wir haben in der Pause mit der Lehrerin gesprochen. Wir haben auch mit dem Jungen gesprochen, um ihn in seiner Wahrnehmung zu stärken und auch zu ermutigen.

Maria Dalhoff: Manchmal gelingt es auch, zu der Lehrperson zu sagen: »Ich möchte in Anwesenheit der Kinder mit Ihnen nicht über sie reden, lassen Sie uns das später am Schulgang machen.«Ich benenne also, dass es mir unangenehm ist und ich es unangebracht finde. Das ist natürlich eine Zurückweisung, aber ich denke, dieses Feedback muss die Lehrperson von mir annehmen können. Wenn nicht, dann ist die Situation ein bisschen unangenehm, aber das versuche ich dann auszuhalten, weil es mir wichtig ist.

Kristin Lerch: Das ist eine sehr hilfreiche Formulierung, finde ich.

\section{Durchkreuzen, Widerstand und Freiwilligkeit in pädagogischen Settings}

Nina Wlazny: Eine Strategie kann sein, gar nicht beim Thema Sexualität anzufangen, sondern eben bei anderen Themen. Welche Themen sind das denn, die von den Adressat:innen angesprochen werden? In meiner Erfahrung ist es so, dass das, was beispielsweise über Rassismuserfahrungen erzählt wird, affirmative Konzepte von Optionenvielfalt oft durchkreuzt. Als ich im Rah- 
men des erwachsenengerechten Pflichtschulabschlusskurses mit meiner sexualpädagogischen Praxis begonnen habe, war es mir sehr wichtig, vielfältige Lebensweisen, Beziehungs- und Begehrensformen sichtbar zu machen, um dann vermitteln zu können, dass jede:r die Freiheit hat, sich selbstbestimmt für die vielfältigsten Varianten zu entscheiden. Dieser positive Zugang wurde dann immer wieder durchkreuzt mit verschiedenen Argumenten, die alle auf Folgendes abhoben: Diese Entscheidungsfreiheit ist ungleich verteilt. Viele haben diese Optionen in der Form nicht, weil sie mit Lebensrealitäten und -notwendigkeiten konfrontiert sind, die von Klassismus, Rassismus etc. durchzogen sind. Z.B., wenn einer wegen der ihm zugeschriebenen kulturellen Zugehörigkeit und dem damit assoziierten Täter-Stigma bei Dates zurückgewiesen wird oder wenn eine durch die Exotisierung und rassistische Bewertung ihrer Sexualität nicht mehr als Person mit individuellen Wünschen und Bedürfnissen wahrgenommen wird. Fallen euch auch Beispiele für Themen ein, die für Jugendliche in dieser Hinsicht relevant sind und die dieses optimistische Vielfaltskonzept erschüttern oder vielleicht sogar in Frage stellen können?

Kristin Lerch: Ich glaube, Vielfaltskonzepte sind oft als Theorie leicht zu vermitteln, aber in unseren Workshops sehen wir in Bezug auf Themen wie Coming-out, dass Jugendliche sie als realitätsferne Utopie erleben. Da erzählen sie oft von ihrer eigenen Familie und sagen so etwas wie: »Wenn meine Eltern oder Familie das wüssten, bringen sie mich um - oder bring ich mich um.«Immer wieder treffe ich Menschen, die das so als ihre Lebensrealität beschreiben. Oft sagen auch Jugendliche - meist hypothetisch, nicht um sich damit vor der Klasse als queer zu outen: »Wäre ich queer, könnte ich das meinen Eltern nicht sagen. Also ich habe kein Problem mit Queer-Sein, aber mit meinen Eltern könnte ich nicht darüber reden.«

Zeynep Önsür-Oluğ: Wenn Gruppen aus außerschulischen Einrichtungen zu uns kommen, geschieht das auf Basis von Freiwilligkeit, und dann ist Coming-out auch Thema: Meistens geht es um die Befürchtung, damit alleingelassen zu werden.

Wenn wir mit Schulgruppen arbeiten, gibt es immer zuerst ein Telefongespräch mit den Lehrenden. Da hören wir viel über die Annahmen der Lehrenden über die Realitäten der Kinder und Jugendlichen. Manche Lehrer:innen beschreiben die muslimischen Schüler:innen als Unwissende, die diesen Workshop brauchen, weil sie aus einem Kontext kämen, in dem sie über Se- 
xualität nichts erfahren und in dem Homosexualität abgelehnt würde. Und dann machen wir eine ganz andere Erfahrung. Die Schüler:innen sind von Anfang bis zum Schluss offen, stellen dutzende Fragen und wissen bereits so viel. Muslimische Schüler:innen sind keine homogene Gruppe. Sie haben unterschiedliche Zugänge, Erfahrungen, Wissen wie auch >Unwissen sehen wir auch sehr oft bei den Workshops.

Nina Wlazny: Kennt ihr auch Widerstände von Seiten der Jugendlichen, weil sie indirekt oder direkt angerufen werden als die, die aufgeklärt werden müssen? Widerstände also gegen die Unterstellung, dass sie möglicherweise keine, eine weniger fundierte oder irgendwie sandere < Idee von Sexualität haben? Ich finde nämlich, dass eine solche - meist implizite - Unterstellung der Sexualpädagogik vor allem im Kontext der Migrationsgesellschaft strukturell eingeschrieben ist.

Maria Dalhoff: Ich nehme schon häufig zu Anfang der Workshops bei uns in der Ausstellung ${ }^{13}$ etwas Unwohlsein oder ein Schweigen wahr, aber natürlich auch weil nicht klar ist, was hier erwartet wird, und die Jugendlichen mit ihnen unbekannten Personen über Sexualität und sexualisierte Gewalt reden sollen. Wir betonen dann immer, dass es freiwillig ist: Wenn ihr nicht wollt, dann setzt euch mit euren Kopfhörern oder einem Buch in die Ecke. Das passiert dann in der Regel nicht, einfach weil man gesagt hat, ihr dürft das, wenn ihr wollt. Die Reaktion ist eher: »Okay, dann guck ich mal und vielleicht setze ich mich in die Ecke.«Wir vermitteln durch die Räumlichkeit und das Setting, dass wir jetzt nicht in so einer schulischen Ihr-müsst-da-jetztdurch-Situation sind. Das hilft.

Zeynep Önsür-Oluğ: Die Freiwilligkeit ist bei dem Thema sehr wichtig. Die Jugendlichen sollen selber bestimmen können, ob sie sich mit dem Thema beschäftigen wollen oder nicht. Und wenn sie sagen, das ist mir alles zu viel, können sie sich zurückziehen. In einem schulischen Setting ist das nicht gegeben, da gibt es nur einen einzigen Klassenraum, keinen Nebenraum oder eine andere Möglichkeit, so dass sie sagen könnten: Ich will das Thema noch werden (siehe https://selbstlaut.org/ausstellung [Zugriff 15.06.2020]). 
nicht, ich bin noch nicht bereit oder ich habe selber schwierige Situationen erlebt, die es schwer machen, sich damit auseinanderzusetzen.

Kristin Lerch: Wir versuchen auch, ein anderes Setting zu schaffen, z.B. mit Gesprächsvereinbarungen. Aber wenn alle gemeinsam in einem Raum sind, merkt man eben die unterschiedlichen Schamgrenzen. Da wollen manche voller Begeisterung über Pornografie und Fetische reden und anderen geht das viel zu weit. Das Gespräch zu leiten, so dass die Grenzen von Menschen gewahrt bleiben ohne gleichzeitig anderen zu sagen, eure Interessen haben hier keinen Platz, das ist schon ein Problem.

Maria Dalhoff: Ja, das ist der Unterschied zu einem geschlossenen Klassenraumsetting. Bei uns in der Ausstellung können sie weggehen und zur nächsten Station weitergehen, ohne dass sie irgendjemand ermahnt.

Zeynep Önsür-Oluğ: Und es gibt in der Ausstellung auch die Möglichkeit, in Kleingruppen bestimmte Fragen zu besprechen. Da können sie noch dutzende Fragen stellen zu einem Thema, das sie interessiert und die anderen, die meinen, das ist mir jetzt zu viel, können etwas anderes tun und bei der nächsten Station steht auch eine Kollegin zur Verfügung. Das ist eine große Ressource für uns, wie auch für die Teilnehmenden. Je nach Gruppengröße (ab 17 Schüler:innen) machen drei bis vier Kolleg:innen den Workshop. Das ist insofern eine Ressource, weil meistens im Workshop oder danach ein Beratungsgespräch mit einzelnen Jugendlichen entsteht. Es ist schön zu sehen, dass sich die Jugendlichen durch dieses niederschwellige Angebot ernst genommen fühlen und auch gerne diese Zeit nutzen.

\section{Team-Zusammensetzung}

Nina Wlazny: Könnt ihr noch etwas zur Zusammensetzung eurer Teams sagen - und welche Bedeutung sie für eure Arbeit hat?

Rafaela Siegenthaler: Die Team-Zusammensetzung halte ich für eine wichtige Frage. Wenn es ein rein homogenes >mehrheitsösterreichisches weißes Team ist, ist es schwieriger, z.B. mit den angesprochenen Vernetzungen. Es ist natürlich problematisch, das so zu verallgemeinern, aber es öffnet Perspektiven, wenn das Team unterschiedlich zusammengestellt ist, was ich jetzt 
nicht nur auf die Diskriminierungserfahrung zurückführen würde. Es sind einfach unterschiedliche Erfahrungsoptionen vorhanden.

Ich merke, in der Arbeit mit männlichen Jugendlichen wird es für mich als Frau manchmal schwierig, gewisse Themen mit ihnen zu besprechen, da ist es hilfreich, wenn ein männlicher Kollege aus seiner genderspezifischen Perspektive z.B. feministische Positionen mit den Jungs bespricht. Ich finde, das zeigt sich besonders in der Arbeit mit männlichen Jugendlichen of Color. Etwa wenn sie mit ihrem Stigma als Täter konfrontiert sind und in Zukunft noch mehr damit konfrontiert werden, wenn sie älter werden - je erwachsener und männlicher sie auftreten und aussehen werden. Es ist auch für mich als rassismuserfahrene Person schwierig, sie abzuholen, weil ich als Frau diese Erfahrungsebene nicht mitbringe. Ich habe beobachtet, dass es für die betroffenen Jugendlichen sehr bestärkend sein kann, männliche Rolemodels of Color als Bezugspersonen zu haben, die rassismuskritische Perspektiven vermitteln, besonders in der sexualpädagogischen Arbeit. Es mangelt in sexualpädagogischen Bildungsprojekten an Männern, die mit einem feministischen Zugang arbeiten. Es mangelt besonders an Schwarzen Pädagogen bzw. Pädagogen of Color. Ich fand den Begriff Optionenvielfalt gut, den du vorhin angesprochen hast, Maria.

Zeynep Önsür-Oluğ: In der Fachstelle Selbstlaut gibt es ein fixes Kernteam von weiblich sozialisierten Mitarbeiterinnen mit unterschiedlichen Professionen. Also ein multiprofessionelles Team, was für unsere Arbeit wiederum eine große Bereicherung ist. Und die Workshops werden von weiteren Personen mit unterschiedlichen sprachlichen wie auch kulturellen Hintergründen unterstützt. Auch männliche Personen arbeiten mit uns als Co-Workshopleiter. So versuchen wir die Optionenvielfalt in unserer Arbeit umzusetzen.

Rafaela Siegenthaler: Das Team von Imagining Desires besteht aus vier Personen mit Festanstellung und, je nach Projektphase, aus wechselnden Mitgliedern mit Werkverträgen. Dazu gehören auch unterschiedliche Kooperationspartner:innen: Schulen, die Schwarze Frauen Community, Selbstlaut. Das Gute an der Zusammensetzung in unserem Projekt war, dass es verschiedene Teilprojekte gab und so viele verschiedene Menschen mit unterschiedlichen Hintergründen dazu geholt werden konnten, was eine Perspektivenvielfalt zuließ und die Diversität im Team erweiterte. 
Kristin Lerch: Wir versuchen auch, in den Teams möglichst unterschiedliche Positionierungen zu inkludieren, nicht ausschließlich Cis-Personen oder ausschließlich männliche oder weibliche. Wir sind schon mehrheitlich weiß und deswegen funktionieren manche Sachen eben auch nicht so, wie es vielleicht ideal wäre. Aber es gibt Menschen mit Migrationserfahrung und viele sind auch bei anderen Vereinen aktiv und wir sind mit Menschen von dort befreundet, bei der Queer Base, bei ARA, in der Türkis Rosa Lila Villa. Klar, es könnte besser sein - ich will die queerconnexion jetzt nicht als etwas darstellen, was sie nicht ist. Aber ich glaube schon, dass in unserer Strategie der Vernetzung Potenzial steckt.

\section{Literatur}

Auma, Maisha-Maureen (2017): Kulturelle Bildung in pluralen Gesellschaften. Diversität von Anfang an! Diskriminierungskritik von Anfang an! In: Schütze, Anja/Maedler, Jens: weiße Flecken. Diskurse und Gedanken über Diskriminierung, Diversität und Inklusion in der Kulturellen Bildung. München: Kopead.

Debus, Katharina (2016): Nicht-diskriminierende Sexualpädagogik. In: Scheer, Albert/El-Mafaalani, Aladin/Yüksel, Emine Gökçen (Hg.): Handbuch Diskriminierung. Wiesbaden: Springer VS, S. 811-833.

Debus, Katharina/Laumann, Vivien (Hg.) (2018): Pädagogik geschlechtlicher, amouröser und sexueller Vielfalt. Zwischen Sensibilisierung und Empowerment. Online unter: https://interventionen.dissens.de/materialien /handreichung [Zugriff: 26.02.2020].

Diallo, Aïcha/Erni, Danja (2017): Wie gesellschaftliche Pluralisierungsprozesse in die Zusammenarbeit zwischen Künstler*innen und Lehrer*innen hineinwirken. In: Schütze, Anja/Maedler, Jens (Hg.): Weiße Flecken: Diskurse und Gedanken über Diskriminierung, Diversität und Inklusion in der Kulturellen Bildung. München: Kopaed, S. 121-128.

Eggers, Maisha-Maureen (2015): Diversity/Diversität. In: Arndt, Susan; Ofuatey-Alazard, Nadja (Hg.): Wie Rassismus aus Wörtern spricht. (K)Ein Erben des Kolonialismus im Wissensarchiv deutsche Sprache. Ein kritisches Nachschlagewerk. Münster: Unrast, S. 256-263. 
Fachstelle Selbstlaut (2017): Interkulturelle Sexualpädagogik und Gewaltprävention mit Eltern und Erziehungsberechtigten nicht deutscher Erstsprache. Handreichung für Lehrer_innen. Wien. Online unter: https://selbs tlaut.org/wp-content/uploads/2017/04/Handreichung_20170403.pdf [Zugriff: 15.06.2020].

Mecheril, Paul/Plößer, Melanie (2011): Diversity und Soziale Arbeit. In: HansUwe, Otto (Hg.): Handbuch Soziale Arbeit. München: Ernst Reinhardt, S. 278-287.

Schmutzer, Karla/Thuswald, Marion (2019): Mit Bildern zu Lust und Begehren arbeiten. Kunst- und sexualpädagogische Methoden und Materialien für Schule und Lehrer*innenbildung. Hannover: fabrico.

Sielert, Uwe/Marburger, Helga/Griese, Christiane (Hg.) (2017): Sexualität und Gender im Einwanderungsland. Öffentliche und zivilgesellschaftliche Aufgaben - ein Lehr- und Praxishandbuch. Oldenburg: De Gruyter.

Thuswald, Marion/Sattler, Elisabeth (2019): Critical Diversity in der kulturellen und sexuellen Bildung. Einblicke in das kunst- und sexualpädagogische Projekt Imagining Desires. In: schulheft Nr. 175, 3/2019, S. 82-96.

Tuider, Elisabeth/Müller, Mario/Timmermanns, Stefan/Bruns-Bachmann, Petra/Koppermann, Carola (2012): Sexualpädagogik der Vielfalt. Praxismethoden zu Identitäten, Beziehungen, Körper und Prävention für Schule und Jugendarbeit. Weinheim/Basel: Juventa. 



\title{
Sexuelle Bildung in der Schule braucht Vielfalt
}

\author{
Gregor Steininger
}

Im Rahmen meines Lehramtsstudiums hatte ich das Glück, mich in einer pädagogischen Lehrveranstaltung mit sexueller Bildung in der Schule auseinandersetzen zu können. ${ }^{1}$ Von Anfang an hat das Thema großes Interesse bei mir geweckt, denn für mich steht fest: Sexualität ist nichts, das vor dem Klassenzimmer einfach mal pausieren kann. Umso wichtiger ist es, dass Lehrer:innen sich in ihrer Verantwortung als Pädagog:innen dem Thema Sexualität von Kindern und Jugendlichen annehmen und entsprechend dafür ausgebildet sind.

Aus meiner Sicht sollte es in der Schule zum einen um die Vermittlung von sexualitätsbezogenem Wissen (z.B. Verhütungsmethoden, sexuell übertragbare Krankheiten, Geschlechtsreife, Zyklus, Schwangerschaftsabbruch, Besuch bei Gynäkolog:innen, Adoption, Gewaltprävention, sexuelle Gewalt, Beratungsstellen etc.) gehen, zum anderen zählt auch Persönlichkeitsentwicklung zu einer ganzheitlichen Sexualpädagogik. Als Lehrpersonen können wir etwa in Form von geeigneten Materialien, (Gruppen-)Übungen, Videoclips, Diskussionen und anderen Methoden Selbstwahrnehmung, Selbstbewusstsein sowie Kommunikations- und Reflexionsfähigkeit stärken.

Sexualität hat immer auch mit gesellschaftlichen (Norm-)Vorstellungen zu tun. Diese mit den Schüler:innen aufzugreifen, zu hinterfragen und zu reflektieren halte ich für zentral. Wir leben in einer pluralistischen Gesellschaft mit verschieden Kulturen, Religionen, Weltanschauungen und eben auch sexuellen Identitäten. Sexuelle Bildung in der Schule soll neben dem Ziel des Wissenserwerbs auch zur Sensibilisierung beitragen und damit ein von Sabine Ziegelwanger im Sommersemester 2019 am Zentrum für Lehrerlnnenbildung der Universität Wien abgehalten wurde. 
Bewusstsein schaffen, dass immer noch vieles in unserem Alltag durch eine sheteronormative Brille< bestimmt ist.

Klarerweise sieht eine sexualpädagogische Arbeit im Unterrichtsfach Deutsch anders aus als in Biologie, Geografie, Englisch oder Religion. Unterschiedliche Blickpunkte können sich jedoch als zielführend erweisen, wenn es darum geht, Schüler:innen verantwortungsvolle und vielseitige Zugänge zu eröffnen, sich mit pluralen Lebensformen und unterschiedlichen Aspekten von Sexualität und Beziehungen auseinanderzusetzen. Diese Aspekte finden sich auch im Grundsatzerlass für Sexualpädagogik, den das österreichische Bundesministerium für Bildung, Wissenschaft und Forschung 2015 neu herausgegeben hat (BMBF 2015). Der Grundsatz hält die Bedeutung von sexueller Bildung in der Schule fest, an der sich alle Unterrichtsfächer beteiligen sollen. Dies bedeutet nun nicht, dass Lehrer:innen bei der Vermittlung sexualpädagogischer Inhalte auf sich allein gestellt sind. Laut Grundsatzerlass können externe Referent:innen an Bord geholt werden, was für bestimmte Themen aus meiner Sicht sehr ratsam, ja sogar notwendig ist.

\section{Sexualität und Behinderung}

Ich arbeite seit mehr als zehn Jahren in einer Peer-Beratungsstelle für Menschen mit Behinderung und sitze seit Geburt selbst im Rollstuhl. Meine Beratungserfahrungen zeigen, dass die Tabuisierung von Sexualität und Behinderung sich einschränkend auf die Selbstbestimmung von Menschen mit Behinderung auswirkt.

Jahrhundertelang wurde behinderten Menschen ihre Sexualität abgesprochen und ihre sexuellen Bedürfnisse wurden tabuisiert. Diese Denkweise ist leider manchmal heute noch zu finden (vgl. Schinagl 2016). Widerstand gegen diese Form der Entmenschlichung und Bewusstseinsarbeit für mehr Selbstbestimmung kommt oft von den Betroffenen selbst. Es braucht jedoch auch Lehrer:innen und Sexualpädagog:innen, um diesen Prozess des Umdenkens voranzutreiben.

Das Problem besteht darin, dass Sexualität immer noch von einem stark normativen Denken geprägt ist, das einer Vielzahl von Menschen nicht gerecht wird. Bei Personen mit Behinderung trifft dies auf Grund der oft mannigfaltigen körperlichen Einschränkungen in besonderer Weise zu. Aufgrund gesellschaftlicher Normen werden ein fehlendes Bein, ein schiefes Rückgrat, mangelnde Muskelkraft und ein geringes Seh- oder Hörvermögen 
häufig als Barrieren für sexuelle Beziehungen wahrgenommen, die sie aber gar nicht sein müssen. Sie sind dennoch für die Betroffenen selbst, wie auch für die Partner:innen, eine große Verunsicherung. Wie mir in Beratungssituationen immer wieder erzählt wird, führt dieser Umstand etwa dazu, dass Menschen mit Behinderung keine Dating-Veranstaltung besuchen oder sehr spät, manchmal sogar nie zu Gynäkolog:innen gehen.

Ich finde es daher wichtig, dass in der Thematisierung von Sexualität auch Behinderung mitbedacht wird, ebenso wie andere Differenzordnungen (Alter, Geschlecht, Nationalität, Religion ...), die zu Benachteiligungen führen können. ${ }^{2}$ Die Verantwortung von sexueller Bildung in der Schule sehe ich darin, sowohl Vorurteile und Berührungsängste gegenüber behinderten Menschen - als gesellschaftlich benachteiligter Gruppe - abzubauen als auch für die sexuelle Selbstbestimmung aller einzutreten.

Didaktisch kann diese Auseinandersetzung je nach Pädagog:in, Unterrichtsfach und Schüler:innen unterschiedlich erfolgen. Neben Erfahrungsberichten können etwa Filme oder Filmausschnitte ein guter Einstieg in die Auseinandersetzung mit Sexualität und Behinderung bieten. Beispielsweise halte ich den Film »Magaritha with a straw« (2014) von Shonali Bose für geeignet, der das Leben einer jungen Rollstuhlfahrerin mit Zerebralparese zeigt. ${ }^{3}$ Eine weitere Möglichkeit wäre der Film »Heute gehe ich allein nach Hause« (Ribeiro 2014), in dem ein sehbehinderter Junge mit seiner Liebesbeziehung zu einem Klassenkameraden und seinem Kampf um Unabhängigkeit von den Eltern im Zentrum steht. Filme wie diese bieten eine gute Grundlage dafür, um anschließend mit Schüler:innen ins Gespräch zu kommen. Sollten Schüler:innen mit Behinderung in der Klasse sein, so ist darauf $\mathrm{zu}$ achten, dass diese nicht auf Grund ihrer eigenen Behinderung zu stark in den Fokus geraten, also z.B. aufgefordert werden: »Erzähl doch mal ...!«

Eine weitere Möglichkeit sich mit dem Thema Sexualität und Behinderung auseinanderzusetzen, stellt das inklusives Schulprojekt "mellow yellow« dar, welches der Verein Mad ins Leben gerufen hat. Durch Methoden der Kontaktimprovisation steht der Körper im Mittelpunkt. Die Workshops zielen darauf $a b$, dass sich Menschen mit all den Unterschiedlichkeiten, Besonderheiten und Möglichkeiten ihres Körpers ausdrücken und durch Tanz als trennend

Zum Anti-Bias-Ansatz vgl. https://www.anti-bias-netz.org.

3 Der Film »Ziemlich beste Freunde« (Nakache/Toledano 2011) ist ebenfalls eine Möglichkeit für den Einstieg ins Thema Behinderung. Er thematisiert jedoch Sexualität nur am Rand. 
wahrgenommene Unterschiede überwunden werden können (vgl. Verein Mad o.J.).

Sexuelle Bildung in der Schule stellt eine besondere Herausforderung für Lehrkräfte da, auf die es in der Ausbildung vorzubereiten gilt. Es braucht also verstärkt Lehrveranstaltungen wie jene, die ich besucht habe, um vielfaltsorientierte sexuelle Bildung zu fördern.

\section{Literatur}

Anti-Bias-Netz (o.J.): Online unter: https://www.anti-bias-netz.org [Zugriff: 13.07.2020].

BMBF - Bundesministerium für Bildung und Frauen (2015): Grundsatzerlass Sexualpädagogik. Online unter: https:/www.bmbwf.gv.at/Themen/schu le/schulrecht/rs/1997-2017/2015_11.html [Zugriff: 18.06.2020].

Leimgruber, Stephan (2011): Christliche Sexualpädagogik. Eine emanzipatorische Neuorientierung für Schule, Jugendarbeit und Beratung. München: Kösel.

Verein Mad (o.J.): Online unter: https://www.mad-dance.eu/mad-projekte/m ellow-yellow [Zugriff: 28.04.2020].

Schinagl, Sarah (2016): Geistige Behinderung und Sexualität im 19. Jahrhundert. Wien: Masterarbeit an der Universität Wien. 


\title{
Geschlechtliche, amouröse und sexuelle Vielfalt in der Sexualpädagogik
}

\author{
Katharina Debus
}

Geschlechtliche, amouröse und sexuelle Vielfalt sind pädagogische Querschnittsthemen (vgl. Debus 2018b) und - explizit oder implizit - Kernthemen von Sexualpädagogik. Dabei meine ich im Folgenden mit Sexualpädagogik nicht nur explizit sexualpädagogische Settings, sondern alle pädagogischen Situationen, in denen Themen rund um Liebe, Partnerschaft, Fortpflanzung, Körpergeschlecht, Erotik, Sexualität etc. im Raum stehen.

Im Artikel Diskriminierungsreflektierte Sexualpädagogik in diesem Band ${ }^{1}$ erläutere ich, dass Sexualpädagogik sich nicht neutral zu Diskriminierung und Vielfalt verhalten kann. In unseren Aussagen, Materialien und Beispielen, unserer Mimik und Körpersprache sowie unserem Umgang mit Fragen, Äußerungen und Verhaltensweisen der Adressat:innen sind Botschaften zum Thema Vielfalt nicht vermeidbar. Entweder reproduzieren wir - gewollt oder ungewollt - einengende und diskriminierende Normalitätsannahmen (vgl. Debus/Laumann 2018a) oder wir bemühen uns darum, vielfaltsorientiert zu handeln. Entsprechend plädiert dieser Artikel nicht für eine sSonderpädagogik` zu geschlechtlicher, sexueller und amouröser Vielfalt, sondern für ein Mainstreaming dieser Themen im gesamten (sexual-)pädagogischen Handeln.

Dabei bewegen wir uns im Spannungsfeld zwischen einer positiven Beschäftigung mit Vielfalt und der kritischen Auseinandersetzung mit Diskriminierung. Beides ist notwendig und kaum zu trennen. Wenn wir einseitig Vielfalt feiern, ignorieren wir Chancen-Ungleichheit und spiegeln de facto falsche Tatsachen vor. Dies kann befördern, dass unsere Adressat:innen ihre Diskriminierungserfahrungen als persönliches Scheitern personalisie- 
ren $^{2}$ mit psychosozialen Folgeerscheinungen wie Schuld- und Schamgefühlen, selbstschädigendem Verhalten, eingeschränkten Selbstwirksamkeitserwartungen und Barrieren, sich Hilfe und Unterstützung zu suchen. Wenn wir hingegen einseitig Diskriminierung in den Blick nehmen und uns nur mit dem Schweren und Belastenden beschäftigen, verpassen wir die Gelegenheit, diskriminierungsbetroffene Adressat:innen in ihren Ressourcen für ein selbstbestimmtes Leben zu stärken und sie dazu zu ermutigen, dass dies trotz Diskriminierung möglich ist, sowie allen Adressat:innen das Thema als ein auch Positives und Lustvolles zugänglich zu machen (vgl. Debus 2018b). ${ }^{3}$

Dieser Beitrag fokussiert einen positiven Blick auf Vielfalt, ohne dabei Diskriminierung auszublenden. ${ }^{4}$ Um Begriffsverwirrungen im Verhältnis zum anderen Artikel in diesem Band zu vermeiden, schreibe ich dennoch von diskriminierungsreflektierter Sexualpädagogik, auch wenn das Adjektiv vielfaltsorientiert ebenso passend wäre.

Der Artikel beginnt mit grundlegenden Überlegungen zu Un_SichtbarkeitsDynamiken, die das Feld geschlechtliche, amouröse und sexuelle Vielfalt tendenziell von manchen anderen Ungleichheitsthemen unterscheiden (1), und zum Einvernehmlichkeitsprinzip als Voraussetzung der Arbeit zu Intimität und Sexualität (2). Danach ist er in zwei Hauptteile gegliedert: Zunächst führe ich in das Thema geschlechtliche Vielfalt ein (3.1) und stelle Praxisableitungen für eine diskriminierungsreflektierte Sexualpädagogik vor (3.2). In ähnlicher Weise führe ich in das Thema sexuelle Vielfalt im engeren Sinne (4.1) sowie amouröse und sexuelle Vielfalt im erweiterten Sinne (4.2) ein und fächere den sexualpädagogischen Praxistransfer auf in die Themen sexuelle und romantische Orientierungen (4.3), Beziehungskonzepte (4.4) sowie eine exemplarische Beschäftigung mit Sexpraktiken anhand von BDSM (4.5). Im Fazit gehe ich auf Potenziale diskriminierungsreflektierter Sexualpädagogik im Kontext von Schule und Regelstrukturen ein (5).

Vgl. zu einer Kritik der Personalisierung von Diskriminierung in der Pädagogik im Kontext von Rassismus Kalpaka 2003.

3 Viele der angerissenen allgemeinpädagogischen Überlegungen zu verschiedenen Dimensionen, Diskriminierungen und pädagogischen Ansätzen im Feld geschlechtlicher, amouröser und sexueller Vielfalt finden sich ausführlicher in Debus/Laumann $2018 \mathrm{~b}$.

4 Der Beitrag ist als gegenseitig ergänzende Lektüre zu meinem Artikel Diskriminierungsreflektierte Sexualpädagogik in diesem Band geschrieben. 
Mir ist bewusst, dass die im Folgenden erarbeiteten Orientierungspunkte diskriminierungsreflektierter Sexualpädagogik im Rahmen üblicher pädagogischer Rahmenbedingungen zum Teil schwer einzulösen sind. Mein Ziel ist nicht, diese als Kriterien aufzustellen, anhand derer Pädagog:innen bewertet werden, sondern damit Weiterentwicklungen $\mathrm{zu}$ motivieren und Mut $\mathrm{zu}$ machen. ${ }^{5}$

\section{Un_Sichtbarkeits-Dynamiken im Kontext geschlechtlicher, amouröser und sexueller Vielfalt}

In einer Welt, in der Diskriminierung in pädagogischen Institutionen an der Tagesordnung ist, verbergen Kinder, Jugendliche, Eltern und Pädagog:innen oft Identitäten, Körper, Begehren und Zugehörigkeiten, die mit Diskriminierungsrisiken verbunden sind. ${ }^{6}$ Wir haben dafür im Projekt Interventionen für geschlechtliche und sexuelle Vielfalt ${ }^{7}$ den Begriff der Un_SichtbarkeitsDynamiken herausgearbeitet (vgl. Laumann 2018). Eine Verheimlichung kann insbesondere in Zwangskontexten wie der Schule oder manchen Familien dem Selbstschutz dienen und somit das geringere Übel für die Betroffenen sein. Pädagog:innen sollten daher darauf achten, nicht durch bewusste oder unbewusste Handlungen einen Coming-out-Sog zu provozieren, der Adressat:innen dazu verleiten kann, sich ohne gute Abwägungen in einer Gruppe z.B. als queer oder trans zu outen, in der sie danach möglicherweise jahrelang Diskriminierung ausgesetzt sind (vgl. Klemm 2018).

Das heißt im Umkehrschluss, dass wir als Pädagog:innen in der Regel nur einen Bruchteil der realen Vielfalt einer Lerngruppe sehen und die präsente geschlechtliche, amouröse und sexuelle Vielfalt oft systematisch unterschätzen. Wir sollten daher in jeder Lerngruppe von der Grundannahme größtmöglicher Vielfalt ausgehen und immer sensibilisierende mit empowernden Lernangeboten mischen (vgl. Laumann 2018; Debus 2018a; Klemm 2018; Bauer 2020b: 585).

Vgl. ausführlicher zu Rahmenbedingungen und anderen Spannungsfeldern diskriminierungsreflektierter Sexualpädagogik Debus i.d.B.

6 Zu Lebenslagen schwuler, lesbischer, bisexueller, transgeschlechtlicher und intergeschlechtlicher Jugendlicher vgl. u.a. Oldemeier 2018; Kleiner 2020.

7 Informationen zu diesem Projekt finden sich online unter https://interventionen.disse ns.de [Zugriff 20.12.2020]. 


\section{Einvernehmlichkeit als Grundlage der Sexualpädagogik}

Einvernehmlichkeit unterscheidet Sexualität von sexualisierter Gewalt. Daher nehme ich in diesem Artikel die normative Setzung vor, nur einvernehmlich $\mathrm{zu}$ verwirklichende Begehren und Sexpraktiken unter einem positiv verstandenen Begriff sexueller Vielfalt zu fassen, also nur solche, die mit der (jederzeit widerrufbaren) Einwilligung aller beteiligten Personen stattfinden und an denen nur einwilligungsfähige Personen beteiligt sind, also beispielsweise keine Personen, die in starker Abhängigkeit zu einer der beteiligten Personen stehen bzw. über sehr viel weniger Macht als diese verfügen (vgl. auch Bauer 2020b: 583ff.). Dies schließt u.a. sexuelles Begehren von Erwachsenen für Kinder aus. Dieses Thema bedarf einer eigenständigen gewaltpräventiven Bearbeitung und sollte m.E. nicht unter sexueller Vielfalt mitverhandelt werden.

Unsere Kultur ist von Bildern in Bezug auf Flirt und Sex geprägt, die Einvernehmlichkeit für Menschen aller Lebensweisen erschweren, z.B. dass es peinlich, unromantisch und/oder abtörnend sei, über Genitalien, Erotik und/oder Sex zu sprechen und sgute Liebhaber:innen nen würden, was das Gegenüber möge. Mädchen gelten oft als `Schlampen<, wenn sie (zu viel) Interesse an Sex zeigen. Jungen sollen >forsch rangehen und im heterosexuellen Kontext ein Nein als Vielleicht oder Ja eines sanständigen Mädchens deuten. Sie sollen immer Sex wollen und es wird als unzumutbar für sie dargestellt, Sex vor ihrem Orgasmus abzubrechen. Eine Erektion bzw. Feucht-Werden werden oft mit Lust und Einvernehmlichkeit gleichgesetzt. Oft wird auch davon ausgegangen, dass partnerschaftliche Liebe und Lust auf Sex ab einem bestimmten Alter korrelierten und es eine automatische Steigerung gäbe, in der ab einem bestimmten Alter/Beziehungsstatus aus >a< immer $>b<$ folge und daher nicht mehr kommuniziert werden müsse etc.

Um Menschen $\mathrm{zu}$ befähigen, eine für sie passende grenzachtende und lustvolle (A-)Sexualität ${ }^{8}$ zu entwickeln, bedarf es einer kritischen Auseinandersetzung mit all diesen Themen, einer Arbeit zur Wahrnehmung der eigenen Grenzen sowie der expliziten Förderung des Zustimmungs-/Einvernehmlichkeitsprinzips in Erotik und Sex ${ }^{9}$ - nicht nur als abstrakte Norm, sondern

$8 \quad$ Zu Asexualität vgl. 3.1.

$9 \quad$ Hierzu gibt es unter dem Stichwort consent (auf Deutsch auch als Konsens übersetzt) relativ viele englischsprachige Materialien, von denen manche auf Deutsch übersetzt 
auch als anwendungsorientierte Förderung. U.a. fördert gewaltpräventive Sexualpädagogik dabei die folgenden Fähigkeiten:

- Umgang mit Wünschen, Gefühlen und Bedürfnissen: über den eigenen Körper, Gefühle, Wünsche und Unsicherheit kommunizieren; mit Einsamkeit und Zurückweisungen grenzachtend und selbstsorgend umgehen;

- Unterscheidung zwischen Fantasien und Realität: verstehen, dass in der Realität das Einvernehmlichkeitsprinzip gilt, während in Fantasien alles erlaubt ist;

- Umgang mit Grenzen: eigene Grenzen wahrnehmen und setzen; Grenzen anderer Menschen wahrnehmen und akzeptieren; verstehen, dass Aufhören im Sex immer erlaubt ist (vgl. Könnecke/Rieske 2017); Grenzsetzungen als Voraussetzung für Vertrauen statt als Zurückweisung oder Ehrkränkung wahrnehmen;

- Umgang mit Problemen: Grenzverletzungen, sexuelle Übergriffe und sexuellen Missbrauch erkennen und benennen (vgl. Enders 2012); mit Grauzonen und mit unbeabsichtigten Grenzüberschreitungen umgehen; sich bei Problemen Unterstützung suchen.

Dabei ist ein dynamischer Gewaltbegriff notwendig. Auflistungen, welche Praktiken gewalttätig sind und welche gewaltfrei, sind aufgrund verschiedener persönlicher Präferenzen und Grenzen, unterschiedlicher Wünsche in verschiedenen Konstellationen sowie schwankender Tagesformen etc. nur begrenzt hilfreich. Es bedarf vielmehr einer dynamischen Unterscheidung entlang der Frage von Einvernehmlichkeit und eines Austauschs darüber, woran (Un-)Wohlsein erkannt werden, wie Einvernehmlichkeit hergestellt und widerrufen werden und wie mit Zweifeln und Hemmungen in der Kommunikation umgegangen werden kann. ${ }^{10}$

wurden, z.B. ein Video, das Einverständnis mit einer Tee-Analogie erklärt: https://www youtube.com/watch?v=2ovcQgIN5C4. Ein für Jugendliche geeignetes Flussdiagramm findet sich in Debus 2021a: 27, weitere Videoclips unter https://imaginingdesires.at/vi deos [Zugriff: 05.02.2021].

10 Je jünger die Adressat:innen und je mehr sie durch die Ausnutzung von Machtgefällen gefährdet sind, desto wichtiger ist es allerdings, bestimmte Regeln doch auch statisch zu setzen, die vor dem Missbrauch von Machtgefällen schützen, also z.B., dass sexuelle Avancen durch Erwachsene gegenüber Kindern oder durch Autoritäten gegenüber ihren Schutzbefohlenen nicht in Ordnung sind. 
Die Debatte um kindliche Sexualität und die Frage der Altersangemessenheit bestimmter Thematisierungen und methodisch-didaktischer Vorgehensweisen ist relevant für Fragen der Grenzachtung in der Sexualpädagogik, würde aber den Rahmen dieses Artikels sprengen. Daher beziehen sich die folgenden Aussagen bei der Thematisierung von Sexpraktiken auf Jugendliche, während ich die Beschäftigung mit Körpern, Lebens- und Liebesformen sowie Einvernehmlichkeit für alle Altersstufen für geeignet halte - bei allen Themen angepasst an die Entwicklungsstände, Bedarfe und Grenzen der konkreten Lerngruppe.

\section{Geschlechtliche Vielfalt in der Sexualpädagogik}

\subsection{Geschlechtliche Vielfalt}

Geschlecht wird gesellschaftlich mit körperlichen Merkmalen, Identität und Ausdrucks-/Verhaltensweisen in Verbindung gebracht. Geschlechtliche Vielfalt lässt sich daher auf drei Ebenen systematisieren: ${ }^{11}$

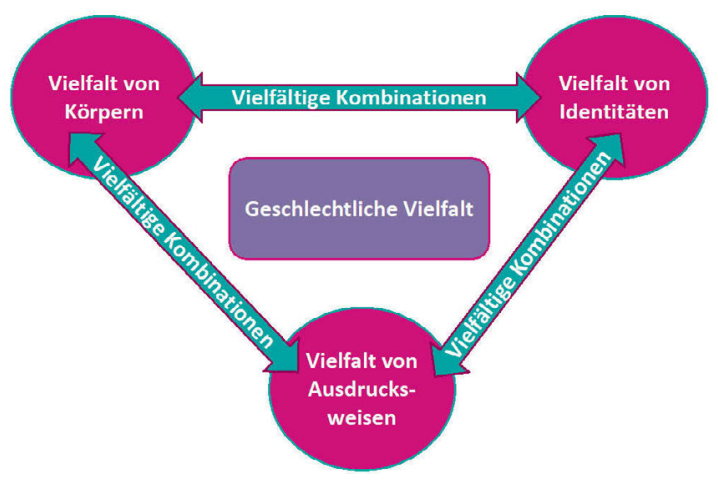

Abb. 3: (c) Katharina Debus

11 Vergleiche vertiefend Debus/Laumann 2018a. Ein unterstützendes Online-Glossar für alle Begriffe, die ich hier nur kurz erkläre oder voraussetze, findet sich bei Debus/Laumann 2020 unter https://interventionen.dissens.de/materialien/glossar [Zugriff: 09.07.2020]. 


\subsubsection{Körper}

Körper sind vielfältig - auch in Bezug auf mit Geschlecht in Verbindung gebrachte Merkmale (auch: Körpergeschlecht bzw. engl. sex) wie Chromosomen, Genitalien, Sexual- und Fortpflanzungsorgane, Zeugungs- und Gebärfähigkeit, Hormone, Brust/Brüste, Körperbehaarung, Körpergröße, Stimmtiefe nach der Pubertät etc. Diese Vielfalt wird durch Normen eines >normalen<, >richtigen`, sschönen` oder `begehrenswerten` männlichen bzw. weiblichen Körpers eingeschränkt, die zudem intersektional funktionieren (vgl. Debus i.d.B.). Sehr viele Menschen entsprechen diesen Normen nicht. Manche haben die Ressourcen, sich darüber hinwegzusetzen, viele leiden darunter u.a. durch soziale Sanktionen, in Bezug auf ihr Selbstverhältnis und bei der Partner:innensuche. Darüber hinaus wird diese Vielfalt derzeit nach hegemonialen biologisch-medizinischen Kriterien (Chromosomen, Hormone, äußere und innere Sexual- und Fortpflanzungsorgane) in weiblich, männlich bzw. intersexuell eingeteilt, was für Letztere oft gravierende medizinische Gewalt und weitere Formen der Diskriminierung nach sich zieht (vgl. u.a. Bauer/Truffer 2016, Barth u.a. 2013; Bauer 2020b: 580f.).

Der Begriff intersexuell wird von einigen Aktivist:innen abgelehnt, weil er von den pathologisierenden Disziplinen Medizin und Biologie geprägt ist und auf Deutsch fälschlich einen Zusammenhang zu Sexualität nahelegt. Manche ziehen die Begriffe intergeschlechtlich bzw. inter*12 vor, andere den Begriff Varianten der Geschlechtsentwicklung. Letzterer hat den Vorteil, dass er deutlich macht, dass Intergeschlechtlichkeit kein einheitliches körperliches Phänomen ist. Es gibt sehr unterschiedliche körperliche Konstellationen aus Hormonen, Chromosomen sowie inneren und äußeren Fortpflanzungs-/Sexualorganen, deren Zusammenfassen in der Sammelkategorie einzig der Logik folgt, die Kategorien (körperlich) männlich oder weiblich als scheinbar einzig snormale، Formen der Körperlichkeit zu erhalten.

Vielfaltsorientierter wäre ein Blick, der menschliche Körperlichkeit als eine Zusammensetzung verschiedener Spektren versteht, die nicht in Norm und Abweichung aufgeteilt werden. Solange aber gesellschaftlich Unterschiede gemacht werden, bietet der Begriff endogeschlechtlich die Möglichkeit, Menschen, die nicht inter* sind, ebenfalls spezifisch zu beschreiben, anstatt sie hierarchisierend als snormak zu benennen. -sexuell. Weitere Informationen zu Inter*: Bauer/Truffer 2016; https://inter-nrw.de [Zugriff: 14.07.2020]; Hechler 2016, 2012. 


\subsubsection{Geschlechtsidentität}

Geschlechtsidentität (auch Gender oder psychisches Geschlecht) beschreibt das Wissen eines Menschen, welchem Geschlecht sie:er angehört. Menschen können beispielsweise von sich wissen, dass sie Mädchen/Frauen oder Jungen/Männer oder keines von beidem sind. Es gibt viele mögliche Identitäten, die nicht männlich oder weiblich sind (vgl. Nonbinary Wiki o.J.). Diese Vielzahl wird oft unter den Begriffen genderqueer bzw. nicht-binär (auch: nonbinary oder abgekürzt enby) zusammengefasst. Menschen, die sich (derzeit oder grundsätzlich) bezüglich ihrer Geschlechtsidentität nicht festlegen wollen oder können, bezeichnen sich manchmal auch als questioning.

All diese Geschlechtsidentitäten können mit allen Körpervariationen einhergehen. Gesellschaftlich werden aber weiterhin Menschen als transsexuell verbesondert, deren Identität nicht der Zuweisung ihres Körpers bei Geburt als männlich oder weiblich entspricht. Aus den oben genannten Gründen lehnen viele (aber nicht alle) trans Menschen den Begriff >transsexuell $a b$. Alternativ werden u.a. die Begriffe transgender, transident oder transgeschlechtlich verwendet. Der Begriff trans* soll alle genannten Begriffsendungen umfassen. Da der Stern derzeit in trans Communitys sehr umstritten ist, wird im Folgenden zusammenfassend von trans geschrieben.

Auch trans Menschen erfahren sehr unterschiedliche Realitäten (vgl. auch Bauer 2020b: 581f.). Manche erleb(t)en körperliche Dysphorie, erleben also ihren Geburtskörper als unpassend und streben z.T. körperliche Angleichungen an oder haben diese bereits umgesetzt. Andere trans Menschen sind mit ihrem Körper zufrieden (oder in ähnlichem Maße unzufrieden wie andere Menschen auch) und erleben vor allem soziale Dysphorie, wenn andere ihnen ein falsches Geschlecht zuweisen. Dysphorie-Erfahrungen, z.B. als Person des falschen Geschlechts behandelt zu werden, fügen trans Menschen bedeutende psychosoziale Belastungen zu. Respekt drückt sich daher u.a. darin aus, trans Menschen als Angehörige des von ihnen als richtig beschriebenen Geschlechts $\mathrm{zu}$ behandeln und mit den gewünschten Namen und Pronomen (z.B. er, sie, er_sie, sie_er, xe, en, they, hen, Vorname statt Pronomen etc.) anzusprechen und $\mathrm{zu}$ beschreiben. ${ }^{13}$ Dabei sollten sie allerdings nicht ohne ihre Einwilli-

So ist beispielsweise ein trans Junge ein Junge, der bei Geburt als Mädchen zugewiesen wurde. Es ist diskriminierend, ihn als Mädchen zu beschreiben, das lieber ein Junge wäre (außer die Person beschreibt sich selbst entsprechend). Es zählt also bei vergeschlechtlichten Begriffen das von der Person selbst als richtig beschriebene Ceschlecht, nicht das Geschlecht, das in die erste Geburtsurkunde eingetragen wurde. 
gung gegenüber anderen Menschen geoutet werden. Eine Nachfrage nach individuellen Wünschen ist grundsätzlich respektvoller als die Suche nach allgemeingültigen Rezepten. ${ }^{14}$

Auch in diesem Themenfeld wurde mit cis ein Begriff entwickelt, um auch mehrheitsangehörige Menschen spezifisch zu beschreiben. Entsprechend ist z.B. ein cis Mädchen ein Mädchen, das bei Geburt aufgrund seiner sichtbaren Genitalien als Mädchen in die Geburtsurkunde eingetragen wurde und sich auch selbst als Mädchen beschreibt. In den meisten Situationen ist die Unterscheidung zwischen cis und trans aber unnötig und damit diskriminierend, außer die Betroffenen äußern entsprechende Wünsche. Fast immer ist es daher am respektvollsten, Mädchen als Mädchen, Jungen als Jungen und nicht-binäre Menschen als nicht-binär zu beschreiben, ohne dabei zwischen cis und trans zu differenzieren.

\subsubsection{Ausdruck und Verhalten}

Wir finden ebenfalls große Vielfalt bezüglich des Ausdrucks (auch Geschlechtsausdruck bzw. engl. gender expression) vor, besser gesagt: bezüglich der geschlechtlich konnotierten Möglichkeiten sich zu verhalten, auszudrücken und in Beziehung $\mathrm{zu}$ anderen $\mathrm{zu}$ treten. Dies betrifft u.a. Körpersprache, Kleidungsstücke und -stile, Körper- und Haarstyling, Farbpräferenzen, Geschmäcker u.a. in Bezug auf Literatur und Medien, Emotionen und ihren Ausdruck, Sozialverhalten und Kommunikationsweisen, Hobbys, Interessen und Kompetenzen, Sexualität, Erotik und Intimität. Individuell unterschiedlich können diese Verhaltensweisen Ausdruck von Geschlechtsidentität sein (Geschlechtsausdruck im engeren Sinne) oder einfach Teile der Persönlichkeit, die für das Individuum unabhängig von seiner:ihrer Geschlechtsidentität sind. Auch hier begegnen Menschen aller Altersstufen gesellschaftlichen Normalitätsannahmen, die die persönlichen Entwicklungsmöglichkeiten einengen und für sogenannte sgendernonkonforme Menschen ${ }^{15}$, also Menschen, die sich untypisch verhalten oder aussehen, $\mathrm{zu}$ Diskriminierung und Mobbing führen können. 
Als Pädagog:innen sollte uns bewusst sein, dass wir in der Regel nicht wissen, ob Menschen mit ihren Ausdrucks- und Verhaltensweisen eine Aussage über ihre Geschlechtsidentität treffen, und/oder ob es um persönlichen Geschmack, Zugehörigkeit zu einer Subkultur oder Ähnliches geht. Wir können also vom Aussehen und Verhalten weder auf die Geschlechtsidentität noch auf den Körper eines Menschen schließen. ${ }^{16}$

\subsubsection{Geschlechternormen, Sexismus, Endosexismus und Cis-Sexismus}

Alle Menschen erleben Einschränkungen ihrer Entfaltungsmöglichkeiten und oft ihrer Lebensqualität durch einengende Geschlechternormen auf den Ebenen Körper, Identität und Ausdruck bzw. Verhalten. Darüber hinaus werden Menschen diskriminierend hierarchisiert in ihrem Zugang zu Selbstbestimmung, Akzeptanz, Ressourcen, Macht, Teilhabe und der Möglichkeit, als >normak anerkannt zu werden sowie Partner:innen zu finden. Diskriminierung betrifft einerseits im Rahmen von Cis-Sexismus/Trans* feindlichkeit und Endosexismus/Inter* feindlichkeit sowie Sanktionen für Gendernonkonformität alle Menschen, die nicht den zweigeschlechtlichen Normen dieser Gesellschaft entsprechen (vgl. Debus i.d.B.; Debus/Laumann 2018a, 2020; Stuve/Debus 2012a; Bauer 2020b). Sie betrifft aber auch cis Frauen und Mädchen, die zwar den zweigeschlechtlichen Normen entsprechen, aber aufgrund von Sexismus Benachteiligung gegenüber Jungen und Männern erleben (vgl. Debus 2012, 2015). Nicht zuletzt erleben auch als >unmännlich markierte cis Jungen und Männer Abwertungen im Kontext von (Cis-)Sexismus (vgl. Debus 2015; Stuve/Debus 2012b).

\subsection{Geschlechtliche Vielfalt in der Sexualpädagogik}

Im Kontext geschlechtlicher Vielfalt lassen sich Orientierungspunkte einer diskriminierungsreflektierten Sexualpädagogik folgendermaßen konkretisieren: 
Diskriminierungsreflektierte Sexualpädagogik verzichtet auf direkte Diskriminierung und Othering-Praxen. ${ }^{17}$ Sie macht die Vielfalt von Geschlechtern sichtbar und trägt ihr in ihren Angeboten Rechnung.

Das heißt u.a., dass sie Normalitäts-Annahmen hinterfragt und verschiedene Geschlechtlichkeiten in den eingesetzten Methoden als selbstverständlich berücksichtigt. ${ }^{18}$ So sollten neben verschiedenen unterschiedlichen cis Mädchen/Frauen und cis Jungen/Männern auch verschiedene trans Menschen und Inter* querschnittsmäßig in den Materialien vorkommen, nicht nur in Sonderkapiteln.

Diskriminierungsreflektierte Sexualpädagogik stellt im Rahmen der Körperaufklärung Informationen über verschiedene Körperlichkeiten in respektvoller und gleichstellungsorientierter Weise bereit. Sie unterstützt ihre Adressat:innen bei der Entwicklung einer grenzachtenden Sprache und Kommunikationskompetenz über Körper und Sexualität. Sie vermittelt Informationen über Körper, die als weiblich, männlich oder inter* klassifiziert werden, sowie über die körperliche Vielfalt innerhalb der verschiedenen Körpertypen u.a. bezüglich der Sexualorgane. Dabei merkt sie an, dass jede Körperform mit unterschiedlichen Geschlechtsidentitäten einhergehen kann.

Diskriminierungsreflektierte Sexualpädagogik nimmt die verschiedenen Körper und ihre sexuellen und erotischen Funktionen gleichermaßen wichtig. U.a. thematisiert sie nicht nur den Penis sowie die sichtbare Eichel der Klitoris (Kitzler), sondern auch deren Schwellkörperfunktion im Inneren des Körpers (vgl. Méritt 2014). Auch die intergeschlechtliche Phalloklitoris (vgl. Costello 2011) ${ }^{19}$ wird als selbstverständlicher Teil des Spektrums zwischen Klitoris und Penis behandelt (vgl. etwa Selbstlaut 2020: 60-63) und kann Anlass bieten, die Entwicklung dieser Schwellkörper aus den gleichen Anlagen zu thematisieren (vgl. Bauer/Truffer 2016: 139ff.; Costello 2011).

17 Zu Othering und Diskriminierung in der Sexualpädagogik vgl. Debus i.d.B.

18 Ein für Jugendliche geeigneter Überblick über geschlechtliche Vielfalt findet sich in Debus 2021a: 16f.

19 Ich habe diesen Begriff auf der Suche nach einer nicht-pathologisierenden Bezeichnung für die intergeschlechtliche Variante des Organs gefunden, das in der Crundanlage gleich ist und sich bei als biologisch männlich klassifizierten Körpern als Penis und bei als biologisch weiblich klassifizierten Körpern als Klitoris ausprägt. Inter* können eine Phalloklitoris, einen Penis oder eine Klitoris haben, da unter dem Begriff Intergeschlechtlichkeit/Intersexualität sehr unterschiedliche körperliche Varianten zusammengefasst werden. 
Die Adressat:innen werden dabei unterstützt, einen selbstbestimmten und kreativen Umgang mit Begriffen für die eigenen Sexualorgane zu entwickeln, sowie einen respektvollen Umgang mit Begriffen für die Sexualorgane anderer Menschen.

Diskriminierungsreflektierte Sexualpädagogik stärkt die sexuelle und reproduktive Selbstbestimmung aller Menschen.

Dabei thematisiert sie Sexualität in menschenrechtsbasierter Weise. Sie vermittelt, dass Grenzen ebenso wie einvernehmlich ausgelebte Lust zwischen einwilligungsfähigen Menschen ohne geschlechtsbezogene Einschränkungen und Doppelbotschaften zu respektieren sind (vgl. Timmermanns 2013: 713). $\mathrm{Zu}$ diesem Zweck werden auch geschlechtsbezogene Normen in Bezug auf Flirt, Sexualität, Beziehungen etc. kritisch bearbeitet (vgl. z.B. Debus/Laumann 2018a; Stuve/Debus 2012b; Debus 2012).

Zur Förderung der reproduktiven Selbstbestimmung informiert diskriminierungsreflektierte Sexualpädagogik über Verhütungsmittel, Notfallverhütungsmittel (vor allem Pille und Spirale danach), Schwangerschaftsabbrüche sowie über den Zugang zu all diesen Maßnahmen. Andererseits vermittelt sie Wissen über verschiedene Wege der Fortpflanzung und Adoption etc. sowie über Unterstützungssysteme bei der Empfängnis, Kindererziehung und -finanzierung.

Diskriminierungsreflektierte Sexualpädagogik weiß um besondere Herausforderungen, denen Menschen mit verschiedenen Körpern und geschlechtlichen Zugehörigkeiten (u.a. durch Normen und Diskriminierung) in der Entwicklung ihrer Sexualität und der Kommunikation über Sexualität ausgesetzt sein können. Sie macht entsprechende Auseinandersetzungs- und Lernangebote.

Dies betrifft u.a. Schönheits- und Körpernormen und die reale Vielfalt der Körper; Körper-Abwertung/-Ekel (z.B. in Bezug auf Menstruation und Ausfluss, Ejakulation, unerwünschte Erektionen oder die Abweichung von Körpernormen); Dysphorie-Erfahrungen sowie normierungs-/diskriminierungsbedingte Schamgefühle und Kommunikationstabus. Wichtig ist auch die kritische Auseinandersetzung mit abwertenden Schlampenbildern, sexuellem Leistungsdruck, Rollenvorgaben sowie geschlechtsspezifischen Normen bezüglich penetrativem/circludierendem ${ }^{20}$ Sex. Auch lustvolle $\mathrm{Zu}$ - 
gänge zu Solo-Sex/Selbstbefriedigung sind relevant sowie das Spannungsfeld zwischen Kritik an Orgasmuszentrierung ${ }^{21}$ einerseits und am sogenannten orgasm gap ${ }^{22}$ andererseits (vgl. u.a. Frederick u.a. 2018).

Darüber hinaus bedarf es eines sensiblen Umgangs mit geschlechtsspezifischen Formen von Gewaltwiderfahrnissen u.a. bezüglich medizinischer, psychiatrischer, psychischer, körperlicher und sexualisierter Gewalt, rechtlicher Diskriminierung, Diskriminierung und Abwertung in der Familie, Alltagsdiskriminierung und -übergriffen sowie diskursiven Abwertungen und Einengungen und deren Auswirkungen auf das persönliche Sexualleben (vgl. z.B. Bauer 2004: 252-253).

Dabei trägt eine diskriminierungsreflektierte Sexualpädagogik der Vielfalt innerhalb der verschiedenen Geschlechtergruppen Rechnung, vermeidet Homogenisierungen und arbeitet ihnen entgegen.

Sie verzichtet auf und dekonstruiert Bilder, die beispielsweise beinhalten,

- dass >normale $J_{\text {Jungen }}^{23}$ >nur an das eine denken< (vgl. Debus 2012: 121122) und simmer können/wollen w würden; es nicht sexuell genießen könnten, einvernehmlich etwas oral oder anal in sich aufzunehmen; heterosexuell seien; (nur) als männlich klassifizierte Körpermerkmale hätten; bei ihnen Erektion und sexuelle Lust gleichzusetzen seien und dass sie normalerweise Täter und nicht Opfer von Übergriffen seien (vgl. Rieske 2016, Schlingmann u.a. 2016), ${ }^{24}$

entdecken, was wem wann Lust bereitet. Der Begriff Circlusion wurde von Bini Adamczak verbreitet (2016) und soll diese Aktiv-Passiv-Zuschreibung verflüssigen: In diesem Sinne kann derselbe Akt als passiv beschrieben werden (penetriert werden, etwas empfangen) oder als aktiv (etwas circludieren bzw. umschließen) und umgekehrt (penetrieren bzw. circludiert werden).

21 Die Kritik an Orgasmuszentrierung will Möglichkeiten eröffnen, Sex mehr lust- als leistungs- und zielorientiert zu gestalten bzw. erleben.

22 Der Begriff orgasm gap (Orgasmuslücke) beschreibt, dass cis Frauen in heterosexuellem Sex deutlich seltener Orgasmen erleben als cis Frauen in gleichgeschlechtlichen Konstellationen oder cis Männer generell.

23 Vertiefend zu Männlichkeitsnormen: Stuve/Debus 2012b; zu homogenisierenden Jungenbildern: Stuve 2012; zu geschlechtsbezogenen Normen bezüglich Sexualität, Verlieben und Partnerschaft: Debus/Laumann 2018a: 56-60.

24 Mehrere dieser Bilder und insbesondere das Letztere spitzen sich in Verschränkung mit rassistischen und klassistischen Bildern gegenüber Jungen mit muslimischen Familienbezügen, Jungen of Color bzw. aus Süd-/Ost-Europa sowie Jungen aus ökonomisch 
- dass >normale`Mädchen ${ }^{25}$ nur zärtlich und in Liebesbeziehungen Sex wollten; weniger Orgasmen und diese nur durch die Stimulation des außenliegenden Teils der Klitoris und nicht durch vaginale Stimulation haben könnten oder umgekehrt; das serste Mak Penetrationssex ihnen wahlweise immer weh tue (traditionelle Sichtweise) oder ausschließlich, wenn eine:r der Beteiligten etwas falsch mache (verkürzte Dekonstruktion des Jungfernhäutchen-Mythos); sie heterosexuell seien und (nur) als weiblich klassifizierte Körpermerkmale hätten (vgl. Busche u.a. 2010); bei ihnen Lust und Feucht-Werden gleichzusetzen seien und sie im Zweifelsfall (auch vor sich selbst) zu schützende $\mathrm{Opfer}^{26}$ seien etc.

- dass alle trans Menschen geschlechtlich binär verortet und heterosexuell seien; ihren Körper als falsch empfänden und eine operative Angleichung anstrebten; sich mit klischeehaften Männlichkeits- bzw. Weiblichkeitsbildern identifizierten etc.

- dass alle Inter* anhand ihrer Genitalien als intergeschlechtlich erkennbar seien; sich als >divers` identifizierten oder Identitätsprobleme hätten und/oder nicht handlungsfähige, zu bemitleidende Opfer seien (vgl. Hechler 2012)

prekarisierten bzw. proletarischen Familien zu: Diese werden oft als patriarchale Täter gesehen, ihre Diversität und Verletzlichkeit werden oft noch massiver unterschätzt als die mehrheitsangehöriger bürgerlicher Jungen und es wird in der Regel noch selbstverständlicher davon ausgegangen, sie seien heterosexuell und cis-geschlechtlich.

Vertiefend zu Weiblichkeitsanforderungen: Debus 2012; zu geschlechtsbezogenen Normen bezüglich Verlieben, Partnerschaft und Sexualität: Debus/Laumann 2018a.

26 Mehrere dieser Bilder und insbesondere das Letztere spitzen sich in Verschränkung mit rassistischen und klassistischen Bildern gegenüber Mädchen mit muslimischen Familienbezügen, Mädchen of Color bzw. aus Süd-/Ost-Europa sowie Mädchen aus ökonomisch prekarisierten bzw. proletarischen Familien zu. Ihnen wird oft pauschal OpferSein unterstellt, insbesondere wenn ihr Verhalten von den oft bürgerlich geprägten Normen von z.B. (auch feministisch geprägten) mehrheitsangehörigen Pädagog:innen abweicht u.a. in einem offenen Ausleben sexuellen Begehrens, durch Abweichen von der bürgerlich aktuell akzeptierten Spannbreite der richtigen Menge an Ent- und Verhüllung als weiblich eingeordneter Körper (z.B. bauchfrei oder Kopftuch) oder auch durch Kinderwünsche in jungem Alter. Oft wird dieses Verhalten nicht im Spannungsfeld zwischen Selbstbestimmung und einem Leben in einer patriarchal geordneten Welt gesehen, in dem wir uns alle orientieren müssen. Vielmehr wird dieses Spannungsfeld häufig vereindeutigend in eine Opferzuschreibung aufgelöst, die es scheinbar legitimiert, in die Selbstbestimmung dieser Mädchen/Frauen bevormundend einzugreifen (vgl. Debus 2021b). Diese Tendenz gilt auch für andere Abweichungen von bürgerlichen Sexualnormen durch Frauen, wie z.B. selbstbestimmte Sex-Arbeit. 
All diese Bilder bedürfen der aktiven Dekonstruktion und Diversifizierung.

\section{Amouröse und sexuelle Vielfalt in der Sexualpädagogik}

\subsection{Sexuelle Vielfalt im engeren Sinne}

Der Begriff sexuelle Vielfalt wurde im Rahmen von Kämpfen gegen die Diskriminierung von Schwulen, Lesben, Bi- und Pansexuellen, ${ }^{27}$ Polysexuellen ${ }^{28}$ sowie Queers geprägt ${ }^{29}$ und bezieht sich primär auf sexuelle Orientierungen, also die Frage, von welchen Geschlechtern sich ein Mensch romantisch und sexuell angezogen fühlt.

Asexuelle Aktivist:innen fordern, Asexualität (die Abwesenheit sexuellen Begehrens für andere Personen - nicht zu verwechseln mit z.B. religiöser Enthaltsamkeit) als sexuelle Orientierung anzuerkennen und gemeinsam mit Aromantik (der Abwesenheit romantischer Anziehung) zu entpathologisieren. Sie differenzieren zwischen sexueller und romantischer Orientierung. In diesem Kontext beschreibt die sexuelle Orientierung die Geschlechter, auf die sich das sexuelle Begehren einer Person richtet - Asexualität beschreibt dann, dass die Person sich zu gar keinem Geschlecht hingezogen fühlt. Die romantische Orientierung fasst, auf welche Geschlechter sich Verliebtheits-

27 Als pansexuell bezeichnen sich u.a. Menschen, für die Ceschlecht bei der Anziehung keine Rolle spielt, bzw. die sich zu allen Geschlechtern hingezogen fühlen und den Begriff bisexuell für sich ablehnen, weil er zu zweigeschlechtlich konnotiert ist. Bisexuelle Aktivist:innen halten dem zum Teil entgegen, dass der Begriff bisexuell Menschen aller Geschlechter einschließe und eine Distanzierung über ein neues Label entsolidarisierend wirke. Es lässt sich festhalten, dass die Unterscheidung zwischen bi- und pansexuell fließend und unscharf und nur von der sich bezeichnenden Person jeweils selbst zu bestimmen ist.

28 Polysexuell bedeutet, dass sich ein Mensch zu Menschen mehr als eines Ceschlechts hingezogen fühlt, aber nicht notwendigerweise zu Menschen aller Geschlechter, z.B. nur zu Frauen und nicht-binären Menschen oder nur zu queeren Menschen aller $\mathrm{Ce}$ schlechter etc.

29 Ein ausführliches Clossar zu diesen und weiteren Begriffen findet sich unter https://in terventionen.dissens.de/materialien/glossar [Zugriff: 14.07.2020]. 
gefühle richten. So kann ein Mensch beispielsweise asexuell und hetero-, homo-, bi-, pan- oder queerromantisch sein. ${ }^{30}$

Es bleibt daher bei der Verwendung z.B. der Begriffe homosexuell oder heterosexuell deutungsoffen, wann damit die generelle Oberkategorie gemeint ist, die nicht zwischen sexueller und romantischer Anziehung unterscheidet, und wann explizit nur die sexuelle Anziehung gemeint ist. ${ }^{31}$

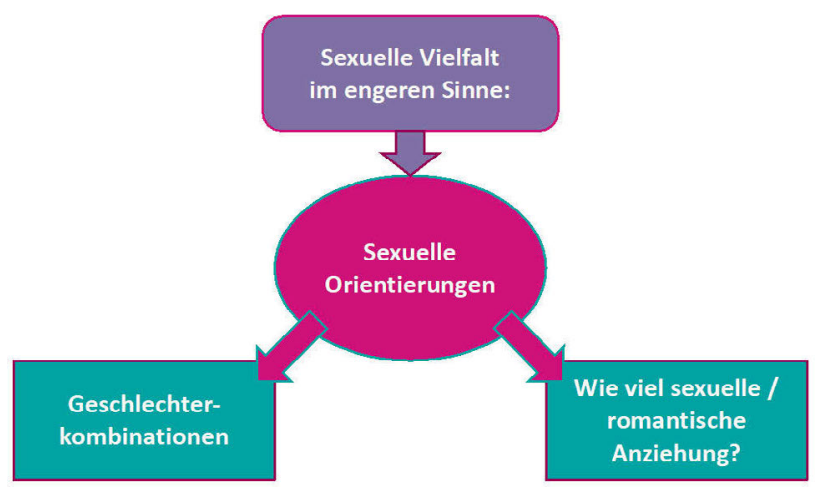

Abb. 4: ๔ Katharina Debus

Sexuelle Vielfalt im engeren Sinne meint also sexuelle Orientierungen, mit anderen Worten: Geschlechterkombinationen, innerhalb derer ein Mensch Anziehung empfindet, sowie das von ihm:ihr empfundene Maß an sexueller und romantischer Anziehung.

Vertiefend: Profus 2016, https://www.asexyqueer.blogsport.de [Zugriff: 20.12.2020]; h ttps://www.aven-info.de [Zugriff: 20.12.2020].

31 Es ist wichtig, diese Deutungsoffenheit beizubehalten, um den Offenbarungs- und Coming-out-Druck nicht weiter zu erhöhen und die Begriffe nicht denen zu enteignen, die sich schon lange mit ihnen beschrieben haben. Die Unterscheidung zwischen sexueller und romantischer Orientierung ist in diesem Sinne als Denkangebot vor allem für Selbstbeschreibungen zu verstehen und als Sensibilisierung dafür, dass nicht bei allen Menschen sexuelle und romantische Orientierung deckungsgleich sind. Viele insbesondere heterosexuelle Menschen sind verwirrt von den vielen verschiedenen Selbstbeschreibungen und Deutungsoffenheiten. Eine wichtige zu fördernde Kompetenz mit hohem Diskriminierungspräventionspotenzial ist die Fähigkeit, mit Unsicherheit, Verwirrung und Mehrdeutigkeit (Ambiguitätstoleranz) umzugehen (vgl. Debus/Laumann 2018a: 91f., zu Spannungsfeldern im Umgang mit Labels ebd.: 33f.). 


\subsection{Amouröse und sexuelle Vielfalt im erweiterten Sinne}

Die Bezeichnung sexuelle Vielfalt lenkt begrifflich die Aufmerksamkeit auf Sexualität. Im Kontext des oben erwähnten Interventionen-Projekts habe ich daher ergänzend eine erweiterte Definition amouröser und sexueller Vielfalt vorgeschlagen (vgl. Debus/Laumann 2018a). Einerseits trägt der Begriff der oben beschriebenen Unterscheidung zwischen sexueller und romantischer Orientierung Rechnung und macht zudem deutlich, dass z.B. Schwul-Sein sich meist nicht nur auf Sexualität bezieht, sondern oft auch auf Verlieben, Partnerschaft und/oder Familie. Zum anderen entsteht in der Differenzierung Raum für weitere Vielfaltsebenen, die keine sexuellen Orientierungen sind, aber relevant in Bezug auf Diskriminierung, Normkritik und die individuelle Lebensgestaltung. Darüber hinaus entspricht diese erweiterte Definition den Assoziationen und Fragen, die viele Jugendliche und Erwachsene haben, wenn sie den Begriff sexuelle Vielfalt hören. Unabhängig davon, ob wir einen engeren oder weiteren Fokus setzen, sollten wir also auf einen diskriminierungsreflektierten Umgang mit Fragen zu allen im Folgenden genannten Themen vorbereitet sein.

Amouröse Vielfalt kann zusätzlich zu Fragen der romantischen Orientierung auch verschiedene Beziehungs- und Bindungsformen sowie die Anzahl der Partner:innen meinen, z.B. Single-Sein, (serielle) Monogamie, offene Beziehungen oder Polyamorie. ${ }^{32}$ Sexuelle Vielfalt im erweiterten Sinne kann sich zusätzlich zu sexuellen Orientierungen auch auf die Anzahl der Sexualpartner:innen, ${ }^{33}$ die Rolle von und Regeln für Sex inner-/außerhalb von Partnerschaften $^{34}$ sowie auf verschiedene Sexpraktiken (z.B. BDSM, siehe 3.5) und damit gegebenenfalls verbundene Identitäten (sexuelle Neigungen) beziehen. Darüber hinaus sind Überschneidungen zwischen sexueller und amouröser Vielfalt und Familien-Vielfalt offensichtlich.

Die Entscheidung, wann eher mit einem engeren Begriff sexueller Vielfalt mit Fokus auf sexuelle (und romantische) Orientierungen gearbeitet werden

32 In polyamoren Beziehungen wird offen mit allen Beteiligten ausgehandelt, dass mehrere verbindliche Beziehungen mit mehreren Personen gelebt werden können, dabei sind verschiedenste Optionen möglich. Vgl. u.a. Baumgartner 2020 sowie die folgenden Grafiken: Veaux 2012 (http://beziehungsgarten.net/blog/alternativen-zur-monoga mie); Rohwer 2014 (https://metakiki.net/relationship-concepts-cartoon).

33 Z.B. Solo-Sex, Sex zu zweit, Sex zu mehreren.

34 Z.B. Partnerschaften mit oder ohne Sexualität, Sex als Stärkung der Partnerschaft und/oder Problemthema, Promiskuität, Vereinbarungen zu Sex mit anderen etc. 


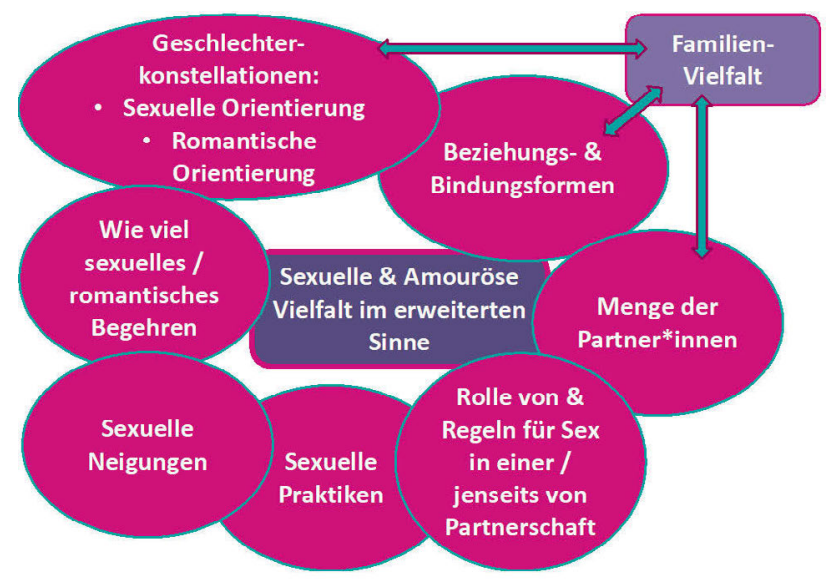

Abb. 5: ๔ Katharina Debus

sollte, und wann eher ein erweiterter Begriff sinnvoll ist, hängt stark vom Kontext ab (vgl. ausführlicher Debus/Laumann 2018a).

Auch hier gilt, wie für geschlechtliche Vielfalt beschrieben, dass einerseits alle Menschen von Normierungen rund um amouröse und sexuelle Vielfalt betroffen sind und andererseits Diskriminierung die Leben nicht-heterosexueller Menschen (Heterosexismus) und weiterer Gruppen besonders belastet (vgl. Debus i.d.B.; Debus/Laumann 2018a: 50-60; Bauer 2020b: 674-579).

Im Folgenden gehe ich im Sinne eines fokussierten Verständnisses sexueller Vielfalt zunächst auf sexuelle und romantische Orientierungen in der Sexualpädagogik ein (4.3) und greife dann zwei zusätzliche Aspekte eines erweiterten Verständnisses sexueller und amouröser Vielfalt heraus: Beziehungskonzepte (4.4) und Sex-Praktiken mit einem Fokus auf BDSM (4.5).

\subsection{Sexuelle und romantische Orientierungen in der Sexualpädagogik}

Im Sinne einer inklusiven Thematisierung verschiedener sexueller und romantischer Orientierungen lassen sich für diskriminierungsreflektierte Sexualpädagogik folgende Orientierungspunkte formulieren: 
Diskriminierungsreflektierte Sexualpädagogik geht anerkennend mit Asexualität und Aromantik um.

Sie geht nicht davon aus, dass jeder (>gesunde`) Mensch sich sexuell und/oder romantisch zu anderen hingezogen fühlt - selbst wenn das eine Provokation gegenüber manchen sexualpädagogischen Dogmen ist. Sie vermittelt, dass viele Menschen sexuelles Begehren und Verliebtheitsgefühle empfinden und Partnerschaften eingehen, dass es aber ebenso selbstverständlich Menschen gibt, auf die das eine und/oder andere phasenweise oder dauerhaft nicht zutrifft, ohne dass dies ein Ausdruck von Krankheit bzw. Beschädigung sein muss (vgl. Liebeck 2014; asexualitätsinklusive Grafik für Jugendliche: Debus 2021a: 17$){ }^{35}$

Für alle Menschen kann es darüber hinaus hilfreich sein, sichtbar und besprechbar zu machen, dass der Zusammenhang zwischen Liebe und sexuellem Begehren individuell sehr unterschiedlich und bei den meisten Menschen und in den meisten Beziehungen dynamisch ist.

Diskriminierungsreflektierte Sexualpädagogik erkennt Identitäten und sexuelle Orientierungen an, schreibt diese aber nicht fest. Sie dekonstruiert homogenisierende Bilder. Sie benennt u.a., dass es viele Menschen gibt, bei denen Identitäten und Orientierungen ein Leben lang gleich bleiben, während sie sich bei anderen ein-

35 Oft fragen Jugendliche, ob sie asexuell seien, wenn sie z.B. mit 16 noch niemanden küssen wollten etc. Es ist wichtig, ihnen den Druck zu nehmen, schnell viele sexuelle Erfahrungen zu machen und sie dabei zu unterstützen, ihr eigenes Tempo zu finden u.a. mit der Anmerkung, dass viele Menschen erst nach der Schulzeit das erste Mal Sex haben oder sich verlieben, dass das also nicht unbedingt ein Hinweis auf Asexualität oder Aromantik sein muss. Hier ist zu betonen, dass das alle für sich selbst herausfinden müssen. Darüber hinaus ist ein Hinweis auf psychotherapeutische Unterstützungsangebote sinnvoll für Fälle, in denen ein Mensch anhaltend unglücklich mit etwas ist u.a. in Bezug auf (Nicht-)Beziehungen/Begehren. Dies gilt für alle Begehren, Beziehungen etc., also auch z.B. für heterosexuelle Menschen. Manchmal kann das Ergebnis z.B. in Bezug auf (Nicht-) Begehren eine Veränderung sein u.a. wenn Traumatisierungen Auslöser waren. Oft wird aber auch klar, dass das Unglücklich-Sein mit Diskriminierung zu tun hat. Eine gute Therapie kann dann in einem besseren Umgang damit und einem Zugang zu Communitys unterstützen. Allerdings kann es schwierig sein, Therapeut:innen zu finden, die nicht mit pathologisierenden Vorannahmen in solche Erkundungen gehen. Wenn es vor Ort Beratungsstellen gibt, die diskriminierungssensible Therapeut:innen vermitteln, ist ein Hinweis auf diese hilfreich. 
mal oder mehrfach verändern. Sie stellt klar, dass das niemandem das Recht gibt, in diese einzugreifen, und weist darauf hin, dass Konversionstherapien sexuelle und romantische Orientierungen (wie auch Geschlechtsidentitäten) nicht verändern, aber oft traumatisierend sind (vgl. Spahn 2018b) und daher zumindest in Deutschland für Minderjährige verboten (Bundesministerium für Gesundheit 2020).

Sie dekonstruiert homogenisierende Bilder, beispielsweise von >den Schwulen, Leben oder Bisexuellen, und wirft den Blick auf Vielfalt innerhalb der verschiedenen Lebensweisen. Und sie kommuniziert, dass es manchmal erleichternd und stärkend sein kann, ein Label für sich zu finden, dass es aber auch einengend sein kann, weil Menschen oft komplexer sind als Labels. Sie vermittelt, dass daher Labels vor allem als Selbstbeschreibungen relevant sind und es legitim und normal ist, diese auch (wieder) zu verwerfen (vgl. Debus/Laumann 2018a: 33-34, 50-52).

Diskriminierungsreflektierte Sexualpädagogik macht die verschiedenen Bedeutungsebenen von Sexualität sichtbar und reduziert die Thematisierung von Sexualität nicht auf die Fortpflanzungsebene.

Sie thematisiert Sexualität nicht nur als Aktivität zum Zweck der Fortpflanzung, sondern auch in ihrer Lust-Funktion, ihrem beziehungsstiftenden und -pflegenden Charakter sowie ihrer Selbstsorge und möglichen Identitätsfunktion (in Anlehnung an Sielert 2015: 47f.; vgl. auch Bauer 202ob: 569ff.). Sexualität wird dadurch in ihrer Vielfalt sichtbar und ihre möglichen Sinnebenen werden für die Beteiligten aushandelbar. ${ }^{36}$

\section{Diskriminierungsreflektierte Sexualpädagogik zeigt verschiedene Fortpflanzungs-} Wege von Menschen auf.

Sie thematisiert nicht nur Befruchtung durch Penis-in-Vagina-Sex, sondern auch verschiedene Methoden künstlicher Befruchtung inklusive der kostengünstig anwendbaren Becher-Methode und informiert über Regenbogenund Co-Eltern-Familien. ${ }^{37}$ Sie informiert damit über Zukunftsoptionen und

36 Darüber hinaus privilegieren Lehreinheiten, die Sexualität mit Fokus auf Fortpflanzung thematisieren, Penis-in-Vagina-Sex und werten zumindest implizit alle anderen Formen der Sexualität sowie alle anderen Partnerkonstellationen ab.

37 Mehr Informationen u.a. unter https://familienportal.de/familienportal/lebenslagen/r egenbogenfamilien; https://planningmathilda.com/was-bedeutet-co-elternschaft-oder -co-parenting-eigentlich-genau [Zugriff: 15.07.2020]. 
ist gleichzeitig inklusiv für Adressat:innen, die oder deren Geschwister aus verschiedenen Gründen über nicht-sexuelle Befruchtung bzw. jenseits von Liebesbeziehungen gezeugt wurden.

Diskriminierungsreflektierte Sexualpädagogik behandelt verschiedene romantische und sexuelle Optionen nicht hierarchisch, sondern inklusiv anhand von Querschnittsthemen.

Sie vermeidet eine Zweiteilung zwischen einerseits snormalen $<$ heterosexuellen (und cis-geschlechtlichen) Themen und andererseits Sonderthematiken unter Begriffen wie >Vielfalt< oder $>$ Minderheiten $\triangleleft{ }^{38}$ Sie stellt verschiedene Optionen von (Nicht-)Verlieben, (Nicht-)Sex, Safer Sex (vgl. Thörner 2016) und (Nicht-)Fortpflanzung gleichwertig nebeneinander (vgl. Timmermanns 2013: 713). Dabei knüpft sie an Gemeinsamkeiten zwischen Menschen unterschiedlicher sexueller und romantischer Orientierungen an, z.B. bezüglich der Sinnebenen von Sexualität oder verschiedener konstellationsübergreifender Sex-Praktiken (z.B. dem Einsatz von Händen, Mündern etc.), ohne Unterschiede zu dethematisieren. Wenn das vorhandene Lernmaterial bestimmte Lebensweisen nicht inklusiv abdeckt, stellt sie ergänzende Materialien bereit $^{39}$ und übt Medienkritik an den Auslassungen der Standardmaterialien.

Diskriminierungsreflektierte Sexualpädagogik weiß um spezifische Formen von Diskriminierung und Herausforderungen, mit denen Menschen verschiedener Liebes- und Lebensweisen konfrontiert sind, und trägt diesen Rechnung.

Sie denkt Folgen orientierungsspezifischer Normierungen und Tabuisierungen sowie von Gewalt- und Diskriminierungswiderfahrnissen für die sexuelle Entwicklung mit und stärkt die Adressat:innen im Umgang damit.

U.a. arbeitet sie pornografisch geprägtem Normierungsdruck entgegen, macht die Vielfalt (auch hetero-)sexueller Möglichkeiten sichtbar und

38 Jutta Hartmann (2018) übt mit ihrem Ansatz Vielfalt von der Vielfalt aus denken Kritik an Othering-Praxen, bei denen >Vielfalt < zur Chiffre wird, mit der doch nur die als >nicht normak konstruierten Lebensweisen gemeint sind. Sie arbeitet eine Herangehensweise aus, die Vielfalt inklusiv denkt, also alle Lebensweisen inklusive der normnahen Lebensweisen gleichermaßen anspricht.

Altersgerechte Informationen zu schwulen, lesbischen, bi- und pansexuellen Lebensweisen (ebenso wie zu Trans und Inter*) sollten für alle Adressat_innen an einem Ort mit geringem Outing-Risiko zugänglich sein, z.B. Dawson 2020. Für eine Überblicksgrafik über verschiedene sexuelle Orientierungen für Jugendliche vgl. Debus 2021a: 17. 
stellt geschlechterstereotype Vorstellungen >richtigen heterosexuellen Geschlechtsverkehrs im Sinne des koitalen Imperativs in Frage (vgl. Ancke 2014). Sie kritisiert also die Annahme, dass nur Penis-in-Vagina-Sex >richtiger Sex und alles andere kein Sex, Vorspiel, exotisch, abenteuerlich, pervers, eine Ersatzhandlung oder Ähnliches sei. Dies ist hilfreich für Menschen verschiedenster sexueller Orientierungen. In diesem Zuge können u.a. lesben- und transfeindlichen Klischees dekonstruiert werden, z.B. dass Konstellationen ohne Penis keinen >richtigen Sex haben könnten ${ }^{40}$ und dass ein Phallus Teil eines Männerkörpers sein sollte. Letzteres ist transfeindlich und führt zur Annahme, wenn Lesben einen Dildo benutzten, wollten sie seigentlich einen Mann.

Sie weiß um Schwierigkeiten der Partnersuche für viele Menschen jenseits der heterosexuellen Norm, insbesondere im ländlichen Raum, und um die Gewaltbedrohungen, denen insbesondere schwule und bisexuelle Jungen/Männer ausgesetzt sind, wenn andere Jungen/Männer sich von ihnen angeflirtet fühlen. Ebenso weiß sie um paradoxe Männlichkeitsanforderungen, wenn Schwul-Sein aus mehrheitsgesellschaftlicher Sicht mit Effeminiertheit verbunden wird, aber in vielen schwulen Communitys traditionelle Männlichkeitsvorstellungen normativ sind. Sie vermittelt ihren Adressat:innen Ressourcen für den Umgang mit diesen Herausforderungen.

Zudem arbeitet diskriminierungsreflektierte Sexualpädagogik der Annahme entgegen, bi-/pan-/polysexuelle Menschen seien unentschlossen, entweder neugierige Heterosexuelle oder versteckte Homosexuelle und suchten immer gleichzeitig Beziehungen bzw. Sex zu Menschen mehrerer Geschlechter (Eisner 2013).

\subsection{Beziehungskonzepte in der Sexualpädagogik}

Diskriminierungsreflektierte Sexualpädagogik zeigt auf, dass es mehr mögliche Formen von (Nicht-)Partnerschaft gibt als die Abfolge von Single-Sein, (seriell) monogamen Beziehungen und gegebenenfalls >Fremdgehen $<$. Sie berücksichtigt zudem u.a. die Möglichkeit, dauerhaft keine Beziehungen zu 
wünschen (Profus 2016: 239-241), freund:innenschaftszentrierte Lebensweisen $^{41}$ und offene sowie polyamore Beziehungen. ${ }^{42}$

Aus einer Beschäftigung mit verschiedenen Möglichkeiten von Beziehungen können alle Menschen unabhängig von ihren eigenen Bindungspräferenzen interessante Impulse ziehen. Diskriminierungsreflektierte Sexualpädagogik ermutigt die Teilnehmenden zum Nachdenken über ihre persönlichen Bedürfnisse an Partnerschaften und übt Auseinandersetzungsfähigkeit, z.B. zu Fragen von Eifersucht, Gefühlen eingeschränkter Freiheit, Bindungs- und Verbindlichkeitswünschen, fairem Umgang mit Interessenkonflikten, Aushandlung von Bedürfnissen, Thematisierung von Unsicherheiten und Ängsten, Umgangsmöglichkeiten mit Zurückweisung, enttäuschten Hoffnungen und unerfüllten Bedürfnissen sowie verschiedenen Möglichkeiten der Herstellung von Verbindlichkeit und der sozialen Verantwortungsübernahme. Verschiedene Beziehungsmodelle werden dabei nicht bewertet, vielmehr werden die Adressat:innen $\mathrm{zu}$ einem selbstbestimmten Umgang mit den aufgeworfenen Fragen ermutigt.

\subsection{Sexuelle Praktiken und Neigungen in der Sexualpädagogik: Fokus BDSM}

Diskriminierungsreflektierte Sexualpädagogik berücksichtigt vielfältige Sexpraktiken in inklusiver Weise, um ihre Adressat:innen zu befähigen, »ihre Sexualität zu verstehen und zu genießen, sichere und erfüllende Beziehungen einzugehen sowie verantwortlich mit ihrer eigenen sexuellen Gesundheit und der ihres Partners umzugehen« (BzgA 2011: 22; vgl. Debus i.d.B.). Wenn einvernehmliche Sexpraktiken, für die sich Adressat:innen interessieren, einseitig problematisiert, beschwiegen oder tabuisiert werden, kann dies diskriminierend wirken, z.B. in Überschneidung zu verschiedenen Diskriminierungen geschlechtlicher Vielfalt und sexueller Orientierungen bei den Themen Analsex, Safer Sex, Solo-Sex, lustvolle Sexualität (u.a. auch mit Sex-Toys) etc. Besonders unerforscht im Kontext von Jugend und Sexualität, Sexualpädagogik sowie Diskriminierung ist das Thema BDSM (vgl. Debus i.E.). Zugleich

\footnotetext{
41 Mehr Informationen und Literaturverweise: https://freundschaftszentriertleben.com [Zugriff: 15.07.2020].

42 Vgl. für eine Überblicksgrafik für Jugendliche über verschiedene Beziehungsformen Debus 2021a: 18.
} 
kommen in der Beschäftigung mit BDSM einige besondere Herausforderungen und gewaltpräventive Erkenntnisse hinzu, sodass hier im Folgenden ein exemplarischer Schwerpunkt gelegt wird.

BDSM steht im engeren Sinne für Bondage (einvernehmliche Spiele mit Bewegungseinschränkung, Fesseln etc.), Dominance and Submission (einvernehmliche Spiele mit Macht) sowie Sadismus und Masochismus (einvernehmliche Spiele mit Lustschmerz). Auch Fetischismus, Rollenspiele sowie einvernehmlicher Voyeurismus und Exhibitionismus können dem BDSM-Spektrum zugerechnet werden. Der Begriff BDSM (auch: kink, Adjektiv: kinky) bezieht sich auf eine Subkultur, die die Einvernehmlichkeit aller Beteiligten als wichtigste Grundbedingung des Auslebens des eigenen Begehrens voraussetzt. Dies wird mit den Akronymen SSC (safe, sane and consensual) oder RACK (risk aware consensual kink) beschrieben. ${ }^{43}$

Für eine diskriminierungsreflektierte Sexualpädagogik sind in Bezug auf BDSM vier Personengruppen zu berücksichtigen: Menschen mit Fantasien im BDSM-Spektrum; Menschen, die gelegentlich BDSM-Praktiken ausüben (möchten); Menschen, für die BDSM integraler Bestandteil ihrer Sexualität ist, und/oder Menschen, die BDSM als Teil ihrer Identität begreifen bzw. für die BDSM-Communitys eine wichtige Bezugsgröße in ihrer Lebensgestaltung darstellen. Die dritte und vierte Gruppe wären als sexuelle Minderheit mit einer sexuellen Neigung zu BDSM/Kink zu beschreiben (vgl. auch Bauer 2020a: 179). ${ }^{44}$

Für alle vier Gruppen ist einerseits direkte Diskriminierung als ‘krank', >pervers etc. relevant ${ }^{45}$ und andererseits die Frage, welche Bedarfe sie an eine inklusive Sexualpädagogik haben. Eine explorative Studie, die ich 2015 in einem BDSM-Online-Forum zu Jugenderfahrungen von Menschen durchgeführt habe, die bereits in ihrer Jugend eine Affinität zu BDSMPraktiken hatten, ${ }^{46}$ ergab auffällige Überschneidungen mit psychosozialen

\footnotetext{
43 Vgl. zu BDSM Debus i.E.; Bauer 2020a; Borkenhagen/Brähler 2016.

44 Vgl. zur Vertiefung und zum besseren Verständnis des jeweils individuell sehr unterschiedlichen Lustgewinns aus BDSM Debus i.E.; Debus/Laumann 2018a: 44-47.

45 Für Erwachsene bestehen zudem Diskriminierungsrisiken im beruflichen Kontext (z.B. Kündigungen bei Bekanntwerden der Neigung) und das Risiko einer Benachteiligung bei Sorgerechtsstreitigkeiten.

Teilgenommen haben 14 Nutzer:innen des Online-Portals zwischen 20 und 56 Jahrenhier werden die Antworten der elf Teilnehmenden zwischen 20 und 36 Jahren berücksichtigt.
} 
Herausforderungen und Bedarfen queerer Jugendlicher, ${ }^{47}$ z.B. in Bezug auf Selbstzweifel und Selbsthass; Isolationsgefühle; Sorge vor Entdeckung, Abwertung und Ausschluss; mangelnde Austauschmöglichkeiten sowie mediale Zerrbilder, die eine positive Identifikation mit möglichen Suchbegriffen und damit den Zugang zu Informationen und Community-Angeboten erschwerten. An anderen Stellen wurden spezifische Bedarfe deutlich, u.a. in Bezug auf den Umgang mit Risiken verschiedener BDSM-Praktiken; Angst bezüglich der eigenen Fantasien; Sorge, unethisch zu handeln, sowie Schuldgefühle und Selbstabwertung aufgrund der Formähnlichkeit ${ }^{48}$ zwischen BDSM und Gewalt.

Als hilfreich erlebten die Teilnehmenden liberale und sexpositive Umfelder; Zugang $\mathrm{zu}$ Informationen u.a. bezüglich (sichererer) Praktiken; Aneignung von Sprache, Begriffen, verbesserter Kommunikationsfähigkeit und Techniken zur Herstellung von Einvernehmlichkeit; Vermittlung von Unterscheidungskriterien zwischen Einvernehmlichkeit und Gewalt; Austausch; Community-Anschluss sowie akzeptierende und gesprächsoffene Reaktionen von Eltern und Freund:innen beim Coming-out.

Bezüglich der grenzachtenden Thematisierung von BDSM-Praktiken in der Sexualpädagogik besteht didaktischer und methodischer Diskussionsund Entwicklungsbedarf. Wenn das Einvernehmlichkeitsprinzip im eingangs beschriebenen Sinne gefördert wird, fördert das die sexuelle Selbstbestimmung und Gesundheit von Menschen mit Vanilla- ${ }^{49}$ wie auch mit BDSM-Präferenzen. Hierbei kann die allgemeine Sexualpädagogik einiges von BDSM-Communitys lernen (vgl. auch Bauer 2004: 261), die sich früh veranlasst sahen, sich mit der Herstellung von Einvernehmlichkeit $\mathrm{zu}$ beschäftigen, um ihre Sexualität von Straftaten abzugrenzen. Darüber hinaus ist es für alle Kinder und Jugendlichen wichtig, Raum für Fragen zu schaffen, und diese respektvoll und informativ zu beantworten.

Spezifisch für BDSM-affine Jugendliche ist zudem die Erkenntnis wichtig, dass es vielfältige BDSM-Praktiken gibt, von denen viele nicht den öf-

47 Zur psychosozialen Situation queerer Jugendlicher vgl. u.a. Oldemeier 2018; Spahn 2018a.

48 Mit Formähnlichkeit meine ich, dass einige BDSM-Praktiken wie Gewalt aussehen, wenn man außer Acht lässt, dass BDSM sich durch Einvernehmlichkeit von Gewalt unterscheidet (vgl. Debus i.E.).

49 >Vanilla< ist der Gegenbegriff zu BDSM, meint also Menschen ohne Affinität zu BDSMPraktiken. 
fentlich sichtbaren Klischees entsprechen, dass nicht für alle genitale Sexualität bedeutsam ist und dass alles individuell ausgehandelt werden muss und niemand durch Behauptungen z.B. srichtiger Submissivität e etc. unter Druck gesetzt werden darf. ${ }^{50}$ Eine weitere Thematisierung von BDSM in allgemeinen sexualpädagogischen Angeboten sollte in Abhängigkeit von Interessen und Grenzen der Gruppe erfolgen, wobei insbesondere aufgrund der Formähnlichkeit zu Gewalt ein Umgang mit der Spannung zwischen den Bedarfen BDSM-interessierter Jugendlicher und möglichen Grenzen anderer Jugendlicher gesucht werden muss (vgl. Debus i.E.). Vertiefte Bedarfe BDSM-affiner Jugendlicher können oft besser durch die SMJG, ${ }^{51}$ einen Verein von und für junge BDSMer:innen, oder andere spezifische Angebote gedeckt werden. Auf diese sollte hingewiesen werden.

\section{Fazit: Potenziale diskriminierungsreflektierter vielfaltsorientierter Sexualpädagogik in Schule und Regelstrukturen}

Im besten Fall kann diskriminierungsreflektierte vielfaltsorientierte (Sexual-)Pädagogik Kindern und Jugendlichen einen besseren Zugang zu ihrer eigenen Sexualität, Identität, ihren Körpern und ihrer Fähigkeit zur Beziehungsgestaltung ermöglichen. Sie kann sowohl die Lebensqualität der Adressat:innen in der Gegenwart als auch ihre Gestaltungsfähigkeit für die Zukunft stärken.

Bei all dem muss sie ein Spannungsfeld beachten: Einerseits ist Freiwilligkeit im Sinne von Grenzachtung ein wichtiges Prinzip der Sexualpädagogik. Andererseits verknüpfen sich Scham und/oder Desinteresse z.T. mit diskriminierenden Strukturen, beispielsweise wenn gleichgeschlechtliche Sexpraktiken als besonders abstoßend gelten, Mädchen in vielen Peergroups oder Familien riskieren, bei Interesse an Sexualität als `Schlampen

50 Wenn die Jugendlichen Shades of Crey ansprechen, sollte darauf hingewiesen werden, dass die Autorin sich mit BDSM nicht auskannte und Beziehungsgewalt und BDSM vermischt, dass also vieles darin mit BDSM wenig zu tun hat. 
werden, oder Jungen beim Zeigen von Unsicherheit die Männlichkeit abgesprochen wird. Nicht zuletzt sollten Respekt, Grenzachtung und Verantwortungsübernahme nicht nur bei persönlichem Interesse, sondern auch zum Schutz anderer gefördert werden. Es gilt also, ein Gleichgewicht im Spannungsfeld zwischen Freiwilligkeit, Orientierung an den (un-)ausgesprochenen Interessen und Bedarfen der Adressat:innen ${ }^{52}$ und der pädagogischen Setzung von Informationen ${ }^{53}$ sowie basalen Präventionsthemen zu finden.

In Anbetracht der genannten Bedarfe und Potenziale haben Schule und andere Regelstrukturen die besondere Chance, Synergieeffekte mit anderen Themen zu nutzen. Es gibt vielfältige Überschneidungen mit psychosozialem Lernen, Identitätsbildung, Familie, Zukunfts- und Lebensorientierung, Gewaltprävention etc. Einige der Themen und Kompetenzen können neben expliziter Sexualpädagogik z.B. gefördert werden im Sport-, Literatur-, Biologie-, Geschichts- oder Politikunterricht, über Aushänge/Plakate, Filmabende, Projekte, Besuche von Kooperationspartner:innen oder Ausstellungen, Diskussionsveranstaltungen, eine Sex-Fragen-und-Antworten-Wand im Foyer, indem Gesprächsanlässe ${ }^{54}$ geschaffen und situativ aufgegriffen werden etc.

Wenn Pädagog:innen aufmerksam in Bezug auf Themen rund um Sexualität, Erotik und Liebe sind, wird oft schnell deutlich, wie viele Fragen viele Kinder und Jugendliche dazu haben. Diese lassen sich in verschiedenste Lerneinheiten integrieren. Ein solches Ernstnehmen der Anliegen der Adressat:innen kommt nicht zuletzt der pädagogischen Beziehung zu Gute.

52 Ausführlicher zur anonymen Fragenkiste und der Regulation von (Scham-)Grenzen per Kopfhörer, weiteren Spannungsfeldern und der Problematik sexualpädagogischer Arbeitsbedingungen: Debus i.d.B.

53 Wichtig ist in diesem Sinne u.a., allen Jugendlichen eine Linkliste mit verschiedenen Anlaufstellen rund um geschlechtliche, sexuelle und amouröse Vielfalt, sexuelle Gesundheit und Cewaltprävention auszuteilen, sodass sie Zugriff auf diese Informationen haben, ohne sich beispielsweise durch Mitnehmen eines Flyers vor den Mitschüler:innen verdächtig zu machen. Linklisten für die deutschen Bundesländer finden sich beispielsweise unter https://interventionen.dissens.de/fuer-jugendliche/links-anlaufst ellen [Zugriff: 13.07.2020].

54 Cesprächsanlässe können durch alle genannten Maßnahmen wie auch kleine Bemerkungen nebenbei etc. geschaffen werden. Es ist wichtig, bei Interesse der Adressat:innen Raum für ein weiteres Gespräch zu schaffen, sei es im Unterricht, auf dem Pausenhof oder in einer Tür- und Angelsituation. Interesse kann sich im Übrigen auch in Albernheit oder Provokationen zeigen. 


\section{Literatur}

Adamczak, Bini (2016): Come on. Über ein neues Wort, das sich aufdrängt - und unser Sprechen über Sex revolutionieren wird. analyse \& kritik 614. Online unter: https://www.akweb.de/ak_s/ak614/04.htm [Zugriff: 15.07.2020].

Ancke, Judith (2014): Veränderungsstrategien zwischen Infragestellung von Normen, Pluralisierung und stärkung des Selbst‘. (Hetero-)Sexualität in der sexualpädagogischen Praxis. Humboldt-Uni zu Berlin.

Bauer, Markus/Truffer, Daniela (2016): Intersex und Selbstbestimmung. In: Katzer, Michaela/Voß, Heinz-Jürgen (Hg.): Geschlechtliche, sexuelle und reproduktive Selbstbestimmung. Gießen: Psychosozial, 137-160. Online unter: https://www.psychosozial-verlag.de/catalog/dispatch-free.php?id $=6799$ [Zugriff: 15.07 .2020 ].

Bauer, Robin (2004): SM, Gender Play und Body Modification als Techniken zur (Wieder-)Aneignung des eigenen Körpers, der eigenen Sexualitäten und Geschlechtsidentitäten. Anregungen für sexualpädagogische Diskussionen aus queer-feministischer Perspektive. In: Timmermanns, Stefan/Tuider, Elisabeth/Sielert, Uwe (Hg.): Sexualpädagogik weiterdenken. Postmoderne Entgrenzungen und pädagogische Orientierungsversuche. Weinheim: Juventa, S. 241-262.

Bauer, Robin (2016): Vom liberalen zum kritischen Konsens. Ein empirischer Blick auf Praxen der Aushandlung von Konsens in queeren BDSMKontexten. In: Borkenhagen, Ada/Brähler, Elmar (Hg.): Wer liebt, der straft? Gießen: Psychosozial, S. 129-142.

Bauer, Robin (2020a): Interdisziplinäre Perspektiven auf BDSM aus queertheoretischer Sicht. In: Timmermanns, Stefan/Böhm, Maika (Hg.): Sexuelle und geschlechtliche Vielfalt. Interdisziplinäre Perspektiven aus Wissenschaft und Praxis. Weinheim: Beltz Juventa, S. 179-193.

Bauer, Robin (2020b): Sexualpädagogik der Vielfalt in der Kinder- und Jugendarbeit. In: Meyer, Thomas/Patjens, Rainer (Hg.): Studienbuch Kinder- und Jugendarbeit. Wiesbaden: Springer, S. 565-592.

Baumgartner, Renate (2020): >Viele Lieben - Polyamorie als Identität und Praxis. In: Timmermanns, Stefan/Böhm, Maika (Hg.): Sexuelle und geschlechtliche Vielfalt. Interdisziplinäre Perspektiven aus Wissenschaft und Praxis. Weinheim: Beltz Juventa, S. 194-210. 
Borkenhagen, Ada/Brähler, Elmar (2016): Wer liebt, der straft? SM- und BDSM-Erotik zwischen Pathologisierung und Anerkennung. Gießen: Psychosozial.

Bundesministerium für Gesundheit (2020): »Es ist ok, so wie du bist.« - Therapien zur »Heilung« von Homosexualität sollen künftig verboten werden. Online unter: https://www.bundesgesundheitsministerium.de/konversio nstherapienverbot.html [Zugriff: 14.07.2020].

Busche, Mart/Maikowski, Laura/Pohlkamp, Ines/Wesemüller, Ellen (2010): Feministische Mädchenarbeit weiterdenken. Zur Aktualität einer bildungspolitischen Praxis. Bielefeld: transcript.

BzgA (2011): Standards für die Sexualaufklärung in Europa. Rahmenkonzept für politische Entscheidungsträger, Bildungseinrichtungen, Gesundheitsbehörden, Expertinnen und Experten. Köln: BzgA. Online unter: https://w ww.bzga-whocc.de/publikationen/standards-sexualaufklaerung [Zugriff: 13.07.2020].

Costello, Cary Gabriel (2011): The Phalloclitoris. Anatomy and Ideology. Online unter: https://intersexroadshow.blogspot.com/2011/01/phalloclitoris -anatomy-and-ideology.html [Zugriff: 13.07.2020].

Dawson, Juno (2020): How To Be Gay. Alles über Coming-out, Sex, Gender und Liebe. Frankfurt a.M.: Fischer.

Debus, Katharina (2012): Und die Mädchen? Modernisierungen von Weiblichkeitsanforderungen. In: Dissens e.V./Debus, Katharina/Könnecke, Bernard/Schwerma, Klaus/Stuve, Olaf: Geschlechterreflektierte Arbeit mit Jungen an der Schule. Berlin: Dissens, S. 103-124. Online unter: http://w ww.jungenarbeit-und-schule.de/material/abschlusspublikation [Zugriff: 15.07.2020].

Debus, Katharina (2015): Du Mädchen! Funktionalität von Sexismus, Postund Antifeminismus als Ausgangspunkt pädagogischen Handelns. In: Hechler, Andreas/Stuve, Olaf (Hg.): Geschlechterreflektierte Pädagogik gegen Rechts. Opladen: Verlag Barbara Budrich, S. 79-99. Online unter: https://www.oapen.org/search?identifier=1004470 [Zugriff: 15.07.2020].

Debus, Katharina (2017): Nicht-diskriminierende Sexualpädagogik. In: Scherr, Albert/El-Mafaalani, Aladin/Yüksel, Gökçen (Hg.): Handbuch Diskriminierung. Wiesbaden: Springer VS, S. 811-833.

Debus, Katharina (2018a): Diskriminierungskritische Sensibilisierung und Förderung von Zivilcourage in heterogenen Lerngruppen. In: Dies./Laumann, Vivien: Pädagogik geschlechtlicher, amouröser und sexueller Vielfalt. Zwischen Sensibilisierung und Empowerment. Berlin: Dissens - In- 
stitut für Bildung und Forschung, S. 131-144. Online unter: https://interve ntionen.dissens.de/materialien/handreichung [Zugriff: 13.07.2020].

Debus, Katharina (2018b): Was heißt das für die Praxis? Konzeptionelle und didaktische Zugänge zu geschlechtlicher, amouröser und sexueller Vielfalt in der Pädagogik. In: Dies./Laumann, Vivien: Pädagogik geschlechtlicher, amouröser und sexueller Vielfalt. Zwischen Sensibilisierung und Empowerment. Berlin: Dissens - Institut für Bildung und Forschung, S. 87-120. Online unter: https://interventionen.dissens.de/materialien/h andreichung [Zugriff: 13.07.2020].

Debus, Katharina (2021a): Was geht? Das Heft über Geschlechter, Liebe und Grenzen. Bonn: bpb. Online unter: https://www.bpb.de/shop/lernen/was -geht [Zugriff: 10.03.2021].

Debus, Katharina (2021b): Bauchfrei und Kopftuch. Eingriffe in die körperliche Autonomie von Mädchen. Online unter: https:/www.ufuq.de/bauch frei-und-kopftuch-eingriffe-in-die-koerperliche-autonomie-von-maedc hen [Zugriff: 25.06.2021].

Debus, Katharina (i.E.): BDSM und Sexualpädagogik. In: Laimbauer, Viktoria/Scheibelhofer, Paul: Sexualität und Pädagogik. Teil 1: Konzepte \& Debatten. Innsbruck: Studienverlag.

Debus, Katharina/Laumann, Vivien (2018a): LSB-was? Geschlechtliche, amouröse und sexuelle Vielfalt - Einführung und Spannungsfelder. In: Dies.: Pädagogik geschlechtlicher, amouröser und sexueller Vielfalt. Zwischen Sensibilisierung und Empowerment. Berlin: Dissens - Institut für Bildung und Forschung, S. 12-70. Online unter: https://interventionen.disse ns.de/materialien/handreichung [Zugriff: 13.07.2020].

Debus, Katharina/Laumann, Vivien (2018b): Pädagogik geschlechtlicher, amouröser und sexueller Vielfalt. Zwischen Sensibilisierung und Empowerment. Berlin: Dissens - Institut für Bildung und Forschung. Online unter: https://interventionen.dissens.de/materialien/handreichung [Zugriff: 13.07 .2020$]$.

Debus, Katharina/Laumann, Vivien (2020): Glossar zu Begriffen geschlechtlicher und sexueller Vielfalt. Online unter: https://interventionen.dissens. de/materialien/glossar [Zugriff: 13.07.2020].

Eisner, Shiri (2013): Bi. Notes for a bisexual revolution. Berkeley, CA: Seal Press.

Enders, Ursula (2012): Grenzen achten. Schutz vor sexuellem Missbrauch in Institutionen. Köln: Kiepenheuer \& Witsch. 
Frederick, David A./John, H. Kate St/Garcia, Justin R./Lloyd, Elisabeth A. (2018): Differences in Orgasm Frequency Among Gay, Lesbian, Bisexual, and Heterosexual Men and Women in a U.S. National Sample. Archives of Sexual Behavior, 47/1, S. 273-288. Online unter: https://doi.org/10.1007/s1 0508-017-0939-z.

Geldermann, Milan/Hamm, Jonas/Hoenes, Josch/Mittelstädt, MaikeSophie/Wörmann, Simo (2018): Trans*-Sexualität. Informationen $\mathrm{zu}$ Körper, Sexualität und Beziehung für junge Trans*menschen. Bremen: Trans Recht. Online unter: https://trans-recht.de/2017/10/22/trans-sexua litaet-informationen-zu-koerper-sexualitaet-und-beziehung-fuer-junge -trans-menschen [Zugriff: 15.07.2020].

Hartmann, Jutta (2018): Theoretisch fundiert handeln! Einführung in die queere genderreflektierte Pädagogik. In: Spahn, Annika/Wedl, Juliette (Hg.): Schule lehrt/lernt Vielfalt. Praxisorientiertes Basiswissen und Tipps für Homo-, Bi-, Trans- und Inter*freundlichkeit in der Schule. Göttingen: Edition Waldschlösschen, S. 44-51. Online unter: http://akzeptanz-fuer-v ielfalt.de/home.html [Zugriff: 14.07.2020].

Hechler, Andreas (2012): Intergeschlechtlichkeit als Thema geschlechterreflektierender Bildung. In: Dissens e.V./Debus, Katharina/Könnecke, Bernard/Schwerma, Klaus/Stuve, Olaf: Geschlechterreflektierte Arbeit mit Jungen an der Schule. Berlin: Dissens, S. 125-136. Online unter: http://w ww.jungenarbeit-und-schule.de/material/abschlusspublikation [Zugriff: 15.07.2020].

Hechler, Andreas (2016): „Was ist es denn?« Intergeschlechtlichkeit in Bildung, Pädagogik und Sozialer Arbeit. In: Katzer, Michaela/Voß, Heinz-Jürgen (Hg.): Geschlechtliche, sexuelle und reproduktive Selbstbestimmung. Gießen: Psychosozial, S.161-186. Online unter: https:// www.psychosozial-verlag.de/catalog/dispatch-free.php?id=6799 [Zugriff 15.07.2020].

Jugendnetzwerk Lambda Bayern (2015): Akzeptrans*. Arbeitshilfe für den Umgang mit transsexuellen Schüler_innen. München: Jugendnetzwerk Lambda Bayern. Online unter: https://www.lambda-bayern.de/lambda/d ownloads [Zugriff: 15.07.2020].

Kalpaka, Annita (2003): Stolpersteine und Edelsteine in der interkulturellen und antirassistischen Bildungsarbeit. In: Stender, Wolfram/Rohde, Georg/Weber, Thomas (Hg.): Interkulturelle und antirassistische Bildungsarbeit. Projekterfahrungen und theoretische Beiträge. Frankfurt a.M.: Brandes \& Apsel, S. 56-79. 
Kleiner, Bettina (2020): Lebenslagen von lesbischen, schwulen, bisexuellen, trans* und inter*"geschlechtlichen sowie genderqueeren (Kindern und) Jugendlichen. In: Timmermanns, Stefan/Böhm, Maika (Hg.): Sexuelle und geschlechtliche Vielfalt. Interdisziplinäre Perspektiven aus Wissenschaft und Praxis. Weinheim: Beltz Juventa, S. 40-54.

Klemm, Sarah (2018): Empowerment von queeren Jugendlichen in heterogenen Lerngruppen. In: Debus, Katharina/Laumann, Vivien: Pädagogik geschlechtlicher, amouröser und sexueller Vielfalt. Zwischen Sensibilisierung und Empowerment. Berlin: Dissens - Institut für Bildung und Forschung, S. 145-155. Online unter: https://interventionen.dissens.de/mater ialien/handreichung [Zugriff: 13.07.2020].

Könnecke, Bernard/Rieske, Thomas Viola (2017): Grundlagen sexualpädagogischen Arbeitens mit männlichen Kindern und Jugendlichen. In: Stiftung Männergesundheit (Hg.): Sexualität von Männern. Dritter Deutscher Männergesundheitsbericht. Gießen: Psychosozial, S. 147-157. Online unter: https://www.psychosozial-verlag.de/2683 [Zugriff: 13.07.2020].

Laumann, Vivien (2018): Queere Un_Sichtbarkeiten. LSBTIQAP+ in heterogenen pädagogischen Settings. In: Debus, Katharina/Laumann, Vivien: Pädagogik geschlechtlicher, amouröser und sexueller Vielfalt. Zwischen Sensibilisierung und Empowerment. Berlin: Dissens - Institut für Bildung und Forschung, S. 71-86. Online unter: https://interventionen.dissens.de /materialien/handreichung [Zugriff: 13.07.2020].

Liebeck, Anne (2014): Asexualitätsinklusive Sexualaufklärung. Online unter: http://asexyqueer.blogsport.de/2014/12/04/asexualitaetsinklusive-sex ualaufklaerung [Zugriff: 13.07.2020].

Méritt, Laura (2014): Frauenkörper neu gesehen. Ein illustriertes Handbuch. Berlin: Orlanda.

Nonbinary Wiki (o.J.): https://nonbinary.wiki [Zugriff: 21.07.2020].

Oldemeier, Kerstin (2018): Ambivalente Coming-Out-Erfahrungen. In: Spahn, Annika/Wedl, Juliette: Schule lehrt/lernt Vielfalt. Praxisorientiertes Basiswissen und Tipps für Homo-, Bi-, Trans- und Inter*freundlichkeit in der Schule. Göttingen: Edition Waldschlösschen, S. 70-75. Online unter: http: //akzeptanz-fuer-vielfalt.de/home.html [Zugriff: 14.07.2020].

Profus, Andrej (2016): Unsichtbares sichtbar machen. Asexualität als sexuelle Orientierung. In: Katzer, Michaela/Voß, Heinz-Jürgen (Hg.): Geschlechtliche, sexuelle und reproduktive Selbstbestimmung. Gießen: Psychosozial, S. 225-242. Online unter: https:/www.psychosozial-verlag.de/catalog/dis patch-free.php?id=6799 [Zugriff: 15.07 .2020 ]. 
Rieske, Thomas Viola (2016): Junge $\neq$ Opfer? Zur (These der) Verleugnung männlicher Betroffenheit von sexualisierter Gewalt im pädagogischen Feld. In: Mahs, Claudia/Rendtorff, Barbara/Rieske, Thomas Viola (Hg.): Erziehung - Gewalt - Sexualität. Zum Verhältnis von Geschlecht und Gewalt in Erziehung und Bildung. Opladen: Verlag Barbara Budrich, S. 79-94.

Rohwer, Kirstin (2014): Die Vielfalt der Liebesbeziehungskonzepte. Online unter: https://metakiki.net/relationship-concepts-cartoon [Zugriff: 15.07.2020].

Sauer, Arn/Meyer, Erik (2016): Wie ein grünes Schaf in einer weißen Herde. Lebenssituationen und Bedarfe von jungen Trans*-Menschen in Deutschland. Berlin: BVT*. Online unter: https://www.bundesverband-trans.de/ portfolio-item/wie-ein-gruenes-schaf [Zugriff: 15.07.2020].

Schlingmann, Thomas/Wittenzellner, Ulla/Könnecke, Bernard/Wojahn, Marcus/Sieber, Anke (2016): Sexualisierte Gewalt. Männliche* Betroffene unterstützen! Mythen, Fakten, Handlungsmöglichkeiten. Berlin: Dissens Institut für Bildung und Forschung. Online unter: https://aup.dissens.de /materialien/praxisbroschuere [Zugriff: 14.07.2020].

Selbstlaut (2020): Ganz schön intim. Sexualerziehung für 6-12 Jährige [sic!]. Unterrichtsmaterialien zum Download. Erstellt im Auftrag des Bundesministeriums für Unterricht, Kunst und Kultur. Aktualisierte Auflage. Online unter: https://selbstlaut.org/publikationen-und-materialien/unserepublikationen [Zugriff: 23.12.2020].

Sielert, Uwe (2015): Einführung in die Sexualpädagogik. Weinheim: Beltz.

Spahn, Annika (2018a): Körperliche und psychische Gesundheit von queeren Jugendlichen. In: Dies./Wedl, Juliette (Hg.): Schule lehrt/lernt Vielfalt. Praxisorientiertes Basiswissen und Tipps für Homo-, Bi-, Trans- und Inter*freundlichkeit in der Schule. Göttingen: Edition Waldschlösschen, S. 80-83. Online unter: http://akzeptanz-fuer-vielfalt.de/home.html [Zugriff: 14.07.2020].

Spahn, Annika (2018b): Sexuelle und geschlechtliche Vielfalt sind nicht behandlungsbedürftig. Konversionstherapien verhindern. In: Dies./Wedl, Juliette (Hg.): Schule lehrt/lernt Vielfalt. Praxisorientiertes Basiswissen und Tipps für Homo-, Bi-, Trans- und Inter*freundlichkeit in der Schule. Göttingen: Edition Waldschlösschen, S. 84-85. Online unter: http://akzep tanz-fuer-vielfalt.de/home.html [Zugriff: 14.07.2020].

Stuve, Olaf (2012): Homogenisierende Bilder von Jungen. Und warum sie dem pädagogischen Handeln im Weg stehen. In: Dissens e.V./Debus, Kathari- 
na/Könnecke, Bernard/Schwerma, Klaus/Stuve, Olaf: Geschlechterreflektierte Arbeit mit Jungen an der Schule. Berlin: Dissens, S. 17-26. Online unter: http://www.jungenarbeit-und-schule.de/material/abschlusspublik ation [Zugriff: 15.07.2020].

Stuve, Olaf/Debus, Katharina (2012a): Geschlechtertheoretische Anregungen für eine geschlechterreflektierte Pädagogik mit Jungen. In: Dissens e.V./Debus, Katharina/Könnecke, Bernard/Schwerma, Klaus/Stuve, Olaf: Geschlechterreflektierte Arbeit mit Jungen an der Schule. Berlin: Dissens, S. 27-42. Online unter: http://www.jungenarbeit-und-schule.de/material /abschlusspublikation [Zugriff: 15.07.2020].

Stuve, Olaf/Debus, Katharina (2012b): Männlichkeitsanforderungen. Impulse kritischer Männlichkeitstheorie für eine geschlechterreflektierende Pädagogik mit Jungen. In: Dissens e.V./Debus, Katharina/Könnecke, Bernard/Schwerma, Klaus/Stuve, Olaf: Geschlechterreflektierte Arbeit mit Jungen an der Schule. Berlin: Dissens, S. 43-60. Online unter: http://w ww.jungenarbeit-und-schule.de/material/abschlusspublikation [Zugriff: 20.11.2018].

Thörner, Daniela (2016): Safer Sex - und wie machst Du das so? praktisch. sicher. sexy. Ein Handbuch. Berlin: Daniela Thörner. Online unter: https://www.daniela-thoerner.de/projekte/safer-sex-handbuch [Zugriff: 13.07 .2020$]$.

Timmermanns, Stefan (2013): Materialien der Sexualerziehung. In: Schmidt, Renate-Berenike/Sielert, Uwe (Hg.): Handbuch Sexualpädagogik und sexuelle Bildung. Weinheim: Beltz Juventa, S. 708-716.

Veaux, Franklin (2012): Alternativen zur Monogamie. Online unter: http ://beziehungsgarten.net/blog/alternativen-zur-monogamie [Zugriff: 15.07.2020]. 



\section{Im Schwimmbad}

\section{Anna Vida}

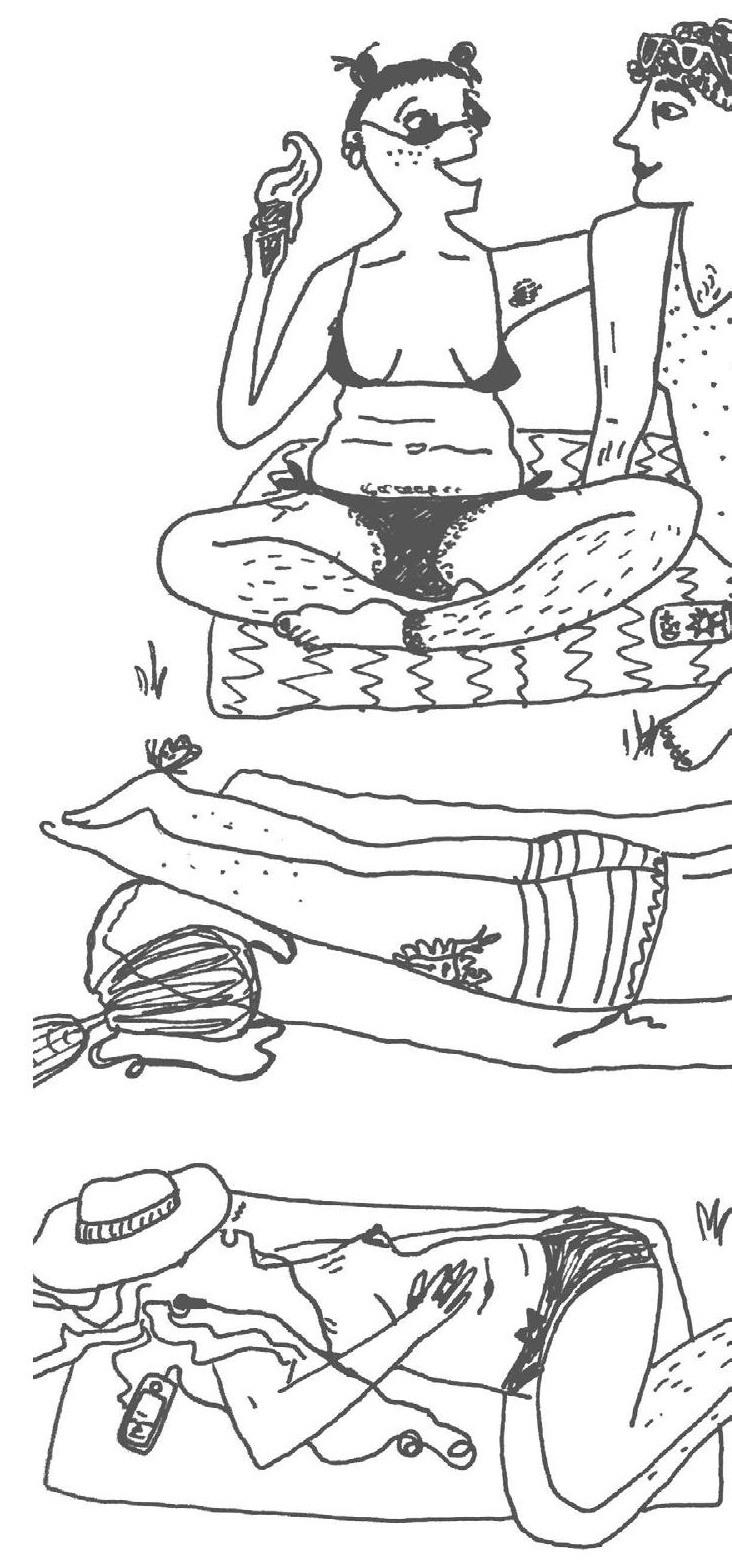






\title{
Sexuelle Übergriffe unter Kindern Eine Herausforderung für den Schulalltag
}

\author{
Ulli Freund
}

Unzweifelhaft zählen sexuelle Übergriffe unter Schulkindern zu den Herausforderungen, denen jede Lehrerin und jeder Lehrer im Schulalltag begegnet. Diese Begegnungen verlaufen aber sehr verschieden und können höchst unterschiedliche Ergebnisse respektive Gefühle zwischen Überforderung, Zufriedenheit, Erleichterung, Frustration, Hilflosigkeit, Überlegenheit, Ohnmacht bei den verschiedenen Beteiligten - der Lehrperson, dem übergriffigen Kind, dem betroffenen Kind - zur Folge haben. Ein professionell gut begleiteter Verlauf befreit das betroffene Kind aus seinem Ohnmachtsgefühl, weil es geschützt wird. Das übergriffige Kind erlebt Erleichterung, weil es die Chance bekommt, sein Verhalten zu verändern, ohne als Täter:in abgestempelt zu werden. Und die Lehrperson empfindet Zufriedenheit, weil sie durch ihren pädagogischen Umgang mit der Situation beiden Kindern gerecht geworden ist.

\section{Sexuelle Übergriffe - (k)ein neues Thema?}

Sexuelle Übergriffe unter Kindern sind kein neues Thema, aber sie finden heute eine gesellschaftliche, mediale und fachliche Aufmerksamkeit wie nie zuvor. Es entsteht der Eindruck und wird insbesondere durch die mediale Berichterstattung geschürt: >Alles wird immer schlimmer<. Aber der Eindruck trügt. Vermutlich gibt es nicht mehr Übergriffe als früher. ${ }^{1}$ Eine Ausnahme stellen die Übergriffe mit digitalem Medieneinsatz dar, wie z.B. sexualisierte

Zwar ist in Deutschland in den vergangenen Jahren in der polizeilichen Kriminalstatistik ein starker Anstieg der kindlichen Tatverdächtigen (die ohnehin erst ab 8 Jahren statistisch erfasst werden) bei Straftaten gegen die sexuelle Selbstbestimmung zu 
Formen von Cybermobbing oder missbräuchliches Sexting, also das unautorisierte Weiterleiten von erotischen Aufnahmen und dergleichen mehr. ${ }^{2}$ Das sind neue Phänomene, die neue pädagogische Antworten brauchen. Aber jenseits des Digitalen hat sich nicht viel verändert. Was sich verändert hat, ist der gesellschaftliche Diskurs zu Gewalt. Gewalterfahrungen werden inzwischen eher als solche bewertet - Erfahrungen, die noch vor wenigen Jahrzehnten nicht problematisiert, sondern als normale und hinzunehmende Erfahrung in Kindheit und Jugend angesehen wurden. ${ }^{3}$ Systematische Demütigung und Ausgrenzung einzelner Kinder durch andere wird nicht mehr als Hänseln bagatellisiert, sondern als Gewalt in Form von Mobbing bezeichnet. Der ehemalige, mit einem Augenzwinkern bedachte `Jungenstreich<, einem Mädchen den Rock hochzuheben, so dass alle ihre Unterhose sehen können, wird heute als sexueller Übergriff bewertet.

Hier hat sich in den letzten Jahren, zeitversetzt zur Enttabuisierung von sexuellem Missbrauch durch Erwachsene, viel bewegt, das angestammte Recht der Stärkeren wird immer hartnäckiger hinterfragt und kritisiert, die Kinderrechte ${ }^{4}$ entfalten Wirkung. Dem Erleben der Betroffenen gilt unsere Aufmerksamkeit, (sexuelle) Gewalt gilt nicht länger als selbstverständliche Erfahrung, als zumutbar. Mit dieser Entwicklung steigen aber auch die Zahlen der bekannt werdenden Fälle.

\section{Nicht jede sexuelle Handlung unter Kindern ist ein sexueller Übergriff!}

Wie die Begegnung einer Lehrperson mit einem sexuellen Übergriff unter ihren Schüler:innen verläuft, hängt ganz entscheidend davon $a b$, ob sie das sexuell Gewalttätige an dem Verhalten überhaupt erkennt und nicht mit einverständlichen Ausdrucksweisen kindlicher Sexualität verwechselt. Anders als bei sexuellem Missbrauch durch Erwachsene, womit ja jede sexuelle Handlung an oder vor Kindern gemeint ist und bei dem das Einverständnis von

verzeichnen, aber es ist nicht auszuschließen, dass sich das Anzeigeverhalten entsprechend der erhöhten Sensibilität verändert hat.

2 Vgl. dazu das Portal wissen-hilft-schuetzen.de [Zugriff: 15.06.2020].

3 Das gilt auch für den nicht-sexuellen Bereich.

$4 \mathrm{Zu}$ Kinderrechten in pädagogischen Beziehungen vgl. Prengl/Winklhofer (2014; 2014a); Krappmann/Petry (2016). 
Kindern keine Rolle spielt, muss man bei sexuellen Handlungen unter Kindern sehr genau unterscheiden: Sind sich die Kinder einig oder handelt es sich um einen sexuellen Übergriff? Wird ein Machtgefälle ausgenutzt?

Bei sexuellen Aktivitäten von Schulkindern ${ }^{5}$ kommt es besonders häufig zu Fehlinterpretationen im Sinne von sexuellen Übergriffen. Ihnen werden im Gegensatz zu Kindergartenkindern, denen Entdeckungs-Lust, Interesse an sexuellem Wissen und Freude an sinnlichen Erfahrungen im Rahmen von sogenannten Doktorspielen zugestanden werden, und anders als bei Jugendlichen, deren erste sexuellen Kontaktaufnahmen akzeptiert werden, oft keine legitimen Ausdrucksweisen von Sexualität zugetraut. Aber auch Schulkinder sind sexuell neugierig, zwar nicht mehr so spontan und unbefangen wie junge Kinder, aber auch noch nicht vergleichbar mit dem sexuellen Begehren von Jugendlichen und Erwachsenen. Im Grundschulalter finden sexuelle Aktivitäten eher im Verborgenen statt, denn Schamgefühle sind schon stärker entwickelt. Um im Sinne des Kinderschutzes zu agieren, ist es aber nicht erforderlich, exakte Kenntnisse dazu zu haben, welche Verhaltensweisen in welchem Alter entwicklungsbedingt bei (den meisten) Kindern auftreten. Erforderlich ist es, zu erkennen, wo ein Kind ein anderes schädigt. Nur bei Handlungen, die Ausdrucksweise erwachsener Sexualität sind, muss grundsätzlich von Übergriffen ausgegangen werden, denn Sex im Sinne von Geschlechtsverkehr und ähnlichen Handlungen gehört nicht zur kindlichen Sexualität. Bei dieser Form der Sexualität werden Entwicklungsschritte ausgelassen bzw. vorweggenommen und Erfahrungen gemacht, die die kindliche Psyche schlicht überfordern. Kinder, die Sex praktizieren, sind nicht etwa ,frühreif^. Ganz im Gegenteil: Sie tun Dinge, für die sie nicht reif sind. Selbst wenn es den Anschein hat, dass die beteiligten Kinder einverstanden sind, ändert sich nichts an der Bewertung als Übergriff. Der Eindruck der Freiwilligkeit entsteht häufig bei Kindern, die Opfer sexualisierter Gewalt durch Erwachsene wurden und sich von Kindern mit ähnlichen Belastungen angezogen fühlen, um mit diesen die sexuelle Gewalterfahrungen im Sinne einer Reinszenierung weiterzugeben bzw. wieder zu erleben.

Die folgenden Beispiele können verdeutlichen, worauf es bei der Abgrenzung von sexuellen Aktivitäten und Übergriffen ankommt: 
1. Wenn zwei Schüler auf Klassenfahrt im gemeinsamen Zimmer die Gelegenheit nutzen, unter der Bettdecke zu vergleichen, wie ihre Penisse aussehen, ist das eine sexuelle Aktivität, die so niemandem schadet.

2. Wenn ein elfähriger Kapitän der Fußballpausenmannschaft eine Mitschülerin, die mitkicken will, vor die Wahl stellt, ihm ihre Brust zu zeigen oder eben nicht mitzuspielen, liegt ein sexueller Übergriff vor. Er nutzt seine Machtposition, um ein sexuelles Interesse umzusetzen, so dass ein Übergriff vorliegt. Dass sie schließlich nachgibt, ändert nichts daran, denn ihre Freiwilligkeit ist nicht authentisch, sondern erpresst.

3. Wenn eine Viertklässlerin mit geistiger Behinderung von einer Mitschülerin, die als ihr Buddy fungiert, in der Pause auf der Schultoilette gezwungen wird, an ihrer Vulva zu lecken, ist das ein intensiver sexueller Übergriff. Die Mitschülerin nutzt ihre Position als vorgebliche Beschützerin und zugleich die Unterlegenheit der anderen aus und sie verlangt eine sexuelle Handlung, die keine Ausdrucksweise von kindlicher, sondern von erwachsener Sexualität ist.

4. Wenn ein 12-Jähriger beim Auftritt seiner Band beim Schulfest vor Aufregung an seinem Penis spielt, ist das eine autoerotische sexuelle Aktivität, jedenfalls solange er es nicht gezielt tut, um andere damit zu beschämen. Dass dieses Verhalten unpassend ist, macht es noch nicht zum Übergriff. Gut wäre es, dem Schüler einen diskreten Hinweis zu geben, denn auch solche Aktivitäten dürfen und müssen nicht immer geduldet werden.

5. Wird ein Schüler von anderen Schülern gezwungen, vor ihnen zu masturbieren, damit sie ein entsprechendes Foto in den Klassenchat schicken können, liegt ein intensiver sexueller Übergriff vor. Die sexuelle Handlung fand unter Zwang statt. Dass den Mitschülern das gelungen ist, bedeutet für sie ein Machterleben, das sich dadurch steigert, dass sie den Betroffenen durch die Verbreitung des Bildes noch weiter demütigen können.

Die Fallbeispiele illustrieren nicht nur den Unterschied zwischen sexuellen Aktivitäten und Übergriffen, sondern verdeutlichen, dass es im Grunde zwei Kategorien von sexuellen Übergriffen gibt, die sich hinsichtlich ihrer zugrundeliegenden Motivation unterscheiden: solche, die zwar ein sexuelles Motiv haben, aber mit Macht und unter Überwindung der Unfreiwilligkeit durchgesetzt werden, wie das Beispiel 2 zeigt. Beispiel 5 hingegen steht für Übergriffe, die zwar sexuelle Handlungen zum Gegenstand haben, aber das dahinterlie- 
gende Motiv ist nicht sexuelles Interesse, sondern das Ziel Macht zu erleben, indem andere abgewertet werden.

\section{Verbale sexuelle Übergriffe}

Wenn sich Schüler:innen mit Begriffen wie »Wichser«, »Bitch«, »Schwuchtel« oder »Hurensohn« beschimpfen, gehen die Meinungen in Schulkollegien meistens auseinander. Manche Lehrer:innen reagieren empört, andere halten es für eine Frage der Zeiten, die sich eben ändern und mit denen man mitgehen muss. Wieder andere vermuten, »die wissen doch gar nicht, was sie da sagen«. Sicher ist: Diese sexuellen Begriffe dienen der Abwertung anderer, Sexualität bekommt eine negative Bedeutung und wird benutzt, um andere zu demütigen. Der Einwand, die Kinder wüssten ja gar nicht, was sie da sagen, geht ins Leere, denn entscheidend ist nicht, ob Zehn- oder Elfährige die genaue Bedeutung dieser Begriffe kennen. Entscheidend ist, dass sie bereits gelernt haben, dass man mit Beleidigungen, die auf die Sexualität zielen, Menschen besonders verletzen kann. Das ist das Rüstzeug, die Mechanik der sexuellen Gewalt, die früh gelernt wird, wenn sie keine Korrektur erfährt. Daraus ergibt sich ein sexualpädagogischer und gewaltpräventiver Auftrag, der sehr niederschwellig anzusetzen ist und nicht erst greift, wenn massive Formen sexueller Übergriffe unter Kindern stattfinden.

\section{Umgang mit Übergriffen: keine Frage der Einstellung}

Bei sexuellen Aktivitäten unter Kindern (vgl. Beispiel 1 und 4) haben Lehrer:innen Entscheidungsspielräume, wie sie damit umgehen wollen, welche Handlungen sie tolerieren und welche sie unterbinden. Sie können sich dabei an ihren persönlichen Einstellungen orientieren, optimal ist jedoch ein sexualpädagogisches Konzept der Schule, das Handlungssicherheit gibt. Es befreit einzelne Lehrer:innen aus der oft verunsichernden Situation, spontan und individuell reagieren zu müssen. Der Teamkonsens, der im sexualpädagogischen Konzept zum Ausdruck kommt, stärkt den einzelnen Lehrer:innen den Rücken.

Ob und wie man mit sexuellen Übergriffen umgeht, ist keine Frage der persönlichen Einstellung, sondern eine Frage des Kinderschutzes. Kinder brauchen den Schutz ihrer Lehrkräfte vor sexuellen Übergriffen durch Mit- 
schüler:innen, denn meist können sie sich nicht allein wehren. Auch ein Nein oder Stopp ist in vielen Fällen kein zuverlässiger Schutz, schließlich finden Übergriffe in aller Regel in einem Machtverhältnis statt, in dem sich der oder die Stärkere leichter über Widerstand hinwegsetzen kann. Von einem Machtgefälle ist immer dann auszugehen, wenn sich die Beteiligten nicht auf Augenhöhe begegnen und Einigkeit herstellen können. Altersunterschiede, unterschiedliche Bedeutung und Beliebtheit in Gruppen und manchmal auch Machtunterschiede aufgrund des Geschlechts zählen zu den häufigsten Machtgefällen. Beim Thema Geschlecht ist jedoch zu beachten, dass Jungen zwar deutlich häufiger übergriffig sind als Mädchen, bei den betroffenen Kindern aber Jungen etwa die Hälfte ausmachen.

In vielen Fällen ist pädagogisch angemessenes Reagieren, wie es in den folgenden Standards skizziert wird, ausreichend. Sexuelle Übergriffe jedoch, die massiv sind, wiederholt stattfinden oder mit Drohungen verbunden sind und die sich durch pädagogische Interventionen allein nicht stoppen lassen, können ein Hinweis auf eine Kindeswohlgefährdung des übergriffigen Kindes sein und brauchen entsprechende zusätzliche Reaktionen. In Deutschland haben schulische Beschäftigte den Anspruch nach $\$ 8$ b Achtes Buch des Sozialgesetzbuches, sich durch eine insoweit erfahrene Fachkraft bei der Einschätzung beraten $\mathrm{zu}$ lassen, ob eine Kindeswohlgefährdung vorliegt, und sind in Fällen, in denen eine Kindeswohlgefährdung bejaht wird, nach den Schulgesetzen des jeweiligen Bundeslandes berechtigt oder sogar verpflichtet, das Jugendamt einzuschalten. ${ }^{6}$

\section{Prioritätensetzung bei der pädagogischen Reaktion}

Das betroffene Kind sollte die erste und ungeteilte Aufmerksamkeit erhalten, denn die Folgen für betroffene Kinder sind umso geringer, je besser und je früher sie Unterstützung erfahren und dadurch die Ohnmachtserfahrung beendet wird. In der Praxis erhalten übergriffige Kinder meist diese erste Aufmerksamkeit, werden zur Rede gestellt oder nach den Ursachen für ihr Verhalten gefragt. Aus fachlichen Gründen sollte man diesen Impuls kontrollieren und dem betroffenen Kind Priorität einräumen. Die Suche nach den Ursachen bleibt oft ohne Ergebnis, bindet aber viel Energie, die der Zuwendung zum betroffenen Kind fehlt. Die Frage nach den Gründen ist deshalb

6 Für Österreich vgl. dazu den Leitfaden des Bundesministeriums für Bildung (2016). 
zurückzustellen, denn selbst wenn man die Hintergründe des übergriffigen Verhaltens kennt, hat man noch nichts für den Schutz des betroffenen Kindes getan.

Von Sechs-Augen-Gesprächen, um mit beiden Beteiligten den Sachverhalt aufzuklären und eine Verständigung zu erreichen, ist dringend abzuraten. Dieses Vorgehen ist eine unnötige Belastung für das betroffene Kind, denn die Übergriffs-Dynamik setzt sich in solchen Gesprächen fort: Aus seiner Sicht ist das übergriffige Kind wieder mächtiger, weil es die gleiche Chance hat, dass man ihm glaubt. Doch die Ausgangslage der Kinder ist nicht gleich: Die Erfahrung zeigt, dass Kinder sich sexuelle Übergriffe nicht ausdenken. Das Thema ist mit zu viel Peinlichkeit besetzt, als dass es sich dafür anbieten würde, sich `damit interessant zu machen fast immer die Chance des gemeinsamen Gesprächs, um sich herauszureden und die Situation anders darzustellen - in ihrer Situation ein durchaus verständliches Verhalten, um der Verantwortung für ihr Handeln zu entgehen. Letztlich erschweren solche Gespräche den übergriffigen Kindern, ihr Fehlverhalten einzusehen. Gemeinsame Gespräche machen möglicherweise zu einem späteren Zeitpunkt Sinn, wenn sie einer Entschuldigung oder der Wiederannäherung der beteiligten Kinder dienen.

\section{Wie das Gespräch mit dem betroffenen Kind gelingt}

Das betroffene Kind soll in dem Gespräch erleben, dass die Lehrperson mit ihrer Macht auf seiner Seite steht, weil so das Gefühl der Unterlegenheit beendet wird. Die bei Konflikten weit verbreitete »Dazu gehören immer zwei!« -Haltung ist bei sexuellen Übergriffen nicht sinnvoll, weil es hier nicht um gleich starke Kontrahent:innen mit unterschiedlichen Interessen geht. Schüler:innen, die einen sexuellen Übergriff erlebt haben, brauchen Trost, Zuwendung und das erleichternde Gefühl, dass ihnen geglaubt wird. Ziel ist zu vermitteln, dass sich das andere Kind falsch verhalten hat und das betroffene Kind keinerlei (Mit-)Schuld trifft - selbst wenn es sich nicht sehr wehrhaft gezeigt hat. Die Lehrperson nimmt dem betroffenen Kind die Verantwortung für das weitere Handeln $a b$ und macht deutlich, dass sie sich darum kümmern wird und dass sich der Übergriff nicht wiederholen darf. Partizipation spielt hier ausnahmsweise nur eine untergeordnete Rolle. Es wäre eine unzulässige Verantwortungsverschiebung, wenn das betroffene Kind gefragt würde, was zu seinem Schutz getan werden soll. Das zu wissen, das Kind darüber zu in- 
formieren und entsprechend zu handeln, ist allein Aufgabe und Zuständigkeit der Lehrer:innen, die sich für das weitere Vorgehen auch durch das Kollegium oder die Schulleitung unterstützen lassen können.

\section{Dem übergriffigen Kind gerecht werden}

Im Anschluss daran spricht die Lehrkraft mit dem übergriffigen Kind, konfrontiert es mit dem Vorfall und verzichtet auf eine Schilderung aus seiner Sicht. Denn in aller Regel sind solche Schilderungen nicht hilfreich, sie dienen meist der Rechtfertigung und verzögern den Prozess der Einsicht. Das übergriffige Verhalten muss bewertet, als sexueller Übergriff bezeichnet und für die Zukunft strikt verboten werden. Das übergriffige Kind darf sich aber nicht als Person abgelehnt fühlen, sondern soll merken, dass sein Verhalten gemeint ist. Damit es sein Verhalten ändert, braucht es Unterstützung und ein Gegenüber, das keinen Zweifel an seiner Entschiedenheit aufkommen lässt. Eine klare und entschiedene Intervention ist letztlich nicht zum Nachteil des übergriffigen Jungens oder Mädchens, sondern gibt die Chance auf Verhaltensänderung. Damit sich das übergriffige Kind wegen der klaren Parteilichkeit für das betroffene Kind nicht ungerecht behandelt fühlt, braucht es die Zusicherung, dass die Parteilichkeit auch ihm gelten wird, wenn es in die Situation kommt Hilfe zu brauchen.

\section{Maßnahmen: mehr als reden}

Wenn der Übergriff nicht intensiv war und die Lehrperson den Eindruck hat, dass dieses ernste Gespräch das übergriffige Kind nachhaltig beeindruckt und es sein Fehlverhalten eingesehen hat, so dass keine weiteren Übergriffe zu befürchten sind, kann dieses Gespräch als Maßnahme genügen. Denn das Ziel, zum Schutz des betroffenen Kindes Wiederholungen zu vermeiden, wird ja erreicht.

In den meisten Fällen sind aber weitergehende Maßnahmen erforderlich. Keinesfalls darf der Eindruck entstehen, >die reden ja nur<. Wichtig ist, dass Maßnahmen nur das übergriffige Kind einschränken und nicht das betroffene. Sie werden befristet, damit sich die Verhaltensänderung lohnt. Beispielsweise könnte man im Fall 2 dem übergriffigen Schüler die Kapitänsfunktion für zwei Wochen entziehen und ihn für die erste Woche vom Pausenfuß- 
ball ganz ausschließen. Wenn er in der zweiten Woche mitspielt - ohne die Macht der Kapitänsposition - kann er zeigen, dass er die Mitspielerin fair behandelt, und nach der zweiten Woche seine angestammte Position zurückbekommen. Das Kollegium und insbesondere die Klassenleitung der beteiligten Schüler:innen werden informiert, denn zur konsequenten Umsetzung der Maßnahmen ist Kommunikation und Einigkeit im Kollegium wichtig. Es empfiehlt sich, Vorfälle und die ergriffenen Maßnahmen zu dokumentieren nicht zuletzt, um bei Wiederholungen nicht auf bloße Erinnerungen angewiesen zu sein.

\section{Eltern informieren und gewinnen}

Der fachliche Umgang mit sexuellen Übergriffen erfordert, die Eltern der beteiligten Kinder zu informieren, ihnen bei der Einordnung und Bewertung des Vorfalls zu helfen und sie für das fachliche Vorgehen der Schule zu gewinnen. Auch hier sind gemeinsame Gespräche zwischen allen Beteiligten nicht anzuraten, zu unterschiedlich sind ihre Interessen. Entsteht bei Eltern betroffener Kinder der Eindruck, dass die Situation ihres Kindes nicht ernst genommen wird, reagieren sie oft sehr emotional und empfinden die Schule schnell als Gegner. Hier ist eine professionelle, besonnene Reaktion gefragt, die sich aktiv um das Vertrauen der Eltern bemüht, indem sie deren Sorgen ernst nimmt.

Die Erfahrungen zeigen, dass Eltern übergriffiger Kinder nur dann bereit sind, an einer Lösung des Problems mitzuwirken, wenn sie nicht befürchten müssen, dass ihr Kind als Täter:in abgestempelt wird. Sonst werden Eltern ihr Kind in Schutz nehmen, indem sie sein Verhalten bagatellisieren. Es empfiehlt sich auf Begriffe wie Opfer und Täter:in zu verzichten und in Gesprächen die Wortwahl »übergriffiges« bzw. »betroffenes Kind« zu praktizieren und auf diese zu bestehen.

Für die Einbeziehung der Eltern gibt es eine Ausnahme: Besteht der Verdacht, dass das Kind zu Hause sexuelle Gewalt erfährt, sind nicht die Eltern $\mathrm{zu}$ informieren, sondern es ist eine Fachberatungsstelle einzuschalten (vgl. Miosga/Schele 2018: 89). Manchmal ist es sinnvoll, einen Elternabend zu veranstalten, um die Unruhe in der Elternschaft aufzufangen, die ein solcher Vorfall oft auslöst. Die Informationen, die innerhalb der Elternschaft und vor allem über soziale Medien geteilt werden, sind nicht immer zutreffend, stiften viel Verunsicherung und führen auch zu Eskalationen. Die Eltern sollen 
erfahren, wie die Schule mit sexuellen Übergriffen umgeht, was als Übergriff gilt, ob die Schüler:innen wissen, dass sie sich beschweren dürfen, und dergleichen mehr. Die Eltern der am Übergriff beteiligten Kinder und vor allem die Kinder selbst haben aber ein Recht auf Diskretion im Sinne des Schutzes der Persönlichkeit. Deshalb darf ein Elternabend nicht zu Diskussionen über konkrete Vorfälle genutzt werden, wohl aber zur Information über das Thema aus Anlass eines Vorfalls.

\section{Sexuelle Übergriffe - ein Thema für die Klasse}

Um in Zukunft sexuelle Übergriffe zu verhindern, sollte man auch in der Klasse darüber sprechen, was vorgefallen ist, und welche Maßnahmen für das übergriffige Kind nun gelten, und somit die Chance zur Prävention nutzen. Die unbeteiligten Kinder lernen, dass es sich lohnt, Bescheid zu sagen, sich Hilfe zu holen und dass das kein Petzen ist. Potenzielle Nachahmer:innen merken schnell, dass sie damit rechnen müssen, dass Übergriffe bekannt werden und unangenehme Konsequenzen drohen. Die Erfahrung zeigt, dass solche Gespräche in der Gruppe eine Atmosphäre entstehen lassen, die andere betroffene Kinder ermutigen kann, sich mit entsprechenden Erfahrungen anzuvertrauen. Manchmal gleicht die Situation einem Dammbruch, weil viele Kinder anfangen zu sprechen und komplexe Übergriffs-Geschehen aufdecken, in die zahlreiche Kinder über lange Zeiträume involviert waren.

Sexuelle Übergriffe unter Schüler:innen gänzlich zu verhindern, ist ein unrealistisches und die Lehrkräfte überforderndes Ziel. Schlimmstenfalls führt es sogar dazu, eher wegzusehen, weil sonst der >Erfolgく, eine Schule ohne sexuelle Übergriffe vorweisen zu können, gefährdet wäre. Die Herausforderung besteht darin, zunächst alle Wege der Prävention zu nutzen, um das Risiko zu verringern, dass es zu solchen Vorfällen kommt. Und wenn es doch geschieht, sexuelle Übergriffe als solche richtig zu erkennen, den beteiligten Schüler:innen gerecht $\mathrm{zu}$ werden und fachlich angemessen mit ihren Eltern und Mitschüler:innen umzugehen. So entsteht Orientierung für alle, die ihre präventive Wirkung entfalten kann. 


\section{Literatur}

Bundesministerium für Bildung (2016): Sexuelle Gewalt. Leitfaden für Pädagoginnen und Pädagogen. Rechtliche Situation. Online unter: https: //www.schulpsychologie.at/fileadmin/upload/psychologische_gesundhei tsfoerderung/Sexualerziehung/sexuellegewalt.pdf [Zugriff: 05.02.2020]. Freund, Ulli/Riedel-Breidenstein, Dagmar (2004/2006): Sexuelle Übergriffe unter Kindern. Handbuch zur Prävention und Intervention. Köln: Mebes \& Noack.

Krappmann, Lothar/Petry, Christian (2016): Worauf Kinder und Jugendliche ein Recht haben. Kinderrechte, Demokratie und Schule: Ein Manifest. Schwalbach/Ts: Wochenschau Verlag.

Miosga, Margit/Schele, Ursula (2018): Sexualisierte Gewalt und Schule. Was Lehrerinnen und Lehrer wissen müssen. Weinheim: Beltz.

Prengl, Annedore/Winklhofer, Ursula (2014a): Kinderrechte in pädagogischen Beziehungen. Bd. 1: Praxiszugänge. Opladen: Verlag Barbara Budrich.

Prengl, Annedore/Winklhofer, Ursula (2014b): Kinderrechte in pädagogischen Beziehungen. Bd. 2: Forschungszugänge. Opladen: Verlag Barbara Budrich. 



\section{Einvernehmlichkeit und Zustimmungsunfälle}

Orlinder Krinkel
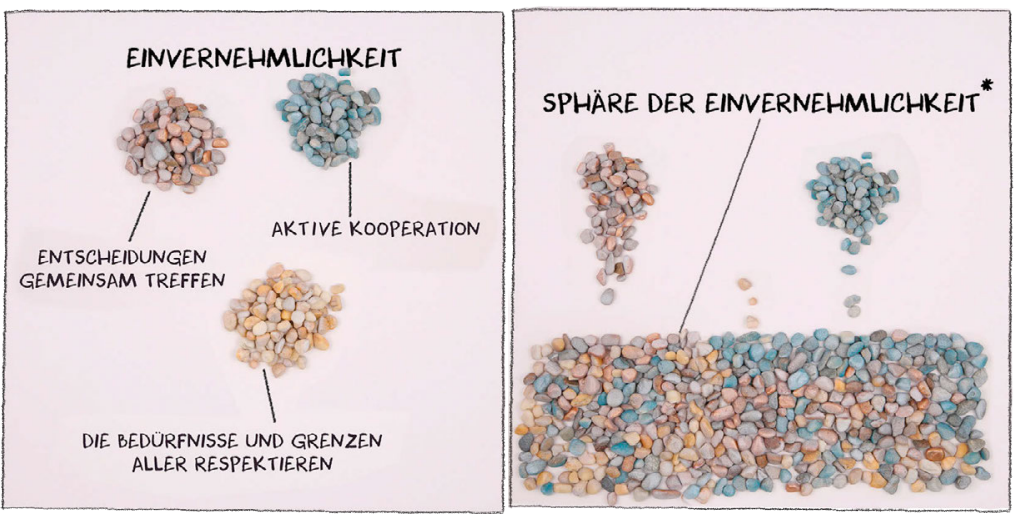

Nehmen wir an, zwei Personen wollen gemeinsam schaukeln.

Hier sind zwei, die Lust haben.

gemeinsam eine Wippe zu baven.

Manche gehen dann zusammen zum

Spielplatz, andere hangen eine Hangematte auf.
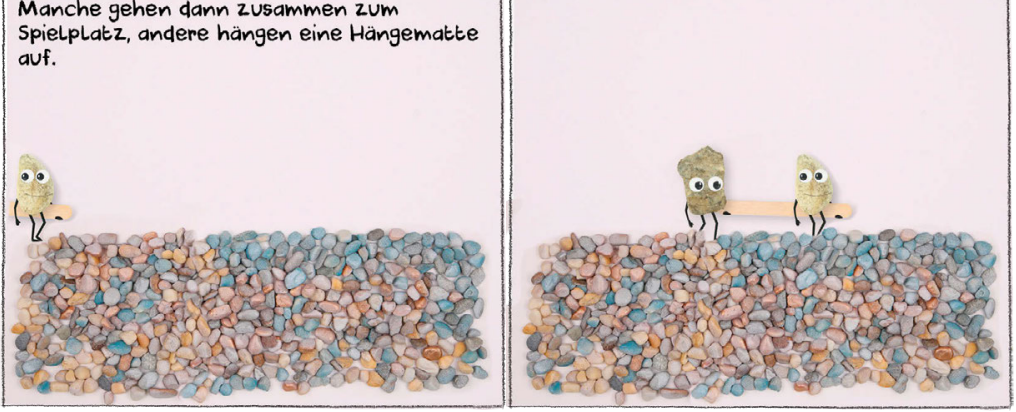

Sie einigen sich darauf, dass sie die

Bedürfnisse und Grenzen beider respektieren.
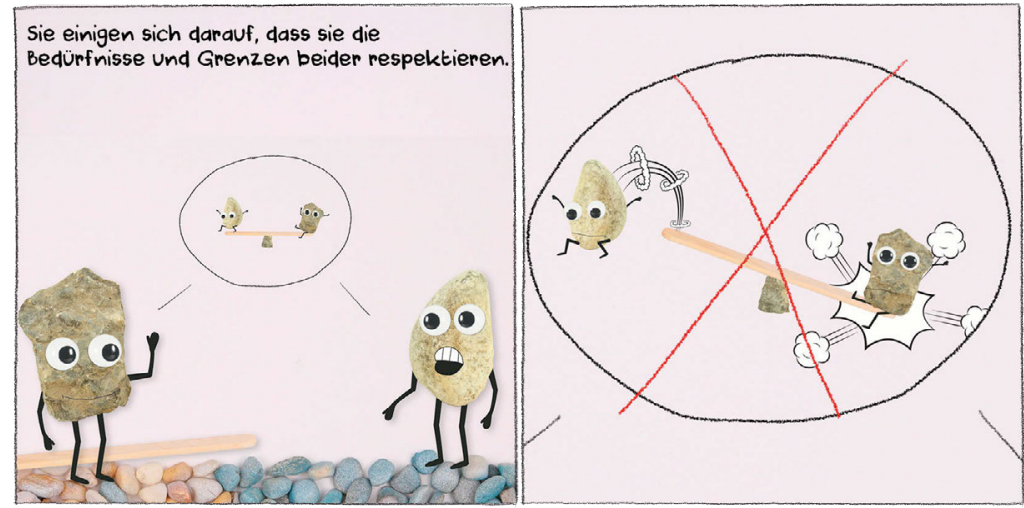
Sie kooperieren.

Es ist ihr gemeinsames Projekt.

Es gibt also zustimmung zum gemeinsamen Wippen.

$W$ as sie tun, machen sie einvernehmbich.
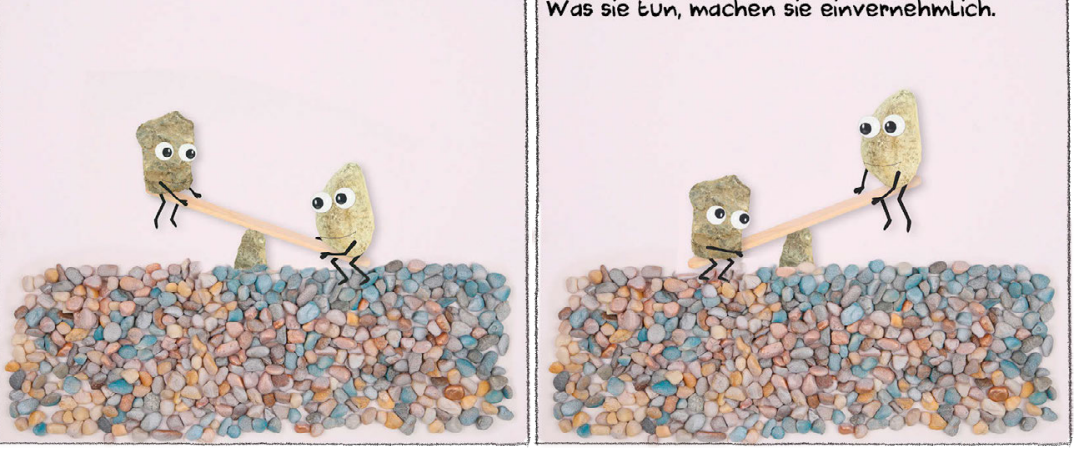

Auch wenn sie sehr achtsam miteinander umgehen, ist der Prozess nicht frei von Zustimmungsunfällen -

auch in der Sphare der Einvernehmlichkeit.

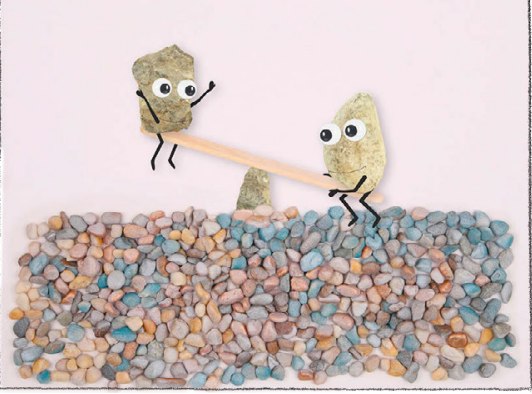

Jede situation ist einzigartig

Und beim Spielen und Experimentieren ist nicht immer vorhersehbar, was passieren wird.

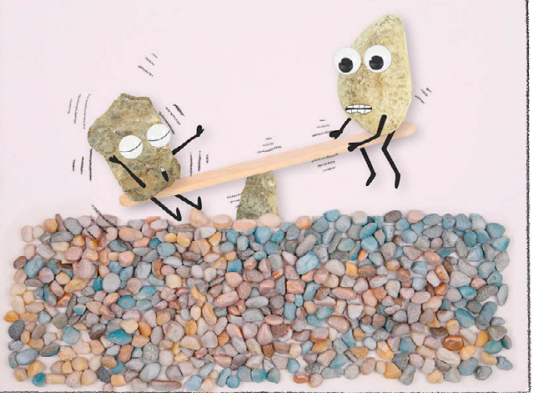

Es kann davon ausgegangen werden, dass es manchmal zu Zustimmungsunfallen kommt.
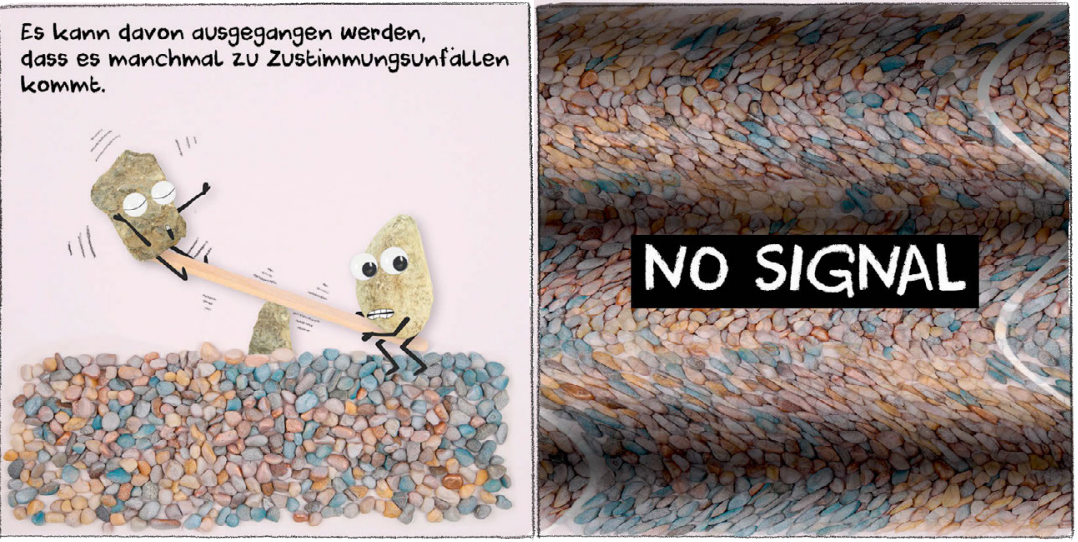

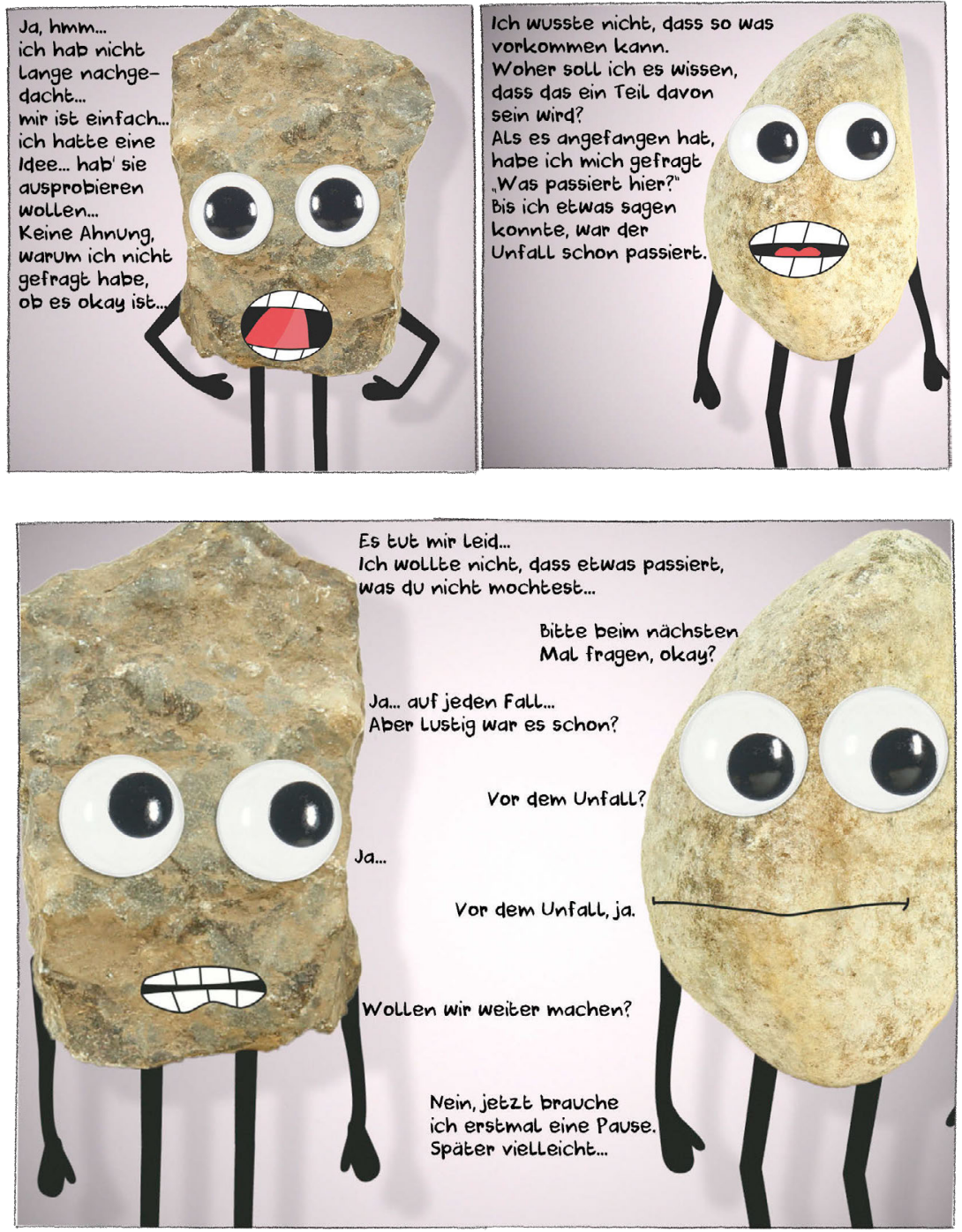

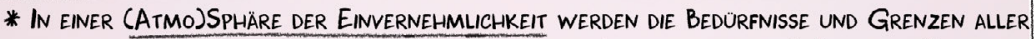
respektiert Und Zustimmungsunfälle als ANLAss zum LeRnen genutzt. Wenn eine Person NUR DIE BEFRIEDIGUNG IHRER EIGENEN WÜNSCHE DURCHSETZEN WIL, SICH NICHT MIT ANDEREN ABSPRICHT, GRENZEN NICHT RESPEKTIERT UND NICHT AUS FEHLERN LERNT, DANN HERRSCHT EINE (ATMO)SPHÄRE DER UBERGRIFFIGKEIT.

Diese ILLustrationen sind Ausschnitte aus dem Video "Wippe: Einvernehmlichkeit und Zustimmungsunfalle", das im Rahmen des Projekts Reflecting Desires unter der Mitarbeit von Maria Dalhoff konzipiert wurde. zu diesem Video und anderen sexualpadagogischen Materialien siehe: wwwimaginingdesires.at 



\section{Nähe in pädagogischen Beziehungen gestalten Von der Notwendigkeit eines integrierten Prozesses von Professionalisierungsstrategien und Organisationsentwicklung für Schulen}

Alexandra Retkowski

Nähe in pädagogischen Beziehungen ist ein hochambivalenter Aspekt pädagogischen Handelns. Allein die Thematisierung des Begriffs im Kontext des Lehrberufes kann - insbesondere durch die Verbindung zu Fragen der sexualisierten Gewalt - kontroverse Diskussionen auslösen; bei jenen, die im pädagogischen Feld tätig sind, wie auch bei Eltern und auch unter den Schüler:innen. Gleichzeitig ist Nähe ein Alltagsphänomen von pädagogischen Beziehungen in allen beruflichen Feldern der Arbeit mit Kindern und Jugendlichen im Bildungs-, Gesundheits- und Sozialsystem. Sie ist ein wichtiger Aspekt pädagogischer Arbeit, denn Erfahrungen von Nähe sind konstitutiv für Individuations- und Sozialisationsprozesse (vgl. Kowalski u.a. 2018). Auch aus Perspektive der Professions- bzw. Professionalisierungstheorie umschreiben die Begriffe Nähe und Distanz Kernanforderungen an die Gestaltung von pädagogischen Beziehungen bzw. von »Arbeitsbündnissen« (vgl. Braun u.a. 2011: 85).

Es geht - wie später noch näher ausgeführt wird - im Kern um das angemessene Verhältnis von Nähe und Distanzierungen zwischen Professionellen und Adressat:innen, also um das Ausfüllen einer formalen Berufsrolle einerseits und vertrauensvolle, affektiv besetzte und nur begrenzt steuerbare Beziehungen andererseits, wobei eine nahe Beziehung ebenso unangemessen sein kann wie eine distanzierte (vgl. ebd.). Nähe und ihr Gegenpol Distanz sind also ein Begriffspaar, das keine eindeutig bestimmbare Bedeutung hat, allerdings im Hinblick auf mögliche Grenzüberschreitungen, vor allem »in seiner sinnlich-sexuellen Leibdimension« sowie mit Blick auf Gefühle und Affekte 
von pädagogisch Tätigen kontinuierlich reflektiert und thematisiert werden muss (vgl. Dörr 2018).

Der Themenkomplex von Nähe und Distanz ist auch im Kontext der Entwicklung von organisationsbezogenen Schutzkonzepten gegen sexualisierte Gewalt (UBSKM 2013; Wolff/Schröer 2018) ein wichtiger Baustein und zwar nicht nur, weil zu viel Nähe die Grenze zu sexualisierten Grenzüberschreitungen überschreitet, sondern auch, weil gute Nähe Vertrauen, Offenbarungsprozesse ${ }^{1}$ und Enttabuisierungen in Bezug auf Sexualität ermöglicht (Christmann/Wazlawik 2018). Um solche Schutzkonzepte umzusetzen, bedarf es für schulische Professionalisierung und Organisationsentwicklung eines fundierten Konzeptes, welches für alle Beteiligten - jedoch besonders für Lehrer:innen - sowohl Orientierungswissen als auch Lern-, Thematisierungs- und Reflexionsräume gewährleistet. Im Folgenden wird zunächst auf Basis eines sprachlich überarbeiteten Interviewausschnitts aus dem berufsschulischen Kontext ein Aspekt möglicher individueller Professionalisierungsbedürfnisse und -bedarfe im Themenbereich Nähe vorgestellt. Hieraus werden Konturen für einen dualen bzw. integrierenden Prozesses von Organisationsentwicklung und personalisierten Strategien in der Personalentwicklung skizziert. $^{2}$

$1 \quad$ Der Begriff der Offenbarung wird synonym verwendet mit den Begriffen Disclosure oder Aufdeckung und bezeichnet »Prozesse des Erinnerns, Einordnens und Offenlegens von sexualisierter Cewalt durch Betroffene selbst« (Rieske u.a. 2018: 700).

Der Fortbildungsansatz wurzelt in den Forschungsprojekten der in den Jahren 20132019 vom deutschen Bundesministerium für Bildung und Forschung (BMBF) an der Universität Kassel geförderten Juniorprofessur »Professionsethik. Sexualität und Macht in Schule und Sozialer Arbeit«, die von Friederike Heinzel, Marianne LeuzingerBohleber, Elisabeth Tuider und Werner Thole beantragt wurde. Wichtiger Impulsgeber war auch das aus Wissenschaftler:innen und Praktiker:innen bestehende Netzwerk »Professioneller Umgang mit Sexualität und Macht im Sozial- und Bildungssektor« (siehe https://www.professionsethik.de [Zugriff 16.06.2020]). Hier wurden z.B. mittels kasuistischer Fallarbeit Dimensionen einer möglichst individualisierten, personenzentrierten und an den spezifischen Bedarfen einzelner pädagogischer Professioneller ausgerichteten institutionellen Reflexionskultur in pädagogischen Einrichtungen verfolgt. 


\section{Professionalisierungsbedürfnisse und -bedarfe im Kontext des Umgangs mit Nähe im Schulalltag}

Viele Lehrer:innen nehmen für sich in erster Linie eine professionelle Identität als Fachlehrer:in in Anspruch, was zur Folge hat, dass sie durch persönliche, teilweise deutlich emotionale und affektive Adressierungen seitens der Schüler:innen stark verunsichert werden können. Wenn diese persönliche Adressierung sexuelle Komponenten aufweist, ist die Verunsicherung häufig besonders groß. Auch in berufsbiografischen Interviews wird dies von Lehrer:innen thematisiert (Hess/Retkowski 2018). Deutet man diese Verunsicherung innerhalb der strukturtheoretischen Professionalisierungstheorie nach Ulrich Oevermann (1996; 2002), in der es im Lehrer:innen-Schüler:innen-Verhältnis um den Umgang mit asymmetrischen Machtrelationen und der Balancierung von rollenförmigen und diffusen Beziehungsanteilen geht, heißt dies, dass aus der Perspektive der jeweiligen Lehrperson die Asymmetrie aus der Balance geraten und es zu Symmetrisierungsprozessen in der pädagogischen Beziehung gekommen ist (Helsper/Reh 2012).

Persönliche Adressierungen mit erotisch-sexueller Komponente durch Schüler:innen sind für Sozialisationsprozesse in der Schule durchaus üblich (vgl. Schmidt 2014). Lehrer:innen sind in diesen Fällen beispielsweise mit kommunikativen und performativen Handlungen von Schüler:innen konfrontiert, die - sei es in schmeichelhafter oder aggressiver Art und Weise eine Nähe durch sexuelles Explorations- und Erprobungsverhalten generieren. Solche Verhaltensweisen können allerdings durch ihr verunsicherndes Potenzial das asymmetrische Verhältnis der Lehrkraft zu den Schüler:innen, welches für viele Lehrkräfte immer auch Sicherheit und Selbstwirksamkeit durch bessere Kontrollierbarkeit vermittelt, verschieben und zu symmetrischen, eher außerschulischen Kommunikations- und Interaktionsformaten führen. Lehrkräfte sind dann herausgefordert, mit diesem Verhalten so umzugehen, dass sie die noch nicht entwickelten Persönlichkeitsanteile von Schüler:innen im Rahmen einer »Wissens- und Normenvermittlung dienende[n] kooperative[n] Interaktion« (Oevermann 1996: 154) aufgreifen und zur Reifung bringen.

Es geht mit anderen Worten darum, die sexuell-erotische Ansprache seitens der Schüler:innen als Anlass für eine pädagogische Intervention zu betrachten, die in einer die Schüler:innen nicht beschämenden Weise ein doppeltes Ziel hat: Es geht darum, die Schüler:innen darauf aufmerksam 
zu machen, dass es sich (1.) um sexuell-erotisches Verhalten handelt, welches wahrgenommen, als legitimes Bedürfnis von Schüler:innen geachtet wird und für das man im Fall von Wissens- oder Beratungsbedarf als Ansprechpartner:in zur Verfügung steht bzw. Personen kennt, die als geeignete fachliche Ansprechpartner:innen dienen können. Und es geht (2.) darum, den Schüler:innen zu verdeutlichen, dass weder man selbst als Lehrkraft die passende Adresse für erotisch-sexuelle Äußerungen ist noch schulische Unterrichtsprozesse den adäquaten Rahmen hierfür darstellen.

Dass solche Situationen für Lehrer:innen eine erhebliche Herausforderung darstellen, illustriert der folgende Interviewausschnitt mit einer 34 Jahre alten Berufsschullehrerin:

»Ich kann nicht gut mit den Anzüglichkeiten der Jungs umgehen. In der Berufsschule habe ich Klassen mit Malern oder Mechatronikern, in denen vor allem junge Männer sind. Die werden schon sehr direkt in ihrem Ton und hauen einem sexistische Sachen um die Ohren, fragen nach der Telefonnummer oder machen anzügliche Bemerkungen über mein Aussehen. Ich werde dann schnell rot im Gesicht und einmal kam es auch vor, dass ich aus der Klasse rausgegangen bin. Die Kolleg:innen raten mir, dass ich selbst einen dummen Spruch raushaue, damit ich zeigen kann, dass mich das nicht trifft und damit ich mich daran gewöhne. Aber ich weiß, dass ich das nicht kann. $\aleph^{3}$

Auf Basis dieses Interviewausschnittes lassen sich vier Dimensionen der notwendigen Bearbeitung der schulischen Handlungsanforderung herausstellen:

1.) Professionalisierungsbedarf der Lehrerin: Die Berufsschullehrerin thematisiert hier Gefühle der Verunsicherung sowie einen Autonomie- und Kontrollverlust über die eigene pädagogische Handlungspraxis. Dieses offene Ansprechen eigener Verunsicherungen ist bereits eine wichtige professionelle Leistung, da es eine gute und hilfreiche Grundlage für die weitere Bearbeitung des Handlungsproblems im schulischen Kontext darstellt. ${ }^{4}$

Das Interview stammt aus dem Forschungsprojekt »Berufsbiographische Identitätskonstruktionen und Sexualität«, welches im Rahmen der oben genannten vom BMBF geförderten Juniorprofessur entstanden ist und von den wissenschaftlichen Mitarbeiter:innen Johanna Hess und Martin Grosse durchgeführt wurde. 
2.) Hinweise auf Nähe-Entgrenzung durch erotisch-sexuelles Verhalten von Schüler:innen: Es zeichnet sich eine gefühlte oder tatsächliche Symmetrisierung im Lehrerin-Schüler:innen-Verhältnis oder sogar die Umkehr der Macht-Asymmetrie innerhalb der pädagogischen Beziehung ab, denn die Berufsschullehrerin sieht sich einer kollektiv agierenden Schüler:innengruppe gegenüber, die sich vorwiegend aus jungen Männern zusammensetzt, von denen sie als Frau sexualisierend positioniert wird und durch die sie verbale Grenzüberschreitungen erleidet. Dieser sozialen Dynamik im Klassenzimmer fühlt sich die Lehrerin allein und zutiefst verunsichert ausgeliefert, so dass sie »einmal« nur noch die Flucht aus dem Klassenzimmer als Exit-Option habe wählen können.

3.) Hinweise auf einen ungenügenden, deprofessionalisierenden und hierarchisch organisierten Reflexionsraum: Über diese Gefühle der Verunsicherung sowie einen Autonomie- und Kontrollverlust in der eigenen Handlungspraxis habe die Berufsschullehrerin Austausch und Beratung im Kollegium gesucht. Ihr sei signalisiert worden, dass ihre Reaktion pädagogisch als deutlich ungeeignet und professionalisierungsbedürftig eingeschätzt werde. Die Empfehlungen hätten in eine Richtung gezielt, die vor allem auf die Notwendigkeit der (Wieder-)Herstellung der Asymmetrie im pädagogischen Verhältnis ausgerichtet waren, wobei ihr eine nicht rollenförmige, auf der Ebene der Peer-Group-Interaktion angesiedelte Reaktion empfohlen worden sei (»selbst einen dummen Spruch raushauen«). Implizit thematisiert die Berufsschullehrerin auch, dass ihr gegenüber eine Auslegung des Umgangs mit grenzüberschreitender Nähe in Form von Sexualisierungen kommuniziert wurde, die diese Phänomene einerseits als pädagogisch und persönlich irrelevant einschätzt (»ich zeigen kann, dass mich das nicht triffe) und andererseits als normal bewertet (»ich mich daran gewöhne«).

4.) Vereinzelung der Berufsschullehrerin: Von dem ihr vom Kolleg:innenkreis eröffneten Lösungsweg distanziert sie sich deutlich: »Aber ich weiß, dass ich das nicht kann«, wobei hier mutmaßlich auf die emotional-körperliche Ebene (z.B. Rot-Werden) abgehoben wird. Daraus ergibt sich eine problematische Vereinzelung der Lehrerin und die Selbstwahrnehmung,

fische Beobachtungsprotokolle dokumentieren (Helsper/Reh 2012). In solchen Fällen muss überlegt werden, wie durch Interventionen seitens der Kolleg:innen und/oder der Schulleitung Veränderungs- und Reflexionsprozesse initiiert werden können. 
anders als die anderen Kolleg:innen zu sein, die im Kollegium nicht produktiv bearbeitet wird.

Zusammengenommen zeigt sich ein Professionalisierungsdefizit der Berufsschullehrerin. Sie ist isoliert und verunsichert und benötigt Unterstützung bezüglich ihrer professionellen Positionierung gegenüber den Schüler:innen wie auch gegenüber den Kolleg:innen. Gleichzeitig wird deutlich, dass sich die Problemstellung nicht allein auf diese Berufsschullehrerin begrenzt, sondern auf eine problematische Auffassung im Kolleg:innenkreis verweist, die weder zu einer Professionalisierung der Kolleg:innen beiträgt noch auf ein adäquates pädagogisches Handeln gegenüber den Berufsschüler:innen hinweist.

Betrachtet man diesen Problemkomplex einmal anhand der Oevermann'schen Professionalisierungstheorie, so stellt sich die Frage, wie der Kern der pädagogischen Aufgabe, nämlich ein »Arbeitsbündnis als [...] widersprüchliche Einheit von diffusen und spezifischen Beziehungskomponenten « (Oevermann 1996: 152) durch die Lehrerin in diesem Schulkontext hergestellt werden kann. Voraussetzung für das Gelingen eines solchen Arbeitsbündnisses ist, dass auch die Lehrkräfte sich selbst komplementär in einer widersprüchlichen Einheit von diffusen und spezifischen Anteilen des Handelns wahrnehmen und dementsprechend am Arbeitsbündnis beteiligen (vgl. ebd.: 155). Demgemäß kann auf der Grundlage der Schilderung der Lehrerin festgehalten werden, dass in diesem berufsschulischen Kontext eine Vereinseitigung der diffusen Komponenten des Lehrer:innenverhaltens im Sinne einer »Verkindlichung« (ebd.: 155) vorliegt - bei der Lehrerin als eine Form regressiven Rückzugs und des Vermeidungsverhaltens und bei ihren Kolleg:innen - gemäß der Beschreibung der Lehrerin - als Form einer Nachahmung jugendlicher Dominanzkultur. Es stellt sich nun die Frage, welcher Unterstützung die Lehrerin bedarf und in welcher Verbindung dies mit komplexen Prozessen der Personalentwicklung im Kontext von Organisationsentwicklungsprozessen steht?

\section{Konsequenzen für ein integratives Konzept von Organisations- und Professionalisierungsstrategien}

Schon diese kurze Fallreflexion zeigt, wie eng die Bedarfslagen für individuelle Professionalisierungsprozesse mit komplexen Organisationsentwick- 
lungsbedarfen zusammenhängen. Daher wird im Folgenden für einen Ansatz plädiert, der die organisationale Auseinandersetzung mit formalisierten Verhaltensnormen (codes of conduct) mit einer personalisierten Personalführung verbindet (Retkowski 2016).

Für das konkrete Fallbeispiel bedeutet dies, dass es bedeutsam wäre, einerseits klare normative Setzungen schulintern zu kommunizieren: So wäre dies die für die Berufsschule deutlich gewordene Notwendigkeit, verbale sexualisierte Grenzüberschreitungen - in diesem Fall mutmaßlich seitens der Schüler:innen - nicht mehr länger zu normalisieren, sondern als Gegenstand eines kommunikativen schulischen Selbstverständigungsprozesses über Verhaltensregeln anzusehen. Daran anschließende weiterführende Fragen der schulinternen Auseinandersetzung wären etwa: Welche Normen setzt die Schule im Umgang mit Nähe und Intimität? Wie werden diese pädagogisch begründet, kommuniziert und institutionalisiert? Wie werden die Schüler:innen von Seiten der Lehrer:innen in Bezug auf Sexualität und Geschlecht angesprochen, wie wird das Verhältnis von Nähe und Distanz gestaltet? Was erwartet wer in diesem Kontext (z.B. die Eltern) und welchen Erwartungen muss wie entsprochen werden? Wie ist die Kommunikationskultur innerhalb des Kollegiums allgemein hinsichtlich Vertrauen, Vertraulichkeit und dem Ausdruck von Gefühlen?

Diese Fragen können auf unterschiedlichen Ebenen der schulischen Organisation - möglichst unter Beteiligung der Schüler:innen - bearbeitet werden, und ebenso können unterschiedliche Mittel und Formate in den Blick genommen werden, die von der Gründung schulinterner AGs zur Ausarbeitung von Richtlinien und Leitbildern über Handouts und andere schriftliche Formen der Handlungsempfehlungen bis hin zu Supervision und Fortbildungsangeboten reichen. Damit einhergehend sollten gleichzeitig die Normen der pädagogischen Zuständigkeit für Fragen von Sexualität, Emotion und Nähe sowie das normative Ziel einer diversitätsorientierten Professionskultur, welche in einem ersten Schritt unterschiedliche Umgangsweisen der Lehrer:innen ernst und als Ausgangspunkt weiterer fachlicher Reflexionen nimmt, etabliert werden. Für den geschilderten Fall bedeutet dies, dass eine komplexe Handlungsnotwendigkeit besteht: Ebenso wie die Berufsschullehrerin sich damit auseinandersetzen müsste, dass sie sich - auch mit der Begründung des Rot-Werdens - nicht ihrer pädagogischen Zuständigkeit entziehen kann, müssten bei den Kolleg:innen Reflexionsprozesse dahingehend initiiert werden, dass auch ihre Ratschläge nicht die Norm der pädagogischen Hand- 
lungspraxis erfüllen, weil sie zur Normalisierung sexuell diskriminierenden Verhaltens beitragen.

Andererseits sollte parallel $\mathrm{zu}$ diesen allgemeinen normativen Setzungen eine personalisierte Personalentwicklungsstrategie verfolgt werden. Demnach sollte der Berufsschullehrerin (wie auch den Kolleg:innen) ein individualisiertes Konzept der Unterstützung angeboten werden, welches ihr die Möglichkeit eröffnet, die individuellen Dimensionen ihrer beruflichen Handlungsblockade, die häufig berufsbiografische Ursachen haben (vgl. Hess/Retkowski 2018), in den Blick $\mathrm{zu}$ nehmen und $\mathrm{zu}$ bearbeiten. Eine solche personalisierte Personalentwicklungsstrategie könnte beispielsweise dabei ansetzen, ihr eine körperorientierte Weiterbildung zur Bearbeitung des Rot-Werdens anzubieten.

Eine andere Strategie wäre, Reflexionsräume zu schaffen, in denen sich die Lehrerin mit ihren eigenen Selbstbestimmungsrechten und -bedarfen im Verhältnis zu den pädagogischen Anforderungen hinsichtlich Nähe und Distanz auseinandersetzen kann. Dies könnte zu einem Klärungsprozess darüber führen, dass auch erwachsene und im Berufsfeld der Pädagogik tätige Menschen nicht immer in der Lage sind, die eigenen persönlichen Grenzen zu schützen, sondern hinsichtlich der (sexuellen) Selbstbestimmungsrechte vulnerabel sein können, was - wie in diesem Fall - zu veränderten Asymmetrien im pädagogischen Verhältnis führt. Zielperspektive eines solchen Reflexionsprozesses müsste sein, dass die Lehrerin nicht an ihrer Eignung als Lehrerin zweifelt, sondern Unterstützung und Beratung erhält, wie sie sich weiterhin als pädagogisch Handelnde verstehen kann und welche Bedingungen notwendig sind, damit sie auch im Fall der Unsicherheit oder gar des Verlusts der asymmetrischen Beziehungsrundlage gegenüber den Schüler:innen ihre eigene Position im Dienste der »Wissens- und Normenvermittlung dienende[n] kooperative[n] Interaktion «(Oevermann 1996: 154) mit den Schüler:innen realisieren kann. ${ }^{5}$ Ein pädagogisches Sprechen und Handeln auch in

Der bei der Berufsschullehrerin in den Worten »aber ich weiß, dass ich das nicht kann « sichtbar werdende Professionalisierungsbedarf lässt sich bei Lehrkräften auch im Kontext von Offenbarungsprozessen sexualisierter Cewalt finden und geht oftmals mit der Gefahr einer Professionalisierungsverweigerung einher (vgl. Retkowski 2018). Dies illustriert das folgende Segment aus einem berufsbiografischen Interview mit einer älteren Grundschullehrerin: „Smalltalk mit Kindern machen, um so was rauszukitzeln, können andere Leute ja unheimlich gut, aber ich denke, das liegt einfach auch an meiner Persönlichkeit, weil ich das generell irgendwie nicht kann, ich kann das mit Erwachsenen auch nicht gut.«Diese Lehrkraft spricht aus, dass sie einer fachlichen oder 
schulischen Situationen, die mit dem Gefühl eigener Unsicherheit und Ohnmacht verknüpft sind, kann gegebenenfalls durch Rollenspiele eingeübt und gefestigt werden - bedarf aber in jedem Fall der reflexiven Auseinandersetzung und der Anerkennung und Unterstützung durch Schulleitung und Kolleg:innen.

\section{Ausblick: Wer ist zuständig?}

Der vorliegende Beitrag zeigt auf Basis eines Fallbeispiels die Notwendigkeit der Integration von Organisationsentwicklungsprozessen mit einer differenzierten Personalentwicklungsstrategie auf. Dies gilt insbesondere für den Umgang mit den vielfältigen Erscheinungsformen von Nähe als einer der Herausforderungen pädagogischen Handelns, die widersprüchliche Einheit von Rollenhandeln und Handeln als ganzer Person umzusetzen. Wichtig ist, dass solche Prozesse nicht als Topdown-Prozess, sondern dialogisch gemeinsam mit den Lehrer:innen umgesetzt werden. Dies bedeutet, dass es wichtig ist, die Heterogenität der Lehrer:innen-Persönlichkeiten als Ressource zu betrachten, die nicht zuletzt für die Autonomisierungsprozesse der Schüler:innen einen wichtigen Resonanzraum darstellt. Jedoch wurde im Beitrag bis dato die Frage ausgeblendet, wer die Akteur:innen eines solchen dualen Prozesses sind. Neben engagierten und reflexionsoffenen Lehrkräften ist es wünschenswert, wenn sich dieser komplexen Aufgabe der Integration von Personal- und Organisationsentwicklung die Schulleitungen verstärkt annehmen. Blickt man in den organisationspädagogischen Diskurs um Führung, so zeigt sich bereits seit einigen Jahren eine Abkehr von eigenschaftstheoretischen Ansätzen der Führungsperson zu Gunsten eines Bedeutungszuwachses von aufgaben- und mitarbeiterorientiertem Führungsverhalten als zentrales Element, um Personalentwicklung und die Gestaltung lernförderlicher organisationaler Kontexte umzusetzen (vgl. Weber u.a. 2011). Wichtig ist, den Schulleitungen entsprechende Ressourcen - wie die Möglichkeit der Nutzung externer Fachberatungen - für diesen Schulentwicklungsweg zur Verfügung

schulkulturellen Norm nicht entsprechen und für sich selbst keinen Weg erkennen kann oder will, dies zu verändern. Auch hier bedarf es einer dualen Prozessorientierung: der klaren Kommunikation der Norm der pädagogischen Zuständigkeit, kombiniert mit diversen individualisierten und kollektiven Strategien zur Ermöglichung der Erfüllung derselben. 
zu stellen. Im besten Fall sollte allerdings eine Aufmerksamkeit für diese Verschränkungen von Professions- und Organisationsperspektive bereits in der hochschulischen Ausbildung von zukünftigen Lehrer:innen verankert werden.

\section{Literatur}

Christmann, Bernd/Wazlawik, Martin (2018): Disclousure in schulischen Settings. In: Retkowski, Alexandra/Treibel, Angelika/Tuider, Elisabeth (Hg.): Handbuch sexualisierte Gewalt und pädagogische Kontexte. Theorie, Forschung, Praxis. Weinheim und Basel: Beltz Juventa, S. 729-736.

Dörr, Margret (2018): Nähe-Distanz-Verhältnisse und sexualisierte Gewalt. In: Retkowski, Alexandra/Treibel, Angelika/Tuider, Elisabeth (Hg.): Handbuch sexualisierte Gewalt und pädagogische Kontexte. Theorie, Forschung, Praxis. Weinheim und Basel: Beltz Juventa, S. 178-187.

Helsper, Werner/Reh, Sabine (2012): Nähe, Diffusität und Asymmetrie in pädagogischen Interaktionen: Herausforderung pädagogischer Professionalität und Möglichkeitsräume sexualisierter Gewalt in der Schule. In: Thole, Werner/Baader, Maike/Helper, Werner/Keppeler, Manfred/Leuzinger-Bohleber, Marianne/Reh, Sabine/Sielert, Uwe/Thompson, Christiane (Hg.): Sexualisierte Gewalt, Macht und Pädagogik. Opladen u.a.: Verlag Budrich, S. 265-290.

Hess, Johanna/Retkowski, Alexandra (2018): Berufsbiographische Identitätskonstruktionen und Sexualität. In: Wazlawik, Martin/Voß, HansJürgen/Retkowski, Alexandra/Henningsen, Anja/Dekker, Arne (Hg.): Sexuelle Gewalt in pädagogischen Kontexten. Aktuelle Forschungen und Reflexionen. Wiesbaden: Springer VS, S. 231-247.

Kowalski, Marlene/Hildebrand, Julia/Marks, Svenja/Retkowski, Alexandra/Schäfer, Dorothee/Behnisch, Michael (2018): Pädagogische Intimität. Zur Untersuchung von Mustern der Gestaltung pädagogischer Beziehungen in unterschiedlichen Handlungsfeldern. Interdisziplinäre Fachzeitschrift für Prävention und Intervention, 21(2), S. 188-201.

Oevermann, Ulrich (1996): Theoretische Skizze einer revidierten Theorie professionellen Handelns. In: Combe, Arne/Helsper, Werner (Hg.): Pädagogische Professionalität. Frankfurt a.M.: Suhrkamp, S. 70-183.

Oevermann, Ulrich (2002): Professionalisierungsbedürftigkeit und Professionalisierbarkeit pädagogischen Handelns. In: Kraul, Margret/Marotz- 
ki, Winfried/Schweppe, Cornelia (Hg.): Biographie und Profession. Bad Heilbrunn: Klinkhardt, S. 19-63.

Retkowski, Alexandra (2016): The Crisis of Pedagogy and the Potentials of Professional Ethics. In: Brand, Cordula (Hg.): Dual-Process Theories in Moralpsychologie: Interdisciplinary Approaches to Theoretical, Empirical and Practical Considerations. Wiesbaden: Springer VS, S. 411-432.

Retkowski, Alexandra (2018): Professionelle, organisationale und schulöffentliche Selbstverständigungsprozesse im Kontext schwebender Verdachtsfälle auf sexualisierte Gewalt. In: Andresen, Sabine/Tippelt, Rainer (Hg.): Zeitschrift für Pädagogik. Sexuelle Gewalt in Kindheit und Jugend. Theoretische, empirische und konzeptionelle Erkenntnisse und Herausforderungen erziehungswissenschaftlicher Forschung. Weinheim/Basel: Beltz Juventa, S. 162-171.

Rieske, Thomas Viola/Scambor, Elli/Wittenzellner, Ulla (2018): Aufdeckungsprozesse - Dimensionen und Verläufe. In: Retkowski, Alexandra/Treibel, Angelika/Tuider, Elisabeth (Hg.): Handbuch sexualisierte Gewalt und pädagogische Kontexte. Theorie, Forschung, Praxis. Weinheim und Basel: Beltz Juventa, S. 700-708.

Schmidt, Renate-Berenike (2014): Schule als Ort sexueller Sozialisation. In: Hagedorn, Jörg (Hg): Jugend, Schule und Identität. Wiesbaden: Springer Fachmedien, S. 249-264.

UBSKM (2013): Handbuch Schutzkonzepte sexueller Missbrauch: Befragungen zum Umsetzungsstand der Empfehlungen des Runden Tisches »Sexueller Kindesmissbrauch«. Unabhängiger Beauftragter der Bundesregierung für Fragen des sexuellen Missbrauchs. Berlin.

Weber, Susanne/Göhlich, Michael/Schiersmann, Christiane (2011): Organisation und Führung - Organisationspädagogische Verhältnisbestimmungen. Wiesbaden: VS Verlag für Sozialwissenschaften.

Wolff, Mechthild/Schröer, Wolfgang (2018): Schutzkonzepte - Schutz und Stärkung persönlicher Rechte. In: Retkowski, Alexandra/Treibel, Angelika/Tuider, Elisabeth (Hg.): Handbuch sexualisierte Gewalt und pädagogische Kontexte. Theorie, Forschung, Praxis. Weinheim und Basel: Beltz Juventa, S. 589-599. 



\title{
Queer in der Schule?
}

\author{
Rosa Wiesauer
}

Das Schulumfeld ist in Bezug auf Geschlecht binär aufgebaut: Frau und Herr Professor, ${ }^{1}$ Mädchen- und Jungen-Umkleiden, geschlechtergetrennte Toiletten; die Vorstellung, dass Mädchen ruhig und besser im Schreiben sind und Buben laut, aber gut im Rechnen. Doch was geschieht, wenn man versucht an diesen rigiden Konstrukten zu rütteln und queere Lebensrealitäten in diesem Umfeld sichtbar werden?

Ich habe mich während der Zeit, in der ich diverse Schulpraktika im Lehramtsstudium absolvierte, ${ }^{2}$ als nicht-binär identifiziert. Nicht-binär bzw. non-binary bedeutet, dass sich eine Person jenseits der Geschlechtergrenzen identifiziert, also weder als Mann noch als Frau. Als mir während des Schulpraktikums die Betreuungslehrerin ${ }^{3}$ die Frage stellte, ob ich als Herr oder Frau Wiesauer angesprochen werden will, wusste ich nicht, was ich dazu sagen sollte: Können die Schüler:innen nicht einfach meinen (gewählten) Vornamen sagen und auf den Geschlechtermarker verzichten?

Die Erfahrungen, die ich als nicht-binäre Lehrperson in der Schule gemacht habe, waren unterschiedlich. Als ich bei einer Co-TeachingUnterrichtspraxis mit zehn Kolleg:innen von einer Gruppe dreizehnjähriger Jungen gefragt wurde, ob ich ein Mann oder eine Frau bin, dachte ich mir nur: Was mache ich hier? Möchte ich mich wirklich in Zukunft diesen Situationen aussetzen? Ich ärgerte mich weniger über die Frage als über die Kollegin, die

$1 \quad$ Es ist an Gymnasien und berufsbildenden höheren Schulen in Österreich üblich, die Lehrpersonen mit »Herr«bzw. »Frau Professor « anzusprechen.

2 Ich habe Bildnerische Erziehung - der österreichische Ausdruck für Kunsterziehung und Textiles Gestalten am Institut für künstlerisches Lehramt an der Akademie der bildenden Künste Wien studiert.

3 Betreuungslehrer:innen sind Lehrpersonen, die Lehramtsstudierende während ihres Studiums im Praktikum an Schulen begleiten. 
diese Gruppe Jungen zu mir geschickt hatte, ohne sich vorher mit mir abzusprechen. Nach der für mich sehr unangenehmen Erfahrung habe ich mit meinen Kolleg:innen darüber gesprochen und Unterstützung verlangt, weil es sonst für mich nicht möglich gewesen wäre, diese Schulpraxis abzuschließen. Konkret haben wir vereinbart, dass wir mit den Schüler:innen nicht über private Angelegenheiten sprechen, und falls nochmals eine unangenehme Situation auftritt, wir kurz den Raum verlassen und uns beraten, wie wir damit umgehen. Vielleicht war es im Rahmen dieser Unterrichtspraxis für die Schüler:innen auch eher möglich, mir diese Frage zu stellen, weil sich das behandelte Themengebiet mit Sexualität, Identität und Gender befasste. Daraus gelernt habe ich, dass es wichtig ist, sich auf solche Situationen vorzubereiten, Antworten parat zu haben und, wenn möglich, sich auch mit Kolleg:innen abzusprechen.

Eine andere Praxiserfahrung habe ich in einem Bundesoberstufenrealgymnasium mit Kunst-Zweig in einer Klasse gemacht, die kurz vor der Matura $^{4}$ stand. Gemeinsam, im Co-Teaching mit meiner Kollegin, haben wir uns ca. zehn Unterrichtseinheiten mit der Technik der Cyanotypie beschäftigt, bei der es sehr viel um das korrekte Mischen von Chemikalien, Lichtempfindlichkeit und Gestaltung geht. Durch das gemeinsame Unterrichten boten sich mehr Möglichkeiten, sich während des Unterrichts mit einer anderen Lehrperson auszutauschen und Pläne eventuell kurzfristig zu ändern. Die Schule hatte auch einen sehr großen Fokus auf individuelle Persönlichkeitsentwicklung von Schüler:innen und Geschlechtersensibilität, was wahrscheinlich auch einen großen Anteil daran hatte, dass ich mich in dieser Umgebung wohl gefühlt habe. Die Schüler:innen haben mich mit meinem gewählten Vornamen angesprochen. Meine Identität und Autorität als Lehrperson wurde, wenn auch nur für ein paar Stunden Unterricht, nicht in Frage gestellt, sondern es ging tatsächlich um ein Vermitteln von Inhalten und künstlerischen Techniken. 5

\footnotetext{
$4 \quad$ Matura ist der österreichische Ausdruck für Abitur.

5 Darüber hinaus ist mir aufgefallen, dass unangenehme Situationen eher aufgetreten sind, wenn ich versucht habe mich anzupassen, also mich weniger auffällig anzuziehen bzw. zu schminken. Sich nicht zu verstellen ist also vielleicht doch eine gute Strategie, um nicht aus dem Konzept gebracht zu werden?
} 


\section{Sensibilisierung und Empowerment}

Am Institut für künstlerisches Lehramt, an dem ich studiere, liegt ein großer Fokus auf gesellschaftskritischen Diskursen, Queer Theory, (Queer) Feminismus, Anti-Rassismus und Post-Colonial Studies. In fast jeder theoretischen Lehrveranstaltung, die ich auf diesem Institut besucht habe, ging es zumindest mehr oder weniger um eines dieser Themen, was ich als gut empfinde, weil Diskriminierungen alle Bereiche der patriarchalen, weißen, heterosexuellen Gesellschaft, in der wir leben, durchziehen. Trotzdem besprachen wir nur im Schnelldurchlauf, wie wir Schüler:innen, die migrantischen Hintergrund haben, aus anderen Klassenverhältnissen kommen, queer oder trans*ident oder homosexuell sind, unterstützen können. Die meisten Themen werden nur theoretisch behandelt. Aufgrund der wenigen Praxiseinheiten und des de facto sehr geringen Kontakts zu Schüler:innen und dem eigentlichen Schulumfeld gab es für mich nicht genug Möglichkeiten die theoretischen Grundlagen im Studium praktisch anzuwenden.

Für queere, trans*idente, nicht-binäre oder auch nicht-weiße Personen ist es oft unausweichlich, sich mit gesellschaftskritischen und (anti-)diskriminierenden Inhalten zu beschäftigen, weil diese oft den Alltag stark prägen. Insbesondere für Lehramts-Studierende, die einer marginalisierten Gruppe angehören, kann das Unterrichten oft eine Mehrfachbelastung darstellen. Wie schützt man sich gegen Diskriminierung und wieviel gibt man Schüler:innen und Kolleg:innen über sich preis? Wie wird mit Diskriminierungserfahrungen umgegangen?

Eine Idee wäre etwa, eine spezielle Lehrveranstaltung für marginalisierte Gruppen anzubieten, wie z.B. für Studierende die sich als trans* oder nichtbinär identifizieren. Diese Veranstaltungen könnten ein Ort sein, an dem sich Studierende und Lehrpersonen austauschen und gegenseitig ermächtigen und unterstützen können und diese Form des Arbeitens auch in ECTSPunkten Ausdruck findet. Es könnte konkret daran gearbeitet werden, wie man mit schwierigen Kommentaren umgeht, welche Rolle Passing ${ }^{6}$ in Gesellschaft und Schule hat, wie man als geschlechter-nicht-konforme Lehrperson

6 Passing bedeutet, als Cis-Person (= nicht trans*) wahrgenommen zu werden, d.h. als Person die sich mit seinem_ihrem bei Geburt zugeschriebenen Ceschlecht identifiziert. Anstatt mit >normak oder >biologisch $<$ zu arbeiten, bietet der Begriff cis ein Cegenstück zum Begriff trans* an. Ein Beispiel: Auf Annas Ceburtsurkunde steht der Ceschlechtsmarker $F$ und sie identifiziert sich und lebt als Frau. 
agieren kann, wie man mit Outing-Situationen umgeht, welche Alternativen es zu »Herr « und »Frau Professor« geben kann, wie queere, nicht-binäre oder trans*idente Schüler:innen unterstützt werden können und wie eine Sensibilisierung stattfinden kann, damit die Verantwortung für antidiskriminierendes Handeln nicht nur Aufgabe der von Diskriminierung Betroffenen bleibt. ${ }^{7}$

\section{Geschlechterreflektiert Unterrichten}

Als queere Person, die vielleicht eines Tages in der Schule unterrichten wird, frage ich mich: Wie wäre wohl meine eigene Entwicklung verlaufen, wenn ich als Teenager in der Schule mit einer kritischen Auseinandersetzung mit Gender und Sexualität in Kontakt gekommen wäre?

Eine geschlechterreflektierte Pädagogik kann neue und alternative Sichtweisen zu Geschlechtsidentität, Sexualität und Lebensrealitäten jenseits von Zweigeschlechtlichkeit bieten, die für die Entwicklung des Selbst maßgeblich sind. Die Beschäftigung mit vielfältigen geschlechtlichen und sexuellen Lebensweisen sollte schon in der Schule einsetzen, z.B. durch Workshops oder auch integriert in den Unterricht. Eine Auseinandersetzung mit diesen Themen kann die nächste Generation in einer möglichst selbstbestimmten Gestaltung ihres Lebens unterstützen. Dafür ist es wichtig, dass geschlechterreflektierte Pädagogik einen hohen Stellenwert in allen Lehramtsstudiengängen bekommt.

\section{Literatur}

Akademie der bildenden Künste Wien (2019): trans. inter*. nicht-binär. Lehrund Lernräume an Hochschulen geschlechterreflektiert gestalten. Online unter: https://www.akbild.ac.at/Portal/universitaet/frauenfoerderung-g eschlechterforschung-diversitaet/non-binary-universities/non-binary-u ni-accessible-300ppi.pdf [Zugriff: 18.06.2020].

7 Wichtige Arbeit hierzu wurde schon geleistet in der Broschüre »trans. inter*. nichtbinär. Lehr- und Lernräume an Hochschulen geschlechterreflektiert gestalten « (Wien 2019), herausgegeben von der Akademie der bildenden Künste Wien. Online unter: https://www.akbild.ac.at/Portal/universitaet/frauenfoerderung-geschlechterforsch ung-diversitaet/non-binary-universities/non-binary-uni-accessible-30oppi.pdf [Zugriff: 18.06.2020]. 


\section{LGBTIOA*-Jugendliche in der Schule Ansätze für einen bestärkenden Umgang mit sexueller und geschlechtlicher Vielfalt im Arbeitsfeld Schule}

Paul Haller und Anton Cornelia Wittmann

Sexuelle und geschlechtliche Vielfalt sind Teil der Lebenswelten von Kindern und Jugendlichen. ${ }^{1}$ »Fragen der Sexualität, Geschlechtsidentität, Partner* innenwahl, Ablösung von den Eltern und Zugehörigkeit zur Peergroup« sind wichtige Themen in der Adoleszenz (Kleiner 2020: 41). Dazu gehören Fragen wie: Wie erlebe ich mich körperlich und meine körperliche Entwicklung? Wie lebe ich meine Geschlechtlichkeit? In wen kann oder darf ich mich verlieben? Von wem fühle ich mich angezogen? Mit wem möchte ich Sexualität, Partner:innenschaft und Lust (er-)leben?

Aus ihrem Umfeld erhalten Kinder und Jugendliche oft unterschiedliche und mitunter widersprüchliche Botschaften in Bezug auf körperliche Normen, Geschlechterperformance oder Begehren. Sie stehen vor der Herausforderung diese Botschaften einzuordnen, in ihr sich entwickelndes Werteund Normensystem zu integrieren und ein positives Selbstbild herauszubilden (vgl. Damisch/Haller 2018: 25f.). Das Besprechbar-Machen von sexueller und geschlechtlicher Vielfalt kann dabei helfen, gesellschaftliche Normen kritisch $\mathrm{zu}$ hinterfragen und vielfältige »Skripte für mögliche Lebensentwürfe« (Kleiner 2020: 43) sichtbar zu machen. Davon profitieren letztlich alle, weil vorliegen und in unserem Praxisbeispiel eine trans* Jugendliche im Mittelpunkt steht. Zu sexueller und geschlechtlicher Vielfalt in der Elementarpädagogik siehe z.B. Kugler (2020), zur praktischen pädagogischen Arbeit die Materialien von Queerformat e.V. (www.queerformat.de) und zu sexueller und geschlechtlicher Vielfalt im Kontext frühkindlicher Inklusionspädagogik Nordt/Kugler (2020). 
»Menschen aller Geschlechter und sexueller Orientierungen [sich] besser entfalten und lernen [können], wenn sie weniger Sorge haben müssen, von geschlechts-, bindungs- und begehrensbezogenen Normen abzuweichen « (Laumann/Debus 2018: 284).

Sexuelle und geschlechtliche Vielfalt wird häufig als >Minderheitenthemar abgetan. Tatsächlich kann davon ausgegangen werden, dass sich LGBTI$\mathrm{QA}^{* 2}$-Jugendliche in jeder Schule und jeder Schulklasse befinden. Schätzungen zufolge sind rund 5-15\% der Gesamtbevölkerung homo- oder bisexuell (vgl. WASt o.J.), 1 \% asexuell (Profus 2016: 226), bis zu 1,7\% intergeschlechtlich $^{3}$ (VIMÖ, o.J.; Office of the High Commissioner of Human Rights 2015: 1) sowie $1 \%$ trans $^{* 4}$ (Güldenring/Sauer 2017: 234). ${ }^{5}$ Aktuelle Studien können als Anzeichen dafür gesehen werden, dass gerade bei Jugendlichen die Übergänge zwischen den Kategorien hetero-, homo- und bisexuell fließender werden (vgl. Timmermanns 2016: 19; Pöge et al. 2020: 4f.). Darüber hinaus kommen Schüler:innen unweigerlich mit Themen rund um sexuelle und geschlechtliche Vielfalt in Berührung, am offensichtlichsten, wenn sie Teil einer Regenbogenfamilie ${ }^{6}$ sind oder wenn sie LGBTIQA*-Personen in ihrem sozialen Um-

2 LCBTIQA* steht für lesbisch, schwul (gay), bisexuell, trans*/transgender/transident, inter"/intergeschlechtlich/intersex, queer/questioning und asexuell/aromantisch. Das Akronym wird in Wissenschaft und Aktivismus in unterschiedlichen Schreibweisen verwendet. Der Stern* bzw. Asterisk soll hier ein Hinweis auf vielfältige Selbstbezeichnungen im Spektrum sexueller und geschlechtlicher Vielfalt sein. Wenn wir Studien zitieren, die sich auf bestimmte Teilgruppen (z.B. »LCBT«) beziehen, verwenden wir die Formulierungen der jeweiligen Quelle.

"Queer « verwenden wir in diesem Text als Regenschirmbegriff für unterschiedliche Selbstbezeichnungen im Spektrum sexueller und geschlechtlicher Vielfalt bzw. als Synonym zu LCBTIQA*. Für die unterschiedlichen Bedeutungen von »queer« siehe z.B. Nagy 2016; Degele 2008: 42.

3 Inter* oder intergeschlechtlich meint Menschen, deren Ceschlechtsmerkmale nicht den engen medizinischen und gesellschaftlichen Normvorstellungen von »männlichen« und »weiblichen« Körpern entsprechen.

4 Als trans*, transgender, transident, nicht-binär oder genderqueer verstehen sich Menschen, deren Geschlechtsidentität nicht dem bei der Ceburt zugewiesenen Ceschlecht entspricht.

5 Diese Zahlen lassen sich nicht einfach addieren, da sie unterschiedliche Ebenen von Geschlecht und Begehren betreffen. Diversen Studien liegen sehr unterschiedliche Konzepte und Erhebungsformen zugrunde, was zu einer großen Bandbreite an quantitativen Ergebnissen führt. Für eine Diskussion des Bevölkerungsanteils von LCBTIPersonen in Deutschland siehe Pöge et al. 2020: $4 \mathrm{ff}$.

6 Damit sind Familien gemeint, in denen mindestens ein Elternteil LGTBIQA* ist. 
feld, im Bekannten- oder Freund:innen-Kreis haben. Aber auch aus popkulturellen Darstellungen und (sozialen) Medien, die Teil der Lebenswelten von Jugendlichen sind, sind queere Lebensweisen nicht mehr wegzudenken. Ein Nicht-Thematisieren von queeren Lebensweisen verkennt somit die Lebensrealitäten von Jugendlichen.

Nicht zuletzt sind die Auseinandersetzung mit »vielfältigen Lebensweisen« (vgl. Hartmann 2002) und ein wertschätzender Umgang mit sexueller und geschlechtlicher Vielfalt in Bildungskontexten auch ein Beitrag zur Prävention ${ }^{7}$ und Aufdeckung von (sexualisierter) Gewalt an Kindern und Jugendlichen: Auch heterosexuelle und cis-geschlechtliche ${ }^{8}$ Schüler:innen können von queer-feindlich motivierter Gewalt betroffen sein, wenn ihnen beispielsweise eine nicht-heterosexuelle Orientierung zugeschrieben wird. Wenn der Geschlechtsausdruck oder das Verhalten von Schüler:innen nicht in die herrschenden Bilder von >Männlichkeit < oder >Weiblichkeit< passen, wird dies häufig (gewaltvoll) sanktioniert (vgl. Krell/Oldemeier 2015: 21).

Tabus, Abwertungen, Diskriminierungen und Sprachlosigkeit schwächen Kinder und Jugendliche und unterstützen täter:innenschützende Strukturen. Täter:innen von sexualisierter Gewalt nutzen fehlendes Wissen ${ }^{9}$ und Sprachlosigkeit von Jugendlichen aus. Queere Jugendliche wiederum können sich im Fall von Grenzverletzungen und sexualisierter Gewalt (durch Erwachsene oder andere Jugendliche) schwerer Hilfe holen, wenn sexuelle Handlungen oder vielfältige Lebensweisen stigmatisiert oder als nicht >normak abgewertet werden.

»Ängste vor zugeschriebener und abgewerteter Homosexualität« (Scambor et al. 2019: 11of.) stellen eine Hemmschwelle für die Offenlegung von sexualisierten Gewalterfahrungen dar. Clemens Fobian und Rainer Ulfers von basis-praevent, einer Hamburger Fachberatungsstelle bei sexueller Gewalt an Jungen, schildern, dass Jungen und Männer, die sexuelle Gewalt erlitten haben, häufig »Gefühle wie Ohnmacht, Vereinsamung, Angst vor Homosexua-

Zum Zusammenhang zwischen sexueller Bildung und Prävention von sexualisierter Gewalt an Kindern und Jugendlichen siehe Henningsen 2016.

8 Menschen, deren Ceschlechtsidentität dem bei der Geburt zugewiesenen Ceschlecht entspricht. Also z.B. eine Person, die bei der Geburt als Mädchen oder Junge klassifiziert wurde und sich damit wohl fühlt.

9 Für eine differenzierte Auseinandersetzung mit»Wissen«als hilfreichem Faktor für die Aufdeckung von sexualisierter Cewalt siehe Scambor u.a. 2019: 116f. 
lität bzw. Irritationen der Geschlechtsidentität sowie Schuld- und Schamgefühle« äußern (2018: 308). Große Verunsicherungen bestünden beim Thema Homosexualität: »entweder befürchten die Jungen, dass sie schwul sein könnten, >weil doch ein Mann etwas mit ihnen gemacht hat von der Gewalterfahrung zu erzählen aus der Unsicherheit heraus, vielleicht als schwul angesehen zu werden« (ebd.: 308f.). Der gesellschaftliche Umgang mit sexueller und geschlechtlicher Vielfalt beeinflusst sowohl LGBTIQA* - als auch heterosexuelle cis-geschlechtliche Jugendliche in der Aufarbeitung von sexualisierter Gewalt - wenn auch auf unterschiedliche Weise. ${ }^{10}$ Prävention von sexualisierter Gewalt kommt daher ebenso wenig wie Sexualpädagogik um ein Hinterfragen gesellschaftlicher Macht- und Geschlechterverhältnisse herum (vgl. Fobian/Ulfers 2018: 322). Heteronormativität ${ }^{11}$ und Zweigeschlechtlichkeit als zentrale gesellschaftliche Ordnungsprinzipien spielen im Kontext von sexualisierter Gewalt ebenso eine Rolle wie andere Herrschaftsverhältnisse.

Mit diesem Beitrag laden wir zu einer Auseinandersetzung mit den Erfahrungen von queeren Schüler:innen und einem professionellen Umgang mit sexueller und geschlechtlicher Vielfalt ein. Da die "Lebenssituationen und Zukunftsoptionen« von LGBTIQA*-Jugendlichen »durch gesetzliche Grundlagen gerahmt « werden (Krell/Oldemeier 2017: 22), greifen wir zunächst die rechtliche Entwicklung in Österreich auf (Abschnitt 1). Danach skizzieren wir in Abschnitt 2 einige zentrale Erkenntnisse zur Lebenssituation und zu den Erfahrungen von LGBTI(QA)*-Jugendlichen. Mit der Diskussion eines Praxisbeispiels aus der Transgender-Beratung der HOSI Salzburg in Abschnitt 3 veranschaulichen wir exemplarisch die Herausforderungen für einen pro-

10 Fobian/Ulfers führen z.B. an, dass homosexuelle Männer eher davon berichten, aufgrund von sexuellen Cewalterfahrungen durch Männer länger mit ihrer eigenen Homosexualität gehadert zu haben (2018: 310f.).

11 »Heteronormativität beschreibt [...] ein natürlich erscheinendes, binäres Ceschlechtersystem, in welchem lediglich und genau zwei Geschlechter akzeptiert sind, die sich in ihrer Sexualität aufeinander beziehen« (Degele 2008: 21). Für eine kritische und praxisorientierte Auseinandersetzung mit Heteronormativität und Zweigeschlechtlichkeit eignet sich die »Pädagogik geschlechtlicher, amouröser und sexueller Vielfalt« (Debus/Laumann 2018) - zu Heterosexismus siehe Debus i.d.B. 
fessionellen, nicht-diskriminierenden und empathischen Umgang mit trans* Jugendlichen in der Schule. ${ }^{12}$

\section{Rechtliche Entwicklungen und Rahmenbedingungen in Österreich}

In den letzten Jahrzehnten haben LGBTI(QA)*-Personen und -Bewegungen auf nationaler und internationaler Ebene wesentliche rechtliche (und gesellschaftliche) Verbesserungen erkämpft - oft über den Weg zu Höchstgerichten. ${ }^{13}$ Nach der Verfolgung und Ermordung von Homosexuellen ${ }^{14}$ und der Durchführung von menschenverachtenden Experimenten $\mathrm{zu}>$ Intersexualität< (vgl. Voß 2014, 2010: 220f.) im Nationalsozialismus herrschte in Österreich noch bis 1971 ein Totalverbot ${ }^{15}$ gleichgeschlechtlicher sexueller Handlungen, die im Gesetzestext als »Unzucht wider die Natur« ( $\$ 129 \mathrm{lb} \mathrm{StG)}$ bezeichnet wurden. Mit der »kleinen Strafrechtsreform« 1971 und der Abschaffung des Totalverbots wurden vier neue »Anti-Homosexuellen-Paragrafen « eingeführt: das Verbot der männlichen homosexuellen Prostitution ${ }^{16}$, ein Vereins- ${ }^{17}$ und

Wir bedanken uns für die hilfreichen Hinweise zu diesem Beitrag bei Andreas Brunner (QWIEN - Zentrum für queere Ceschichte) und Hans-Peter Weingand (RosaLila PantherInnen) sowie bei Tinou Ponzer (Verein intergeschlechtlicher Menschen Österreich, VIMÖ) und Luan Pertl (OII Europe).

13 Aus Platzgründen beschränken wir uns auf einen groben Überblick über rechtliche Entwicklungen in Österreich. Für einen Überblick über die rechtliche Entwicklung in Deutschland siehe Krell/Oldemeier (2017: 22f.), spezifisch zur Einführung der Eingetragenen Partnerschaft und der Öffnung der Ehe für gleichgeschlechtliche Paare in Deutschland siehe Mangold (2018). Den rechtlichen Umgang mit Trans*Geschlechtlichkeit beleuchten Güldenring/Sauer (2017: 241ff.). Zu geschlechtsnormierenden Eingriffen an intergeschlechtlichen Kindern und Jugendlichen und historischen Hintergründen siehe Klöppel (2017; 2019), zur rechtlichen Entwicklung der »dritten Option « beim Ceschlechtseintrag in Deutschland siehe Plett (2019). Zu Selbst- und Fremdbildern homosexueller Männer in der Zeit des Nationalsozialismus in Österreich siehe Bauer et al. 2018.

15 Zwischen 1922 und 1971 kam es in Österreich zu ca. 25.000 Verurteilungen aufgrund des $\$ 129 \mathrm{lb}$ des Strafgesetzes von 1852 (StC) (vgl. Weingand 2011: 87).

$16 »[$ G] ewerbsmäßige gleichgeschlechtliche Unzucht«, §210 StCB, aufgehoben 1989.

17 »Verbindungen zur Begünstigung gleichgeschlechtlicher Unzucht«, §221 StCB, aufgehoben 1997. 
ein Werbeverbot ${ }^{18}$ sowie ein höheres Schutzalter für männliche homosexuelle Sexualkontakte $^{19}$ (Weingand 2011).

Bis 2006 galt in Österreich ein Scheidungszwang für verheiratete trans* Personen, die eine Personenstandsänderung durchführen wollten, und bis $2010^{20}$ mussten sich trans* Personen einer Sterilisation unterziehen, um ihren Personenstand und ihren Vornamen ändern zu lassen (TransX o.J.).

Gerade in den letzten Jahren gab es wesentliche rechtliche Verbesserungen in Österreich: Von der Umsetzung des Diskriminierungsschutzes in der Arbeitswelt (2004-2006) und der Einführung der Eingetragenen Partnerschaft (2010) über die Öffnung medizinisch unterstützter Fortpflanzung z.B. für lesbische Paare (2015), die Möglichkeit der Adoption (2016) und der Pflegeelternschaft durch gleichgeschlechtliche Paare (seit 2017 in allen Bundesländern) bis hin zur Ehe-Öffnung ${ }^{21}$ (2019). 2018 erkämpfte die intergeschlechtliche Person Alex Jürgen vor dem Verfassungsgerichtshof (VfGH) das Recht auf eine »dritte Option« beim Geschlechtseintrag neben »männlich« und »weiblich«. Seither wird um eine menschenrechtskonforme Umsetzung der VfGHEntscheidung gerungen. ${ }^{22}$ Davon unberührt bleibt die Hauptforderung von Inter*-Organisationen: ein sofortiger Stopp und ein gesetzliches Verbot von geschlechtsnormierenden medizinischen Eingriffen an intergeschlechtlichen Menschen ohne vorherige persönliche, freiwillige und voll informierte Einwilligung. ${ }^{23}$

Diese rechtlichen Entwicklungen gehen mit einem gesellschaftlichen Wandel und einer grundlegenden Neubewertung von sexueller und geschlechtlicher Vielfalt einher, aber auch mit queer-feindlichen und anti-fe-

18 »Werbung für Unzucht mit Personen des gleichen Ceschlechts oder mit Tieren«, §220 StCB, aufgehoben 1997.

19 »[C]leichgeschlechtliche Unzucht mit ]ugendlichen«, §209 StCB, aufgehoben 2002.

20 Die Aufhebung des Operationszwangs erfolgte durch den Österreichischen Verwaltungsgerichtshof $(\mathrm{VwCH}, 2018 / 17 / 0054)$ am 27.02.2009, die rechtskonforme Umsetzung durch die zuständigen Behörden dauerte bis 2010.

21 Diese Darstellung soll nicht davon ablenken, dass das Rechtsinstitut Ehe aus (queer-)feministischer Sicht problematisiert werden muss (siehe dazu z.B. Haller/Schönpflug 2018: 52).

22 Siehe dazu z.B. die Stellungnahme des Vereins intergeschlechtlicher Menschen Österreich vom 23.10.2018 zur Umsetzung eines dritten Ceschlechtseintrags im österreichischen Personenstand (VIMÖ: 2018).

23 Siehe dazu das Konzept des »personal, prior, free and fully informed consent« (Chattas 2019: 16f.). 
ministischen Gegenreaktionen und mitunter heftigen Angriffen gegen emanzipatorische und vielfaltsorientierte Sexualpädagogik (vgl. Laumann/Debus 2018). ${ }^{24}$ Mit dem Grundsatzerlass "Sexualpädagogik« des Bildungsministeriums (2015) fand nach 20 Jahren eine umfassende Neuformulierung des Sexualerziehungs-Erlass aus den 1990er Jahren statt (Vasold 2016: 1), die Teilen der gesellschaftlichen und rechtlichen Veränderungen Rechnung trägt. Darin heißt es:

»Sexualpädagogik soll altersgerecht, an der Lebensrealität von Kindern und jungen Menschen orientiert sein und auf wissenschaftlich gestützten Informationen basieren. Sie soll einen positiven Zugang zur menschlichen Sexualität darstellen und eine positive Grundhaltung sich selbst gegenüber sowie das eigene Wohlbefinden befördern. Sie soll sich am Prinzip der Gleichstellung der Geschlechter sowie der Vielfalt der Lebensformen (z.B. sexuelle Orientierung, Geschlechteridentitäten) orientieren, soll Kompetenzen (z.B. kritisches Denken, Kommunikationsfähigkeiten) vermitteln und an internationalen Menschenrechten ausgerichtet sein.« (BMB 2018 [2015]: 4).

Der im Grundsatzerlass »Sexualpädagogik« formulierte klare Auftrag zur Auseinandersetzung mit vielfältigen sexuellen Orientierungen und $\mathrm{Ge}$ schlechtsidentitäten in der schulischen Sexualpädagogik deckt sich mit den "Standards der Sexualaufklärung in Europa der Weltgesundheitsorganisation und der deutschen Bundeszentrale für gesundheitliche Aufklärung (WHO-Regionalbüro für Europa und BZgA 2011). Dennoch sind sexuelle und geschlechtliche Vielfalt im Bildungssystem strukturell zu wenig verankert. In Österreich fehlen beispielsweise verpflichtende Lehrer:innen-Fortbildungen, die LGBTIQA*-Themen behandeln, es gibt keine nationale Anti-BullyingStrategie, die LGBTIQA* beinhaltet, und es hängt immer noch weitgehend von einzelnen Lehrkräften $\mathrm{ab}$, ob und wie sexuelle und geschlechtliche Vielfalt im Unterricht behandelt wird (vgl. IGLYO o.J.).

24 Henningsen u.a. sprechen in diesem Zusammenhang von »widersprüchlichen Cleichzeitigkeiten« (2016: 8-10). 


\section{Forschungsarbeiten zu Erfahrungen von LGBTI(OA)*-Jugendlichen}

LGBTI(QA)* -Jugendliche haben neben den alterstypischen Entwicklungsaufgaben aller Jugendlichen oft zusätzliche Herausforderungen zu bewältigen. ${ }^{25}$ Sie machen später erste romantische Beziehungserfahrungen als ihre heterosexuellen und cis-geschlechtlichen Peers, die nicht denselben >Findungsprozess durchlaufen müssen (Watzlawik 2020: 31). In unterschiedlichen Lebensbereichen erfahren sie Gewalt, Differenzierung und Ausgrenzungen, die durch ein Zusammenwirken unterschiedlicher gesellschaftlicher Differenzordnungen (z.B.: Heteronormativität, Rassismus, Ableismus, Klassismus) geprägt sind. ${ }^{26}$

»Im Schulalltag kommt es, wie verschiedene Studien zeigen, in der Tendenz zu einer Reproduktion stereotyper, diskriminierender und potenziell verletzender gesellschaftlicher Vorstellungen von Geschlecht und Begehren« (Kleiner 2020: 47). Queere Jugendliche werden damit konfrontiert, dass Wörter wie »lesbisch«, »schwul«, »Zwitter« oder »Transe« als Schimpfwörter ${ }^{27}$ verwendet werden (vgl. Klocke 2020: 357), und machen häufig die Erfahrung, dass Lehrkräfte bei Abwertungen nicht (ausreichend) einschreiten (Krell/Oldemeier 2015: 21). In Schulbüchern finden sie kaum Repräsentationen von LGBTI(QA*)-Lebensweisen (Bittner 2012, 2015). Ihre »Lebens- und Erfahrungsweisen « werden in Unterrichtsdiskursen und Lehrmaterialien unsichtbar gemacht (Kleiner 2020: 49). Oft fehlen Vorbilder aus dem nahen sozialen Umfeld. Dies gilt auch für die Schule: So gehen laut einer ersten deutschlandweiten Erhebung nur vier von zehn LGBTIQ*-Lehrer:innen ${ }^{28}$ an ihrer Schule offen mit ihrem LGBTIQ*-Sein um (Antidiskriminierungsstelle des Bundes

25 In Rahmen dieses Kapitels erscheint es uns wichtig, einige zentrale Problemstellungen von LGBTIQA*-Jugendlichen zu benennen. Zur Notwendigkeit, die spezifischen Ressourcen von LGBT(IQA)*-Jugendlichen verstärkt in den Blick zu nehmen, siehe z.B. Oldemeier/Timmermanns (2020).

26 Nach Kleiner 2020: 44 wird die Verstrickung von Heteronormativität mit anderen Differenzordnungen (Rassismus, Ableismus und Klassismus) in Studien zu den Erfahrungen von $\mathrm{LCBTI}(\mathrm{QA})^{*}$-Jugendlichen unzureichend berücksichtigt.

27 Praktische Hinweise zum Umgang mit Beschimpfungen bietet die Broschüre »Schwule Sau!`>Du Transe!`>Kampflesbe`-Was tun bei Beschimpfungen und diskriminierenden Äußerungen?«(Queerformat e.V. 2015).

28 Der Verein Ausgesprochen! LCBTIQ* Lehrer*innen bietet Austausch und Information (www.verein-ausgesprochen.at [Zugriff 19.12.2020]). 
2017). Dies wirkt sich nicht nur negativ auf das Wohlbefinden und die Arbeitszufriedenheit der Lehrkräfte aus, sondern bedeutet auch, dass queeren Schüler:innen potenzielle Vorbilder verloren gehen.

Wie Annika Spahn (2018) beschreibt, machen auch asexuelle Menschen weitreichende Diskriminierungserfahrungen in Bildungskontexten und werden mit spezifischen Vorurteilen ${ }^{29}$ konfrontiert. Über die Erfahrungen von inter* Personen im Schulsystem ist kaum etwas bekannt. ${ }^{30}$ Aktuell forscht dazu Mart Enzendorfer anhand von biografischen Interviews am Institut für Bildungswissenschaft der Uni Wien im Rahmen eines Dissertationsprojekts. ${ }^{31}$ Selbstvertretungsorganisationen wie der Verein intergeschlechtlicher Menschen Österreich (VIMÖ) berichten u.a. von Scham, Gefühlen von Isolation und Einsamkeit sowie Selbst-Pathologisierung als zentralen Themen.

Wenn LGBTIQA*-Jugendliche in ihrer Schulzeit mit Ängsten, Diskriminierungs- oder Gewalterfahrungen konfrontiert sind, kann dies negative Auswirkungen auf ihre Gesundheit haben. Im Minoritätenstress-Modell unterscheidet Ilan H. Meyer vier Ebenen, auf denen Diskriminierung und Vorurteile negativ auf die psychische und körperliche Gesundheit von Menschen mit einer nicht-heterosexuellen Orientierung wirken: (1) die Erfahrung von Diskriminierung und Gewalt, (2) die Befürchtung von Diskriminierungsund Gewalterlebnissen, (3) das Verbergen der eigenen sexuellen Orientierung und Lebensweise sowie (4) die Verinnerlichung negativer gesellschaftlicher Bewertungen gegenüber lesbischen und schwulen Lebensweisen (vgl. Meyer 2007 zit.n. Dennert o.J.).

Ängste und Erfahrungen von Abwertung, Diskriminierung und Vorurteilen können verinnerlicht und gegen die eigene Person gerichtet werden. Diese »internalisierte Queer-Feindlichkeit ${ }^{32}$ hat wiederum negative Auswirkungen

29 Als Vorurteile benennt Spahn u.a.: »Du bist eigentlich nur schwul/lesbisch und hast Angst, es zuzugeben «, »Du kannst niemanden lieben, ohne mit ihm:ihr Sex zu haben.«, »Du hast nur noch nicht den“die Richtige*n gefunden.«,»Das ist nur eine Phase.«(2018: 170).

Die australische quantitative Studie von Jones et al. (2016) berichtet u.a. von massiven Diskriminierungserfahrungen und einer hohen Zahl von Bildungsabbrüchen. Erste Erkenntnisse aus dem Dissertationsprojekt finden sich bei Enzendorfer/Haller (2020) sowie Enzendorfer (i.E.).

Ähnliche Bezeichnungen sind z.B. »internalisierte Homo- oder Transnegativität« (vgl. Oldemeier/Timmermanns 2020: 345). Wir verwenden den Begriff »QueerFeindlichkeit«, da er uns möglichst einschließend erscheint. 
auf Selbstwert und Gesundheit. In der Forschung ist ein Zusammenhang zwischen internalisierter Homo-Feindlichkeit und gesteigertem Risikoverhalten bekannt (Warwick/Douglas 2001 zit.n. Gualdi et al. 2008: 16). Zu den dramatischsten gesundheitlichen Beeinträchtigungen für LGBT(IQA*)-Jugendliche gehören sicherlich die gegenüber ihren heterosexuellen und cis-geschlechtlichen Peers erhöhten Raten von Suizidgedanken und Suizidversuchen (vgl. Plöderl 2016, 2020; Pöge et al. 2020). ${ }^{33}$

\subsection{Coming-out}

Ein zentrales Thema queerer Jugendlicher kann ein Coming-out-Prozess sein, ${ }^{34}$ bei dem häufig zwischen innerem und äußerem Coming-out unterschieden wird (vgl. etwa Watzlawick 2004: 54f.; Krell/Oldemeier 2017; Oldemeier 2020; Kleiner 2020: 46). Das innere Coming-out umfasst dabei die Phase der inneren Bewusstwerdung über das eigene Geschlecht, die eigene Geschlechtsidentität und/oder sexuelle Orientierung. Das äußere Comingout bezeichnet das >Anvertrauen b bzw. das Erzählen gegenüber anderen Personen. Das Coming-out ist kein einmaliges Ereignis, sondern prozesshaft $\mathrm{zu}$ verstehen. Bei jeder neuen Bekanntschaft, jedem Schul-, Lehrstellenoder Jobwechsel wird erneut darüber entschieden, welche Information preisgegeben wird. Die Notwendigkeit eines Coming-outs bzw. ssich outen zu müssen, ist Ausdruck einer heteronormativen Gesellschaft. Anders gesagt: Nur wenn Heterosexualität und Zweigeschlechtlichkeit die Norm sind, macht es Sinn bzw. kann es notwendig sein, sich zu outen. Gleichzeitig kann ein selbstbestimmtes (äußeres) Coming-out eine empowernde Erfahrung für LGBTIQA* -Jugendliche sein. ${ }^{35}$

33 »Das Ceschlecht beziehungsweise die sexuelle Orientierung selbst sind nicht ursächlich für höhere Prävalenzen von Depressivität und Suizidalität. Vielmehr sind mangelnde Akzeptanz, Diskriminierung und Cewalterfahrungen psychisch belastend und können zu Erkrankungen führen « (Pöge et al. 2020: 19f.). Außerdem ist darauf hinzuweisen, dass trotz erhöhter Raten die überwiegende Mehrheit der LCBTI-Personen kein erhöhtes Suizidrisiko aufweist, um »unnötige Pathologisierungen und Stereotypisierungen « zu vermeiden (Plöderl 2020: 296f.).

34 Für eine kritische Betrachtung von Coming-out-Konzepten und zur Verstrickung von Coming-out, Heteronormativität und Rassismuserfahrungen siehe Kleiner 2020: 44-47.

35 Differenziert hierzu Kleiner 2020: 44f. 
Nach Krell/Oldemeier (2017), die erstmals in einer deutschlandweiten Studie $^{36}$ über 5.000 LGBTQ-Jugendliche im Alter von 14 bis 27 Jahre befragten, vergehen zwischen innerem und erstem äußeren Coming-out oft mehrere Jahre. Die Spanne reicht von durchschnittlich 1,7 Jahren bei lesbischen und bisexuellen weiblichen Jugendlichen über knapp drei Jahre bei schwulen und bisexuellen männlichen Jugendlichen bis hin zu rund vier Jahren bei trans* männlichen und fast sieben Jahren bei trans* weiblichen Jugendlichen (vgl. ebd.: 84, 151). In dieser Zeit können sie mit unterschiedlichen Ängsten konfrontiert sein. Ein Großteil der befragten LGB-Jugendlichen und jungen Erwachsenen gibt an, dass sie in der Phase zwischen innerem und äußerem Coming-out Angst vor Ablehnung durch Freund:innen $(74,4 \%)$ und Familienmitglieder (69\%) hatten (vgl. ebd.: 79). Für trans* und gender*diverse Jugendliche steht vor allem die Angst, nicht ernst genommen zu werden, mit knapp $80 \%$ im Vordergrund, gefolgt von der Befürchtung, Ablehnung durch Familienmitglieder (77,0\%) zu erleben (vgl. ebd.: 148).

LGBT-Jugendliche überlegen sich oft sehr gut, wem sie was, wann und wie anvertrauen. In der Phase zwischen innerem und äußerem Coming-out kann ein hoher Leidensdruck entstehen, der schließlich zum ersten äußeren Coming-out führt (vgl. Krell/Oldemeier 2015: 15). Unter den befragten trans* Personen nennt rund ein Viertel den Beginn der körperlichen Transition ${ }^{37}$ als Grund für das erste äußere Coming-out (vgl. ebd.: 151).

\section{Trans* im Schulalltag: Praxisbeispiel und theoriegeleitete Reflexion}

Anhand eines Praxisbeispiels wollen wir nun spezifische Herausforderungen von trans* Jugendlichen aufzeigen und diskutieren. Das Beispiel basiert auf Erfahrungen der Peer-Beratung des Transgender-Referats der HOSI Salzburg. Wir wählen bewusst ein Fallbeispiel aus, in dem Erfahrungen von trans* Jugendlichen sichtbar gemacht werden. Einerseits weil diese oft $\mathrm{zu}$ wenig berücksichtigt werden, andererseits weil wir in den letzten Jahren

36 Eine vergleichbare Studie liegt in Österreich bis dato nicht vor.

37 Transition beschreibt den Prozess der Angleichung an das individuelle Geschlechtserleben. Oftmals wird damit allein auf die körperliche Angleichung verwiesen. Transitionsprozesse finden aber auch auf rechtlicher und sozialer Ebene statt (vgl. Franzen/Sauer 2010: 95). 
nicht nur einen großen Anstieg an Beratungen im Transgender-Referat der HOSI Salzburg verzeichnen, sondern auch weil wir gerade im Umgang mit trans*, inter* und genderdiversen Jugendlichen mitunter große Verunsicherungen unter Pädagog:innen und sozialen Fachkräften wahrnehmen. In der Diskussion des Fallbeispiels verbinden wir wissenschaftliche Erkenntnisse mit Erfahrungen aus der Peer-Beratung sowie Wissen aus Selbstvertretung und aktivistischen Zusammenhängen.

\subsection{Praxisbeispiel: Julia Kusz, 14, trans* Schülerin}

Wie viele trans* Jugendliche beschäftigt sich Julia Kusz ${ }^{38}$ über einen längeren Zeitraum intensiv mit ihrer eigenen Geschlechtsidentität, bevor sie sich anderen Menschen anvertraut. In dieser Zeit begleiten sie Unsicherheiten und Ängste. Neben der Angst vor Ablehnung und negativen Reaktionen durch das Umfeld können bei trans* Jugendlichen Ängste rund um körperliche Veränderungen und Pubertätsverläufe hinzukommen.

Nachdem Julia Kusz durch ihr soziales Umfeld kaum Wissen zu nichtheteronormativen Geschlechtsidentitäten erhält und auch nicht auf die Erfahrungen von anderen trans* Menschen zurückgreifen kann, sucht sie online ${ }^{39}$ nach Informationen und Anlaufstellen. Das Transgender-Referat der HOSI Salzburg erlebt immer wieder, dass die Selbstaneignung von Wissen zu Trans* sowie das Aufbauen von Kontakten zu anderen trans* Jugendlichen empowernde Erfahrungen sein können. ${ }^{40}$ Online findet Julia Kusz den Weg zur Peer-Beratung. Es ist immer wieder erstaunlich, wie viel Wissen sich queere Jugendliche aneignen, bevor sie Beratungstermine wahrnehmen. Gleichzeitig bietet die persönliche Beratung die Gelegenheit, gängige Mythen ${ }^{41}$ sowie normative bis pathologisierende und sogar abwertende Darstellungen von Trans* zu thematisieren. Wissenslücken können im persönlichen

38 Name und Alter sowie einzelne Aspekte der Fallbeschreibung wurden verändert, um die Anonymität der Schülerin zu wahren.

$39 \mathrm{Zu}$ den (ambivalenten) Erfahrungen von LCBTIQ*-Jugendlichen im Internet siehe Krell/Oldemeier 2018: 22-26 und Oldemeier 2020: 64f.

40 Oldemeier/Timmermanns argumentieren, dass LSBTQ* [sic!] Jugendliche » [d]urch einen selbstständig organisierten passenden Erfahrungs- und Informationsaustausch « defizitäre Bedingungen bewältigen können (2020: 350).

41 Häufige Mythen, denen das Transgender-Referat in der Beratung begegnet, sind z.B.: »Alle trans" Personen wollen eine operative Angleichung. «»Medizinische Maßnahmen sind notwendig, um im Ceschlecht anzukommen.«»Alle trans* Personen wussten 
Gespräch geschlossen oder zumindest angesprochen und fehlerhafte sowie veraltete Informationen aufgeklärt werden.

Auch die gängige und falsche Vorstellung, dass die Transition für alle trans* Menschen als linearer Prozess verläuft, der mit >dem`Coming-out beginnt und mit Operation(en) als erstrebenswertem Ziel endet (siehe Abbildung), kann im Beratungsprozess reflektiert werden.

\section{TRANSIIION}

... WIE ES SICH CIS-PERSONEN MEIST VORSTELLEN

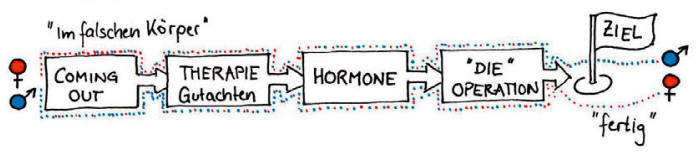

... WIE ES VIELE TRANS MENSCHEN ERLEBEN

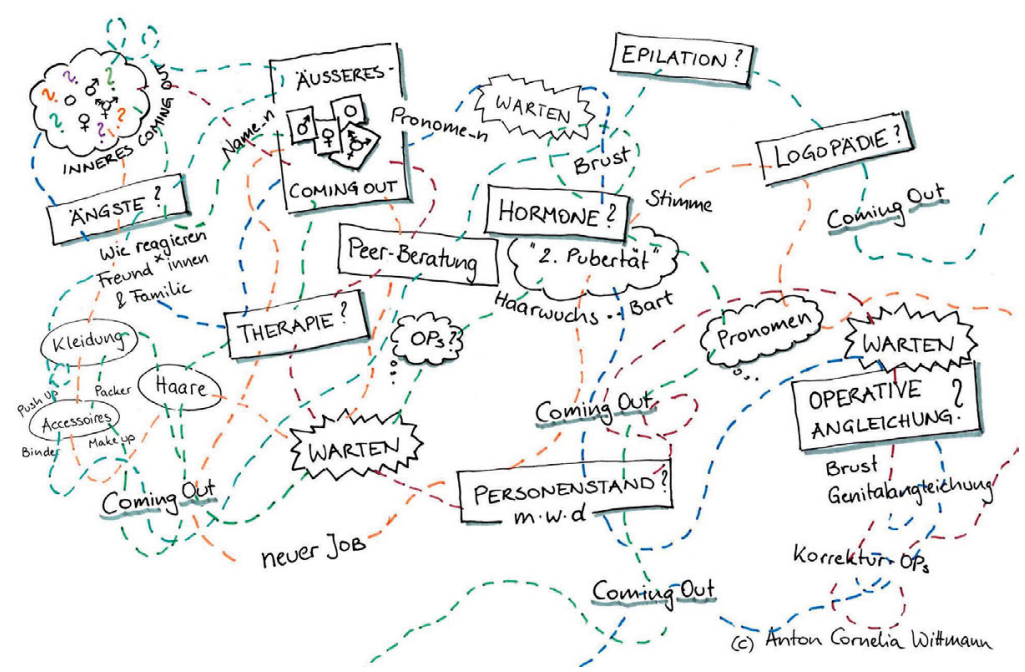

Abb. 6: ๑ Anton Cornelia Wittmann

schon in früher Kindheit, dass sie sich in ihrem zugewiesenen Ceschlecht nicht zugehörig fühlen.« 
Julia Kusz schildert, dass ihre Geschlechtsidentität innerhalb der Familie lächerlich gemacht wird. In der Peer-Beratung erzählen Jugendliche und junge Erwachsene immer wieder, dass ihnen ihre Geschlechtsidentität bzw. ihr Trans*-Sein abgesprochen wird. Ein Teil berichtet, dass ihr (selbst gewählter) Vorname und ihr (selbst gewähltes) Pronomen absichtlich ignoriert werden. Fast alle machen die Erfahrung, dass über sie mit dem falschen Vornamen, Pronomen und Bezeichnungen wie »Sohn/Tochter« gesprochen wird, was sie als verletzend erleben.

Die körperlichen Veränderungen in der Pubertät, insbesondere aber die Veränderung ihrer Stimme und der sichtbare Adamsapfel, sind für Julia Kusz schwer auszuhalten. Sie wünscht sich endlich als Mädchen akzeptiert zu werden, während ihr Körper sich in eine Richtung entwickelt, die von ihr, aber auch einem Großteil der Gesellschaft, als männlich wahrgenommen wird. Wie sie empfinden manche trans* Menschen »Teile ihres angeborenen Körpers als falsch oder nicht stimmig« (Debus/Laumann 2018: 26). Daraus resultiert für viele von ihnen auch der Wunsch nach Angleichungen ihres Körpers an ihr Geschlechtserleben. Die Ambivalenz zwischen innerem Erleben und äußerem Erscheinen einerseits und zwischen der Angst vor dem äußeren Coming-out und dem Wunsch nach Anerkennung andererseits macht die Zeit vor Beginn der Transition zu einer besonders vulnerablen Phase. Güldenring/Sauer sprechen in diesem Zusammenhang von einem »suizidgefährdeten Lebensabschnitt« (2017: 247). In der Coming-out-Studie von Krell/Oldemeier berichten trans* Jugendliche von psychischen Schwierigkeiten und ambulanter und stationärer Therapieerfahrung (2017: 146).

Julia Kusz entscheidet sich für ein Coming-out in der Klasse. Die Lehrerin steht ihr zur Seite und bietet den Rahmen für ein Gespräch mit den Mitschüler:innen. Dennoch erlebt Julia Kusz das Coming-out als belastend, da ihr durch die exponierte Stellung vor der Klasse die geballte Aufmerksamkeit zuteilwird. Die Angst die Lehrerin zu enttäuschen oder ihre Unterstützung $\mathrm{zu}$ verlieren, macht es ihr unmöglich, eigene Forderungen und Vorstellungen zu ihrem Coming-out einzubringen. Im Unterricht geht die Lehrerin (aus zeitlichen Gründen) nicht näher auf grundlegende Informationen zu Trans* ein. Das trägt dazu bei, dass die Mitschüler:innen unzureichend sensibilisiert sind. In weiterer Folge ist Julia Kusz zum Teil sehr persönlichen Fragen einiger Schüler:innen ausgesetzt. Andere Mitschüler:innen hingegen sind unsicher und meiden sie. War sie vorher schon eher zurückgezogen, fühlt sie sich nun noch mehr als Außenseiterin. 
Auch nach dem Coming-out in der Klasse ergeben sich neue Schwierigkeiten. So sind die Schulleitung und die Klassenlehrerin zunächst verunsichert, ob der gewünschte Vorname schon vor einer offiziellen Namensänderung (z.B. in Klassenlisten) verwendet werden darf und ab wann der Name auch in den Stammdaten und somit auf dem Zeugnis geführt werden kann. Verunsicherungen bzw. Hilflosigkeit von Lehrkräften aufgrund von fehlendem Wissen zu trans* Schüler:innen zeigt auch der Forschungsbericht zu dem Jugendprojekt »TRANS* - JA UND?!« in Deutschland auf (Sauer/Meyer 2016: 25). Hinzu kommt, dass Schule wie kaum ein anderer Bereich zweigeschlechtlich geprägt ist (ebd.: 53). So wird die Teilnahme an geschlechtergetrenntem (Sport-)Unterricht sowie der Zugang zu getrennten Sanitär- und Umkleideräumen für trans* und intergeschlechtliche Schüler:innen zur Herausforderung (vgl. Ghattas 2016: 12; Focks 2014: 10f.). ${ }^{42}$

In Julia Kusz' Geschichte versuchen die Schulleitung sowie Lehrkräfte eine Störung der gewohnten Geschlechterordnung zu umgehen, indem sie ihr nahelegen, sich bis zur Personenstandsänderung ${ }^{43}$ vom Sportunterricht befreien zu lassen. Damit forcieren sie weitere Ausgrenzungserfahrungen der Schülerin.

Im Laufe des Schuljahres erfolgt die Personenstandsänderung von Julia Kusz. Erst jetzt ist die Schule rechtlich gezwungen, sie am Sportunterricht der Mädchen teilnehmen zu lassen. An der sozialen Situation von Julia Kusz ändert sich - insbesondere in Bezug auf die Nutzung von Sanitär- und Umkleideräumen - durch die Personenstandsänderung allerdings kaum etwas. Ängste und Verunsicherungen z.B. beim Umziehen in der Garderobe bestehen sowohl bei Julia Kusz als auch bei ihren Mitschüler:innen. Erst nach unterschiedlichen Interventionen sucht die Schule nach Möglichkeiten, um die Partizipation am Unterricht zu ermöglichen, ohne neue Ausschlüsse und Diskriminierungserfahrungen zu produzieren.

42 Dan Christian Chattas schreibt dazu im Kontext von intergeschlechtlichen Personen: »Orte, an denen der eigene Körper sichtbar für Andere wird, wie Toiletten oder Umkleideräume, werden in der Regel als angstbesetzt und als Tatorte von Quälereien beschrieben - unabhängig davon, ob an dem betroffenen intergeschlechtlichen Menschen ein sogenannter normalisierender chirurgischer Eingriff vorgenommen wurde oder nicht« (Chattas 2016: 12).

43 Änderung des Geschlechtseintrags im Personenstandsregister. 


\section{Ausblick: ein weiter Weg zur queer-freundlichen Schule}

LGBTIQA*-Schüler:innen profitieren von Pädagog:innen und sozialen Fachkräften, die professionell, empathisch und diskriminierungskritisch mit sexueller und geschlechtlicher Vielfalt umgehen können. Dafür benötigen sie Wissen, einen (selbst-)reflektierten Umgang mit eigenen Werten, Vorurteilen und Vorstellungen von Körperlichkeit, Geschlecht und Sexualität sowie spezifische Sozial- und Methoden-Kompetenzen im Kontext sexueller und geschlechtlicher Vielfalt. ${ }^{44}$

Wie an Studien und unserem Praxisbeispiel veranschaulicht, wird auf die Bedürfnisse, Herausforderungen und Gesundheitsrisiken von LGBTIQA*Schüler:innen nicht ausreichend eingegangen. Die Geschichte von Julia Kusz zeigt, wie im System Schule mit Störungen der heteronormativen Geschlechterordnung durch eine trans* Schülerin umgegangen wird.

Wir plädieren für kreative, individuelle Lösungen in der schulischen Praxis unter Einbeziehung der jeweiligen Schüler:innen und in Absprache gegebenenfalls mit Mitschüler:innen, Eltern und Erziehungsberechtigten. Werden starre Lösungen vorgegeben, können auch >gut gemeinte Interventionen von Lehrkräften und Schulleitung Diskriminierungs- und Ausschlusserfahrungen fördern. Um das zu vermeiden, erscheinen uns Offenheit, Fehlerfreundlichkeit, die Anerkennung von Selbstbestimmung und Selbstreflexion als zentrale Bestandteile einer professionellen Haltung von Pädagog:innen und sozialen Fachkräften. ${ }^{45}$

In der Praxis erleben wir, dass die Bedürfnisse von queeren Jugendlichen in der Schule - wie hier am Beispiel von Julia Kusz gezeigt - häufig erst durch ein Coming-out in den Mittelpunkt rücken. Gerade bei trans* Jugendlichen verstellen rechtliche Fragen oft den Blick auf die (meist dringlichere) soziale Situation von Schüler:innen. Schulen sind gefordert Rahmenbedingungen und sichere soziale Räume zu schaffen, in denen sich alle Schüler:innen angstfrei entfalten können. Unterstützung finden Lehrkräfte und Schulleitung dazu beispielsweise bei Selbstvertretungsorganisationen oder queeren

\footnotetext{
44 Siehe dazu das Konzept der »Regenbogenkompetenz« von Ulrike Schmauch 2015; 2016; 2020.

45 Für eine Auseinandersetzung mit pädagogischer Professionalität im Umgang mit sexueller und geschlechtlicher Vielfalt siehe Hartmann 2020. Für eine Auseinandersetzung mit pädagogischem Handeln und Intergeschlechtlichkeit siehe Hechler 2016.
} 
Bildungs- und Anti-Diskriminierungsprojekten. Neben Workshops für Schüler:innen und Informationsmaterialien bieten diese oft auch individuelle Beratungen oder Fort- und Weiterbildungen an.

In der Gesellschaft und ihrem Teilsystem Schule sind daher auch strukturelle Veränderungen notwendig, etwa Änderungen von Lehrplänen, von Schulbüchern, von Lehrer:innen-Aus- und Fortbildung. ${ }^{46}$ Letztendlich führt kein Weg vorbei an einer auch in der Schule $\mathrm{zu}$ führenden Auseinandersetzung mit vielfältigen Lebensweisen sowie an einer grundlegenden Infragestellung von Heteronormativität und Zweigeschlechtlichkeit als gesellschaftlichen Ordnungsprinzipien in ihrer Interdependenz $\mathrm{zu}$ anderen Ordnungsprinzipien und Machtverhältnissen.

\section{Literatur}

Antidiskriminierungsstelle des Bundes (2017): LSBTIQ*-Lehrkräfte in Deutschland. Diskriminierungserfahrungen und Umgang mit der eigenen sexuellen und geschlechtlichen Identität im Schulalltag. Berlin: Antidiskriminierungsstelle des Bundes. Online unter: https://www.antid iskriminierungsstelle.de/SharedDocs/Downloads/DE/publikationen/Um fragen/LSBTIQ_Lehrerkraeftebefragung.pdf [Zugriff: 17.05.2020].

Bauer, Manuela/Brunner, Andreas/Salzenbacher, Hannes/Treiblmayer, Christopher (2018): »Warme vor Gericht«. Zu Selbst- und Fremdbildern homosexueller Männer in der Zeit des Nationalsozialismus in Österreich. In: Österreichische Zeitschrift für Geschichtswissenschaften, Bd. 29, Nr. 2 (2018), S. 87-110.

BMBF - Bundesministerium für Bildung und Frauen (2015): Grundsatzerlass Sexualpädagogik. Online unter: https://www.bmbwf.gv.at/Themen/schu le/schulrecht/rs/1997-2017/2015_11.html [Zugriff: 17.05.2020].

Bittner, Melanie (2012): Geschlechterkonstruktionen und die Darstellung von Lesben, Schwulen, Bisexuellen, Trans* und Inter* (LSBTI) in Schulbüchern. Eine gleichstellungsorientiere Analyse [mit einer Materialsamm-

46 Es braucht zudem rechtliche und politische Schritte, z.B. die Umsetzung von wirkungsvollen Maßnahmen gegen sogenannte »Konversionstherapien « und gegen alle Arten von LCBTIQA*-feindlich motivierter Cewalt sowie ein Verbot von geschlechtsnormierenden Eingriffen an intergeschlechtlichen Kindern und Jugendlichen ohne vorherige persönliche, voll informierte und freie Einwilligung. 
lung für die Unterrichtspraxis]. Frankfurt a.M.:. Gewerkschaft Erziehung und Wissenschaft.

Bittner, Melanie (2015): Die Ordnung der Geschlechter in Schulbüchern. Heteronormativität und Genderkonstruktionen in Englisch- und Biologiebüchern. In: Schmidt, Friederike/Schondelmayer, Anne-Christin/Schröder, Ute B. (Hg.): Selbstbestimmung und Anerkennung sexueller und geschlechtlicher Vielfalt. Lebenswirklichkeiten, Forschungsergebnisse und Bildungsbausteine. Wiesbaden: Springer VS, S. 247-260.

Damisch, Kerstin/Haller, Paul (2018): Sexuelle und geschlechtliche Vielfalt in der Schulsozialarbeit. Warum Regenbogenkompetenzen zu einer Kernkompetenz der Schulsozialarbeit werden müssen. SiÖ - Zeitschrift für Soziale Arbeit in Österreich, 2018 (1), S. 23-29.

Debus, Katharina/Laumann, Vivien (2018): Pädagogik geschlechtlicher, amouröser und sexueller Vielfalt. Zwischen Sensibilisierung und Empowerment. Dissens - Institut für Bildung und Forschung e.V. Online unter: https://interventionen.dissens.de/fileadmin/Interventionen/reda kteure/Dissens___PädagogikGeschlechtlicheAmouröseSexuelleVielfalt.p df [Zugriff: 17.05.2020].

Degele, Nina (2008): Gender/Queer Studies. Eine Einführung. Paderborn: Wilhelm Fink Verlag.

Dennert, Gabriele (o.J.): Gesundheit und Gesundheitsversorgung lesbischer und schwuler MigrantInnen in Deutschland. Heinrich-Böll-Stiftung. Online unter: https://heimatkunde.boell.de/2009/04/18/gesundheit-undgesundheitsversorgung-lesbischer-und-schwuler-migrantinnen-deutsch land [Zugriff: 17.05.2020].

Enzendorfer, Mart/Haller, Paul (2020): Intersex and Education: What Can Schools and Queer School Projects Learn from Current Discourses on Intersex in Austria? In: Francis, Dennis A./Kjaran, Jón Ingvar/Lehtonen, Jukka. (Hg.): Queer Social Movements and Outreach Work in Schools. Queer Studies and Education. Cham: Palgrave Macmillan, S. 261-284.

Enzendorfer, Mart (i.E.): »Das haben halt dann ein paar Lehrer gewusst« Inter* in pädagogischen Diskursen und ihre Grenzen des Sagbaren. In: Groß, Melanie/Niedenthal, Katrin (Hg.): Geschlecht: divers. »Dritte Option« im Personenstandsgesetz. Perspektiven für die Soziale Arbeit. Bielefeld: transcript.

Focks, Petra (2014): Lebenswelten von intergeschlechtlichen, transgeschlechtlichen und genderqueeren Jugendlichen aus Menschenrechtsperspektive. Expert"inneninterview. Berlin. Online unter: http://www.meingeschlech 
t.de/MeinGeschlecht/wp-content/uploads/Focks_Lebenswelten_Experti nneninterviews-_2014.pdf [Zugriff: 17.05.2020].

Franzen, Jannik/Sauer, Arn (2010): Benachteiligung von Trans*Personen, insbesondere im Arbeitsleben. Antidiskriminierungsstelle des Bundes. Berlin. Online unter: https://www.antidiskriminierungsstelle.de/Shared Docs/Downloads/DE/publikationen/Expertisen/Expertise_Benachteiligu ng_von_Trans_personen.pdf [Zugriff: 17.05.2020].

Fobian, Clemens/Ulfers, Rainer (2018): Präventionsarbeit und Beratung männlicher Betroffener sexueller Gewalt unter den Eindrücken anti-feministischer Diskurse. In: Lang, Juliane/Peters, Ulrich (Hg.): Antifeminismus in Bewegung. Aktuelle Debatten um Geschlecht und Sexuelle Vielfalt. Hamburg: Marta Press, S. 303-323.

Ghattas, Dan Christian (2016): Die Menschenrechte intergeschlechtlicher Menschen schützen - wie können Sie helfen? Herausgegeben von OII Europe und OII Germany. Online unter: https://oiigermany.org/wp-conten t/uploads/2017/11/toolkit_deutsch.pdf [Zugriff: 17.05.2020].

Ghattas, Dan Christian (2019): Protecting Intersex People in Europe: A Toolkit for Law and Policy Makers. With Digital Appendix and Checklist. OII Europe \& ILGA-Europe. Online unter: https://oiieurope.org/protectingintersex-people-in-europe-a-toolkit-for-law-and-policy-makers [Zugriff: 17.05.2020].

Gualdi, Miles/Martelli, Matteo/Wilhelm, Wolfgang/Biedroń, Robert/Graglia, Maghertia/Petrantoni, Luca (2008): Bullying in der Schule. Ein Leitfaden für LehrerInnen und Schulpersonal. Schoolmates. Online unter: ht tps://www.wien.gv.at/menschen/queer/pdf/bullying-schule.pdf [Zugriff: 17.05.2020].

Güldenring, Anette/Sauer, Arn (2017): Trans* ... inklusiv? Geschlechtsidentitäten in Medizin, Recht und Gesellschaft. In: Diehl, Elke (Hg.): Teilhabe für alle?! Lebensrealitäten zwischen Diskriminierung und Partizipation. Bonn: Bundeszentrale für politische Bildung, S. 231-257.

Haller, Paul/Schönpflug, Karin (2018): Ehe-Öffnung und »dritter Geschlechtseintrag«: Auftrag für die Soziale Arbeit? «In: SiÖ - Zeitschrift für Soziale Arbeit in Österreich, 2018/1, S. 51-54.

Hartmann, Jutta (2002): Vielfältige Lebensweisen. Dynamisierungen in der Triade Geschlecht - Sexualität - Lebensformen. Wiesbaden: Springer VS.

Hartmann, Jutta (2020): Heteronormativitätskritische Jugendarbeit - Pädagogische Professionalisierung zum Themenfeld >geschlechtliche und sexuelle Vielfalt‘. In: Timmermanns, Stefan/Böhm, Maika (Hg.): Sexuelle 
und geschlechtliche Vielfalt. Interdisziplinäre Perspektiven aus Wissenschaft und Praxis. Weinheim/Basel: Beltz Juventa, S. 136-153.

Hechler, Andreas (2016): »Was ist es denn?« Intergeschlechtlichkeit in Bildung, Pädagogik und Sozialer Arbeit. In: Katzer, Michaela/Voß, HeinzJürgen (Hg.): Geschlechtliche, sexuelle und reproduktive Selbstbestimmung. Praxisorientierte Zugänge. Gießen: Psychosozial Verlag, S. 161-185.

Henningsen, Anja (2016): Sexuelle Bildung und Gewaltprävention. Eine systematische Reflexion zur Prävention sexualisierter Gewalt in pädagogischen Kontexten. In: Henningsen, Anja/Tuider, Elisabeth/Timmermanns, Stefan (Hg.): Sexualpädagogik kontrovers. Weinheim/Basel: Beltz Juventa, S. 120-141.

IGLYO, International Lesbian, Gay, Bisexual, Transgender, Queer \& Intersex Youth and Student Organisation (o.J.): LGBTQI Inclusive Education Index. Austria. Online unter: https://www.education-index.org/country/austria [Zugriff: 17.05.2020].

Jones, Tiffany/Hart, Bonnie/Carpenter, Morgan/Ansara, Gavi/Leonard, William/Lucke, Jayne (2016): Intersex. Stories and Statistics from Australia. Cambridge: Open Book Publishers.

Kleiner, Bettina (2015): subjekt bildung heteronormativität. Rekonstruktion schulischer Differenzerfahrungen lesbischer, schwuler, bisexueller und Trans* Jugendlicher. Opladen/Berlin/Toronto: Verlag Barbara Budrich.

Kleiner, Bettina (2020): Lebenslagen von lesbischen, schwulen, bisexuellen, trans* und inter* geschlechtlichen sowie genderqueeren (Kindern und) Jugendlichen. In: Timmermanns, Stefan/Böhm, Maika (Hg.): Sexuelle und geschlechtliche Vielfalt. Interdisziplinäre Perspektiven aus Wissenschaft und Praxis. Weinheim/Basel: Beltz Juventa, S. 40-54.

Krell Claudia/Oldemeier, Kerstin (2015): Coming-out - und dann...?! Ein DJIForschungsprojekt zur Lebenssituation von lesbischen, schwulen, bisexuellen und trans* Jugendlichen und jungen Erwachsenen. München: Deutsches Jugendinstitut e.V. Online unter: https://www.dji.de/fileadmin/user _upload/bibs2015/DJI_Broschuere_ComingOut.pdf [Zugriff: 17.05.2020].

Krell, Claudia/Oldemeier, Kerstin (2017): Coming-out - und dann ...?! Coming-out-Verläufe und Diskriminierungserfahrungen von lesbischen, schwulen, bisexuellen, trans* und queeren Jugendlichen und jungen Erwachsenen in Deutschland. Opladen u.a.: Verlag Barbara Budrich.

Krell, Claudia/Oldemeier, Kerstin (2018): Queere Freizeit. Inklusions- und Exklusionserfahrungen von lesbischen, schwulen, bisexuellen, trans* und *diversen Jugendlichen in Freizeit und Sport. Deutsches Jugendinstitut 
e.V. Online unter: https://www.dji.de/fileadmin/user_upload/bibs2018/2 6869_DJI_QueereFreizeit.pdf [Zugriff: 17.05.2020].

Klöppel, Ulrike (2017): Der Dritte-Option-Beschluss und die Praxis kosmetischer Genitaloperationen an Kindern, 13.11.2017. Verfassungsblog.de. Online unter: https://verfassungsblog.de/der-dritte-option-besc hluss-und-die-praxis-kosmetischer-genitaloperationen-an-kindern [Zugriff:17.05.2020].

Klöppel, Ulrike (2019): Brennpunkt »uneindeutiges« Geschlecht - Beharrungsvermögen und Anpassungsfähigkeit der Medizin. In: Greif, Elisabeth (Hg.): No Lessons from the Intersexed? Anerkennung und Schutz intergeschlechtlicher Menschen durch Recht. Linzer Schriften zu Gender und Recht, Bd. 62. Linz: Trauner Verlag, S. 149-179.

Kugler, Thomas (2020): Geschlechtervielfalt in der Kita - Theorie und Praxis: Inklusion und Kinderrechte als menschenrechtlich fundierter Zugang einer genderbewussten Pädagogik. In: Timmermanns, Stefan/Böhm, Maika (Hg.): Sexuelle und geschlechtliche Vielfalt. Interdisziplinäre Perspektiven aus Wissenschaft und Praxis. Weinheim/Basel: Beltz Juventa, S. 122-135.

Mangold, Anna Katharina (2018): Stationen der Ehe für alle Deutschland, 09.08.2018. Bundeszentrale für politische Bildung. Online unter: https://www.bpb.de/gesellschaft/gender/homosexualitaet/274019/sta tionen-der-ehe-fuer-alle-in-deutschland [Zugriff: 17.05.2020].

Meyer, Illan H. (2007): Prejudice and Discrimination as Social Stressors. In: Meyer, Illan H./Northridge, Marry E. (Hg.): The Health of Sexual Minorities. Public Health Perspectives on Lesbian, Gay, Bisexual and Transgender Populations. New York: Springer, S. 242-267.

Nagy, Andrea (2016): Soziale Arbeit >queer denken. Zur Notwendigkeit der Auseinandersetzung mit Heteronormativität in der Ausbildung sozialer Professionen. In: soziales_kapital. Nr. 15 (2016). Online unter: https://sozia les-kapital.at/index.php/sozialeskapital/article/viewFile/427/763.pdf [Zugriff: 17.05.2020].

Nordt, Stephanie/Kugler, Thomas (2020): Sexuelle und geschlechtliche Vielfalt im Kontext frühkindlicher Inklusionspädagogik. In: Timmermanns, Stefan/Böhm, Maika (Hg.): Sexuelle und geschlechtliche Vielfalt. Interdisziplinäre Perspektiven aus Wissenschaft und Praxis. Weinheim/Basel: Beltz Juventa, S. 110-121.

Office of the High Commissioner of Human Rights (2015): Free \& equal campaign fact sheet: Intersex. United Nations. Online unter: http 
s:/www.unfe.org/wp-content/uploads/2017/05/UNFE-Intersex.pdf [Zugriff: 17.05 .2020$]$.

Oldemeier, Kerstin (2020): Coming-out-Verläufe und Freizeiterfahrungen von jungen lesbischen, schwulen, bisexuellen, trans* und divers* geschlechtlichen Menschen. In: Timmermanns, Stefan/Böhm, Maika (Hg.): Sexuelle und geschlechtliche Vielfalt. Interdisziplinäre Perspektiven aus Wissenschaft und Praxis. Weinheim/Basel: Beltz Juventa, S. 55-72.

Oldemeier, Kerstin/Timmermanns, Stefan (2020): Defizite und Ressourcen in den Lebenswelten von LSBTQ*-Jugendlichen und jungen Erwachsenen: zwei Seiten einer Medaille. In: Timmermanns, Stefan/Böhm, Maika (Hg.): Sexuelle und geschlechtliche Vielfalt. Interdisziplinäre Perspektiven aus Wissenschaft und Praxis. Weinheim/Basel: Beltz Juventa, S. 343-356.

Plett, Konstanze (2019): Männlich, weiblich, divers oder »ohne« - zum Personenstand intergeschlechtlich Geborener im deutschen Recht. In: Greif, Elisabeth (Hg.): No Lessons from the Intersexed? Anerkennung und Schutz intergeschlechtlicher Menschen durch Recht. Linzer Schriften zu Gender und Recht, Bd. 62. Linz: Trauner Verlag, S. 10-33.

Plöderl, Martin (2016): Out in der Schule? Bullying und Suizidrisiko bei LGBTI Jugendlichen. Suizidprophylaxe. 43, S. 7-13.

Plöderl, Martin (2020): Suizidrisiko bei LSBTI*. In: Henningsen, Anja/Tuider, Elisabeth/Timmermanns, Stefan (Hg.): Sexualpädagogik kontrovers. Weinheim/Basel: Beltz Juventa, S. 17-31.

Pöge, Kathleen/Dennert, Gabriele/Koppe, Uwe/Güldenring, Anette/Matthigack, Ev B./Rommel, Alexander (2020): Die gesundheitliche Lage von lesbischen, schwulen, bisexuellen, sowie trans- und intergeschlechtlichen Menschen. ... Online unter: https://www.rki.de/DE/Content/Gesundheit smonitoring/Gesundheitsberichterstattung/GBEDownloadsJ/JoHM_S1_ 2020_Gesundheitliche_Lage_LSBTI.pdf?_blob=publicationFile [Zugriff: 17.05.2020].

Profus, Andrzej (2016): Unsichtbares sichtbar machen. Asexualität als sexuelle Orientierung. In: Katzer, Michaela/Voß, Heinz-Jürgen (Hg.): Geschlechtliche, sexuelle und reproduktive Selbstbestimmung. Praxisorientierte $\mathrm{Zu}$ gänge. Gießen: Psychosozial Verlag, S. 225-242.

Queerformat e.V. (2015): Wie sie vielfältige Lebensweisen an Ihrer Schule unterstützen können. Teil 3: »Schwule Sau! « »Du Transe! « »Kampflesbe!« Was tun bei Beschimpfungen und Äußerungen. 4. überarbeitete Auflage. Online unter: https://www.queerformat.de/wp-content/uploads/mat _Broschuerenquartett_03_15.pdf [Zugriff: 17.05.2020]. 
Sauer, Arn/Meyer, Erik (2016): Wie ein grünes Schaf in einer weißen Herde. Lebenssituationen und Bedarfe von jungen Trans*-Menschen in Deutschland. Online unter: https://www.bundesverband-trans.de/portfolio-item /wie-ein-gruenes-schaf [Zugriff: 17.05.2020].

Scambor, Elli/Rieske, Thomas Viola/Wittenzellner, Ulla/Schlingmann, Thomas/Könnecke, Bernard/Puchert, Ralf (2019): Was hilft? Aufdeckungsprozesse bei männlichen Betroffenen von sexualisierter Gewalt in Kindheit und Jugend. In: Wazlawik, Martin/Voß, Heinz-Jürgen/Retkowski, Alexandra/Henningsen, Anja/Dekker, Arne (Hg.): Sexuelle Gewalt in pädagogischen Kontexten. Aktuelle Forschungen und Reflexionen. Wiesbaden: Springer VS, S. 109-123.

Schmauch, Ulrike (2015): Sexuelle Vielfalt und Regenbogenkompetenz in der Sozialen Arbeit. In: Bretländer, Bettina/Köttig, Michaela/Kunz, Thomas (Hg.): Vielfalt und Differenz in der Sozialen Arbeit. Perspektiven auf Inklusion. Stuttgart: Kohlhammer, S. 100-110.

Schmauch, Ulrike (2016): Sexualpädagogisches Handeln in der Sozialen Arbeit. In: Henningsen, Anja/Tuider, Elisabeth/Timmermanns, Stefan (Hg.): Sexualpädagogik kontrovers. Weinheim/Basel: Beltz Juventa, S. 32-45.

Schmauch, Ulrike (2020): Regenbogenkompetenz in der Sozialen Arbeit. In: Timmermanns, Stefan/Böhm, Maika (Hg.): Sexuelle und geschlechtliche Vielfalt. Interdisziplinäre Perspektiven aus Wissenschaft und Praxis. Weinheim/Basel: Beltz Juventa, S. 308-325.

Schönpflug, Karin u.a. (2015): „Queer in Wien«. Stadt Wien Studie zur Lebenssituation von Lesben, Schwulen, Bisexuellen, Transgender und Intersex Personen (LGBTIs). Projektbericht, WASt, IHS. Online unter: http s://www.wien.gv.at/menschen/queer/pdf/studie-queer-in-wien.pdf [Zugriff: 17.05.2020].

Spahn, Annika (2018): A_sexualität im Fachunterricht thematisieren. In: Spahn, Annika/Wedl, Juliette (Hg.): Schule lehrt/lernt Vielfalt. Praxisorientiertes Basiswissen und Tipps für Homo-, Bi-, Trans- und Inter*freundlichkeit in der Schule. Edition Waldschlösschen Materialien, S. 170-171. Online unter: https://www.waldschloesschen.org/files/Downloads/AWS_ MAT18_Schule_lehrt_lernt_Vielfalt_Bdi.pdf [15.05.2020].

Timmermanns, Stefan (2016): Vielfalt erwächst aus Freiheit. Zur theoretischen Verortung einer Sexualpädagogik der Vielfalt. In: Henningsen, Anja/Tuider, Elisabeth/Timmermanns, Stefan (Hg.): Sexualpädagogik kontrovers. Weinheim/Basel: Beltz Juventa, S. 17-31. 
TransX, Verein für Transgender Personen (o.J.): Rechtsentwicklung. Online unter: https://www.transx.at/Pub/Rechtsentwicklung.php [Zugriff: 17.05.2020]

Vasold, Stefanie (2016): Hemmungslos gegen Gleichheit. Wie reaktionäre Gruppen das Feld der sexuellen Bildung zum Austragungsort gegen gesellschaftlichen Fortschritt inszenieren. In: medien implse. Beiträge zur Medienpädagogik. Online unter: http://selbstlaut.org/wp-content/u ploads/2016/11/Medienimpulse_Hemmungslos_gegen_Gleichheit_Vasold _20160614.pdf [Zugriff: 17.05.2020].

VIMÖ - Verein intergeschlechtlicher Menschen Österreich (o.J.): Inter*, Intergeschlechtlich, Intersex, Zwischengeschlecht ...- was ist das? Online unter: https://vimoe.at/faq [Zugriff: 17.05.2020].

VIMÖ - Verein intergeschlechtlicher Menschen Österreich (2018): Stellungnahme des Vereins intergeschlechtlicher Menschen Österreich (VIMÖ) zur Umsetzung eines dritten Geschlechtseintrags im österreichischen Personenstand. Online unter: https:/vimoe.at/wp-content/up loads/2018/10/2018-10_Stellungnahme_VIMÖ_dritteOption.pdf [Zugriff: 17.05.2020].

Voß, Heinz-Jürgen (2010): Making Sex Revisited. Dekonstruktion des Geschlechts aus biologisch-medizinischer Perspektive. Bielefeld: transcript. Voß, Heinz-Jürgen (2014): Intergeschlechtlichkeit - Aktivismus und Forschung, ihre Verzahnung und intersektionale Fortentwicklung. In: Bundesstiftung Magnus Hirschfeld (Hg.): Forschung im Queerformat - Aktuelle Beiträge der LSBTI*-, Queer- und Geschlechterforschung. Bielefeld: transcript, S. 117-131.

Watzlawick, Meike (2020): Sexuelle Orientierungen und Geschlechtsidentitäten: Thinking outside the box. Überlegungen aus entwicklungspsychologischer Perspektive. In: Timmermanns, Stefan/Böhm, Maika (Hg.): Sexuelle und geschlechtliche Vielfalt. Interdisziplinäre Perspektiven aus Wissenschaft und Praxis. Weinheim/Basel: Beltz Juventa, S. 22-39.

WASt (o.J.): Definition sexueller Orientierungen: Homosexualität, Bisexualität, Heterosexualität. Online unter: https://www.wien.gv.at/menschen/q ueer/sexuelle-orientierung/definitionen.html [Zugriff: 17.05.2020].

Watzlawick, Meike (2004): Uferlos? Jugendliche erleben sexuelle Orientierungen. Aachen: Lambda: nrw.

Weingand, Hans-Peter (2011): Homosexualität und Kriminalstatistik in Österreich. In: Invertito - Jahrbuch für die Geschichte der Homosexualitäten, Jg. 13 (2011), S. 40-87. 
WHO-Regionalbüro für Europa und BZgA, Bundeszentrale für gesundheitliche Aufklärung (2011): Standards für die Sexualaufklärung in Europa. Rahmenkonzept für politische Entscheidungsträger, Bildungseinrichtungen, Gesundheitsbehörden, Expertinnen und Experten. Online unter: https://www.oif.ac.at/fileadmin/user_upload/p_oif/andere_ Publikationen/WHO-Standards_DE_Endfassung_11_10_2011pdf.pdf [Zugriff: 17.05.2020]. 



\section{Sexualbiografische Reflexionsarbeit als Element pädagogischer Professionalisierung}

Julia Kerstin Maria Siemoneit

Der vorliegenden Artikel plädiert für (sexual-)biografische Reflexionsarbeit als Teil pädagogischer Professionalisierung und widmet sich den Möglichkeiten und Grenzen biografischer Arbeit in der Lehrer:innenbildung. Hierzu wird anhand des Sprechens über Körper der Einfluss der eigenen Sexualbiografie auf pädagogisches Deuten, Verstehen und Handeln verdeutlicht und anhand des Begriffs der Lehrer:innenprofession konkretisiert.

Lehrer:innen verstehen und deuten den sexuellen Gehalt unterschiedlicher Situationen im Schulalltag auch auf der Basis ihrer eigenen Sexualbiografie. Die individuelle Sexualbiografie kann dann als Faktor für das Verstehen, die Bewertung von und den Umgang mit als sexuell oder intim wahrgenommenen Situationen, von Körperlichkeit sowie der Gestaltung pädagogischer Beziehungen in der Schule gelten. Dabei wird der Anteil der eigenen Erfahrung in der Deutung von Situationen nicht immer reflektiert. Angelehnt an das dargelegte Sexualitätsverständnis werden mögliche Reflexionsanstöße für die (sexual-)pädagogische Professionalisierung gegeben. Abschließend folgen Überlegungen zu den Chancen und Herausforderungen sexualbiografischer Reflexionsarbeit für die Aus- und Fortbildung von Lehrer:innen.

\section{Zur Verschränkung von biografischen Erfahrungen, kulturellen Deutungsmustern und pädagogischem Deuten und Verstehen}

Die sexualpädagogische Reflexionsarbeit als fortlaufende, die praktische Arbeit vorbereitende und begleitende Tätigkeit gehört zum professionellen Selbstverständnis der Sexualpädagogik dazu. Durch die Exploration der eigenen Sexualbiografie im Hinblick auf die Beziehungs-, Körper-, Bedürfnisund Geschlechtererfahrungen (s.u.) sollen die eigenen Normen und Werte, 
Fantasien und moralischen Vorstellungen expliziert und damit sichtbar gemacht werden (vgl. Osbar u.a. 1999: 83). Mittels der Begegnung mit den eigenen Handlungsmotiven, die das pädagogische Verstehen und Handeln bestimmen, wird reflexiv zugänglich, ob etwa das Verhalten von Kindern und Jugendlichen angemessen interpretiert und verstanden und die Deutungen in angemessene (sexual-)pädagogische Handlungsweisen überführt wurden. Damit soll das sheimliche bzw. versehentliche Hineinwirken persönlicher Ansichten moderiert werden, die vielleicht im Hinblick auf die eigene biografische Gewordenheit Sinn ergeben, aber nicht auf alle pädagogischen Situationen bzw. den speziellen pädagogischen Fall übertragbar sind.

Eine Biografie >existiert « nicht aus sich selbst heraus (s. Abschnitt 2). Biografien sind eingelassen in einen historisch, kulturell und sozial spezifischen gesellschaftlichen Rahmen, der etwa im Hinblick auf Konventionen, Normen und Werte orientierungsgebend ist. Diese Normen, Werte und Deutungsmuster finden Eingang in das Denken und Handeln von Gesellschaftsmitgliedern, fühlen sich >wahr $<$ an und lassen sich daher möglicherweise nur schwer einer kritischen Auseinandersetzung unterziehen. So bilden Deutungsmuster gewissermaßen die Folie, auf der Schulalltag interpretiert und verstanden wird, und somit das schulische Miteinander beeinflussen kann.

In von mit durchgeführten Interviews mit Gymnasiallehrkräften fiel etwa auf, dass Schülerinnenkörper als Bezugspunkt für Sexualität im schulischen Raum herangezogen und problematisiert wurden. ${ }^{1}$ Besonders deutlich war dabei, dass gerade Lehrer im Vergleich zu ihren Kolleginnen die Kleiderwahl einiger Schülerinnen als besonders »unangenehm« und »unangebracht« bewerteten. So befürchteten die Lehrkräfte zum einen auf der Folie einer heterosexuellen Begehrensmatrix ${ }^{2}$ in der Bekleidungspraxis der Schülerinnen ein Risiko für die Konzentrationsfähigkeit der Jungen und damit einhergehend deren Bildungsbenachteiligung. Darüber hinaus sahen sich Lehrer

$1 \quad$ Hierbei handelt es sich um ein Zwischenergebnis aus meinem Forschungsprojekt, in welchem anhand von Lehrer:inneninterviews das Verhältnis von Schule und Sexualität untersucht wird (vgl. Siemoneit 2021).

2 Dies bezeichnet die Norm, dass Menschen jeweils als Mann oder Frau gekennzeichnet werden und jeweils gegengeschlechtlich begehren. Damit bleiben sämtliche Geschlechter und Begehrensformen, die nicht der heterosexuellen Norm entsprechen, unberücksichtigt (vgl. Butler 2014 [1991]: 22ff.). 
durch die Schülerinnenkörper in der Gestaltung ihrer pädagogischen Beziehung im Hinblick auf das Aufrechterhalten von Distanz eingeschränkt, weshalb in der Folge Schülerinnen bisweilen mit beschämenden Bemerkungen oder direktiven Ermahnungen zu einer anderen Bekleidungspraxis diszipliniert wurden.

Beides, sowohl die Aufrechterhaltung der Leistungsfähigkeit der Jungen als auch der pädagogischen Beziehung zu den Lehrern, setzten jeweils am Körper der Schülerinnen an. Hier haben sich geschlechtsspezifische soziale Deutungsmuster in das Verstehen und Handeln von Lehrkräften eingearbeitet, die an gewohnte Denktraditionen zu Geschlecht anschließen und damit die Sexualisierung von Situationen durch Sozialität erst ermöglichen.

Lehrer markieren Schülerinnenkörper als ein pädagogisches Problem und stellen damit den Körper in der Schule als ein bearbeitungswürdiges Problem her. Denn Körper an sich können kein >Problem`sein, sondern werden dies erst, wenn sie auf der Folie sozial verfügbarer Wissensbestände zu Geschlecht gelesen und interpretiert und etwa als »unangenehm « oder »unangebracht« erlebt werden. So ist der Körper bedeutungslos ohne den sexualisierenden Blick auf die Mädchen- bzw. Schülerinnenkörper. Ohne diese Lesart würde er auch nicht mit den Leistungsanliegen und den Beziehungsformationen von Schule in Konflikt geraten. Damit wird der Bekleidungs- und Entscheidungsautonomie von Mädchen gegenüber `blickenden Mitschülern und Lehrern ein geringerer Wert eingeräumt. Was sich auf diese Weise in der Schule vermitteln könnte, sind Geschlechterkonstruktionen, die Jungen- bzw. Männersexualität als unkontrollierbar und striebhaft aktualisieren und mit der Forderung nach >entsexualisierender Disziplinierung des weiblichen Körpers einhergehen.

Über sexualbiografische Reflexionsarbeit ist es möglich, sich solche Geschlechterkonstruktionen vor dem Hintergrund der eigenen biografischen Erfahrungen in einer spezifisch verfassten Gesellschaft zu vergegenwärtigen und diese für den eigenen sexualisierenden Blick und damit auch das eigene pädagogische Tun kritisch zu befragen. 


\section{Berufs- und Sexualbiografien von Lehrer:innen als konstitutives Element der pädagogischen Beziehung und des Sprechens über Sexualität}

Die Bearbeitung von sexualitätsbezogenen Themen - hierunter kann etwa das Sprechen über Sexualität im (Sexualerziehungs-)Unterricht verstanden werden oder aber auch der Umgang mit Körpern in der Schule (s.o.) u.v.m. - kann das pädagogische Verhältnis in ein intimisiertes, diffuses und partikularistisches Adressierungsgefüge verschieben, was mit der Norm der Affektneutralität pädagogischer Beziehungen brechen könnte. Die Herausforderung besteht darin, dass Sexualität der privaten und intimen Sphäre zugerechnet wird, demnach als besonders nähestiftend gilt und mit den institutionalisierten Rollen von Lehrer:innen und Schüler:innen im öffentlichen Raum konfligiert. Der Anspruch an professionelles Handeln im pädagogischen Kontext ist im Kontrast zu laienhaftem Alltagshandeln jedoch, »Nähe und Distanz zu seinen Adressaten und deren Problemen auf kunstvolle Weise zu verschränken und miteinander zu vermitteln« (Müller 2012: 145).

Im Folgenden wird einem Verständnis der Lehrer:innenprofession gefolgt, welches zunächst das (1) Unterrichten als Kerngeschäft beschreibt, d.h. an der erfolgreichen Umsetzung dieser Kernaufgabe bemisst sich im Letzten die Qualität einer Lehrperson. Darüber hinaus wird das pädagogische Tun, zu dem auch, aber nicht nur das Unterrichten zählt, als (2) unhintergehbar verschränkt mit der Person der Lehrperson selbst angesehen sowie (3) eine klient:innenorientierte Unterstützung in Krisen- und Umbruchsituationen angeboten (vgl. Bonnet/Hericks 2014; Oevermann 1996). Im Folgenden werden vor allem die pädagogisch umfassenden Merkmale der Unhintergehbarkeit der eigenen Person sowie der Klient:innenzentrierung mit den jeweiligen sexualpädagogischen Anforderungen verbunden und im Hinblick auf ihre pädagogischen Implikationen ausgelotet. Sichtbar wird dabei, dass professionalisiertes pädagogisches Tun auch bedeutet, Rückgriffe auf die Sexualbiografie und sexualbiografische Wissensbestände kontinuierlich zu beobachten und zu moderieren.

Charakteristisch für die Lehrer:innenprofession ist, dass das pädagogische Denken und Tun unhintergehbar mit der (Privat-)Person selbst verwoben ist. In der Vermittlungspraxis schlagen sich das Denken, Sprechen und Fühlen von Lehrer:innen nieder. Bezogen auf Sexualität bzw. Sexualerziehung bedeutet dies, dass im pädagogischen Handeln Normen und Werte, persönli- 
che Standpunkte und Anliegen sichtbar werden, die für jene Lehrperson von Bedeutung sind, kurz: Das pädagogische Tun leitet sich (auch) aus den eigenen Überzeugungen $a b$. Von Sexualität als intimem und persönlichem Thema sind Lehrkräfte stets >betroffen`. Ihr pädagogisches Handeln läuft insofern Gefahr zu deprofessionalisieren, als sich das Handeln auf das biografisch >Eigene bezieht und die Bedürfnislagen von Schüler:innen dahinter zurücktreten könnten. Erst die reflexive Begegnung und Auseinandersetzung mit dem >Eigenen< ermöglicht auch die Unterscheidung zum >Anderen .

Aus psychoanalytisch orientierter Perspektive stehen Pädagog:innen immer vor zwei Kindern zugleich: dem Kind vor ihnen, das erst noch in die Logik des rollenförmigen Handelns hineinsozialisiert werden müsse, ohne etwa Zurückweisungen als Kränkungen seiner Gesamtperson aufzufassen, und dem Kind in ihnen mit den eigenen (unerfüllten) Bedürfnissen und Wünschen: »Die eigene Anerkennungsgeschichte [...] bildet genau insofern einen Resonanzboden für drohende Verstrickungen in der Interaktion mit Kindern und Jugendlichen, die diffuse Beziehungsmuster befördern und emotionale Beziehungsdynamiken der Lehrperson aktualisieren können.« (Helsper/Reh 2012: 273)

Wenn Lehrer:innen also kein reflektiertes Verhältnis zu ihrer eigenen Beziehungs- und Sexualbiografie pflegen, besteht das Risiko, dass sie ihre eigene (berufs- und sexual-)biografische Anerkennungsgeschichte fortlaufend re-inszenieren und ihre pädagogischen Beziehungen vorwiegend im Modus ihrer eigenen biografischen Erfahrung gestalten. Umgekehrt können auch die biografischen Erfahrungshaushalte ressourcenorientierter als "privates biografisches Kapital (Brüsemeister 2004: 57) perspektiviert werden. So können etwa biografische Parameter wie Herkunft, Geschlecht, Begehren usw. pädagogisch reflektiert in die pädagogische Beziehung oder das Sprechen über Sexualität eingebracht werden. Aufgrund dieser Unhintergehbarkeit der eigenen Person, ihrer Überzeugungen, Normen und Werte und nicht zuletzt ihrer Wissensbestände ist es umso bedeutender, sich im Rahmen einer wissensbasierten (sexual-)biografischen Reflexionsarbeit der eigenen Geschichte zuzuwenden und somit das >Eigene $<$ vom >Anderen $<\mathrm{zu}$ trennen. Das bedeutet, eigene biografische Themen sowohl aus den pädagogischen Beziehungen als auch aus dem Sprechen über Sexualität herauszulösen oder aber dieses umschriebene Erfahrungswissen pädagogisch reflektiert einzusetzen. In diesem Sinne leitet sich die besondere Herausforderung sexualpädagogischer Professionalisierung sowohl aus der pädagogischen 
Praxis und Beziehungsführung als auch aus der Besonderheit der Sexualität selbst ab (vgl. Burchardt 2000: 190).

Daraus ergibt sich ein zweifaches Deprofessionalisierungsrisiko: Es betrifft zum einen die Gestaltung der pädagogischen Beziehung und zum anderen das biografische, verfügbare sexualitätsbezogene (Erfahrungs-)Wissen über Sexualität, in das sich implizite gesellschaftliche Deutungsmuster oder persönliche Erfahrungen unbemerkt eingearbeitet haben, die Einfluss nehmen auf das Deuten und Verstehen von Schüler:innenverhalten sowie auf die Entwicklung von Handlungsantworten.

Das pädagogische Tun ist unhintergehbar mit der Lehrperson als Individuum und Privatperson verknüpft und zugleich repräsentieren und verkörpern Lehrkräfte mit ihrer hegemonialen Sprecher:innenposition gegenüber den Schüler:innen - sowie gegenüber allen weiteren schulischen Akteur:innen wie etwa Eltern - (symbolisch) die Schule sowie das Bildungssystem. Letztlich definieren Lehrkräfte über ihre Deutungshoheit in der Schule und den schulischen Interaktionen, was in der Schule in Hinblick auf Sexualität als (un-)sagbar, verhandelbar oder sanktionierbar gilt. So ist es schon zum Allgemeinplatz geworden, dass Sexualaufklärungsunterricht an deutschen Schulen vorwiegend über genuin biologische Themen wie etwa Geschlechtsorgane, Empfängnisverhütung und sexuell übertragbare Krankheiten aufklärt, moralisch aufgeladene und umkämpfte Themen wie Homosexualität, Schwangerschaftsabbruch, Zärtlichkeit, Liebe und Lust sowie emotional belastende und potenziell überfordernde Themen wie sexuelle Gewalt und Missbrauch demgegenüber etwa $50 \%$ seltener thematisiert werden (vgl. Heßling/Bode 2015: 36). Diese Dethematisierung kann dazu führen, dass Schule von Heranwachsenden nicht als Ort erlebt wird, an dem sich solcher mitunter emotional belastenden Themen und der Verantwortung für den Schutz von Heranwachsenden angenommen wird (Themenbereich: sexuelle Gewalt, Diskriminierung etwa von nicht-heterosexuellem Begehren) oder Heranwachsende als sexuelle Wesen mit sexuellen Bedürfnissen anerkannt werden (Themenbereich: Zärtlichkeit, Liebe, Lust).

Ein weiteres Professionsmerkmal von Lehrer:innen sei, dass das Lehrer:innenhandeln klient:innenzentriert auf die Befähigung von Schüler:innen im Umgang mit Krisen- bzw. Umbruchsituationen abgestellt ist (vgl. Bonnet/Hericks 2014: 4f.). Dabei kann Lehrer:innenhandeln selbst auch krisenauslösend sein: So können Lehrer:innen als Krisenauslöser:innen eine Aufgabe stellen, die etwa zu Frust auf der Schüler:innenseite führen kann, oder Leistungsbewertungen können von Schüler:innen als Attacke auf 
ihre Gesamtperson gedeutet und als verletzend und entwürdigend erlebt werden (vgl. Helsper 2012: 28). Darüber hinaus begleiten Lehrer:innen ihre Schüler:innen aber auch bei der Problem- bzw. Aufgabenbewältigung.

Zentral für das Merkmal der Klient:innenzentrierung ist zudem, die pädagogische Beziehung bei der Krisenbewältigung als Vertrauens- und Abhängigkeitsverhältnis zu denken, weshalb der Abstinenzregel eine besondere Bedeutung beigemessen wird (vgl. Oevermann 1996: 177ff.). Somit müssen Lehrkräfte im Rahmen der Krisenbewältigung Fragen, Anliegen und Verhalten von Heranwachsenden stets im Horizont der entwicklungsspezifischen Bedürfnisse und Aufgaben ihrer Schüler:innen rekonstruieren und anerkennen, eigene Bedürfnisse und Anliegen treten dahinter zurück. Würde nach eigenen Bedürfnissen und persönlichen Anliegen gedeutet und gehandelt, wäre dies vor dem Hintergrund des Macht- und Abhängigkeitsverhältnisses als unprofessionell einzustufen. Gerade vor dem Hintergrund vielfältiger sexuell konnotierter Situationen im Schulalltag sind sie fortlaufend gefordert, beobachtetes Verhalten, Gefühle, Bedürfnisse etc. altersangemessen einzuordnen und zugleich die schulischen Abläufe zu sichern (vgl. Siemoneit/Verlinden 2021).

Unter der Prämisse der Befähigung zur Krisenbewältigung und Abstinenzregel verfügen Lehrkräfte über ein »lizensiertes Interventions- und Eingriffsrecht (Radtke 1999: 15) in die Lebenspraxis und personale Integrität von Heranwachsenden, wodurch die pädagogische Beziehung mitunter deutlich an Nähe gewinnen kann. Dies bedeutet, dass Lehrkräfte z.B. bei Liebeskummer trösten, bei sexualisierter Gewalt begrenzen bzw. schützen oder amourös-diffundierende Näheanfragen gegenüber ihrer Person von Seiten der Schüler:innen erkennen und benennen, aber verständnisvoll abweisen. Die dadurch entstehende Nähe ist allein durch die Klient:innenzentrierung und die Unterstützung bei ihrer Krisenbewältigung zu rechtfertigen und damit notwendige Voraussetzung für ebendiese.

Mit der Bearbeitung sexualitätsbezogener Themen in der pädagogischen Beziehung kann Intimität entstehen, wodurch das Nähe-Distanz-Verhältnis potenziell aus dem Gleichgewicht geraten und zu diffus und nah werden kann. Angesichts dieser Deprofessionalisierungsgefahr kommt in der pädagogischen Beziehung der Abstinenzregel eine besondere Bedeutung $\mathrm{zu}$, die die auf die Erwachsenenwelt angewiesenen Schutzbefohlenen vor Nähebedürfnissen, welche Pädagog:innen über ihre Schüler:innen zu stillen suchen könnten, schützen soll (vgl. Helsper 2012: 29; Oevermann 1996: 118). 


\section{Theoriegeleitete sexualbiografische Reflexionsanstöße}

Sexualität existiert nicht allein aus den Selbstverständlichkeiten einer unverrückbaren >Natur und Biologie heraus, sondern ist immer eingelassen in kulturell verfügbare Deutungsmuster und Wissensbestände, auf deren Grundlage Individuen ihre Sexualität gestalten. Darüber entscheidet sich, was als sexuell erlebt wird, welchen Handlungen welche Bedeutungen zugewiesen und welche Riten, Kleidung und Praktiken mit Sexualität verknüpft werden. Wie Körper und Fantasien für Sexualität >nutzbar gemacht bzw. als erotisiert und sexuell erlebt werden, wird damit kulturell und individuell sehr verschieden definiert (vgl. Sigusch 2011; 2013: 24f.).

Im Wechselspiel zwischen Biologie und Kultur sind für das Individuum vier Erfahrungsbereiche herauszustellen, die als konstitutiv für eine Sexualbiografie gelten: die Erfahrungen, die Menschen mit ihren Bedürfnissen, der Moderation von Spannungsaufbau und -abbau sowie der Kommunikation ihrer Bedürfnisse machen, und wie hierauf von der Umwelt Bezug genommen wird (Bedürfnisgeschichte); das Erleben des Körpers und Leibes, der Sinne sowie der (Un-)Möglichkeiten, den Körper für sinnliche Wahrnehmung und Genuss zu nutzen (Körpergeschichte); die Erfahrungen, die aus jeder Beziehung - bereits auch den frühesten - hervorgehen und zwischen den Polen von Bindung und Exploration, von Nähe und Distanz, Sorge vor dem Verlassenwerden und Verschmelzungsdrängen aufgespannt sind (Beziehungsgeschichte).

Schließlich werden die Erfahrungen im Rahmen der je spezifischen geschlechtlichen und sexuellen Identität gemacht, die sich im Spannungsfeld gesellschaftlich (un-)verfügbarer Geschlechtervorstellungen bewegt und ausgestaltet wird (Geschlechtergeschichte) (vgl. Schmidt 1986: 77ff.; Schmidt 2004). Diese Erfahrungen werden erst im Laufe der Pubertät sexualisiert, d.h. es bilden sich auf dem Boden dieser Erfahrungsgeschichte sexuelle Skripte heraus, Fantasien, Wünsche, Vorlieben etc., die eine sexuelle Bedeutung erlangen und ein Leben lang veränderbar bleiben (vgl. Gagnon/William 1973/2005).

In Orientierung an diesen vier sexualbiografischen Erfahrungsbereichen möchte ich im Folgenden erste Denkanstöße und -richtungen für die Einzelarbeit vorstellen. Sie können aber auch als Gesprächsanlässe für die angeleitete Arbeit in Kleingruppen dienen, da diese mehr Möglichkeiten zur Entdeckung und Erkundung des >Eigenen`, gerade in Abgrenzung zu sanderen` (Sexual-)Biografien, eröffnen können. Alle folgenden Segmente können als Explorationsfelder für die übergeordnete Frage, wie innerhalb der eigenen Herkunftsfamilie mit Sexualität, Körpern und Sinnlichem umgegangen wur- 
de, gelesen werden. Daraus ergibt sich auch, welche Themen als besprechbar und welche - vielleicht bis heute - als unsagbar galten.

- Beziehungsgeschichte: Wie ist das Beziehungsverhalten der eigenen Eltern $\mathrm{zu}$ beschreiben? Welche Parallelen gibt es $\mathrm{zu}$ dem eigenen Beziehungsleben und wie sind vor allem Abweichungen davon zu erklären? Welche Hürden wurden genommen und was ist herausfordernd geblieben? Wie viel Nähe und wie viel Autonomie sind subjektiv erwünscht? An welche Mythen über Beziehung hat man eine Zeit lang geglaubt und wie ist das aus heutiger Sicht zu erklären?

- Körpergeschichte: Wie wurde mit Körper, Nacktheit, Körperformen und Körperberührungen in der Herkunftsfamilie umgegangen? Welche Normen von und welcher Umgang mit Körper haben sich vermittelt? Welche Botschaften haben gefehlt und woher wurden bzw. werden diese Botschaften bezogen? Welche Grenzen wurden gewahrt bzw. überschritten?

- Geschlechtergeschichte: Welche Eigenschaften sind subjektiv mit dem eigenen Geschlecht verknüpft? An welchen Alltagspraktiken macht sich dies bemerkbar? Was bedeutet dies für die Perspektive auf Beziehung, Begehren und Sexualität? Welche Möglichkeiten und Grenzen ergeben sich aus dieser Verknüpfung?

- Bedürfnisgeschichte: Welche Erfahrungen wurden im Umgang mit der Erfüllung eigener Bedürfnisse in der Herkunftsfamilie gemacht? Welche Erfahrungen mit Genuss, Kontrollverlust, Selbstbefriedigung etc. wurden gemacht? Das Stillen welcher Bedürfnisse ist gegenwärtig (un-)möglich und warum? (Vgl. Wanzeck-Sielert 2004: 15; Osbar u.a. 1999: 89f.)

Darauf aufbauend kann ferner gefragt werden, inwieweit die entwickelten Antworten in die pädagogische Arbeit hineinwirken, den Blick auf das schulalltägliche Geschehen beeinflussen und ob in diesem Verstehen auch die entwicklungsspezifischen Bedürfnislagen anerkannt und von eigenen Bedürfnissen getrennt werden. Um noch einmal auf das eingangs aufgegriffene Beispiel aus den Interviews mit Lehrkräften zurückzukommen: Unter Rückgriff auf Diskurse aus feministischer Theorie und Politik könnte sich aus einer wissensfundierten Distanz mit dem sexualbiografisch gewordenen eigenen Blick auf Körper auseinandergesetzt werden. Denn diese sexualbiografische Gewordenheit in einer spezifisch verfassten Gesellschaft bringt bestimmte Lesarten von Geschlecht hervor, die nicht identisch sein müssen mit dem, was etwa Schülerinnen mit ihrer Kleidung zum Ausdruck bringen möchten. 
Nicht zuletzt liegt hierin ein Potenzial, verschiedene Facetten von Sexualität sprechbar zu machen, ohne eigene Grenzen zu überschreiten.

Auch wenn sexualbiografische Reflexionsarbeit für andere Professionen wie etwa Psychotherapeut:innen die Regel ist, fehlt ihr im Bereich der Lehrer:innenbildung bislang die Tradition, weshalb ihr auch ein gewisser »Zumutungscharakter« (Valtl 2003: 84) attestiert wird. Angesichts der Debatten um sexuelle Gewalt in pädagogischen Kontexten, der Erarbeitung institutioneller Schutzkonzepte sowie der Notwendigkeit professionsethischer Überlegungen und Anforderungen an Pädagog:innen ist jedoch fraglich, ob es sich die (universitäre) Lehrer:innenbildung leisten kann, die Verwobenheit von Sexualbiografie mit pädagogischem Handeln weiterhin auszusparen. Es ist zu vermuten, dass die meisten angehenden Lehrer:innen mit ihrem Studium eher die fachdidaktische Vorbereitung auf das Unterrichten verbinden und daher die Bereitschaft zu sexualbiografischer Reflexionsarbeit nicht unbedingt vorauszusetzen ist. Daher ist zumindest zu diskutieren, ob ohne Freiwilligkeit sexualbiografische Reflexionsarbeit zum einen an ethische Grenzen stößt, weil sie auch auf die intimste, persönliche Lebenswelt zurückgreift und die betreffende Person damit konfrontiert, oder ob dies zum anderen in einem angemessenen Verhältnis zu Deprofessionalisierungsrisiken steht, die wahlweise auf Kosten der Schüler:innen (s.o.) oder der Lehrer:innen selbst gehen können, wenn etwa eigene Erfahrungen reinszeniert werden und auch zu einer hohen Belastung führen können.

Die außergewöhnlich hohe Nachfrage bei sexualbiografischen bzw. sexualpädagogischen Seminaren, die sich etwa durch die hohen Bewerbungszahlen und das Bedauern über die insgesamt geringe Anzahl solcher Seminare in den Seminarevaluationen ausdrückt, könnten als Hinweis auf eine prinzipiell hohe Bereitschaft zur freiwilligen Teilnahme an Seminaren dieser Art gedeutet werden. Gerade durch das Festlegen von Regeln für freiwillige Beteiligungen sowie einen wertschätzenden, diskriminierungsfreien Umgang miteinander zu Beginn der Seminare einerseits und Möglichkeiten zu geschützteren Reflexionsarbeiten andererseits - z.B. durch reflective writing sessions, in denen Studierende textgebundene Reflexionsarbeiten verfassen - werden verschiedene Dimensionen von Beteiligung ermöglicht.

Pädagogische Beziehungen zwischen Lehramtsstudierenden und Lehrenden - ob an der Universität oder den schulpraktischen Zentren - sind durch leistungsbezogene Benotungsverfahren strukturiert, die den Prozess der Biografiearbeit irritieren können. Hier könnten von Beurteilung losgekoppelte Angebote durch externe Lehrbeauftragte eine Alternative schaffen. Der Fort- 
bildungsbereich bietet dabei den Vorteil, dass sich Seminarleitung und Lehrkräfte eher auf Augenhöhe begegnen können.

Sexualbiografische Reflexionsarbeit als Element (sexual-)pädagogischer Professionalisierung ist ein voraussetzungsvolles Unterfangen. Gleichzeitig liegt unter Berücksichtigung der genannten Einwände hierin die Chance, das >Eigene als solches zu erkennen und in ein Verhältnis zu dem >Anderen $\mathrm{zu}$ setzen, sodass diese beiden möglichst nicht unbewusst ineinanderfließen. Die Präzisierung pädagogischer Deutungsprozesse und pädagogischen Fallverstehens erfolgt auf dem Boden einer verbesserten Orientierung innerhalb der eigenen Sexualbiografie und ist damit als Professionalisierungsgelegenheit für das pädagogische Tun zu verstehen.

\section{Literatur}

Barbaras, Friedrich (2013): Jugendrecht und Sexualerziehung. In: Schmidt, Renate-Berenike/Sielert, Uwe (Hg.): Sexualpädagogik und sexuelle Bildung 2. Auflage. Weinheim/Basel: Beltz Juventa, S. 536-546.

Bonnet, Andreas/Hericks, Uwe (2014): Professionalisierung und Deprofessionalisierung im Lehrer/innenberuf. Ansätze und Befunde aktueller empirischer Forschung. In: Zeitschrift für interpretative Schul- und Unterrichtsforschung, Nr. 3, S. 3-13.

Brüsemeister, Thomas (2004): Das andere Lehrerleben. Lehrerbiographien und Schulmodernisierung in Deutschland und in der Schweiz. Bern/Stuttgart/Wien: Haupt.

Burchardt, Eva (2000): Persönlichkeitslernen. In: Sielert, Uwe/Valtl, Karlheinz (Hg.): Sexualpädagogik lehren. Didaktische Grundlagen und Materialien für die Aus- und Fortbildung. Weinheim/Basel: Beltz, S. 190-232.

Butler, Judith (1991/2014): Das Unbehagen der Geschlechter, 17. Auflage. Frankfurt a.M.: Suhrkamp.

Gagnon, John H./Simon, William (2005 [1973]): Sexual conduct. The Social Sources of Human Sexuality. New Brunswick/London: Aldine Transaction.

Gudjons, Herbert/Wagener-Gudjons, Birgit/Pieper, Marianne (2008): Auf meinen Spuren. Übungen zur Biografiearbeit, völlig neu überarb. u. akt. Auflage. Bad Heilbrunn: Julius Klinkhardt.

Helsper, Werner (2012): Die Antinomie von Nähe und Distanz in unterschiedlichen Schulkulturen. Strukturelle Bestimmungen und empirische Ein- 
blicke. In: Nerowski, Christian/Hascher, Tina/Lunkenbein, Martin/Sauer, Daniela (Hg.): Professionalität im Umgang mit Spannungsfeldern der Pädagogik. Bad Heilbrunn: Julius Klinkhardt, S. 27-46.

Helsper, Werner/Reh, Sabine (2012): Nähe, Diffusität und Asymmetrie in pädagogischen Interaktionen. Herausforderung pädagogischer Professionalität und Möglichkeitsräume sexualisierter Gewalt in der Schule. In: Thole, Werner/Baader, Meike/Helsper, Werner/Kappeler, Manfred/Leuzinger-Bohleber, Marianne/Reh, Sabine/Sielert, Uwe/Thompson, Christiane (Hg.): Sexualisierte Gewalt, Macht und Pädagogik. Opladen u.a.: Verlag Barbara Budrich, S. 265-290.

Heßling, Angelika/Bode, Heidrun (2015): Jugendsexualität 2015. Die Perspektive der 14- bis 25-Jährigen. Ergebnisse einer aktuellen repräsentativen Wiederholungsbefragung. Köln: BZgA.

Hilgers, Andrea (2004): Richtlinien und Lehrpläne zur Sexualerziehung. Eine Analyse der Inhalte, Normen, Werte und Methoden zur Sexualaufklärung in den sechszehn Ländern der Bundesrepublik Deutschland. Köln: Bundeszentrale für gesundheitliche Aufklärung.

Kultusministerkonferenz (KMK) (1968): Sammlung der Beschlüsse der Ständigen Konferenz der Kultusminister der Länder in der Bundesrepublik Deutschland. 3. Auflage, Bd. 3, Nr. 659. Neuwied/Darmstadt: Hermann Luchterhand.

Maß, Reinhard (2011): Evolutionspsychologie der Sexualität. In: Zeitschrift für Sexualforschung, Jg. 24, Nr. 2, S. 105-110.

Müller, Burkhard (2012): Nähe, Distanz, Professionalität. Zur Handlungslogik von Heimerziehung als Arbeitsfeld. In: Dörr, Margret/Müller, Burkhard (Hg.): Nähe und Distanz. Ein Spannungsfeld pädagogischer Professionalität, 3. akt. Auflage. Weinheim/München: Beltz Juventa, S. 145-162.

Oevermann, Ulrich (1996): Theoretische Skizze einer revidierten Theorie professionalisierten Handelns. In: Combe, Arno/Helsper, Werner (Hg.): Pädagogische Professionalität. Untersuchungen zum Typus pädagogischen Handelns. Frankfurt a.M.: Suhrkamp, S. 70-182.

Osbar, Christian/Specht, Ralf/Wanzeck-Sielert, Christa/Bartels, Ilme/Beck, Heinz-Peter/Großkreutz, Ellen/Waltke, Michael (1999): Sexualpädagogik zwischen Persönlichkeitslernen und Arbeitsfeldorientierung. Unterrichtsmaterialien für die sozialpädagogische Ausbildung. Köln: Bundeszentrale für gesundheitliche Aufklärung.

Radtke, Frank-Olaf (1999): Autonomisierung, Entstaatlichung, Modularisierung. Neue Argumente in der Lehrerbildungsdiskussion? In: Ders. (Hg.): 
Lehrerbildung an der Universität. Zur Wissensbasis pädagogischer Professionalität. Frankfurter Beiträge zur Erziehungswissenschaft. Frankfurt a.M.: Fachbereich Erziehungswissenschaft der Johann-WolfgangGoethe-Universität, S. 11-25.

Schmidt, Gunter (1986): Das große Der Die Das. Über das Sexuelle. Hemsbach über Weinheim: März.

Schmidt, Gunter (2004): Kindersexualität - Konturen eines dunklen Kontinents. In: Zeitschrift für Sexualforschung, Jg. 17, S. 312-312.

Siemoneit, Julia Kerstin Maria (2021): Sexualität und Schule. Eine Deutungsmusteranalyse von Interviews mit Gymnasiallehrer*innen, Bielefeld: transcript.

Siemoneit, Julia Kerstin Maria/Verlinden, Karla (2021, i.E.): Sexuelle Situationen in der Schule - Ergebnisse einer Befragungsstudie in NRW. In: Eiberger, Meike/Masurek, Martina/Nikodem, Claudia (Hg.): Diskurse zu Geschlecht, Sexualität und sexualisierter Gewalt. Heterogene Beiträge aus Wissenschaft und Praxis. Opladen u.a.: Verlag Barbara Budrich.

Sigusch, Volkmar (2011): Kritik evolutionspsychologischer Sexualforschung. In: Zeitschrift für Sexualforschung, Jg. 24, Nr. 3, S. 279-291.

Sigusch, Volkmar (2013): Sexualitäten. Eine kritische Theorie in 99 Fragmenten. Frankfurt a.M./New York: Campus.

Valtl, Karlheinz (2003): Sexuelle Biographie. In: Bundeszentrale für gesundheitliche Aufklärung (BZgA) (Hg.): Rahmencurriculum Sexualpädagogische Kompetenz. Qualifizierungsmaßnahmen im Bildungs-, Sozial- und Gesundheitswesen. Köln: BZgA, S. 83-110.

Wanzeck-Sielert, Christa (2004): »Eigentlich ist es der schönste Unterricht überhaupt!« Die Rolle des >Lehr-Körpers in der Sexualerziehung. In: Die Grundschulzeitschrift, Nr. 178, S. 12-15. 



\section{Wie hast du dir in deiner Kindheit Sexualität vorgestellt? Erfahrungen und Zeichnungen aus einem Workshop zu Körper, Sexualität und Intimität}

Christine Aebi

Die Zeichnungen zu diesem Textbeitrag stammen aus dem Workshop »DAS machen? DAS zeichnen?«, der im Mai 2019 am Institut für das künstlerische Lehramt der Akademie der bildenden Künste Wien stattfand. Die halbtägige Veranstaltung war Teil des Projekts Imagining Desires und richtete sich an (angehende) Lehrer:innen, Sexualpädagog:innen, Kunstvermittler:innen und andere Interessierte.

Die Idee des Workshops bestand darin, zweierlei zu verbinden: Zum einen sollte er einen Einblick in den Schaffensprozess zum Buch »DAS machen? Projektwoche Sexualerziehung in der Klasse 4C« (Axster/Aebi 2012) ermöglichen. Zum anderen zielte er darauf ab, die Teilnehmenden dazu zu ermutigen, sich Themen wie Körperlichkeit, Sexualität und Intimität zeichnerisch anzunähern. ${ }^{1}$

\section{Sexualität zeichnen?}

In der Planung des Workshops war es mir wichtig, den gemeinsamen Arbeitsprozess des Zeichnens in den Mittelpunkt zu stellen. Ein Ausgangspunkt war die Annahme, dass es für viele Menschen nicht leicht ist, über Themen wie Körper, Sexualität und Intimität zu sprechen. Zudem vermutete ich, dass es

1 Auf dieser Basis - so die Überlegung - lassen sich Ideen entwickeln, wie das Medium Zeichnen in der sexual- und kunstpädagogischen Praxis eingesetzt werden könnte. 
bei manchen Teilnehmenden Hemmschwellen beim Zeichnen geben könnte - insbesondere in einer Gruppe, in der sich nicht alle kennen. Gleichzeitig hoffte ich, dass das gemeinsame Interesse am Thema Verbindungen schaffen würde. Meine Vorbereitung war an der Frage orientiert, wie Vertrauen entstehen und zeichnerische Experimentierfreude geweckt werden kann.

Konzeptionell orientiert sich der Workshop am Bilderbuch »DAS machen? «, dessen Entstehungsprozess von dem Wunsch getragen wurde, ein Buch zu machen, das wir Verfasserinnen als Kind gerne in die Hand genommen hätten. Im Fokus des Buches stehen Fragen zu kindlicher Sexualität, wobei es jedoch nicht darum geht, zu erklären und Wissen zu vermitteln, sondern Räume zum Entdecken aufzumachen. ${ }^{2}$ Auch der Workshop ist nicht vorrangig darauf ausgerichtet, Expertinnenwissen zur Verfügung $\mathrm{zu}$ stellen, sondern Suchbewegungen $\mathrm{zu}$ initiieren und gemeinsam auf Entdeckungsreise zu gehen. Ziel ist, sich auf Augenhöhe zu begegnen.

Wir starten mit einer Vorstellungsrunde in den Workshop: »Was führt mich hierher und mit welchen Fragen bin ich gekommen? ${ }^{3}$ Die Antworten zeigen, dass die Teilnehmenden sich aus unterschiedlichen Beweggründen für diesen Workshop entschieden haben. Sexualpädagoginnen sind daran interessiert, zu erfahren, wie das Zeichnen in ihre Arbeit einbezogen werden kann, andere eher an den Illustrationen und am Entstehungsprozess eines Bilderbuchs. Manche Teilnehmer:innen äußern Bedenken gegenüber dem bevorstehenden Zeichnen, weil sie sich darin ungeübt fühlten. ${ }^{4}$ Explizite Widerstände sind keine zu spüren, vielmehr eine vorsichtige Bereitschaft, sich auf unbekanntes Terrain zu begeben.

Die Autorin Lilly Axster und ich haben das Bilderbuch prozesshaft und über Jahre erarbeitet, indem wir uns gegenseitig Bild- und Textentwürfe hin- und hergeschickt, aufeinander reagiert, uns gegenseitig inspiriert und kritisiert haben.

Die eingebrachten Fragen werden auf einem Flipchart festgehalten. Manche Fragen beantworten sich im gemeinsamen Tun von selbst, die übriggebliebenen werden am Schluss des Workshops beantwortet - entweder von mir oder von Teilnehmenden mit fachspezifischem Wissen. mischt. Während einige aus ihrem Studium Erfahrungen mitbrachten, hatten andere keinerlei Vorkenntnisse. 


\section{Erste Zeichenrunde: Was habe ich mir als Kind unter S.E.X. vorgestellt?}

Bevor wir ins Zeichnen einsteigen, erzähle ich von den ersten Schritten der Entstehung des Bilderbuchs »DAS machen? - Projektwoche Sexualkunde in der Klasse 4C«: Ich habe zunächst versucht herauszufinden, was ich mit kindlicher Sexualität verbinde und was mich bezüglich der Darstellung von Körpern, Sexualität und Intimität interessieren würde. ${ }^{5}$ Dabei habe ich mich auch gefragt, welche Vorstellungen ich mir als Kind gemacht habe. Genau diese Frage ist der Ausgangspunkt für die erste Erinnerungssammlung und die erste Zeichenrunde im Workshop: "Was habe ich mir als Kind unter S.E.X. ${ }^{6}$ vorgestellt? Was habe ich damit in Verbindung gebracht?« Ich bitte die Teilnehmenden, ihre Erinnerungen zu diesen Fragen in ein bis zwei Sätzen auf kleine Zettel zu schreiben. Die eingesammelten Zettel werden gemischt und auf Tischblöcke verteilt, an denen die Teilnehmenden Platz nehmen. Nach dem Zufallsprinzip ziehen die Teilnehmenden nun Zettel mit Sätzen und sind aufgefordert, diese spontan auf Kartonkarten im DIN-A5-Format zu illustrieren.

Nach einer Zeichenphase von etwa 40 Minuten legen die Teilnehmenden ihre Zeichnungen mit den dazugehörigen Sätzen im Raum aus. Jede Person wählt ein bis zwei Skizzen aus, die sie besonders ansprechen, und die im Plenum besprochen werden. Deutlich wird dabei die sichtbare Vielfalt der Erinnerungen wie auch erstaunliche Gemeinsamkeiten. So erinnern sich z.B. mehrere Teilnehmende an Teddybären, mit denen sie in der Kindheit Vorstellungen von Sex spielerisch in Szene gesetzt haben. Der Austausch in der Gruppe ist von Ernsthaftigkeit und Tiefe geprägt, während gleichzeitig eine heitere Leichtigkeit spürbar ist.

In der Auseinandersetzung mit diesen Fragen ist umfangreiches Skizzenmaterial entstanden. Lilly Axster sichtete und ordnete es und entwickelte daraus eine Grundstruktur für die Erzählung: Zum einen stellen wir uns die Frage nach den Themen und dem `Fahrplan der Lehrerin, die eine Projektwoche Sexualerziehung für ihre Klasse plant. Zum anderen gab es die Figur der Ich-Erzählerin, eine Schülerin, die von der Projektwoche erzählt. Wir haben uns gefragt, woran sie sich erinnert und was ihr wichtig geworden ist. Welche Fragen stellt sie sich? Was beschäftigt sie? Im Aufbau des Buches selber werden diese beide Ebenen - die Ebene der Ich-Erzählung und jene des geplanten Ablaufs der Lehrerin - miteinander verknüpft.

6 Die Schreibweise S.E.X. ist dem Bilderbuch »DAS machen?« entnommen. S.E.X. wird häufig von Kindern benutzt, um das Wort »Sex« nicht aussprechen zu müssen. 


\section{Zweite Zeichenrunde: Welche Fragen zum Thema Sexualität haben mich als Kind beschäftigt?}

Nach einer Pause stelle ich anhand ausgewählter Entwürfen vor, wie sich einzelne Buchseiten aus »DAS machen?« entwickelt haben. Mit den ersten Doppelseiten des Buches, in denen es explizit um kindliche Vorstellungen von Sexualität geht, zeige ich, welche Lösungen wir als Autorinnen zu diesem Thema gefunden haben. ${ }^{7}$

Eine weitere Doppelseite, die sich mit Kinderfragen zum Thema Sexualität befasst, ist der Anknüpfungspunkt für die Frage zur zweiten Erinnerungsund Zeichenrunde: »Welche Fragen zum Thema Sexualität haben mich als Kind beschäftigt? « Die Fragen, die den Teilnehmenden einfallen, werden wiederum auf kleine Zettel notiert, gemischt und auf die Tische verteilt. Gezeichnet werden nun entweder die Fragen oder mögliche Antworten.

Abschließend haben die Teilnehmer:innen die Möglichkeit ihre Zeichnungen zu den Fragen aufzulegen und gemeinsam zu sichten. Eine Art von Vernissage-Stimmung kommt auf, die Vielfalt der Arbeiten ist inspirierend. Ich bin überrascht über das umfangreiche und vielschichtige Material, das wir in kurzer Zeit zusammengetragen haben.

Die folgende Schlussrunde des Workshops ermöglicht einen Austausch darüber, was allen Beteiligten an diesem Nachmittag wichtig gewesen oder geworden ist. Im Mittelpunkt steht, was zeichnerisch sichtbar und auch besprechbar gemacht werden kann. Die Teilnehmer:innen bringen ihre Zufriedenheit zum Ausdruck, dass eine vertiefte Auseinandersetzung mit einem anspruchsvollen Thema stattgefunden hat. Sie äußern, dass es hilfreich gewesen ist, nicht die eigenen Erinnerungen illustrieren zu müssen. Das Zufallsprinzip hat auch den Vorteil, dass die Notizen auf den Zetteln anonymisiert werden. Wichtig ist im Austausch nicht, wer was geschrieben und gezeichnet hat. Im Mittelpunkt stehen vielmehr die gemeinsam zustande gekommenen Skizzen, die gedanklichen Verknüpfungen und die Anlässe für Austausch und Reflexion, die durch die Zeichnungen möglich werden.

7 Die ausgewählten Entwürfe machen den Entwurfsprozess hinter einzelnen Illustrationen bzw. Doppelseiten sichtbar. Ich habe dabei Ausdrucksmöglichkeiten gesucht für die Vielstimmigkeit einer Kindergruppe, für kindliche Körper, Kleidung und Nacktheit, für Intimität und Schamgrenzen, ohne voyeuristisch zu werden und den Kindern zu nahe zu treten. 


\section{Fazit}

Der Austausch war lebendig, ernsthaft und doch auch heiter. In einer offenen und vertrauensvollen Atmosphäre konnte sich die thematische Arbeit entfalten. Dass dies möglich geworden ist, liegt wohl auch am spielerischen Charakter der Zeichenaufgaben, die sowohl einen Rahmen geboten als auch Spielraum gelassen haben. Die Teilnehmenden fanden Zugang zu eigenen Erinnerungen und Bildern. Sie wurden dazu ermutigt, zeichnerische Fertigkeiten im Zusammenhang mit den Themen »Körper, Sexualität und Intimität« als spielerisches Mittel einzusetzen. Das unmittelbare Sichtbar- und Besprechbarmachen der eigenen Erinnerungen und Vorstellungen stand im Vordergrund und nicht der Anspruch auf den perfekten zeichnerischen Ausdruck.

Wichtig ist die Erkenntnis, dass Illustrationen auch dann aussagekräftig sind, wenn man scheinbar nur über wenig Erfahrungen und Mittel verfügt. Manche Illustrationen bekommen ihre Prägnanz oder ihren Witz gerade dadurch, dass sie stark vereinfacht und auf Wesentliches reduziert sind.

Die folgenden Zeichnungen sind im Workshop »DAS machen? DAS zeichnen?« von Christine Aebi am 17. Mai 2019 am Institut für das künstlerische Lehramt an der Akademie der bildenden Künste Wien entstanden.

Abb. 7: Zeichung von Cornelia Lindner

Abb. 8: Zeichnung von Sarah Fichtinger

Abb. 9-11: Zeichnungen von Workshopteilnehmer:innen

Abb. 12: Zeichnung von Anna Scheer

Abb. 13: Zeichnung von Workshopteilnehmer:innen

Abb. 14: Zeichnung von Stanislaus Medan

Abb. 15: Zeichnung von Marion Bugelnig-Berger

Abb. 16: Zeichnung von Workshopteilnehmer:innen

Abb. 17-18: Zeichnungen von Johanna Fürlinger

Abb. 19: Zeichnung von Stanislaus Medan

Abb. 20: Zeichnung von Sarah Fichtinger

Abb. 21-22: Zeichnungen von Workshopteilnehmer:innen

Abb. 23: Zeichnung von Marion Bugelnig-Berger

Abb. 24: Zeichnung von Sarah Fichtinger

Abb. 25: Zeichnung von Johanna Fürlinger

Abb. 26: Zeichnung von Luca Haibach

Abb: 27: Zeichnung von Workshopteilnehmer:innen 


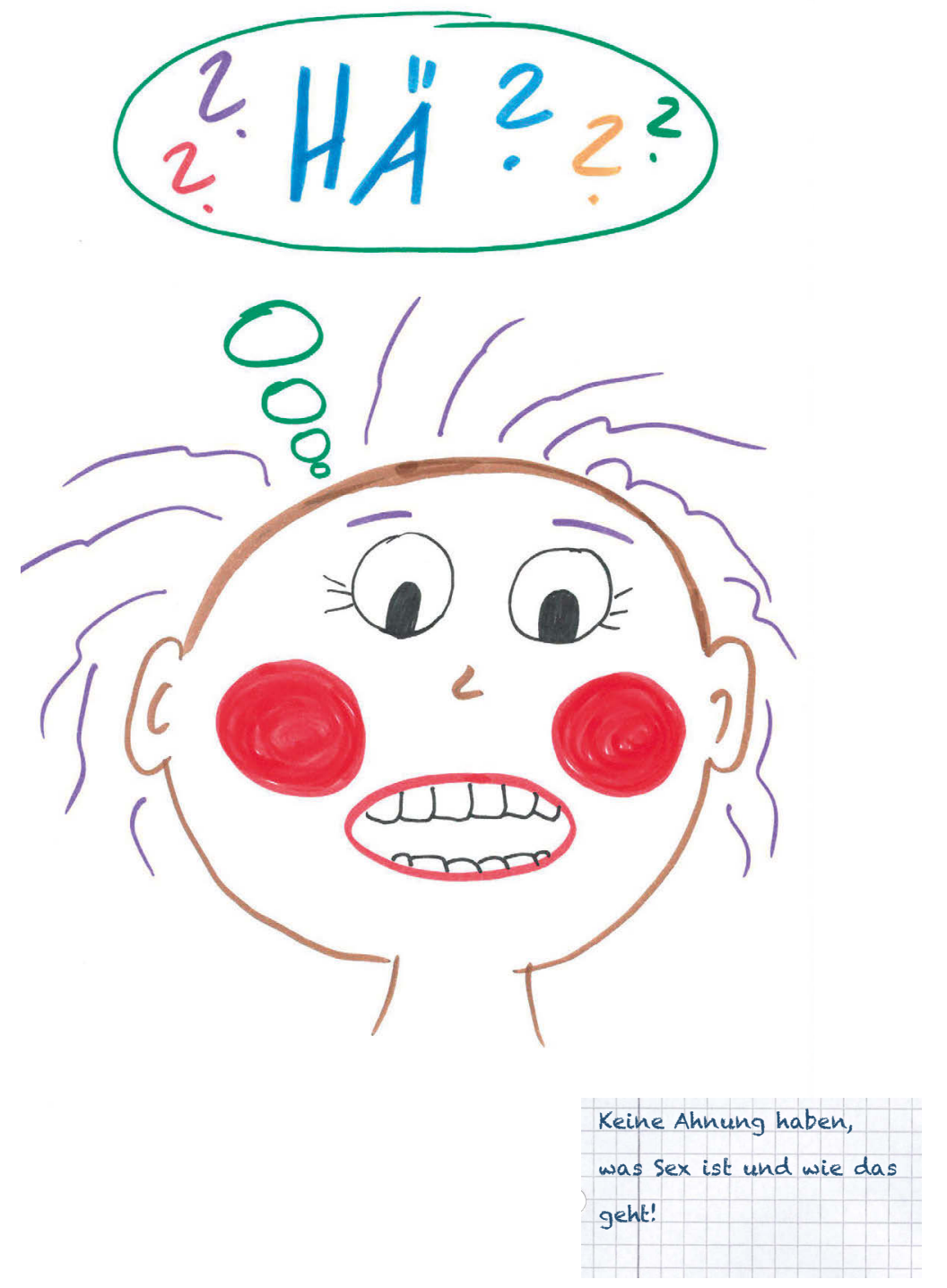

Abb. 7 


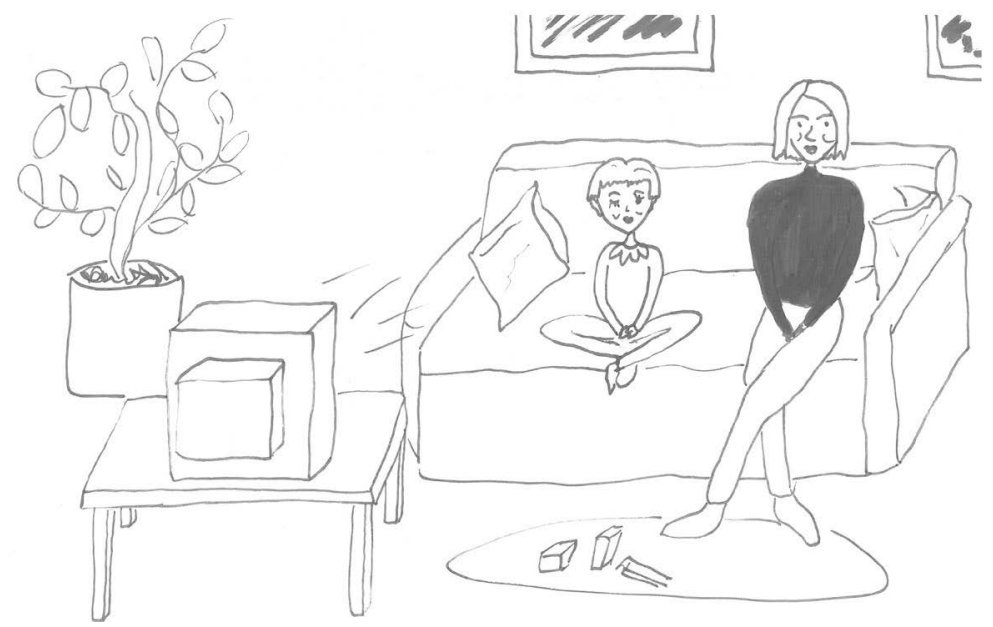

Abb. 8

\section{Bei der Sexszene von Titanic so ge-}

tan, als würde ich schlafen. (Aber

fasziniert zugeschaut)
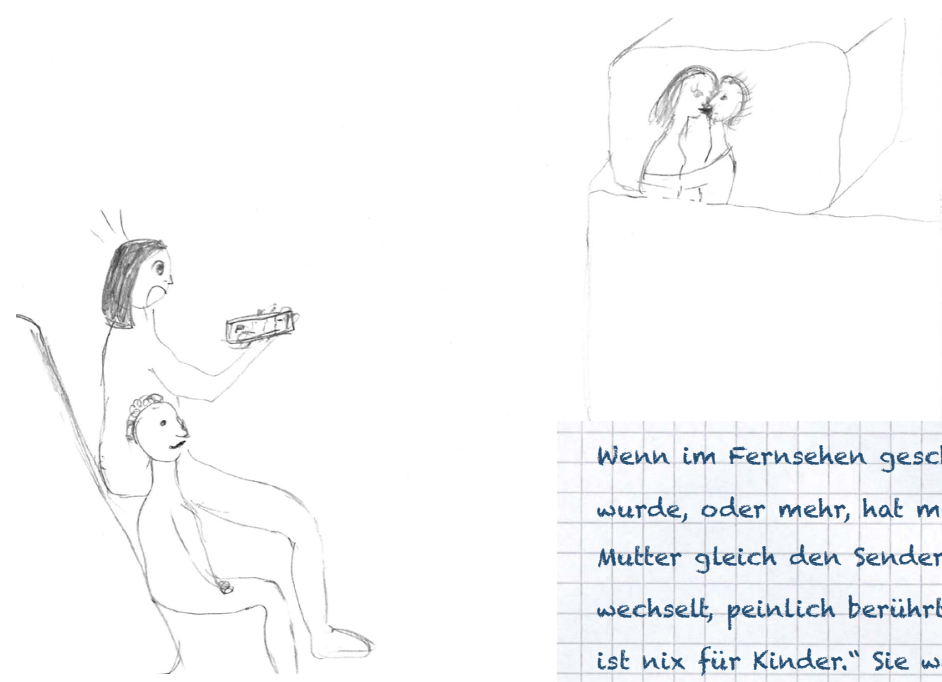

Abb. 9

Wenn im Fernsehen geschmust wurde, oder mehr, hat meine Mutter gleich den Sender gewechselt, peinlich berührt? "Das ist nix für Kinder." Sie wollte uns schützen und so lange wie möglich Kind sein Lassen. 


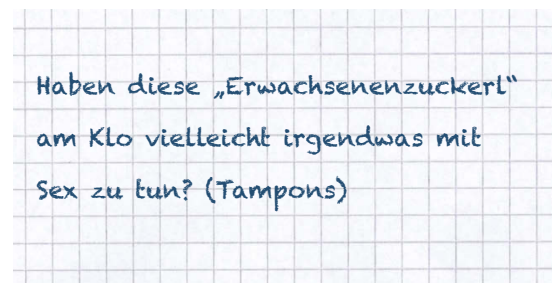



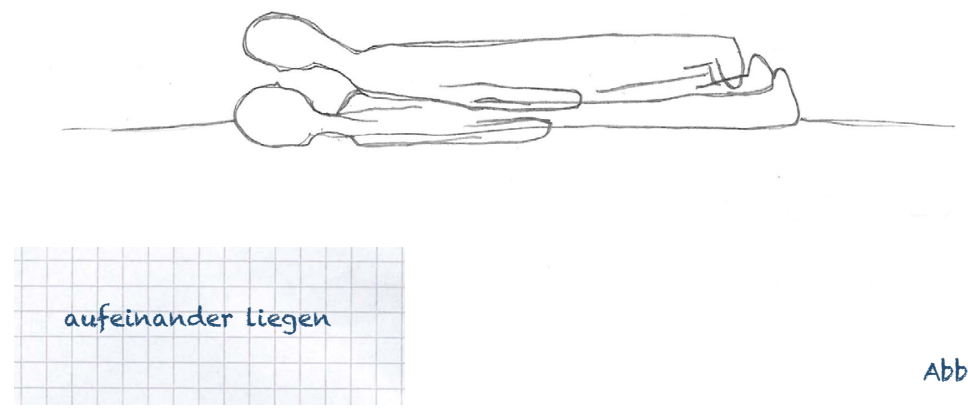

Abb. 11
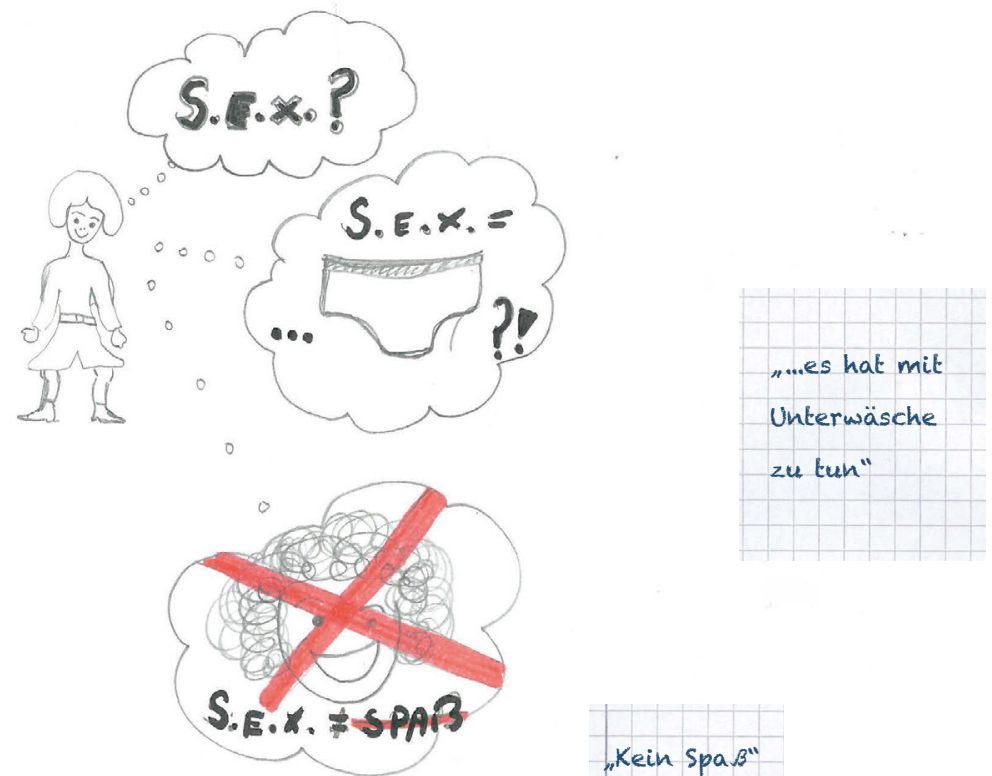

Abb. 12 


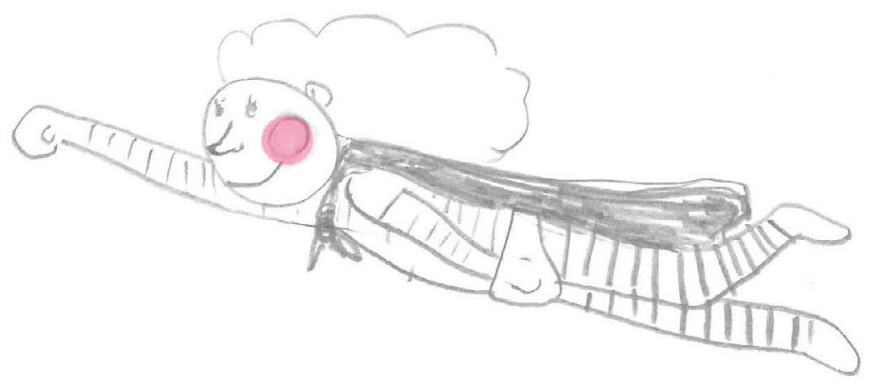

Abb. 13

Ich habe schon recht früh zu masturbieren begonnen

(Ende Kindergarten/Anfang

Volksschule) und wusste

dabei gar nicht, was das ist.

Deshalb dachte ich, dass nur

ich das kann und es sowas wie eine Superkraft sein muss.

(Wobei es auch manchmal schambesetzt war, da eben nie jemand darüber geredet hat) 

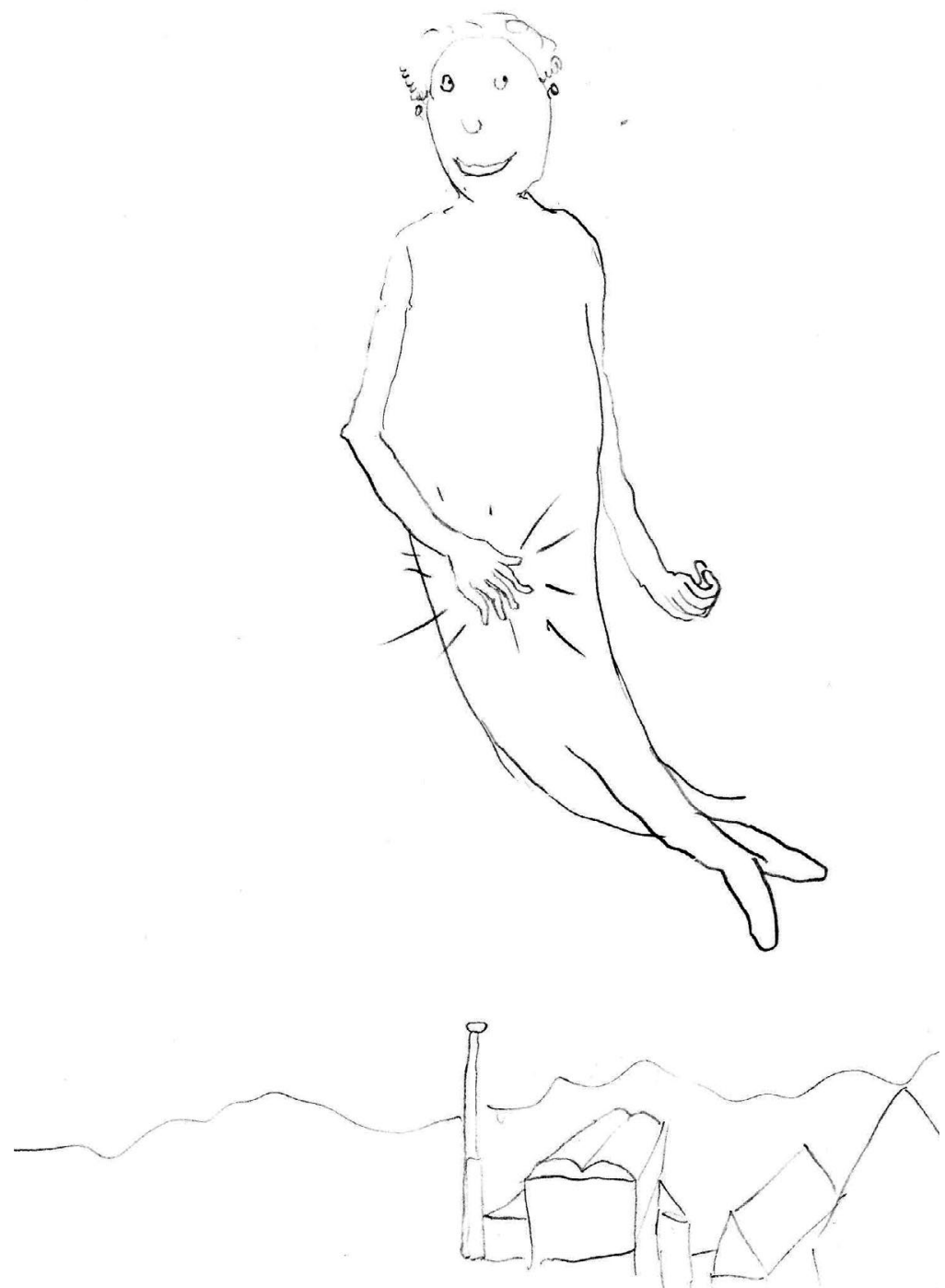

Abb. 14 


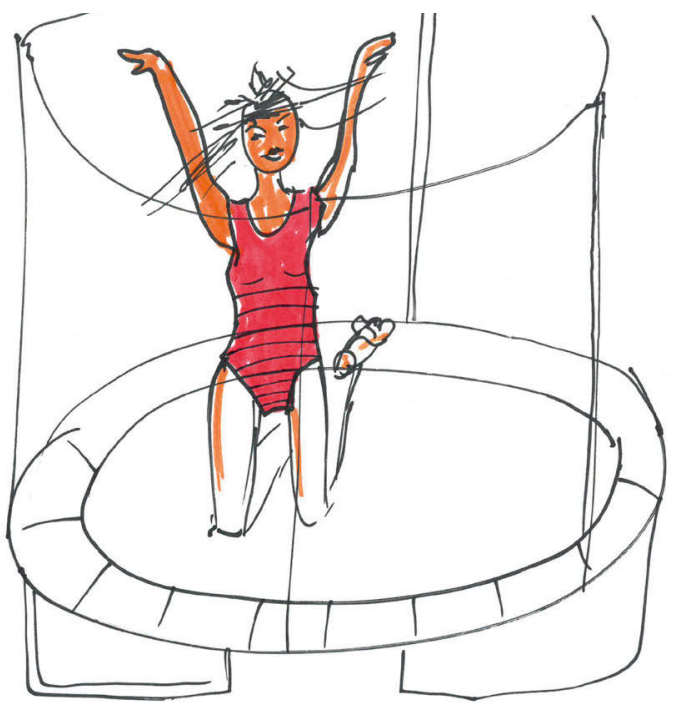

Abb. 15

Warum fühlt es sich beim

Trampolin-springen im

Gummiahzug so gut ah?

Whirlpool - Wasserdrüsen

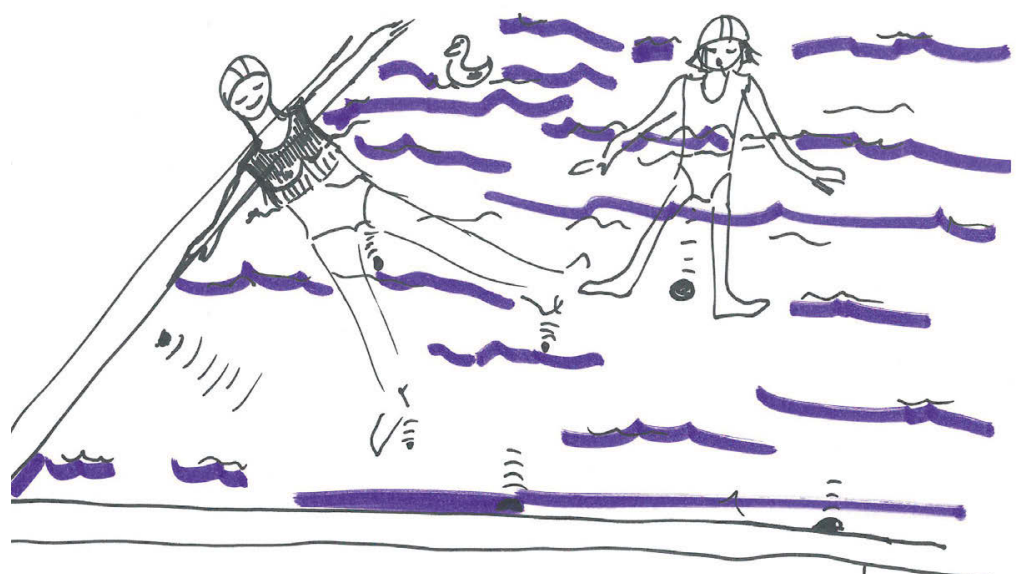

Abb. 16 


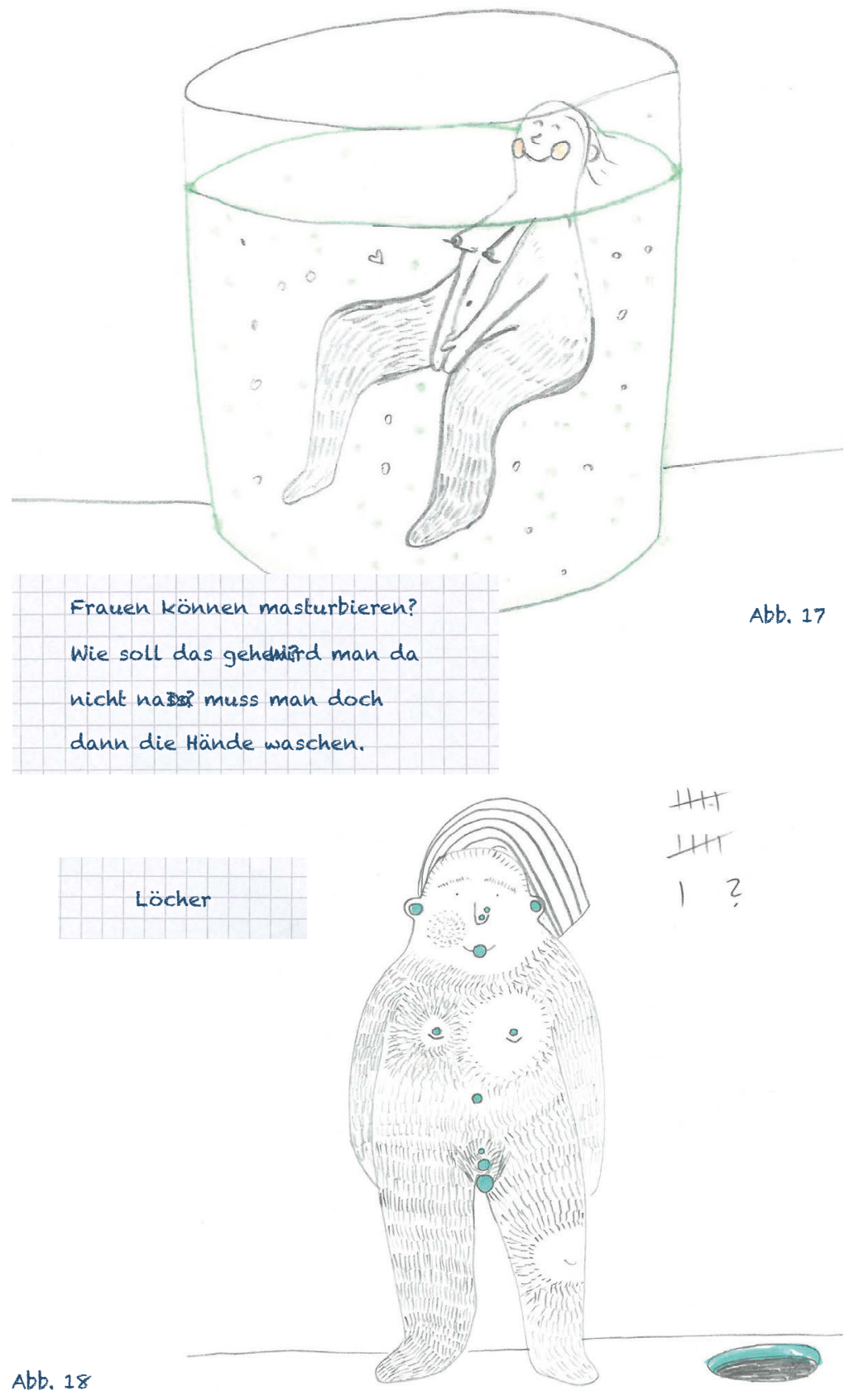




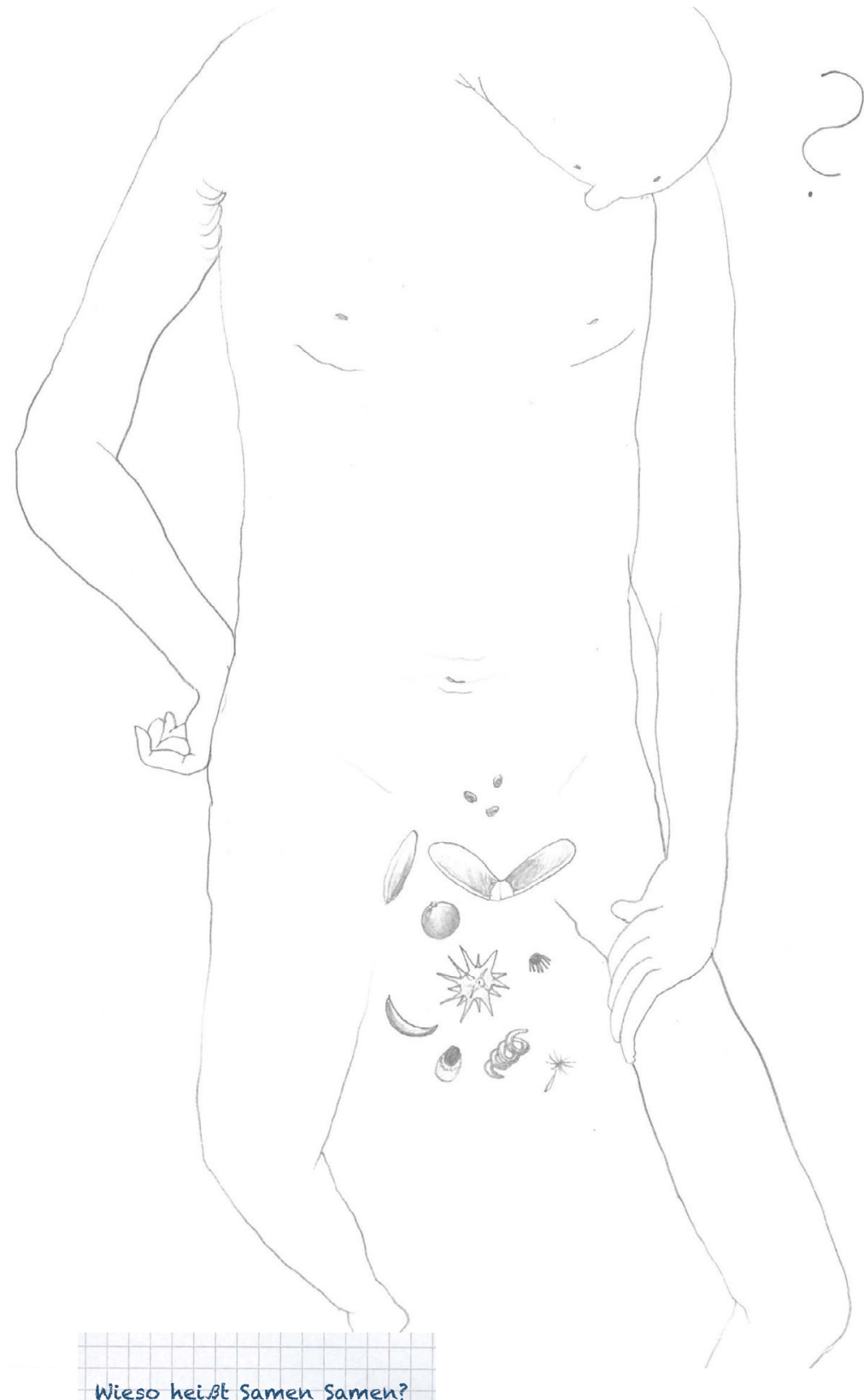

Abb. 19 


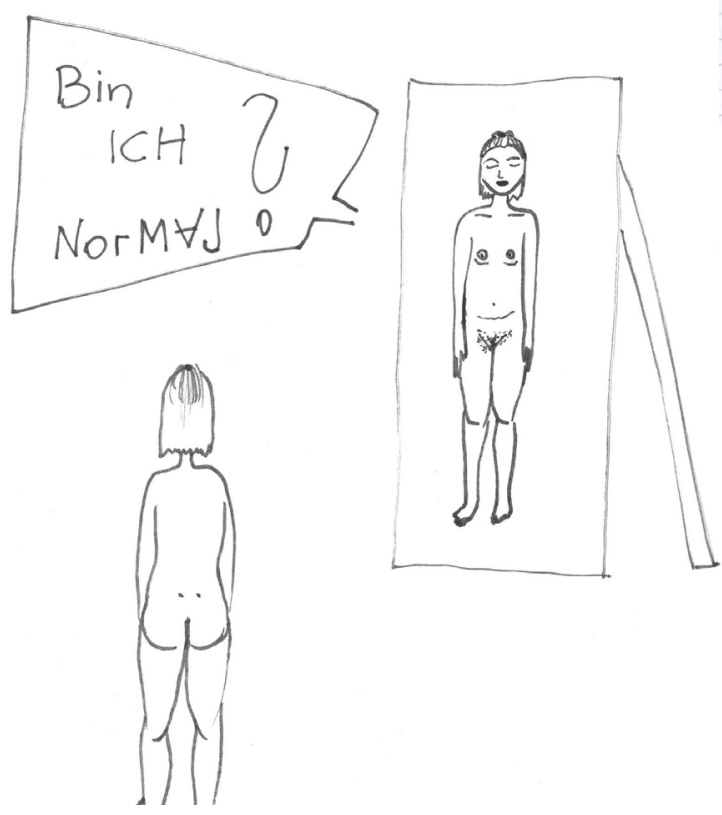

Bin ich normal?

"mileinander schlafen" als "im selben Bett schlafen" - dadurch ganz logisch auch 2 Frauen, 2 Männer...

Abb. 20

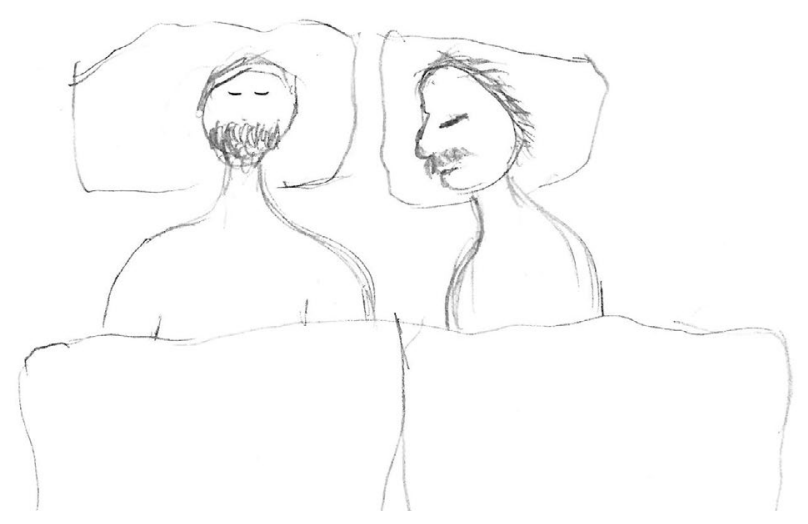

Abb. 21 


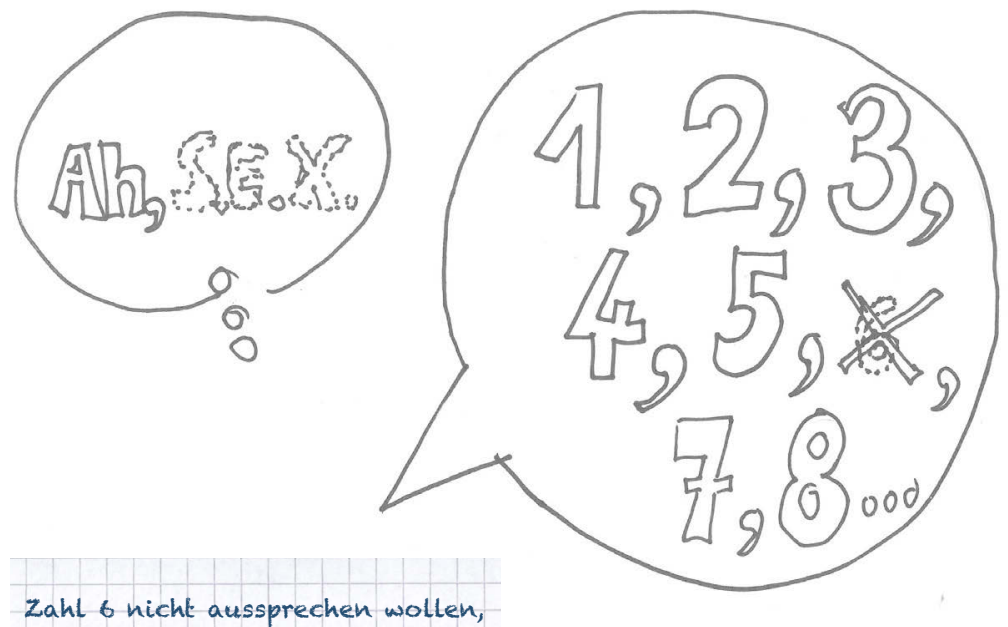

weil es sich wie S.E.X. anhört

Abb. 22

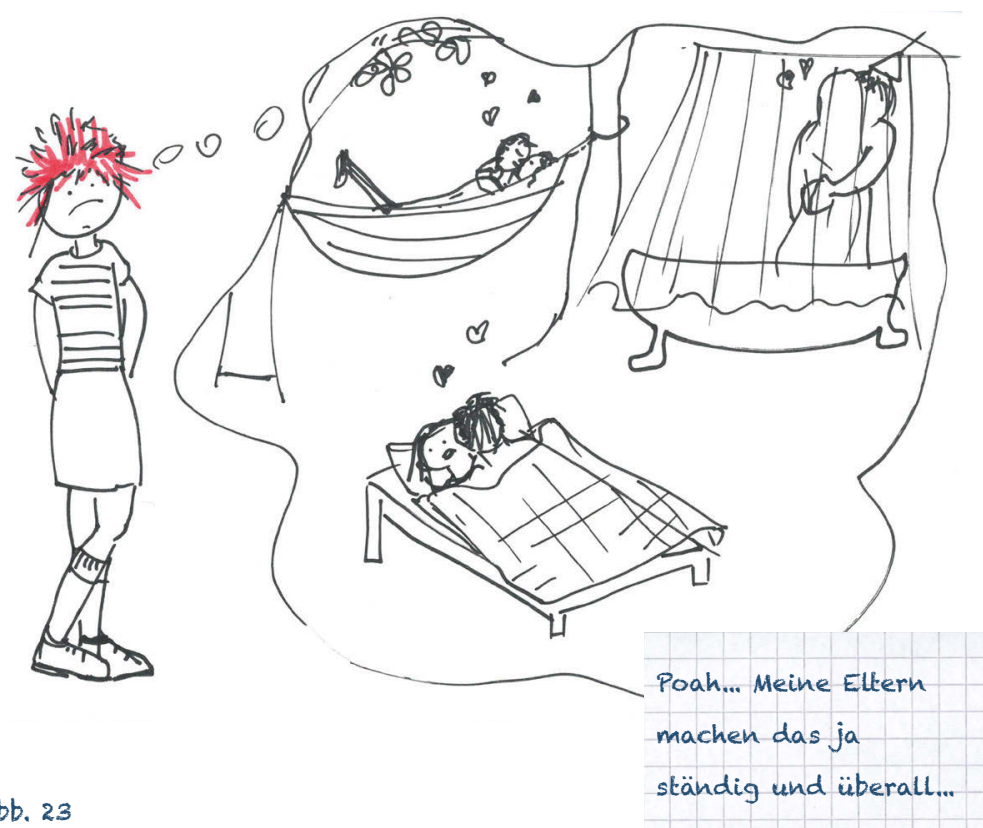




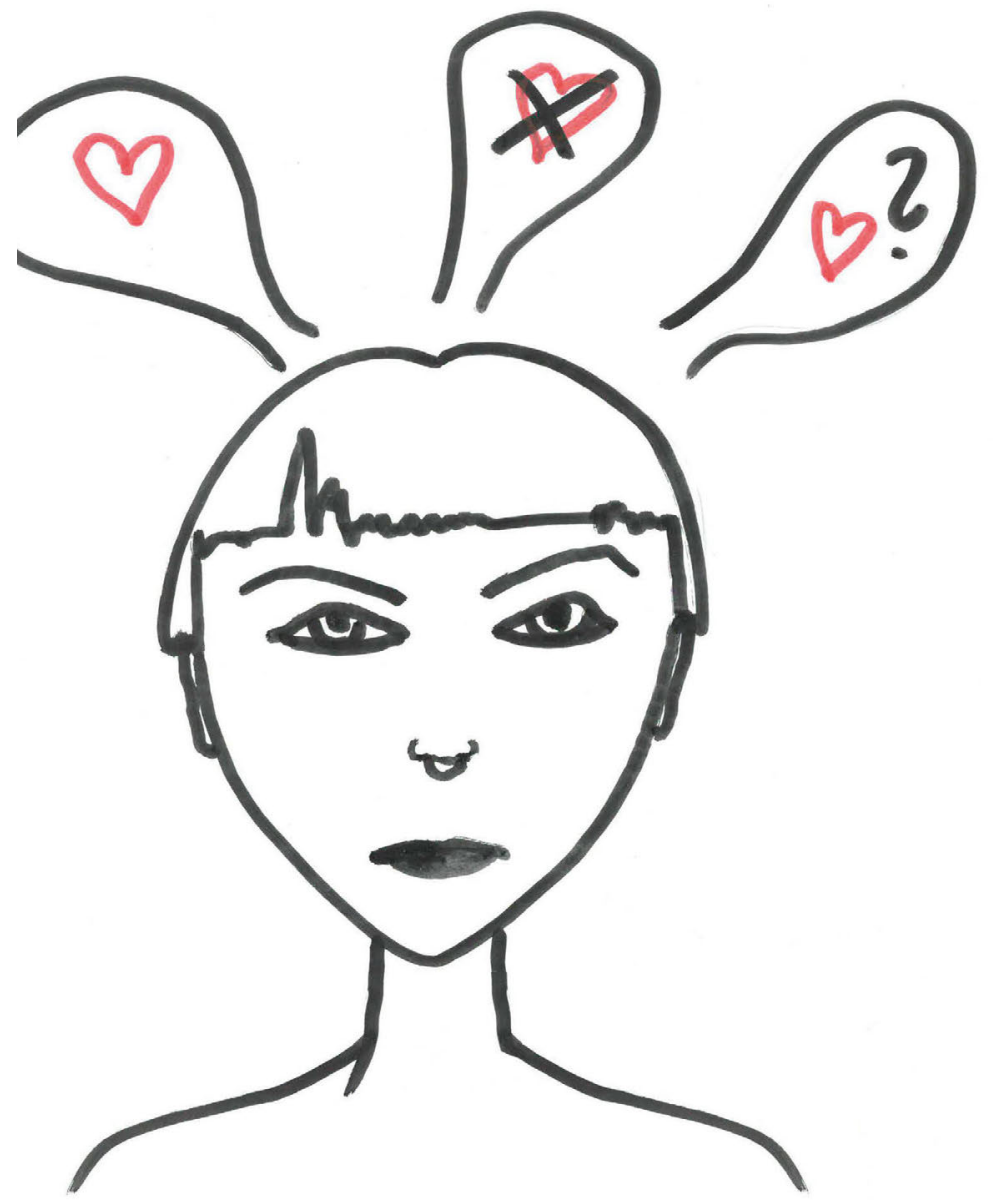

Abb. 24 


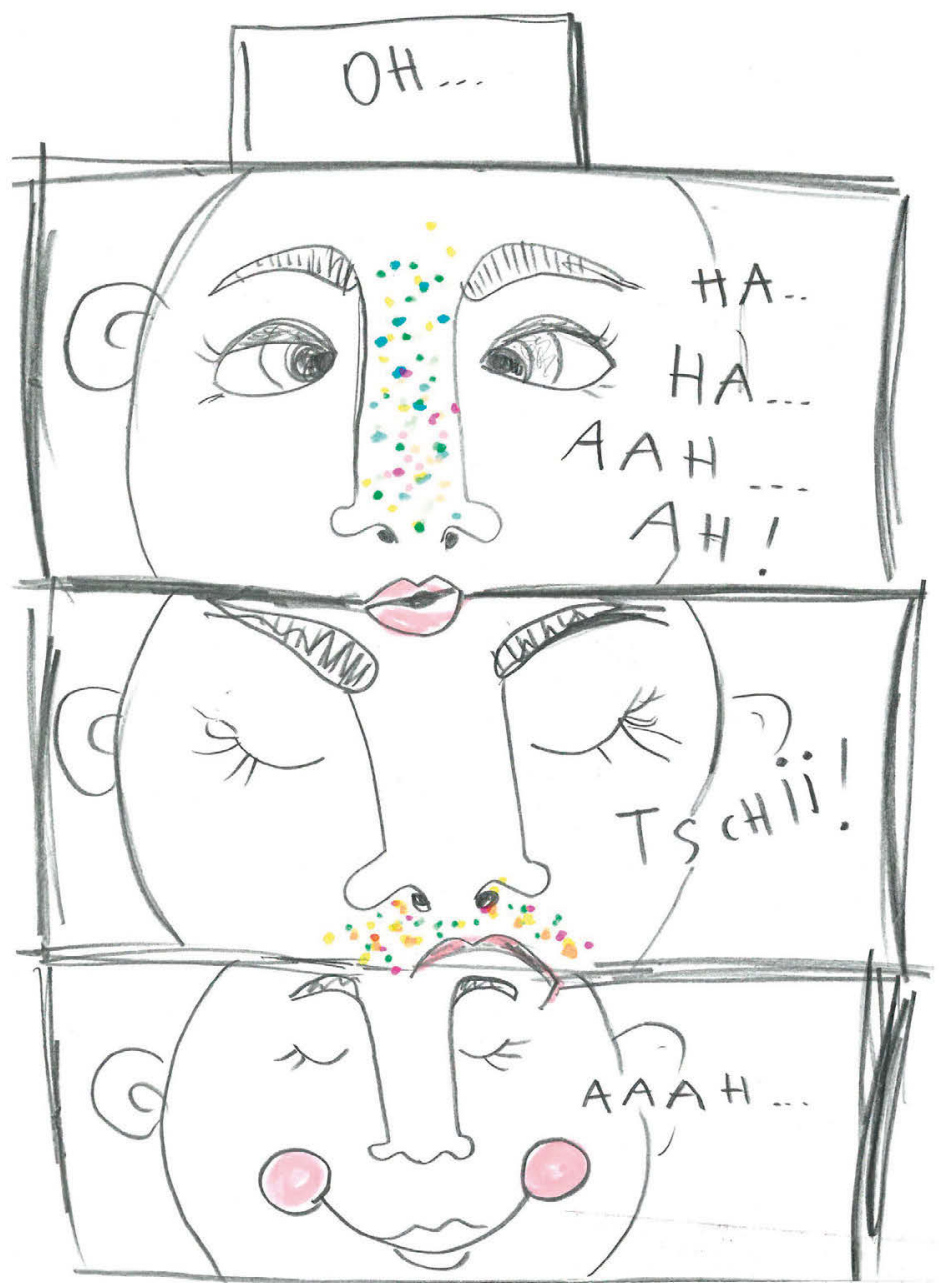

In einem Kinderbuch hatte ich gelesen, dass ein Orgasmus sich so anfühlt, wie wenh es in der Nase kitzelt und man dann niesen muss. Ich dachte mir zwar gleich, dass dies ein Blödsinn ist, aber die vorstellung hat mich noch lange beAbb. 25 gleitet und mich gewundert. 
Warum küsseh sich

Menschen? Da werden doch

Bakterien übertragen.

Abb. 26
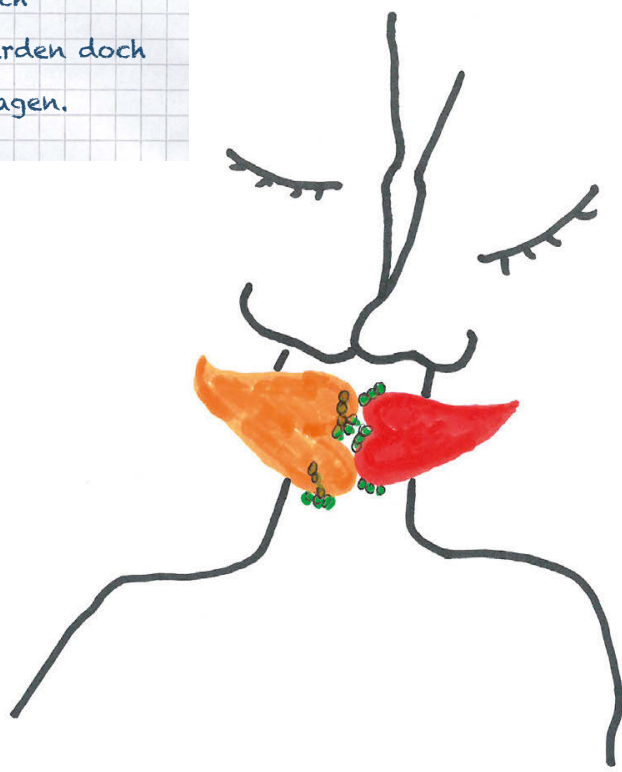

zungenverbercitung für den Zungenkuss:

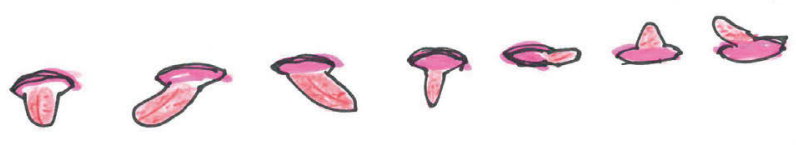

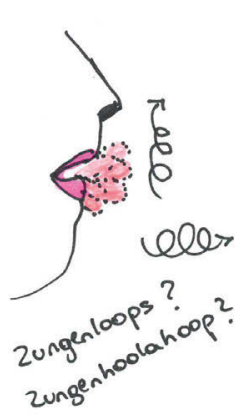

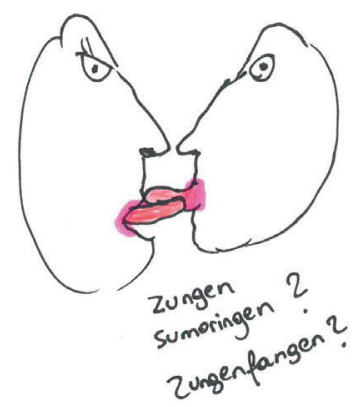

Abb. 27

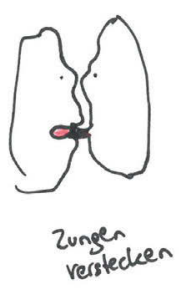

Was macht man mit

seiner zunge bei einem

Zungenkuss? 



\section{Das Ungreifbare greifbar machen Handlungsmöglichkeiten von Pädagog:innen gegen sexuelle Gewalt an Schüler:innen}

Stefanie Vasold

Gerüchte ... eine Freundin ... in der WhatsApp-Gruppe ... ein aufgebrachter Vater ... Stimmt das alles überhaupt? Anzeige? Gefährdungsmeldung?

Sexuelle Gewalt erschüttert und verunsichert einzelne Personen und ganze Systeme. Dies führt häufig zum Verlust der Handlungsfähigkeit, die Vorwürfe werden geleugnet oder ignoriert. Oder übereiltes Handeln sorgt für große Aufregung, verläuft dann aber ergebnislos. Worauf Lehrer:innen im Hinblick auf sexuelle Gewalt achten können, wie bei einem Verdacht zu handeln ist, was beim Gespräch mit Betroffenen wichtig ist und welche besonderen Dynamiken wirken, wenn eine Lehrperson unter Verdacht steht, Übergriffe zu setzen - all das sind Fragen, denen sich dieser Artikel widmet.

Sexueller Missbrauch ist dann gegeben, wenn Erwachsene oder deutlich ältere Jugendliche ein Kind dazu benutzen, eigene sexuelle Bedürfnisse $\mathrm{zu}$ befriedigen. Die Täter(:innen) ${ }^{1}$ nutzen ihre Autoritätsposition und die $A b$ hängigkeit von Kindern und Jugendlichen aus und ignorieren ihre Grenzen. dien, die sich mit Frauen als Täterinnen beschäftigen, kommen zu Zahlen von 1-25\% (Lamnek \& Ottermann, 2004; Enders 2001; Kavemann 1996; Elliott 1995; Berner 2013). Insofern ist es bedeutsam, auch Frauen als mögliche Täterinnen im Blick zu haben, gleichzeitig soll mit der Schreibweise dem Ceschlechterverhältnis Rechnung getragen werden und es sollen eben nicht alle Ceschlechter gleichberechtigt benannt werden. Die Klammer soll zum Ausdruck bringen, dass sexualisierte Gewalt nach wie vor zu einem großen Teil von Männern begangen wird. 
Sexuelle Gewalt kann mit oder ohne Berührung stattfinden und geschieht immer gegen den Willen der Kinder und Jugendlichen, da sie aufgrund der körperlichen, emotionalen, sozialen, kognitiven und sprachlichen Unterlegenheit die Folgen und Konsequenzen sexueller Handlungen mit Erwachsenen nicht absehen und diesen nicht wissentlich zustimmen können (vgl. Selbstlaut 2014:22). ${ }^{2}$

Es gibt im Alltag wie im fachlichen Diskurs viele unterschiedliche Begriffe wie sexueller Missbrauch, sexuelle oder sexualisierte Gewalt, sexuelle Ausbeutung, Aggression oder sexuelle Übergriffe u.a. Jeder Begriff stellt unterschiedliche Aspekte in den Vordergrund. Im Folgenden werden deshalb unterschiedliche Begriffe verwendet (vgl. ebd.).

Nicht nur die Begrifflichkeiten machen das Thema kompliziert. Sexuelle Gewalt unterscheidet sich von vielen anderen Formen von Gewalt auch deshalb, weil es kaum Beweise gibt und vieles unklar ist, manchmal auch bleibt. Fast immer findet sexuelle Gewalt im Geheimen statt, allermeistens ohne Zeug:innen. Körperliche Hinweise wie etwa Hämatome, sexuell übertragbare Krankheiten oder DNA-Spuren sind zwar eindeutige Hinweise auf sexuellen Missbrauch, stellen aber die große Ausnahme dar und sind von Pädagog:innen im Schulalltag kaum feststellbar.

Sofern also nicht Bild- oder Videomaterial vorliegt oder die Handlung zufällig von jemandem beobachtet wurde, sind es immer die Betroffenen, die über erlebte sexuelle Gewalt Auskunft geben müssen. Aus verschiedenen Studien ist bekannt, dass nur ein Viertel bis ein Drittel der Betroffenen kurz nach einem erlebten sexuellen Übergriff darüber berichten (vgl. Ungar u.a. 2009 zit.n. Amyna 2011: 31). Durchschnittlich benötigen Betroffene zwischen drei und zehn Jahre, um von sexuellem Missbrauch zu erzählen. $26 \%$ der Mädchen und sogar fast $40 \%$ der betroffenen Jungen vertrauen sich überhaupt niemandem an (vgl. Priebe/Svedin 2008 zit.n. Amyna 2011: 31). »Selbst bei Vorliegen unabhängigen Beweismaterials für einen sexuellen Missbrauch (z.B. ärztlicher Befund einer sexuell übertragbaren Krankheit oder Video-Material) scheint etwa die Hälfte der Kinder im Erstinterview einen Missbrauch zu verneinen« (Amyna 2011: 31).

Neben dem Tabu Sexualität können Gefühle von Verwirrung, Scham und Schuld und das ambivalente Verhältnis zu Täter(:innen) Gründe sein,

2 Dem Umfang des Artikels geschuldet bleibt das Thema sexuelle Übergriffe unter Kindern und Jugendlichen hier ausgespart. Zu diesem Thema siehe Selbstlaut 2009 sowie den Beitrag von Ulli Freund in diesem Band. 
die das Erlebte unsagbar machen. Vielleicht fehlen auch tatsächlich die Worte und/oder Betroffene befürchten, es würde ihnen nicht geglaubt. Viele Betroffene senden allerdings bewusst oder unbewusst Signale aus, um aufmerksam zu machen, um Hilfe zu bekommen oder um mit dem Schmerz umzugehen. Diese Signale können individuell sehr verschieden, zum Teil sogar widersprüchlich sein und lassen zumeist nicht eindeutig auf sexuelle Gewalt schließen, sondern sind oft unspezifische Anzeichen, dass es Kindern oder Jugendlichen nicht gut geht.

\section{Betroffene setzen vielfältige Zeichen}

Oft stehen Verhaltensveränderungen oder starke Stimmungsschwankungen am Anfang. Manche Schüler:innen werden ganz still, leiden vielleicht unter Depressionen, ziehen sich zurück, andere sind unruhig, extrem reizbar oder aggressiv. Viele leiden zudem unter (psychosomatischen) Beschwerden, z.B. Bauchschmerzen, Kopfschmerzen, chronischer Verstopfung oder ständigen Blasenentzündungen, Hautkrankheiten und Schmerzen, für die sich keine organischen Ursachen finden lassen. Häufig sind Betroffene chronisch erschöpft und können sich nicht konzentrieren, weil sie Schlafstörungen oder Albträumen haben oder in der Nacht sexuelle Übergriffe erleben.

Manche verhalten sich auffällig sexualisiert, z.B. mittels Bilder, Ausdrücken, Zeichnungen, Andeutungen, oder setzen bewusst Gerüchte in Umlauf. Einige Betroffene verhalten sich selbstverletzend, entwickeln Essstörungen oder andere Formen von Sucht. Manche Betroffenen sind suizidgefährdet.

Bei schwerer und langanhaltender sexueller Gewalt können auch Zeichen von Traumatisierung sichtbar werden, z.B. wenn Schüler:innen im Unterricht dissoziieren, wie >weggebeamt « wirken oder Flashbacks mit heftigen Reaktionen erleben. Auch permanente Anspannung im Sinne unaufhörlicher Wachsamkeit kann auf Traumatisierung hinweisen. ${ }^{3}$

Die möglichen Signale Betroffener sind also insgesamt so vielfältig, dass sie in keiner Aufzählung erschöpfend erfasst werden könnten. Gleichzeitig kann jede Auffälligkeit für sich genommen auch auf etwas ganz anderes hinweisen. Klar ist aber bei vielen Punkten, dass es den Kindern und Jugendlichen nicht 
gut geht. Das ernst zu nehmen ist oft genug für einen ersten Schritt, etwas $\mathrm{zu}$ unternehmen, selbst wenn noch unklar ist, worum es eigentlich geht.

Als Mitarbeiterin der Fachstelle Selbstlaut-gegen sexualisierte Gewalt an Kindern und Jugendlichen zeigt meine Erfahrung, dass selten ein einzelnes Signal, sondern vielmehr eine ganze Reihe von anhaltenden und wiederkehrenden Irritationen, Merkwürdigkeiten, Sorgen um ein Kind und das Fehlen anderer Erklärungen Pädagog:innen an sexuelle Gewalt als Möglichkeit denken lässt.

\section{Die Strategien der Täter(:innen)}

Wo immer langjähriger sexueller Missbrauch bekannt wird, stellt sich die Frage, wie dieser so lange unbemerkt geschehen konnte, warum Kinder und Jugendliche nicht viel früher davon erzählt haben und wieso das Umfeld - die Eltern oder in Institutionen die Kolleg:innenschaft - nicht eher aufmerksam wurden, Verdacht geschöpft und den Missbrauch gestoppt haben.

Eine Antwort liegt in der Vorgehensweise der Täter(:innen). Ihnen gelingt es, nicht nur die Kinder und Jugendlichen $\mathrm{zu}$ manipulieren, sondern vor allem auch die Bezugspersonen rund um das Kind so zu täuschen, dass sie lange unentdeckt bleiben. Dementsprechend ist ein Ausgangspunkt für das Handeln gegen sexuelle Gewalt das Wissen um die Vorgehensweise von Missbrauchs-Täter(:innen) und die sich daraus entwickelnde Psychodynamik. Die Beschäftigung damit gründet nicht in der Faszination an schrecklichen Taten oder Lust am Gruseln, sondern in der Tatsache, dass dort der Schlüssel für wirkungsvolle Prävention und Intervention mit Blick auf das Kindeswohl $\mathrm{zu}$ finden ist.

Sexueller Missbrauch wird immer geplant vollzogen und geht oft mit einer akribischen Vorbereitung einher. Nachdem alle Täter(:innen) wissen, dass das, was sie tun, verboten ist und ein Auffliegen ernstzunehmende Konsequenzen hat, fließt viel Aufmerksamkeit in die Planung, um niemanden auch nur eine Idee davon zu geben, was tatsächlich vor sich geht.

\subsection{Manipulation von Kindern und Jugendlichen: Grooming}

Grooming ist der englische Begriff für das Vorbereiten und Anbahnen sexueller Handlungen mit Kindern und Jugendlichen. Sofern der Kontakt zu Kindern oder Jugendlichen nicht ohnehin über das direkte familiäre Umfeld besteht, nützen Täter:innen den Bekanntenkreis, die Nachbarschaft oder Orte, 
an denen Kinder und Jugendliche sich gerne aufhalten, wie Vereine, Spielplätze oder Schwimmbäder, oder knüpfen Kontakte über das Internet. Manche arbeiten auch in pädagogischen Institutionen wie Kindergärten und Schulen, um Zugang zu Kindern und Jugendlichen zu haben. So wird in perfider Weise ein Vertrauensverhältnis aufgebaut: Die Annäherung beginnt meist damit, dass dem Kind oder Jugendlichen besondere Aufmerksamkeit geschenkt wird und sich Täter(:innen) sehr beliebt machen. Sie verbringen gemeinsam Zeit, interessieren sich für das Kind, geben dem Jugendlichen einen besonderen Stellenwert, Zuwendung, machen Geschenke, bieten sich als Ansprechperson für Schwierigkeiten an usw. Dann werden nach und nach die Grenzen verschoben, auch die körperlichen. Zuerst geht es vielleicht um sexuelle Gesprächsthemen. Dann taucht >zufällig` ein Porno auf, ein >Austausch über sexuelle Praktiken wird angeboten, bis nach und nach anzügliche Bemerkungen und - zu Beginn harmlose - Berührungen damit einhergehen.

Für Jugendliche ist dieses >Angebot< vielleicht am Anfang spannend, möglicherweise auch körperlich erregend. Geschickt verstehen es Täter(:innen) bei erstbester Gelegenheit den Kindern und Jugendlichen die Verantwortung für das eigene Handeln zu geben: Sie hätten es ja schließlich gewollt oder mitgemacht oder nach diesem und jenem gefragt oder dieses und jenes Geschenk angenommen. Oder Täter(:innen) erpressen Jugendliche, wenn diese sich z.B. überreden ließen, ein Nacktfoto von sich zu schicken. Gedroht wird damit, dieses zu veröffentlichen, an Eltern oder Freund:innen weiterzuschicken oder als Fotomontage zu verändern und ins Netz zu stellen.

Gleichzeitig bleiben die als positiv erlebten Anteile der Täter(:innen) oft aufrecht. Für Täter(:innen) ist das wichtig, um sich die Loyalität der Betroffenen und deren Stillschweigen weiterhin zu sichern. Für Kinder und Jugendliche ist sexuelle Gewalt deshalb oft sehr verwirrend. Die sexuellen Handlungen können als ekelig erlebt werden und gleichzeitig körperlich erregend sein. Auch emotional kann gleichzeitig Ablehnung und eine starke emotionale Verbindung bestehen, es können eventuell sogar Verliebtheits-Gefühle und Freude über das besondere Geheimnis bei den Betroffenen präsent sein.

Täter(:innen) zerstreuen Hoffnungen, dass den Jugendlichen geglaubt oder geholfen würde, wenn sie jemanden davon erzählten. Je länger solche Situationen dauern, desto schwieriger wird es für Jugendliche, davon zu erzählen. Sie denken, sie hätten schon viel früher etwas erzählen müssen und sind spätestens jetzt im Erklärungsnotstand, warum sie so lange >mitgetan<, 
sich nicht ausreichend >gewehrt oder nicht zumindest früher davon erzählt haben.

\subsection{Manipulation des Umfeldes: Täter(:innen) als Profis im Impression Management}

Für Täter(:innen) ist die Meinung und Haltung des Umfeldes und eine gute Beziehung zu den erwachsenen Bezugspersonen der Kinder bedeutsam, um ihre Handlungen verschleiern zu können. Insofern unternehmen Täter(:innen) einen erheblichen Aufwand gegenüber dem sozialen Umfeld von Kindern, um gut dazustehen und vom eigenen Handeln abzulenken. Täter(:innen) sind meisterhaft in diesem Impression Management (vgl. Goffman 2003).

Im familiären und sozialen Umfeld der Kinder suchen sich Täter(:innen) z.B. Personen in schwierigen Lebenssituationen, denen sie finanziell oder in der Bewältigung des Alltags zu Hilfe kommen, z.B. bei Erledigungen, Reparaturen, Kinderbetreuung, Nachhilfe o.Ä., und geben sich sehr engagiert und kinderlieb.

Möglicherweise werden darüber hinaus gegenüber Eltern oder Bezugspersonen schon vor der Tat Gerüchte über das Kind gestreut, um die eigene Glaubwürdigkeit zu stärken und die des Kindes in Zweifel zu ziehen, z.B. indem über angebliche >Lügengeschichten oder über sunpassendes` sexualisiertes Verhalten des Kindes berichtet wird. So wird vorgebaut: Sollte den Bezugspersonen etwas merkwürdig vorkommen oder sollten Kinder Andeutungen machen, gibt es dafür auch schon eine andere Erklärung. ${ }^{4}$

\section{Wenn Lehrer:innen sexuell missbrauchen}

In den vergangen zehn Jahren ist es vielen ehemaligen Schüler:innen, die sexuelle Gewalt erleben mussten, gelungen, eine öffentliche Debatte auszulösen, in der klar wurde: Das, was man sich aufs Erste weder vorstellen kann noch möchte, nämlich sexuelle Gewalt durch Lehrpersonen und andere pädagogisch Tätige, findet in einem viel größeren Ausmaß in Schulen statt, als 
vermutet. Die Enthüllungen erreichten, ausgehend von den USA und Australien, im Jahr 2010 Europa (Bundschuh 2010: 17ff.).

Im deutschsprachigen Raum gerieten einige bis dahin namhafte Einrichtungen ins Wanken, etwa kirchliche wie das Canisius-Kolleg in Berlin (Bundschuh 2010: 18), aber auch angesehene reformpädagogische Schulen wie die Odenwaldschule in Hessen. Dort wurden die ersten Missbrauchs-Vorwürfe bereits 1998 öffentlich, aber es brauchte mehr als zehn Jahre, bis Schritte der Aufarbeitung unternommen wurden (vgl. Budschuh 2010: 20). In Österreich kamen nach 2010 ebenfalls mehrere kirchliche, aber auch städtische Einrichtungen in die Schlagzeilen. ${ }^{5}$ Auch in der Schweiz geriet 2017 eine reformpädagogische Einrichtung in Embrach in den Fokus, als ein ehemaliger Schüler den jahrelangen Missbrauch durch einen Lehrer öffentlich machte (vgl. Zangger 2017).

Die meisten dieser öffentlich gewordenen Skandale behandelten Fälle sexueller Gewalt, die viele Jahrzehnte zurückliegen. Dennoch war es für viele Betroffene das erste Mal, dass sie von der erlebten Gewalt berichteten. Jüngere Befragungen (vgl. Deutsches Jugendinstitut 2011: 60) zeigen, dass das Problem leider nicht nur eines der Vergangenheit ist: In $40 \%$ der befragten Schulen, $49 \%$ der Internate und $70 \%$ der stationären Jugendhilfeeinrichtungen gab es innerhalb der letzten drei Jahre Verdachtsfälle von sexueller Gewalt (mit Körperkontakt) (vgl. ebd.). ${ }^{6}$ Diese Zahlen machen deutlich, dass Hand-

In Österreich gab es bereits Mitte der 1990er Jahre erste Berichte über sexuellen Missbrauch durch den Kardinal Hans Hermann Croër. Nachdem in den folgenden Jahren immer wieder Missbrauchs-Vorwürfe in kirchlichem Zusammenhang laut wurden, gründete die katholische Kirche 2010 die »Klasnic-Kommission «zur Aufarbeitung und Entschädigung von Missbrauchs-Fällen (vgl. Hoesele 2013: 295f.; Österreichische Bischofskonferenz 2019: 1f.). Im selben Jahr gingen ehemalige Bewohner:innen des städtischen Kinderheims Wilhelminenberg in Wien an die Öffentlichkeit und berichteten von massiven Misshandlungen und sexuellem Missbrauch. Die Stadt Wien initiierte daraufhin eine Historiker:innenkommission zur Untersuchung (vgl. Heilige u.a. 2013). Über 2000 Betroffene erhielten Entschädigungszahlungen von insgesamt 52 Millionen Euro (vgl. Weißer Ring 2019; ORF Wien 12.11.2019).

6 Dabei wurden sowohl Übergriffe unter Jugendlichen als auch Verdachtsfälle auf sexuelle Gewalt im sozialen, familiären Nahraum und sexuelle Gewalt durch Mitarbeiter:innen der Schule erfragt. Am häufigsten hatten die Einrichtungen mit einem Verdacht auf sexuellen Missbrauch durch jemanden außerhalb der Einrichtung (31\%) zu tun, gefolgt von Übergriffen unter Jugendlichen (17\%). Sexuelle Übergriffe durch Lehrer:innen waren aber immerhin in $4 \%$ der Einrichtungen ein Thema (vgl. Deutsches Jugendinstitut 2011: 60). 
lungsbedarf in pädagogischen Einrichtungen besteht, umso mehr vor dem Hintergrund einer hohen Dunkelziffer, von der im Bereich sexueller Gewalt immer auszugehen ist (vgl. Krahé/Scheinberger-Olwig 2002: 18f.).

Bei sexuellem Missbrauch durch Lehrpersonen verschärfen sich die ohnehin bestehenden Dynamiken und das Machtgefälle, das zwischen Erwachsenen und Kindern grundsätzlich besteht. Lehrer:innen beurteilen schließlich die Leistungen der Schüler:innen, können den täglichen Schulaufenthalt ohne große Mühe zur Qual machen, sind vielleicht beliebt bei den anderen Lehrer:innen und der Schulleitung und haben eventuell viel Einfluss auf die Eltern u.a.m.

Lehrer(:innen), die Gelegenheit für sexualisierte Übergriffe suchen, können den Schulalltag vielseitig für ihre Pläne ausnützen. Gegenüber den (oder manchen) Schüler:innen machen sie sich beliebt, z.B. durch Benotung und Bevorzugung, das Verraten von Prüfungsaufgaben oder durch Geschenke, private Nachhilfe, durch das Erlauben von Dingen, die eigentlich verboten sind, oder durch einen besonders >coolen « Unterrichtsstil. Vielleicht verbreiten sie gleichzeitig Angst und Schrecken in der Klasse durch cholerische Ausbrüche.

Vor diesem Hintergrund und darin verwoben finden dann Grenzverletzungen statt, etwa unpassende Kommentare, nicht adäquate, scheinbar unabsichtliche Berührungen; es werden uneindeutige Situationen und EinzelSettings geschaffen, Umkleide-, Dusch- und WC-Räume der Schüler:innen betreten oder versperrbare Nebenräume in der Schule als eigene Räume genützt usw.

Wenn sexuelle Übergriffe durch Lehrpersonen gesetzt werden, befürchten Schüler:innen zudem noch mehr, dass ihnen ihre Eltern womöglich nicht glauben. Denn Kinder klagen häufig, oft alltäglich, über Lehrer:innen, und Eltern tun das oft ab oder geben zu verstehen, dass der Schulerfolg das einzig Wichtige ist. Auch von den Klassenkolleg:innen gibt es selten Rückhalt, geht doch dem Missbrauch oft eine lange Phase der bewussten Spaltung der Klassengemeinschaft voraus, z.B. durch Bevorzugung Einzelner.

Das sexuelle Interesse einer Lehrperson muss aber nicht immer gleich Widerwillen bei Schüler:innen hervorrufen. Im Gegenteil: Manchmal treffen die Annäherungen vielleicht sogar auf eine Schwärmerei seitens der Schüler:innen für diese Lehrperson. Pubertäre Fantasien gehören zur psychosexuellen Entwicklung von Jugendlichen. Die Verantwortung zur Einhaltung der Grenze liegt eindeutig bei den Erwachsenen, insbesondere wenn diese Lehrer:innen sind. Allerdings ist es in dieser Konstellation für die Schüler:innen noch 
schwieriger, das Erlebte als Gewalt einzuordnen und davon zu erzählen (vgl. Münder/Kavemann 2010: 11f.).

Wenn Lehrer(:innen) sexuelle Gewalt ausüben, wenden sie ähnliche Strategien wie für den privaten Bereich skizziert im schulischen Umfeld an. Oft machen sie sich unentbehrlich, übernehmen wichtige, aber unbeliebte Arbeitsbereiche oder kümmern sich um besonders >schwierige< Kinder. Sie stellen sich gut mit der Leitung und/oder übernehmen selbst Leitungs- oder Personalvertretungsfunktionen. Das Engagement wird bis in den privaten Bereich ausgedehnt, privater Kontakt über soziale Netzwerke rund um die Uhr angeboten, Nachhilfe in der Freizeit gegeben oder sich ehrenamtlich in einem Kinder- und Jugendverein engagiert.

Auf Ebene der Kolleg:innenschaft werden Seilschaften, Loyalitäten und Abhängigkeiten hergestellt, z.B. durch Decken von Fehlern oder Versäumnissen anderer oder es werden Geheimnisse, Gerüchte und Verwirrung verbreitet. Vielleicht werden kritische Kolleg:innen bewusst schlechtgemacht und der Eindruck erweckt, diese würden lügen oder Mobbing betreiben. Auch gegenüber Kolleg:innen werden professionelle und private Grenzen verschoben, z.B. indem ausgiebig über private Details oder sexuelle Probleme berichtet wird. Oder es werden Affären im Kollegium oder in der Elternschaft begonnen. Das Klima in der Institution wird nach und nach sexualisiert und Grenzverletzungen werden bagatellisiert.

Manche Täter(:innen) geben sich als >Dauerjugendlicheく aus, die besonders gut mit Schüler:innen zurechtkommen, anderen werden viele Dinge verziehen, weil sie sich als besonders arm und unbeholfen darstellen und damit im Kollegium Mitleid erwecken. Wieder andere kommen nicht unter Verdacht, weil sie aktiv als Kinderschützer(:innen) auftreten oder sich als besonders fortschrittliche Sexualpädagog(:innen) in Szene setzen. Andere sind gänzlich unauffällig, so dass niemand auf die Idee kommt, sich über diese Person nähere Gedanken zu machen. Wenn Hinweise oder Kritik aufkommen, werden diese unter großer Empörung als Missverständnis und Fehldeutung zurückgewiesen und andere Erklärungen gefunden, z.B. dass Männer in diesem Kontext immer ungerecht beurteilt und besonders kritisch betrachtet werden. Es gibt also eine Reihe von Strategien, die Täter(:innen) gegenüber dem Umfeld anwenden, um nicht aufzufallen und andere über sich zu täuschen. 
Wenn sexuelle Gewalt aufgedeckt wird, ist es für Kolleg:innen meist schwer zu glauben, dass das wahr sein könnte. ${ }^{7}$ Fast immer führt das Aufdecken von sexueller Gewalt auch im Umfeld der betroffenen Kinder und Jugendlichen zu massiven Schuldgefühlen. Vor allem Menschen, die nahe an den Kindern oder Täter(:innen) dran sind, machen sich Vorwürfe, nicht schon früher Verdacht geschöpft zu haben. Noch schlimmer sind die Schuldgefühle für alle Beteiligten, wenn es vielleicht schon einzelne Anhaltspunkte gab, denen aber - aus welchen Gründen immer - nicht nachgegangen wurde.

Das Handeln gegen sexuellen Missbrauch ist vor diesem Hintergrund nicht nur gekennzeichnet von fehlenden Beweisen, fehlender Klarheit, dem Stehen von Aussage gegen Aussage, sondern auch von einer Psychodynamik, die die Betroffenen und das Helfer:innensystem beschämt, täuscht und spaltet und Täter(:innen) schützt. Das Wissen darum kann diese Dynamiken nicht aussetzen, aber es ermöglicht, sie zu erkennen und Handlungsspielräume im Umgang damit zu eröffnen.

\section{Was Pädagog:innen bei Verdacht auf sexuelle Gewalt tun können}

Sexualisierte Gewalt in ihren verschiedenen Formen, Zusammenhängen und mit unterschiedlichen Beteiligten erfordert ein fallspezifisches, durchdachtes und koordiniertes Vorgehen im pädagogischen Helfer:innensystem. Im Zentrum des Handelns steht das Kindeswohl. Jeder Schritt muss sich letztlich daran ausrichten, ob er dem Kindeswohl dienlich ist. Das Ziel der Intervention ist die nachhaltige Beendigung sexueller Gewalt. Es ist weder sinnvoll noch hilfreich zu dramatisieren oder zu bagatellisieren. Vernetzung und sensible Kommunikation ist notwendig, Getratsche muss hingegen möglichst unterbunden werden.

Im Folgenden werden konkrete Handlungsschritte dargestellt und Tipps gegeben, einerseits bei einer sehr vagen, ersten Vermutung, ande-

Die Lehrperson unter Verdacht ist ja >Eine(:)r von uns«. Oft gibt es ein Vertrauensverhältnis oder zumindest eine Identifikation mit der Position (»lch könnte die:der Nächste sein«). Oder Kolleg:innen trauen es der Person einfach nicht zu. Das trägt ebenso dazu bei wie die Scheu, Fehlverhalten anderer zu benennen, aus Kollegialität und/oder aus Sorge vor eigenen Unzulänglichkeiten. Häufig gibt es Angst, einen Ceneralverdacht auszulösen oder jemanden zu Unrecht zu beschuldigen u.a. (vgl. Enders 2012: 85). 
rerseits wenn ein:e Schüler:in von sexueller Gewalt berichtet, bis hin zu einer Gefährdungsmeldung. Abschließend in diesem Kapitel geht es um die Besonderheiten, wenn eine Lehrkraft beschuldigt wird, sexuelle Gewalt auszuüben.

\subsection{Erster Verdacht}

Wenn sich Irritationen nicht auflösen oder wiederholt auftreten oder wenn Schüler:innen Andeutungen machen, Trauma-Anzeichen bzw. beunruhigende Signale senden oder Mitschüler:innen von angeblichen Übergriffen berichten, dann ist es gut, diese Zeichen ernst zu nehmen und dem möglicherweise unguten Bauchgefühl zu trauen. Es ist hilfreich, Ruhe zu bewahren und sich einer vertrauenswürdigen verbündeten Person anzuvertrauen, mit der ein Austauschen möglich ist, ohne vorschnell ins Handeln zu kommen. Dieser Prozessschritt erfordert mitunter viel Zeit und Geduld. Möglichst früh professionelle Unterstützung beizuziehen, ist umso ratsamer.

Ziel in einer solchen Situation ist es dann, Gesprächsangebote gegenüber den Schüler:innen zu setzen, vertrauensvolle Rahmenbedingungen zu schaffen und Hilfsmöglichkeiten vorzustellen, in der Hoffnung, dass eventuell betroffene Schüler:innen diese (gleich oder später) annehmen. Denn ohne nähere Informationen und die Bereitschaft der Kinder oder Jugendlichen, etwas $\mathrm{zu}$ berichten, sind weitere Schritte der Intervention sehr schwierig oder versanden schnell ergebnislos.

Wichtig ist es auch in diesem Prozessschritt, die Rollenklarheit zu bewahren: Es geht nicht darum, ein möglicherweise geschehenes Verbrechen $\mathrm{zu}$ beweisen, jemanden zu überführen oder ganz genau herauszufinden, was >wirklich passiert ist; noch viel weniger - und da ist große Achtsamkeit geboten - geht es darum, Schüler:innen die eigenen Assoziationen einzureden. Die Rolle von Pädagog:innen im Interventionsprozess ist immer an der Seite der Kinder. Hier eine verlässliche Vertrauensperson zu sein, ist die wichtigste Aufgabe. Es gilt, behutsam vorzugehen und vor allem eine Vertrauens- und Gesprächsbasis mit den möglicherweise betroffenen Schüler:innen aufzubauen. Je nach Situation kann es sinnvoll und/oder wichtig sein, Einzelgespräche zu suchen oder z.B. bei Gerüchten entsprechend in Kleingruppen nachzufragen, die involviert waren. Gleichzeitig kann präventiv mit der ganzen Klasse gearbeitet werden: Für alle Schüler:innen ist es hilfreich, Informationen zum Thema zu bekommen sowie Anlaufstellen zu kennen. Für Betroffene kann dies 
eine Hilfe sein, das Erlebte einzuordnen und gleichzeitig ein Signal senden, dass es möglich ist, darüber zu sprechen ${ }^{8}$.

Einbetten lässt sich das Thema im Unterricht mit der ganzen Klasse z.B. in einen Schwerpunkt zu allgemeinen Kinder- und Jugendrechten, zu Jugendschutzgesetzen, unterschiedlichen Formen von Gewalt sowie Einrichtungen und Personen, die Kindern und Jugendlichen helfen können. Darüber hinaus gibt es zahlreiche andere Themenbereiche, die mit der ganzen Klasse bearbeitet werden können und die präventiv für alle Schüler:innen stärkend sind und jenen, die eventuell sexuelle Gewalt erlebt haben oder derzeit erleben, helfen, das Geschehene einzuordnen und zu benennen. Darüber hinaus erfahren Betroffene, dass sie nicht die Einzigen sind, die sexuellen Gewaltverhältnissen unterworfen sind. Außerdem ist es ein Signal, dass der Pädagoge:die Pädagogin sich mit solchen Themen auskennt und auch für Gespräche zur Verfügung steht ohne die Souveränität zu verlieren.

\subsection{Hinweise für Gespräche mit betroffenen Schüler:innen}

Wenn Schüler:innen von sexueller Gewalt berichten oder diese andeuten, benötigen sie vor allem ein Gegenüber, das aufmerksam zuhört, verstehen will, glaubt und tröstet. Es geht nicht darum, fertige Lösungen aus dem Ärmel zu schütteln oder Versprechungen zu machen, die nicht einhaltbar sind, sondern sich zu allererst den Schüler:innen zu widmen und für diese emotional da zu sein. Ziel eines Gesprächs mit Betroffenen ist es, Raum für Erfahrungen $\mathrm{zu}$ geben und dabei zu unterstützen, über möglicherweise erlebte Gewalt zu berichten.

Auch wenn Pädagog:innen das Gefühl haben, sie seien die falschen Personen für ein solches Gespräch, für diese Rolle oder diese Schüler:innen: Die betroffene Person hält sie augenscheinlich im Moment als Gesprächspartner:in für geeignet - oder zumindest für die geeignetste Person, die aktuell verfügbar ist. Diese Rolle lässt sich in dieser Situation nicht an jemanden anderen

In einer breit angelegten Studie nach schweren Cewaltvorkommen in Institutionen konnten mehrere Aspekte herausgefiltert werden, die es Kindern und Jugendlichen erleichtern, von sexuellen Grenzverletzungen zu erzählen. So ist es hilfreich, wenn sexuelle Cewalt bereits Thema war und die Haltung der Pädagog:innen einschätzbar ist, wenn das Thema ernst genommen, aber nicht dramatisiert wird, wenn nachgefragt wird, ohne Druck aufzubauen, und wenn ein unterstützendes Klima geschaffen wird und Kinder und Jugendliche informiert und einbezogen werden, welche Schritte gesetzt werden (vgl. Amyna 2011: 33). 
delegieren. Es ist wichtig, in der Situation dranzubleiben und das Gespräch möglichst nicht aufzuschieben. Denn am nächsten Tag hat der Mut die Schüler:innen vielleicht schon wieder verlassen.

Wenn Betroffene erzählen, gilt es aktiv zuzuhören und sie möglichst nicht zu unterbrechen, Nachfragen eher zu vermeiden oder auf später zu verschieben. Wenn Fragen gestellt werden, dann sollten es offene Fragen sein wie »Erzähl mir mehr!«, »Was ist dann passiert?« oder W-Fragen wie Wann?, Wer?, Wo?, Was?, Wie? und Womit? Wichtig ist möglichst bei den gewählten Worten der Kinder oder Jugendlichen zu bleiben und bestenfalls wörtlich zu wiederholen, was sie formuliert haben. Darüber hinaus können folgende Sätze als unterstützend erlebt werden: »Ich weiß, dass es mehrere Kinder gibt, die so etwas erleben. Gemeinsam können wir nach einer Lösung suchen.« Ebenfalls hilfreich kann es sein, die Gefühle zu spiegeln und empathisch zu sein. Wichtig erscheint es auch, Kinder und Jugendliche für ihren Mut zu erzählen explizit zu loben und das Gespräch als Vertrauensbeweis zu verstehen. ${ }^{9}$

Über den Grad der Detailliertheit der Erzählung entscheiden die Betroffenen. Nur dann, wenn es grobe Verständnisprobleme gibt, soll das Gesagte hinterfragt werden, z.B.: »Das habe ich nicht ganz verstanden. Könntest du das noch einmal erzählen?« Wichtig für die weiteren Schritte ist es, zu klären, ob die sexuellen Übergriffe in der Vergangenheit liegen oder nach wie vor stattfinden.

Jedenfalls wichtig ist es, mit den Schüler:innen die nächsten Schritte zu besprechen und gegebenenfalls Lösungen $\mathrm{zu}$ finden, wie das Kind in der nächsten Zeit vor sexuellen Übergriffen geschützt werden kann. Es ist möglich anzukündigen, dass (möglicherweise vorerst anonym) Unterstützung bei einer Beratungsstelle eingeholt, im Anschluss das Kind darüber informiert und dann gemeinsam die nächsten Schritte besprochen werden.

Zu vermeiden sind ]a-/Nein-Fragen, Erwartungsfragen (»Dann bist du doch sicher weggelaufen?«); Voraussetzungsfragen (»Hast du ihn auch einmal anfassen müssen?«); Wiederholungsfragen (also bereits gestellte Fragen erneut stellen) und das Wort »Warum«, weil es Rechtfertigungsdruck erzeugt. Ebenfalls kontraproduktiv sind Ceheimhaltungsversprechungen, die nicht gehalten werden können. Auch auf gut gemeinten Körperkontakt und sichtbare Betroffenheit sollte verzichtet werden, sie machen es für die Betroffenen noch schwieriger. Außerdem ist es nicht hilfreich, Täter:innen abzuwerten. Je näher die gewalttätige Person zum Kind steht, desto mehr ist eine Beurteilung des:der Täters: Täterin auch eine Beurteilung des Kindes (Balloff 2017 zit.n. Birchbauer 2019: 43). 
Insbesondere was die Information und Einbindung der Eltern betrifft, ist wichtig, die Betroffenen einzubeziehen. Meist gibt es einen Grund dafür, dass Schüler:innen sich einer Lehrkraft anvertrauen und nicht zuerst zuhause von dem Erlebten erzählen. Das kann an der befürchteten Reaktion der Eltern liegen, an Scham und Peinlichkeits-Gefühlen oder aber daran, dass die Person, die die Übergriffe setzt, Teil des familiären Systems ist. Selbst wenn das nicht der Fall ist, ist es für Eltern schwer auszuhalten, von sexueller Gewalt gegenüber dem eigenen Kind zu hören. Eltern brauchen Begleitung und Unterstützung; dementsprechend muss dieser Schritt gut überlegt und abgestimmt sein. Auf jeden Fall muss das Kind darüber informiert werden, wenn es Gespräche mit den Eltern gibt.

Möglichst unmittelbar nach dem Gespräch sollte von der pädagogisch tätigen Person, die als Vertrauensperson gewählt wurde, ein detailliertes Gedächtnisprotokoll angefertigt werden. Je genauer es am Wortlaut des Kindes ist, desto besser, denn sollte es zu einer Anzeige oder einem Gerichtsverfahren kommen, sind die ersten Aussagen von Kindern und deren Wortwahl oft entscheidend für die Beurteilung der Glaubwürdigkeit. Vor diesem Hintergrund ist es auch wichtig, gestellte Fragen oder Sätze der Pädagog:innen so festzuhalten, wie sie gestellt wurden - selbst dann, wenn die Lehrperson manches rückblickend vielleicht als nicht so optimal formuliert einschätzt.

Je nach Situation kann es notwendig sein, die Kinder- und Jugendhilfe einzuschalten oder auch eine Anzeige zu machen. Dafür ist es unabdingbar, die Kinder und Jugendlichen vorab $\mathrm{zu}$ informieren und $\mathrm{zu}$ erklären, warum diese erfolgt. Wichtig ist es, dabei herauszustreichen, dass die Schüler:innen nichts falsch gemacht haben und sie keine Schuld trifft, sondern dass es im Gegenteil mutig war, sich Hilfe zu holen und dass es in der Verantwortung der Erwachsenen liegt, Kindern zu helfen, wenn sie so etwas erleben. Auch für diese Schritte ist eine fachliche Beratung und Begleitung für die Betroffenen sowie die helfenden Pädagog:innen sehr zu empfehlen.

\subsection{Werden Lehrer:innen beschuldigt ...}

Besonders schwierig wird die Situation in der Schule, wenn die beschuldigte Person aus der Kolleg:innenschaft kommt. Werden Lehrpersonen oder andere Schulangestellte beschuldigt, sexuelle Übergriffe gesetzt zu haben, ist es unvermeidbar, die Schulleitung zu informieren. Um diesen Schritt im Detail durchzudenken, ist es ratsam, sich Unterstützung von einer Beratungsstelle zu holen. Auch darüber sollen die betroffenen Schüler:innen Bescheid wissen. 
Es ist dann die Aufgabe von Schulleiter:innen den Interventionsprozess bestenfalls gemeinsam mit einem internen Krisenteam und externer Begleitung $\mathrm{zu}$ koordinieren. ${ }^{10}$

Mitunter entsteht ein Verdacht nicht (nur) aufgrund von Äußerungen von Schüler:innen, sondern es beobachten Kolleg:innen oder Eltern irritierende Situationen, unpassendes Verhalten oder grenzverletzende Sprüche. Oft sind es scheinbare Kleinigkeiten, mit denen übergriffiges Verhalten beginnt. Wenn Grenzverletzungen verbal und im täglichen Miteinander Normalität sind, fällt es weder besonders auf, wenn sie jemand auch gegenüber Schüler:innen setzt, noch ist es thematisierbar, weil ja ohnehin snormak. Vor diesem Hintergrund empfiehlt es sich, bereits auf kleinere Grenzverletzungen und auf pädagogisch irritierendes Verhalten proaktiv zu antworten. So gut es geht, kann das von den beobachtenden Kolleg:innen oder Eltern direkt angesprochen werden, bei wiederholten Irritationen oder fehlender Bereitschaft zur Veränderung ist es aber bei solchen vermeintlich harmloseren Formen von Übergriffen ratsam, die Schulleitung $\mathrm{zu}$ informieren. Letztlich obliegt ihr die Verantwortung pädagogische Standards herzustellen und durchzusetzen.

\section{Nachhaltige Prävention von sexueller Gewalt}

Dieser Beitrag zielt darauf ab, auf sexuelle Gewalt aufmerksam zu machen und Lehrpersonen $\mathrm{zu}$ sensibilisieren und in ihrer Handlungssicherheit $\mathrm{zu}$ stärken, gegen sexuelle Gewaltverhältnisse vorzugehen. Vor diesem Hintergrund wurde dargestellt, welche Anzeichen auf sexuelle Gewalt bei Schüler:innen hindeuten können, wie Täter(:innen) das Umfeld und die Betroffenen manipulieren, welche Handlungsschritte bei einem vagen Verdacht gesetzt werden können, und es wurden wichtige Hinweise für die Gesprächsführung mit Betroffenen gegeben.

Es liegt in der Verantwortung Erwachsener, Kinder und Jugendliche vor Gewalt zu schützen. Viel zu häufig findet diese statt, ohne dass sie bemerkt und ohne dass den Betroffenen geholfen wird. Diesen Zustand zu verändern, ist auch Aufgabe von Schule und Pädagog:innen.

10 Wie ein solcher Prozess aussehen kann und vor allem, was in den Schulen getan werden kann, um sexuelle Gewalt bestmöglich zu verhindern, findet sich in Selbstlaut 2020 . 
Viele Schüler:innen erleben im Alltag Diskriminierung, Abwertung und Ausgrenzung. Viele erfahren, dass ihre Meinung letztlich nichts zählt. Viele sehen, dass gegen Gewalt - und sei es >nur< verbale Gewalt - die sich z.B. jeden Tag im Schulhof wiederholt, von Erwachsenen nicht eingeschritten wird. Wenn Erwachsene Kinder und Jugendliche vor (sexueller) Gewalt schützen wollen, dann sind nicht nur verlässliche, vertrauensvolle, respektvolle und ehrliche Beziehungen gefragt, sondern auch das konsequente Handeln und Eintreten gegen jede Form der Gewalt und Abwertung. Wie gut das jeweils gelingt, hat neben den Akteur:innen auch mit den schulischen Rahmenbedingungen, der Kultur in der Schule und dem Umgang miteinander zu tun. Präventive Strukturen aufzubauen, ist angesichts der bekannten Fälle sexueller Gewalt in Schulen und Internaten höchst an der Zeit.

Sexuelle Gewalt zeigt sich als eingebunden in Systeme, Regeln, Hierarchien, gesellschaftliche und institutionelle Abläufe, die dann individuell erfahrbar werden: z.B. wenn Kinder zu spüren bekommen, dass sie wegen ihrer Familienkonstellation, ihrer sozialen und ethnischen Herkunft oder ihrer sexuelle Orientierung weniger wert sind. Oder dann, wenn Kinder erleben, dass sie gegenüber Erwachsenen nichts zu sagen haben. Oder dann, wenn sie im Alltag erleben, dass ihre Bedürfnisse, Wünsche oder Beschwerden von Erwachsenen ignoriert werden. Oder dann, wenn Kinder erleben, dass das Einschalten staatlicher Einrichtungen zu häuslichen Gewaltausbrüchen führt u.v.a.m.

Gewaltprävention muss, wenn sie nachhaltig sein will, immer sowohl am Verhalten als auch an den Verhältnissen ansetzen. Letztlich geht es darum, eine Gesellschaft $\mathrm{zu}$ entwickeln, in der alle Kinder und Jugendlichen wissen und im Alltag erleben, dass sie angenommen werden, so wie sie sind, und dass ihnen niemand Gewalt antun darf.

\section{Literatur}

AMYNA e.V. (Hg.) (2011): Wirksamkeit von Maßnahmen zur Prävention und Intervention im Fall sexueller Gewalt gegen Kinder. Expertise im Rahmen des Projektes »Sexuelle Gewalt gegen Mädchen und Jungen in Institutionen«. Online unter: https://www.dji.de/fileadmin/user_upload/sgmj/Exp ertise_Amyna_mit_Datum.pdf [Zugriff: 10.07.2020]

Arbeitsstab des Unabhängigen Beauftragten für Fragen des sexuellen Kindesmissbrauchs (2016): Was muss geschehen, damit nichts geschieht? 
Schutzkonzepte helfen, Schülerinnen und Schüler vor sexueller Gewalt zu schützen. Informationen zu den Bestandteilen von Schutzkonzepten. Online unter: https://www.schule-gegen-sexuelle-gewalt.de/fileadmin/Inha lte/PDF/Downloads/Broschuere/Broschuere.pdf [Zugriff: 10.07.2020].

Berner, Wolfgang (2013): Sexueller Missbrauch - Epidemiologie und Phänomenologie. In: Stompe, Thomas/Laubichler, Werner/Schanda, Hans (Hg.): Sexueller Kindesmissbrauch und Pädophilie, Wiener Schriftenreihe für Forensische Psychiatrie. Berlin: Medizinisch Wissenschaftliche Verlagsgesellschaft, S. 1-14.

Birchbauer, Petra (2019): Von der Vermutung zur Verdachtseinschätzung. In: Wiener Netzwerk gegen sexuelle Gewalt an Mädchen, Buben und Jugendlichen (Hg.): »Thank you for hearing me« (Sinéad O'Connor). Ein Versuch des Zuhören und Verstehens. Dokumentation der 17. Fachtagung vom 18.11.2019, Wien. Online unter: http://wienernetzwerk.at/dokumente/ne tzwerktagungsdoku_2019.pdf [Zugriff: 10.07.2020].

Bundschuh, Claudia (2010): Sexualisierte Gewalt gegen Kinder in Institutionen. Nationaler und internationaler Forschungsstand. Expertise im Rahmen des Projekts "Sexuelle Gewalt gegen Mädchen und Jungen in Institutionen«. Online unter: https://www.dji.de/fileadmin/user_upload/sgm j/Expertise_Bundschuh_mit_Datum.pdf [Zugriff: 10.07.2020].

Deutsches Jugendinstitut e.V. (Hg.) (2011): Sexuelle Gewalt an Mädchen und Jungen in Institutionen. Abschlussbericht des DJI-Projekts im Auftrag der Unabhängigen Beauftragten zur Aufarbeitung des sexuellen Kindesmissbrauchs, München. Online unter: https://www.dji.de/fileadmin/us er_upload/izkk/IzKK_DJIAbschlussbericht_Sexuelle_Gewalt.pdf [Zugriff: 10.07.2020].

Deegener, Günther (2014): Kindesmissbrauch. Erkennen - helfen - vorbeugen. Weinheim/Basel: Beltz.

Enders, Ursula (Hg.) (2003): Zart war ich, bitter war's. Handbuch gegen sexuellen Missbrauch. Köln: Kiepenheuer \& Witsch.

Enders, Ursula (2003): Missbrauch durch Mitarbeiter und Mitarbeiterinnen aus Institutionen. Online unter: https://www.zartbitter.de/o/Eltern_und _Fachleute/6060_missbrauch_in_Institutionen.pdf [Zugriff: 10.7.2020].

Enders, Ursula (Hg.) (2012): Grenzen achten. Schutz vor sexuellem Missbrauch in Institutionen. Ein Handbuch für die Praxis. Köln: KiWiTaschenbuch.

Fegert, Jörg/Hoffmann, Ulrike/König, Elisa/Niehues, Johanna/Liebhardt, Hubert (Hg.) (2015): Sexueller Missbrauch von Kindern und Jugendlichen. Ein 
Handbuch zur Prävention und Intervention für Fachkräfte im medizinischen, psycho-therapeutischen und pädagogischen Bereich. Berlin/Heidelberg: Springer.

Goffman, E. (2003): Wir alle spielen Theater. Die Selbstdarstellung im Alltag. München: Piper.

Heilige, Barbara/John, Michael/Schmucker, Helge/Wörgötter, Gabriele/Wisinger, Marion (2013): Endbericht der Kommission Wilhelminenberg. Online unter: https://www.kommission-wilhelminenberg.at/presse/jun2013 /Bericht-Wilhelminenberg-web_code.pdf [Zugriff 10.07.2020].

Heiliger, Anita (2001): Täterstrategien bei sexuellem Missbrauch und Ansätze der Prävention. In: beiträge zur feministischen theorie und praxis 56/57, S. 71-82.

Hoesele, Herwig (2013): Kindesmissbrauch: Bewältigung in Kirche und Staat. In: Kohl, Andreas/Karner, Stefan/Halper, Dietmar (Hg.): Österreichisches Jahrbuch für Politik. Wien: Böhlau, S. 295-309.

Krahé, Barbara/Scheinberger-Olwig, Renate (2002): Sexuelle Aggression. Göttingen u.a.: Hogrefe.

Ohlmes, Judith (2006): Pädosexuelle Täter. Merkmale und Strategien als Ansatzpunkte präventiver Maßnahmen. Wettenberg: Johannes Hermann.

ORF Wien (2019): Missbrauch in Heimen: Kritik an Verjährung. 12.11.2019. Online unter: https://wien.orf.at/stories/3021309 [Zugriff: 10.07.2020].

Österreichische Bischofskonferenz (2019): Maßnahmen der Katholischen Kirche in Österreich gegen Missbrauch und Gewalt. Presseerklärungen zur Frühjahrsvollversammlung der Österreichischen Bischofskonferenz 18. bis 21. März 2019 in Reichenau an der Rax. Online unter: https://www.ombudsstellen.at/dl/LqlmJmoJMLKJqx4KJKJKJKklmKL /Ma_nahmen_gegen_Missbrauch.pdf [Zugriff: 10.07.2020].

Petze - Institut für Gewaltprävention (Hg.) (2010): Sexuelle Übergriffe in der Schule. Leitfaden für Schulaufsicht und Kollegien zur Wahrung des sexuellen Selbstbestimmungsrechts von Schülerinnen und Schülern. Online unter: https://www.petze-institut.de/wp-content/uploads/2014/07/2010_ 11_04_sexuelle_uebergriffe.pdf [Zugriff: 10.07.2020].

Selbstlaut (2009): Spiel, Lust \& Regeln. Sexuelle Übergriffe unter Kindern Prävention und Intervention im Schulalltag. Online unter: https://selbs tlaut.org/publikationen-und-materialien/unsere-publikationen [Zugriff: 23.12.2020].

Selbstlaut (2020): Ganz schön intim. Sexualerziehung für 6-12 Jährige [sic!]. Unterrichtsmaterialien zum Download. Erstellt im Auftrag des Bundes- 
ministeriums für Unterricht, Kunst und Kultur. Aktualisierte Auflage. Online unter: https://selbstlaut.org/publikationen-und-materialien/unserepublikationen [Zugriff: 23.12.2020].

Selbstlaut (2014): Handlung, Spiel \& Räume. Leitfaden für Pädagoginnen und Pädagogen zum präventiven Handeln gegen sexuelle Gewalt an Kindern und Jugendlichen. Online unter: https://selbstlaut.org/publikationen-un d-materialien/unsere-publikationen [Zugriff: 23.12.2020].

Selbstlaut (2020): Achtsame Schule. Strukturelle Prävention von sexueller Gewalt. Online unter: https://www. https://selbstlaut.org/publikationen-un d-materialien/unsere-publikationen [Zugriff: 23.12.2020].

Stompe, Thomas/Laubichler, Werner/Schanda, Hans (Hg.) (2013): Sexueller Kindesmissbrauch und Pädophilie. Wiener Schriftenreihe für Forensische Psychiatrie. Berlin: Medizinisch Wissenschaftliche Verlagsgesellschaft.

Weißer Ring (Hg.) (2019): Hilfe für Opfer von Gewalt in Einrichtungen der Wiener Jugendwohlfahrt. Abschlussbericht. Online unter: https://ww w.wien.gv.at/kontakte/ma11/pdf/abschlussbericht-gewaltopfer.pdf [Zugriff: 10.07.2020].

Zangger, Markus (2017): Jürg Jegges dunkle Seite. Gockhausen: Wörtherseh. 



\title{
Sexualpädagogik - betrifft mich das?
}

\author{
Eva Zürcher
}

Vor dem Wahlmodul "Sexualpädagogik in der Schule« sah ich mich im Laufe meines Lehramtsstudiums an der Universität Innsbruck nie mit diesem Thema konfrontiert. Ich hatte die Einstellung, dass für Sexualpädagogik in der Schule speziell ausgebildete Personen von außen herangezogen werden sollten, weshalb ich mich als angehende Lehrerin davon nicht betroffen fühlte. Als Schülerin waren meine Erfahrungen mit Sexualpädagogik auf den klassischen Aufklärungsunterricht im Fach Biologie beschränkt, den ich nicht als mitreißend erlebt hatte.

Als Lehramtsstudierende schrieb ich mich für die Lehrveranstaltung ein, da ich das Gefühl hatte, sehr wenig über Sexualität im Zusammenhang mit Schule zu wissen. Ich war mir weder über sexualpädagogischen Unterricht noch über die rechtliche Situation zu sexueller Gewalt im Klaren. In der Lehrveranstaltung "Sexualpädagogik in der Schule« wurden viele Themen, von queeren Lebensweisen über das Hinterfragen von traditionellen Rollenbildern bis hin zu sexueller Gewalt, angesprochen. Es gab interessante Diskussionen, beispielsweise zu dem `Outing-Usus $<$, dem homosexuell orientierte Menschen in unserer Gesellschaft unterworfen sind: Es wird von ihnen erwartet, ihre sexuelle Orientierung kundzutun. Des Weiteren wurde etwa auch über Aufklärung von Teenagern durch Teenager und über Toleranzförderung gesprochen, um nur einige Themen zu nennen. Besonders die große Vielfalt an Bereichen, die im Seminar behandelt wurde, war für mich beeindruckend.

In der Lehrveranstaltung zeigte sich, dass bei dem Thema Sexualität oft auch eine persönliche Komponente mitschwingt und es sich deshalb nicht so einfach neutral und losgelöst von Persönlichem wie andere Themen betrachten lässt. Vorstellungen davon, was >normak ist, können dazu führen, dass andere Lebens- und Liebensweisen abgewertet werden. Die fehlende Akzeptanz kann beispielsweise damit zu tun haben, dass andere Lebensweisen nicht verstanden werden oder es an Wissen darüber mangelt. Deshalb finde ich es 
wichtig, durch Unterrichtsgespräche Wissen zu vermitteln, das zu mehr Verständnis und Akzeptanz beitragen kann.

Sexualpädagogische Themen können im Schulalltag jederzeit auftreten. Lehrer:innen sind in ihrem Unterricht etwa dann mit sexualpädagogischen Fragen konfrontiert, wenn es um die Sprache der Schüler:innen geht. Es kommt sehr oft vor, dass Wörter wie homosexuell oder schwul als Beschimpfung verwendet werden. Mir ist es ein Anliegen, dafür zu sensibilisieren, dass Wörter eine große Macht haben und unsere Vorstellungen auch unbewusst mitprägen. Ziel eines sensibilisierenden Unterrichts könnte sein, dass Schüler:innen klar wird, dass andere Lebensweisen nicht abzuwerten sind, nur weil sie nicht der Mehrheit oder den dominanten Normen entsprechen.

Eine weitere Herausforderung, mit der alle Lehrkräfte konfrontiert werden können, sind sexuelle Gewalterfahrungen von Kindern und Jugendlichen. Für Lehrer:innen ist es wichtig, aufmerksam für Veränderungen im Verhalten der Schüler:innen zu sein und um die Optionen professioneller Ansprechpersonen ebenso Bescheid zu wissen wie über die (Grenzen der) eigenen professionellen Zuständigkeiten.

Vor dem Seminar war ich nicht der Meinung, dass Sexualpädagogik auch im Regelunterricht stattfinden kann. In der Lehrveranstaltung konnte ich lernen, dass das Thema auch mich in meinen Fächern betrifft. Im Deutschunterricht könnte anhand von Textbeispielen wie Romanen, Kurzgeschichten oder Gedichten diskutiert werden, welche Macht Worte haben und wie sie unsere Vorstellungen prägen. Wichtig bei Textarbeiten ist auch ein anschließender Diskussionsteil, bei dem die Schüler:innen Gedanken und Fragen austauschen und Gerüchte und Mythen rund um Sexualität besprochen werden können. Des Weiteren bietet es sich im Deutschunterricht auch an, über Rollenbilder zu sprechen, wobei besonders Werbetexte interessant sein können, um Stereotype aufzudecken und Darstellungen kritisch zu beleuchten. Eine andere Möglichkeit wäre auch, Texte aus verschiedenen Zeiten zu vergleichen, um zu sehen, wie sich Rollenbilder genderspezifisch verändert haben.

Auch in meinem Zweitfach, Englisch, lässt sich Sexualpädagogik gut integrieren. So könnte etwa mit englischen Songtexten gearbeitet werden, anhand derer über kontroverse Themen gesprochen werden kann. Einerseits finden sich in manchen Musikgenres gehäuft frauenfeindliche wie auch homophobe Texte. Andererseits gibt es auch viele Liedtexte, die queere Lebensweisen unterstützen. In beiden Fällen kann durch die Arbeit an den Songtexten eine Diskussion angeleitet werden, die Schüler:innen dazu anregt, einen kritischen Blick einzunehmen. 
Zusammenfassend lässt sich sagen, dass mir die Lehrveranstaltung aufzeigt hat, inwiefern mich sexualpädagogische Fragen im schulischen Altag betreffen können und an wie vielen Stellen Sexualpädagogik in den Unterricht eingebaut werden kann. 



\section{Rechtliche Aspekte in der Sexualpädagogik}

Heidemarie König und Adriane Krem

Um mit jungen Menschen zum Themenbereich Sexualität zu arbeiten, braucht es interdisziplinäre Fachkompetenzen. Neben (medien-)pädagogischem, biologischem, medizinischem, psychologischem, sexualwissenschaftlichem, gruppendynamischem und methodisch-didaktischem Knowhow ist auch Wissen hinsichtlich rechtlicher Aspekte erforderlich, die das Arbeitsfeld der Sexualpädagogik betreffen. Zum einen sind juristische Informationen von Interesse für die Zielgruppe, wie beispielsweise zu den Themen Verhütung, Schwangerschaftsabbruch oder Schutzalter. ${ }^{1}$ Zum anderen brauchen pädagogisch Tätige einschlägige Kenntnisse für das Arbeitsfeld Schule, wie etwa zur rechtlichen Relevanz pornografischer Bilder, zum schulischen Auftrag in der Sexualpädagogik oder zur Aufsichtspflicht bei sexualpädagogischen Workshops. Der folgende Beitrag bietet - ohne Anspruch auf Vollständigkeit - eine Auswahl an sexualpädagogisch relevanten rechtlichen Bestimmungen, die für den Schutz und die Unterstützung der begleiteten Menschen wie auch für die eigene Person und Arbeit relevant sein können. Er bietet rechtliche Orientierung $\mathrm{zu}$ ausgewählten Themen und will damit auch anregen, sich gegebenenfalls weiterführend mit juristischen Themenbereichen zu beschäftigen.

Prinzipiell haben jeweils die Gesetze jenes Landes Gültigkeit, in dem sich eine Person gerade befindet. Pädagog:innen werden darauf spätestens dann verweisen, wenn eine jugendliche Zielgruppe darauf beharrt, dass einvernehm-

Bisweilen sind einschlägige Inputs für eine Zielgruppe auch seinfach nur spannend und interessant ohne unmittelbare Bedeutung für den eigenen Lebenszusammenhang, so etwa die Frage nach der Strafbarkeit öffentlicher sexueller Handlungen oder rechtlich relevanter Altersunterschiede zwischen Sexualpartner:innen. Öffentliche sexuelle Handlungen sind im §218 des Strafgesetzbuches (StCB) geregelt und könnten auch laut §81 des Sicherheitspolizeigesetzes (SPC) die öffentliche Ordnung stören. 
licher Geschlechtsverkehr zwischen einer 18-jährigen und einer 15-jährigen Person strikt verboten sei, da sie derartige Szenarien, wie sie in anderen Ländern gelten, via Medien aufnehmen. Solche rechtlichen Informationen werden von Jugendlichen oft nicht länderspezifisch differenziert.

Da die Autorinnen dieses Artikels in Österreich sexualpädagogisch tätig sind, beziehen sich die jeweiligen Ausführungen - wenn es nicht explizit anders angegeben wird - auf die österreichische Rechtslage. Die beschriebenen Themenbereiche und rechtlichen Regelungen stehen exemplarisch für die sexualpädagogische Arbeit im deutschsprachigen Raum und finden sich in Deutschland und der Schweiz oftmals in ähnlicher Form wieder.

\section{Schutzalter}

Eine Person gilt $\mathrm{ab}$ einem bestimmten Alter als sexuell mündig und damit hinsichtlich sexueller Handlungen als einwilligungsfähig. Dieses Alter wird üblicherweise als Schutzalter bezeichnet. Dieser Begriff wird in den Gesetzestexten selbst nicht verwendet, verweist aber darauf, dass die entsprechenden Gesetze dem Schutz jener dienen, die dieses Alter noch nicht erreicht haben. Sexuelle Handlungen von strafmündigen Personen mit solchen unterhalb des Schutzalters sind strafrechtlich relevant. In Österreich und Deutschland beginnt die Strafmündigkeit im Alter von 14 Jahren (vgl. Häßler/Schepker 2016: 255).

\subsection{Schutzalter in Österreich}

Der Altersunterschied bei einvernehmlichen sexuellen Begegnungen zwischen Personen ab 14 Jahren ist strafrechtlich prinzipiell irrelevant. ${ }^{2}$ Unter 14 Jahren gilt man als unmündige minderjährige Person. Personen zwischen 14 und 18 (BMASGK 2019: 6) gelten als mündige Minderjährige. Sexuelle Kontakte von mündigen Minderjährigen mit Unmündigen im Alter von 12 oder 13 Jahren, $\mathrm{zu}$ denen ein geringer Altersunterschied besteht, können unter die Alterstoleranzklauseln ${ }^{3}$ fallen und demnach straffrei sein. ${ }^{4}$

$2 \S 206 \mathrm{StCB}$, Bundesgesetzblatt I (BCBI.) Nr. 116/2013. und § $207 \mathrm{StCB}, \mathrm{BCB}$. I Nr. $116 / 2013$.

$3 \S 206$ StCB 2013 und § 207 StCB 2013 sowie BMDW (o.j.).

4 Die Regelungen zum Schutzalter führen bisweilen zu der Interpretation, dass Menschen erst ab einem bestimmten Alter mit anderen sexuell aktiv sein dürfen. Das Ge- 
Abgesehen davon widmen sich bestimmte Strafrechtsparagrafen dem besonderen Schutz mündiger Minderjähriger. ${ }^{5}$ Die Verbote beziehen sich auf Ungleichverhältnisse zwischen den beteiligten Personen. Das Ausnutzen einer altersbedingten Überlegenheit gegenüber Personen unter 16 Jahren, die aufgrund mangelnder Reife die Bedeutung einer sexuellen Handlung nicht einsehen können, ist strafrechtlich relevant. ${ }^{6}$ Diese Regelung dürfte $\mathrm{zu}$ der häufigen Fehlannahme beitragen, dass das Schutzalter erst mit 16 Jahren ende. Sie wird nicht oft vollzogen und bedarf eines psychologischen Sachverständigengutachtens. Besonders geschützt sind Jugendliche unter 18 Jahren auch bei Ausnutzung einer Zwangslage ${ }^{7}$ und entgeltlicher Verleitung zum Sex. ${ }^{8}$ Das Verbot intimer Kontakte von Lehrkräften mit Schüler:innen wird in der sexualpädagogischen Arbeit gerne von Jugendlichen thematisiert. Tatsächlich ist die strafrechtliche Relevanz bei mündigen Minderjährigen hier nur bei Ausnutzung des Autoritätsverhältnisses gegeben, also bei durch Druck zustande gekommenen sexuellen Begegnungen. ${ }^{9}$ Abgesehen davon kann das Dienstrecht zum Tragen kommen: Die sexuelle Handlung kann als Dienstrechtsverletzung der Lehrperson gewertet werden und als solche Konsequenzen haben. ${ }^{10}$

Das Geschlecht der beteiligten Personen ist laut Gesetz irrelevant. In Österreich wurde das allgemeine Verbot homosexueller Kontakte 1971 aufgehoben. Bis in die 2000er Jahre enthielten die Regelungen zu den Altersgrenzen ein erhöhtes Schutzalter von 18 Jahren für homosexuelle männliche Begegnungen. Dieses wurden 2002 egalisiert und geschlechtsneutral gestaltet.

setz richtet sich jedoch ausschließlich an Jugendliche und Erwachsene, also an mündige Personen, um Kinder vor sexuellen Begegnungen mit diesen zu schützen.

Zusammenfassend zur Strafbarkeit von sexuellen Handlungen mit Kindern und Jugendlichen vgl. auch Staffe 2009: 114f.

$6 \S 207$ b Abs. 1, StCB, BCBI. I Nr. 112/2015.

$7 \S 207$ b Abs. 2, StCB, BCBI. I Nr. 112/2015.

8 §207b Abs. 3, StCB, BCBI. I Nr. 112/2015.

$9 \S 212 \mathrm{StCB}, \mathrm{BCBI}$. I Nr. 117/2017.

10 Eine Dienstpflichtverletzung kann Kündigung, Suspendierung, Versetzung oder ein Disziplinarverfahren nach sich ziehen (siehe $\S 69$ Landeslehrer-Dienstrechtsgesetz 1984 und folgende), § 69 Landeslehrer-Dienstrechtsgesetz (LDC), BCBI. Nr. 302/1984. 


\subsection{Schutzalter in Deutschland}

Ähnlich wie in Österreich spielt auch im deutschen Strafrecht ${ }^{11}$ der Altersunterschied bei freiwilligen sexuellen Begegnungen keine juristische Rolle, sofern die Personen mindestens 14 Jahre alt sind. Es gibt auch in der deutschen Rechtsprechung einige untersagte Sonderfälle, wie etwa das Ausnutzen einer Zwangslage, in der sich eine unter 18-jährige Person befindet, oder das Verleiten von Personen unter 18 Jahren zu sexuellen Handlungen gegen Entgelt. Somit wird auch in Deutschland zum Schutz jugendlicher Personen geregelt, dass sexuelle Handlungen unter Ausnutzen der fehlenden Selbstbestimmung oder eines Abhängigkeitsverhältnisses verboten sind.

\section{Verhütung}

Jene kontrazeptiven Methoden, für die eine ärztliche Verschreibung (hormonelle Verhütung) oder Intervention (z.B. das Legen einer Spirale) notwendig ist, sind mündigen Personen ab 14 Jahren vorbehalten oder brauchen eine explizite Überprüfung der Einsichtsfähigkeit. Andere Verhütungsmethoden, wie das Kondom oder die Muttermundkappe, unterliegen keinen rechtlichen Bestimmungen.

\subsection{Hormonelle Verhütungsmittel}

Die Verschreibung eines hormonellen Verhütungsmittels (z.B. Pille, Verhütungspflaster) ist ab der Einsichtsfähigkeit der minderjährigen Person ohne Einwilligung der Eltern bzw. der Erziehungsberechtigten möglich. Ab 14 Jahren wird von einer Einsichtsfähigkeit ausgegangen, bei 13-Jährigen ist eine Verschreibung möglich, nachdem Einsicht und Verständnis von ärztlicher Seite überprüft wurden.

Hormonelle Verhütungsmittel sind sowohl in Deutschland als auch in Österreich verschreibungspflichtig. In Österreich sind hormonelle Verhütungsmittel allerdings nie eine Kassenleistung und können daher nicht mit der

$11 \S 176$ Strafgesetzbuch (StGB) (1871) in der Fassung vom 3.3.2020, Bundesgesetzblatt (BCBI.) 2020 Teil I Nr. 11 S. 431. und § 176a StCB (1871) in der Fassung vom 3.3.2020, BCBI. I, Nr. 11, S. 431. und $\S 176$ b StGB (1871) in der Fassung vom 3.11.1998, BCBI. I, Nr. 75, S. 3372. und § 182 StCB (1871) in der Fassung vom 21.1.2015, BCBI. 2015 I, Nr. 2, S. 10. 
Krankenkasse abgerechnet werden. In Deutschland erhalten Frauen seit dem 29. März 2019 bis zum vollendeten 22. Lebensjahr verschreibungspflichtige Verhütungsmittel auf Kassenrezept.

\subsection{Nicht-hormonelle Verhütungsmethoden}

Rein rechtlich ist das Legen eines Intrauterinpessars (z.B. Kupferspirale) ab Beginn der Mündigkeit möglich. In der Praxis wird diese Form der Verhütung zumeist erst nach dem Auswachsen der Gebärmutter angewendet. Sterilisation und Vasektomie als irreversible Methoden der Verhütung sind im Gesetz ${ }^{12}$ extra geregelt; ein derartiger Eingriff ist für Personen unter 25 Jahren nicht möglich.

\subsection{Die Pille danach}

Für die Pille danach gibt es in Österreich keine gesetzliche Regelung bezüglich Abgabe und Altersbeschränkung. Auf Empfehlung der Apothekerkammer und auf Grundlage eines Bescheids aus dem Bundesamt für Sicherheit im Gesundheitswesen (BASG o.J.) werden alle Apotheker:innen angehalten, die Pille danach allen Frauen im gebärfähigen Alter auf deren Verlangen auszuhändigen.

\subsection{Sexualpädagogische Überlegungen zum Thema Verhütung}

Das Thema Verhütung ist in vielen sexualpädagogischen Interventionen das zentrale Thema, es soll aber nicht das alleinige sein: Die Erklärung von einzelnen Verhütungsmitteln ersetzt nicht das grundsätzliche Gespräch über Sexualität. Unumgänglich ist, dass die angebotenen Informationen zu den einzelnen Verhütungsmitteln in einem lebens- und handlungsnahen Kontext thematisiert und zielgruppen- und altersgerecht präsentiert werden. Unsere Erfahrung in der Arbeit mit Jugendlichen zeigt, dass es wichtig ist, mit Jugendlichen ganz konkret zu besprechen, wie beispielsweise eine regelmäßige Pilleneinnahme in den persönlichen Alltag integrierbar sein könnte. Konkretheit braucht es auch, wenn es um Ausnahmesituationen wie z.B. eine Kondompanne geht. Klare und verständliche Informationen zu Verhütung können die Handlungsmöglichkeiten der Zielgruppe unterstützen, vor allem 
dann, wenn sie respektvoll und ohne moralisierende Botschaften vermittelt werden. Letzteres gilt auch und besonders für die Auseinandersetzung mit dem Thema Safer Sex, ${ }^{13}$ das sich im Zusammenhang mit Informationen zur Verhütung anbietet.

\section{Schwangerschaftsabbruch}

Grundsätzlich ist der Abbruch einer Schwangerschaft in Österreich verboten, ${ }^{14}$ bleibt unter bestimmten Umständen aber straffrei. ${ }^{15}$ Dies gilt für eine Schwangerschaftsunterbrechung innerhalb der ersten drei Monate nach Beginn der Schwangerschaft, wenn die Schwangere von ärztlicher Seite beraten und behandelt wurde. Der Beginn der Schwangerschaft wird mit der Einnistung der Eizelle gleichgesetzt. In der Praxis wird der Beginn der dreimonatigen Frist, innerhalb derer ein Abbruch möglich ist, vom ersten Tag der letzten Monatsblutung an gerechnet. Für eine betroffene Person bedeutet dies, dass sie wahrscheinlich frühestens im zweiten Monat von der Schwangerschaft erfährt und abhängig davon einen mehr oder weniger begrenzten Zeitraum für die Entscheidungsfindung zur Verfügung hat, falls ein Weiterführen der Schwangerschaft nicht feststehen sollte.

Die rechtliche Regelung zum straffreien Schwangerschaftsabbruch wird auch als Fristenlösung oder Fristenregelung bezeichnet. Straffrei bleibt eine Abtreibung auch über die gesetzliche Frist von drei Monaten hinaus, wenn eine ernste Gefahr für die Schwangere besteht, wenn eine schwere geistige oder körperliche Beeinträchtigung des Kindes vorliegt, die mit dem Leben nicht vereinbar ist oder wenn die werdende Mutter zum Zeitpunkt der "Schwängerung « ${ }^{16}$ das 14. Lebensjahr noch nicht vollendet hat. Indikationen für einen sogenannten Spätabbruch entscheiden ausschließlich Ärzt:innen.

Eine Abtreibung durchzuführen, ohne die Einwilligung der Schwangeren einzuholen, ist nicht gesetzeskonform, es sei denn, der Abbruch dient der Rettung der Schwangeren aus einer nicht anders abwendbaren Lebens-

13 D.h. mit Maßnahmen, die dazu geeignet sind, das Risiko einer Ansteckung mit sexuell übertragbaren Infektionen zu verhindern bzw. reduzieren.

$14 \S 96 \mathrm{StCB}, \mathrm{BCBI}$. I Nr. 112/2015.

$15 \S 97 \mathrm{StCB}, \mathrm{BCB}$. I Nr. 112/2015.

$16 \S 97$ Abs. 2, StCB, BCBI. I Nr. 112/2015. 
gefahr. ${ }^{17}$ Dies ist auch der einzige Grund, der Ärzt:innen zur Durchführung einer Abtreibung verpflichtet. Ein Schwangerschaftsabbruch ist in Österreich ab 14 Jahren ohne Einwilligung einer erziehungsberechtigten Person möglich. Nicht zuletzt weil von ärztlicher Seite bei sehr jungen mündigen Minderjährigen mitunter dennoch auf eine solche Einwilligung bestanden wird, kann elterliche bzw. erwachsene Unterstützung in einer solchen Situation notwendig sein.

Die einschlägigen deutschen Regelungen sind jenen in Österreich ähnlich. In Deutschland sind allerdings eine Schwangerschaftskonfliktberatung, die nicht von Ärzt:innen durchgeführt wird, und eine dreitägige Wartefrist zwischen Beratung und Eingriff gesetzlich vorgeschrieben (vgl. Pro familia 2017: 9).

Die medizinischen Methoden sind in Deutschland und Österreich ident. Ein Schwangerschaftsabbruch kann chirurgisch durchgeführt werden und bis zur 9. Woche kann die Schwangerschaft auch medikamentös abgebrochen werden. Die Kosten für einen Schwangerschaftsabbruch sind unterschiedlich hoch. Während diese in der Schweiz beispielsweise von der Krankenkasse übernommen werden, übernimmt in Deutschland das Sozialamt die Kosten, wenn das Nettoeinkommen der Schwangeren unter 1.000 Euro liegt. In Österreich muss der finanzielle Aufwand, der zwischen 400 und 700 Euro liegt, grundsätzlich privat bezahlt werden. Eine Ausnahme stellt der Abbruch nach einer Indikation dar, bei der die medizinische Leistung von der Krankenkasse übernommen wird. Frauen müssen in Österreich keine Gründe für den Abbruch angeben, auch werden ihre personenbezogenen Daten nicht weitergegeben.

\subsection{Sexualpädagogische Überlegungen zum Thema Schwangerschaftsabbruch}

Das Thema Schwangerschaftsabbruch ist zweifelsohne ein sehr wertebesetztes (vgl. Weidinger/Kostenwein/Dörfler 2007: 84ff.). Es gibt unterschiedliche Haltungen dazu und die Debatten werden mitunter emotional geführt. Wie bei anderen Inhalten auch geht es nicht um den persönlichen Zugang der Pädagog:in, sondern um sachliche Information und respektvolle Auseinandersetzung. Das Thema kann gerade aufgrund seiner (moralischen) Aufgeladenheit exemplarisch dafür stehen, wie im Unterricht wertschätzend mit 
unterschiedlichen Meinungen und Haltungen umgegangen werden kann und wie diese nebeneinander stehen können, ohne dass auf der Werteebene ein allgemeingültiges $>$ Richtig< oder $>$ Falsch $<$ identifiziert werden muss.

\section{Heilbehandlungen}

Außer bei unmittelbarer Gefahr für Leben oder Gesundheit brauchen Heilbehandlungen ${ }^{18}$ die wirksame Einwilligung der betroffenen Patient:innen. ${ }^{19}$ Für unmündige Minderjährige (Kinder zwischen 0 und 14 Jahren) muss diese durch die Zustimmung von Eltern bzw. Erziehungsberechtigten ersetzt werden. Bei mündigen Minderjährigen (zwischen 14 und 18 Jahren) wird die nötige Einsichts- und Urteilsfähigkeit für eine Einwilligung in einfache Behandlungen angenommen (vgl. Staffe 2009: 113f.). Als einfache, hier zustimmungsfreie Eingriffe gelten alltägliche, risikoarme Behandlungen, wie z.B. eine Warzenentfernung, Zahnfüllung oder Grippeimpfung (vgl. Gleixner-Eberle 2014: 71). Dazu gehören auch Untersuchungen auf sexuell übertragbare Infektionen. Für schwerwiegende Eingriffe ${ }^{20}$ brauchen mündige Minderjährige die Zustimmung einer erziehungsberechtigten Person.

\subsection{Schönheitsoperationen}

Eine ästhetische Operation ${ }^{21}$ gilt nur dann als Heilbehandlung, wenn eine medizinische Indikation vorliegt (etwa bei orthopädischen Beschwerden aufgrund der Brustgröße) und wird ohne eine solche nicht von der Krankenkasse bezahlt. Minderjährige dürfen sich ab dem vollendeten 16. Lebensjahr und nach einer Beratung durch Klinische Psycholog:innen, Fachärzt:innen für Psychiatrie und psychotherapeutische Medizin oder Fachärzt:innen für

18 Als Heilbehandlungen gelten sämtliche medizinische Maßnahmen, die nach medizinischer Indikation vorgenommen werden, um Krankheiten, Leiden, körperliche Schäden, körperliche Beschwerden oder seelische Störungen zu erkennen, zu heilen, zu lindern oder zu verhüten (vgl. Cleixner-Eberle 2014: 18ff.).

$19 \S 173$ Allgemeines Bürgerliches Gesetzbuch (ABCB), BCBI. I Nr. 59/2017.

20 Als schwerwiegende Behandlungen gelten risikoreichere, beeinträchtigende Eingriffe wie größere Operationen oder eine Strahlentherapie (vgl. Gleixner-Eberle 2014: 71f.).

21 Laut $\$ 4$ Bundesgesetz für die Durchführung von ästhetischen Behandlungen und Operationen (ÄsthOpC), BCBI. I Nr. 80/2012 z.B. Brustkorrekturen, Fettabsaugungen oder Lippenaufpolsterungen. 
Kinder- und Jugendpsychiatrie einer Schönheitsoperation unterziehen. Bis zum 18. Lebensjahr braucht es dafür die Zustimmung der Eltern oder Erziehungsberechtigten. Zudem müssen bestimmte Fristen zwischen Aufklärung, Einwilligung und Operation eingehalten werden. ${ }^{22}$

\subsection{Tattoos und Piercings}

Das Piercen von unter 14-Jährigen ist verboten, bei mündigen Minderjährigen braucht es die Zustimmung der Eltern bzw. der Erziehungsberechtigten. Wenn jedoch zu erwarten ist, dass die gepiercte Stelle innerhalb von 24 Tagen verheilt, ist die Zustimmung nicht notwendig. Das Tätowieren ist für Minderjährige ab 16 Jahren erlaubt. Bis zum Alter von 18 Jahren braucht es dafür die Zustimmung einer erziehungsberechtigten Person (vgl. BMDW o.J.).

\section{Pornografie}

Regelungen $\mathrm{zu}$ pornografischem Material finden sich in Österreich im Pornographiegesetz (PornoG), ${ }^{23}$ im Sexualstrafrecht ${ }^{24}$ zur pornografischen Darstellung Minderjähriger und in den Jugendschutzgesetzen der einzelnen Bundesländer. Der Begriff der Pornografie ist gesetzlich nicht eindeutig definiert. Der $\$ 1$ des PornoG bezieht sich auf »unzüchtige Schriften, Abbildungen, Laufbilder ...«; im $\$ 2$ geht es um »eine Schrift, Abbildung oder sonstige Darstellung, die geeignet ist, die sittliche oder gesundheitliche Entwicklung jugendlicher Personen durch Reizung der Lüsternheit oder Irreleitung des Geschlechtstriebes zu gefährden «. ${ }^{25}$ Welches Material unter diese Definitionen fällt, unterliegt zeitlichen und kulturellen Veränderungen und im Einzelfall einer richterlichen Entscheidung.

$22 \S 7$ ÄsthOpG, BGBI. I Nr. 59/2018.

$23 \S 10$ Pornographiegesetz (PornoG), BCBI. Nr. 97/1950; § 11 PornoC, BCBI. Nr. 97/1950; $\S 12$ PornoG, BGBI. Nr. 97/1950; § 13 PornoG, BCBI. Nr. 97/1950; § 14 PornoG, BGBI. Nr. 97/1950.

$24 \S 207$ a StGB, BCBI. I Nr. 117/2017.

25 Im Duden findet sich folgende Definition: »sprachliche, bildliche Darstellung sexueller Akte unter einseitiger Betonung des genitalen Bereichs und unter Ausklammerung der psychischen und partnerschaftlichen Aspekte der Sexualität« (Dudenredaktion, o.J.) 


\subsection{Pornografiegesetz und Sexualstrafrecht}

Nach dem Pornografiegesetz ist das Anbieten, Überlassen und Zugänglichmachen jeglicher Art von Pornografie an Personen unter 16 Jahren verboten. Ein allgemeines Verbot gilt für sogenannte »harte Pornografie« (Graupner 2006: 16). Nach herrschender Rechtsprechung fallen Kinderpornografie, Gewalt- und Tierpornografie unter diese Definition. Das Verbot von harter Pornografie gilt nach dem Pornografiegesetz nur für den gewerblichen Umgang mit Gewinnabsicht, nicht für Konsum und Besitz. Was Kinderpornografie betrifft, ist laut Sexualstrafrecht auch der Besitz und das wissentliche Zugreifen strafbar. Der Begriff der Kinderpornografie bezeichnet Kindesmissbrauchsdarstellungen und generell die Darstellung von "geschlechtlichen Handlungen ${ }^{26}$ an oder mit Personen unter 18 Jahren, unabhängig davon, in welchem Setting diese zustande gekommen sind, oder ob Gewalt mit im Spiel war. Letzteres schlägt sich jedoch im Strafausmaß nieder).

\subsection{Pornografie in den Jugendschutzgesetzen}

Die Regelungen in den Landesjugendschutzgesetzen sind länderspezifisch leicht unterschiedlich formuliert. ${ }^{27}$ Generell gilt, dass die Weitergabe von pornografischen, diskriminierenden oder gewaltverherrlichenden Inhalten an Jugendliche unter 18 verboten ist und Jugendliche solche Inhalte nicht erwerben, besitzen oder verwenden dürfen.

Das Besuchen von Sexshops, Nachtclubs und Bordellen ist Jugendlichen unter 18 Jahren verboten, ebenso die Inanspruchnahme und das Anbieten sexueller Dienstleistungen.

\subsection{Sexualpädagogische Überlegungen zum Thema Pornografie}

Aus unserer Sicht zielen sexualpädagogische Inputs zur Altersfreigabe pornografischer Inhalte nicht auf Abschreckung oder Drohung mit juristischen

\footnotetext{
$26 \S 207 a$ Abs. 4, StCB, 2017.

27 Eine Übersicht über die Jugendschutzgesetze der österreichischen Bundeländer findet sich online unter https://de.wikipedia.org/wiki/Jugendschutzgesetze_in_Österreich [Zugriff: 15.04.2020].
} 
Konsequenzen, sondern auf Information zur Sache und die Reflexion des Themas. Neugier auf sexuelle Inhalte in Wort und Bild ist Teil der Entwicklung von Kindern und Jugendlichen. Die Förderung der Medienkompetenz junger Menschen steht im Vordergrund und vollzieht sich zum einen durch eine differenzierte Auseinandersetzung mit medialen Inhalten, zum anderen auch durch Unterstützung von kognitiven, emotionalen, körperlichen und sozialen Kompetenzen der Zielgruppen (vgl. Kostenwein 2009: 61ff.). Das Wissen um persönliche und generell menschliche sexuelle Möglichkeiten und Grenzen jenseits virtueller Inszenierung sowie eine gute körperliche und emotionale Selbstwahrnehmung unterstützen das sexuelle Selbstbewusstsein und eine Orientierung an eigenen Bedürfnissen und Sehnsüchten im Gegensatz zu äußeren Bildern und Handlungsvorgaben. Das respektvolle Einlassen auf eine sexuelle Begegnung mit all ihren Überraschungen ist dann leichter möglich, ohne bestimmte Handlungen (durch-)setzen zu müssen.

Wenn Jugendliche pornografische Inhalte konsumieren, zieht es in der Regel keine rechtlichen Konsequenzen nach sich. Dies gilt jedoch nicht für das Verbreiten pornografischer Inhalte unter Minderjährigen und vor allem nicht für Abbildungen von Genitalien oder Darstellungen sexueller Handlungen, in die Minderjährige involviert sind. Neben dem Download entsprechender Inhalte oder dem "wissentlichen Zugriff ${ }^{28}$ darauf kann auch das Verschicken solcher Abbildungen oder Videos - etwa im Freundeskreis - bei einer Anzeige rechtliche Konsequenzen nach sich ziehen. Dies gilt auch, wenn eine minderjährige Person ein Selfie, auf dem sie bei einer sexuellen Handlung oder dem Zeigen der eigenen Genitalien zu sehen ist, verbreitet oder veröffentlicht.

Neben dem Hinweis, dass solche Inhalte zu löschen sind und etwaige Funde im Netz gegebenenfalls gemeldet werden können, ${ }^{29}$ bietet sich in der sexualpädagogischen Arbeit eine Auseinandersetzung mit Themen wie Selbstdarstellung (im Netz) oder der Bedeutung von Attraktivität an. Attraktivität durch >Sexyness< ist Teil unserer Kultur und Jugendliche bedienen sich deren Versatzstücke in der Selbstpräsentation.

$28 \S 207 a$ Abs. 3a StCB, 2017. Ein »wissentlicher Zugriff« auf einschlägige Inhalte wird bei wiederholtem Zugriff angenommen.

29 Anonyme Meldungen sind online möglich unter https://www.stopline.at [Zugriff 19.12.2020]. 


\subsection{Sexting}

Prinzipiell ist das Versenden von eigenen Nacktaufnahmen durch Minderjährige verboten. Jedoch ist Sexting, also das einvernehmliche private Versenden von erotischem Bildmaterial des eigenen Körpers, für Jugendliche ab 14 Jahren seit 2016 entkriminalisiert. ${ }^{30}$ Das Verschicken oder Tauschen und damit auch Besitzen von pornografischen Fotos bzw. Filmen untereinander für den Eigengebrauch ist unter mündigen Minderjährigen damit straffrei. Solche Aufnahmen Dritten zu zeigen oder weiterzuleiten ist jedoch nicht erlaubt.

\subsection{Sexualpädagogische Überlegungen zum Thema Sexting}

In der Lebensrealität Jugendlicher kann Sexting ein Teil der Kommunikation sein, genauso wie unter Erwachsenen. Oft ergeben sich dadurch keine Probleme, doch kann die Handhabe über das eigene Bild oder den Film verloren gehen, wenn etwa nach einem Streit oder einer Trennung Material weitergesendet oder gar veröffentlicht wird. Jenseits von Dramatisierungen kann in der sexualpädagogischen Arbeit eine Sensibilisierung für den Umgang mit eigenem Bildmaterial angeregt und über die Gestaltung erotischer Bilder diskutiert werden.

Landet ein heikles Bild letztlich ungewollt im Netz oder erleben Jugendliche andere Probleme mit den sogenannten Neuen Medien, wenden sie sich oft aus Scham oder in der Erwartung von Unverständnis und möglichen Vorwürfen nicht an Erwachsene um Unterstützung. Bei einschlägigen Fragestellungen gibt es verschiedene Informations- und Anlaufstellen, die sowohl weiterführende rechtliche Informationen, etwa zum Cybermobbing-Paragrafen oder anderen Regelungen, als auch Tipps für den persönlichen Umgang und die Unterstützung Betroffener bieten. ${ }^{31}$

$30 \S 207 a$ Abs. 5, StGB, 2017.

31 Für Österreich siehe etwa: https://www.saferinternet.at, https://www.ombudsmann.at, für Deutschland: https://www.klicksafe.de [Zugriff 19.12.2020]. 


\section{Aufsichtspflicht}

Die Aufsichtspflicht gegenüber Kindern und Jugendlichen ist nicht explizit in einem eigenen Gesetz geregelt (vgl. Nademleinsky 2019). Sie ergibt sich aus diversen Erwähnungen in unterschiedlichen gesetzlichen Regelungen wie etwa der Obsorgepflicht im Allgemeinen Bürgerlichen Gesetzbuch (ABGB) und den Jugendschutzgesetzen sowie durch die Rechtsanwendung der Gerichte. In diesem Sinne wird retrospektiv beurteilt, ob in bestimmten Fällen von Personenschaden oder Sachbeschädigung eine Aufsichtspflicht verletzt wurde. Die Aufsichtspflicht ist also abhängig vom Setting und vom Kompetenzprofil der zu beaufsichtigenden Person(en). Aufsichtspflicht gilt für jene Personen, denen sie gesetzlich zukommt (Obsorgepflichtige, Lehrkräfte während der Unterrichtszeit), die sich vertraglich dazu verpflichtet haben (im Kindergarten, im Ferienlager) und für jene, die sie faktisch übernommen haben (beim Besuch vom Nachbarskind, im Jugendzentrum).

Für externe Sexualpädagog:innen im Schulsetting bedeutet dies, dass die geltende Hausordnung eingehalten werden muss. Anderslautende Regelungen, etwa für Pausen, sind klar zu vereinbaren. Schüler:innen dürfen nicht aus der Klasse geschickt werden, es sei denn, eine Lehrkraft übernimmt die Aufsichtspflicht. Das kurzfristige Verlassen der Klasse in üblicher Weise (für den Gang auf die Toilette) ist davon ausgenommen. Bei Unwohlsein empfiehlt es sich, mündige Minderjährige nicht ohne Begleitung zu Schulärzt:innen gehen zu lassen und Unmündige in die Hände einer Lehrkraft zu übergeben.

Die Folgen von verletzter Aufsichtspflicht sind rechtlich belangbar und können eine Schadenersatzpflicht nach sich ziehen.

\section{Sexualpädagogik als Unterrichtsprinzip}

Das Thema Sexualität an sich und vor allem die sexualpädagogische Begleitung von Kindern und Jugendlichen wird nicht selten kontrovers diskutiert und ist von Missverständnissen geprägt. Unterschiedliche Vorstellungen über das Ob und Wie der Etablierung des Themenbereichs in der Schule befeuern bisweilen das Aufflammen von Kontroversen.

In Österreich ist der sexualpädagogische Auftrag der Schulen im Grundsatzerlass Sexualpädagogik geregelt: "Schule als Teil des Entwicklungsfeldes von Kindern und Jugendlichen hat im Bereich der Sexualpädagogik klare Aufgaben, die sich auf die unterschiedlichen Ebenen der sexuellen Kompe- 
tenzentwicklung beziehen. Altersadäquat beginnt Sexualpädagogik in der Schule mit dem Schuleintritt und endet mit Austritt aus dem Schulbereich.« (BMBF 2015: 5) Im Rahmen einer Haltung, die »einen positiven Bezug zur sexuellen Entwicklung von Kindern und Jugendlichen spürbar macht«, sollen unterschiedliche Ressourcen wie Persönlichkeitsentwicklung, Ausdrucksund Auseinandersetzungsfähigkeit, Fakten-Wissen, Körperkompetenz und der Umgang mit sozialen Regeln unterstützt werden (vgl. BMBF 2015: 6ff.). Dem Sexualerziehungserlass gemäß ist Sexualpädagogik als Bildungsund Lehraufgabe in allen Lehrplänen als Unterrichtsprinzip verankert und postuliert die dahingehende Zusammenarbeit mit Erziehungsberechtigen, Schulpsycholog:innen, Schulärzt:innen sowie außerschulischen Einrichtungen (vgl. BMBF 2015: 10).

Die deutsche Bundeszentrale für gesundheitliche Aufklärung (BZgA) hat zusammen mit dem WHO-Regionalbüro für Europa Standards für die Sexualaufklärung in Europa herausgegeben (WHO/BZgA 2011), auf die auch der Grundsatzerlass in Österreich Bezug nimmt. Die Umsetzung der Regelungen für deutsche Schulen obliegt den einzelnen Ländern und ist in deren Schulgesetzen verankert (vgl. BZgA 2004).

\section{Rechtliche Inputs als Teil ressourcenorientierter sexualpädagogischer Arbeit}

Rechtliche Fragestellungen können sich in der sexualpädagogischen Gruppenarbeit spontan ergeben, z.B. aufgrund des Interesses der Zielgruppe, oder für einen vorbereiteten Input entwickelt werden. Letzteres kann beispielsweise auch im Rahmen einer Übung oder eines Spiels Platz finden. So könnte etwa für die Thematik der Altersgrenzen für sexuelle Kontakte eine Fallgeschichte mit einer Quizfrage konstruiert werden. ${ }^{32}$ Anhand dieser können die Fragen nach >dem optimalen Zeitpunkt< und dem >Bereit-Sein für Sex , die Jugendliche häufig interessieren, besprochen werden. Dies bietet eine Gelegenheit zur Reflexion eigener Emotionen, Wahrnehmungen und Körperempfindungen, die es erleichtern kann, sich innerhalb mitunter ambivalenter Gefühlslagen zu orientieren (vgl. Kapella/Kostenwein/Weidinger 2009: 
187ff.). Hinweise auf Gesetzeslagen stellen nur einen Aspekt innerhalb der ressourcenorientierten sexualpädagogischen Arbeit mit jungen Menschen dar. Es gilt, sie in einen Gesamtzusammenhang einzubetten, der den Lebenswelten der Zielgruppe gerecht wird.

Zur Unterstützung der Pädagog:innen gibt es Anlaufstellen, die rechtliche Belange inhaltlich abdecken und ein Broschürenservice ${ }^{33}$ bereitstellen, das auf die Zielgruppe zugeschnitten ist. ${ }^{34}$ Rechtliches Wissen ermöglicht Pädagog:innen eine Themenauswahl für ihre Zielgruppe zu treffen, auf einschlägige Fragen einzugehen und begründet zu entscheiden, wann es sinnvoll ist, Jugendliche mit ihren Fragen an Beratungsstellen oder juristische Expert:innen zu verweisen.

\section{Abkürzungsverzeichnis}

ABGB Allgemeines Bürgerliches Gesetzbuch

BGBI. Bundesgesetzblatt

Ästh0pg Bundesgesetz über die Durchführung von ästhetischen Behandlungen und Operationen

LDG Landeslehrer-Dienstrechtsgesetz

PornoG Pornografiegesetz

StGB Strafgesetzbuch

SPG Sicherheitspolizeigesetz

33 Es empfiehlt sich vor der Verwendung von Materialien im Unterricht persönlich zu eruieren, ob sie aktuell sind und inhaltlich bzw. umfänglich den eigenen Anforderungen entsprechen.

34 So bietet etwa die Jugendinfo von wienXtra Broschüren zu Jugendrechten, eine Taschenanwältin u.v.m. zum Download oder im kostenlosen Bestellservice. Die Seite richtet sich an verschiedene Zielgruppen. Es gibt auch Materialien für Multiplikator:innen und Eltern (vgl. Jugendinfo o.].). 


\section{Literatur}

BASG - Bundesamt für Sicherheit im Gesundheitswesen (o.J.): Vikela (»Pille danach«) rezeptfrei. Online unter: https://www.basg.gv.at/marktbeob achtung/amtliche-nachrichten/detail/vikela-pille-danach-rezeptfrei [Zugriff: 14.05.2020].

BMASGK - Bundesministerium für Arbeit, Soziales, Gesundheit und Konsumentenschutz (2019): Die Geschäftsfähigkeit Jugendlicher. Online unter: https://broschuerenservice.sozialministerium.at/Home/Download?p ublicationId=116 [Zugriff: 14.05.2020].

BMBF - Bundesministerium für Bildung und Frauen (2015): Grundsatzerlass Sexualpädagogik. Online unter: https://www.bmbwf.gv.at/Themen/schu le/schulrecht/rs/1997-2017/2015_11.html [Zugriff: 05.05.2020].

BMDW - Bundesministerium für Digitalisierung und Wirtschaftsstandort (o.J.a): Piercing und Tattoo. Online unter: https://www.oesterreic h.gv.at/themen/jugendliche/jugendrechte/5/Seite.1740309.html [Zugriff: 03.05.2020].

Bundesministerium für Digitalisierung und Wirtschaftsstandort (o.J.b): Sexuelle Kontakt unter Jugendlichen. Online unter https:/www.oesterreich .gv.at/themen/jugendliche/sexuelle_kontakte.html [Zugriff: 03.05.2020]. BZgA - Bundeszentrale für gesundheitliche Aufklärung (2004): Richtlinien und Lehrpläne zur Sexualerziehung. Eine Analyse der Inhalte, Normen, Werte und Methoden zur Sexualaufklärung in den sechzehn Ländern der Bundesrepublik Deutschland. Online unter: https://www.bzga.de/infom aterialien/fachpublikationen/band-04-richtlinien-und-lehrplaene-zur-se xualerziehung [Zugriff: 03.05.2020].

Dudenredaktion (Hg.). (o.J.): Pornografie. Online unter: https://www.duden. de/rechtschreibung/Pornografie [Zugriff: 10.05.2020].

Gleixner-Eberle, Elisabeth (2014): Die Einwilligung in die medizinische Behandlung Minderjähriger. Eine arztrechtliche Untersuchung im Rechtsvergleich mit Österreich und der Schweiz sowie mit Blick auf das Internationale Privat- und Strafrecht. Berlin/Heidelberg: Springer-Verlag.

Graupner, Helmut (2006): Das späte Menschenrecht. Sexualität im Recht. Innsbruck: Universität Innsbruck. 
Häßler, Frank/Schepker, Renate (2016): Strafrechtliche Verantwortung von jugendlichen Straftätern. In: Völkl-Kernstock, Sabine/Kienbacher, Christian (Hg.): Forensische Arbeit mit Kindern und Jugendlichen. Wien: Springer Verlag, S. 253-268.

Internet Ombusmann Österreich (o.J.): Online unter: https://www.ombudsm ann.at [Zugriff: 15.04.2020].

Johannes Kepler Universität Linz (2017): Multimediale Studienmaterialien GmbH. Strafrecht. Paragraph. Seitenweise österreichische Rechtstexte für Studium und Praxis. 12. Auflage. Wien: Manz Verlag.

Jugendinfo von wienXtra (o.J.): Diverse Broschüren. Online unter: https://ww w.wienxtra.at/jugendinfo [Zugriff: 05.05.2020].

Kapella, Olaf/Kostenwein, Wolfgang/Weidinger, Bettina (2009): Fragen und Antworten. In: Österreichisches Institut für Sexualpädagogik (Hg.): Sex we can?! Das Manual zum Film. Wien: Bundesministerium für Unterricht, Kunst und Kultur, S. 166-192. Online unter: https://sexualpaedagogik.at/s ex-we-can [Zugriff: 02.06.2020].

Klick Safe (o.J): klick safe. Initiative des Telecom Programm der Europäischen Union. Online unter: https://www.klicksafe.de [Zugriff: 15.04.2020].

Kostenwein, Wolfgang (2009): Informationsquellen - Massenmedien und Pornografie. In: Österreichisches Institut für Sexualpädagogik (2009), Sex we can?! Das Manual zum Film. Wien: Bundesministerium für Unterricht, Kunst und Kultur, S. 59-65.

Nademleinsky, Marco (2019): Aufsichtspflicht. Was Kinder- und JugendbetreuerInnen wissen müssen (E-Book). Wien: Manz Verlag.

ÖIAT - Österreichisches Institut für angewandte Telekommunikation (o.J): Safer internet. Online unter: https://www.saferinternet.at [Zugriff: 15.04.2020].

Pro familia (2017): Schwangerschaftsabbruch. Fakten und Hintergründe. Frankfurt a.M.: Pro familia. Online unter: https://www.profamilia.de/fil eadmin/publikationen/Fachpublikationen/Schwangerschaftsabbruch/Hi ntergrund-Schwangerschaftsabbruch.pdf [Zugriff: 03.05.2020].

Staffe, Martina (2009): Juristische Fragen im Umgang mit Kindern und Jugendlichen. In: Österreichisches Institut für Sexualpädagogik (2009), Sex we can?! Das Manual zum Film. Wien: Bundesministerium für Unterricht, Kunst und Kultur, S. 113-117. Online unter: https://sexualpaedagogik.at/s ex-we-can [Zugriff: 02.06.2020].

Stopline Meldestelle Österreich (o.J.): Online unter: https://www.stopline.at [Zugriff 10.05.2020]. 
Weidinger, Bettina/Kostenwein, Wolfgang/Dörfler, Daniela (2007): Sexualität im Beratungsgespräch mit Jugendlichen. Wien: Springer Verlag. WHO-Regionalbüro für Europa/BZgA (2011): Standards für die Sexualaufklärung in Europa. Rahmenkonzept für politische Entscheidungsträger, Bildungseinrichtungen, Gesundheitsbehörden, Expertinnen und Experten. Online unter: https:/www.bzga.de/infomaterialien/sexualaufklaeru ng/who-regionalbuero-fuer-europa-und-bzga-standards-fuer-die-sexua laufklaerung-in-europa [Zugriff: 18.01.2020]. 


\section{Sexualpädagogische Elternarbeit in und rund um Schule \\ Eine Gesprächsrunde zu Haltungen, Herausforderungen und Rahmenbedingungen in der Elternarbeit}

Claudia Schneider

Die schulische sexualpädagogische Arbeit mit Kindern und Jugendlichen wird u.a. davon beeinflusst, was die Herauswachsenden an Wissen und Erfahrungen aus ihren Familien bzw. primären Bezugsgruppen mitbringen, also auch davon, wie ihre Eltern und Erziehungsberechtigten Sexualität wahrnehmen, definieren und bewerten. Familien haben unterschiedliche Eigenheiten und Gewohnheiten in Bezug auf Körpergrenzen, Liebes- und Lebensformen, in Bezug auf Sprache oder Wünsche oder das Sprechen oder Nicht-Sprechen über Gefühle. All diese Bereiche sind verbunden mit Intimität und Körper, Lust und Begehren und damit Aspekten von Sexualität.

Mit Eltern im Rahmen von sexualpädagogischer Elternarbeit ins Gespräch zu kommen und auf ihre Fragen und Sorgen einzugehen, kann als ein Auftrag von Sexualpädagog:innen betrachtet werden. Um über Haltungen, Herausforderungen und Rahmenbedingungen ins Gespräch zu kommen, wurden sexualpädagogisch Tätige zu einer Gesprächsrunde über Erfahrungen mit Elternbildungs- und -beratungsarbeit rund um Sexualität geladen. An dem Gespräch teilgenommen haben fünf Expert:innen: Peter Boden und Katrin Lehner arbeiten als Sexualpädagog_innen u.a. für die Fachstelle für Suchtprävention und Sexualpädagogik Niederösterreich, Robert Steiner ist als Sexualpädagoge in der Fachstelle Selbstbewusst in Salzburg tätig und Sevil Eder ist selbstständige Mitarbeiterin der Fachstelle Selbstlaut in Wien. Alle drei genannten Organisationen bieten in Schulen sexualpädagogische Workshops an. Diesen Workshops für Schüler:innen geht ein Elternabend voraus, im Rahmen dessen die sexualpädagogische Tätigkeit im Allgemeinen und das Programm des Workshops mit den Schüler:innen im Konkreten vorgestellt wird. Se- 
vil Eder spricht darüber hinaus auch im Rahmen des Programms »Mama lernt Deutsch« mit Müttern zu Sexualerziehung. Danijela Cicvarić arbeitet als Sozialarbeiterin im Romano Centro, einem Verein, der sich in Bildungs-, Beratungs- und Kulturarbeit für die Verbesserung der Lebensbedingungen von Roma einsetzt. Sie ist im Bereich Schulmediation und Lernhilfe tätig. Sexualität ist nicht das Hauptthema ihrer Arbeit, spielt jedoch etwa in Gesprächen mit Eltern oder Projekten wie der »Mädchen-Woche« eine wichtige Rolle. $^{1}$

Die Gesprächsrunde ${ }^{2}$ wurde von Claudia Schneider, Lektorin am Institut für das künstlerische Lehramt an der Akademie der bildenden Künste Wien, moderiert und aufgenommen. Der vorliegende Text basiert auf dem Transkript und ist eine von der Gesprächsleiterin gekürzte und - mit Zustimmung der Gesprächsteilnehmer:innen - umstrukturierte Fassung, die nicht immer dem chronologischen Ablauf des Gesprächs folgt. Dadurch ergibt sich eine inhaltliche Konzentration auf zentrale Themen, die durch die Zwischenüberschriften hervorgehoben werden.

Im Gespräch wird deutlich, dass Sexualerziehung viel mehr umfasst als die Bezeichnung von Genitalien und Wissen über Zeugung, Schwangerschaft und Geburt von Kindern. Die Gesprächsteilnehmer:innen betonen, wie wichtig Humor ist, dass Enttabuisierung Druck reduzieren kann, und erläutern, welche Bedeutung der Sexualerziehung für die Prävention von sexueller Gewalt zukommt. Sie sprechen über die Herausforderungen im Kontakt mit dieser Zielgruppe der Eltern und Erziehungsberechtigten und berichten von spannenden und gelingenden Momenten in der Elternarbeit. ${ }^{3}$

Die »Mädchen-Woche« ist ein Projekt für Roma-Mädchen, bei dem zu unterschiedlichen Themen wie etwa auch Sexualität und Lebensplanung gearbeitet wird.

2 Die Gesprächsrunde fand im September 2019 am Institut für das künstlerische Lehramt an der Akademie der bildenden Künste Wien statt.

3 Sowohl die Fachstelle Selbstlaut als auch die Fachstelle Selbstbewusst haben online verfügbare Broschüren für Eltern erstellt (vgl. Fachstelle Selbstlaut 2017; Fachstelle Selbstbewusst o.J.). 


\section{Fragen und Antworten als Geschenk}

Claudia Schneider: Ihr arbeitet in unterschiedlichen Settings mit Eltern mehr oder weniger explizit zu sexualpädagogischen Themen. Was ist euch in der Elternarbeit besonders wichtig?

Katrin Lehner: Mir ist in der Elternarbeit Transparenz sehr wichtig. Das bedeutet, dass wir die Eltern darüber aufklären, was wir im Workshop machen, wie wir bestimmte Themen bearbeiten und dass wir alle Fragen der Kinder beantworten, die das Thema betreffen. Eltern äußern besonders bei jüngeren Kindern immer wieder Bedenken und empfinden Fragen als nicht altersgerecht. Wir beantworten diese trotzdem, wenn auch sehr vereinfacht.

Mit dem Thema kindliche Sexualität können Eltern teilweise gar nichts anfangen, manche erleben es als Grenzüberschreitung, darüber zu sprechen. Viele wissen nichts über kindliche Sexualität und haben Angst, Kinder durch zu viele Informationen und/oder zu frühe Aufklärung zu überfordern.

Möglicherweise liegt das auch daran, dass manche generell ungern in einer größeren Gruppe über Sexualität sprechen. Es ist für manche Eltern schwierig, dass die eigenen Kinder, die vielleicht idealisiert werden, Fragen zu einem Thema haben, die für eine:n selbst schwierig zu beantworten wären. Deswegen ist es mir beim Elternabend wichtig, dass die Eltern für sich einen $>$ Rucksack mit Inputs bekommen, die sie dann auch selber zu Hause verwenden können. Der >Rucksack enthält Vorschläge, andere Blickwinkel auf das Thema einzunehmen und Hinweise, wie das Thema besprochen werden kann.

Peter Boden: Das ist für mich eine ganz wichtige Botschaft an die Eltern: Wenn sie eine Frage von einem Kind oder einem Jugendlichen zum Thema Sexualität bekommen, sollen sie sie als Geschenk wahrnehmen und ein Geschenk zurückgeben. Das Geschenk, das man zurückgeben kann, ist schlichtweg eine Antwort. Es geht darum, den Knopf im Kopf zu lösen - sollte es ihn geben -, auf dem steht, dass es >die perfekte Antwort< braucht. Zum Thema Sexualität gibt es keine perfekten Antworten. Es ist - unabhängig vom eigenen Alter - eine schöne Erfahrung, wenn man zum Thema Sexualität etwas fragt und die andere Person sich Zeit nimmt und sich eine Antwort dazu überlegt. Das ist, glaube ich, etwas ganz Wichtiges. Jüngere Kinder lernen dadurch, dass es okay ist, dass sie dazu Fragen stellen.

Ich nehme Unterschiede zwischen der Arbeit in Volksschulen und Mittel- 
schulen ${ }^{4}$ wahr. In der Volksschule fragen mich die Eltern oft: »Was kann ich machen, wenn mich mein Kind etwas fragt? « Und in der Mittelschule fragen mich die Eltern oft: »Was kann ich machen, dass mich mein Kind etwas fragt? «Ich glaube, es ist zentral die Lernerfahrung zu machen »Hey, es ist einfach okay, dazu eine Frage zu stellen«, auch wenn die andere Person sagt: »Du, da brauche ich jetzt mal zwei Tage, ich muss mich da einfach noch schlau machen«. Wenn das in frühen Jahren gelingt, kann die Chance höher sein, dass die Kinder, auch wenn sie älter sind, das Gespräch suchen.

Sevil Eder: Sexualerziehung ist das A und O für Prävention und den Schutz vor sexueller Gewalt. Darauf legen wir von der Fachstelle Selbstlaut besonders großen Wert wie auch auf Transparenz in Bezug darauf, wie wir mit den Kindern arbeiten. Wir stellen beim Elternabend unseren Workshop ganz genau vor und erklären die Gründe, warum wir dieses oder jenes machen. Die Eltern haben Bilder im Kopf, und häufig heißt Sexualerziehung für sie Geschlechtsverkehr. Sexualerziehung oder Sexualität sind Tabu-Themen und emotionale Themen. Wenn wir ihnen die Einladung schicken, dann steht da auch unser Name »Fachstelle gegen sexuelle Gewalt«, und das macht manchmal Angst. Wenn die Eltern beim Elternabend sitzen, sieht man, dass sie nervös sind und sich fragen, warum sie überhaupt hier sind. Es ist schön zu sehen, wenn sie danach mit einer gewissen Erleichterung nach Hause gehen und die Erkenntnis mitnehmen, dass sie mit ganz einfachen alläglichen Dingen ihre Kinder schützen und stärken können.

Robert Steiner: Wir in der Fachstelle Selbstbewusst in Salzburg versuchen in Elternabenden auch immer wieder Schwerpunkte $\mathrm{zu}$ aktuellen Themen zu setzen - sei es das Internet, sei es Pornografie oder bestimmte Apps, die bei Jugendlichen sehr beliebt sind und bei denen die Eltern gar nicht wissen, welche Gefahren von diesen ausgehen könnten. Dieses Anbieten von Schwerpunkt-Themen wird von den Eltern umso besser angenommen, je jünger die Kinder sind.

Katrin Lehner: Wir besprechen mit den Eltern auch Themen wie Beziehung und Körperwahrnehmung. Ich glaube, dass vielen Eltern nicht bewusst ist,

4 Die österreichische Volksschule entspricht der deutschen Grundschule und umfasst die 1. bis 4. Schulstufe; die österreichische Mittelschule umfasst die 5. bis 8. Schulstufe und entspricht in etwa der deutschen Hauptschule. 
wie sie durch verschiedene Alltagshandlungen und Abläufe in der Familie ihre Kinder prägen. Ich nenne da immer das Beispiel, wie mit Körperhygiene umgegangen wird: Es gibt Familien, bei denen es gang und gäbe ist, dass die Tür offen ist und man miteinander quatscht, wenn jemand am Klo sitzt oder dass beim Duschen die Badezimmertür offen ist. Bei anderen Familien wird die Tür zugesperrt. Das ist weder positiv noch negativ - wir machen einfach die unterschiedlichen Gewohnheiten bewusst. Auch das Frauen- und Männerbild in der Familie und Rollenbilder generell haben etwas mit Sexualität zu tun, sie sind auch Teil von sexueller Bildung und prägen die Kinder.

Sevil Eder: Wir betonen, dass es dabei nicht darum geht, irgendwelche Ideologien gegeneinander auszuspielen, sondern dass das Ziel der Schutz vor sexueller Gewalt ist.

\section{Über Gefühle reden ist Präventionsarbeit}

Robert Steiner: Wir sagen den Eltern, dass niemand etwas Persönliches über sich erzählen muss. Das ist ein sexualpädagogisches Prinzip, wie wir alle wissen, und das Gleiche sagen wir auch zu den Eltern: »Wenn Ihnen das Kind eine Frage stellt, die Sie selber betrifft und die Ihnen vielleicht $\mathrm{zu}$ intim oder $\mathrm{zu}$ peinlich ist, dann ziehen Sie einfach eine Grenze.« Dabei lernt das Kind auch, »Okay, ich muss nicht alles jedem auf die Nase binden.«Aber die Frage soll trotzdem allgemein beantwortet werden.

Peinlichkeit darf natürlich auch sein, da braucht man sich nicht zu verstellen. Man kann auch sagen: »Das ist mir ein bisschen peinlich, da muss ich erst schauen, wie ich das mache.«Wichtig ist es, das Gefühl zu benennen und als Erwachsene:r nicht um dieses Gefühl herumzulavieren, sondern zu sagen, das ist mir unangenehm oder peinlich, das weiß ich jetzt nicht.

Sevil Eder: Manchen fällt es leichter über Sexualität zu reden, anderen nicht, das ist vollkommen in Ordnung. Wenn es schwerer fällt, ist es besser, nicht vom Thema abzulenken, sondern darüber offen zu sprechen: »Ich weiß nicht, wie ich deine Frage beantworten soll, das ist mir zu viel.« Das Reden über diese Gefühle ist auch Prävention. Oder Eltern können andere Wege oder Alternativen finden, wie sich die Kinder informieren können, z.B. indem sie entsprechende Bücher anschaffen. 
Danijela Cicvarić: Mir ist in meiner Arbeit mit Roma-Familien aufgefallen, dass über Sexualität nicht so oft gesprochen wird. Sexualität ist ein TabuThema. Aber es wird, glaube ich, sehr oft daran gedacht. Eltern möchten Mädchen davor bewahren, vor der Ehe ihre Jungfräulichkeit zu verlieren. Das ist ein sehr wichtiges Thema. Andererseits habe ich oft erlebt, dass einige - obwohl sie verheiratet sind und Kinder haben - sehr wenig Ahnung vom eigenen Körper haben. Wenn ein Mädchen sagt, dass sie Eierstock-Schmerzen hat, hat die Mutter schon den Verdacht, sie hätte ihre Jungfräulichkeit verloren, weil sie meint, dass diese Schmerzen nur verheiratete Frauen haben können oder Frauen, die Kinder bekommen haben. Hier ist Aufklärung sehr wichtig.

Ich rede sehr viel mit den Müttern darüber, dass auch die Frauen ein Recht auf Sexualität haben, und dass sie damit nicht bis zum Alter von 21 oder 22 warten müssen. Ich glaube, dass sehr viele Mädchen aus Angst davor, die Jungfräulichkeit vor der Ehe zu verlieren, die Schule abbrechen und heiraten, wenn sie sich verliebt haben. Ich versuche die Mädchen dabei zu unterstützen, dass sie einen Abschluss machen und ihre Sexualität ausleben können. Ich denke, dass ich einen Vorteil in der Arbeit habe, weil ich aus der Community komme und deshalb von den Eltern und Jugendlichen akzeptiert werde.

\section{Sprache hat etwas mit Wertschätzung zu tun}

Peter Boden: Man kann so mancher Peinlichkeit auch begegnen, indem man sagt: »Es ist wichtig und okay über das Thema Sexualität zu sprechen und zusätzliche Infos zu erhalten." Man kann es niemandem vorschreiben und sagen, du musst jetzt über das Thema sprechen. Aber wenn ich z.B. vor einer Gruppe afghanischer junger Männer stehe, die 15 bis 17 Jahre alt sind, und sie wollen sich gerade alle vergraben, weil sie sagen, »Hey, Moment, eigentlich reden wir über dieses Thema nicht «, dann kann ich ihnen sehr wohl sagen, es ist wichtig, dass man über dieses Thema spricht, und dann kann ich meinerseits die Gestaltung übernehmen. Und das können Eltern, glaube ich, auch. Als Sexualpädagoge habe ich klipp und klar einen Auftrag. Als Eltern hat man ja den Vorteil, dass man länger mit diesen jungen Menschen zu tun hat. Man muss nicht alles in einem Gespräch oder in einem Aufeinandertreffen erledigen. Ich habe genau diese drei Stunden Zeit. 
Katrin Lehner: Sprache ist generell ein wichtiges Thema. Es ist bedeutsam, Gefühle benennen zu lernen; sowohl solche, die als positiv eingeschätzt werden, als auch solche, die eher als negativ angesehen werden. Eltern können dabei unterstützen, indem sie selbst sagen, wie es ihnen mit dem Thema Sexualität geht, etwa, »das ist mir jetzt unangenehm« oder "peinlich« oder »das ist jetzt eine lustige Frage, da werde ich selber ganz rot«. Das vermittelt ein Stück Normalität und zeigt, dass Eltern - und auch wir als Sexualpädagog:innen - nicht allwissend sein müssen.

Wir geben Kindern auch eine Sprache für Geschlechtsorgane. Ich als Sexualpädagogin verwende dabei das Wort Schamlippen sehr ungern. Sprache hat etwas mit Wertschätzung zu tun. Wir verwenden z.B. das Wort »Scheidenlippen«. Ich finde, das macht einen Unterschied. Einmal hat eine Mutter gesagt, das sei Wortklauberei. Ich habe geantwortet, dass wir uns mit dem Thema sehr viel beschäftigen und wir es als Sexualpädagog:innen als wichtig empfinden, stimmige Begriffe zu verwenden.

Danijela Cicvarić: Manche Menschen aus Ex-Jugoslawien, mit denen wir im Romano Centro arbeiten, verwenden viele sexuelle Schimpfwörter. Sie würden aber nie zu ihrem Sohn oder ihrer Tochter sagen, »dein Penis« und »deine Vagina«. Sie verwenden stattdessen eine Art Koseformen - »Pippi«z.B. Ich halte es für wichtig, sie zu unterstützen, auch über den eigenen Körper ganz normal reden zu können, also »Salz, Kaffee, Penis, Vagina ...«. Die Geschlechtsteile $\mathrm{zu}$ benennen ist für viele schwierig.

Peter Boden: Manchmal ist es auch für Jugendliche peinlich, wenn Eltern fragen: »Na, und wie ist's? Hast du jetzt schon einen Freund?« Vielleicht haben die Jugendlichen einen Freund oder eine Freundin, aber es ist jetzt noch nicht der Zeitpunkt, das zu offenbaren. Manchmal ist es auch gut, Eltern zu beruhigen, dass viel mehr Dinge im Kopf anderer passieren und sich die Situation der betroffenen Personen selbst oft deutlich anders darstellt als die Phantasie darüber. Wenn ein jugendliches 14-jähriges Paar ins Kino geht, dann werden sie wahrscheinlich den Film anschauen. Es kann auch sein, dass sie sich im Kinosaal küssen. Es kann sein, dass sie ein halbes oder ein ganzes Jahr ein Paar sind und noch nicht miteinander geschlafen haben. Dabei ist das vielleicht im Kopf der Eltern schon viel früher passiert, und sie denken: »Na, hoffentlich passen sie eh auf! Jetzt müssen wir aber wirklich über Verhütung reden. Nicht, dass was passiert!« Mir geht es nicht darum, diese Ängste lä- 
cherlich zu machen, sondern bewusst zu machen, dass viele Jugendliche sehr gut auf sich achten, und dadurch die Eltern zu beruhigen.

Robert Steiner: Fakten sind oft erleichternd für Eltern. Es nimmt ihnen Stress, wenn wir ihnen sagen, dass das »Erste Mal« laut Studien ${ }^{5}$ durchschnittlich mit knappen 17 Jahren stattfindet und in den letzten Jahrzehnten das Alter des »ersten Mal« nahezu unverändert geblieben ist. Das entspannt die Eltern, die meist annehmen, dass es viel früher >passiert<. Dieses Wissen entspannt aber auch die Jugendlichen, die häufig auch Druck haben: einerseits Druck in der Peergruppe, sschon mal gehabt haben zu müssen<, und andererseits Druck durch die leicht zugänglichen Pornografie-Bilder, die zeigen, wie und was `man alles machen sollte . Diese Vorstellungen muss man mit ihnen besprechen und gemeinsam richtig einordnen.

\section{Jede:r ist Expert:in der eigenen Sexualität}

Peter Boden: Manchmal müssen wir klare Grenzen ziehen. Auf einem Elternabend hat mir eine Mutter erklärt, es wäre wider den Willen des Schöpfers das waren ihre Worte -, dass wir zum Thema Sexualität arbeiten. Ich habe mich auf den Bildungsauftrag 6 berufen.

Ich respektiere es, wenn eine Person ihre religiöse Ausrichtung hat, so wie eine Person ihre ideologische Ausrichtung haben kann. Wir müssen als Sexualpädagoginnen und Sexualpädagogen unsere Haltung deutlich machen, wenn jemand - wie auch immer motiviert - meint, es gebe nur eine richtige Sichtweise und Lebensweise. Ich betone z.B. immer, dass jede:r Experte und Expertin der eigenen Sexualität ist. Dieses Expertin- und Experte-Sein umfasst beispielsweise die Bereiche der sexuellen Orientierung, der Wahrnehmung und Definition des eigenen Geschlechts und verschiedenste Möglichkeiten von gelebten Beziehungskonstellationen. Wenn eine Person für sich

5 Viele Studien kommen auf ähnliche Ergebnisse, z.B. die Dr.-Sommer-Studie (Bauer Media Group 2016), Statista $\mathrm{CmbH}$ (2016) oder die BzgA-Studie (Bode/Heßling 2016).

6 Sexualpädagogik ist in Österreich eines von mehreren Unterrichtsprinzipien, das fächerübergreifend für alle Schulstufen und Schulformen gilt. Der Grundsatzerlass zum Unterrichtsprinzip Sexualerziehung wurde 1970 veröffentlicht und 2015 unter dem Titel Sexualpädagogik überarbeitet (vgl. BMBF 2015). 
weiß, »ich möchte verheiratet sein, bevor ich meine ersten sexuellen Erfahrungen im Sinne von Geschlechtsverkehr mache«, dann kann die Person das für sich leben und darf gleichzeitig akzeptieren, dass andere Menschen andere Entscheidungen treffen. Es gibt vielleicht Leute, die finden heiraten genauso wichtig, können das aber gut trennen von ihrem Wunsch, ihre ersten sexuellen Erfahrungen zu machen.

Mir geht es nicht darum, bestimmte religiöse Richtungen schlechtzureden oder gutzureden. Aber sobald jemand erklärt: "Das ist richtig und das ist falsch in der Sexualität«, wird es schwierig. Da entwerfen wir ein Lebenskonzept für alle, das funktioniert nicht. Auch in der Elternarbeit müssen wir als Professionelle im Fall der Fälle sagen »Nein, stopp! Da ist eine klare Grenze«, wenn von einzelnen Personen versucht wird, die eigenen ideologischen Ideen in Bezug auf Sexualität als die einzig gültige Größe und Wahrheit zu etablieren. Vor allem wenn diese Ansichten alle anderen Arten der Gestaltung von Sexualität diffamieren und diesen die Berechtigung absprechen.

Robert Steiner: Es gibt gewisse Werte, die sind nicht verhandelbar, das stimmt. Z.B. beim Thema LGBTIQ*: Menschen sind vielfältig, das ist Fakt. Uns ist es wichtig, diese Vielfalt als gleichwertig anzuerkennen. Im 21. Jahrhundert kann es nicht sein, dass Menschen, die einer sexuellen oder geschlechtlichen Minderheit angehören, gemobbt werden. An den Elternabenden laden wir die Eltern auch ein, einmal darüber nachzudenken, was denn ihr Kind brauchen würde, wenn es einer dieser Minderheiten angehören würde. Und da wird schnell klar: genau, Elternliebe und Unterstützung.

\section{Gut, dass ihr das macht}

Katrin Lehner: Es entstehen während der Elternarbeit immer wieder Diskussionen oder Gespräche zwischen den Eltern. Ich finde das gerade dann spannend, wenn Menschen mit unterschiedlichen kulturellen Hintergründen und religiösen Bekenntnissen aufeinandertreffen. Viele sind sehr offen für das Thema Sexualität im Allgemeinen. Es kommt oft auch zum Austausch darüber, wie unterschiedlich Familien aufgrund ihrer Wertvorstellungen, ihrer religiösen Einstellung usw. funktionieren und wie sie mit der sexuellen Entwicklung ihrer Kinder umgehen. Das kann Nähe schaffen. Man sieht, dass alle mit ihren Kindern ihre Themen haben, und es für viele ein sehr sensibles Thema ist. In diesen Gesprächen wird spürbar, dass die Eltern sehr froh 
sind, dass das Thema auch von anderen, nämlich von speziell ausgebildeten Pädagog:innen, übernommen wird. Sie sagen, sie machen das nach bestem Wissen und Gewissen, aber es gibt oft eine Erleichterung, wenn das auch noch jemand außerhalb der Familie macht.

Robert Steiner: Es gibt Eltern, die streng religiös sind - etwa muslimisch oder katholisch - und zu Verhütung, >Jungfräulichkeit< und Masturbation eine sehr strenge Sichtweise haben. Da macht es meist keinen Unterschied, um welche Religion es geht, sondern es geht darum, wie streng oder offen diese ausgelegt bzw. gelebt wird.

Wir haben muslimische Eltern gehabt, vor allem Mütter, die gesagt haben: "Gut, dass ihr das macht. Ich habe diese Aufklärung nicht erfahren. Und ich kann das meiner Tochter auch nicht beibringen. Es ist gut, dass ihr das macht." Auch aus diesem streng Religiösen kommt dann die Erkenntnis: "Hoppala, eigentlich ist das nicht gut, was ich erlebt habe. Jetzt gibt es jemanden, der das mit meiner Tochter super macht.« Wir haben da auch schon guten Rückenwind bekommen.

Sevil Eder: Manchmal sitzen Eltern beim Elternabend, die nur da sind, um uns zu kritisieren. Es geht bei denen nicht darum, irgendetwas aufzunehmen oder das Beste für die Kinder zu wollen, sondern darum, provokativ zu sein. Das ist immer anstrengend. Manche Eltern stoßen sich daran, dass wir unterschiedliche sexuelle Orientierungen und verschiedene Identitäten gleichberechtig einbeziehen. Angesichts von Kritik oder Provokationen betonen wir, dass es uns um Schutz der Kinder geht und Sexualerziehung Schutz bedeutet. Über diese Argumentation versuchen wir die Eltern zu erreichen bzw. zu gewinnen. Unser Ansatz findet meistens auch Unterstützung von Eltern und wird gut aufgenommen. Alle Kinder und Jugendliche, unabhängig davon, wie sie sind, wie sie aussehen und wie sie sich fühlen, sollen gleichberechtigt sein und ernst genommen werden. Auch das ist Prävention. Wie eine Kollegin von mir sagt: Prävention heißt, jedem Kind das Gefühl zu geben, »richtig« zu sein.

\section{Zumindest einmal lachen}

Claudia Schneider: Was sind für euch gelungene Momente in der Elternarbeit, was war bisher schön oder spannend? 
Danijela Cicvarić: Bei mir war es so, dass ich einer Mutter erklären konnte, dass ihr Sohn nicht krank ist, weil er schwul ist, und dass er deswegen nicht medizinisch behandelt werden muss. Es war schön, dass sie ihren Sohn dann so akzeptiert hat, wie er ist.

Robert Steiner: Ich kann mich an einen Rückmeldebogen erinnern, in den eine Mutter geschrieben hat: »Wenn ich ein ähnliches Projekt als Schülerin gehabt hätte, dann wäre mir als Jugendliche viel erspart geblieben.« Da habe ich mir gedacht: »Wow, die Arbeit macht Sinn.«

Peter Boden: Also für mich ist Elternarbeit gelungen, wenn die Eltern zumindest einmal an diesem Abend auch herzhaft lachen konnten. Weil es auch wichtig ist, bewusst zu machen: Das Thema Sexualität hat auch etwas mit Leichtigkeit zu tun, es ist mehr als nur etwas Schweres. Natürlich ist es auch wichtig, über bestimmte Bereiche von Sexualität sehr klar und sehr seriös zu sprechen. Aber es gibt andere Dinge, die darf man auch mit einer gewissen Lockerheit und Leichtigkeit wahrnehmen. Da ist es schön, wenn sie zumindest einmal lachen konnten.

Was ich den Eltern auch immer sage: Ich bin davon überzeugt, dass die allermeisten Eltern wirklich ihr Bestmögliches tun und schon viel richtig machen. Und wenn sie aus dem Elternabend noch ein, zwei Ideen mitgenommen haben, die sie vielleicht in Zukunft anwenden können, dann ist es gut gegangen.

Robert Steiner: Sexuelle Bildung der Eltern wäre uns eigentlich auch wichtig. Aber nach unserer Erfahrung kommen die Eltern, um sich zu informieren. Sie sind es von anderen Elternabenden gewohnt, dass das nach einer halben Stunde erledigt ist. Wenn der Elternabend zu lange dauert, dann gehen die Eltern irgendwann weg. Meistens kommen auch genau die Eltern nicht, die eigentlich eine starke Abneigung gegen das Thema haben. Je älter die Schüler:innen werden, desto weniger Eltern kommen. In der Volksschule sind die Eltern fast zu hundert Prozent da, und dann in der Unterstufe sitzen wir oft fast im Vieraugen-Gespräch mit zwei, drei Eltern da. Den anderen ist es egal.

Peter Boden: Es gibt ja die Elternsprechtage, um den Stand der Kinder in den einzelnen Schulfächern zu besprechen. Was wäre, wenn es dabei die freiwillige Möglichkeit für die Eltern gebe, sich von professionellen Sexualpädagog:innen beraten zu lassen? Es gibt ja ganz allgemeine Anliegen, Eltern 
fragen: »Ist das normal?«, und wollen z.B. Hintergrundinformationen zu sexuellen Entwicklungsphasen. Oder sie wollen wissen, ob es Unterstützungsmöglichkeiten für ihre Kinder gibt. Manchmal geht es um eine Aufklärung über mögliche rechtliche Konsequenzen. Manchmal geht es einfach auch ein Stück weit um Beruhigung. Wenn ich mir das jetzt so durchdenke, mag das am Anfang wahrscheinlich ein einsames Dasein sein, aber ich glaube, es würde angenommen werden, wenn man das für zwei, drei Jahre an den entsprechenden Elternsprechtagen durchziehen würde. Das wäre etwas, bei dem viele Eltern in der Schule anwesend sind, weil sie sich dazu verpflichtet fühlen. Ich fände es zumindest einen Versuch wert, diese Gelegenheit langfristig für Beratungsangebote zu nutzen.

\section{Weiterhin über Sexualität sprechen}

Claudia Schneider: Wie wirken sich aktuelle oder vergangene politische oder mediale Diskussionen auf eure Arbeit aus?

Robert Steiner: Wenn wir es positiv betrachten: Conchita Wurst z.B. war und ist medial sehr präsent. Das war total positiv. Ich kann mich erinnern, als ich um 2009 die ersten Workshops gemacht habe, war Homosexualität - und Transgeschlechtlichkeit sowieso - für viele Eltern ein rotes Tuch. Das hat sich durch dieses mediale Thematisieren und durch das mediale Aufarbeiten positiv verändert. Jetzt gibt es nicht mehr so viele Leute, die sagen, das ist krankhaft. Es gibt sie noch, aber nicht mehr in dieser Masse.

Die aktuelle Berichterstattung darüber, dass Sexualpädagogik nicht mehr zugelassen wird ${ }^{7}$ - so wird es ja oft transportiert -, erzeugte sehr viel Verun-

2018 wurde in Österreich öffentlich bekannt, dass ein Verein an Schulen sexualpädagogisch tätig ist, der bedenkliche Inhalte verbreitet und etwa Homosexualität als heilbar ansieht. Bildungsminister Heinz Faßmann empfahl den Schulen im Frühling 2018, nicht mehr mit diesem Verein zusammenzuarbeiten und kündigte ein Akkreditierungsverfahren für Vereine an, die an Schulen tätig sind. Dazu kam es aufgrund des Regierungswechsels 2019 nicht mehr. In der Zeit der Übergangsregierung im Frühsommer 2019 nutzten Abgeordnete von ÖVP und FPÖ den Anlassfall für einen Schlag gegen alle sexualpädagogischen Vereine. Sie brachten einen sogenannten Entschließungsantrag im Parlament ein, der im Eilverfahren beschlossen wurde und darauf abzielte, alle sexualpädagogischen Angebote an Schulen zu verbieten und Schulen die Zusammenarbeit mit sexualpädagogischen Vereinen zukünftig zu unter- 
sicherung bei den Schulleiter:innen: „Dürfen wir euch überhaupt noch buchen?« Die Verunsicherung gab es auch bei den Eltern. Andererseits haben sehr viele Pädagog:innen angerufen und gesagt: "Das ist eine Katastrophe, was gerade passiert, ich buche euch trotzdem und erst recht! Egal, was herauskommt, es ist wichtig, und ihr sollt kommen!«

Sevil Eder: Ich weiß nicht, was auf uns zukommen wird, aber wir bieten auch bei uns im Büro sexualpädagogische Workshops an. Vielleicht wäre eine Möglichkeit, dass wir in Zukunft nicht mehr in die Schulen gehen, sondern dass die Schüler:innen zu uns kommen.

Danijela Cicvarić: Ich glaube, dass die Politik allgemein einen Einfluss auf meinen Job hat. Aber was Sexualität betrifft: Ich habe jetzt keine Angst vor Konsequenzen, ich werde weiterhin über Sexualität reden.

\section{Literatur}

Bauer Media Group (2016): Bravo-Dr.-Sommer-Studie 2016. Hamburg: o.V. BMBF - Bundesministerium für Bildung und Frauen (2015): Grundsatzerlass Sexualpädagogik. Online unter: https://www.bmbwf.gv.at/Themen/schu le/schulrecht/rs/1997-2017/2015_11.html [Zugriff: 18.06.2020].

Bode, Heidrun/Heßling, Angelika (2015): Jugendsexualität 2015. Die Perspektive der 14- bis 25- Jährigen. Ergebnisse einer aktuellen repräsentativen Wiederholungsbefragung. Köln. Online unter: https://www.forschung.s exualaufklaerung.de/projekt/jugendsexualitaet-2015-repraesentative-wi ederholungsbefragung-die-perspektive-der-14-bis-25-jaehrige/abstract [Zugriff: 28.04.2015].

Fachstelle Selbstlaut (2017): Interkulturelle Sexualpädagogik und Gewaltprävention mit Eltern und Erziehungsberechtigten mit nicht deutscher Erstsprache. Voraussetzungen, Möglichkeiten, Hindernisse und Empfehlungen. Eine Handreichung für Lehrerinnen und Lehrer. Online

sagen. Dies geschah gegen die entschiedenen und breiten Proteste von Fachkräften wie auch ÖVP-nahen Organisationen und Politiker:innen. Aufgrund der Neubildung der Regierung im Herbst 2019 wurde der Entschließungsantrag hinfällig. Weitere Infos auf: https://mein.aufstehn.at/petitions/redmadruber-initiative-fur-qualitatsvolle-se xualpadagogik [Zugriff: 18.06.2020] sowie auf der Plattform sexuelle Bildung http://se xuellebildung.at [Zugriff: 18.06.2020]. 
unter: https://selbstlaut.org/wp-content/uploads/2017/04/Handreichung _20170403.pdf [Zugriff: 18.06.2020].

Statista GmbH (2016): Alter beim ersten Sex in Österreich nach Geschlecht 2016. Online unter: https:/de.statista.com/statistik/daten/studie/583571/ umfrage/alter-beim-ersten-sex-in-oesterreich-nach-geschlecht [Zugriff: 08.05.2020].

Fachstelle Selbstbewusst (o.J.): Darüber reden?! Sexualerziehung und Prävention von sexuellem Missbrauch. Online unter: https://www.selbstbewuss t.at/portfolio/elternbroschuere [Zugriff: 18.06.2020]. 


\section{Masturbate}

Anna Vida

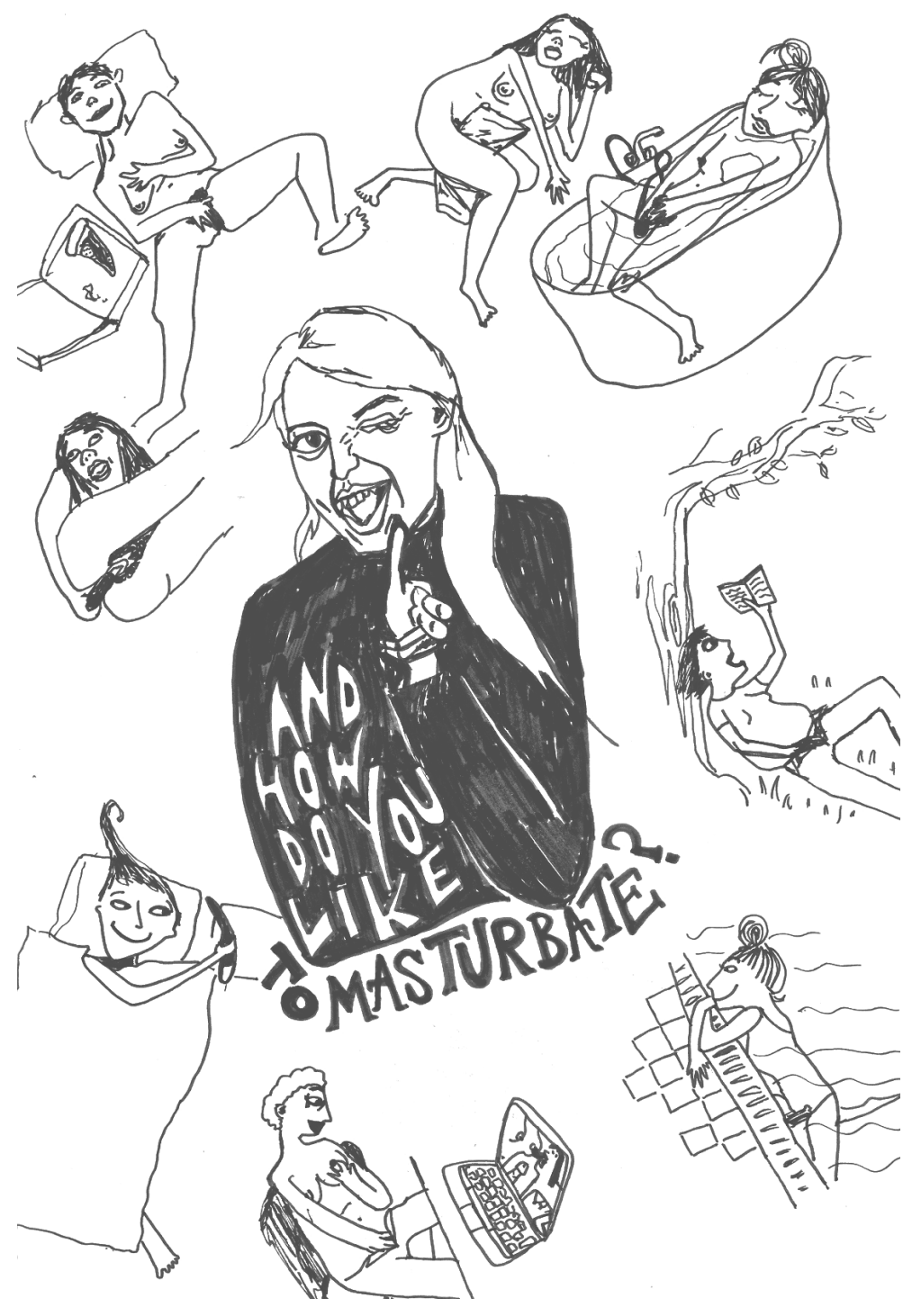


Körperlichkeiten

Anna Vida
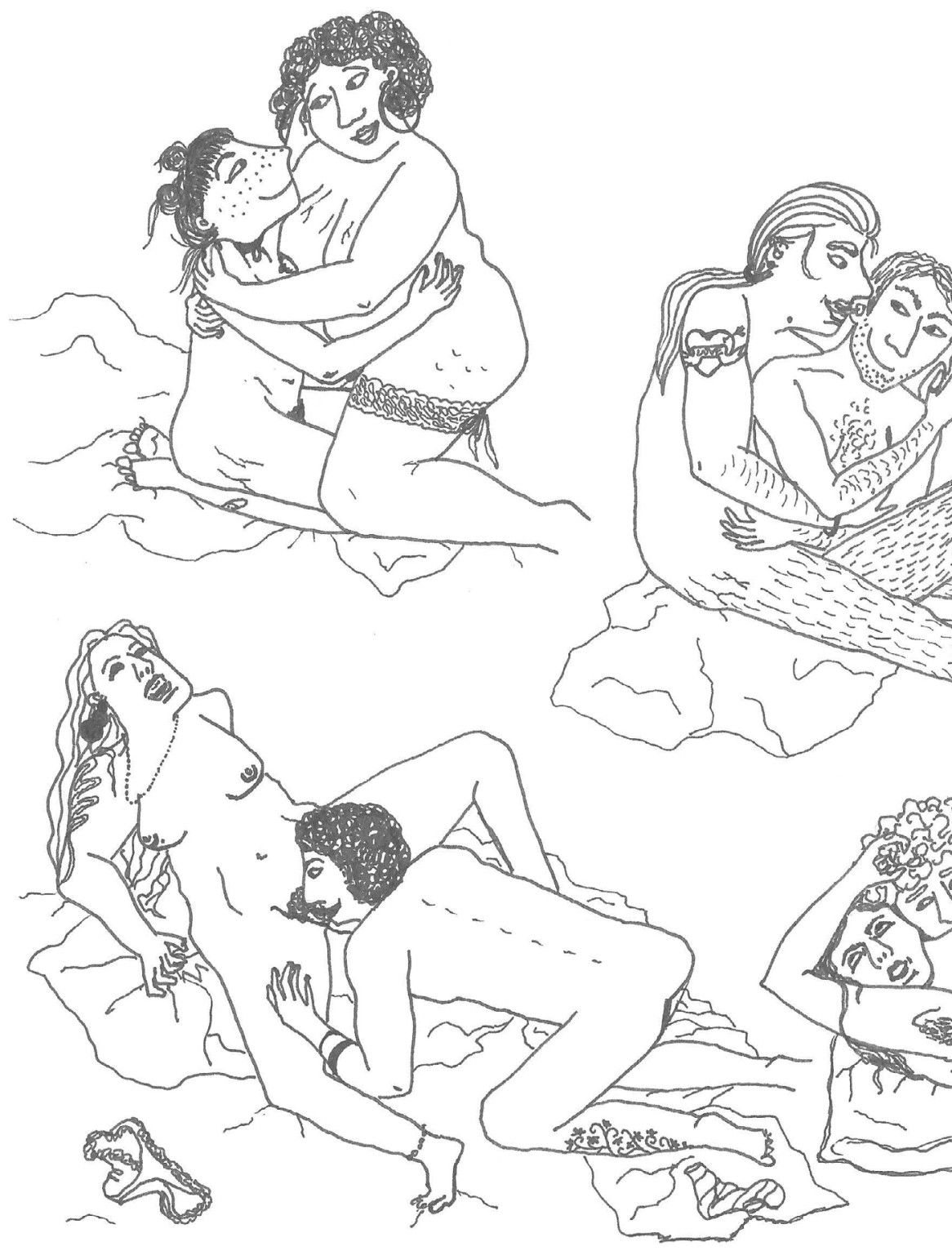


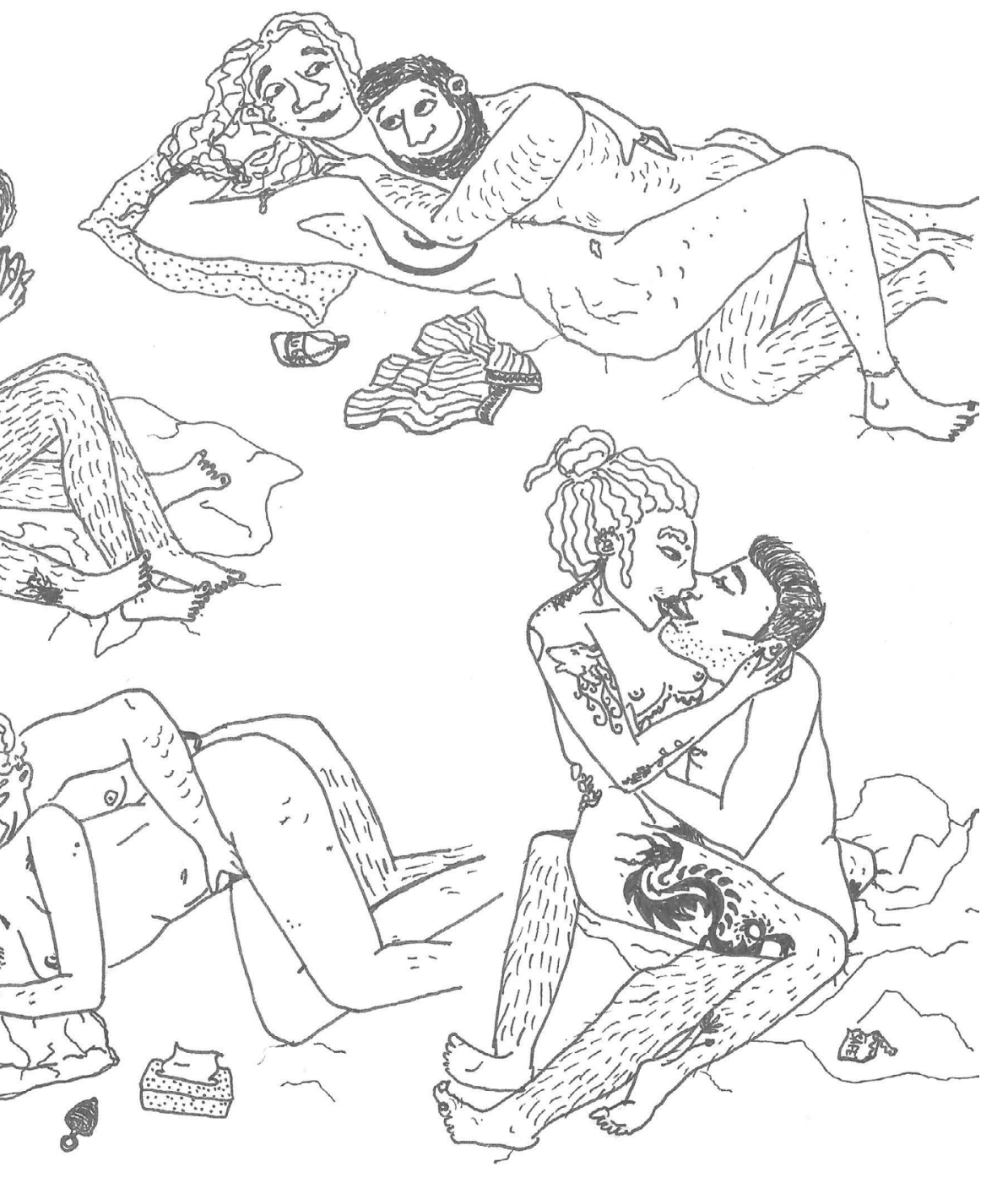





\section{Autor:innenverzeichnis}

Christine Aebi hat Bildende Kunst/Malerei und Grafik an der Universität für angewandte Kunst Wien studiert und ist als freischaffende Künstlerin und Illustratorin tätig. Seit 2002 lehrt sie etwa an der Ecole d'Humanité (CH) oder der New Design University (Ö) und ist seit 2010 Lehrbeauftragte an der Schule für Gestaltung St. Gallen $(\mathrm{CH})$. Gemeinsam mit Lilly Axster hat sie mehrere Bilderbücher veröffentlicht u.a. 2012 »DAS machen? Projektwoche Sexualerziehung in der Klasse $4 \mathrm{C} «$ (www.dasmachen.net). Alle Bücher wurden ausgezeichnet u.a. mit dem österreichischen Kinder- und Jugendbuchpreis.

Angelika Atzinger ist Politik- und Translationswissenschaftlerin. Sie arbeitet seit Jahren in mädchen- und frauenrelevanten Kontexten sowie in der Erwachsenenbildung und setzt sich im Zuge dessen mit Bildungsthemen und feministischer Arbeit auseinander. Im Verein Amazone (www.amazone.or.at) ist sie als Geschäftsführerin tätig.

Peter Helmut Boden ist Magister (FH) für soziale Arbeit, zertifizierter Sexualpädagoge, Anhänger des FK Austria Wien und selbstständiger Referent im Bereich Sexualpädagogik und Suchtprävention. Sein derzeitiges Hauptbetätigungsfeld sind Workshops in Schulen, Elternabende, Weiterbildungen für Pädagog:innen und sexualpädagogische Einzelcoachings für Menschen mit besonderen Bedürfnissen. Authentizität ist für ihn die Grundlage seiner professionellen Angebote. Das bedeutet für ihn eigene Meinung und Haltung in klarer Sprache zu verdeutlichen und anderen und sich selbst gegenüber ehrlich zu sein.

Marlies Brommer ist Absolventin des Instituts für das künstlerische Lehramt der Akademie der bildenden Künste Wien. Sie unterrichtet Bildnerische Erziehung, Werkerziehung, Medienpraxis sowie Bildnerisches Gestalten und 
Werken in der Sekundarstufe I und II an der Wiener Mittelschule/Realgymnasium/Oberstufen-Realgymnasium antonkriegergasse. Ihre Arbeitsschwerpunkte sind Institutionskritik und Heterogenität im schulischen Feld.

Katharina Buhri ist Diplom-Sozialpädagogin sowie Jugend- und Erziehungsberaterin. Im Verein Amazone (www.amazone.or.at) leitet sie verschiedene Projekte zu Medien, Körperbildern und Suchtprävention. Sie leitet das Projekt \#followme - Sicherheit durch Medienkompetenz und entwickelt in diesem Zuge gemeinsam mit Jugendlichen Workshops zu digitalen und sozialen Medien.

Danijela Cicvarić ist Sozialarbeiterin und Pädagogische Leiterin im Verein Romano Centro. Sie führt Frauenberatung für Romnja durch, leitet das Projekt Roma Schulmediation an Wiener Schulen sowie die Lernhilfe für Schüler:innen und zwei Mädchenprojekte (Mädchen Woche und Zurale Seja-Starke Mädchen). Im Romano Centro, 1991 als einer der ersten Roma-Vereine Österreichs gegründet, sind Roma aus unterschiedlichen Gruppen vertreten, um sich gemeinsam für die Verbesserung der Lebensbedingungen von Roma und gegen deren Diskriminierung einzusetzen. Schwerpunkte der Tätigkeit von Danijela Cicvarić sind Bildung, Beratung und Kultur.

Maria Dalhoff ist Traumapädagogin/traumaspezifische Fachberaterin, Erwachsenenbildnerin und Sexualpädagogin. Seit 2011 arbeitet sie bei Selbstlaut-Fachstelle gegen sexualisierte Gewalt an Kindern und Jugendlichen in Wien (www.selbstlaut.org). Ihr aktueller Interessenschwerpunkt sind machttheoretische Einbettungen sexueller Zustimmungskonzepte. Sie promoviert zu Politiken der Einvernehmlichkeit in der sexuellen Bildung an der Akademie der bildenden Künste Wien.

Katharina Debus, Diplom-Politologin und Sexualpädagogin (ISP), arbeitet freiberuflich u.a. für Dissens - Institut für Bildung und Forschung in der Fachkräfte-, Erwachsenen- und Jugendbildung, Forschung, Evaluation und Fachberatung. Ihre inhaltlichen Schwerpunkte sind Geschlechterverhältnisse; Sexismus; geschlechtliche, amouröse und sexuelle Vielfalt; Intersektionalität; geschlechterreflektierte Pädagogik; Sexualpädagogik sowie die diskriminierungsreflektierte Gestaltung von Lernprozessen. Eine thematisch relevante Veröffentlichung, gemeinsam mit Vivien Laumann: Pädagogik geschlechtlicher, amouröser und sexueller Vielfalt. Zwischen Sensibilisierung und Empowerment. Berlin 2018. 
Tobias Dörler unterrichtet an der Berufsbildenden Höheren Schule in der Herbststraße in Wien im Fachbereich Kunst und ist Lektor an der Akademie der bildenden Künste Wien. Er beschäftigt sich mit privilegierungs- und diskriminierungskritischer inklusiver Kunstdidaktik und -pädagogik, mit Handlungs(spiel)räumen im Bildungswesen sowie Kritischer Schulentwicklung.

Sevil Eder ist Soziologin und Grundschulpädagogin. Sie ist u.a. auf Geschlechterforschung, soziale Struktur und soziale Integration spezialisiert. Im Zuge ihres Studiums absolvierte sie zahlreiche Praktika in Schulen und in der außerschulischen Arbeit mit Kindern. Seit 2011 ist sie Mitarbeiterin bei Selbstlaut - Fachstelle gegen sexualisierte Gewalt an Kindern und Jugendlichen (www.selbstlaut.org). Im Rahmen ihrer Tätigkeit arbeitet sie mit Kindern und Jugendlichen präventiv gegen sexualisierte Gewalt. Sie hält Seminare und Workshops zur Sexualpädagogik sowie zur Vorbeugung von sexualisierter Gewalt für pädagogisches Personal.

Caroline Estrada-Steiger ist Kunst- und Kulturpädagogin, Fotografin und Medienkünstlerin. Sie unterrichtete bis zu ihrer derzeitigen Elternkarenz Bildnerische Erziehung sowie Textiles und Technisches Werken am Bundesrealgymnasium und Bundesoberstufenrealgymnasium Henriettenplatz in Wien. Ihr Schwerpunkt liegt in den letzten Jahren auf dem Thema Flucht und dem inkludierenden Arbeiten mit Geflüchteten im Kunst- und Werkunterricht.

Ulli Freund, Diplompädagogin, ist freiberufliche Referentin, Fachberaterin und Autorin zu den Themen sexuelle Übergriffe unter Kindern, pädagogische Prävention von sexuellem Missbrauch sowie Schutzkonzepte gegen sexualisierte Gewalt an Kindern und Jugendlichen für Einrichtungen und Organisationen. Zudem ist sie in Teilzeit im Arbeitsstab des Unabhängigen Beauftragten für Fragen des sexuellen Kindesmissbrauchs der deutschen Bundesregierung beschäftigt.

Ariane Grabherr ist Montessori- und Sexualpädagogin. Im Verein Amazone (www.amazone.or.at) leitet sie verschiedene Projekte zu feministischer Mädchenarbeit, Gesundheit sowie Technik und Handwerk. Seit Jahren leitet sie sexualpädagogische Workshops im Rahmen von Klipp \& Klar-das sexualpäd- 
agogische Projekt und \#followme - Sicherheit durch Medienkompetenz im Verein Amazone.

Paul Haller ist Sozialarbeiter, Sexualpädagoge und Fachkraft für Prävention und Intervention bei sexualisierterGewalt an Kindern und Jugendlichen. Er studierte Internationale Entwicklung und arbeitet als Fachreferent für LGBTIQ-Angelegenheiten eines Klubs im österreichischen Parlament sowie als Projektmitarbeiter bei oii Europe. Zuvor war er bei der HOSI Salzburg und der Fachstelle Selbstbewusst tätig. Er ist Mitglied der Plattform Intersex Österreich, macht queere Bildungsarbeit und unterrichtet angehende Sexualpädagog:innen im Bereich sexuelle und geschlechtliche Vielfalt.

Heidemarie König ist Klinische- und Gesundheitspsychologin und seit über zehn Jahren Sexualpädagogin im Team des Österreichischen Instituts für Sexualpädagogik und Sexualtherapien (ISP) in Wien. Neben sexualpädagogischen Workshops mit Kindern und Jugendlichen und der Sexualberatung umfasst ihre berufliche Tätigkeit auch das Abhalten von Seminaren, Workshops und sexualpädagogische Fortbildungen für Multiplikator:innen u.a. zu rechtlichen Aspekten in der Sexualpädagogik. Sie ist in der Koordination und fachlichen Begleitung des Lehrgangs Praxisorientierte Sexualpädagogik am ISP tätig, hat eine Zusatzausbildung in Neurofeedback und ist diplomierte Legasthenie-Trainerin.

Adriane Krem ist diplomierte Lebens- und Sozialberaterin, Sexualpädagogin (ISP) und Klinische Sexologin nach Sexocorporel. Sie ist seit zehn Jahren im Team des Österreichischen Instituts für Sexualpädagogik und Sexualtherapien tätig und unterrichtet im Lehrgang Praxisorientierte Sexualpädagogik u.a. zu rechtlichen Aspekten im Berufsfeld der Sexualpädagogik. Neben der sexualpädagogischen Gruppenarbeit mit Kindern und Jugendlichen hält sie Seminare und Workshops zu den Themenbereichen Sexualität und sexuelles Lernen, sexualpädagogische Fortbildungen für Multiplikator:innen und bietet Sexualberatung für Einzelpersonen und Paare an.

Orlinder Krinkel ist ein offenes Animationsstudio (orlinderkrinkel.net), das 2015 von bildenden Künstler:innen gegründet wurde, die sich an der Akademie der bildenden Künste Wien kennengelernt hatten. Seither wurden verschiedene Kunstprojekte insbesondere in den Bereichen Animationskunst und Konzeptkunst durchgeführt. Die wichtigsten Arbeiten aus dem Studio 
sind KASSA TRACK (sixpackfilm.com/de/catalogue/2433), EMERGENCY TURNED UPSIDE-DOWN in Regie von Oliver Ressler (ressler.at/emergency_turned_upside_down) und die Animatiosserie DIE NORMALE SHOW in Regie von Stefanie Sargnagel (youtube.com/dienormaleshow). Seit 2019 werden von Adnan Popović und Georg Oberlechner im Rahmen des Projekts Reflecting Desires sexual- und kunstpädagogische digitale Lernmittel erarbeitet.

Katrin Lehner ist Sexualpädagogin, arbeitet als Referentin für die Fachstelle Suchtprävention und Sexualpädagogik in Niederösterreich und hält in diesem Rahmen Fortbildungen, Schul-Workshops und Elternabende ab. Sie hat eine Ausbildung im Bereich der klinischen Sexologie und bietet in ihrer eigenen Praxis Einzelcoaching, Workshops und Supervision zu Themen rund um Körper, Liebe, Lust und Sexualität an. Als diplomierte Sozialpädagogin ist sie in der Freizeitbetreuung von Menschen mit Beeinträchtigung für die Caritas der Diözese St. Pölten tätig.

Kristin Lerch studiert Science and Technology Studies sowie Biologie. Sie ist ehrenamtlich im Verein queerconnexion (www.queerconnexion.at) aktiv und leitet Workshops mit Jugendlichen. Außerdem beschäftigt sie sich als Aktivistin und Künstlerin mit dem Zusammenwirken von Körpern, Identitäten und sozialen Positionierungen.

Stanislaus Medan studiert künstlerisches Lehramt an der Akademie der bildenden Künste Wien. Er hat bis 2016 Kunst in der Klasse für textuelle Bildhauerei studiert und drei Jahre lang Bildnerische Erziehung an einem Oberstufengymnasium unterrichtet. Momentan schreibt Medan seine Diplomarbeit über die Möglichkeiten abweichenden Handelns im Kunstunterricht.

Michaela Moosmann ist Diplom-Sozialarbeiterin, Sexualpädagogin und Systemische Beraterin. Im Verein Amazone leitet sie die Mädchenberatung und Klipp \& Klar - das sexualpädagogische Projekt. Sie arbeitet außerdem als Referentin in der Erwachsenenbildung und ist Gründungs- und langjähriges Vorstandsmitglied der österreichweiten Plattform Sexuelle Bildung.

Zeynep Önsür-Oluğ ist Sozialpädagogin und arbeitet seit 2016 bei SelbstlautFachstelle gegen sexualisierte Gewalt an Kindern und Jugendlichen (www.selbstlaut.org). U.a. betreut sie Schulklassen und Jugendgruppen in der Ausstellung 
»Ganz schön intim«. Sie studiert Bildungswissenschaft an der Universität Wien und absolviert die Ausbildung zur Psychotherapeutin.

Rosemarie Ortner ist Bildungswissenschaftlerin und Lehrbeauftragte am Institut für pädagogische Professionalisierung (Universität Graz) und am Institut für Bildungswissenschaft (Universität Wien). Sie beschäftigt sich in Forschung und Lehre mit Gender und Queer Studies, Migrationspädagogik und pädagogischer Professionalisierung. Außerhalb der Academia ist sie im Verein Queer Base (www.queerbase.at) aktiv und als Shiatsu-Praktikerin tätig.

Anna Pritz lehrt und forscht im Fachbereich Kunst- und Kulturpädagogik am Institut für das künstlerische Lehramt an der Akademie der bildenden Künste Wien. Ihr Schwerpunkt in pädagogischer Theorie und Praxis ist die Schnittstelle zwischen Kunstuniversität und Schule. Sie ist im Autor:innenteam des Schulbuchs für Bildnerische Erziehung »KUNST« des Wiener hpt-Verlags.

Dorottya Rédai hat ein Doktorat in Genderstudies an der Central European University in Budapest absolviert und arbeitet als freiberufliche Wissenschaftlerin im Bereich von Gender, Sexualität und Bildung in Ungarn. $\mathrm{Zu}$ ihren Forschungsschwerpunkten gehören Gender Equality und Bildung, Sexualpädagogik sowie die Reproduktion sozialer Ungleichheit im Bildungswesen. Neben ihrer Tätigkeit in der Forschung und Fortbildungsarbeit ist sie in Bildungsprojekten von LGBTQ- und frauenspezifischen NGOs engagiert. Gemeinsam mit Boldizsar Nagy veröffentlichte sie 2020 das vielfaltsorientierte und inklusive Kinder-Märchenbuch »Meseorszag mindenkie«/»A Fairy Tale for Everyone«.

Magdalena Rest arbeitet vor allem als Geschichts- und Kulturvermittlerin und studiert seit 2015 am Institut für das künstlerische Lehramt der Akademie der bildenden Künste Wien. Ihr Beitrag in diesem Buch bezieht sich auf ein Unterrichtsprojekt, das in einer Lehrveranstaltung zu Schulforschung im Sommersemester 2018 im Rahmen des Forschungsprojekts Imagining Desires (www.imagingdesires.at) gemeinsam mit ihrer Studienkollegin Denise Sobota erarbeitet und durchgeführt wurde.

Alexandra Retkowski ist Professorin für Soziale Dienstleistungen für strukturschwache Regionen an der Fakultät für Soziale Arbeit, Gesundheit, Musik an der Brandenburgischen Technischen Universität Cottbus Senftenberg. 
Ihre Forschungsschwerpunkte sind sozial-ökologische Transformationsforschung; sexualisierte Gewalt und Kinderschutz; Alters-, Geschlechter-, und Generationenforschung sowie Theorie-Praxis-Relationen.

Christin Sager arbeitet als wissenschaftliche Mitarbeiterin am Institut für Erziehungswissenschaft in der Abteilung Allgemeine Erziehungswissenschaft der Universität Hildesheim. Sie lehrt und forscht zu Sexualitätsdiskursen, Kinder- und Jugendsexualität sowie zu (historischer) Kindheits-, Jugend- und Familienforschung. Thematisch relevante Publikation: Das aufgeklärte Kind. Zur Geschichte der bundesrepublikanischen Sexualaufklärung (1950-2010). Bielefeld 2015.

Elisabeth Sattler arbeitet als Bildungswissenschaftlerin am Institut für das künstlerische Lehramt an der Akademie der bildenden Künste Wien. Sie lehrt und forscht zu Bildung, Lehren und Lernen, pädagogischer Anthropologie und Subjektivitätstheorien u.a.m. Aktuelle thematisch relevante Publikation gemeinsam mit Marion Thuswald: Teaching desires. Möglichkeitsräume sexueller Bildung im künstlerisch-gestalterischen Unterricht. Wien 2016. Derzeit arbeitet sie in den Projekten Imagining Desires und Reflecting Desires (www.imaginingdesires.at).

Claudia Schneider ist Kultur- und Sozialwissenschaftlerin. Sie arbeitet im Verein EfEU (www.efeu.or.at), einem queer-feministischen Bildungsverein in Wien, in der Beratung und Qualifizierung von Pädagog:innen und als Lektorin an mehreren österreichischen Universitäten und Hochschulen vor allem in der Pädagog:innenaus- und -weiterbildung. Ihre Arbeitsschwerpunkte sind Gender- und Diversitätstheorien und Intersektionalität.

Julia Kerstin Maria Siemoneit, Diplom-Pädagogin, Kinder- und Jugendlichenpsychotherapeutin, ist wissenschaftliche Mitarbeiterin an der Universität zu Köln. Sie arbeitet in der sexualaufklärerischen Praxis an Schulen und zu sexueller Bildung von Lehrkräften und anderen pädagogisch Tätigen an der Universität zu Köln. Arbeitsschwerpunkte sind (sexual-)pädagogische Professionalisierung, Geschlechterforschung, projektbasiertes Studieren sowie Theorie und Praxis der Kinder- und Jugendlichenpsychotherapie. Thematisch relevante Publikation: Schule und Sexualität. Pädagogische Beziehung, Schulalltag und sexualerzieherische Potenziale. 
Rafaela Siegenthaler ist Sozialarbeiterin und Kultur- und Sozialanthropologin. Sie war von 2017-2019 Mitarbeiterin im Projekt Imagining Desires (www.imaginingdesires.at) am Institut für das künstlerische Lehramt an der Akademie der bildenden Künste Wien. Im diesem Rahmen arbeitete sie ein Semester lang mit Jugendlichen vom Jugendcorner, einer Gruppe der Schwarze Frauen Community (www.schwarzefrauencommunity.at), zu sexueller Bildung. Die künstlerischen Arbeiten der Jugendlichen wurden in der Ausstellung Black Excellence (www.imaginingdesires/ausstellungblack-excellence) gezeigt.

Robert Steiner ist ausgebildeter Sexualpädagoge, Jugendarbeiter und Coach und hält seit 2009 sexualpädagogische Workshops für die Fachstelle Selbstbewusst (Salzburg), die er seit 2017 mit einer Kollegin leitet. Davor war er in der allgemeinen Jugendarbeit sowie als Trainer und Coach tätig. Schwerpunkte seiner sexualpädagogischen Arbeit sind missbrauchspräventive Schutzkonzepte für Institutionen, Fortbildungen für Fachkräfte sowie Sexualpädagogik im kulturellen Kontext. Eine qualitätsvolle sexualpädagogische Arbeit ist für ihn einer der wichtigsten Grundsteine der Prävention von sexueller Gewalt. Robert Steiner hat eine Ausbildung in systemischer Aufstellungsarbeit und Beratung und befindet sich derzeit berufsbegleitend in der Ausbildung zum Psychotherapeuten.

Gregor Steininger ist seit Geburt Rollstuhlfahrer. Er lebt in Wien und ist glücklich verheiratet. Nach der Handelsakademie folgte ein Studium der katholischen Religionspädagogik in Wien und Berlin. Er ist seit 2009 in einer Peerberatungsstelle für Menschen mit Behinderung tätig. Als Betroffener und Pädagoge stehen Themen wie Sexualität, Diversität, Selbstbestimmung und Inklusion im Fokus seiner Arbeit. Er sieht besonders im Kontext Schule eine Notwendigkeit der verstärkten Auseinandersetzung mit ebendiesen Themen.

Marion Thuswald arbeitet als Bildungswissenschaftlerin am Institut für das künstlerische Lehramt an der Akademie der bildenden Künste Wien. Sie lehrt und forscht zu Themen wie sexuelle Bildung und Kunstpädagogik, pädagogische Professionalisierung und partizipative Forschung etwa in den Forschungs- und Bildungsprojekten Facing the Differences, Imagining Desires und Reflecting Desires (www.imaginingdesires.at). Sie promovierte $2020 \mathrm{mit}$ einer Arbeit zu Sexualpädagogik in der Lehrer:innenbildung unter dem Titel 
Möglichkeitsräume sexueller Bildung ausloten. Sprachlosigkeit, Lust, Verletzbarkeit und Emanzipation als Herausforderungen pädagogischer Professionalisierung.

Stefanie Vasold hat Politikwissenschaft studiert, ist Mediatorin und hat eine Ausbildung zur traumazentrierten Fachberatung absolviert. Sie arbeitet seit mehr als 13 Jahren bei Selbstlaut - Fachstelle gegen sexualisierte Gewalt an Kindern und Jugendlichen (www.selbstlaut.org). Dort ist sie in der Beratung und Verdachtsbegleitung, der Erwachsenenbildung sowie in Präventionsprojekten mit Kindern und Jugendlichen tätig und publiziert Fachartikel und Leitfäden. Ihre derzeitigen Arbeitsschwerpunkte sind die Entwicklung und Implementierung von Kinderschutzkonzepten in pädagogischen Einrichtungen und institutionellen Interventionsprozessen bei Verdacht auf sexuelle Gewalt durch Mitarbeitende.

Anna Vida studiert Kunst und Bildung am Institut für das künstlerische Lehramt der Akademie der bildenden Künste Wien sowie Germanistik und Genderstudies an der Universität Wien. In ihrer künstlerischen Praxis bewegt sie sich u.a. in den Medien Zeichnung, Text, Collage und Video. Aktuell befasst sie sich insbesondere mit grafischen Narrativen sowie der Konzeption und Umsetzung partizipativer Projekte. Ihre künstlerisch-theoretischen Auseinandersetzungen drehen sich um verschiedene gesellschaftspolitische Fragestellungen, wobei queerfeministische Perspektiven zentraler Angelpunkt sind.

Rosa Wiesauer studierte Bildende Kunst (Video- und Videoinstallation) und künstlerisches Lehramt an der Akademie der bildenden Künste Wien und ist Künstlerin, Performerin und Kostümbildnerin. In ihrer künstlerischen Praxis beschäftigt sie sich mit Femininität, der Sichtbarkeit von trans Identität und queeren Aspekten in japanischer Pop-Kultur.

Kerstin Witt-Löw ist Psychologin, Journalistin, Trainerin und Sozialwissenschaftlerin mit dem Fokus auf Empowerment von Frauen. Die letzte Studie ihres Forschungsinstituts Sofia (www.institut-sofia.at) befasste sich mit der Situation von Frauen 50+ und führte zur Bildung eines Netzwerks von Frauen dieser Zielgruppe. Seit über 30 Jahren lehrt Kerstin Witt-Löw am Institut für Bildungswissenschaft und am Zentrum für Lehrer:innenbildung der Universität Wien. 
Anton Cornelia Wittmann studierte Soziale Arbeit. En ist Geschäftsführung und Transgender-Referent:in der HOSI Salzburg, wo en sich in der Beratung und queeren Bildungs- und Anti-Diskriminierungsarbeit engagiert. En ist in der sexualpädagogischen Bildungsarbeit mit Kindern und Eltern in der Fachstelle Selbstbewusst tätig, lehrt zu sexueller und geschlechtlicher Vielfalt an der Sigmund Freud Privatuniversität Wien und ist Mitglied der Plattform Intersex Österreich.

Nina Wlazny ist Bildungswissenschaftlerin und Erwachsenenbildnerin. Seit 2017 arbeitet sie als Trainerin bei WUK m.power (Pflichtschulabschlusskurs für Jugendliche und junge Erwachsene) in Wien, wo sie sexualpädagogische Inhalte in ihre Bildungsarbeit miteinbezieht.

Eva Zürcher studiert in Innsbruck Englisch und Deutsch auf Lehramt und ist vielseitig interessiert. Sie setzt sich mit Lyrik auseinander und liest sehr viel, am liebsten altmodische Krimis im Miss-Marple-Stil. Zürcher arbeitet neben dem Studium im Tourismussektor und macht in ihrer Freizeit Yoga. Es ist ein Traum von ihr, einmal ein Buch zu schreiben und zu veröffentlichen. 


\section{Pädagogik}

Kay Biesel,

Felix Brandhorst,

Regina Rätz,

Hans-Ullrich Krause

Deutschland schützt

seine Kinder!

Eine Streitschrift zum Kinderschutz

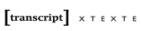
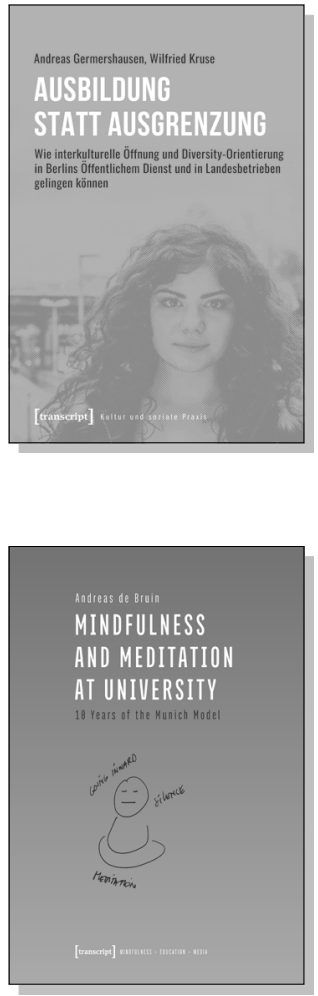

Kay Biesel, Felix Brandhorst, Regina Rätz, Hans-Ullrich Krause Deutschland schützt seine Kinder!

Eine Streitschrift zum Kinderschutz

2019, 242 S., kart., 1 SW-Abbildung

22,99€ (DE), 978-3-8376-4248-3

E-Book: 20,99 € (DE), ISBN 978-3-8394-4248-7

EPUB: $20,99 €$ (DE), ISBN 978-3-7328-4248-3

Andreas Germershausen, Wilfried Kruse

\section{Ausbildung statt Ausgrenzung}

Wie interkulturelle Öffnung und Diversity-Orientierung in Berlins Öffentlichem Dienst und in Landesbetrieben gelingen können

April 2021, 222 S., kart., Dispersionsbindung, 8 Farbabbildungen 25,00€ (DE), 978-3-8376-5567-4

E-Book: kostenlos erhältlich als Open-Access-Publikation

PDF: ISBN 978-3-8394-5567-8

Andreas de Bruin

Mindfulness and Meditation at University

10 Years of the Munich Model

April 2021, 216 p., pb.

25,00€ (DE), 978-3-8376-5696-1

E-Book: available as free open access publication

PDF: ISBN 978-3-8394-5696-5 


\section{Pädagogik}

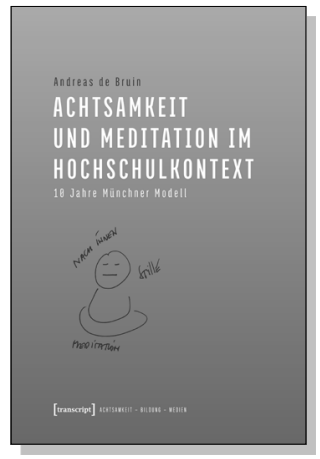

Andreas de Bruin

Achtsamkeit und Meditation

im Hochschulkontext

10 Jahre Münchner Modell

Februar 2021, 216 S., kart., durchgängig vierfarbig 20,00€ (DE), 978-3-8376-5638-1

E-Book: kostenlos erhältlich als Open-Access-Publikation PDF: ISBN 978-3-8394-5638-5

$€$

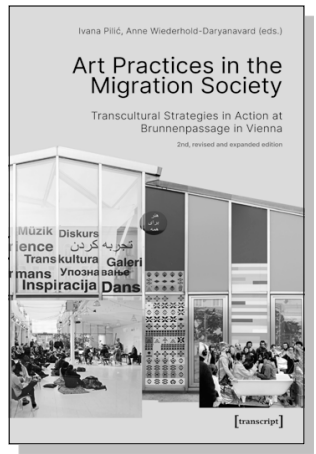

Ivana Pilic, Anne Wiederhold-Daryanavard (eds.)

Art Practices in the Migration Society Transcultural Strategies in Action

at Brunnenpassage in Vienna

March 2021, 244 p., pb.

29,00€ (DE), 978-3-8376-5620-6

E-Book:

PDF: $25,99 €(D E)$, ISBN 978-3-8394-5620-0

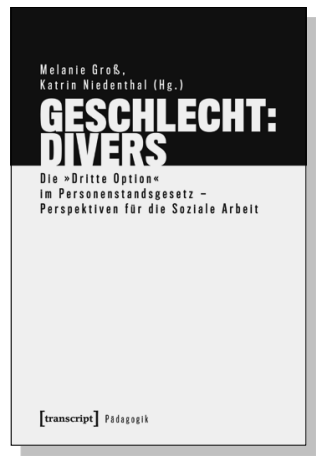

Melanie Groß, Katrin Niedenthal (Hg.)

\section{Geschlecht: divers}

Die "Dritte Option" im Personenstandsgesetz -

Perspektiven für die Soziale Arbeit

Februar 2021, 264 S., kart., Dispersionsbindung, 1 SW-Abbildung

$34,00 €(D E), 978-3-8376-5341-0$

E-Book:

PDF: 33,99€ (DE), ISBN 978-3-8394-5341-4

[transcript] pitusik 